\title{
Compact integrated optical devices for optical sensor and switching applications
}

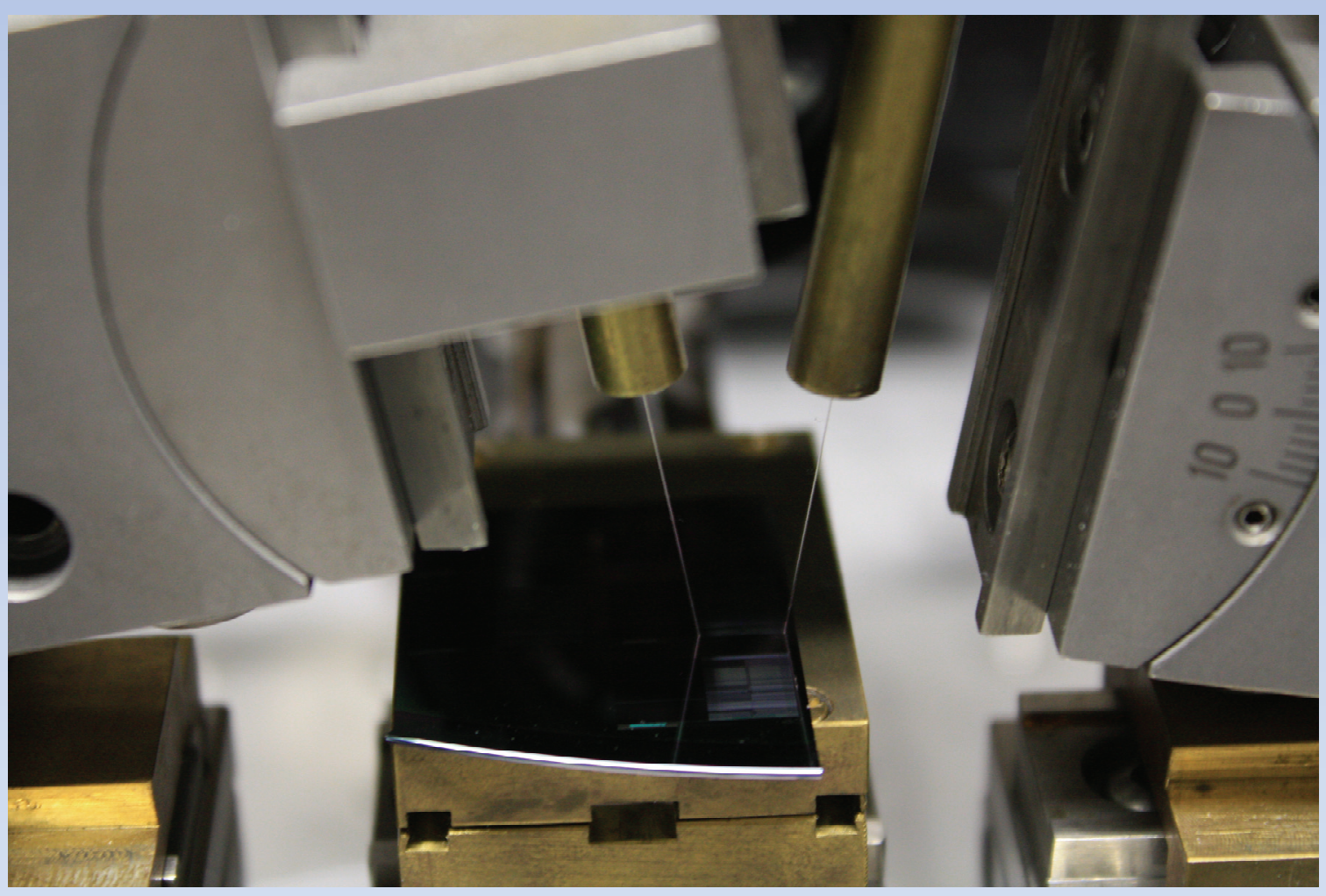

Lasse Kauppinen 
Compact integrated optical devices for optical sensor and switching applications 
Graduation committee:

Chairman and Secretary:

Prof. Dr. Ir. A.J. Mouthaan

Promoter:

Prof. Dr. M. Pollnau

Assistant Promoter:

Dr. Ir. R.M. de Ridder

\section{Members:}

Prof. Dr. K.J. Boller

Dr. H.J.W.M. Hoekstra

Prof. Dr. G.J.M. Krijnen

Prof. Dr. H.W.M. Salemink

\section{University of Twente}

University of Twente

University of Twente

University of Twente

University of Twente

University of Twente

TU Delft

The research described in this thesis was carried out at the Integrated Optical MicroSystems (IOMS) Group, Faculty of Electrical Engineering, Mathematics and Computer Science, MESA+ Institute for Nanotechnology, University of Twente, P.O. Box 217, 7500 AE Enschede, The Netherlands.

This work was financially supported by the Dutch Technology Foundation (STW) for the first 18 months and the last 30 months by the nanotechnology investment program NanoNed, by the Dutch Ministry of Economic Affairs.

Cover design:

Front: Vertical fiber coupling to a silicon photonics chip.

Reverse: Scanning electron microscope (SEM) image of a microcantilever on top of silicon waveguides (top), SEM image of a photonic crystal membrane (bottom).

Printed by Wöhrmann Print Service, Zutphen, The Netherlands.

ISBN: 978-90-365-3088-0 


\title{
COMPACT INTEGRATED OPTICAL DEVICES FOR OPTICAL SENSOR AND SWITCHING APPLICATIONS
}

\section{DISSERTATION}

\author{
to obtain \\ the degree of doctor at the University of Twente, \\ on the authority of the rector magnificus, \\ prof. dr. H. Brinksma, \\ on the account of the decision of the graduation committee, \\ to be publicly defended \\ on Thursday the $7^{\text {th }}$ of October 2010 at 16:45
}

\section{Lasse Juhana Kauppinen}

Born on the $2^{\text {nd }}$ of March 1978

in Anjalankoski, Finland 
This dissertation is approved by:

the promoter: Prof. Dr. M. Pollnau

the assistant promoter: Dr. Ir. R.M. de Ridder 




\section{Contents}

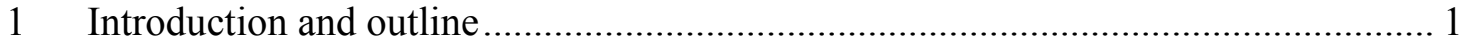

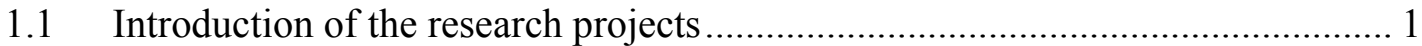

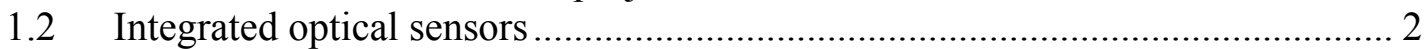

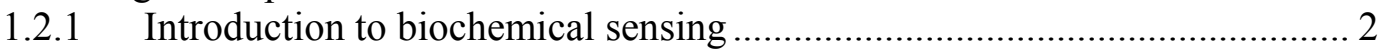

1.2.1.1 Label-free integrated optical sensors ............................................. 3

1.2.2 Integrated optical read-out of microcantilever sensors ............................... 8

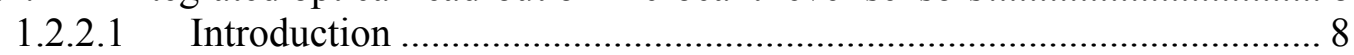

1.2.2.2 Optical read-out methods.................................................... 8

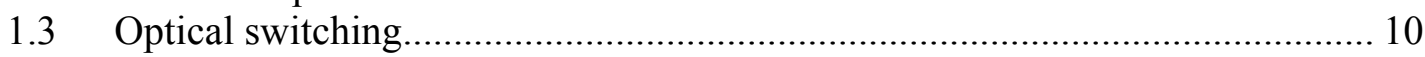

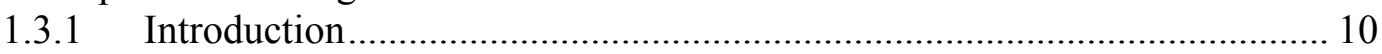

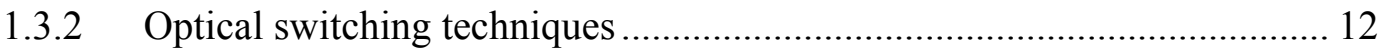

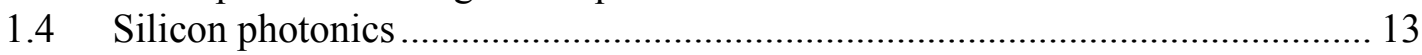

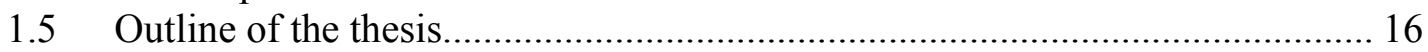

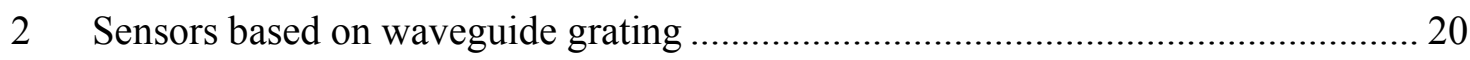

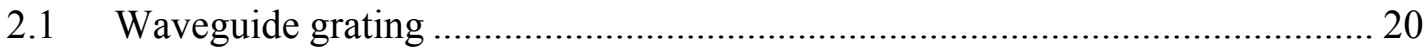

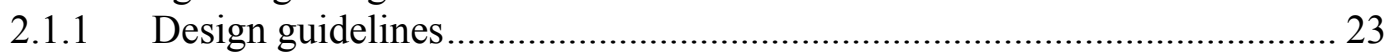

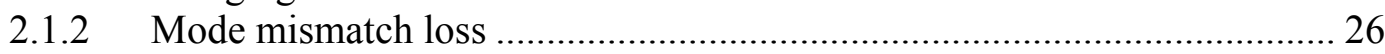

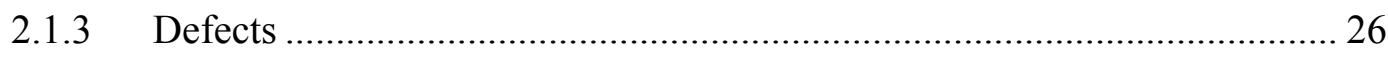

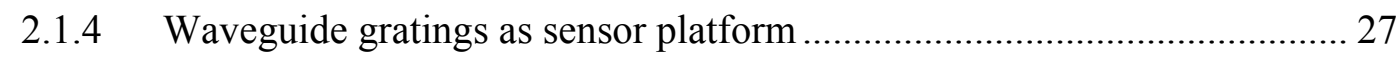

2.2 Design and characterization of the grated silicon photonic wire sensor ............ 31

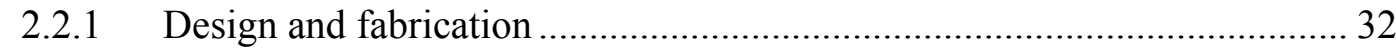

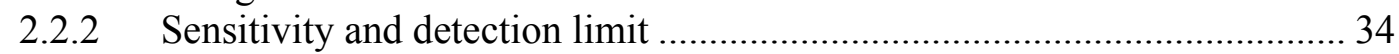

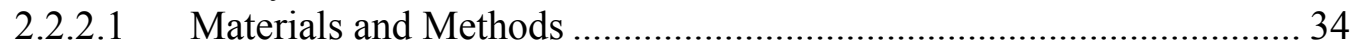

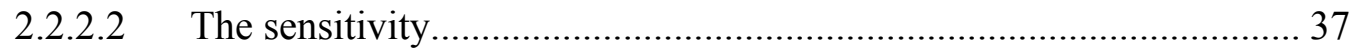

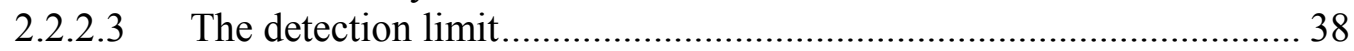

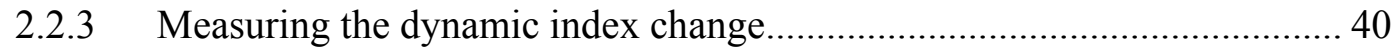

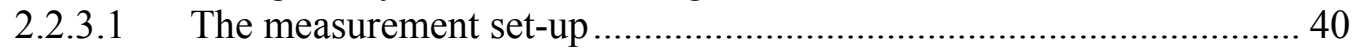

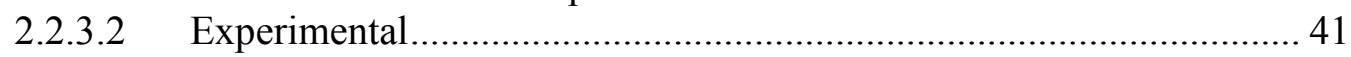

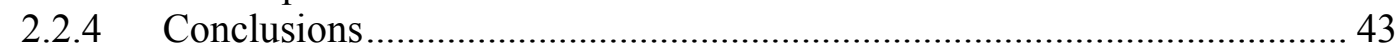

2.3 Label-free enzyme sensing with grated silicon photonic wire ......................... 43

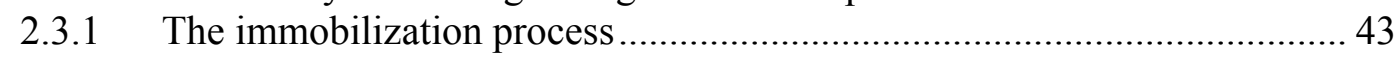

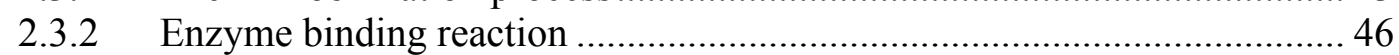

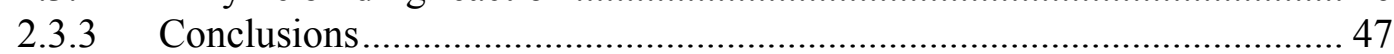

2.4 Grated Waveguide Optical Cavity as a Compact Sensor for Sub-nanometre

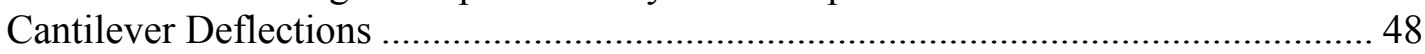

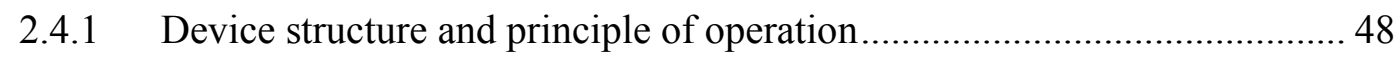

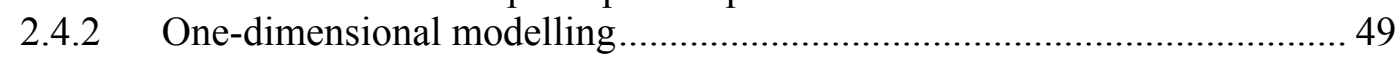

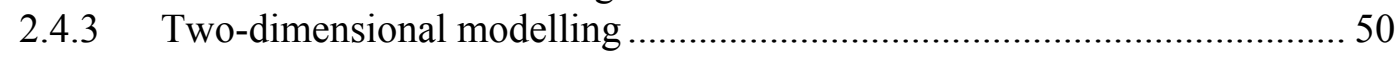

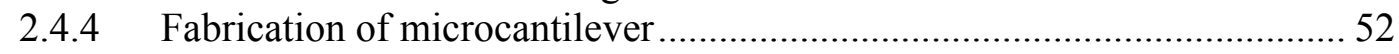

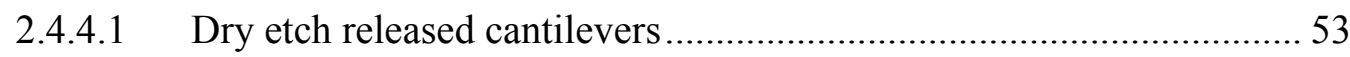

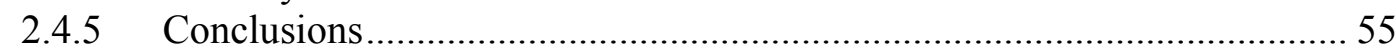

3 Compact silicon photonic devices for MEMS-actuated optical switching .............. 59

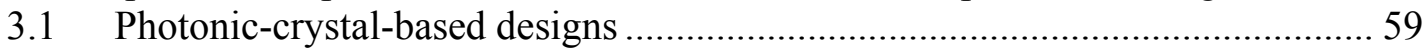




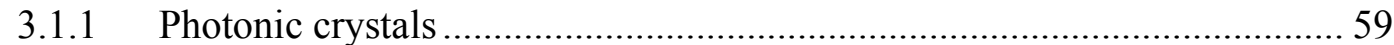

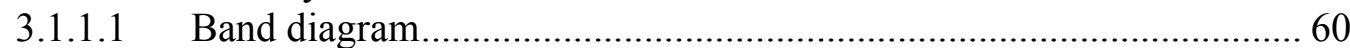

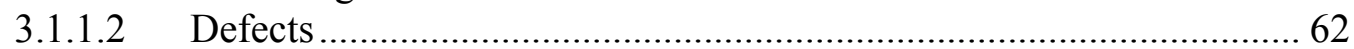

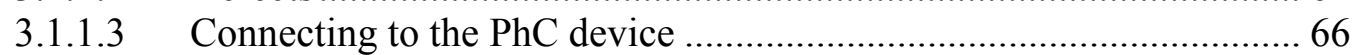

3.1.2 Photonic-crystal-cavity-based channel drop filter ..................................... 66

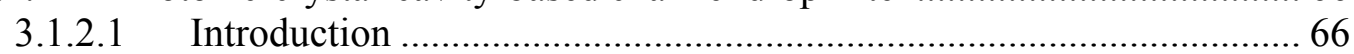

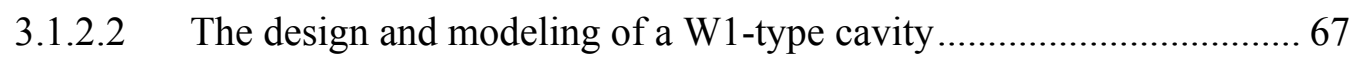

3.1.2.3 The design and modeling of a W3-type cavity ................................. 70

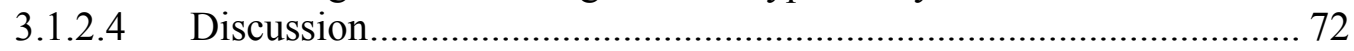

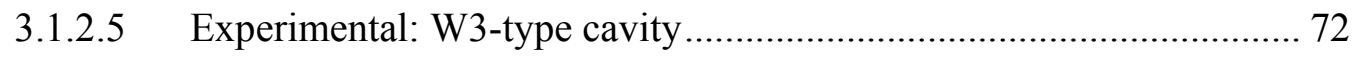

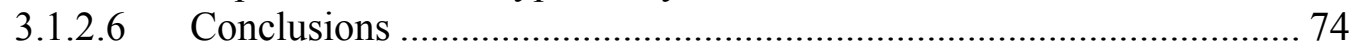

3.1.3 Photonic-Crystal-Cavity-based Y Splitter ........................................... 74

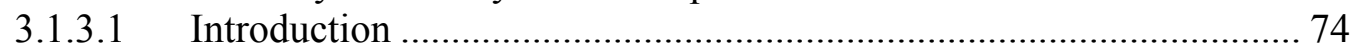

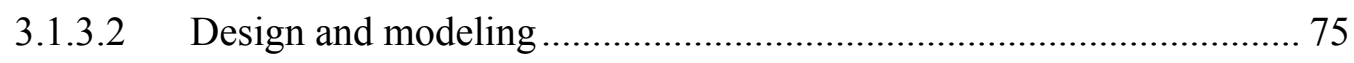

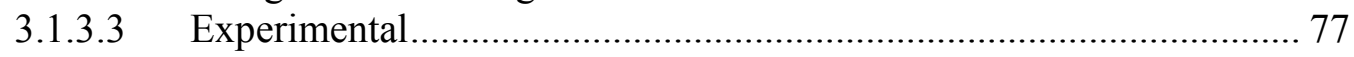

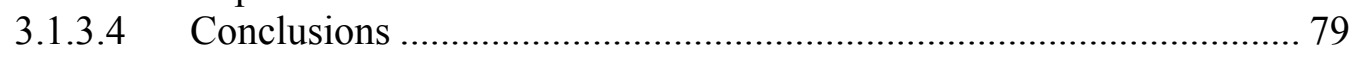

3.2 Waveguide grating and ring-resonator-based designs .................................. 79

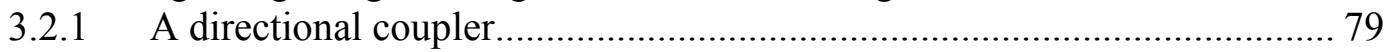

3.2.2 Waveguide-grating-based add-drop multiplexer ...................................... 80

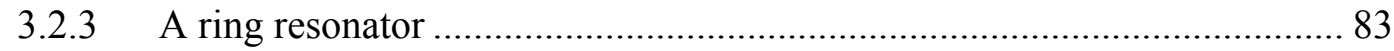

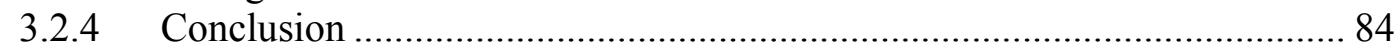

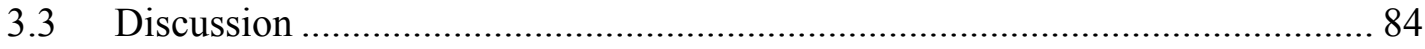

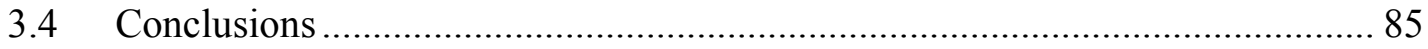

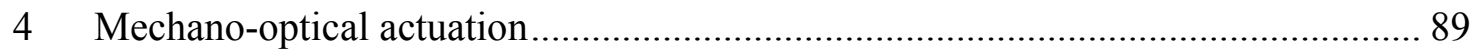

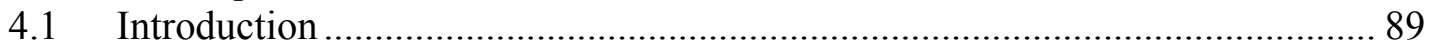

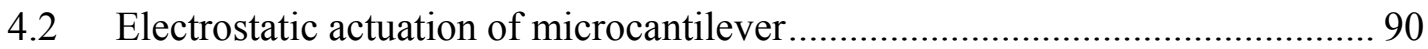

4.3 Monolithic integration of the microcantilever ................................................. 93

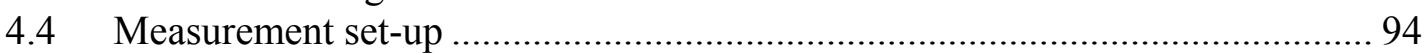

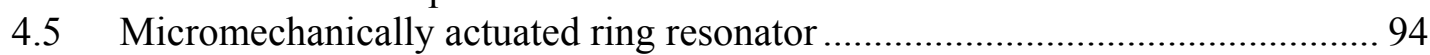

4.6 Micromechanically actuated photonic crystal waveguide ............................... 98

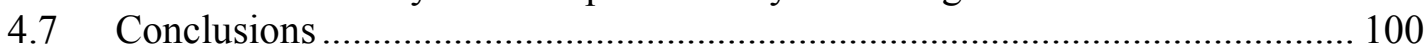

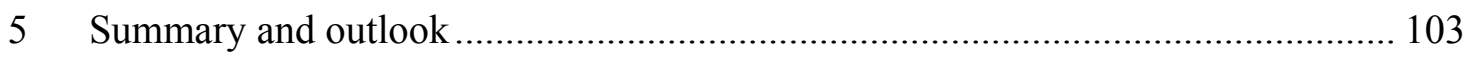

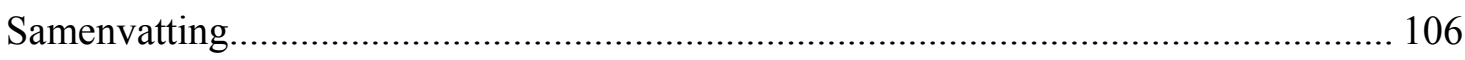

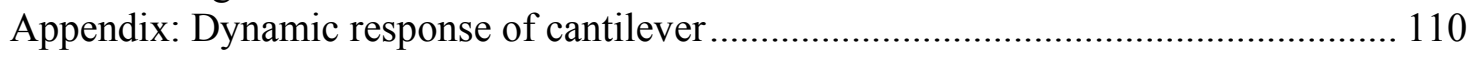

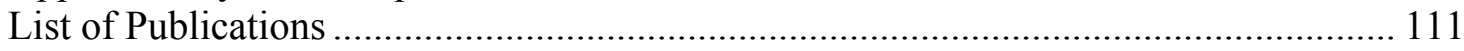

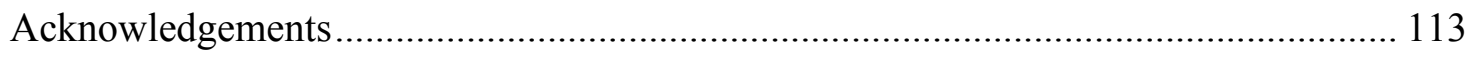






\section{Introduction and outline}

This chapter presents a general introduction of the thesis. An introduction of the research projects is first given as background information. The motivation for the development of the sensor and switching devices is given in the introduction sections. Also a brief introduction to optical switching and sensing is provided. Furthermore, an introduction of the silicon photonics fabrication platform, that was used to fabricate most of the devices reported in this thesis, is given, as well as the motivation for the use of silicon photonics. The chapter ends with the outline of the thesis.

\subsection{Introduction of the research projects}

The work reported in this thesis focuses on two topics; optical sensing and optical switching. The first 18 months of my $\mathrm{PhD}$ period were funded by the STW project called "Multi-sensing arrays of separately accessible optics sensors". The remaining 30 months I worked in the project titled "Optical switching by NEMS-actuated resonator arrays", funded by the nanotechnology investment program NanoNed, by the Dutch Ministry of Economic Affairs.

The aim of the multi-sensing project was to realize a compact and highly sensitive sensor unit that could be used for multi-analyte sensing. The focus was on utilizing waveguide grating based sensors. The work can be divided into two sub-projects; in the cantilever sensor project a novel optical detection method to measure nanodisplacements of microcantilevers was developed while in the biosensing project the sensing properties of deep UV lithography fabricated grated silicon photonic wires were studied. The cantilever project was later split off as a new $\mathrm{PhD}$ project funded by the Dutch Ministry of Economic Affairs through the Point-One MEMSland project, and is continued by So Van Pham in the IOMS group.

The aim of the optical switching project was to realize a compact mechano-optically actuated optical switching device. The project was a joint research effort by the AAMP, IOMS and TST groups of the University of Twente. In the project I continued the work initiated by my predecessor Wico Hopman, whose contribution is reported in his $\mathrm{PhD}$ thesis [1]. My contribution was to provide the optical devices that could be mechanooptically tuned with a monolithically integrated microcantilever. The project was conducted in close co-operation with the TST group, particularly with Shahina Chakkalakkal Abdulla who was responsible for the microcantilever design and fabrication.

In the following sections the introductions to the research topics covered in this thesis are given. Following the division that originates from two separate projects, the introduction and the remaining part of the thesis are each divided in two sections. In chapter 2 the sensor work is reported; chapters 3 and 4 deal with the optical switching work, where chapter 3 discusses the optical designs implemented for the mechano-optical switching and chapter 4 reports the experimental results obtained from the monolithically integrated switching devices. 


\subsection{Integrated optical sensors}

In this section an introduction to integrated optical (IO) sensors is given. As the sensor work in this thesis focuses on implementing a label-free biochemical sensor and nanodisplacement sensor, an introduction of both topics is given.

First the optical biochemical sensors are discussed. A brief introduction of biochemical sensing is given, followed by section that introduces some of the most common IO labelfree biosensor types. The other sensor section (1.2.2) focuses on IO devices that are used to measure small displacements of microcantilevers. These types of devices can be used as an optical read-out method for cantilever sensors that are based on observing reactions that cause the cantilever to deflect.

\subsubsection{Introduction to biochemical sensing}

Biosensing aims at recognizing some specific biomolecules (analytes) from a sample. For this purpose a biosensor consists of two basic components: a biological recognition system (bioreceptor) and a transducer.

An example of a bioreceptor is an antibody. It is a complex biomolecule that immune system cells produce when exposed to molecules called antigens. The specific structure of the antibody targets only an antigen that matches to its structure. As a result of this, the antibody and the antigen can bind together. For more detailed discussion about bioreceptors and they properties we refer to [2].

A transducer is needed to convert the biorecognition event (e.g. binding of an antibody and an antigen) into a measurable effect. Typical transducer types used in biosensors rely on optical, electrochemical or mass-based methods. In electrochemical detection, see e.g. [3], the transduction principle is the detection of an electric current caused by oxidation or reduction of species at the electrode. Mass-based detection methods exploit the fact that a mechanical resonance frequency of a piezoelectric element changes as its mass changes. A possible implementation of this detection scheme involves the use of microcantilevers, see e.g. [4]. Optical transduction methods can be roughly divided in two categories; labeled and label-free methods. Next we will briefly discuss the general properties of both of these optical methods and motivate our focus on label-free sensors.

In the labeled detection scheme either the analytes to be detected or the bioreceptors are labeled with e.g. fluorescent tags, such as dyes. Upon excitation with light, the fluorescent dye will emit photons at a certain wavelength that is specific to the dye. The emitted light can be detected with a sensitive photodetector, and based on the intensity of the emitted light the concentration of the analyte is obtained.

In the label-free sensing scheme the analytes to be measured are not labeled, but typically the receptors are immobilized on the sensor's surface. The surface layer containing the receptors changes thickness upon capturing an analyte. The sensor's transduction mechanism translates the thickness change to a measurable signal.

In label-free detection the target molecules are detected in their natural form, allowing quantitative and kinetic analysis, which is typically not possible by using analytes that are labeled with fluorescent tags. This is due to two main effects: first, it is difficult to control the number of fluorescent tags attached to each molecule, and second, the labeling procedure changes the properties of the analyte. Although the fluorescence-based detection is highly sensitive, the fluorescence light signal scales with the sample volume 
and is typically very weak, requiring photon counting methods. In label-free methods the signal is related to sample concentration and good sensitivity can be obtained with ultrasmall sample volumes.

In label-free sensing much attention has been given to sensors that are capable of multianalyte detection. A straightforward way to implement this type of sensor is to arrange the sensing elements into an array layout, in which each element is functionalized to detect a specific molecule. The following medical diagnostic case illustrates the attractiveness of using a label-free sensor array, capable of multi-analyte detection.

A patient has a virus infection in his central nervous system. In most of the cases one of 15 possible viruses is causing the infection. The infection can be analyzed from cerebrospinal fluid, which is taken with a Lumbar puncture method. Due to the nature of this method it cannot be repeated for some time, which implies that this diagnostic sample should be used as efficiently as possible. The sample contains a very small amount of viruses, and in order to identify them, a real-time polymerase chain reaction (PCR) is used to multiply the genome of the virus. In a real-time PCR procedure, often a dye is used that fluoresces strongly when bound to the multiplied genome, allowing the detection of the multiplication. Typically only one (or in some cases a few) virus(es) can be indentified with one PCR based test.

It would clearly be advantageous to be able to cover all 15 viruses simultaneously in a single diagnostic test. Also, considering the good sensitivity provided by detection with immobilized receptors, the virus could be indentified directly from the diagnostic sample without the PCR step. The main aim of the development of label-free integrated optical sensors for this kind of tests is to add value compared to conventional methods (such as the real-time PCR), as measured by factors such as the time and workforce required to perform the test, the cost of test equipment, and the cost of a single test. The portability of the test equipment may also play an important role.

\subsubsection{Label-free integrated optical sensors}

In this section we introduce integrated optical sensor structures, used for label-free refractive-index-based sensing, that have received most attention in recent research work reported in the literature. These sensor structures can be divided in three categories: surface-plasmon-based, interferometric and resonance-based sensors.

For a comprehensive review of optical biochemical sensors we refer to review papers [57].

In order to evaluate and compare the performance of the optical sensors we introduce two important parameters that are often used for this purpose: sensitivity $\left(S_{n}\right)$ and detection limit $(D L)$. The sensitivity is an intrinsic property of a sensor, defined as the change of the transduction signal $\left(\Delta T_{t s}\right)$ per unit change of analyte concentration $(\Delta A)$.

$$
S_{n}=\frac{\Delta T_{t s}}{\Delta A} \text {. }
$$


In many cases, the concentration of the analyte is deduced from a change in refractive index (either directly caused by the analyte, or through its interaction with a receptor). For optical sensors that respond to a change in refractive index by wavelength-shifting some spectral feature, the sensitivity is specified as wavelength shift per refractive index unit (RIU) change caused by the analyte. For example, addition of sugar to water causes the refractive index to change and this change can then be measured with an optical sensor that has a certain sensitivity to the index change. The detection limit depends on the signal-to-noise ratio (SNR) of the transduction signal and is defined as:

$$
D L=\frac{S N R}{S_{n}} .
$$

Often used transduction mechanism is to observe analyte caused changes in the effective modal index $\left(n_{e f f}\right)$ of the waveguide. This effective index is defined as:

$$
n_{\text {eff }}=\frac{\beta \lambda_{0}}{2 \pi},
$$

in which $\beta$ is the propagation constant and $\lambda_{0}$ is the vacuum wavelength. The effective index depends, among many things, on the refractive index of the materials that form the waveguide structure.

\section{$\underline{\text { Surface plasmon }}$}

Probably the most well-known label-free optical transduction mechanism is surface plasmon resonance (SPR) [8]. The surface plasmon originates from the interaction between photons and the free electron cloud of the metal. Integrated optical waveguides can be used to excite the SPR wave (SPW), as shown in Fig. 1. At a certain wavelength the propagation constant of the waveguide mode matches that of the SPW; this is known as the phase-matching condition. As power transfer from the waveguide mode to the lossy SPW mode is maximum at this phase-matched condition, the light transmission through the waveguide has a dip at this wavelength.

The wavelength for phase matching depends on the dielectric material that is adjacent to the metal, and therefore index changes in this dielectric can be monitored. Moderate to good detection limits have been reported for waveguide based SPR devices; e.g. $1 \cdot 10^{-4}$ RIU [9] and $1.2 \cdot 10^{-6}$ RIU [10], although the interaction lengths are relatively long; 0.7 $\mathrm{mm}$ and $1.8 \mathrm{~mm}$, respectively.

The SPR imaging type sensor, see e.g. [11], is an array type sensor responding to the need of simultaneous multi-analyte detection. Arrays of SPR strips are illuminated and the intensity reflected by each element is measured with a 2D array detector (e.g. CCD image sensor). Detection limits of $3 \cdot 10^{-5}$ [12] and $3 \cdot 10^{-6}$ [13] RIU have been reported. Although this type of sensor is capable of multi-analyte detection it uses bulky optics and the SPR element is nothing more than an array of microfabricated metal strips. Today commercially available SPR sensors are far from being handheld multi-analyte devices. 


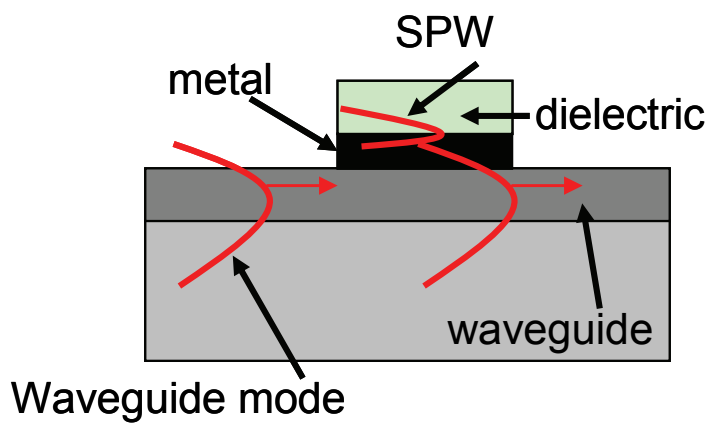

Fig. 1. A schematic cross-section of a waveguide coupled surface plasmon device.

\section{$\underline{\text { Interferometer based }}$}

Interferometer-based sensors measure the optical path length changes caused by the analyte. Probably the most well-known type in this class of sensors is the Mach-Zehnder interferometer (MZI), see Fig 2. Light from an input waveguide is distributed, usually with equal power, over two waveguide branches, often using a so-called 3-dB coupler. In a sensing branch, the analyte causes a certain effective index change (e.g. by binding to the immobilized receptor layer on the waveguide surface) that in turn introduces an optical path length difference between the branches. The light from the two waveguide branches is recombined by a $3 \mathrm{~dB}$ coupler. The distribution of light over the output waveguides depends on the path length difference. By introducing phase modulation to increase sensitivity, a detection limit of the order of $5 \cdot 10^{-8}$ RIU has been achieved [14]. However, in order to introduce sufficient optical path length difference to detect a very small index change, the required length of the MZI device is typically quite long; ranging from a few millimetres to several centimetres. As the device dimensions increase, the undesirable sensitivity to temperature gradients increases as well. A spiral layout has been explored allowing a design that is insensitive to temperature gradients, and that reduces the footprint of the MZI, enabling a compact array configuration for multisensing [15-16].

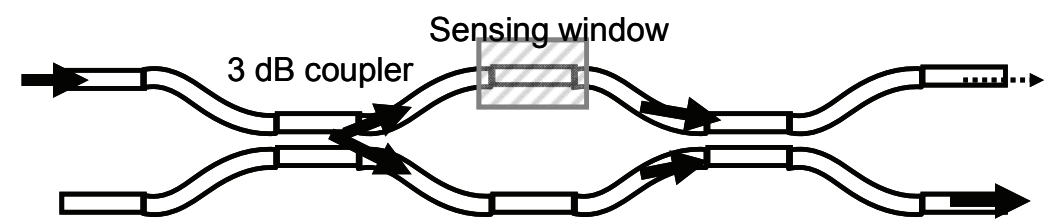

Fig. 2. Top view of Mach-Zehnder interferometer. The input light is split over two waveguide branches. The sensing window defines the region in which the analyte solution is applied. The index change in the sensing branch causes a change in the optical path length difference between the two branches.

\section{Resonance based}

The resonance wavelength of integrated guided-wave optical resonators depends on the effective refractive index of the waveguiding medium. Since in the refractive index-based label-free sensing scheme the analyte causes the effective index to change, the presence of the analyte can be detected by monitoring the change of the resonance wavelength. The crucial feature in achieving a small detection limit is the spectral sharpness of this 
resonance, which is related to the so-called quality factor $Q$. The higher the quality factor is, the smaller index change can be detected [17].

In ring-resonator-based sensors, the sample is applied to a sensing window covering the ring waveguide (Fig. 3a). The effective-index change of the ring waveguide causes the resonance wavelength to change, which can be observed by measuring the transmission spectrum, see e.g. [18-19]. A detection limit of $10^{-5}$ RIU has been reported [18]. The footprint of a ring resonator is typically considerably smaller than that of an MZI (e.g. $500 \mu \mathrm{m}^{2}$ vs. $2 \mathrm{~mm}^{2}$ ), and the integration into an array layout is easier. Unfortunately the quality factor of ring resonators is typically limited to $\sim 10^{4}[18]$, due to optical loss of the ring waveguide, which is mostly caused by surface roughness that results in out-of-plane scattering. This imposes a lower bound on the achievable detection limit $[5,17]$.

Sensors based on resonators in a photonic crystal $(\mathrm{PhC})$ (see Fig. 3b) operate in a similar way as ring-resonator sensors: the resonance frequency changes according to index change. An impressive set-up involving an array of $\mathrm{PhC}$ resonators for multianalyte sensing is reported in [20]. The array element is a 1D PhC structure with a defect, to which the light is coupled from the access waveguide, see Fig. 3b. Each array element has a unique resonance wavelength and by monitoring the transmission spectrum of the access waveguide the response of the individual sensor element can be extracted. Multi channel sensing with a detection limit of $7 \cdot 10^{-5}$ RIU is reported in [20].
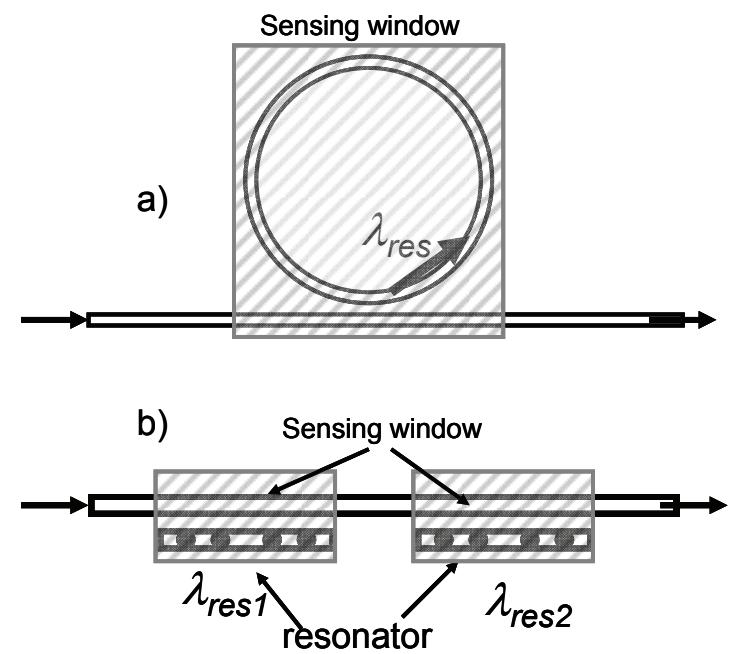

Fig. 3. a) Top view of a ring resonator. Light at a resonance wavelength is coupled from the access waveguide to the ring. The resonance wavelength depends on the effective index of the ring waveguide. $b$ ) A top view of a sensor based on a 1D photonic crystal resonator. The resonance wavelength depends on the effective index of the resonator.

The waveguide gratings and photonic crystal waveguides are resonator-based devices as well. However, these devices do not have a separate resonance cavity to which the light is coupled from the waveguide. For this reason these devices are less suitable for array layout, e.g. due to possible reflections between cavities. Nevertheless, the spectral features of e.g. waveguide gratings typically have a larger dynamic range (stopband attenuation) than e.g. ring resonators.

In a waveguide grating, see Fig. $4 \mathrm{a}$, the spectral position of the photonic stopband depends on the effective index, and by monitoring the changes of the stopband position the index change of the cladding medium can be measured. Typically, waveguide 
gratings are somewhat larger in size than ring resonators. Detection limits of $4 \cdot 10^{-4} \mathrm{RIU}$ [21] and $2.8 \cdot 10^{-5} \mathrm{RIU}$ (this thesis, section 2.2.) have been reported for waveguide-grating based-sensors. The detection limit of a waveguide grating can be increased by tuning the grating parameters, although at some point optical losses and non uniformities of the grating structure due to fabrication technology will set a lower limit.

The operating principle of a photonic crystal waveguide sensor, see Fig. $4 \mathrm{~b}$, is based on the fact that the cut-off wavelength of the waveguide depends on the effective index. The index changes are therefore measured by observing the spectral shift of the cut-off wavelength. Additional sensitivity is expected from the field profile of the waveguide mode near the surface and from liquid penetrating into the holes of the $\mathrm{PhC}$ [22]. By assuming a spectral resolution of $5 \mathrm{pm}$, a detection limit of $8 \cdot 10^{-5}$ RIU can be estimated for the device reported in [22].

a)

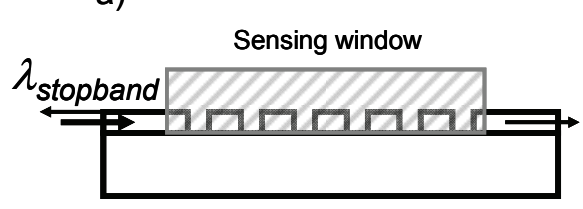

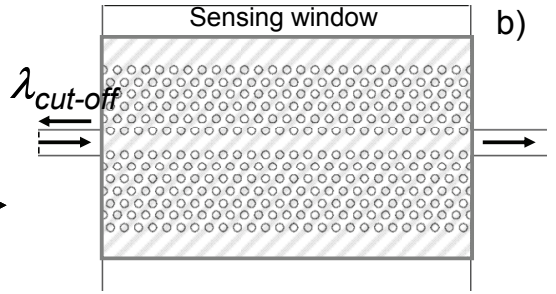

Fig. 4. a) Side view of a grated waveguide. Spectral position of the photonic stopband of a grating depends on the index of the cladding material. b) Top view of a line-defect waveguide in a 2D photonic crystal. The cut-off wavelength of the waveguide depends on the effective index of the environment.

Resonance-based sensor devices, such as ring resonators and $1 \mathrm{D} \mathrm{PhC-cavities,} \mathrm{have} \mathrm{the}$ advantage that they can be easily arranged into array layout for multianalyte sensing. However, on-chip integration of a spectrometer that can resolve a wavelength e.g. at a resolution of $5 \mathrm{pm}$ is challenging. Also, as the spectral sharpness of e.g. the ring resonator depends on the coupling condition between the access waveguide and the ring waveguide, it might be altered by the presence of the molecules in this region.

\section{Methods to increase sensitivity}

Apart from obvious parameter optimization, there are some ways to improve the performance of the sensor.

A slot waveguide based designs attempt to increase the sensitivity with a special waveguide design, see Fig 5. In a slot waveguide the highest field intensity is between two high-index strips, where a sensing medium can be, and hence a high sensitivity can be expected. Indeed, impressive sensitivity of $212 \mathrm{~nm} / \mathrm{RIU}$ has been reported for a slot waveguide ring-resonator, although the detection limit of $2.3 \cdot 10^{-4}$ is only moderate [23]. Porous silicon can be used as a waveguiding material. Due to porosity the surface area of the sensor is considerably increased and improved sensitivity is expected [24].

Gold nanoparticles can be used to greatly increase the SPR reflectivity shift caused by the binding of DNA. 1000-fold increase in sensitivity is reported in [25]. 


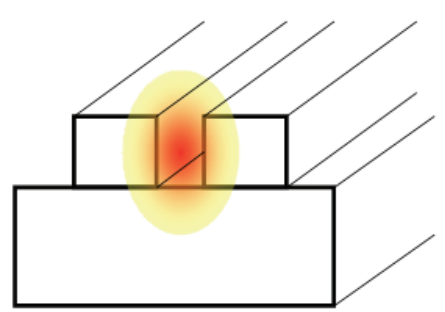

Fig. 5. Slot waveguide geometry. In a slot waveguide the highest field intensity is in the cladding between two high index strips. Therefore the effective index of this waveguide depends strongly on the refractive index of the material that is between the strips.

\subsubsection{Integrated optical read-out of microcantilever sensors}

This section discusses microcantilever-based sensors, in particular integrated optical read-out methods suitable for detection of the deflection of a microcantilever. The physical principles of cantilever sensors are briefly described. A brief summary is given of the integrated optical read-out methods that are reported in the recent literature. Our proposed read-out method, which involves measuring the wavelength shift of an optical resonance of a grated waveguide, is discussed in detail in chapter 2.

\subsubsection{Introduction}

Microcantilever-based sensors can be used to detect the effect of adsorption or absorption of a species by a sensitive layer that is deposited on a micromachined cantilever. These effects cause changes in the surface stress [26], leading to deflection of the cantilever.

A large number of microcantilever-based sensors are reported in the literature. Fritz et al. functionalized Si cantilevers on one side with different oligonucleotide base sequences [27]. DNA hybridization depending on the base sequence was demonstrated. Baller et al. [28] report a cantilever-array-based artificial nose. Different polymer coated cantilevers respond to gaseous analytes due to the swelling effect as the gas is absorbed by the polymer. Artificial flavours, such as lemon, cherry, rum, vanilla, orange and bitter almond, were successfully detected with the sensor.

An interesting type of cantilever-based sensor is the use of cantilevers as pixel elements in an uncooled infrared (IR) imaging system [29-30]. In this case the cantilever response is based on thermal expansion which depends on the amount of IR light absorbed.

Hydrogen gas detection with palladium-coated cantilevers is a widely studied topic, e.g. [31-32].

Several electric methods exist for measuring the deflection of a microcantilever, e.g. piezoelectric, piezoresistive and capacitive. Here we will focus on a comparison of integrated optical read-out methods that have the advantage that no electric connection to the cantilever is required.

\subsubsection{Optical read-out methods}

Often, the displacement of a free-space optical beam is detected as a measure of the cantilever deflection [29-30, 33]. Although the method is simple and accurate, it is bulky, and therefore dense and compact monolithic integration of cantilever sensors is not possible with this method. In addition, the slow response of the large position-sensitive 
photodetectors that are needed for measuring optical beam displacement limits the bandwidth of this method.

Different designs for integrated optical read-out of microcantilever deflection have been proposed and demonstrated. These read-out methods can be roughly divided into two categories. The first one involves exploiting a structural change of the optical device, e.g. displacement of a waveguide attached to the cantilever, which changes the amount of light that can propagate through the device. The second one uses the effect caused by perturbation of the evanescent field of a waveguiding structure. The cantilever, moving in close proximity to the optical device changes its effective refractive index, which, in turn, modulates the light transmission through the device. Table 1 summarizes some of the reported integrated read-out methods and their deflection sensitivities (change of power transmission coefficient $\Delta T$ per unit cantilever deflection $\Delta z$ ).

Table 1.

Different integrated optical designs to detect cantilever deflection and their performance.

\begin{tabular}{|l|l|}
\hline Optical read-out device & Sensitivity $\Delta \mathbf{T} / \Delta \mathbf{z}$ \\
\hline Waveguiding cantilever \#1 & $1 \mu \mathrm{m}^{-1}[34]$ \\
\hline Waveguiding cantilever \#2 & $0.24 \mu \mathrm{m}^{-1}[35]$ \\
\hline Photonic crystal cavity & $0.1 \mu \mathrm{m}^{-1}[37]$ \\
\hline Multi-mode interference (MMI) couplers & $5 \mu \mathrm{m}^{-1}[36]$ \\
\hline Waveguide grating & $60 \mu \mathrm{m}^{-1}[38]$ \\
\hline Slot waveguide resonator & $33000 \mu \mathrm{m}^{-1}[39]$ \\
\hline Ring resonator & $500 \mu \mathrm{m}^{-1}[40]$ \\
\hline
\end{tabular}

The principle of waveguiding-cantilever devices $[34,35]$ is shown in Fig. 6. A segment of an optical waveguide is under-etched to form the cantilever. A small gap between the cantilever waveguide and the non-under-etched (fixed) part of the waveguide allows the cantilever to move. Deflection of the cantilever changes the coupling between the cantilever waveguide and the fixed waveguide.

The read-out methods reported in [36] and [37] are variations of this type of design. In [36] 10 cantilever waveguides couple the outputs of a $1 \times 5$ MMI splitter to the inputs of a $5 \times 1$ MMI combiner. In [37], a 2D photonic crystal, containing a resonance cavity, is patterned on the top surface of the cantilever. Bending of the cantilever causes deformation of the cavity, which results in a shift of resonance wavelength.

Although the read-out methods based on structural change are relatively simple, sensitive and pose no serious challenges in fabrication, it is a disadvantage that the light propagates in a cantilever structure, which severely limits the options for cantilever design and choice of the receptor layer, in particular in case of absorbing receptor layers. Also the deflection sensitivities are not as high as the ones obtained with methods using perturbation of the evanescent field. 


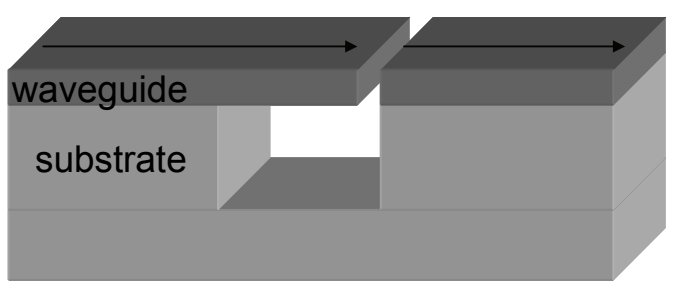

Fig 6. Schematic picture of the waveguiding-cantilever type of sensor. The deflection of the cantilever changes the transmission through the small air gap.

A sensor, in which a microbridge is bonded to an optical chip and located above a waveguide grating, is reported in [38]. This type of read-out exploits the wavelength shift of the photonic stopband of the waveguide grating as the air gap between the grating and the mechanical element changes. Therefore, the size of the mechanical element should at least be equal to the size of the grating. Barrios [39] presents a theoretical study on a nanomechanical sensor employing a disk resonator in a waveguide structure where a slot is present parallel to the disk surface. A deflection of the disk changes the width of the slot, which, in turn, changes the resonance wavelength. Although an extremely high deflection sensitivity is predicted, it poses serious fabrication challenges, e.g. regarding the required etch selectivity between layers.

It is also possible to use an optical ring resonator as a sensor platform for a cantilever deflection read-out [40-41]. In this case the cantilever deflection causes a shift of the resonance wavelength as the presence of the cantilever increases the optical path length of the ring resonator. The biggest challenge for fabricating sensors that are based on perturbation of the evanescent field, is the monolithic integration of the cantilevers and the optical readout circuit, as will be discussed in section 2.4.4.1.

\subsection{Optical switching}

In this section we will introduce applications for optical switches and then briefly discuss some of the most common switching principles.

\subsubsection{Introduction}

Switching functions for optical signal can be realized either in the optical or the electrical domain. In electrical-domain switching, the optical signal is first converted to an electronic signal to which the switching function is applied. Then, the switched electrical signal is converted back to an optical signal. In optical-domain switching, the signal remains optical, although electronic circuits are often used to drive such an optical switch.

At present, it depends on the switching application which one of these two switching domains (electrical or optical) should be used. It is argued that e.g. in optical telecom networks the optical switches are useful in circuit switching applications while for packet switching the technology is not mature enough, due to the fact that an optical random access memory does not exist and all optical packet header processing techniques are primitive, giving advantage to the electrical switches [42]. In general the conversion from optical to electrical signal should be avoided for trivial signal functions due to the high cost of signal conversions (optical-to-electrical-to-optical) [43].

The main application areas for optical switches in the networks are provisioning (e.g. reconfiguring lightpaths), and protection switching. The required switching speeds for these 
applications are in the range of milliseconds, which is well within reach of optical switching technologies [43]. The total number of ports required for provisioning and protection switching can be higher than 1000 [43]. The high number of ports requires a huge amount of switches. This favors optical switching, since the power consumption of a typical $64 \times 64$ electronic integrated circuit switch is $25 \mathrm{~W}$, whereas an optical MEMS based switch would need only a fraction of this [43].

The other main advantage of optical switching is that it allows waveband switching, i.e. selectively switching certain wavelength ranges that are contained in a broadband multiwavelength signal. This is a particularly attractive property for switching applications in optical networks that use wavelength division multiplexing (WDM) to achieve high data bandwidth. WDM systems replace a single carrier wavelength, possibly modulated with an ultrahigh bandwidth signal, by several carrier wavelengths, called channels, each modulated at a much lower rate. There can be tens of channels in a single fiber, each operating at a data rate of e.g. $40 \mathrm{~Gb} / \mathrm{s}$. At a network node there may be a need to switch some of these channels to a different light path. If this is done electronically, all the channels are first demultiplexed and converted to electrical signals for switching. Apparently this causes unnecessary processing for the channels that are not switched, and due to high cost of electrical switches, optical switching is preferred [44].

Apart from their use in optical networks, optical switches could be used for on-chip switching and modulation. A possible application is to access individual optical sensor elements on an integrated sensor array chip, see Fig 7. Other potential applications are in optical instruments (e.g. in an optical spectrum analyzer).

An important future application area is in on-chip optical networks that may replace some of the electrical interconnections, for example for distributing high-speed clock signals and handling massive data streams in processors.

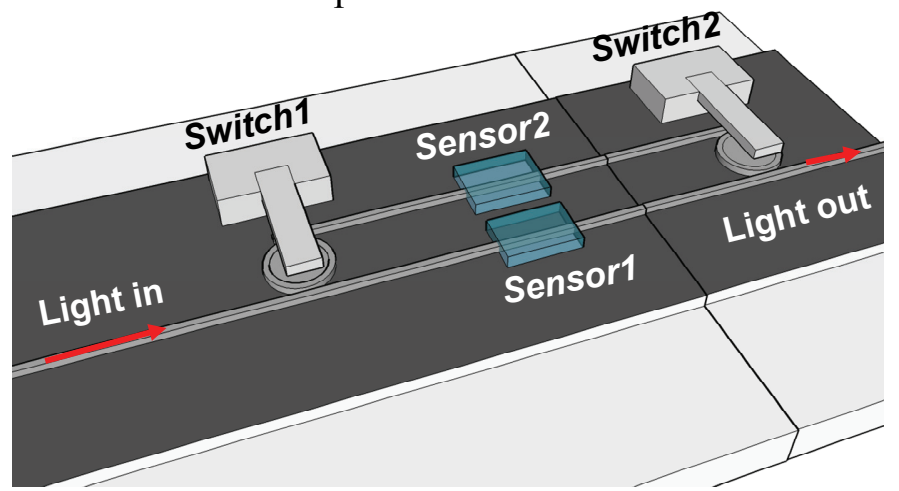

Fig. 7. A conceptual picture; an optical switch is used to access a sensor element. The switch1 controls to which sensor the light is coupled while the switch2 can be used to control from which sensor the signal is read-out. 


\subsubsection{Optical switching techniques}

Several transduction principles can be used for obtaining optical switching, some of which will be briefly discussed below.

\section{Thermo-optic (TO) switching:}

The refractive index of a material depends on its temperature. Therefore it is possible to change the effective index of a waveguide by heating or cooling it, which can be exploited for obtaining a temperature-induced optical switching function in properly designed optical devices. Thermo-optical IO switches are typically designed very similar to the ones used for sensor applications, presented in section 1.2.1.2. The thermo-optic coefficient of the material determines how large an index change is caused per unit temperature change. Compared to other commonly used waveguide materials silicon has a high thermo-optic coefficient of the order of $10^{-4} /{ }^{\circ} \mathrm{C}$. This enables the achievement of relatively large index changes, compared to those attainable with other relevant effects, such as the electro-optic effect. However, the thermal conductivity limits the switching time, typical values in silicon-nitride-based waveguide devices being around $0.1 \mathrm{~ms}$ [45], whereas in silicon, having a much larger thermal conductivity, switching times down to 1 $\mu \mathrm{s}$ have been demonstrated [46]. The biggest disadvantage of TO devices is the power consumption, which is needed even in the static case, as at least one of the on or off states of a TO switch requires continuous local heating. Additionally heat dissipation from a switch element to another may cause cross-talk problems in a TO switch array.

\section{Electro-optic (EO) switching:}

In some materials the (linear) Pockels effect or the (quadratic) electro-optic Kerr effect causes the refractive index to change upon the application of an electric field [47-48]. As the Pockels effect is stronger, thus requiring smaller operation voltages, it is preferred, although it occurs only in non-centrosymmetric crystalline materials. Lithium niobate is a commonly used material for EO switches.

The electro-optic effect is fast, allowing sub-nanosecond switching times [43] with small power consumption; yet the attainable index changes are much smaller than with the TO effect.

\section{Plasma dispersion:}

The presence of free charge carriers in a material causes optical absorption loss, represented by the imaginary part of the refractive index [49]. According to the KramersKronig relation, the real part of the refractive index will change as well. Hence, by injecting electrons (e.g. with an electric current) into some specific region, it is possible to locally change the refractive index. In this way very fast $(>1 \mathrm{~ns})$ and low power $(\sim 10$ $\mu \mathrm{W})$ switching can be achieved [50]. The trade-off is that the phenomena inherently include optical absorption loss, which becomes especially noticeable if a large tuning range is to be realized. 


\section{Mechanical switching:}

As already mentioned in 1.2.2.2, the mechano-optical interaction, which can be used for optical switching, can be roughly divided into two categories: the ones where the mechanical elements are introduced into the optical path and the other ones where the mechanical element is interacting with the evanescent field of the guided light.

This type of switching is not yet as established as the other techniques discussed above, however some related work on the topic has been published. In the late 90's these types of devices were implemented with bonding techniques, i.e. the mechanical part was attached to the optics chips [38, 51]. Later there have been few demonstrations with monolithically integrated cantilevers. In [52] MEMS-actuated switching of a ring resonators is demonstrated with an aluminum microbridge that, when pulled close to the ring, dispatches the resonance due to increased optical loss. Modulation of a photonic crystal waveguide with a polysilicon cantilever is demonstrated in [53]. Also in this case the cantilever causes absorption of light when pulled close to the waveguide. In addition, there have been demonstrations of the AFM cantilever interaction with nanophotonic devices [54-55]. These types of integrated devices aim at providing low power consumption, wide actuation range, and a switching speed at least comparable to thermooptic devices. The micromechanical actuation of an IO device is a major topic of this thesis, which is discussed in chapter 4.

The devices that rely on introducing a mechanical element into the optical path can be seen as miniaturized versions of the bulk mechano-optical switches. An example of such a device is a MEMS mirror that is typically a movable thin film fabricated on a siliconon-insulator wafer. It is possible to fabricate a compact array of thousands of MEMS mirror switch elements [43]. As the required power consumption of an electrostatically actuated mechanical switch is low, this switching technique is attractive for applications requiring a high number of ports. The switching speed of mirror-based devices is on the order of $10 \mathrm{~ms}$ [43].

\subsection{Silicon photonics}

Although very expensive processing equipment is needed to fabricate state-of-the-art silicon complementary metal-oxide-semiconductor (CMOS) devices, the investments have been justified by the economy of mass production which has proven to bring down the price per device to a sufficiently low level so as to open markets to the devices. The ability to use the same standardized process to produce a wide variety of devices can be claimed to be at least partly the reason behind the CMOS glory path. Silicon can be seen as an ideal material for optical-electrical integration, due to the maturity of Si based technology. It has already been demonstrated that the tools that are used for the modern CMOS technology can be used to fabricate micro- and nanophotonic devices as well [6]. The research described in this thesis is partly motivated by the desire to explore the interesting possibility to fabricate state-of-the-art photonic devices with potentially low cost by sharing the equipment with electronics industry and research, and by applying the same standardized processes. 


\section{Silicon photonics fabrication platform, ePIXfab}

Most of the optical devices that are presented in this thesis were fabricated using the silicon photonics platform, known as ePIXfab, established at IMEC, Leuven. This fabrication platform allows the realization of submicrometre features on silicon by using the same state-of-the-art lithographic tools that are used to make modern CMOS transistors. We used two different deep-UV lithographic processes to realize our silicon photonic devices, viz. an older one, employing a 248-nm light source, and a newer one, operating at $193 \mathrm{~nm}$. The specified minimum feature sizes of these processes are given in table 2.

Table 2. Minimum feature sizes of ePIXfab deep UV lithography processes

\begin{tabular}{|l|l|l|}
\hline \multirow{2}{*}{ Feature } & \multicolumn{2}{|c|}{ Minimum size } \\
\cline { 2 - 3 } & $\mathbf{1 9 3} \mathbf{~ n m}$ DUV & $\mathbf{2 4 8} \mathbf{~ n m}$ DUV \\
\hline Periodic structure, pitch & $300 \mathrm{~nm}$ & $400 \mathrm{~nm}$ \\
\hline Holes, diameter & $100 \mathrm{~nm}$ & $200 \mathrm{~nm}$ \\
\hline Holes, spacing between & $90 \mathrm{~nm}$ & $120 \mathrm{~nm}$ \\
\hline Lines, width & $120 \mathrm{~nm}$ & $200 \mathrm{~nm}$ \\
\hline Lines, spacing between & $100 \mathrm{~nm}$ & $150 \mathrm{~nm}$ \\
\hline Trenches, width & $100 \mathrm{~nm}$ & $200 \mathrm{~nm}$ \\
\hline
\end{tabular}

The processing is done on 200-mm SOI wafers, supplied by SOITEC [56], having a 220$\mathrm{nm}$ thick silicon device layer on top of a $2-\mu \mathrm{m}$ thick silicon dioxide layer, all on a $\sim 700$ $\mu \mathrm{m}$ thick silicon handle wafer. The process provides two possible etching depths: $70 \mathrm{~nm}$ and $220 \mathrm{~nm}$. The shallow etch is mainly used to define grating couplers at the ends of the access waveguides. These couplers allow efficient coupling of light to and from the outside world without need for dicing and polishing waveguide end facets. The $220-\mathrm{nm}$ etch depth provides high index contrast, which allows the realization of compact devices. To use this fabrication platform the designer reserves a certain die area, called a mask allocation block, for his/her devices. The size of these blocks is standardized in the platform. The typical size of such a block is $6 \mathrm{~mm}$ by $2.5 \mathrm{~mm}$. The blocks from several designers are combined into an area of roughly $12 \mathrm{~mm}$ by $7.5 \mathrm{~mm}$ to form a master die, as depicted in Fig. 8. A photograph of a fabricated SOI wafer is shown in Fig. 9. Many copies of this master die are then replicated on the wafer with stepper lithography. This allows one to vary the lithographic exposure dose for these dies, which, in turn, means that size variations are obtained. Thus, it is possible to have different waveguide widths in each die, although the designed structures are identical for each die.

The so-called target exposure dose should give exactly the same dimensions on a wafer as designed on the mask. This target exposure dose depends on the structure and its dimensions, and the designer should carefully take this into account.

For a more detailed discussion about the process we refer to [57-58]. 


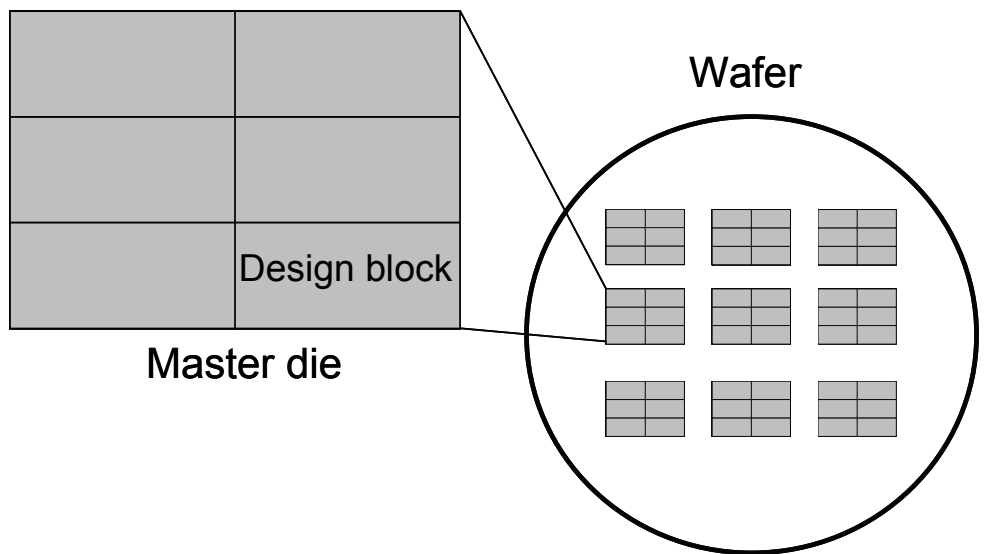

Fig. 8. A Schematic picture of a wafer. A master die that is repeated many times on a wafer is composed of design blocks.

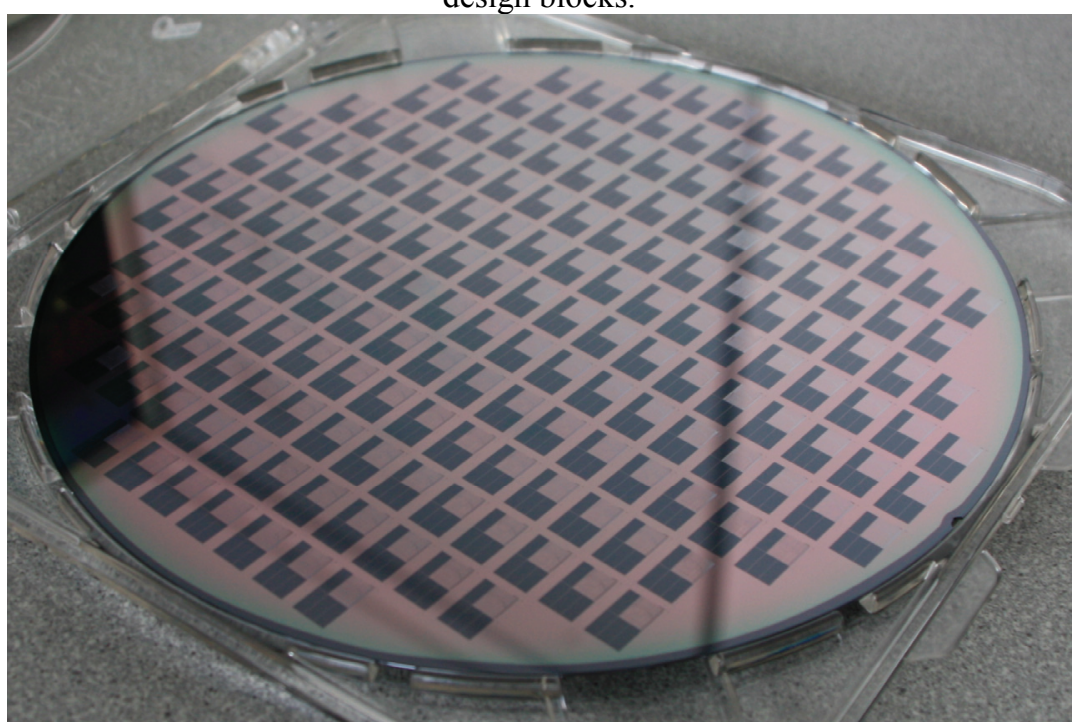

Fig. 9. A photograph of a processed 8 inch SOI wafer. In total there are 186 dies (of size $12.1 \mathrm{~mm}$ by 8.6 $\mathrm{mm})$ on the wafer. 


\subsection{Outline of the thesis}

The current chapter, 1, gives a general introduction to research topics covered in this thesis. These are biosensing, integrated read-out of microcantilever deflection, and optical switching.

Chapter 2 presents the sensor work. The chapter focuses on sensors based on waveguide gratings. An introduction of the optical properties and design issues of waveguide gratings is given. The experimental results of grated silicon photonic wire sensors, applied to bulk refractive-index sensing and label-free enzyme sensing, are given. A novel optical detection method to monitor nanodisplacements of a microcantilever is presented, and the method's potential use as a sensor is discussed.

Chapter 3 discusses the optical designs that were made for MEMS-actuated optical switching. These include cavities in photonic crystals, as well as waveguide gratings and ring resonators. The design procedure for each device is given, and the results of optical characterization are shown.

Chapter 4 gives the experimental results of MEMS-actuated devices. Mechano-optical tuning of a ring-resonator and a photonic crystal waveguide is demonstrated with an electrostatically actuated integrated microcantilever.

Chapter 5 presents an outlook on possible future developments and applications. 


\section{References}

[1] W.C.L. Hopman, "Light-flow characterization and manipulation in 1 and 2 dimensional photonic crystals", PhD thesis, University of Twente, 2007.

[2] T. Vo-Dinh, "Biomedical photonics handbook", CRC Press 2003.

[3] R.E. Gyurcsanyi, Z. Vagfoldi, K. Toth, and G. Nagy, "Fast response potentiometric acetylcholine biosensor", Electroanalysis, vol. 11, pp. 712-718, 1999.

[4] G.A. Campbell, D. Delesdernier, and R. Mutharasan, "Detection of airborne Bacillus anthracis spores by an integrated system of an air sampler and a cantilever immunosensor", Sensors and Actuators BChemical, Vol. 127, pp. 376-382, 2007.

[5] X. Fan, I.M. White, S.I. Shopova, H. Zhu, J.D. Suter, and Y. Sun, "Sensitive optical biosensors for unlabeled targets: A review", Analytica Chimica Acta, vol. 620, pp. 8-26, 2008.

[6] V.M.N. Passaro, F. Dell'Olio, B. Casamassima, and F. De Leonardis, "Guided-Wave Optical Biosensors", Sensors, vol. 7, pp. 508-536, 2007.

[7] P.V. Lambeck, "Integrated optical sensors for the chemical domain," Measurement Science \& Technology, vol. 17, pp. 93-116, 2006.

[8] B. Liedberg, C. Nylander, and I. Lunström, "Surface plasmon resonance for gas detection and biosensing", Sensors and Actuators, vol. 4, pp. 299-304, 1983.

[9] R.D. Harris, B.J. Luff, J.S. Wilkinson, J. Piehler, A. Brecht, G. Gauglitz, and R.A. Abuknesha, "Integrated optical surface plasmon resonance immunoprobe for simazine detection", Biosensors \& Bioelectronics, vol. 14, pp. 377-386, 1999.

[10] J. Dostálek, J. Čtyroký, J. Homola, E. Brynda, M. Skalský, P. Nekvindová, J. Špirková, J. Škvor, and J. Schröfel, "Surface plasmon resonance biosensor based on integrated optical waveguide", Sensors and Actuators B: Chemical, vol. 76, pp. 8-12, 2001.

[11] C.E.H. Berger, T.A.M. Beumer, R.P.H. Kooyman, and J. Greve, "Surface Plasmon Resonance Multisensing", Analytical Chemistry, vol. 70, pp. 703-706, 1998.

[12] E. Fu, T. Chinowsky, J. Foley, J. Weinstein, and P. Yager, "Characterization of a wavelength-tunable surface plasmon resonance microscope", Review of Scientific Instruments, vol. 75, pp. 2300-2304, 2004.

[13] M. Piliarik, H. Vaisocherová, and J. Homola, "A new surface plasmon resonance sensor for highthroughput screening applications", Biosensors and Bioelectronics, vol. 20, pp. 2104-2110, 2005.

[14] R.G. Heideman and P.V. Lambeck, "Remote opto-chemical sensing with extreme sensitivity: design, fabrication and performance of a pigtailed integrated optical phase-modulated Mach-Zehnder interferometer system", Sensors and Actuators B-Chemical, vol. 61, pp. 100-127, 1999.

[15] M. Hoekman, M. Dijkstra, and H.J.W.M. Hoekstra, "Design of a Spiral-Shaped Mach-Zehnder Interferometric Sensor for Refractive Index Sensing of Watery Solutions", IEEE/LEOS Benelux Annual Workshop, Eindhoven, The Netherlands, 25. May 2007.

[16] A. Densmore, D.-X. Xu, S. Janz, P. Waldron, T. Mischki, G. Lopinski, A. Delâge, J. Lapointe, P. Cheben, B. Lamontagne, and J. H. Schmid, "Spiral-path high-sensitivity silicon photonic wire molecular sensor with temperature-independent response", Optics Letters, vol. 33, pp. 596-598, 2008.

[17] I.M. White and X. Fan, "On the performance quantification of resonant refractive index sensors", Optics Express, vol. 16, pp. 1020-1028, 2008.

[18] K. De Vos, I. Bartolozzi, E. Schacht, P. Bienstman, and R. Baets, "Silicon-on-Insulator microring resonator for sensitive and label-free biosensing", Opt. Express, vol. 15, pp. 7610-7615, 2007.

[19] C. Chao, W. Fung, and L.J. Guo, "Polymer microring resonators for biochemical sensing applications", IEEE Journal of Selected Topics in Quantum electronics, vol. 12, pp. 134-142, 2006.

[20] S. Mandal and D. Erickson, "Nanoscale optofluidic sensor arrays", Optics Express, vol. 16, pp. $1623-$ $1631,2008$.

[21] W.C.L. Hopman, P. Pottier, D.Yudistira, J. van Lith, P.V. Lambeck, R.M. De LaRue, A. Driessen, H.J.W.M. Hoekstra, and R.M. de Ridder, "Quasi-one-dimensional photonic crystal as a compact building-block for refractometric optical sensors”, IEEE J. Select. Topics Quantum Electron, vol. 11, pp. 11-16, 2005. 
[22] N. Skivesen, A. Têtu, M. Kristensen, J. Kjems, L.H. Frandsen, and P.I. Borel, "Photonic-crystal waveguide biosensor", Optics Express, vol. 15, pp. 3169-3176, 2007.

[23] C.A. Barrios, K.B. Gylfason, B. Sánchez, A. Griol, H. Sohlström, M. Holgado, and R. Casquel, "Slotwaveguide biochemical sensor", Optics Letters, vol. 32, pp. 3080-3082, 2007.

[24] G. Rong, A. Najmaie, J.E. Sipe, and S.M. Weiss, "Nanoscale porous silicon waveguide for labelfree DNA sensing", Biosensors \& Bioelectronics, vol. 23, pp. 1572-1576, 2008.

[25] L. He, M.D. Musick, S.R. Nicewarner, F.G. Salinas, S.J. Benkovic, M.J. Natan, and C.D. Keating, "Colloidal Au-enhanced surface plasmon resonance for ultrasensitive detection of DNA hybridization", Journal of the American Chemical Society, vol. 122, pp. 9071-9077, 2000.

[26 ] C. Ziegler, "Cantilever-based biosensors", Analytical and Bioanalytical Chemistry, vol. 379, pp. 946959, 2004.

[27] J. Fritz, M. K. Baller, H.P. Lang, H. Rothuizen, P. Vettiger, E. Meyer, H.-J. Güntherodt, Ch. Gerber, and J.K. Gimzewski, "Translating biomolecular recognition into nanomechanics", Science, vol. 288, pp. 316-318, 2000.

[28] M.K. Baller, H.P. Lang, J. Fritz, Ch. Gerber, J.K. Gimzewski, U. Drechsler, H. Rothuizen, M. Despont, P. Vettiger, F.M. Battiston, J.P. Ramseyer, P. Fornaro, E. Meyer, and H.-J. Güntherodt, "A cantilever array-based artificial nose", Ultramicroscopy, vol. 82, pp. 1-9, 2000.

[29] Y. Zhao, M. Mao, R. Horowitz, A. Majumdar, J. Varesi, P. Norton, and J. Kitching, "Optomechanical uncooled infrared imaging system: design, microfabrication, and performance", Journal of Microelectromechanical systems vol. 11, pp. 136-146, 2002.

[30] F. Dong, Q. Zhang, D. Chen, Z. Miao, Z. Xiong, Z. Guo, C. Li, B. Jiao, and X. Wu, "Uncooled infrared imaging device based on optimized optomechanical micro-cantilever array", Ultramicroscopy, vol. 108, pp. 579-588, 2008.

[31] S. Okuyama, Y. Mitobe, K. Okuyama, and K. Matsushita, "Hydrogen gas sensing using a Pd-coated cantilever" Japanese Journal of Applied Physics, vol 39, pp. 3584-3590, 2000.

[32] D. Iannuzzi, M. Slaman, J.H. Rector, H. Schreuders, S. Deladi, and M.C. Elwenspoek, "A fibertop cantilever for hydrogen detection", Sensors and Actuators B, vol. 121, pp. 706-708, 2007.

[33] N.V. Lavrik, M.J. Sepaniak, and P.G. Datskos, "Cantilever transducers as a platform for chemical and biological sensors", Review of Scientific Instruments, vol. 75, pp. 2229-2253, 2004.

[34] K. Zinoviev, C. Dominguez, J.A. Plaza, V.J.C. Busto, and L.M. Lechuga, "A novel optical waveguide microcantilever sensor for the detection of nanomechanical forces", Journal of Lightwave Technology, vol. 24, pp. 2132-2138, 2006.

[35] M. Nordström, D.A. Zauner, M. Calleja, J. Hübner, and A. Boisen, "Integrated optical readout for miniaturization of cantilever-based sensor system", Applied Physics Letters, vol. 91, p. 103512, 2007.

[36] M. Li, W.H.P. Pernice, and H. X. Tang, "Broadband all-photonic transduction of nanocantilevers", Nature Nanotechnology, vol. 4, pp. 377-382, 2009.

[37] C. Lee and J. Thillaigovindan, "Optical nanomechanical sensor using a silicon photonic crystal cantilever embedded with a nanocavity resonator", Applied Optics, vol. 48, pp. 1797-1803, 2009.

[38] W. Gabathuler and W. Lukosz, "Electro-nanomechanically wavelength-tunable integrated-optical Bragg reflectors Part II: Stable device operation”, Optics Communications, vol. 145, pp. 258-264, 1998.

[39] C.A. Barrios, "Ultrasensitive nanomechanical photonic sensor based on horizontal slot-waveguide resonator", IEEE Photonics Technology Letters, vol. 18, pp. 2419-2421, 2006.

[40] P.T. Rakich, M. A. Popovic', M.R. Watts, T. Barwicz, H.I. Smith, and E.P. Ippen, "Ultrawide tuning of photonic microcavities via evanescent field perturbation", Optics Letters, vol. 31, 2006.

[41] G.N. Nielson, D. Seneviratne, F. Lopez-Royo, P.T. Rakich, Y. Avrahami, M.R. Watts, H.A. Haus, H.L. Tuller, and G. Barbastathis, "Integrated wavelength-selective optical MEMS switching using ring resonator filters”, IEEE Photonics Technology Letters, vol. 17, pp. 1190-1192, 2005.

[42] R. Ramaswami, "Optical Networking Technologies: What Worked and What Didn't" IEEE Communications Magazine, pp. 132-139, September 2006.

[43] R. Ramaswami and K.N. Sivarajan, "Optical networks: a practical perspective", second edition, Morgan Kaufmann 2002. 
[44] M. Maier and M. Reisslein, "Trends in Optical Switching Techniques: A Short Survey," IEEE Network, vol. 22, pp. 42-47, 2008.

[45] E.J. Klein, "Densely integrated microring-resonator based components for fiber-to-the-home applications", PhD thesis, University of Twente, 2007.

[46] G. Cocorullo, M. Iodice, I. Rendina, and P.M. Sarro, "Silicon Thermooptical Micromodulator with 700-kHz - 3-dB Bandwidth", IEEE Photonics Technology Letters, vol. 7, pp. 363-365, 1995.

[47] A. Schneider, I. Biaggio, and P. Günther, "Teraherz-induced lensing and its use for the detection of teraherz pulses in a birefringent crystal," Applied Physics Letters, Vol. 84, pp. 2229-2231, 2004.

[48] R. Dekker, A. Driessen, T. Wahlbrink, C. Moormann, J. Niehusmann, and M. Först, "Ultrafast Kerrinduced all-optical wavelength conversion in silicon waveguides using $1.55 \mu \mathrm{m}$ femtosecond pulses," Optics Express, Vol. 14, pp. 8336-8346, 2006.

[49] J. Singh, "Semiconductor optoelectronics: physics and technology", McGraw-Hill 1995.

[50] Q. Xu, B. Schmidt, S. Pradhan, and M. Lipson, "Micrometre-scale silicon electro-optic modulator", Nature, vol. 435, pp. 325-327, 2005.

[51] G.J. Veldhuis, T. Nauta, C. Gui, J.W. Berenschot, and P.V. Lambeck, "Electrostatically Actuated Mechanooptical Waveguide ON-OFF Switch Showing High Extinction at a Low Actuation-Voltage", IEEE Journal of Selected Topics in Quantum Electronics, vol. 5, pp. 60 - 66, 1999.

[52] G.N. Nielson, D. Seneviratne, F. Lopez-Royo, P.T. Rakich, Y. Avrahami, M.R. Watts, H.A. Haus, H.L. Tuller, and G. Barbastathis, "Integrated wavelength-selective optical MEMS switching using ring resonator filters", IEEE Photonics Technology Letters, vol. 17, pp. 1190-1192, 2005.

[53] P.T. Rakich, M. A. Popović, M.R. Watts, T. Barwicz, H.I. Smith, and E.P. Ippen, "Ultrawide tuning of photonic microcavities via evanescent field perturbation", Optics Letters, vol. 31, pp. 1241-1243, 2006.

[54] W.C.L. Hopman, K.O. van der Werf, A.J.F Hollink, W. Bogaerts, V. Subramaniam, and R.M. de Ridder, "Nano-mechanical tuning and imaging of a photonic crystal micro-cavity resonance," Optics Express, vol. 14, pp. 8745-8752, 2006.

[55] I. Märki, M. Salt, and H.P. Herzig, "Tuning the resonance of a photonic crystal microcavity with an AFM probe," Optics Express 14, pp. 2969-2978, 2006.

[56] Soitec, S.A.Parc Technologique des Fontaines, 38190, Bernin, France. (www.soitec.com)

[57] W. Bogaerts, "Nanophotonic Waveguides and photonic crystals in silicon-on-insulator", PhD thesis, Universiteit Gent 2004.

[58] P. Dumon,W. Bogaerts, R. Baets, J.-M. Fedeli, and L. Fulbert, "Towards foundry approach for silicon photonics: silicon photonics platform ePIXfab", IEEE Electronics Letters, vol. 45, no. 12, 2009. 


\section{Sensors based on waveguide grating}

In this chapter waveguide grating based sensors for bio- and nanodisplacement sensing are presented. First the optical properties of waveguide gratings are discussed with a focus on modeling and design issues. The use of a waveguide grating as a sensor platform for refractometric sensing and for sensing of nanodisplacements is discussed and important sensor parameters are introduced. The properties of a grated silicon photonic wire for label- free biochemical sensing applications are discussed and sensitivity, as well as detection limit, of this device is experimentally determined. A new integrated optical detection method to observe the displacement of a microcantilever is presented and options to fabricate this device are discussed.

\subsection{Waveguide grating}

In this section some properties of waveguide gratings are discussed. The focus is on modeling and design issues related to optical losses that are specific to waveguide gratings. Design boundaries to obtain low loss operation are presented. A grating defect and some of its potential applications are shortly described. The use of waveguide gratings as sensors to detect refractive index changes in the proximity of the grating is also discussed.

If the properties of periodic structures are to be exploited in integrated optics, the $2 \mathrm{D}$ lateral confinement provided by channel waveguides needs to be combined with a periodic variation in the third dimension. The most common way to do this is to periodically vary the thickness or width of the waveguide. An example of such a "grated waveguide" or "waveguide grating" is schematically depicted in Fig. 1a.

Exact modelling of such essentially 3-dimensional structures is rather involved, requiring huge computing resources. Depending on the waveguide geometry the model can be reduced to $2 \mathrm{D}$, see Fig. 1b, without losing much information. However, this is not always the case. In particular, the strong modal dispersion of strip waveguides in high-indexcontrast material systems causes a large uncertainty in the parameters of a 2D model [1], see also section 2.2.1.

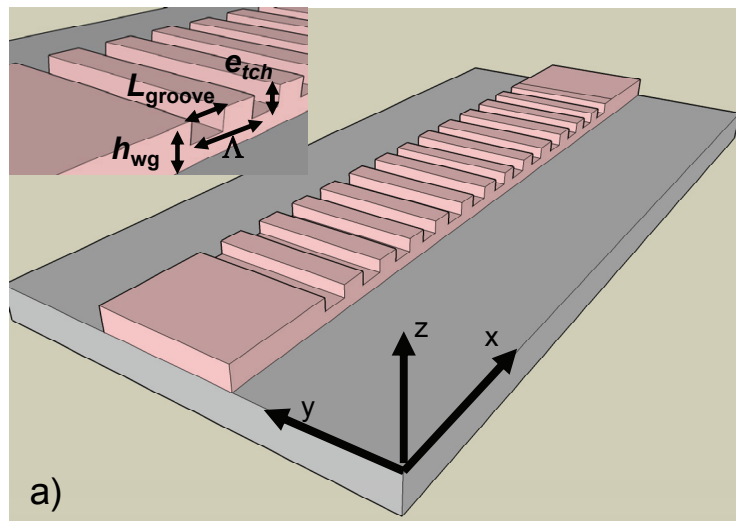



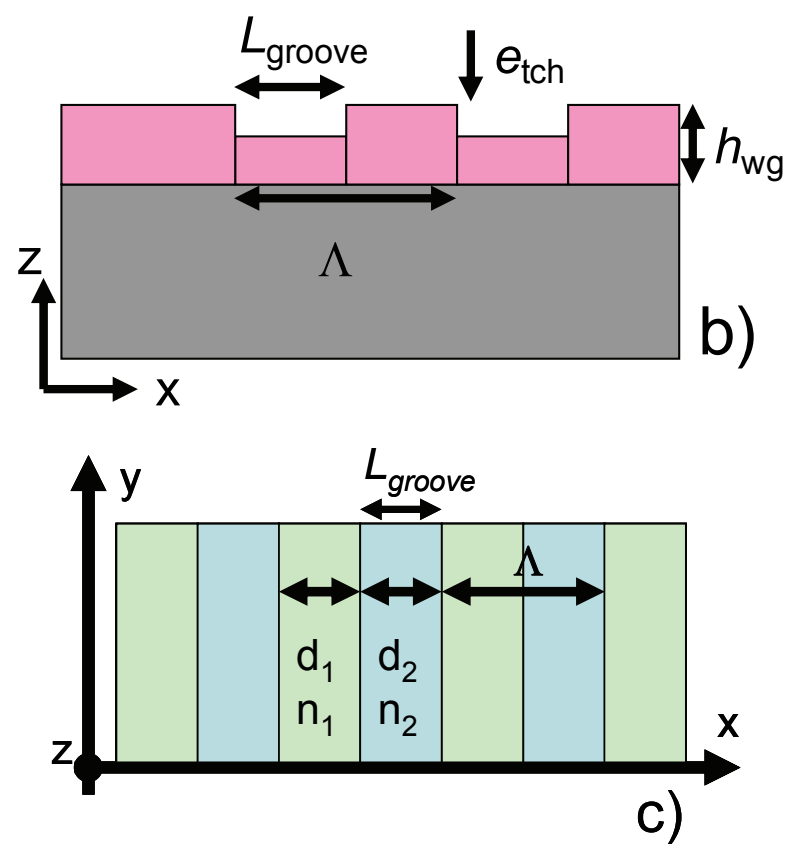

Fig. 1. A schematic 3D picture of a waveguide grating. The grating is formed by periodically altering the thickness of the waveguide. The depth of the groove depends on the etch depth $\left(\mathrm{e}_{\mathrm{tch}}\right)$. b) Schematic picture of waveguide grating reduced to $2 \mathrm{D}$. c) A schematic picture of waveguide grating reduced to $1 \mathrm{D}$. The equivalent $1 \mathrm{D}$ indices $\left(n_{1}\right.$ and $\left.n_{2}\right)$ are calculated using e.g. a mode solver. The layer thicknesses $d_{1}$ and $d_{2}$ correspond to groove length $\left(L_{\text {groove }}\right)$ and ridge length $\left(\Lambda-L_{\text {groove }}\right)$.

The 2D structure can be reduced further to $1 \mathrm{D}$, see Fig. 1c, for efficient modeling. However, in this case some crucial information is lost. In 1D modeling, out-of-plane losses are ignored and the effect of the waveguide geometry is not fully taken into account.

We will use the reduced $1 \mathrm{D}$ and 2D models to illustrate some properties of light propagation in a waveguide grating. Wave propagation in a periodic structure, such as a waveguide grating, can be formulated according to Bloch's theorem [2]. According to it, the wave function in a $1 \mathrm{D}$ periodic structure can be expressed as:

$$
\psi(x, t)=e^{j(\omega t-\beta x)} u(x) .
$$

In which $u$ is the amplitude of the wave, $\beta$ is the wave-vector (also known as propagation constant), $\omega$ is the angular frequency and $t$ is the time. From the periodicity of the structure it follows that the wave-vectors that differ by integer multiples of $2 \pi / \Lambda$ (where $\Lambda$ is the period) are equal; i.e. the wave-vector repeats itself [3]. This implies that the wave-vector of this Bloch state can be reduced to the so-called Brillouin zone $[-\pi / \Lambda$, $\pi / \Lambda]$. A dispersion graph of a grating is obtained by solving the wave-vector values as function of angular frequency in the Brillouin zone. The dispersion graph is useful in analyzing the properties of gratings (and other periodic structures).

As an example, the dispersion properties of a waveguide grating are calculated. We consider a shallow ridge waveguide with a ridge height $\left(h_{\mathrm{wg}}\right)$ of $5 \mathrm{~nm}$ defined in a $275 \mathrm{~nm}$ thick layer of $\mathrm{Si}_{3} \mathrm{~N}_{4}(n=1.981)$ that is on top of a $\mathrm{SiO}_{2}$ cladding $(n=1.445)$. If a grating is defined to this waveguide by etching a periodic pattern of $55 \mathrm{~nm}$ deep grooves (in the 
z-direction) having a length ( $\left.L_{\text {groove }}\right)$ of $240 \mathrm{~nm}$ and a period of $490 \mathrm{~nm}$, the equivalent 1D layer stack indices (as calculated with a 2D mode solver) are $n_{1}=1.59289$ and $n_{2}=$ 1.53209. Figs $2 \mathrm{a}$ and $2 \mathrm{~b}$ show the dispersion diagrams for the infinite layer stack, obtained by computing definite frequency eigenstates of Maxwell's equations using the MIT Photonics Band (MPB) package [4]. As seen from Fig. 2b, for some wavelengths there is no propagating mode in a grating as there is no wave-vector for these wavelengths. This wavelength range is the so-called photonic stopband. In a specific case the energy of the propagating wave is coupled from the forward propagating wave to the backward propagating wave. In terms of wave-vectors phase matching is required; $\beta_{f}-\beta_{b}$ $=m 2 \pi / \Lambda$, in which $m$ is an integer and subscripts $f$ and $b$ refer to backward and forward directions, respectively. In this case the light is reflected by the grating. In the stopband the Bloch mode is named as evanescent Bloch mode [5].

The dispersion graph also shows the so-called lightlines, which are the dispersion curves of plane waves travelling parallel to the waveguide in the corresponding cladding material, given by $\omega / \beta=c / n_{\text {clad }}$.

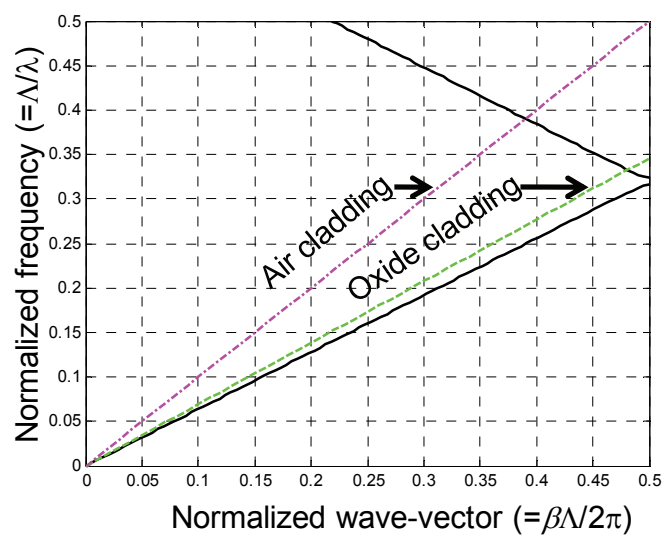

a)

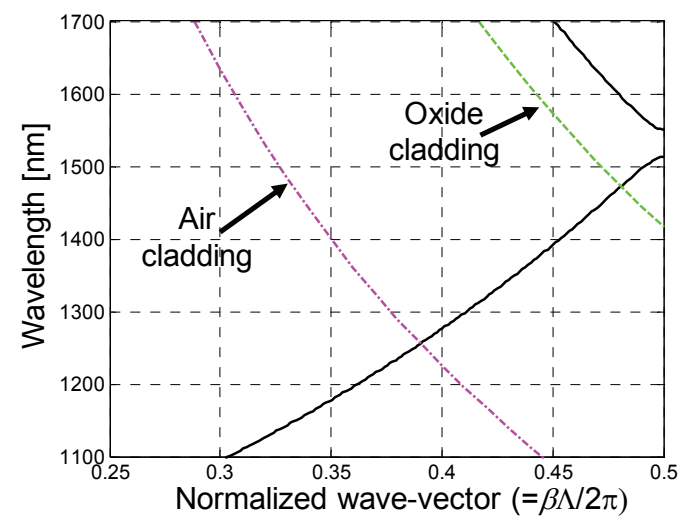

b)

Fig. 2. Dispersion graph of a 1D grating, also showing the lightlines for air and the oxide cladding (coloured dashed lines). a) Normalized dispersion graph. b) Magnified part of Fig. 2a, around the lowestorder stopband, with a real wavelength scale instead of normalized frequency.

If, for a given propagation constant of the grating mode, the frequency is above the lightline, part of the light radiates from the grating. This is because in that case the grating mode $(\beta)$ can always phase-match to a plane wave propagating at an angle $\theta$ to the surface normal in the cladding $\left(\beta_{\text {clad }} \sin \theta\right)$, according to [6]:

$$
\beta_{\text {clad }} \sin \theta=\beta-\frac{m 2 \pi}{\Lambda} \text {. }
$$

For our grating below $1470 \mathrm{~nm}$ the loss starts to increase as the lower dispersion curve crosses the lightline of the oxide cladding at this point (see Fig. 2b).

To obtain more quantitative data of the properties of the grating, we have performed 2D calculations. Fig. 3 shows a simulated transmission spectrum, obtained using the 2D finite difference time domain (FDTD) method [7], of the grating structure that is shown in the inset of Fig. 3. This is the 2D reduced model of the grating and the parameters are the same as the ones that were used for the 1D calculations to obtain the data shown in Fig. 2. For the 2D simulation a grating length of 100 periods was chosen. 
From the transmission spectrum we can see the effect of crossing the lightline for this grating. For wavelengths below $1470 \mathrm{~nm}$ the loss increases with the wavelength difference from this lightline crossing point, as predicted from the dispersion graph. In the simulated transmission spectrum (see Fig. 3) there are peaks in the transmission band. These resonance peaks are explained next.

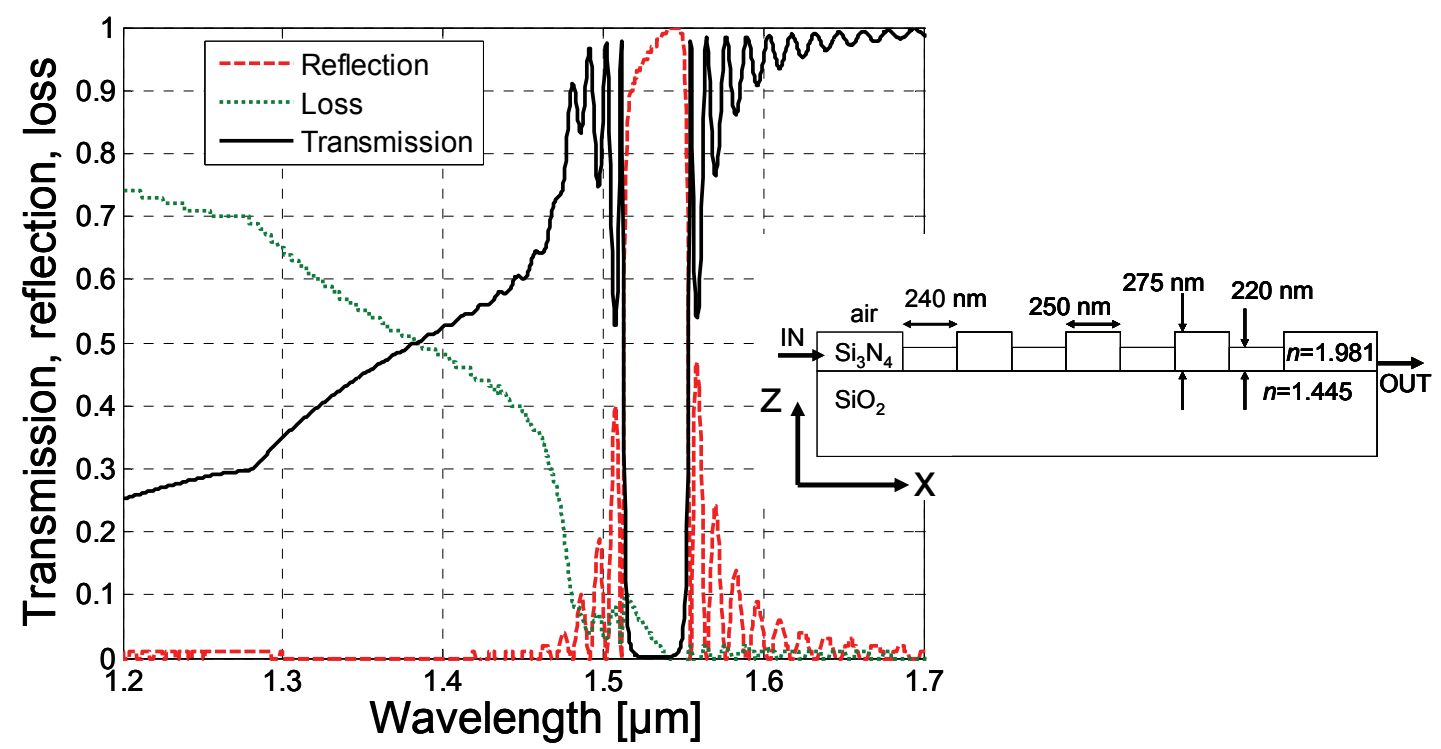

Fig. 3. Simulated transmission spectrum using the same grating parameters as for Fig. 2. The loss increases as the wavelength becomes shorter than the wavelength of $1470 \mathrm{~nm}$, where the dispersion curves of the cladding and the grating modes cross (see Fig. 2b). Inset: schematic picture of the grating cross-section used in the calculation.

If the grating is finite in the $\mathrm{x}$-direction, resonances can be observed in the transmission band. They are similar to Fabry-Pérot cavity resonances of the Bloch modes [8]. Propagation constants of these (forward and backward propagating) modes are given by [8-9];

$$
\pm \beta=\frac{\pi}{\Lambda}-\frac{\pi q}{N \Lambda}, q=[1, N],
$$

in which $N$ is the number of periods in the grating.

When constructive interference occurs, these modes create a standing (electromagnetic) wave, and the nodes of these standing waves are located at $x=N \Lambda s / q$, with $s=[0, q]$.

In sensor applications, discussed in this book, we have exploited the sharp spectral features of the resonating Bloch modes in the design of a compact and highly sensitive sensors for the detection of nanodisplacements (section 2.4) and for refractometric sensing (sections 2.2 and 2.3).

\subsubsection{Design guidelines}

In this section some design guidelines are given to avoid the losses originating from the lightline crossing. 
Based on the fact that the normalized frequency $(\Lambda / \lambda)$ of the cladding lightline is $1 / 2 n_{\text {clad }}$ at the Brillouin zone edge, it is possible to write a simple equation for maximum period $\Lambda_{\max }$, in order to have the stopband at the lossless side of the lightline:

$$
\Lambda_{\text {max }}=\frac{\lambda_{\text {edge }}}{2 n_{\text {clad }}},
$$

where $\lambda_{\text {edge }}$ is the wavelength at the lower wavelength stopband edge. For a silicon oxide cladding $n_{\text {clad }}=1.445$ (at infrared wavelengths), and assuming the band edge of $\lambda_{\text {edge }}=1500 \mathrm{~nm}$, a maximum period of $520 \mathrm{~nm}$ is obtained from equation (17).

An estimate can be given of the minimum operating wavelength $\lambda_{\text {Omin }}$ that is the shortest wavelength at which the grating is still on the lossless side of the lightline, by just considering the properties of a 1D periodic structure (which of course cannot have radiation loss). It is the wavelength corresponding to the crossing of the light line and the dispersion curve of the grating mode of an equivalent 1D structure (see Fig. 2a). The crossing point of two linear curves can be calculated, if the slope and the value of the curves are known in one point. By assuming that the slope of the dispersion curve of the grating mode can be approximated to be $-1 / n_{a v}$, where $n_{a v}$ is the average index of the grating, and the slope of the cladding mode (lightline) is $1 / n_{\text {clad }}$, an equation that gives an approximate value for $\lambda_{O \min }$ is obtained:

$$
\lambda_{\text {Omin }}=\frac{2 \lambda_{\text {edge }} \Lambda\left(n_{a v}+n_{\text {clad }}\right)}{2 n_{a v} \Lambda+\lambda_{\text {edge }}}
$$

Equation (5) gives a good approximation only for weak gratings. Fig. 4 shows a comparison of the minimum lossless operating wavelength as calculated from equation (5) and as obtained using the MPB [3]. The following grating parameters were used: $\Lambda=490 \mathrm{~nm}, d_{1}=250 \mathrm{~nm}, n_{1}=1.55 \ldots 1.98, d_{2}=240 \mathrm{~nm}$ and $n_{2}=1.53$.

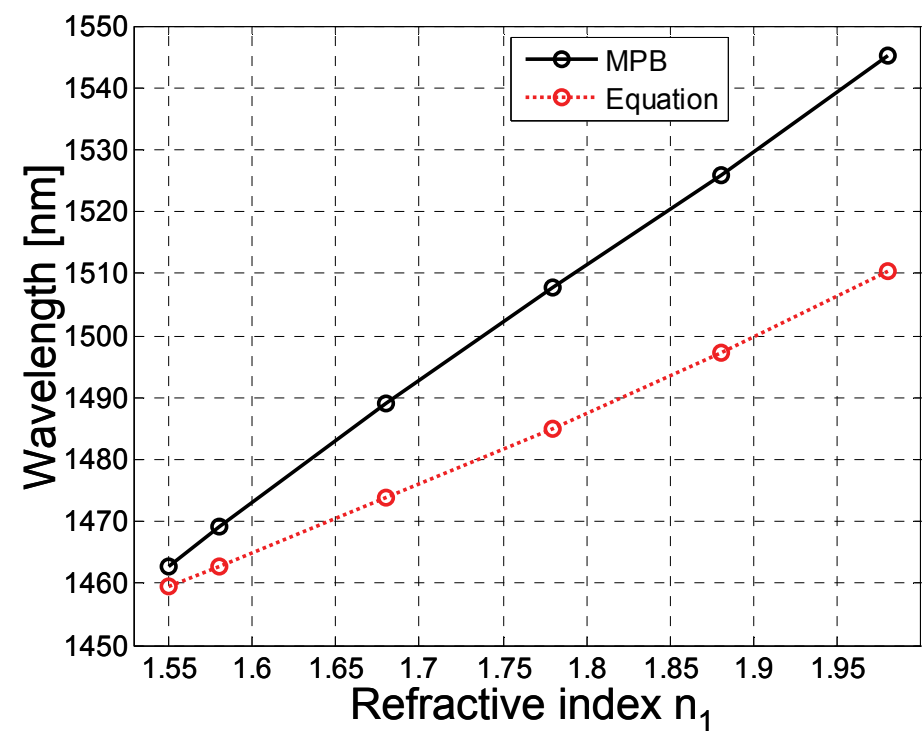

Fig. 4. Minimum operating lossless wavelength for a waveguide grating. Comparison between the data obtained from equation 5 and from MPB calculations. 
It can be seen that the equation gives the minimum operating wavelength $\lambda_{O \min }$ at better than $15 \mathrm{~nm}$ accuracy if $n_{1}-n_{2}<0.1$. For strong gratings the simple approximation is no longer valid as the nonlinearity of the dispersion curve increases. For these cases, the minimum operating wavelengths obtained using the MPB are considerable larger. Nevertheless equation (5) is a useful design tool by providing a quick and rough estimate of the minimum wavelength for lossless operation.

As an example, let us assume that a grating is designed to have $\lambda_{\text {edge }}=1500 \mathrm{~nm}$, and that it is to be fabricated on a waveguide having $n_{\text {eff }}=1.6$ and $n_{\text {clad }}=1.445$. We wish to find a design boundary for the grating period and see what is the minimum wavelength for lossless operation. By inserting the parameters into equation (5) (using $n_{a v}=n_{\text {eff }}$ ), a minimum operating point as a function of period is obtained as shown in Fig. 5. The maximum period can be calculated from equation (4) as well as the minimum period by placing $n_{\text {clad }} \rightarrow n_{a v}$. For the calculation of minimum period it is assumed that maximum $n_{a v}$ is used $\left(n_{e f f}\right)$ and keeping in mind that we set $\lambda_{\text {edge }}=1500 \mathrm{~nm}$. It can be seen from Fig. 5 that it is not possible to design a grating that would be lossless for wavelengths below $\lambda_{\text {Omin }}=1425 \mathrm{~nm}$.

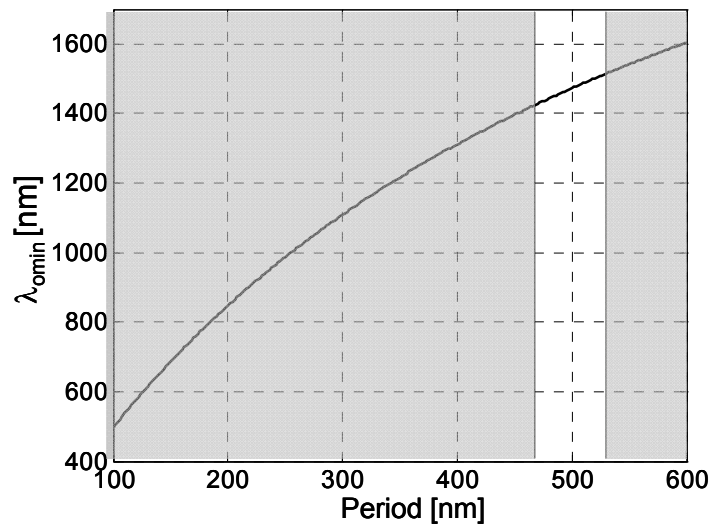

Fig. 5. Minimum lossless operating point as a function of the grating period. The shaded regions correspond to periods that are outside the boundaries set by equation (4), see text.

This type of design rule is useful, e.g. in the design of a waveguide laser that uses grating(s) as mirror(s). The grating operates as a mirror for the wavelengths that are in the photonic stopband of the grating. The wavelength of the pump light, used to excite the laser operation, is often much shorter than the laser wavelength and may therefore operate the grating at the lossy side of the lightline. For sensor applications low loss operation is important to get the best possible signal-to-noise ratio. Also to realize a compact sensor, the period should be small, which favors high index materials $(n>2)$.

It should be noted that in some cases it is not possible to have the periodic devices operating at the lossless side of the lightline, e.g. for some $\mathrm{PhCs}$ defined in III-V group materials [10]. However, if the device length is kept short, as can be the case for the $\mathrm{PhC}$ based devices, the absolute loss may still be acceptable.

The phenomenon of light radiating from a grating operating above the lightline is exploited in grating couplers [11], which are waveguide gratings that are intentionally 
designed to operate in this regime, such that the light in a given wavelength range is coupled into or out of a waveguide at a coupling angle $\theta$, given by (2).

\subsubsection{Mode mismatch loss}

Apart from radiation loss caused by crossing the lightline, there can be additional loss, depending on the geometry of the grating structure. For example, if the low-effectiveindex parts of the grating period are defined by etching as shown in Fig. 1, increasing the etch depth increases the loss. The origin of this loss is modal field mismatch between the incoming waveguide mode and the Bloch mode of the grating [12]. This type of loss affects a wide spectral band, including the stopband. The loss in the stopband is seen e.g. in a simulated transmission spectrum of Fig. 3: the loss is smaller at the longest wavelength.

The loss for the Bloch modes near the band edge is also due to mode mismatch between the Bloch mode and the waveguide mode, which leads to coupling to radiation modes. In order to minimize the mode mismatch loss the grating should be introduced gradually in tapered manner [12-13].

It should be noted that in the ideal grating the Bloch modes are lossless. In real gratings fabrication imperfections cause loss, which is particularly large for the slow Bloch modes due to increased life time of the resonating photons.

\subsubsection{Defects}

A defect is a structural imperfection in a periodic structure. In a waveguide grating a defect can be e.g. a waveguide segment or a grating section that has a different period than the surrounding grating. In optics such defects are often called cavities. Fig. 6a shows a defect in a waveguide grating. Light can be trapped in a cavity as a localized state if the frequency is in the photonic stopband of the surrounding grating. Light can be coupled from the input waveguide to the defect mode e.g. via an evanescent Bloch mode. If the cavity length is well chosen, a Fabry-Pérot resonator type operation is achieved; the surrounding grating sections function as mirrors for the cavity. Due to phase matching at resonance, energy is fed from the input side to the cavity while at the output side a fraction of energy escapes from the cavity via an evanescent Bloch mode. In the steady state, for a lossless structure, the power coupled to the cavity equals to the power transferred to the output. In a grating the reflection comes from distributed Bragg reflections. Therefore, in order to minimize the losses one should take into account the mode mismatch loss discussed above. Fig. 6b shows the transmission spectrum of a grated waveguide that has a waveguide segment with length $0.9 \Lambda$ in the middle of the grating. In this case the cavity loss is about $5 \mathrm{~dB}$.

At resonance, the field intensity is enhanced in a cavity. This phenomenon can be exploited in opto-optical devices. An intense light pulse at a wavelength corresponding to a defect mode resonance can be used to create an index change in a material whose index depends strongly on light intensity. This index change in turn changes the resonance peak position by some amount $\Delta \lambda$. Thanks to the cavity enhanced intensity, a required index change $\Delta n$ can be induced with a less intense pulse than would be needed without exploiting the resonant field enhancement. This kind of design can be used e.g. as an optical switch [14], in which the switching is done with intense optical pulses. Other 
grating cavity applications are e.g. optical delay lines [15], sensors [16 and 17] and waveguide lasers [18].
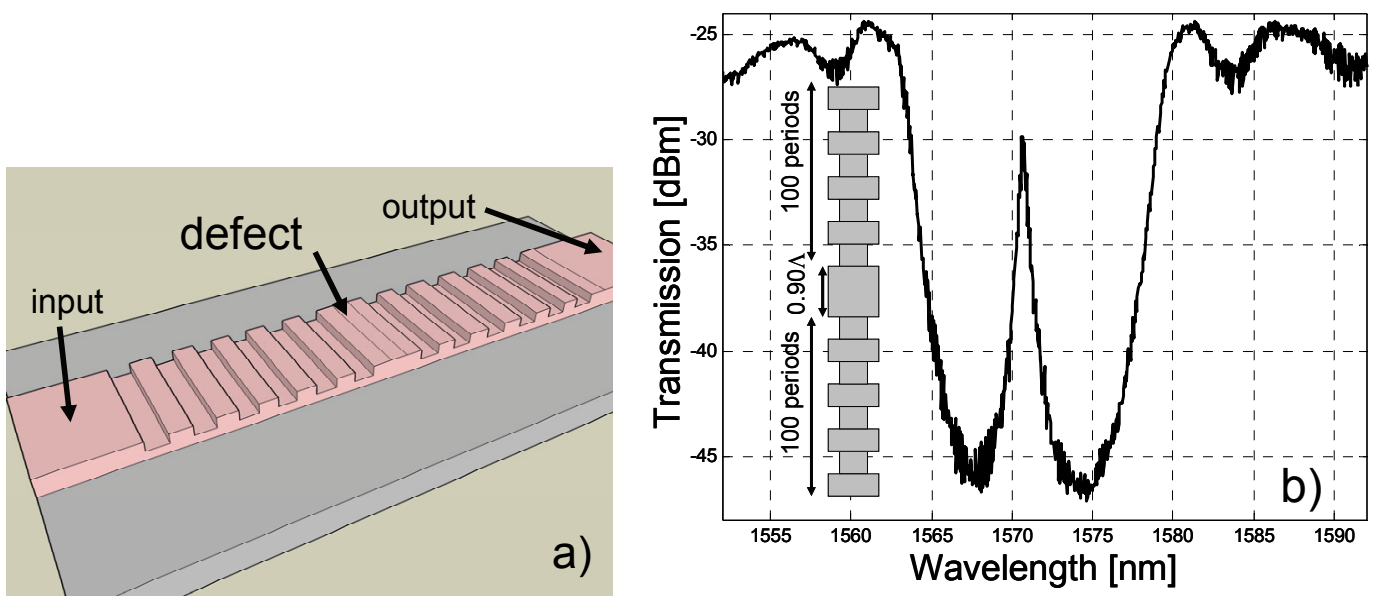

Fig. 6. a) Schematic picture of a defect in a waveguide grating b) An example of a measured transmission spectrum of a waveguide grating that has a defect cavity in the center. The peak in the middle of the stopband originates from the defect cavity. Inset: Schematic top view picture of the measured defective grating.

\subsubsection{Waveguide gratings as sensor platform}

In this section we discuss the use of waveguide gratings as sensors to detect refractive index changes in the proximity of the grating. The sensitivity of the grating to the index changes can be exploited to make sensors for biosensing and nanodisplacement.

\section{Refractometric sensing}

The effective index of the grated waveguide depends on the cladding material index. Therefore, if it is possible to observe the effective index change, it is also possible to measure the change in the cladding material index. For a waveguide grating the effective index change causes the spectral position of the stopband to shift, and this shift is often used to measure the cladding index change. A schematic picture of a waveguide grating sensor is shown in Fig. 7a.

The sharp spectral phenomena of a grated waveguide near the stopband edge exhibit a strong sensitivity to changes in the ambient refractive index. A small cladding-index change $\Delta n_{\text {clad }}$ causes the first-order Bragg wavelength $\lambda_{B}[6]$ of a grating to shift in accordance with:

$$
\Delta \lambda_{B} \approx 2 \eta \Delta n_{\text {clad }} \Lambda
$$

in which $\eta$ is the fraction of energy density in the cladding. 

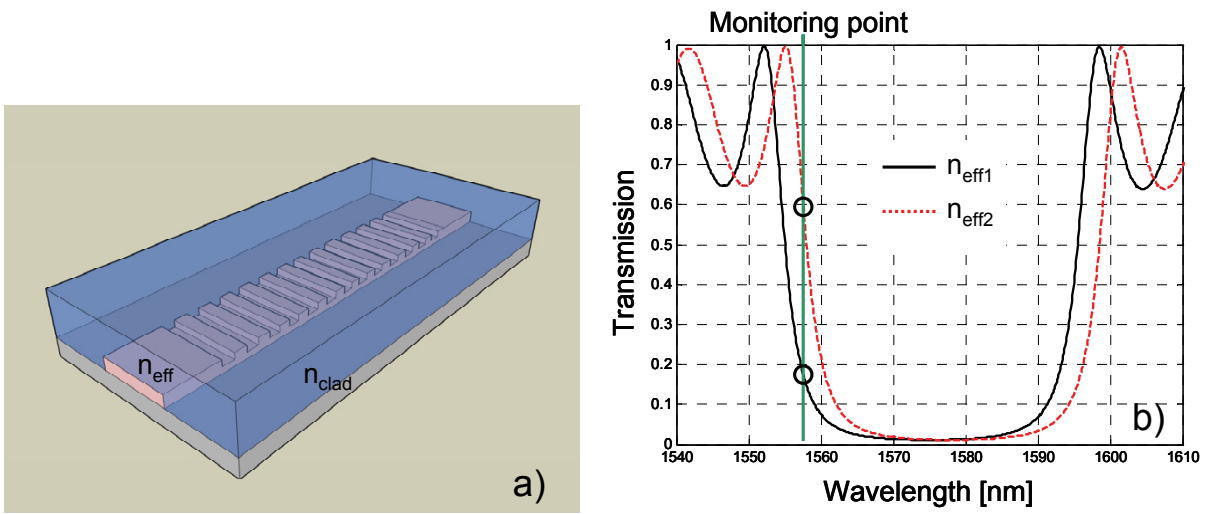

Fig. 7. a) Waveguide grating as a sensor; the index change in the cladding index $n_{\text {clad }}$ causes a change in the effective index of the grating $n_{\text {eff }}$ that in turn shifts the stopband. B) The stopband shift due to index change; $n_{\text {eff } 2}>n_{\text {eff } 1}$. By measuring at a fixed wavelength (monitoring point) at the band edge, a large change in the transmission, corresponding to a small index change, can be observed.

Hence by detecting a wavelength shift, a change in the cladding index can be observed. To fully exploit the sharp spectral features of such a waveguide grating, we monitor the change in transmitted power at a stopband edge, see Fig. 7b. As the cladding index changes, the spectral position of this edge shifts according to (6). A change in transmitted power $\Delta T$ depends on both the slope of the stopband edge and the cladding-index change according to:

$$
\Delta T \approx 2 \eta \Lambda \frac{\partial T}{\partial \lambda} \Delta n_{c l a d}
$$

Therefore, a steep stopband edge and a large energy density fraction in the cladding $(\eta)$ will result in high sensitivity to cladding-index changes. The energy density fraction $(\eta)$ depends on the geometry of the structure and the material properties. The slope of the stopband edge depends on the length and strength (i.e. index contrast between high- and low-index regions) of the grating [20].

The detection limit of the sensor depends on how accurately the small power change $\Delta T$ can be measured, which depends on, among many things, laser power fluctuation and dark current noise of the photodetector.

The accuracy, at which the small power change $\Delta T$ can be measured, sets the detection limit of the sensor. Therefore many things such as laser power fluctuation and dark current noise of the photodetector contribute to detection limit.

\section{Nanodisplacement sensing}

The waveguide grating's response to an effective index change can be exploited to observe nanodisplacements of a micromechanical element (ME) that is in the proximity of the grating [21]. In [21] the ME is attached to the optical chip by bonding. From the fabrication point of view monolithic integration of a micromechanical structure that covers the entire grating is very challenging (see sections 2.4.4.1 and 4.3). However, recalling the spatial positions of the resonating Bloch modes and taking into account that the electromagnetic field strength is enhanced at the resonance wavelength (leading to the situation where also light matter interaction is enhanced) one can exploit these resonances for nanodisplacement sensing. Due to localization of these resonances much smaller ME 
can be used, allowing monolithic integration. Although this comes at the expense of sensitivity, a sub-nanometre deflection sensitivity is achievable as will be discussed in section 2.4. Spatial distributions of some resonance modes are shown in Fig. 8. If a perturbation, say a dielectric microcantilever, is placed such that it is in the evanescent field region of a resonance mode, a shift in the resonance wavelength is observed.
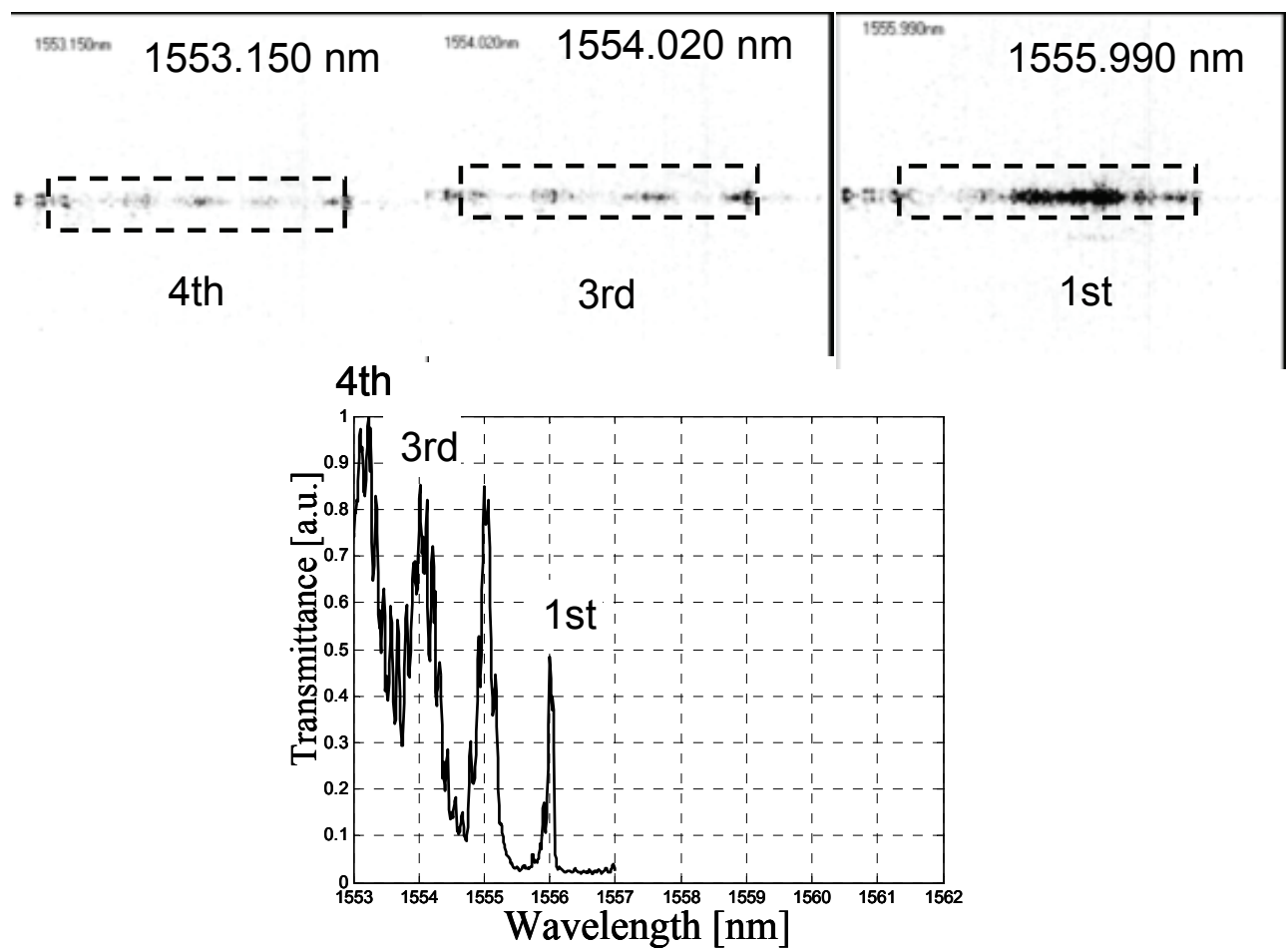

Fig. 8. Transmission spectrum of the grated waveguide, showing the four near band edge resonances. Also shown are the (top views of) spatial distributions of these resonances as captured with the infrared camera. The dashed line shows the position of the grated waveguide, light propagates from left to right.

By placing a cantilever above a waveguide grating, as shown in Fig. 9, the deflection of the cantilever by $\Delta z$ causes a change in the resonance wavelength. By monitoring the transmittance of the grating at the edge of the resonance wavelength, a small deflection can be deducted from a small transmission power change $\Delta T$. Optical deflection sensitivity is defined as:

$$
S_{o d}=\frac{\Delta T}{\Delta z}
$$

The deflection sensitivity, in case of waveguide grating device, depends on many factors, such as the spectral sharpness of the resonance, waveguide geometry and the cantilever parameters. We will next briefly discuss how the deflection $\Delta z$ relates to other measurable quantities like gas concentration. The factors related to $S_{o d}$ are discussed in section 2.4 via a modeling study of a sensor device.

As discussed in chapter 1, the integrated optical read-out methods for nanodisplacements have applications in microcantilever based sensors. These types of sensors, see e.g. Fig. 9 , can be used to detect the effect of adsorption or absorption. The adsorption causes 
changes in the surface stress [22], leading to deflection of the cantilever. In such scheme one side of the cantilever is coated with a material to which some specific molecule binds. The adsorption induces a differential surface stress between the top and the bottom side of the cantilever, and the cantilever will deflect according to Stoney's equation [23];

$$
\Delta z=3 \Delta \sigma L^{2}(1-v) /\left(Y h^{2}\right),
$$

in which $\Delta z$ is the tip displacement of a cantilever having length $L$, thickness $h$, Poisson's ratio $v$ and Young's modulus $Y$, and $\Delta \sigma$ is the differential surface stress.

Apart from adsorption also absorption can cause cantilever to bend. The absorption causes volume expansion, which in turn causes a bimaterial cantilever to deflect according to [24]:

$$
\Delta z=3\left(\alpha_{1}-\alpha_{2}\right)\left(\frac{h_{1}+h_{2}}{h_{2}^{2} K}\right) C L^{2},
$$

where

$$
K=4+6\left(\frac{h_{1}}{h_{2}}\right)+4\left(\frac{h_{1}}{h_{2}}\right)^{2}+\left(\frac{Y_{1}}{Y_{2}}\right)\left(\frac{h_{1}}{h_{2}}\right)^{3}+\left(\frac{Y_{2}}{Y_{1}}\right)\left(\frac{h_{2}}{h_{1}}\right),
$$

$C$ is the ambient parameter change that causes the volume expansion and $\alpha_{\mathrm{i}=1,2}$, are the expansion coefficients with the subscripts referring to the layers of the structure.

In case of for example hydrogen gas absorption the $C$ is the gas concentration, which is, according to Sieverts' law, directly proportional to the square root of the external pressure of the gas molecules [24]. Also temperature difference between the cantilever top and bottom side, or an ambient temperature change (in case the thermal expansion coefficients are different) can cause cantilever to deflect, according to equation (2).

From the equations (9) and (10), it can seen that the mechanical sensitivity of the cantilever to adsorption or absorption induced deflection can be increased by increasing the length of the cantilever, reducing its thickness or by selecting the cantilever materials such that the Young's modulus is small.

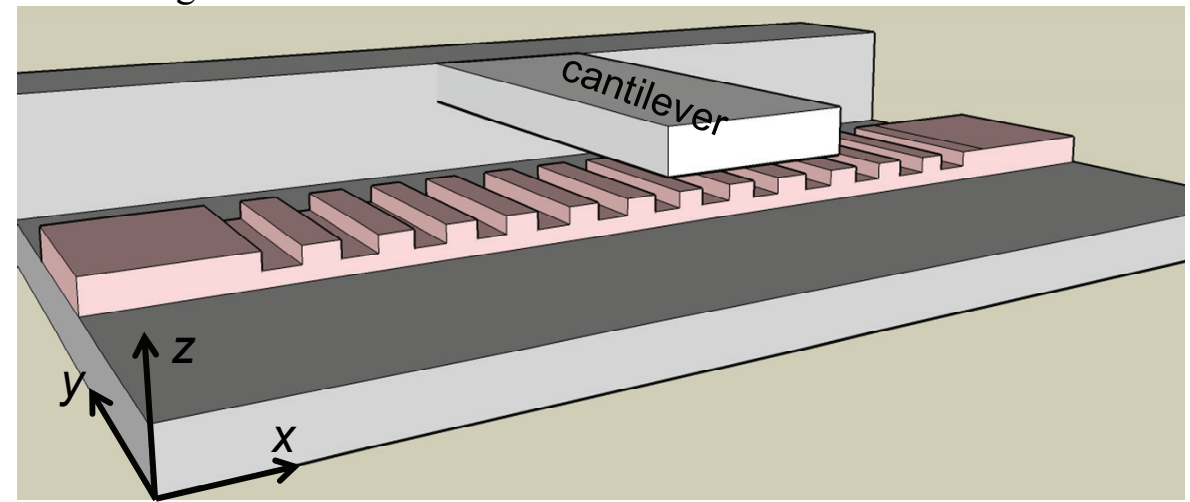

Fig. 9. The grated waveguide-cantilever sensor device. The (up and down) movement of the cantilever changes the transmission of light through the grating.

In the next sections we will discuss in detail the sensor devices that we have implemented based on waveguide gratings. In section 2.4 we present $1 \mathrm{D}$ and 2D modelling results of 
our proposed waveguide grating-cantilever sensor. Also the fabrication issues are shortly discussed based on preliminary test fabrication. Sections 2.2 and 2.3 focus on refractometric sensing by evaluating the sensing performance of the silicon waveguide gratings fabricated in SOI technology.

\subsection{Design and characterization of the grated silicon photonic wire sensor}

In this section the design issues related to a deep UV lithography fabricated grated silicon waveguide are discussed. The sensor's properties are characterized with waterisopropanol and sucrose solutions. The detection limit and the sensitivity of the grated waveguide based sensor to bulk index changes are measured, and the factors that affect on these sensor parameters are discussed. We also explore the properties of the sensor to monitor on-chip chemical reaction by monitoring the evaporation of isopropanol from a watery solution. This evaporation is a dynamic process owning to the fact that the refractive index of the liquid is in a state of continuous change and it therefore mimics the label-free biosensing which will be discussed in section 2.3 .

Various types of silicon-based integrated optical (IO) sensors for label-free sensing have recently been reported, including designs based on the Mach-Zehnder interferometer [25], the ring-resonator [26], waveguide gratings [27-28], porous silicon waveguides [29] and photonic crystals [30-31]. These sensors are based on a change in transmission due to change of refractive index in the proximity of the sensor. To meet the requirements of a small sample volume and to enable dense integration of sensor elements, these sensor designs can be made more compact than in (for example) silicon nitride based devices, because of the high refractive index of silicon. The key advantage of the silicon-based IO sensors can be claimed to be the huge potential to achieve high levels of integration in a cost-effective way by utilizing the CMOS infrastructure. Recent advances in silicon photonics, such as the waveguide photodetectors [32] and the hybrid light sources [33] are examples of building blocks that will allow further miniaturization of these sensors. Apart from pure bulk index sensing, Si photonic wires can be functionalized to versatile biosensor by immobilizing biointerface on the silicon surface [26]. In such a sensor, the sensitivity on the upper cladding index change and compact size are key factors.

It is well-known that a partly grated waveguide defines a cavity in the grated region for wavelengths outside the photonic stopband. For wavelengths near the stopband edge, particularly sharp spectral features can be observed, which are related to the strong modal dispersion and to the increased modal reflectivity in that wavelength region [34], (see section 2.1). These sharp spectral phenomena exhibit a strong sensitivity to changes of the ambient refractive index, which can be exploited in sensor applications.

This section has been published in: Kauppinen, L.J. and Hoekstra, H.J.W.M. and de Ridder, R.M. (2009) A compact refractometric sensor based on grated silicon photonic wires. Sensors and actuators B (Chemical), 139 (1). pp. 194-198. 


\subsubsection{Design and fabrication}

The grated silicon photonic wires (GSPW) have been fabricated using the standard 248$\mathrm{nm}$ deep-UV lithography process for photonic crystals and photonic wires established at IMEC, Leuven [35]. In this process the photonic devices are patterned in a $220 \mathrm{~nm}$ thick top layer of a silicon on insulator (SOI) wafer, using inductively coupled plasma (ICP) etching. The design of the optical structures is constrained mainly by the used waveguide material (silicon) which is transparent at wavelengths $>1100 \mathrm{~nm}$ with a refractive index of 3.45 in the near infrared, as well as by the process limitations requiring a grating period $>400 \mathrm{~nm}$, silicon feature size $>200 \mathrm{~nm}$, and air feature size (gap between two Si features) $>150 \mathrm{~nm}$. The grid size used in a mask design is $5 \mathrm{~nm}$, which determines the minimum feature resolution.

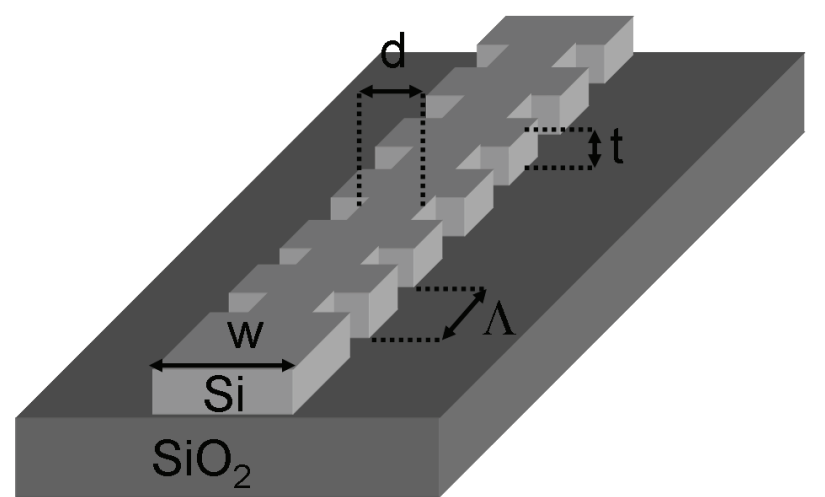

Fig. 10. Lay-out of a grated silicon photonic wire. Waveguide widths $w$ and $d$ are of the order of 400-300 $\mathrm{nm}, \Lambda=450 \mathrm{~nm}$ and the waveguide thickness $t$ is $220 \mathrm{~nm}$.

We have realized the grating structure by periodically varying the waveguide width as shown in Fig. 10. In order to have the first order photonic stopband in the $1.5 \mu \mathrm{m}$ wavelength region of the available equipment, the dimensions for the wide $(w)$ and narrow $(d)$ segments need to be carefully chosen. Although only time-consuming 3dimensional calculations can provide full and accurate information on the transmission properties of the structure [36], we have chosen to use a highly efficient 2-dimensional method. The central wavelength of the stopband is given by the well-known equation:

$$
\lambda_{\mathrm{B}}=2 n_{\mathrm{av}} \Lambda,
$$

where $\lambda_{\mathrm{B}}$ is the Bragg wavelength, $\Lambda$ the period of the grating, and $n_{\mathrm{av}}$ the average modal index of the grating, found by averaging modal indices of the narrow $\left(n_{\mathrm{d}}\right)$ and wide $\left(n_{\mathrm{w}}\right)$ waveguide section over one grating period. If the length ratio of the narrow to the wide waveguide sections is $\xi$ (duty cycle), the average index can be calculated as $n_{\mathrm{av}}=$ $\xi n_{\mathrm{d}}+(1-\xi) n_{\mathrm{w}}$. A 2D modesolver using multi grid finite difference method [37] was used to calculate the allowable range for $w$ and $d$, such that the waveguiding sections are single mode for a TE mode at wavelength of $1520 \mathrm{~nm}$. The same mode solver also produced the corresponding effective refractive indices $n_{w}$ and $n_{d}$. 


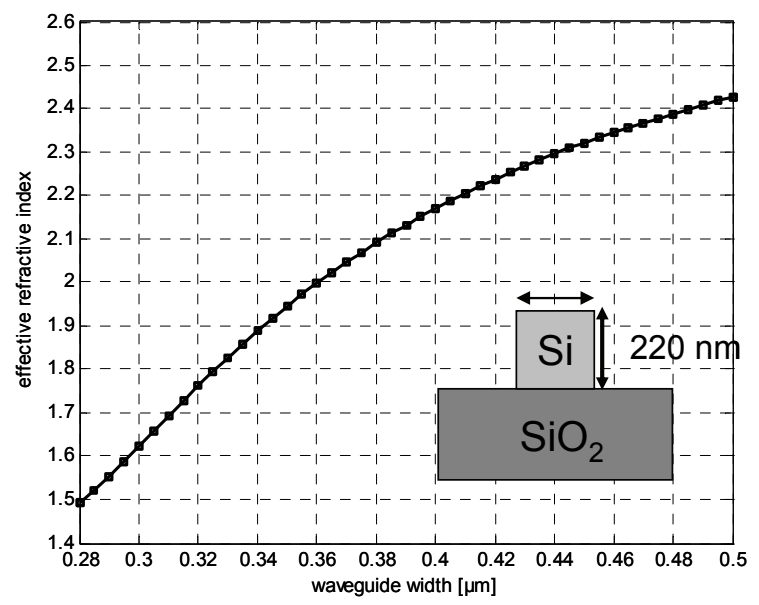

Fig. 11. The effective index versus waveguide width at $1520 \mathrm{~nm}$ wavelength. The height of the waveguide is $220 \mathrm{~nm}$.

The effective refractive index as a function of waveguide width (at wavelength of 1520 $\mathrm{nm})$ is shown in Fig. 11. The graph is used to choose the waveguide widths $d$ and $w$ leading to the desired $\lambda_{B}$. The period $\Lambda$ and the duty cycle are restricted by the process limitations. Because of the high index material (Si) and a minimum period of $400 \mathrm{~nm}$, relatively narrow waveguides are needed.

Since the effective index of the waveguide also depends on the wavelength, see Fig 12, the $\lambda_{B}$ values (in general) cannot be accurately calculated from (12) by just using the indices of Fig.11. However, we can use it to choose $w$ and $d$ for $\lambda_{B}=1520 \mathrm{~nm}$. By keeping in mind the minimum resolution $(5 \mathrm{~nm})$, we choose $w=320 \mathrm{~nm}$ and $d=300$ $\mathrm{nm}$. To obtain design variations, we have chosen the structural dimensions $d$ and $w$ to be between 300 and $400 \mathrm{~nm}$, while the grating period $\Lambda$ is fixed to $450 \mathrm{~nm}$, and the duty cycle $\xi$ to 0.45 . The difference $w-d$ is varied from $20 \mathrm{~nm}$ to $90 \mathrm{~nm}$.

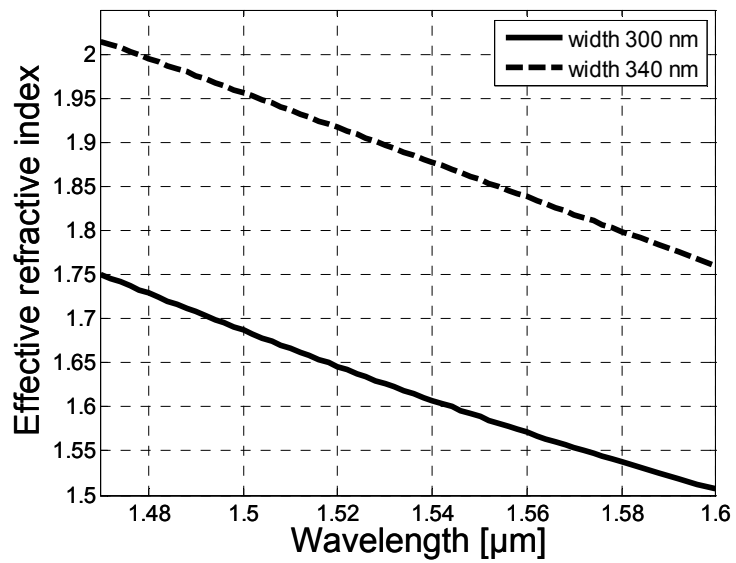

Fig. 12. Effective index as a function of wavelength for two waveguide widths.

In the fabrication process an exposure dose sweep is performed across the wafer. The sweep will give additional variety of the fabricated gratings, as the size of small features on a wafer depends on the exposure dose, shown in Fig. 13. 

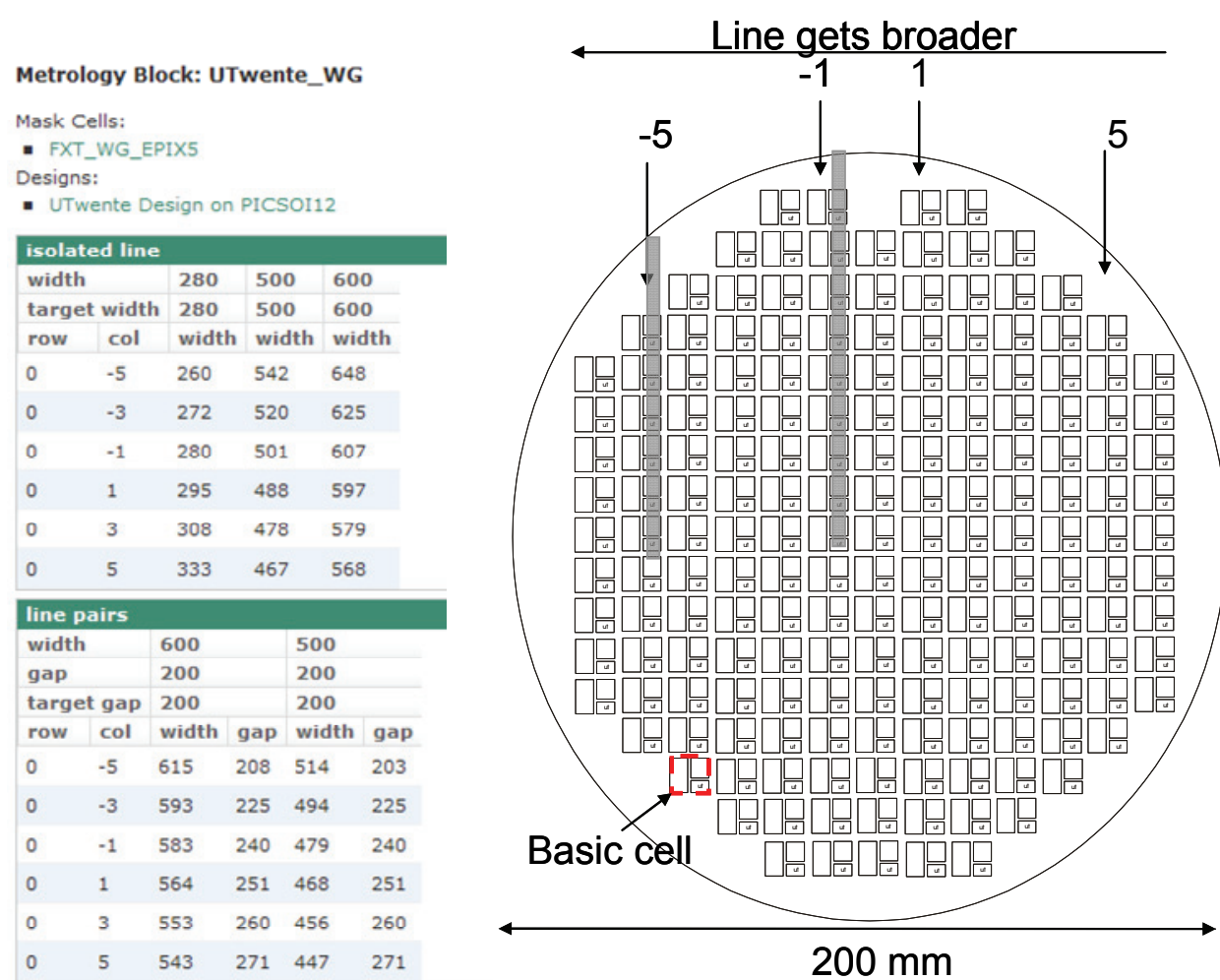

Fig. 13. The effect of exposure dose sweep on feature size. On the left the tables show the feature sizes as measured in IMEC [35]. On the right is a schematic picture of the wafer. A basic cell, of size $12 \mathrm{~mm} \cdot 7$ $\mathrm{mm}$, containing the photonic structures, is repeated across the wafer using stepper lithography. Due to exposure dose sweep, different cell columns get different feature sizes. The columns named as $-5,-1,1$ and 5 are pointed with arrows. The gray shaded areas are the columns that were chosen for first

characterization. The gratings in column -1 have stopbands in the desired wavelength range (1500-1600 $\mathrm{nm}$ ) when the top cladding index is 1.32 (e.g. water) while the gratings in column -5 have the stopbands in same the range for top cladding index of 1 (air).

\subsubsection{Sensitivity and detection limit}

To evaluate the sensitivity and bulk index detection limit of the GSPW, we have implemented a flow cuvette system that allows an easy way to apply different solutions for sensing. It also eliminates the evaporation of liquid hence the index of the solution remains constant during the measurement. We have aimed to get as good signal-to-noiseratio (SNR) as possible, while keeping the signal integration time short, to not limit the measurement scheme for slow reactions only. For this purpose we have used signal modulation. The purpose of the experiment is to determine the achievable detection limit of GSPW sensor in a laboratory conditions and indentify the factors that set the detection limit.

\subsubsection{Materials and Methods}

The detection limit of the GSPW sensor on bulk refractive index changes is evaluated by using water at various sugar concentrations. The refractive index of the water-sugar solution depends linearly on the sugar concentration with a ratio of $1.4 \cdot 10^{-3} \mathrm{RIU} / \mathrm{wt} . \%$ (percent by weight) [38]. For the experiment, a solution with a sugar concentration of 1 $\mathrm{mg} / \mathrm{ml}$ was produced by adding $200 \pm 5 \mathrm{mg}$ of sugar to $200 \pm 2 \mathrm{ml}$ of water. In this way, a 
concentration accuracy of $\pm 0.05 \mathrm{mg} / \mathrm{ml}$ was obtained. Solutions of lower concentrations were produced by diluting from a $1 \mathrm{mg} / \mathrm{ml}$ solution to the desired sugar concentration, resulting in good accuracy, e.g. $0.2 \pm 0.015 \mathrm{mg} / \mathrm{ml}\left(\Delta n=2.8 \pm 0.2 \cdot 10^{-5} \mathrm{RIU}\right)$. All the solutions were produced from same water bottle in order to avoid a case where water obtained on a different day might have a slightly different refractive index.

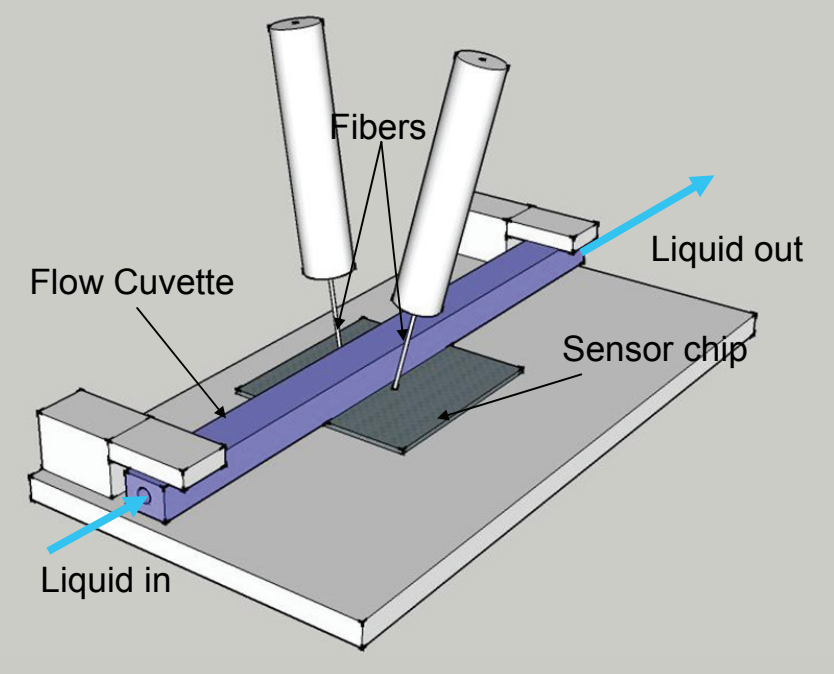

Fig. 14. Schematic picture of the measurement setup. Small opening in a cuvette allows liquid to touch the surface of the sensor chip.

A schematic of our measurement setup is shown in Fig. 14. We used grating couplers at the ends of the waveguides to couple light in and out from the sensor. With this coupling method the losses are 15-25 dB (depending on the wavelength), which is good enough for characterization. However, for a packaged sensor array chip different coupling scheme should be used to ensure that each sensor element receives enough power for high detection limit.

As a light source, we used a tunable infrared laser (Agilent 8164B) and as a photodiode Agilent's 81634B photodiode module. The flow cuvette was clamped on top of the sensor chip. The cuvette has a small opening of $1 \mathrm{~mm} \cdot 3 \mathrm{~mm}$ that allows liquid to contact the surface of the sensor chip. Attention was paid to cuvette design to ensure that the liquid refreshment at the cuvette opening is sufficient. The entire liquid volume of the cuvette system, including the input and output pipes, is approximately $2 \mathrm{ml}$. However, to ensure refreshment of the liquid during the experiments, several millilitres of solution is slowly passed through the cuvette.

If high detection limit is required, the effect arising from the high thermo-optic coefficient of silicon $\left(16-18.5 \cdot 10^{-5} /{ }^{\circ} \mathrm{C}\right)$ should be taken into account. We used active temperature control with a thermo-electric cooling unit that was mounted under the sensor chip. Considering the high thermal conductivity of silicon and the presence of a metallic heat sink under the chip, the temperature of the sensor chip was expected to remain constant. Liquids passing the sensor might cause temperature shifts if the liquid has a temperature that is different to that of the sensor. To reduce this effect, we kept the liquids in a temperature controlled laboratory for 1 day immediately before the experiment. During the experiment, the whole set-up was enclosed in a box which 
reduced the vibration of the coupling fibers due to air flow. This box also reduced possible background light that might reach the output fiber, although in our experience such an effect was shown to be small.

The refractive index sensing properties of the sensor were evaluated by flowing sucrose solutions at various concentrations through the sensor. We first determined an operating point at which the sensitivity of the sensor is high for the index changes. This operating point is near the stop-band edge of the grating. Once the operating point is set, the wavelength of the laser source is kept fixed and the changes in transmission power can be measured according to equation (7).

To improve the SNR and better detect small transmission power changes, the laser signal was modulated with a sine wave at a frequency of $3 \mathrm{kHz}$. The sampling frequency was chosen to be $10 \mathrm{kHz}$ and a total of 32760 samples were recorded, which is the maximum number of samples that our equipment can store. Since the laser is modulated at a specific frequency, we can focus on just this frequency and consider all the other frequency components as noise. The amplitude of the $3 \mathrm{kHz}$ frequency component is proportional to the transmission power going through the sensor.

The slope of the stopband edge is one of the factors that determine the detection limit of the GSPW to refractive index changes, as discussed in section 2.1.4. As the difference $w$ $d$ increases, the index difference $n_{w}-n_{d}$ increases as well. This leads, next to a wider stopband, to a higher modal reflectance at the onsets of the grating, but also to higher losses on reflection at these onsets. The higher reflectance leads to sharper spectral features on the one hand, which are obscured on the other hand by the increased losses [36]. To choose the optimum GSPW for the envisioned sensor experiment an experimental study of the stopband spectral features has been performed.

Fig. 15a shows measured transmission spectra of three different gratings that are all 200 periods long while the designed $w-d$ is varied by increasing the $w$ in $20 \mathrm{~nm}$ steps. The measured spectrum is normalized with respect to a straight photonic wire waveguide including the coupling loss. The uncertainty in coupling loss, due to small changes in coupling conditions and measurement noise, is assumed to be $\pm 2 \mathrm{~dB}$. The effect of the increased grating strength can be seen from the spectra; the stopband edges get steeper and the stopband gets deeper for larger values of $w$. As the average index of the grating is also increased, the stopband shifts to a longer wavelength.

Fig. 15b shows the effect of the grating length; as the length is increased the edges get steeper and the stopband gets deeper. According to our measurements, the stopband edge slope of a grated silicon photonic wire with $w-d=120 \mathrm{~nm}$ and a length of 200 periods equal to the slope of the 800 periods long grating with $w-d=20 \mathrm{~nm}$. The period of the strong grating has to be reduced to $400 \mathrm{~nm}$ to avoid a too narrow waveguide width and to have the stopband at the same wavelength range as the 800 periods long grating. The benefit of strong grating in reducing the size of the sensors, and still maintaining high sensitivity, is obvious.

The grating parameters for sensing should be chosen depending on the application. If small index changes are measured, then the slope of the stopband edge should be as steep as possible. For large index changes less steep stopband edge is beneficial as it provides larger measurement rage (at the cost of sensitivity). 

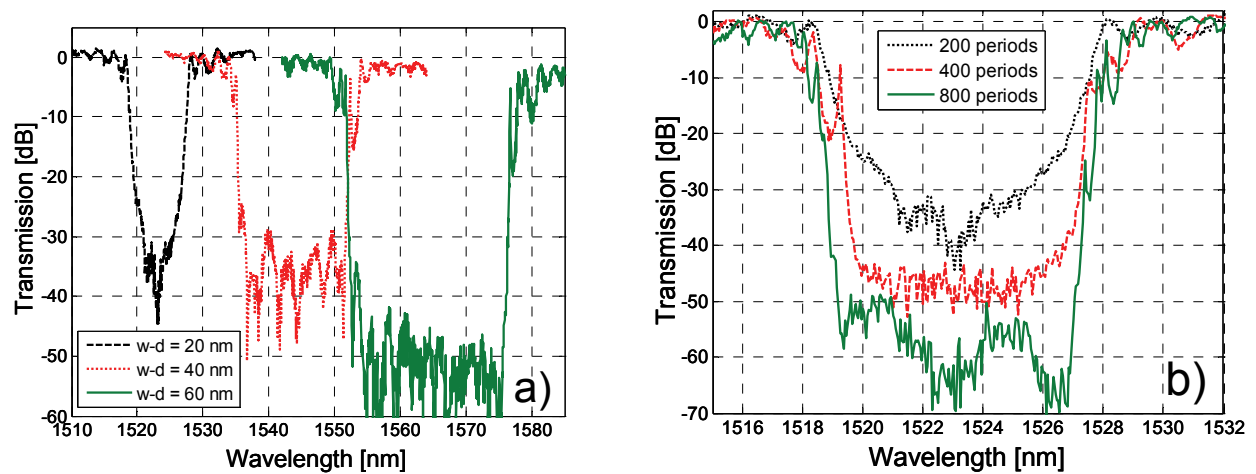

Fig. 15. a) the effect of grating strength on transmission spectrum. The dimension $w$ is changed in steps of $20 \mathrm{~nm}$, while $d$ is fixed. b) The effect of the grating length on transmission spectrum. The grating length varied from 200 periods to 800 periods, for a grating with $\Lambda=450 \mathrm{~nm}$ and $w-d=20 \mathrm{~nm}$.

Due to the fundamental limitations of the photolithographic process, sharp corners will not be faithfully reproduced, resulting in smoothened corners, in particular in narrow structures. This smoothening effect is clearly visible in Fig. 16, showing that the grating shape, which had been designed to consist of rectangular segments, has deteriorated to a sinusoidal-like one. The corner smoothening effect reduces the index contrast between high- and low-index segments. This effect likely reduces the obtainable detection limit as a higher effective index contrast of a segment would provide a steeper band edge slope, although the effect of reduced losses due to lower contrast should be considered [36].

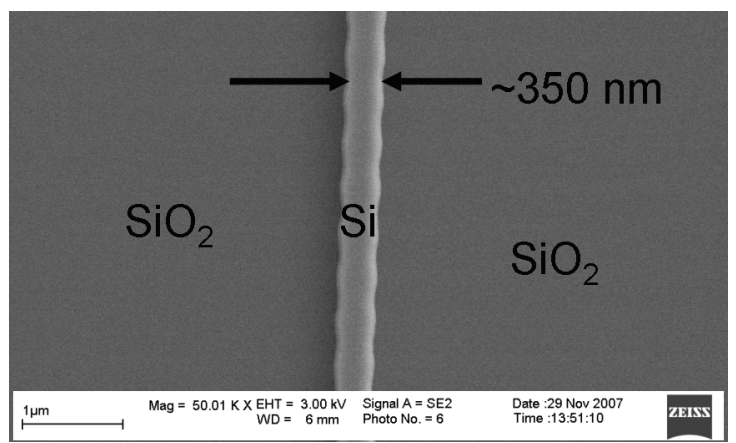

Fig. 16. SEM picture of $350 \mathrm{~nm}$ wide grated photonic wire. Lithography smoothens the rectangular shape and a sinusoidal-like grating is obtained.

\subsubsection{The sensitivity}

To get the estimates of the sensitivity and the index resolution, the stopband position shift and the band edge slope were measured. The stopband shift of a 400 period grating was determined by dropping a water droplet on the sample area $(10 \mathrm{~mm} \times 5 \mathrm{~mm})$. The water droplet $\left(n_{\text {clad }}=1.32\right)$ provides an index shift of 0.32 in the upper cladding. The measured stopband positions before and after depositing the water droplet are shown in Fig. 17. 


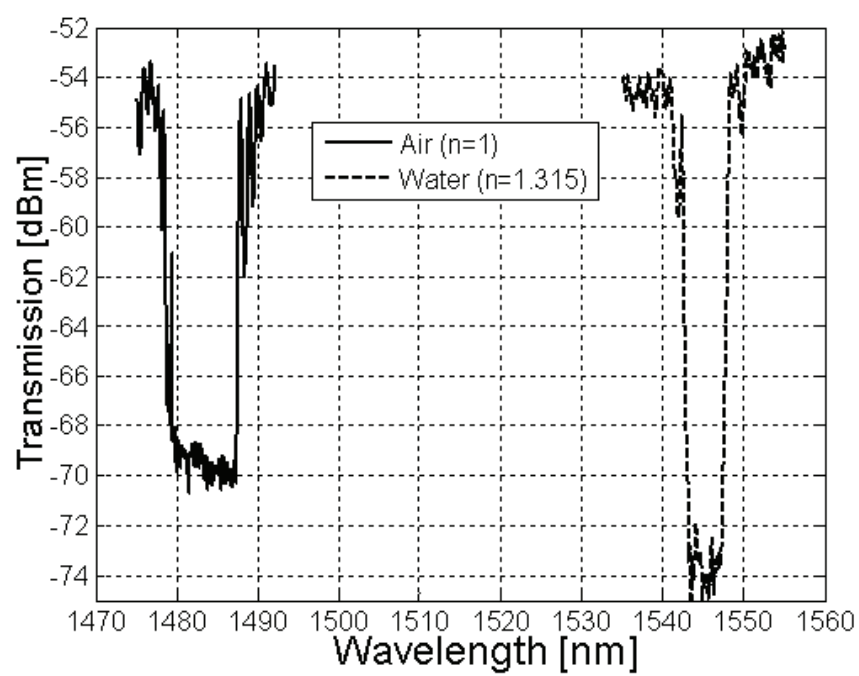

Fig. 17. Stopband positions with air (left) and water (right) claddings.

It can be seen that the shift is roughly $60 \mathrm{~nm}$, which gives a sensitivity of $188 \mathrm{~nm} / \mathrm{RIU}$ according to (6). The slope of the stopband edge at a measured power level (1 nW) is 20 $\mathrm{nW} / \mathrm{nm}$. Assuming that the noise level enables a measurement resolution $\Delta T$ of $10^{-2}$, a resolution of $\Delta \mathrm{n}_{\text {clad }}=10^{-6}$ can be obtained. However, better estimate of the index resolution and limiting factors are obtained by directly measuring it, as will be discussed in the next section.

\subsubsection{The detection limit}

For the determination of the bulk index detection limit, experiments were performed using sucrose solutions, at varying concentrations from $1 \mathrm{mg} / \mathrm{ml}$ to $0 \mathrm{mg} / \mathrm{ml}$ in steps of $0.2 \mathrm{mg} / \mathrm{ml}$. We recorded the transmission power as a function of time as we pumped sucrose solutions through the sensor. From the results, as shown in Fig 18, it is clear that a concentration of $0.2 \mathrm{mg} / \mathrm{ml}$ can be detected. Also, concentration changes in steps of 0.2 $\mathrm{mg} / \mathrm{ml}$ are clearly resolvable. 

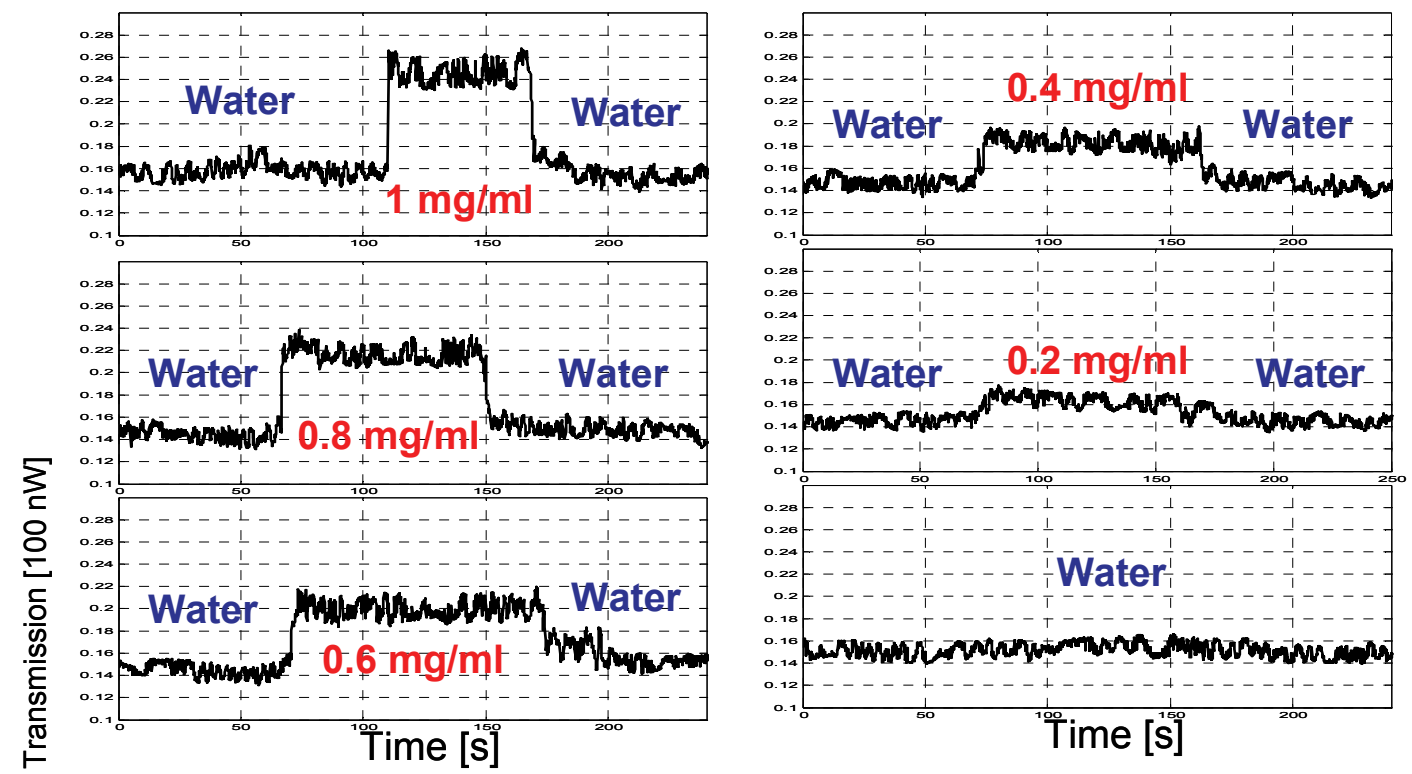

Fig. 18. Sensors response on various sugar concentrations.

The short-term stability of the signal is low. From the results, it can be seen that noise accounts for approximately $10 \%$ of the signal. However, the medium term stability is good, i.e. signal level does not change much over a time periods ranging from one minute to ten minutes. Therefore an integration time of few minutes could be set to improve the $\mathrm{SNR}$, if slow reactions are measured.

As mentioned above to achieve an improved SNR, we repeated the experiment by modulating the laser at a frequency of $3 \mathrm{kHz}$. With this method, the short-term stability of the signal does not play a role as only a single frequency component is considered. However, due to the limited number of samples that can be stored with our equipment, the signal integration time remained short, at $\sim 3.3$ seconds. From the recorded $3 \mathrm{kHz}$ frequency components, shown in Fig.19a, we can clearly observe the concentration changes at a resolution of $0.2 \mathrm{mg} / \mathrm{ml}$. However, the amplitudes of the frequency components corresponding to water appear to vary slightly due to signal variations in time period that is longer than our integration time (i.e. low frequency noise is still present).

The experiment was repeated again about 30 minutes later. This time, the order at which the solutions were applied was changed. When we compare the results, see Fig 19b, there appears to be a systematic difference between the results. We believe that this difference is due to temperature change. Based on the thermo-optic coefficient of silicon, this systematic difference corresponds to a temperature change of $\sim 0.1{ }^{\circ} \mathrm{C}$.

By using the modulation the SNR is better than $20 \mathrm{~dB}$ and the sensors' index resolution is essentially limited only by thermal stability. Sugar concentrations of $0.2 \mathrm{mg} / \mathrm{ml}$ can be detected. This concentration corresponds to refractive index change of $2.8 \cdot 10^{-5}$. To the author's best knowledge, detection limit beyond $10^{-4}$ in bulk refractive index change has not yet been reported with a grated waveguide type sensor.

The results clearly indicate the need for a temperature control element and the necessity of a reference GSPW sensor, if a detection limit better than $10^{-5}$ on refractive index change is required. A reference sensor can be used to compensate the effect of 
temperature fluctuation on refractive index and geometry of the sensor structure, as well as the refractive index of the main solvent for the species to be measured.
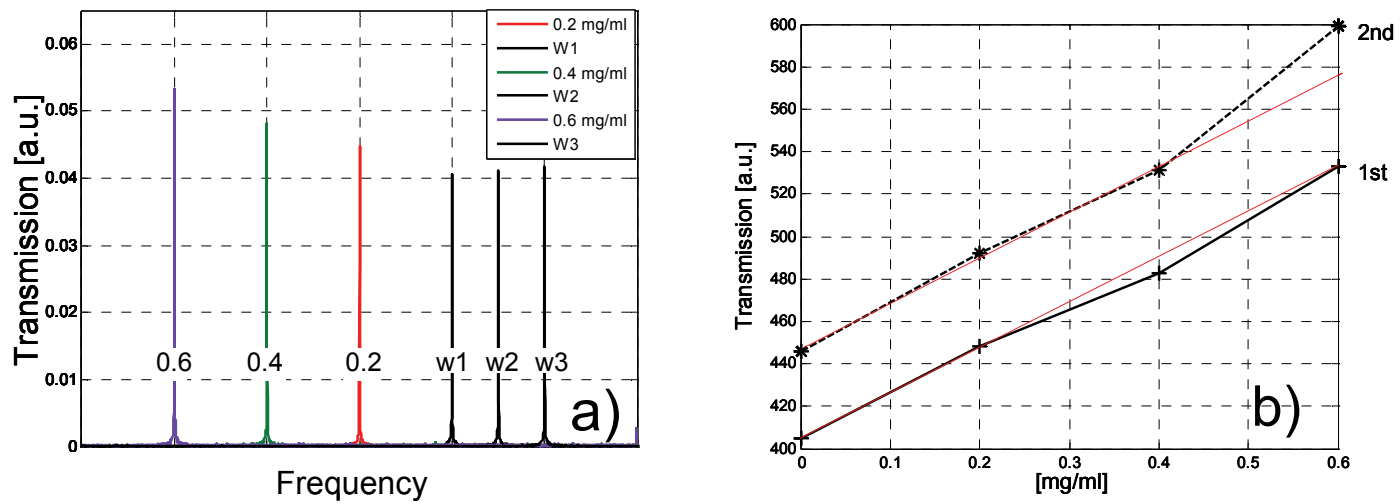

Fig. 19. a) Measured $3 \mathrm{kHz}$ frequency components (see text). Components have been shifted in frequency axis for better visualization. Order of solutions: $0.2 \mathrm{mg} / \mathrm{ml} \rightarrow>$ water $1->0.4 \mathrm{mg} / \mathrm{ml}->$ water2 -> $0.6 \mathrm{mg} / \mathrm{ml}$ $>$ water3. b) Sensors response on different sugar concentrations. The $2^{\text {nd }}$ curve was measured approximately 30 minutes after the first one. Order of solutions for the $2^{\text {nd }}$ curve: $0.4 \mathrm{mg} / \mathrm{ml}->$ water $->0.2$ $\mathrm{mg} / \mathrm{ml}->$ water $->0.6 \mathrm{mg} / \mathrm{ml}->$ water. The linear curves are fitted lines with slope $0.1 \mathrm{mg} / \mathrm{ml}$ per 20 transmission units.

\subsubsection{Measuring the dynamic index change}

As discussed earlier (see e.g. section 1.2.1), the main application area for the GSPW sensor is to follow the progress of on-chip chemical or physical processes, provided that these processes cause a change of the refractive index. As a prelude towards label-free biosensing we performed an experiment in which we followed evaporation of isopropanol from a watery solution.

\subsubsection{The measurement set-up}

A schematic picture of the measurement set-up is shown in Fig. 20. A tunable laser is used as a light source from which the light is coupled with the help of microscope objectives to the waveguide. The objectives and the sample are on 3D translation stages that can be aligned for optimum light coupling. Depending on which objectives are used, different input spot sizes are obtained. The selection of the objectives has been done by experimentally trying different objectives to obtain optimum coupling to the silicon photonic wires. For optimal coupling the spot size (and shape) should match the mode profile of the waveguide.

The typical coupling loss for our end fire setup is about $25 \mathrm{~dB}$. However, when the sample is diced for the end-fire coupling the waveguide end facets may get damaged and the coupling loss can accordingly be much higher. 


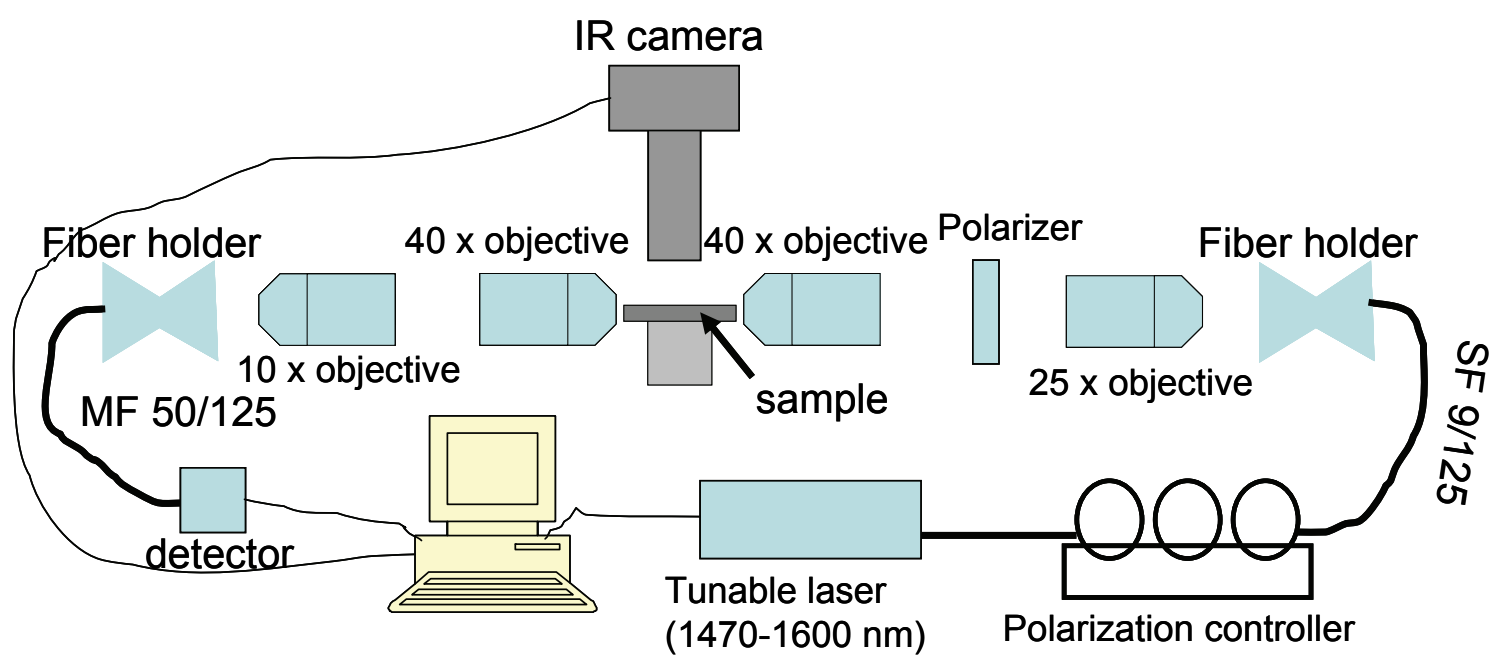

Fig. 20. A schematic picture of the end-fire set-up used to characterize the devices. Light from the laser is guided with a single mode fiber (SF) to the fiber holder from which it propagates in free space and is focused to the end faced of the waveguide by using microscope objectives. At the output side multi mode fiber (MF) is used to collect the light and guide it to the photodetector. The infrared (IR) camera helps in coupling procedure by visualizing the infrared light.

\subsubsection{Experimental}

The suitability to use the GSPW to measure small refractive index changes at a fixed wavelength, and thereby exploiting the spectral sharpness of the stopband edge, was evaluated by using a water-isopropanol solution. Isopropanol evaporates faster than water in ambient temperature and pressure conditions (air at $20^{\circ} \mathrm{C}$ and 1 atm pressure). Therefore the refractive index of a water-isopropanol droplet will decrease with time at some rate. Consequently, when a droplet of water-isopropanol solution is applied to a grating, a gradual stopband shift to lower wavelengths can be observed.

To demonstrate this effect, the droplet of approximately 9 wt. \% isopropanol solution was applied to the grating, and transmitted power at the stopband edge was recorded as a function of time, as shown in Fig. 21. The wavelength was fixed at $1550 \mathrm{~nm}$. The data gap between 400 and 500 seconds was caused by a limitation of the measurement set-up. Fig. 22 shows two transmission spectra, one taken immediately after applying the solution and the other after 14 minutes had passed. The time required for a spectral scan is about 2 minutes. 


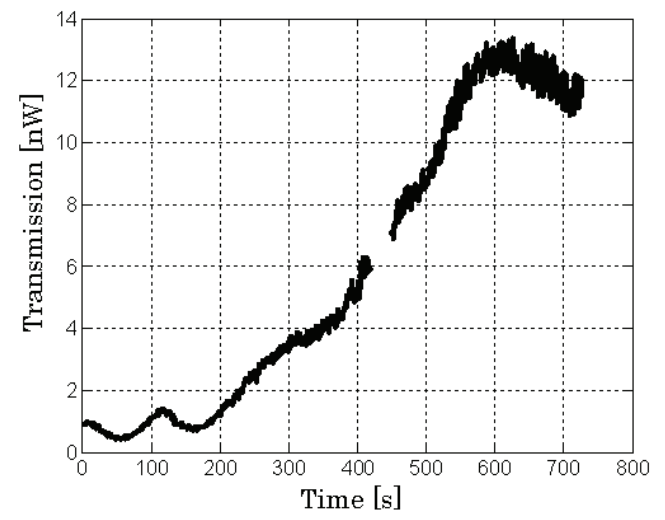

Fig. 21. Transmitted power measured at the stopband edge $(1550 \mathrm{~nm})$ as a function of time during the evaporation of isopropanol from solution.

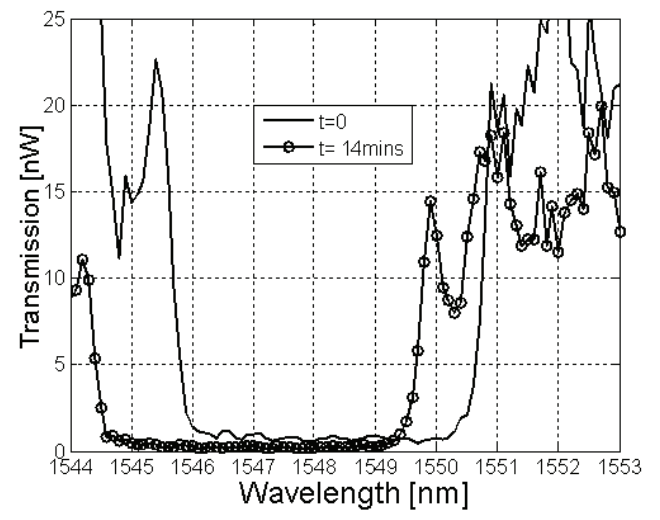

Fig. 22. Transmission spectra, measured at the beginning and at the end of the experiment.

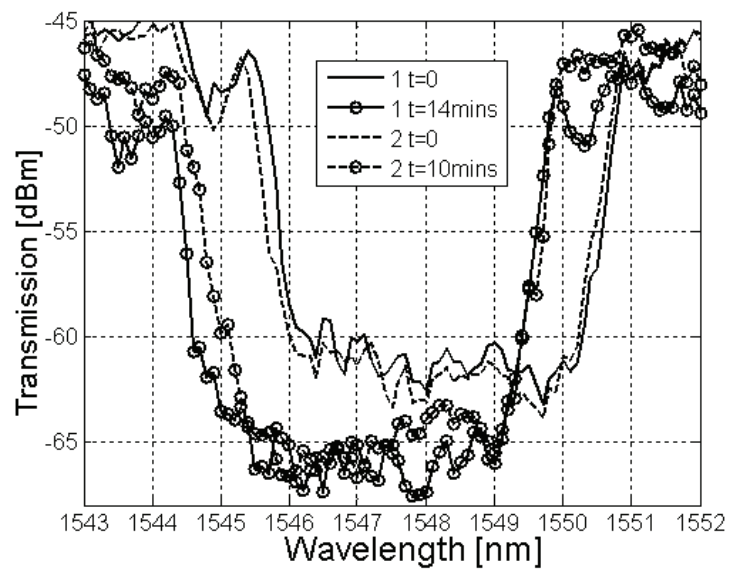

Fig. 23. Repeated measurement of the stopband position shift due to the evaporation of isopropanol. A fresh droplet of solution was used in the second experiment. Solid lines correspond to the first experiment.

The refractive index of the droplet was measured with an Abbe refractometer before and after the experiment. The index of the solution had changed by $5 \cdot 10^{-3}$ over the 16 minutes duration of the entire experiment. The measured index change agrees with the index change that can be calculated from the stopband shift: the stopband shift shown in Fig. 22 is roughly $1 \mathrm{~nm}$, while from Fig. 9 it can be deduced that a shift of $1 \mathrm{~nm}$ corresponds to a refractive index change of $5.3 \cdot 10^{-3}$ in the upper cladding material.

The experiment was repeated by dropping a new droplet of the same solution. The initial and the final stopband positions of the first and the second experiment are nearly equal, as shown in Fig. 23.

The power levels of the transmission spectra measured at the beginning and at the end of the isopropanol evaporation experiment are different; see Figs. 22 and 23. This is due to alignment drift of the measurement set-up. A stabilization period before the experiment can be used to reduce the drift. However, in our end-fire set-up, the coupling conditions change when the droplet is applied to the sample, and due to evaporation of the isopropanol such a stabilization period cannot be applied in this experiment. 


\subsubsection{Conclusions}

It was experimentally shown that using the near stopband edge phenomena of the GSPW, a resolution of the order of $10^{-6}$ to bulk index change is feasible assuming $1 \%$ noise level. It was also demonstrated that the device is suitable to measure the index change of a liquid cladding in real time, which can be used to monitor on chip chemical reactions. Signal modulation -- in combination with a typical photodiode -- is sufficient to achieve a signal-to-noise ratio at which the detection limit of a GSPW sensor is limited only by thermal stability. A detection limit of $2.8 \cdot 10^{-5}$ on bulk index changes has been measured. Lower detection limits call for thermal stability of better than $0.1{ }^{\circ} \mathrm{C}$ or a reference GSPW sensor to compensate for the thermal effects.

Finally we note that low-loss light coupling into silicon waveguides needs careful attention. Especially when considering a packaged chip that houses arrays of sensor waveguides, the optical power reaching the detector should remain well above the noise level of the detector. Robust and efficient fiber-chip coupling solutions exist, for example involving an intermediate waveguide [39].

\subsection{Label-free enzyme sensing with grated silicon photonic wire}

An interesting application field for IO sensors is label-free bio-sensing. In this sensing scheme, the target bio-molecules are attracted to the surface of the IO sensor. Due to exponential nature of the evanescent field, the sensitivity is high near the surface and much smaller concentrations, than with the pure bulk index sensing, can be detected. The surface sensing scheme using immobilized receptors also enables selectivity; the sensor is practically only sensitive to the specific molecules that attach to the surface.

As a demonstration of label-free bio-sensing, we have monitored the binding process of enzymes on an immobilized antibody layer that we applied to a surface of a GSPW sensor (see section 2.2). Also we have used the sensor to monitor the antibody immobilization process.

\subsubsection{The immobilization process}

To obtain an antibody layer for the bio-sensing experiment, an immobilization process is required. We followed the standard immobilization process developed by Imenz b.v. [40]. This process consists of five main steps as shown in Fig 24. The first two steps were performed at chip-scale in a cleanroom. The remaining three steps were done at sensor scale by using the flow cuvette (see Fig. 24). This allowed us to use the sensor to monitor the immobilization process.

This section has been published in: Kauppinen, L.J. and Dijkstra, M. and Hoekstra, H.J.W.M. and de Ridder, R.M. (2009) Label-free Enzyme Sensing with Grated Silicon Photonic Wire. In: Proceedings of the 2009 Annual Symposium of the IEEE Photonics Benelux Chapter, 05-06 Nov 2009, Brussels, Belgium. pp. 41-44. VUBPress Brussels University Press. 


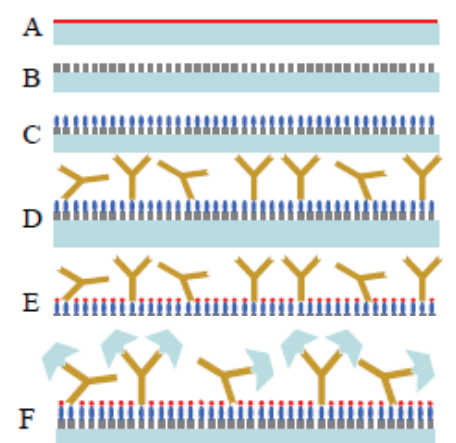

Fig. 24. Immobilization process (A-E) and enzyme binding (F). (A) Surface cleaning and activation, (B) Silanization, (C) Glutaraldehyde, (D) Antibody immobilization and (E) Blocking of reactive carbonyl groups.

The immobilization procedure begins by cleaning the chip with acetic acid and $\mathrm{H}_{2} \mathrm{O}_{2}$ (ratio 4:1). Silanization is performed using a silane solution that will result in a monolayer of silane on the waveguide surface. After silanization, the sensor chip is washed eight times with ethanol and once with phosphate buffered saline (PBS). Silane forms a surface to which glutaraldehyde molecules attach. Glutaraldehyde is used as a surface for anti-bodies selective to an enzyme known as PepN. Blocking solution is required to block non-specific binding on carbonyl groups in glutaraldehyde interface.

Initially, we measured the stop-band position of the GSPW after the silization. In this case, the GSPW sensor is covered by a monolayer of silane, and then air. Next, a PBS solution was flowed into the cuvette. This solution replaces air as top cladding, and the stopband shift of approximately $45 \mathrm{~nm}$ was observed, as shown in Fig. 25. We measured the refractive index of the PBS solution using an Abbe refractometer $(n=1.3348)$. The stopband shift was roughly $10 \mathrm{~nm}$ smaller than we expected from a bulk index sensing experiment (reported in the previous section) for this type of grating. This indicates that the sensor had become less sensitive to bulk index changes after silanization. This, in turn, could mean that silanization had formed a thicker silane layer than just the desired monolayer.

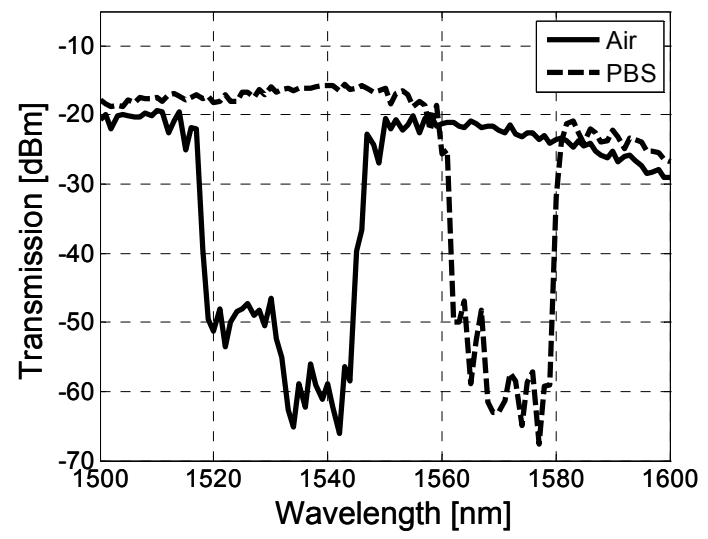

Fig. 25. Stopband positions after silanization in air (solid line) and in PBS solution (dashed line)

After the PBS solution, a glutaraldehyde solution is flowed into the cuvette to form a surface for the anti-bodies to attach to. As the glutaraldehyde solution has a higher 
refractive index $(n=1.3480)$ than the PBS, we expected the shift in the stop-band position to continue. Surprisingly, the shift was in the "wrong" direction: into a lower wavelength region. We have measured the same unexpected stopband shift several times. The reaction time for the glutaraldehyde process step is 1 hour. During this time, we noticed a stopband shift, of few nanometres, back to higher wavelengths, see Fig. 26a. Fig. 26b shows the measured reaction curve of glutaraldehyde binding, as recorded in the monitoring point, shown in Fig 26a, after 240 seconds from injecting the solution into the cuvette. The reaction has saturated after 60 minutes, which corresponds to specified reaction time. PBST $(n=1.3345)$ was flowed in as an intermediate step; however the stopband position remained at lower wavelengths than before the glutaraldehyde step.

Monitoring point
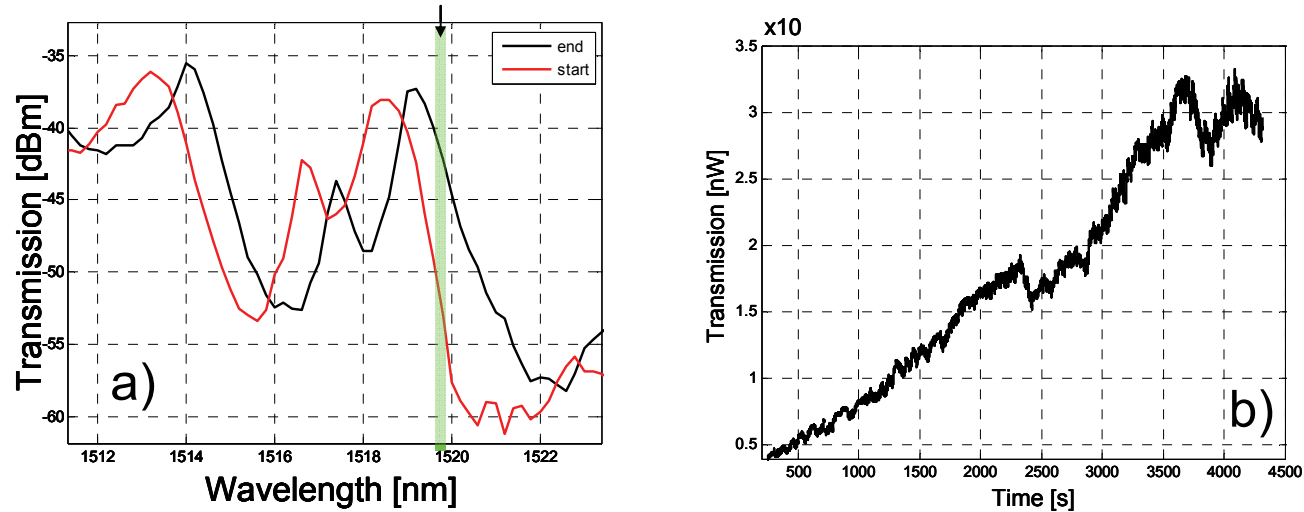

Fig. 26. a) Glutaraldehyde reaction. a) Transmission spectrum at the band edge showing the stopband edge position initially (start) and after $1 \mathrm{~h}$ (end). b) The reaction curve as measured after 4 minutes from injecting the Glutaraldehyde solution into cuvette. The reaction has saturated after 60 minutes.

The PepN antibodies were immobilized on the glutaraldehyde surface by flowing an antibody solution into the cuvette. The reaction time for this step is specified as one hour at $37{ }^{\circ} \mathrm{C}$; however, to avoid complications due to the thermo-optic effect, we performed the step at room temperature $\left(19^{0} \mathrm{C}\right)$. To allow more time for antibodies to bind, we kept the solution in cuvette for 1.5 hours. We measured deformed stopband shapes while the antibody solution was in a cuvette; see Fig 27 . One possible reason for this is that clustering of the antibody molecules near the surface may destroy the uniformity of the grating.

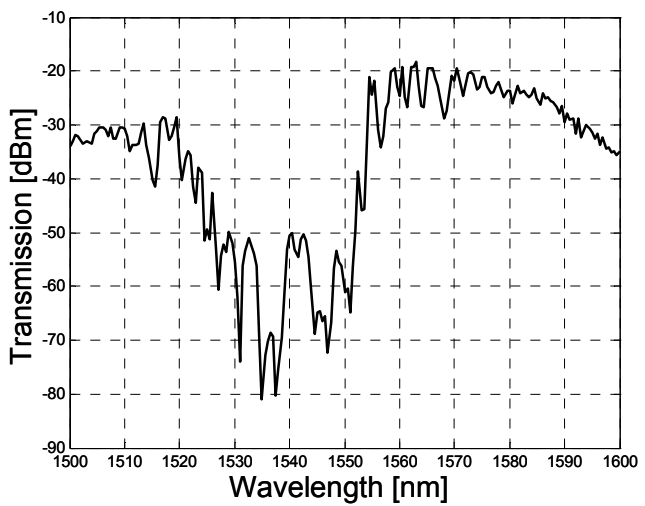

Fig. 27. Deformed stopband stop-band shape due to antibody solution. 
Adding the blocking solution restored the shape of the spectrum, see Fig. 28a. Also, a slight shift to the higher wavelengths was observed, indicating an index increase due to both immobilized antibodies and the attached blocking molecules. The reaction of blocking solution is rapid and strong, as shown in Fig.s 28b. The stopband edge passes the monitoring point in few minutes. After 30 minutes the reaction appears to be saturated as no further stopband shift is measured.
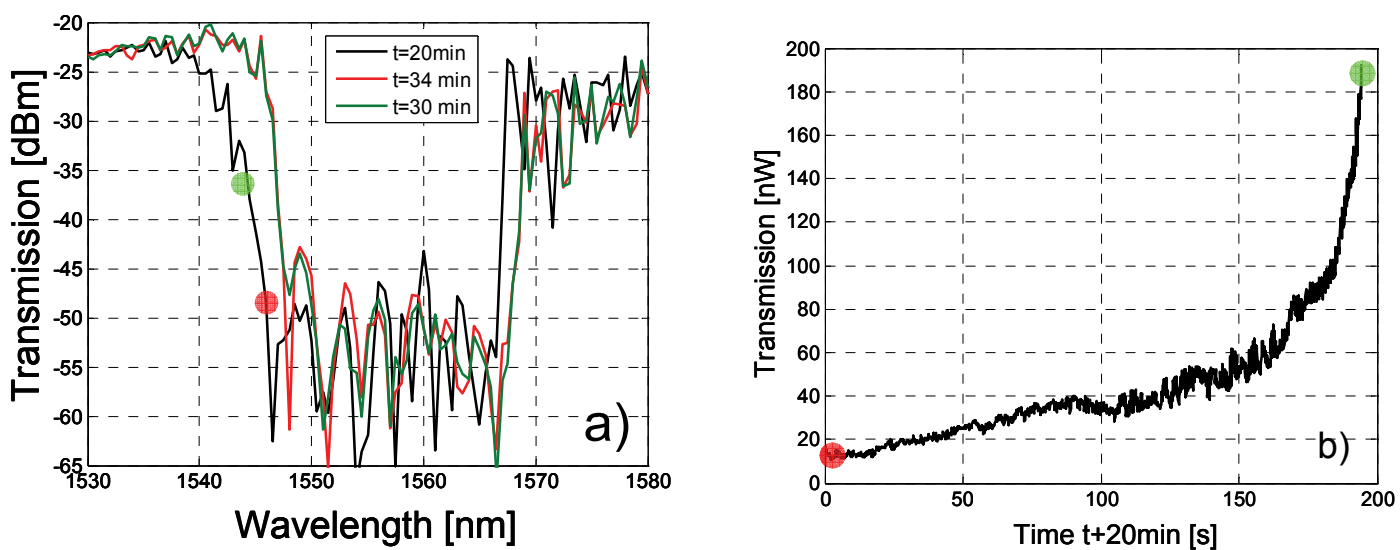

Fig. 28. a) Transmission spectrum with blocking solution. After 30 mins the reactions appears to have saturated. b) The reaction curve of carbonyl group blocking. The reaction is rapid and the stopband edge slides quickly through the monitoring point $(\lambda=1546.1 \mathrm{~nm})$. The corresponding points indentified in the transmission spectrum (Fig. 21a)

\subsubsection{Enzyme binding reaction}

The PepN enzyme binding on the antibody interface was monitored by measuring the transmittance using a couple of operation points near the stopband edge. The reaction time for enzyme binding is one hour. Based on measurement results, the enzyme binding appeared to be active during the first 30 minutes after which the activity fell to a very low level; see Fig 29. The response of the enzymes depends on the number of antibody sites. If there are fewer antibody sites than enzymes, the response saturates more quickly and the total signal change is smaller compared to the situation where there is excess amount of antibody sites.

The total wavelength shift caused by the enzyme binding is estimated to be $230 \mathrm{pm}$, which corresponds to bulk index change of $\sim 10^{-3}$. This index change is high, and given the sensor's detection limit of $10^{-5}, 100$ times smaller enzyme concentration than in our sample, can potentially be detected. Due to the compact size of the GSPW sensor, the sample volume can be very small, down to the picolitre level. 


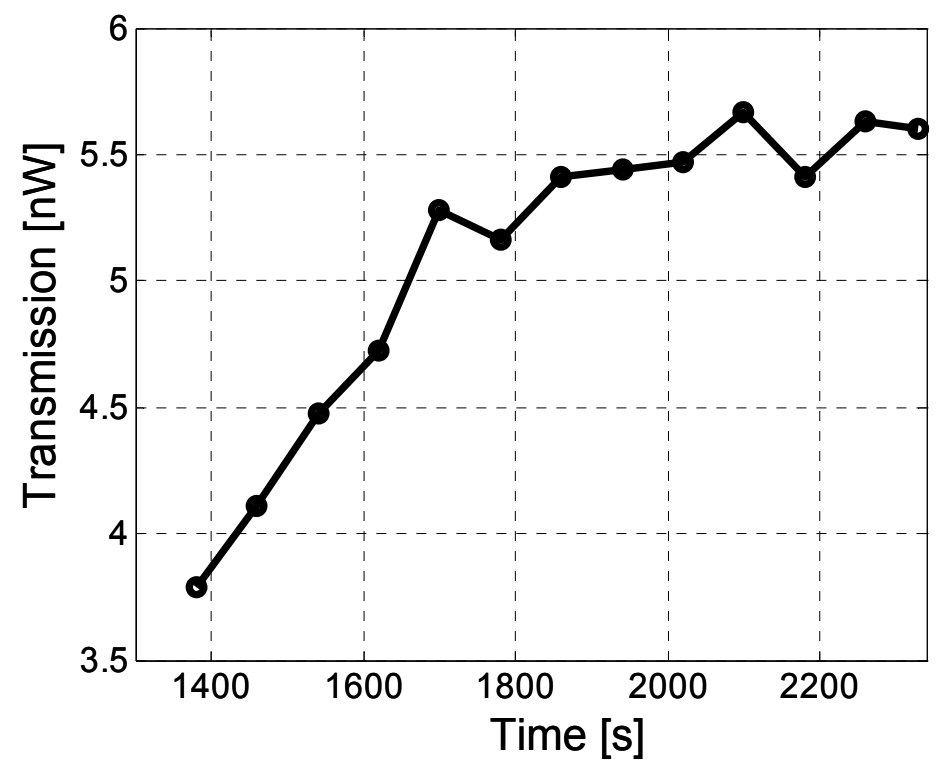

Fig. 29. Reaction curve of enzyme binding process. The saturation in enzyme binding happens after about 30 minutes from injecting the solution.

\subsubsection{Conclusions}

Label-free enzyme sensing, using a GSPW sensor, was demonstrated by immobilizing the enzyme antibody interface on a surface of the sensor. Enzyme binding on this interface was measured. Also the immobilization process was monitored with the sensor. This can deliver calibration data that may be crucial considering variations in different immobilization processes. The compact size of the device enables the use of $<1 \mathrm{pl}$ sample volumes. The CMOS compatible fabrication process, mass production potential and compact size make these devices a very attractive sensor platform. 


\subsection{Grated Waveguide Optical Cavity as a Compact Sensor for Sub-nanometre Cantilever Deflections}

We propose a compact, novel and highly sensitive integrated read-out scheme to detect small deflections of a cantilever in close proximity to a grated waveguide (GWG) structure. In optical resonator (such as $G W G$ ) the electromagnetic field strength is enhanced at the resonance wavelength. This leads to the situation where also light matter interaction is enhanced.

We discuss modelling results for an $\mathrm{SiO}_{2}$ cantilever to be integrated with an optical cavity defined by a grated $\mathrm{Si}_{3} \mathrm{~N}_{4}$ waveguide. The aim is not to provide theoretically optimized design, but to provide discussion of relevant topics and provide information how different parameters affect on sensor performance. Motivation for the work is to get parameter range to fabricate $1^{\text {st }}$ batch of sensors using a safe design.

\subsubsection{Device structure and principle of operation}

We consider a grating defined in a shallow ridge silicon nitride $\left(\mathrm{Si}_{3} \mathrm{~N}_{4}\right)$ waveguide $(\mathrm{WG})$, as shown in the inset of Fig. 30. A waveguide width of $5 \mu \mathrm{m}$, a ridge height of $5 \mathrm{~nm}$ and a core height of $275 \mathrm{~nm}$ were chosen, leading to single mode operation. The grating can be realized on the WG e.g. with laser interference lithography [41].

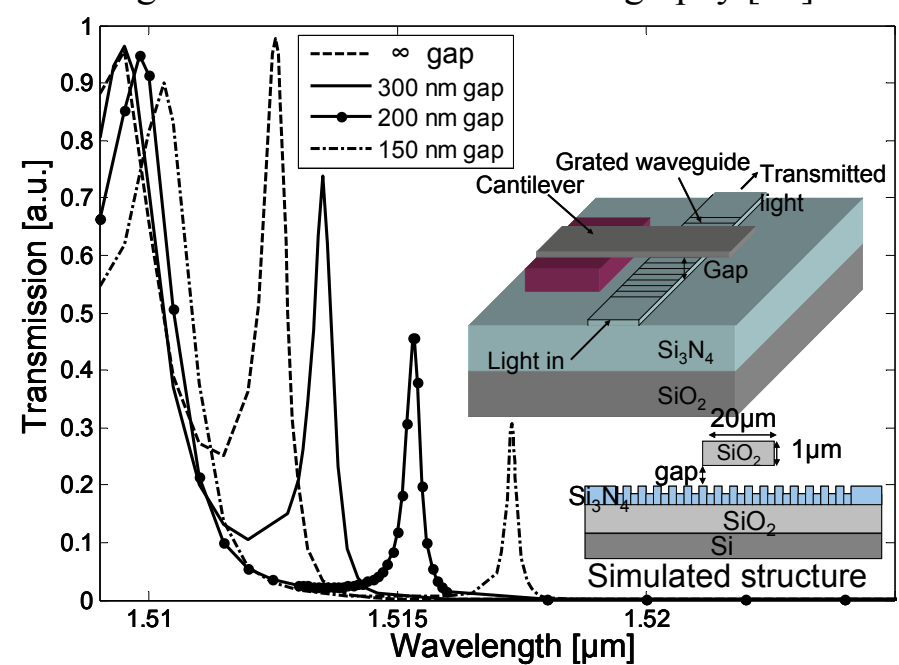

Fig. 30. Simulated transmission spectra of a 200-period grating, with the cantilever position as parameter, using a 2D bidirectional eigenmode propagation method [42]. Insets: device structure and its 2D model.

A very compact and stable sensor element can be realized by monolithically integrating a microcantilever structure with the GWG, using conventional layer deposition and sacrificial layer etching techniques. The device can be functionalized by depositing a sensitive layer on top of the cantilever, e.g. palladium $(\mathrm{Pd})$ for hydrogen $\left(\mathrm{H}_{2}\right)$ sensing. Absorption of $\mathrm{H}_{2}$ into $\mathrm{Pd}$ will cause the cantilever to bend [43]. This bending of the cantilever can then be optically detected by exploiting the properties of the GWG.

This section has been published in: Kauppinen, L.J. and Hoekstra, H.J.W.M. and Dijkstra, M.

and de Ridder, R.M. and Krijnen, G.J.M. (2008) Grated waveguide optical cavity as a

compact sensor for sub-nanometre cantilever deflections. In: Proceedings of the 14th

European Conference on Integrated Optics (ECIO), 11-13 June 2008, Eindhoven, The

Netherlands. pp. 111-114. Eindhoven University of Technology. 
The presence of a dielectric object, in this case a cantilever, in the evanescent-field region of the GWG may lead to the occurrence of propagating modes for wavelengths inside the stopband of the unloaded grating, and so to resonances (defect modes) inside the stopband, as shown in Fig. 30. The first near band edge resonance of a grated waveguide has its field maxima at a center of the grating [44]. If perturbation, say dielectric cantilever, is placed such that it is in the evanescent field region of this resonance mode, a shift in resonance frequency will occur. As the cantilever approaches the grating, the first near band-edge resonance peak is pulled inside the stopband and its spectral width decreases. This effect can be used for the detection of cantilever displacements.

\subsubsection{One-dimensional modelling}

The optical deflection sensitivity (dT/dgap) depends strongly on the maximum slope of the transmission peak of the mode that the cantilever pulls into the stopband. To analyze the effect of cantilever width on the slope of the transmission peak, 1D calculations were performed applying the transfer matrix method to the cantilever-loaded grating structure. The cantilever-induced effective-index change was calculated with a $2 \mathrm{D}$ mode solver, and the obtained values were used in the 1D calculations. The modelled 1D grating is composed of layers arranged as HLHL...H'L'H'L'...HLHL, where H and L represent high- and low-index layers, respectively, and $\mathrm{H}^{\prime}$ and $\mathrm{L}$ ' are the corresponding indices in the cantilever induced defect region. The period of the modelled grating is $490 \mathrm{~nm}$ and the refractive indices of the layers $H$ and $L$ are 1.5928 and 1.53211 , respectively. The proximity of the cantilever increases the indices below it by $\sim 0.5 \%$. A cantilever induced defect region width of 20 to 30 periods in a 100-period grating produces the steepest slope, as shown in Fig. 31. The slope also depends strongly on the grating length. Using the same method as above, the slope was calculated as a function of grating length. Two cases were studied: (a) the cantilever width is fixed at 20 periods, and (b) the relative cantilever width is constant, in this case at $20 \%$ of the total grating length. Doubling the grating length provides more than one order of magnitude slope improvement, as shown in Fig. 32. A large spectral slope means a high quality factor $Q$ of the defect mode. However, the 1D method does not account for scattering loss that is often large for a high Q resonance [44]. Because of noise considerations, the detection limit for cantilever deflection depends on the peak amplitude. 


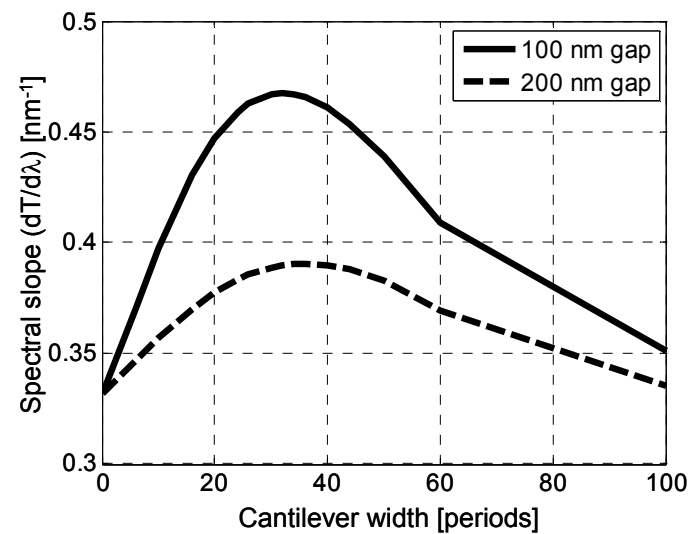

Fig. 31. Spectral slope of defect-mode transmission peak in a 100 -period grating versus of cantilever width.

\subsubsection{Two-dimensional modelling}

A 2D bidirectional eigenmode propagation (BEP) method [42] was applied to the model shown in Fig. 33, to analyze the effect of cantilever thickness on the deflection sensitivity and to account out of plane losses that affect on the detection limit. The defect-mode spectra corresponding to various cantilever thicknesses are shown in Fig. 34. Thinner cantilevers induce defect modes closer to the stopband edge and with higher transmittance than thicker ones. The difference in slope is small and thus the difference in deflection sensitivity comes mainly from the spectral shift. Since the defect modes of the thicker cantilevers are deeper in the stopband, they experience a larger spectral shift when the gap decreases from infinity to $200 \mathrm{~nm}$. This suggests that the optical sensitivity is higher for the thicker cantilevers at this gap range ( $\infty$ to $200 \mathrm{~nm}$ ).

The optical deflection sensitivity of the grating was calculated with two different cantilever thicknesses, $200 \mathrm{~nm}$ and $1 \mu \mathrm{m}$. Fig. 35 shows the transmitted power versus the cantilever deflection for 2 different initial gaps, $200 \mathrm{~nm}$ and $300 \mathrm{~nm}$ where the wavelength is fixed at the corresponding resonance peak of the defect mode. From Fig. 35 it can be seen that the deflection sensitivity at an initial $300 \mathrm{~nm}$ gap is higher for the thick cantilever, although the difference is not large. However, at $200 \mathrm{~nm}$ gap the thin cantilever is preferred due to a higher transmission power and a slightly higher sensitivity.

The theoretical deflection sensitivity can be estimated from the graphs in Fig. 35. The sensitivity slope of the $200 \mathrm{~nm}$ thick cantilever at $200 \mathrm{~nm}$ initial gap is $0.058 / \mathrm{nm}$. By assuming that the noise level allows power detection at an accuracy of $10^{-2}$ (e.g. transmission unit is in $\mu \mathrm{W}$ and noise level is $<10 \mathrm{nW}$ ), the deflection can be detected with a resolution of $0.17 \mathrm{~nm}$. Higher sensitivity is possible, e.g. with a longer grating, provided that the losses remain low. 


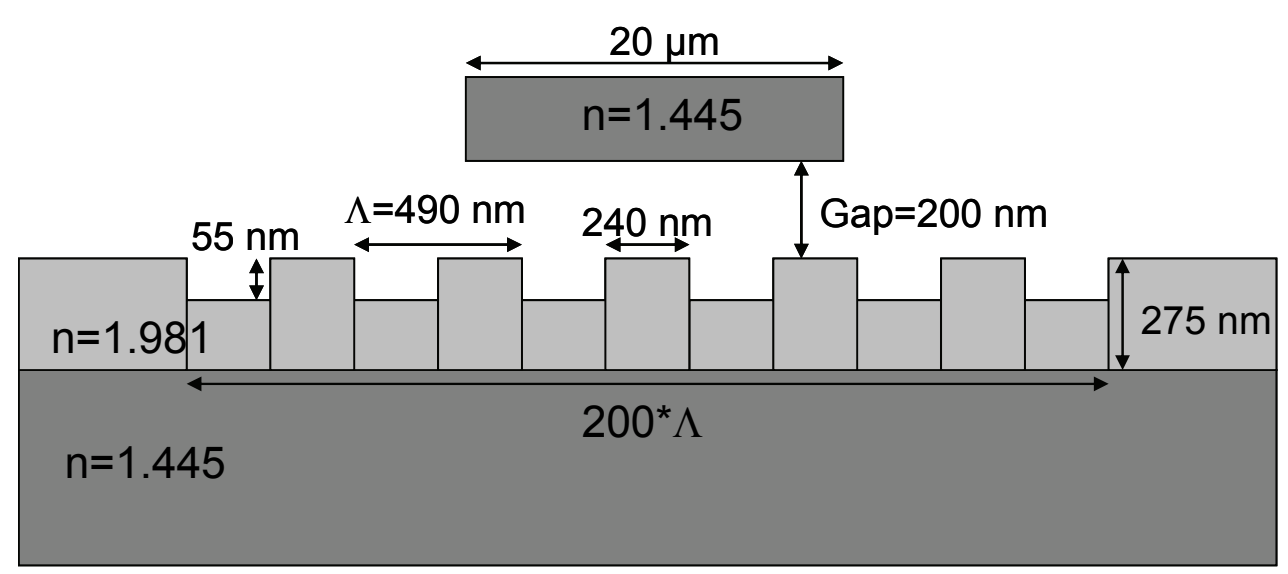

Fig. 33. 2D cross-sectional model of the device used in simulations.

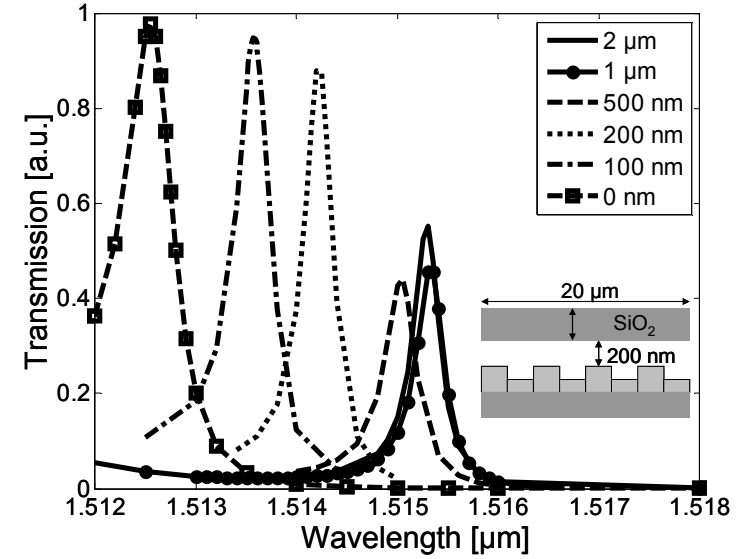

Fig. 34. Defect mode positions obtained with different cantilever thicknesses. Thinner cantilevers induce defect modes that are closer to the stopband edge.

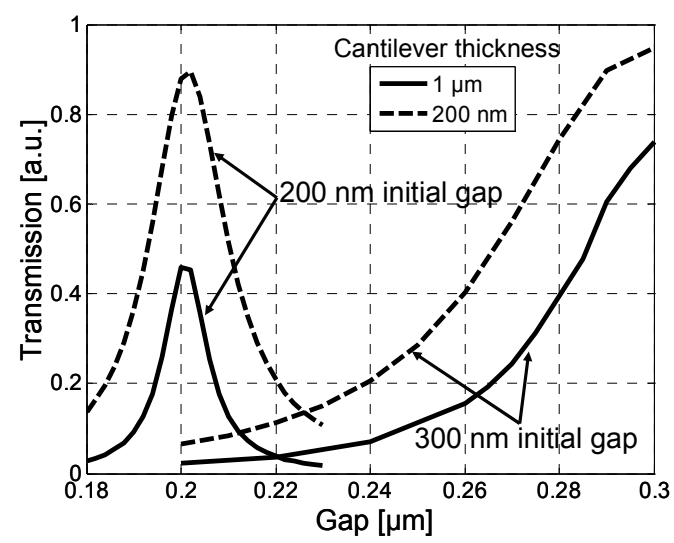

Fig. 35. Simulated transmitted power versus cantilever deflection, calculated for two cantilever thicknesses and two fixed wavelengths corresponding to resonances at 200 and $300 \mathrm{~nm}$ initial gap values.

The choice of cantilever thickness should be carefully considered to obtain maximum sensitivity and stable operation. Thinner cantilevers are more sensitive to mechanical bending that arises from differential surface stress, as follows from Stoney's model [23], see equation (1). However, the thermal and mechanical stability of thin cantilevers is low due to this high sensitivity. Also, if a thin cantilever is coated with a metal layer, the evanescent field of the WG may reach the metal layer through the thin silicon dioxide layer, increasing optical loss. Partial metal coating can be used to avoid such a loss, but at the expense of smaller sensitive area and therefore smaller surface stress.

From the fabrication point of view, it is convenient to have the initial gap between the WG and the cantilever as large as possible. For the considered WG design, the maximum gap for getting an optical response on downward deflection is around $400 \mathrm{~nm}$. For this initial gap there is not a large difference in optical sensitivity between thin $(200 \mathrm{~nm})$ and thick $(1 \mu \mathrm{m})$ cantilevers. 
For maximum sensitivity, a thin cantilever with a small initial gap should be selected, whereas a safer design calls for a thick cantilever with a large initial gap.

\subsubsection{Fabrication of microcantilever}

Below we will briefly describe in general a typical fabrication procedure of surface micromechanical cantilever. The main issues to fabricate the GWG cantilever sensor are discussed as well as the properties of dry etch method to release the cantilevers.

On substrate (which in our case has the waveguiding structure) so-called sacrificial layer is deposited and on top of this is deposited a layer to which the cantilever is patterned, e.g. using photolithography. After the cantilever structure is patterned, it is released by etching the sacrificial layer. The cantilever can be anchored to the substrate simply by defining it's geometry such that the release etch will not release the whole structure, as shown in Fig. 36. It is also possible to etch an anchor hole into the sacrificial layer prior to depositing the cantilever layer. The later anchoring option requires additional mask and photolithography steps, but results in well defined cantilever length.

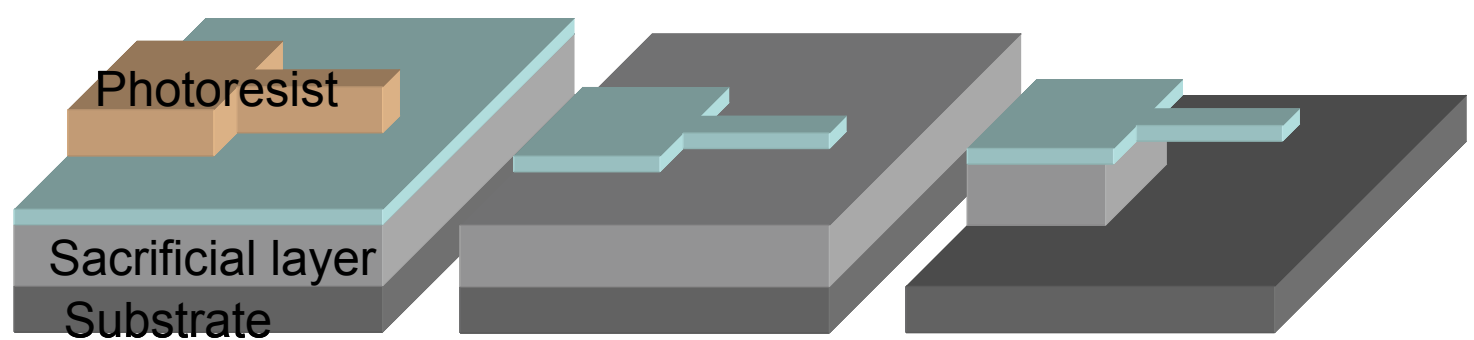

Fig. 36. Cantilever fabrication steps; definition of cantilever structure and release by etching the sacrificial layer.

The sacrificial material needs to be selected such that high selectivity in etching can be achieved between the surround layers. Commonly the sacrificial layer is either $\mathrm{Si}$ or $\mathrm{SiO}_{2}$. Several etchants have high selectivity between $\mathrm{Si}$ and $\mathrm{SiO}_{2}$. Silicon can be etched with high selectivity e.g. using wet etchants $\mathrm{KOH}, \mathrm{TMAH}, \mathrm{EDP}$. The $\mathrm{SiO}_{2}$ can be etched e.g. with BHF or by dry etching with gaseous HF.

If wet etching is used to release the cantilever, one needs to take stiction into account. Stiction occurs after wet etching due to capillary forces and it limits the cantilever length, $L$, according to equation 4 [45];

$$
L<L_{\text {crit }}=\sqrt[4]{\frac{3}{8} \frac{Y h^{3} g^{2}}{\gamma_{s}}},
$$

in which $Y$ is the Young's modulus, $h$ the thickness of the cantilever, $g$ the gap between the cantilever and the substrate and $\gamma_{s}$ is the adhesion energy per unit area. If the cantilever length exceeds the critical length $L_{c r i t}$ stiction will occur.

For integrated optics applications, in which the evanescent field perturbation is exploited, the cantilever should be within reach of this field. Typically this means that a distance of smaller than $400 \mathrm{~nm}$ is required. To avoid stiction after wet etching special drying methods can be used, e.g. freeze drying [46] or critical point drying [47]. Since the 
reduction of adhesion energy $\gamma_{s}$ allows longer cantilever length, a liquid that has a lower surface tension than normally used water, can be selected for rinsing. Also stiction (surface) area can be reduced by introducing bumps or by increasing the surface roughness. Dry etching has a clear benefit, since no liquid is involved and the stiction risk is negligible. However, depending on material selection, dry etching may not be possible or wet etching may provide much higher selectivity between different layers.

Initial stress in the layer(s), that form(s) the cantilever, may cause significant initial bending after the cantilever is released. This is unwanted effect, if the initial gap increases such that the evanescent field is not affected by the cantilever. Intrinsic stress inside the thin film originates from growth mechanism that has resulted in density or compositional gradients. In a cantilever that is composed of several layers the stress may additionally originate due to mismatch between the thermal expansion coefficients of the layers [48]. However, it is also possible to use layer stack such a way that different film stresses compensate one another, effectively reducing the stress and initial bending [49].

\subsubsection{Dry etch released cantilevers}

Dry etching is attractive option to release cantilevers since no additional steps are required to minimize the stiction risk during the release etch, as no liquid is involved. This etch method is versatile as it can be used to etch either anisotropic or isotropic etch profile by just changing the etch parameters, see e.g. [49]. In order to study the suitability of this etch technique as an etch step to release the microcantilevers, we performed an etch test by using the isotropic etch properties of an inductively coupled plasma (ICP) etch.

The layer stack required for the sensor that is presented in section 2.2. is: $\mathrm{Si}_{3} \mathrm{~N}_{4}$, poly-Si and $\mathrm{SiO}_{2}$. The poly-Si was selected as sacrificial layer as $\mathrm{Si}$ is the main target for the etchant gas used in the ICP, and therefore provides the best possible etch selectivity between the layers. The $200 \mathrm{~nm}$ thick stochiometric silicon nitride layer was deposited using low pressure chemical vapor deposition (LPCVD) method. This layer is the layer in which the optical devices for the cantilever deflection read-out can be patterned. The sacrificial layer, $400 \mathrm{~nm}$ of polysilicon was deposited as well by using the LPCVD method. The device layer for the microcantilevers is a $1 \mu \mathrm{m}$ thick plasma enhanced chemical vapor deposited (PECVD) silicon dioxide layer. The cantilevers were patterned by means of photolithography on the $\mathrm{SiO}_{2}$ layer and etched in $\mathrm{BHF}$. The $\mathrm{SiO}_{2}$ is etched over 600 times faster than the sacrificial poly-Si layer, meaning that the sacrificial layer is an excellent etch stop layer in this step. Finally the microstructures were released using the isotropic plasma etch using Sulfur hexafluoride $\left(\mathrm{SF}_{6}\right)$ gas as an etchant.

In Fig. 37a microscope image is shown of the etch hole after etching and resist strip off. The total under etch is approximately $20 \mu \mathrm{m}$. From the gradient it can be concluded that the silicon nitride is attacked by the etchant (as expected). 


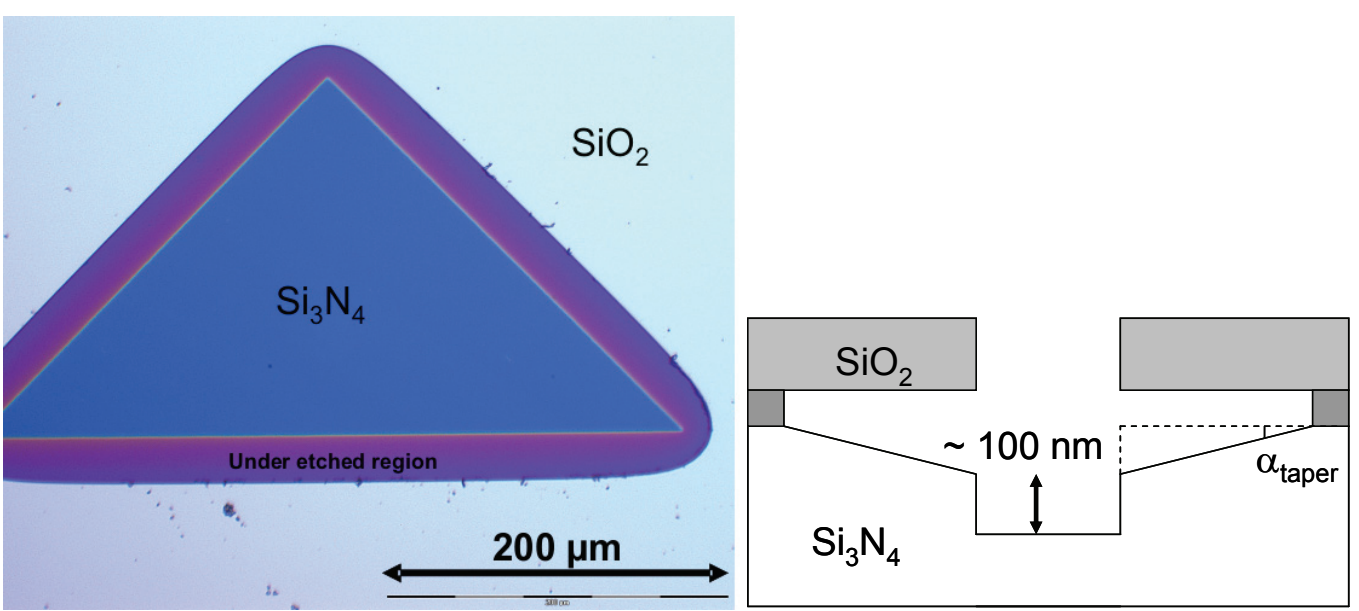

Fig. 37. a) Microscope image of the etch hole. An under etch of approximately $22 \mu \mathrm{m}$ is seen from the image. Color changes indicate tapered etch profile in $\mathrm{SiN}$ layer. b) schematic of the etch profile in a $\mathrm{Si}_{3} \mathrm{~N}_{4}$ layer. Under the etch hole the nitride is etched much faster than under the $\mathrm{SiO}_{2}$ roof, where $\alpha_{\text {taper }}=0.05^{\circ}$.

Directly under the etch hole, the nitride was etch by $\sim 100 \mathrm{~nm}$, whereas under the $\mathrm{SiO}_{2}$ roof the etch profile is tapered, as shown in the schematic picture in Fig 37b. Based on the Dektak surface profile measurement, the horizontal etch speed of the poly-Si is 1200 times faster than the vertical etch rate for the $\mathrm{Si}_{3} \mathrm{~N}_{4}$ under the $\mathrm{SiO}_{2}$ roof. For the integrated device this means that the waveguide should be under a protective (photoresist) roof during this etch step. The sacrificial material could be removed through such an etch windows as shown in Fig 38.

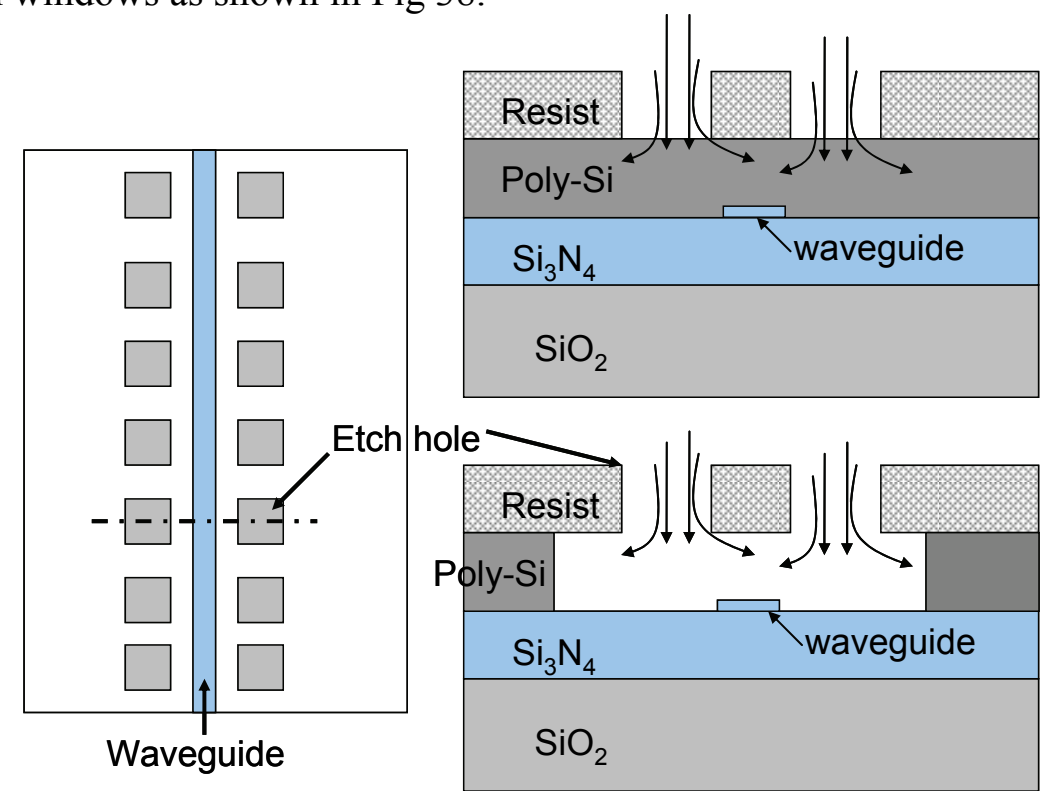

Fig. 38. Etch holes to remove the sacrificial material; on left schematic top view, on right schematic crosssection.

The unavoidable result is that the tapered etch profile (see Fig. 37b) would most likely affect the optical properties of the waveguide grating, although the taper angle $\alpha_{\text {taper }}$ is very small; 0.05 degrees. The SEM image in Fig. 39 gives a hint about the biggest disadvantage of this etch method; high surface roughness of the $\mathrm{Si}_{3} \mathrm{~N}_{4}$ layer after the dry 
etch. Later AFM study revealed that the surface roughness is of the order of the waveguide ridge $(\sim 5 \mathrm{~nm})$ [50]. Given the disadvantages of this release etch method, a wet etchant (TMAH) in combination with a freeze drying method was chosen; with successful results [50]. However, it should be noted that the isotropic dry etching with another etchant gas, Xenon difluoride $\left(\mathrm{XeF}_{2}\right)$, might yield better results than $\mathrm{SF}_{6}$, as etch selectivity between $\mathrm{Si}$ and $\mathrm{Si}_{3} \mathrm{~N}_{4}$ is much higher than with the $\mathrm{SF}_{6}$. Unfortunately this etchant was not available at time being.

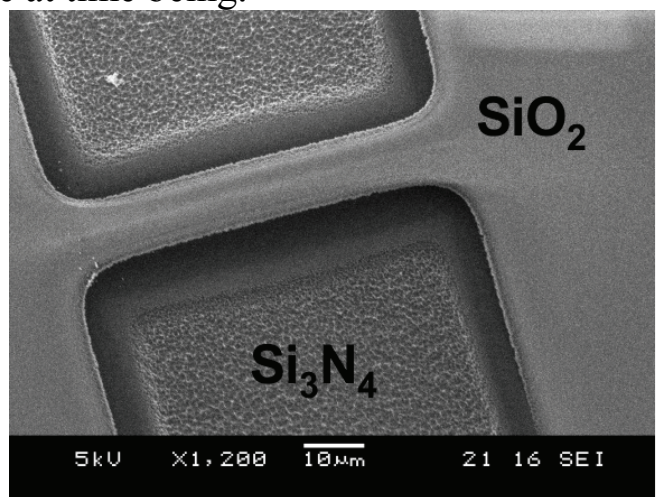

Fig. 39. SEM image of the released microbridge. The isotropic etching with $\mathrm{SF}_{6}$ results in high surface roughness in $\mathrm{Si}_{3} \mathrm{~N}_{4}$ layer. Although the roughness is smaller under the microbridge, it is still in the order of the planned waveguide ridge $(5 \mathrm{~nm})$.

\subsubsection{Conclusions}

Based on our simulations we have shown that GWG can be used to sense deflections of micromechanical cantilevers at sub-nanometre resolution. The presented read-out scheme is a good candidate to enable dense integration of cantilever sensors, providing an accurate and stable optical detection of cantilever bending. Issues related to cantilever fabrication and integration with the optical design have been discussed. The initial bending of the cantilever and the selection of the proper etch process have been identified as key issues for further process integration study. 


\section{References}

[1] M. Gnan, G. Bellanca, H.M.H. Chong, P. Bassi, and R.M. de la Rue, "Modelling of photonic wire Bragg gratings", Optical and Quantum Electronics, vol. 38, pp. 133-148, 2006.

[2] J. Singh, "Semiconductor optoelectronics: physics and technology", McGraw-Hill 1995.

[3] J.D. Joannopoulos, S.G. Johnson, J.N. Winn, and R.D. Meade, "Photonic crystals: molding the flow of light", Princeton University Press, 2008.

[4] S.G. Johnson and J.D. Joannopoulos, "Block-iterative frequency-domain methods for Maxwell's equations in a planewave basis," Optics Express, vol. 8, pp. 173-190, 2001.

[5] P. Yeh, "Optical waves in layered media", Wiley, 1988.

[6] C. Pollock and M. Lipson, "Integrated photonics", Kluwer Academic Publishers 2003.

[7] A. Farjadpour, D. Roundy, A. Rodriguez, M. Ibanescu, P. Bermel, J.D. Joannopoulos, S.G. Johnson, and G. Burr, "Improving accuracy by subpixel smoothing in FDTD," Optics Letters, vol. 31, pp. 29722974, 2006.

[8] H.J.W.M. Hoekstra, W.C.L. Hopman, J. Kautz, R. Dekker, and R.M. de Ridder, "A simple coupled mode model for near band-edge phenomena in grated waveguides", Optical and Quantum Electronics, vol. 38, pp. 799-813, 2006.

[9] Alex Figotin and Ilya Vitebskiy, "Slow light in photonic crystals", Waves in Random and Complex Media, vol. 16, No. 3, pp. 293-382, 2006.

[10] A.A.M. Kok, Jos J. G. M. van der Tol, Roel Baets, and Meint K. Smit, "Reduction of propagation loss in pillar-based photonic crystal waveguides", Journal of Lightwave Technology, vol. 27, pp. $3904-$ 3911, 2009.

[11] D. Taillaert, F. van Laere, M. Ayre, W. Bogaerts, D. van Thourhout, P. Bienstman, and R. Baets, "Grating couplers for coupling between optical fibers and nanophotonic waveguides", Japanese Journal of Applied Physics, vol. 45, pp. 6071-6077, 2006.

[12] M. Palamaru and P. Lalanne, "Photonic crystal waveguides: Out-of-plane losses and adiabatic modal conversion", Applied Physics Letters, vol. 78, pp. 1466-1468, 2001.

[13] C. Sauvan, G. Lecamp, P. Lalanne, and J.P. Hugonin, "Modal-reflectivity enhancement by geometry tuning in photonic crystal microcavities", Optics Express, vol. 13, pp. 245-255, 2005.

[14] G.M. Jie Shen, Z. Zhang, Z. Hua, and S.H. Tang, "Ultrafast all-optical switching in onedimensional photonic crystal with two defects", Optics Express, vol. 14, pp. 858-865, 2006.

[15] S. Zhu, N. Liu, H. Zheng, and H. Chen, "Time delay of light propagation through defect modes of onedimensional photonic band-gap structures", Optics Communications, vol. 174, pp. 139-144, 2000.

[16] H. Alatas, H. Mayditia, H. Hardhienata, A.A. Iskander, and M.O. Tjia, "Single-frequency refractive index sensor based on a finite one-dimensional photonic crystals with two defects", Japanese Journal of Applied Physics, vol. 45, pp. 6754-6758, 2006.

[17] P. Prabhathan, V.M. Murukeshan, Z.Jing, and P.V. Ramana, "Compact SOI nanowire refractive index sensor using phase shifted Bragg grating", Optics Express, vol. 17, pp. 15330-15341, 2009.

[18] F. Ay, L.J. Kauppinen, J.D.B. Bradley, K. Wörhoff, R.M. de Ridder, and M. Pollnau, "Focused ion beam milled on-chip resonator nanostructures for applications in rare Earth-ion-doped $\mathrm{Al}_{2} \mathrm{O}_{3}$ active waveguides", IEEE Photonics Society Annual Meeting, Antalya, Turkey, 2009.

[19] W.C.L. Hopman, H.J.W.M. Hoekstra, R. Dekker, L. Zhuang, and R.M. de Ridder, "Far-field scattering microscopy applied to analysis of slow light, power enhancement, and delay times in uniform Bragg waveguide gratings", Optics Express, vol. 15, pp. 1851-1870, 2007.

[20] W.C.L. Hopman, P. Pottier, D.Yudistira, J. van Lith, P.V. Lambeck, R.M. De LaRue, A. Driessen, H.J.W.M. Hoekstra, and R.M. de Ridder, "Quasi-one-dimensional photonic crystal as a compact building-block for refractometric optical sensors”, IEEE J. Select. Topics Quantum Electron, vol. 11, pp. 11-16, 2005.

[21] W. Gabathuler and W. Lukosz, "Electro-nanomechanically wavelength-tunable integrated-optical Bragg reflectors - Part II: Stable device operation", Optics Communications, vol. 145, pp. 258-264, 1998. 


\section{Sensors based on waveguide grating}

[22] C. Ziegler, "Cantilever-based biosensors", Analytical and Bioanalytical Chemistry, vol. 379, pp. 946959, 2004.

[23] N.V. Lavrik, M.J. Sepaniak, and P.G. Datskos, "Cantilever transducers as a platform for chemical and biological sensors", Review of Scientific Instruments, vol. 75, pp. 2229-2253, 2004.

[24] Z. Hu, T. Thundat, and R.J. Warmack, "Investigation of adsorption and absorption-induced stresses using microcantilever sensors", Journal of Applied Physics, Vol. 90, pp. 427-431, 2001.

[25] A. Densmore, D.-X. Xu, S. Janz, P. Waldron, T. Mischki, G. Lopinski, A. Delâge, J. Lapointe, P. Cheben, B. Lamontagne, and J. H. Schmid, "Spiral-path high-sensitivity silicon photonic wire molecular sensor with temperature-independent response", Optics Letters, vol. 33, pp. 596-598, 2008.

[26] K. De Vos, I. Bartolozzi, E. Schacht, P. Bienstman, and R. Baets, "Silicon-on-Insulator microring resonator for sensitive and label-free biosensing", Opt. Express, vol. 15, pp. 7610-7615, 2007.

[27] L.J. Kauppinen, H.J.W.M. Hoekstra, and R.M. de Ridder, "A compact refractometric sensor based on grated silicon photonic wires", Sensors and Actuators B: Chemical, vol. 139, pp. 194-198, 2009.

[28] A.S. Jugessur, J. Dou, J.S. Aitchison, R.M. De La Rue, and M. Gnan, "A photonic nano-Bragg grating device integrated with microfluidic channels for bio-sensing applications", Microelectronic Engineering, vol. 86, pp. 1488-1490, 2009.

[29] G. Rong, A. Najmaie, J.E. Sipe, and S.M. Weiss, "Nanoscale porous silicon waveguide for labelfree DNA sensing”, Biosensors \& Bioelectronics, vol. 23, pp. 1572-1576, 2008.

[30] S. Mandal and D. Erickson, "Nanoscale optofluidic sensor arrays", Optics Express, vol. 16, pp. 1623 $1631,2008$.

[31] N. Skivesen, A. Têtu, M. Kristensen, J. Kjems, L.H. Frandsen, and P.I. Borel, "Photonic-crystal waveguide biosensor", Optics Express, vol. 15, pp. 3169-3176, 2007.

[32] V. Sorianello, M.Balbi, L.Colace, G.Assanto, L.Socci, L.Bolla, G.Mutinati, and M.Romagnoli, "Guided-wave photodetectors in germanium on SOI optical chips", Physica E, vol. 41, pp. 1090-1093, 2009.

[33] A.W. Fang, R. Jones, H. Park, O. Cohen, O. Raday, M.J. Paniccia and J.E. Bowers, "Integrated AlGaInAs-silicon evanescent racetrack laser and photodetector", Optics Express, vol. 15, pp. 23152322, 2007.

[34] H.J.W.M. Hoekstra, W.C.L. Hopman, J. Kautz, R. Dekker, and R.M. de Ridder, “A simple coupled mode model for near band-edge phenomena in grated waveguides", Optical and quantum electronics, vol. 38, pp. 799-813, 2006.

[35] Silicon Photonics Platform, http://www.epixfab.eu/

[36] M. Gnan, G. Bellanca, H.M.H. Chong, P. Bassi, and R.M. de la Rue, "Modelling of photonic wire Bragg gratings", Opt. Quantum Electron., vol. 38, pp. 133-148, 2006.

[37] OlympIOs Integrated Optics Software. C2V, P.O. Box 318, 7500 AH Enschede, The Netherlands,(http://www.c2v.nl/software/)

[38] M-K. Kwon and W.H. Steier, "Microring-resonator-based sensor measuring both the concentration and temperature of asolution", Optics Express, vol. 16, pp. 9372-9377, 2008.

[39] R. Costa, G. Cusmai, A. Melloni, and M. Martinelli, "TE-TM coupling of a standard fiber to a Si-wire waveguide", in: Proceedings of the 13th European Conference on Integrated Optics (ECIO'07), Copenhagen, Denmark, 2007, ThC3.

[40] IMEnz Bioengineering BV, L.J. Zielstraweg 1, 9713 GX Groningen, The Netherlands.

[41] W.C.L. Hopman, R. Dekker, D. Yudistira, W.F.A. Engbers, H.J.W.M. Hoekstra, and R.M. de Ridder, "Fabrication and characterization of high-quality uniform and apodized $\mathrm{Si}_{3} \mathrm{~N}_{4}$ waveguide gratings using laser interference lithography”, IEEE Photonics Technology Letters, vol. 18, pp. 1855-1857, 2006.

[42] OlympIOs Integrated Optics Software. C2V, P.O. Box 318, 7500 AH Enschede, The Netherlands, (http://www.c2v.nl/software/)

[43] Y.I. Chou, H.C. Chiang, and C.C. Wang, "Study on Pd functionalization of microcantilever for hydrogen detection promotion", Sensors and Actuators B, vol. 129, pp. 72-78, 2008. 


\section{Sensors based on waveguide grating}

[44] W.C.L. Hopman, H.J.W.M. Hoekstra, R. Dekker, L. Zhuang, and R.M. de Ridder, "Far-field scattering microscopy applied to analysis of slow light, power enhancement, and delay times in uniform Bragg waveguide gratings", Optics Express, vol. 15, pp. 1851-1870, 2007.

[45] N. Tas, T. Sonnenberg, H. Jansen, R. Legtenberg, and M. Elwenspoek, "Stiction in surface micromachining", Journal of Micromechanics and Microengineering, 6, pp. 385-397, 1996.

[46] H. Guckel, J.J Sniegowski, T.R. Christenson, S. Mohney, and T.F Kelly, "Fabrication of micromechanical devices from polysilicon films with smooth surfaces", Sensors Actuators, vol. 20 pp. $117-22,1989$.

[47] G.T. Mulhern, D.S. Soane, and R.T. Howe, “carbon dioxide drying of microstructures", Proc. 7th Int. Conf. on Solid-State Sensors and Actuators (Transducers'93) (Yokohama, 1993) pp. 296-9, 1993.

[48] D. Resnik, U. Aljanc ic $^{`}$, D. Vrtačnik, M. Možek and S. Amon, "Mechanical stress in thin film microstructures on silicon substrate", Vacuum, vol. 80, pp. 236-240, 2005.

[49] P. Griss and G. Stemme, "Side-opened out-of-plane microneedles for microfluidic transdermal liquid transfer", Journal of Microelectromechanical systems, vol. 12, pp. 296-301, 2003.

[50] Pham Van So, L.J. Kauppinen, H.J.W.M. Hoekstra, M. Dijkstra, H.A.G.M. van Wolferen, G.J.M. Krijnen, and R.M. de Ridder, "Fabrication of microcantilever-based IO grated waveguide sensors for detection of nano-displacements", In: Proceedings of the 13th Annual Symposium of the IEEE LEOS Benelux Chapter, pp. 155-158, 2008. 


\section{Compact silicon photonic devices for MEMS-actuated optical switching}

In this chapter we present the optical structures that were designed and fabricated for MEMS-actuated switching. Also the results of optical characterization of these devices are presented and discussed.

The design work aimed at realizing compact devices that can be actuated with a cantilever-type MEMS. In the design work we have applied and used the existing body of knowledge on design and theory that is available in textbooks and journal publications. By using computer simulations the designs have been tailored such that they can be realized in ePIXfab fabrication platform.

All studied devices were designed to perform wavelength routing; light of a certain wavelength is routed through the device to a wavelength-specific output port. By perturbing the device with the MEMS actuator, either the wavelength for a given route is tuned, or this routing is cancelled (on/off switch). The perturbation effect of the cantilever on the optical structure is similar to the one discussed in section 2.4; it changes the effective index in a section of the optical device.

We have focused on four different designs. Judging by its theoretical specifications, a photonic-crystal-cavity-based channel drop filter (4-port) is an ideal device; as a PhCbased device it is compact and the cavity provides a mechanism for strongly wavelength selective routing. Another PhC-cavity-based device is a Y-splitter-type device (3-port) in which a certain wavelength is routed to one of the two output waveguides via cavity. A waveguide-grating-based device represents another 3-port device that can route a broad wavelength band from the input to the desired output port. The last design is the wellknown ring resonator, a 4-port device that has been widely studied for wavelength routing. However, very little effort has so far been made to realize a mechanically tuned ring resonator. We have implemented compact racetrack-type ring resonators where the resonance, and hence the drop wavelength, can be tuned with MEMS.

The discussion and conclusion sections at the end of the chapter summarize the performance of the devices.

\subsection{Photonic-crystal-based designs}

In this section we present photonic-crystal-based devices for optical switching. First the general properties of PhCs are discussed, followed by sections that present the switching structures in detail.

\subsubsection{Photonic crystals}

In this section we will discuss the basic properties of photonic crystals (PhCs). We focus on the most common photonic crystal structure: the two-dimensional (2D) slab-type photonic crystal, usually called "photonic crystal slabs" (PCSs), with a triangular lattice of air holes. The energy band diagram -also known as the photonic band structure-, the photonic bandgap, and guiding and trapping of light with crystal defects will be briefly explained. 
The propagation of light in $\mathrm{PhCs}$ has some mathematical similarities to the electron transport in atomic crystals. From intuition one would think that since in the atomic crystal there are so many atoms, at least a few of them would be on the electron's way and the electron would lose some of its kinetic energy in a series of collisions with the crystal atoms. However, the electron can go through such a perfect crystal without scattering at all. The explanation of this phenomenon is that for certain energy ranges, called bands, the electrons can move from unit cell to unit cell as described by a so-called Bloch wave, according to Bloch's theorem [1]. Note that in real atomic crystals the vibrations disturb the crystal structure and electrons do scatter.

As photons do not carry electric charge, like electrons do, they cannot be guided or "trapped" in the crystal by electric potential distributions. Photons can be guided and trapped by crystal defects, as will be discussed in more detail in section 3.1.1.2.

As the periodicity of structures supporting Bloch waves is typically of the order of the wavelength, and the wavelength of light is larger than that of an electron, photonic crystal structures are considerably larger than, e.g., the atomic crystals of semiconductor materials. Nevertheless, for integrated optics (IO), photonic crystals provide a way to realize ultracompact optical devices due to light confinement by structures in the crystal. Ideally the photons would propagate without scattering in a photonic crystal as the electrons do in an atomic crystal. Unfortunately the available fabrication technologies are not mature enough to produce such high-quality $\mathrm{PhCs}$.

The fabrication of 3D photonic crystal structures is challenging, as well as their integration with 'conventional' IO devices that rely on planar fabrication techniques. For this reason the most popular IO photonic crystals are the slab-type photonic crystals in which the crystal is formed by introducing a 2D-periodic refractive index pattern in the slab plane, as shown in Fig. 1.

The propagating modes in photonic crystals are characterized by dispersion curves, sometimes called photonic bands or energy bands, which correspond to propagating modes in the crystal. In contrast to the dispersion curves of waveguide gratings, in $\mathrm{PhCs}$ all possible propagation directions in the slab plane need to be considered. Due to the periodicity PhCs may also have energy (wavelength) ranges at which no (running) wave propagation is possible in the crystal.

\subsubsection{Band diagram}

Structures such as shown in Fig. 1, having two-dimensional periodicity, also have structural variation in the third dimension, thus requiring three-dimensional optical modelling for finding accurate solutions of Maxwell's equations. Since full 3D modelling of such structures requires huge computing resources, several approaches have been taken to reduce the problem to 2D. The first one is simply ignoring the third $(z)$ dimension, which is equivalent to extending the structure infinitely into the $z$ direction to a purely $2 \mathrm{D} \mathrm{PhC}$, and considering only waves that travel parallel to the $x-y$ plane. A second approach is to include one effect of the finite $z$-dimension, by replacing the refractive index of the "background" material by an effective index of the (unperturbed) slab, often simply calculated using the effective index method (EIM) [2]. This type of 2D modeling in general produces similar dispersion diagrams as full 3D methods. However, out-off-plane losses are neglected; in addition, the 2D method introduces a considerable red shift to the spectral phenomena [3]. 


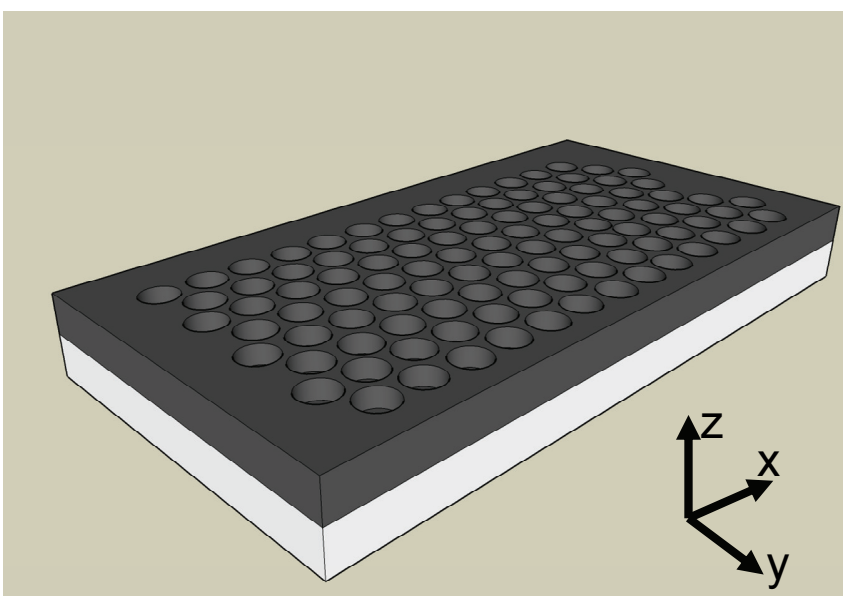

Fig. 1. Schematic picture of a photonic crystal slab formed by a triangular lattice of air holes in the slab.

Fig. 2 shows the band diagram of a PCS with parameters: $n_{\text {slab }}=3.479$, Si layer thickness $220 \mathrm{~nm}$, semi-infinite oxide layer, lattice period $\Lambda=440 \mathrm{~nm}$ and hole diameter $270 \mathrm{~nm}$. The inset of Fig. 2 shows the irreducible Brillouin zone of the triangular lattice and the high symmetry points of the zone. The irreducible Brillouin zone is the smallest region within the Brillouin zone for which the normalized frequency values as a function of wave-vector are not related by symmetry [4]. The Brillouin zone (BZ) can be constructed from the irreducible zone. Also shown in Fig. 2 is the band diagram of the corresponding $2 \mathrm{D}$ PhC obtained by using the effective index method $\left(n_{\text {eff }}=2.85\right)$. It can be seen that the bands calculated with the 3D method are at higher frequencies than the ones obtained with the 2D method. Although the finite thickness of the Si layer is taken into account by the effective index, the calculation of the field distribution in the 2D method assumes infinite thickness. The frequency shift can be understood by considering the fact that the energy of a mode is concentrated in a high-index material (being orthogonal to lower modes) in order to minimize the frequency [4]. In the actual 3D structure the mode extends into low index material in the $z$-direction (which, in the 2D approximation, is assumed uniform), which tends to increase the frequency of the mode(s). 


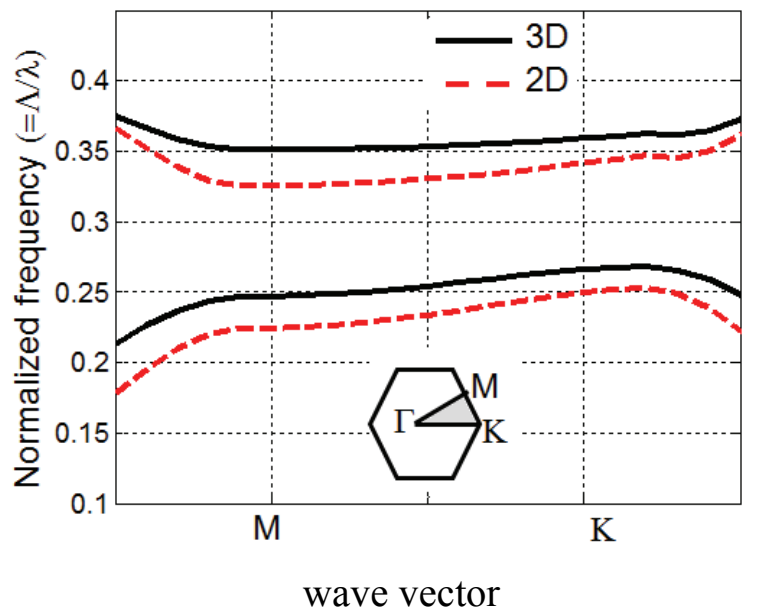

Fig. 2. Band diagram of a photonic crystal slab with $n_{\text {slab }}=3.479$, Si layer thickness $220 \mathrm{~nm}$, oxide layer thickness of $2 \mu \mathrm{m}$, triangular lattice of holes with period $\Lambda=440 \mathrm{~nm}$ and hole diameter $270 \mathrm{~nm}$.

Calculations have been done for TE polarized light with MIT Photonic-Bands (MPB) [5] for a full 3D

structure (solid lines) and for the 2D-reduced structure (dashed line). Inset: The Brillouin zone of the triangular lattice, the irreducible zone gray-shaded. Also shown are the high symmetry points $(\Gamma, \mathrm{K}$ and $\mathrm{M})$.

Dispersion curves, and hence photonic stopbands are polarization dependent. Only when the stopbands for both polarizations overlap for all possible propagation directions, one can speak of a (full) photonic bandgap (PBG). This overlap of stopbands for TE and TM polarization depends for 2D PhCs with a triangular lattice of air holes on the ratio of the lattice period to the hole radius. Unfortunately this PBG occurs typically (depending on the slab index) at normalized frequencies around 0.4. From Fig. 2a of chapter 2, it can be seen that the lightlines of the oxide cladding and the air cladding are at the normalized frequencies below 0.35 and 0.5 , respectively. Note that the same wave-vector range $(0-$ $\pi / \Lambda$ ) holds for a reduced $\mathrm{BZ}$ of a typical $\mathrm{PhC}$ waveguide, as will be discussed in the next section. To avoid out-of-plane diffraction losses that may arise for modes above the lightline the $\mathrm{PhC}$ should be designed such that the bandgap is at the lossless side of the lightlines. Based on our discussion above, this is more difficult to realize if operation at both polarization states is required.

\subsubsection{Defects}

The presence of defects in a $\mathrm{PhC}$ structure may introduce additional energy levels within the original bandgap. Light can be confined to these defects, provided that the energy of the photons matches the energy level of the defect.

\section{Line defects, acting as waveguides}

A particularly useful type of defect is created if an entire row of holes is removed. In this case a PhC waveguide called W1 is formed. Light in such a waveguide is confined in the z-direction by total internal reflection (TIR) and in other transverse direction by the photonic crystal due to the presence of a bandgap (gap guided). If a PCS is formed with 
air holes in a high-index material, such as silicon $(n \cong 3.5)$, only a few rows of holes around the W1 waveguide are sufficient to virtually confine the light to the waveguide.
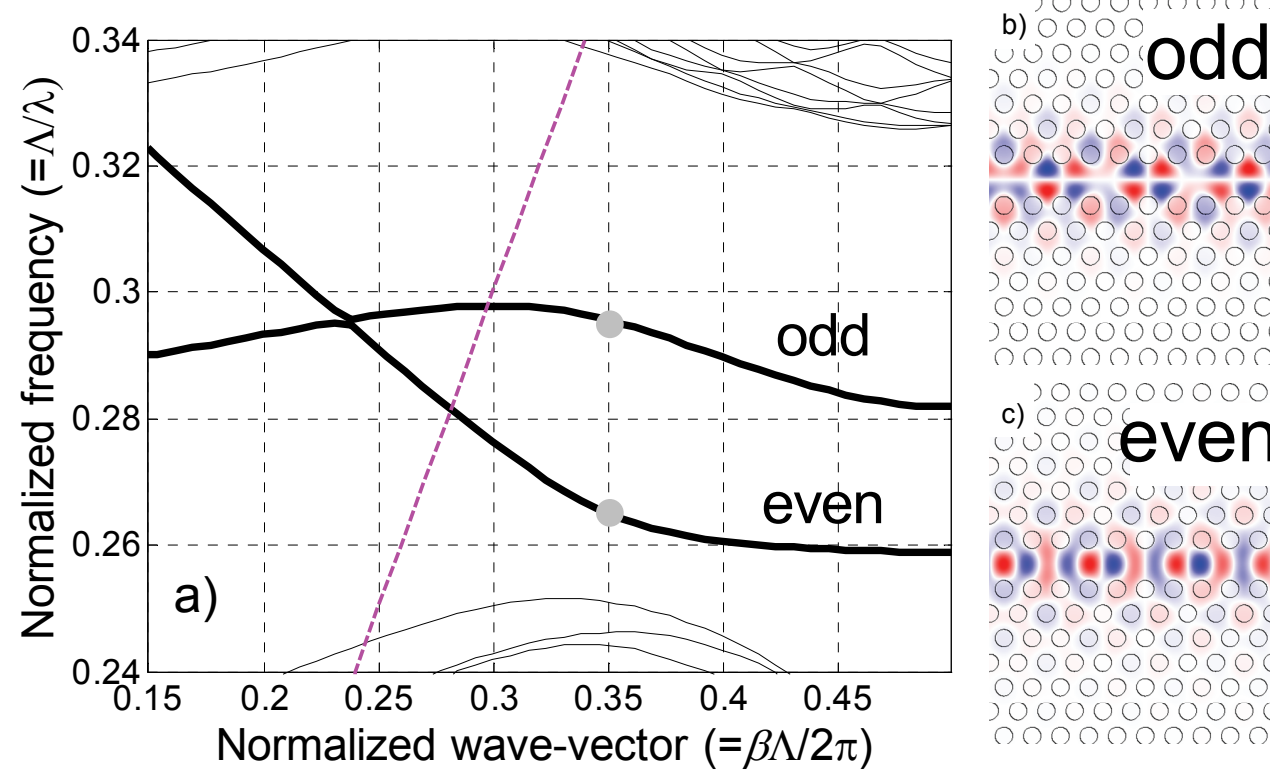

Fig. 3. a) Calculated dispersion diagram of a photonic crystal W1 waveguide in the $\Gamma$-K-direction, showing two H-like (TE-modes), I and II. The dashed line is the light line of the air cladding. b) and c) The H-field profiles of the W1 modes at a normalized wave-vector value of 0.35 .

A calculated dispersion graph of a W1 waveguide in a 2D photonic crystal with a period / hole radius ratio of $440 / 135$ and $n_{\text {eff }}=2.85$ is shown in Fig. 3a. This type of graph is also called a projected band diagram as the $k$-vectors belonging to the $\mathrm{BZ}$ of the $\mathrm{PhC}$ are projected to the reduced $\mathrm{BZ}$ of the waveguide. The mathematical details of this projection procedure are discussed e.g. in [6]. From Fig. 3 it can be seen that the waveguide has two H-like modes, i.e., modes with the only H-component along $z$ [6], that are in the photonic bandgap. Figs. $3 \mathrm{~b}$ and $3 \mathrm{c}$ show the corresponding $\mathrm{H}$-fields of these localized Bloch states (i.e. the guided modes) at the normalized wave-vector value of 0.35. Based on these field plots, the modes are identified as odd (antisymmetric) and even (symmetric), with respect to the centre-line of the waveguide. For practical reasons, related to coupling light into a $\mathrm{PhC}$ structure using single-mode channel waveguides, the even mode is preferred. As seen from Fig. 3a, single mode operation at telecom wavelengths (e.g. 1530-1565 nm) requires a small period (of the order of $400 \mathrm{~nm}$ ). From the fabrication point of view, the small period is a challenge and a number of ways to make the W1-type waveguide single mode and/or with improved dispersion profile have been proposed. Some of these designs and their properties are discussed in detail in e.g. [7]. In general the improved designs are based on reducing the effective index of the $\mathrm{PhC}$ waveguide by reducing the width or the index of the core region.

\section{Losses in $\mathrm{PhC}$ waveguides}

Fig. 4 shows the transmission spectra of three different photonic crystal waveguides. The spectra have been normalized with respect to the measured transmission spectrum of a 
photonic wire waveguide, with a cross-section of $500 \mathrm{~nm}$ (width) by $220 \mathrm{~nm}$ (height). Typical propagation loss of such a waveguide is $0.24 \pm 0.16 \mathrm{~dB} / \mathrm{mm}$ [7].

The PhC WG1 is a W1-type waveguide with a slab hole diameter of $270 \mathrm{~nm}$. The $\mathrm{PhC}$ WG2 and WG3 are W1-type waveguides with increased boundary hole diameter (BHD), see section 3.1.2.2. The PhC WG2 has a slab hole diameter of $250 \mathrm{~nm}$ and BHD of 330 $\mathrm{nm}$ and the PhC WG3 has a slab hole diameter of $255 \mathrm{~nm}$ and BHD of $340 \mathrm{~nm}$. All three $\mathrm{PhCs}$ have the same period, $440 \mathrm{~nm}$, and the same length, 24 periods.

It can be seen that the additional loss of these straight $\mathrm{PhC}$ waveguides compared to a conventional waveguide is about $5 \mathrm{~dB}$ with increasing trend near the mode cut-off wavelength. At a wavelength of $\sim 20 \mathrm{~nm}$ below the mode cut-off the loss is $14 \mathrm{~dB} \pm 2 \mathrm{~dB}$. The loss near the mode cut-off is high due to slow light propagation [8-9].

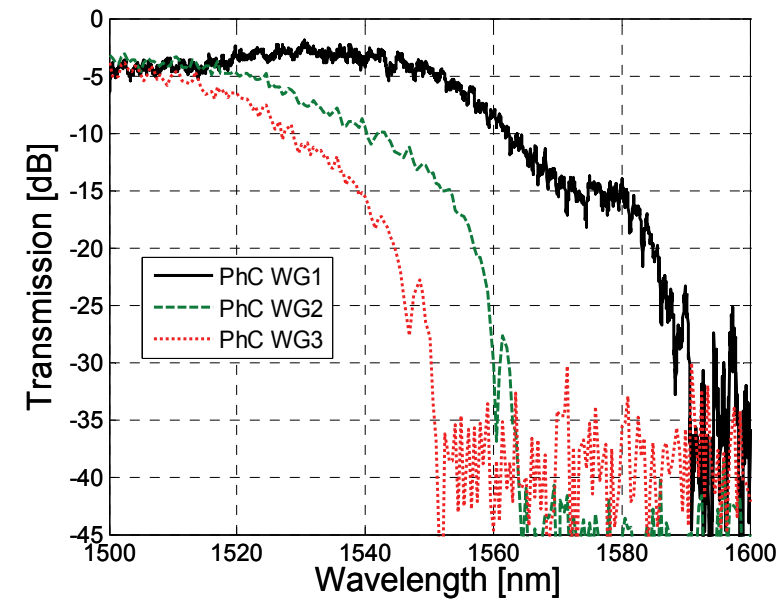

Fig. 4. The transmission spectra of three different photonic crystal waveguides (see text).

\section{Photonic crystal cavity}

An example of another interesting defect-type is shown in Fig. 5. It is an isolated defect that can act as a resonant optical cavity. Photons having a wavelength inside the PBG of the crystal and matching a resonant mode of the defect, are trapped inside this cavity in the $x-y$-plane by the surrounding crystal structure and in the $z$-direction due to TIR. Unlike the case of $\mathrm{W} 1$ defects, where a continuity of modes exists over a certain wavelength range, a cavity only supports certain discrete modes. Light can be coupled from a waveguide (e.g. W1) to the cavity, by locating the waveguide close to the cavity, separated by just a few holes, so that there is some (generally small) overlap between the evanescent fields of waveguide and cavity modes. The resonating cavity mode has to interfere constructively with the input light that feeds the energy to the cavity. As the cavity loss and out-coupling increase with the stored energy, at some point a steady state is achieved where the power fed into the cavity equals the lost power. In the steady state for a given input power a constant amount of electromagnetic energy is stored in the cavity. This amount can be calculated from the so-called Q-factor, which is defined as the ratio of the time-average energy stored at a resonance frequency to the energy dissipated in one period of this frequency. 


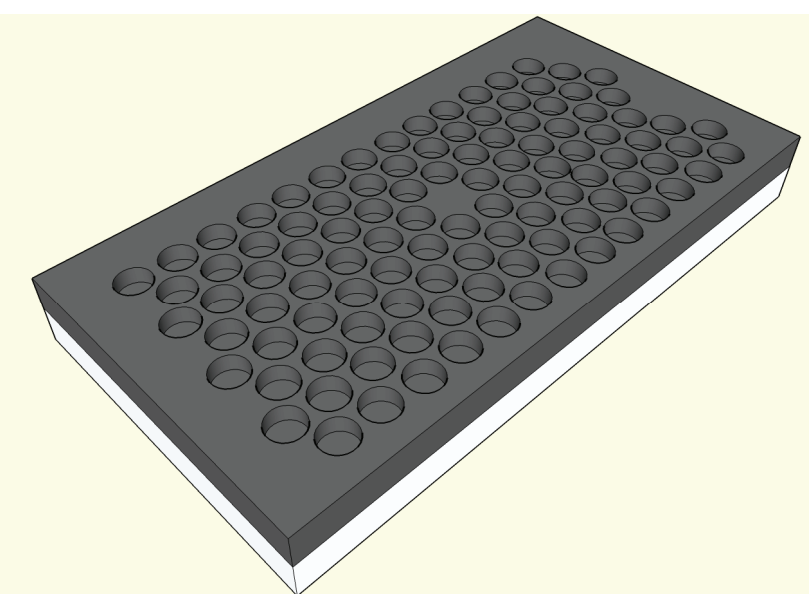

Fig. 5. Point defect in a 2D slab photonic crystal.

One of the key advantages of cavity resonators in a $\mathrm{PhC}$ is that they can be made compact. However, as the cavity size gets smaller, the losses increase. This can be explained by decomposing the resonant mode into its spatial Fourier components (i.e. plane waves). A large number of such plane wave components are needed for a strongly confined mode in a small cavity (e.g. below the wavelength). Losses arise when such components can couple to freely propagating plane waves in the cladding regions above and below the slab. In order to prevent this coupling, all the plane wave components should fulfill the TIR condition. If the absolute value of the tangential component of the $k$-vector of the plane wave is between 0 and $2 \pi / \lambda$, the light leaks from the surface of the microcavity to radiation modes. In order to obtain a low-loss, high-Q cavity, the amount of light radiating from the surface should be minimized. This can be accomplished by designing the cavity structure in such a way that the number of plane wave components is minimized and that there are no tangential components in the range from 0 to $2 \pi / \lambda[10]$. The shape of the cavity can be tuned, e.g. by shifting the position of the holes around the cavity. Shifting the position of the holes (in a $\mathrm{PhC}$ ) requires special accuracy in the fabrication process, as the typical shift is only a few tens of nanometers. As the shape of the cavity is tuned to obtain the maximum Q-factor, also the resonance wavelength is tuned. For these reasons it is challenging to fabricate a high-Q cavity operating at a specific wavelength for a given PCS; both the Q-factor and the resonance wavelength of such cavities are extremely sensitive to the process variations, which may cause serious problems with reproducibility.

Microcavities can be used in several applications. The development of cavities in compound semiconductor materials (such as InGaAsP) is motivated by their application for low-threshold (nano)lasers. The wavelength-selectivity of microcavities makes them interesting for application in ultracompact wavelength drop filters in optical network applications. In this configuration the cavity is placed in the proximity of the $\mathrm{PhC}$ waveguide. Light at a resonant wavelength is coupled to the cavity and can then be further coupled to the drop (output) channel.

Several coupled microcavities, arranged in line, form a special type of waveguide, where light will propagate by tunneling from cavity to cavity at low group velocity propagation. By exploiting the rotational symmetry of the field inside each cavity, very sharp bends 
can be achieved with this kind of waveguide [11]. The disadvantage is that this type of waveguide operates only for a narrow wavelength band.

\subsubsection{Connecting to the $\mathrm{PhC}$ device}

The PhC structure has to be connected to the outside world somehow. In the most common case waveguides are used. Due to the fundamental difference in light guiding properties, the coupling of a conventional waveguide mode to a $\mathrm{PhC}$ waveguide mode introduces coupling loss. Efficient coupling schemes (discussed e.g. in [12-13]) have been developed that considerably reduce the coupling loss.

\subsubsection{Photonic-crystal-cavity-based channel drop filter}

This section introduces design principles for PhC-cavity-based channel drop filters. Computer simulations have been performed in order to obtain device parameters that on the one hand would give the wavelength selective channel drop performance and on the other hand would result in a device that can be realized on the selected fabrication platform. Two variations of the device have been designed; one optimized for performance and one with better tolerance to fabrication process variations. In a discussion section our design is compared to similar designs reported in the literature. The section ends with a presentation of the experimental results of the $\mathrm{PhC}$ channel drop device.

\subsubsection{Introduction}

A schematic picture of the cavity-based channel drop filter is shown in Fig 6. In the ideal case, the light of a specific wavelength, propagating along the bus channel is transmitted to the drop port (out2) while the light at the other wavelengths continues traveling through the bus channel to output port out 1 . The cavity should be designed such that the power at the drop wavelength is fully coupled to the drop port. A fundamental challenge is that if the cavity supports only one mode, it decays the power to all the possible output ports. However, with an appropriate design $100 \%$ of the input power, at a resonance wavelength of the cavity, can be dropped from the bus channel to the drop port [14-15].

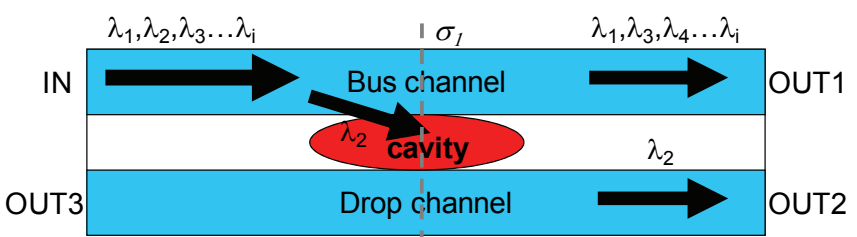

Fig. 6. A Schematic picture of a cavity-based wavelength selective channel drop filter.

This kind of performance requires (quasi)degenerate resonant modes with different field symmetries [14-16] at the operation wavelength. These resonant modes should have opposite symmetry with respect to the mirror plane centered on the cavity and perpendicular to the waveguides ( $\sigma_{l}$ in Fig. 6). The odd resonant mode excites forward and backward traveling guided modes in the $\mathrm{PhC}$ waveguides with $\pi$ phase shift, whereas the even resonant mode excites them in phase, thus causing destructive interference for the backward traveling waves [15-16]. 
It is possible to achieve such a complete directional channel dropping by applying a design of two coupled cavities or a design based on single cavity. The two cavity approach requires careful design work to balance the direct and indirect coupling rates between the cavities [14]. As pointed out in [15] the single-cavity-type of design has a clear benefit as the requirements to fabricate two cavities with exactly tailored structure is more challenging. For this reason, we have chosen to focus on channel drop filter designs that are based on a single cavity. In our design work, we have followed the principles described in [15] and [16] to design photonic-crystal-cavity-based channel drop filters.

\subsubsection{The design and modeling of a W1-type cavity}

The PhC structure under study, shown in Fig. 7, consists of two symmetrically arranged modified W1-type waveguides, separated by 7 rows of holes, with a cavity in between. The diameters of the boundary holes adjacent to the waveguides are made larger than the rest of the slab holes, in order to obtain single mode operation [17] and to adjust the dispersion curves for the cavity cross-section such that the desired wavelength drop performance is achieved. The cavity is formed by omitting a row of holes at the center of the structure between the waveguides, as shown in Fig. 7. In addition the boundary holes around the cavity are made smaller than the slab holes in order to adjust the dispersion properties of the structure as will be discussed below.

As suggested in [15] the wavelength dropping properties of this type of $\mathrm{PhC}$ structure can be analyzed by calculating the dispersion characteristics of the structure. They can be obtained by computing definite frequency eigenstates of Maxwell's equations using the MIT Photonics Band (MPB) package [5]. For computational efficiency, a so-called supercell approach is used, which assumes that a sub-structure (the supercell, shown as a slice in Fig. 8b) is repeated periodically. In this calculation, which cannot directly model the finite-length resonator, the cavity defect is treated as an intermediate waveguide.

The diameter of the holes surrounding the cavity can be chosen such that the cavity supports the quasi-degenerate modes needed for directional channel dropping. Based on MPB calculations we have chosen a 440-nm lattice period, a 200-nm diameter for the cavity boundary holes, and a 340-nm diameter for the boundary holes around the waveguides. The remaining "interior" holes, further denoted as "slab holes" have a diameter of $270 \mathrm{~nm}$. The calculated dispersion graphs of the structure are shown in Fig. 8a. The slow-light-regime of the intermediate waveguide section (that forms the cavity) provides the desired dispersion characteristics, showing bimodal behavior over an extended frequency range. The requirement for opposite symmetry with respect to the plane perpendicular to the propagation direction $\left(\sigma_{1}\right)$, implies that the propagation constants of the modes should differ by $(2 q+1) \pi / L_{\text {eff }}[15]$, in which $q$ is an integer and $L_{\text {eff }}$ is the effective length of the cavity. The symmetry with respect to the mirror plane parallel to the propagation direction $\left(\sigma_{2}\right)$, determines whether the dropped power propagates to the forward or backward direction [15]. 


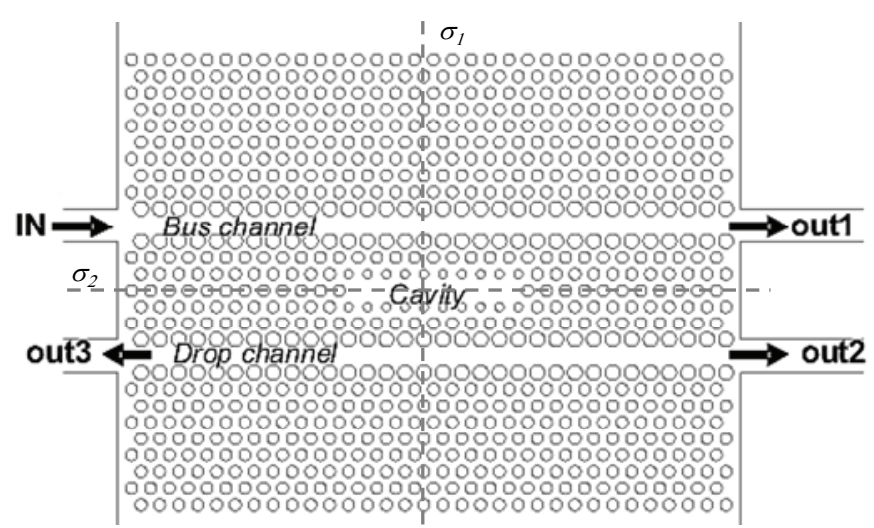

Fig. 7. A schematic picture of the PhC-cavity-based channel drop filter. Mirror plane perpendicular to propagation direction $\sigma_{l}$, mirror plane parallel to propagation direction

$\sigma_{2}$.
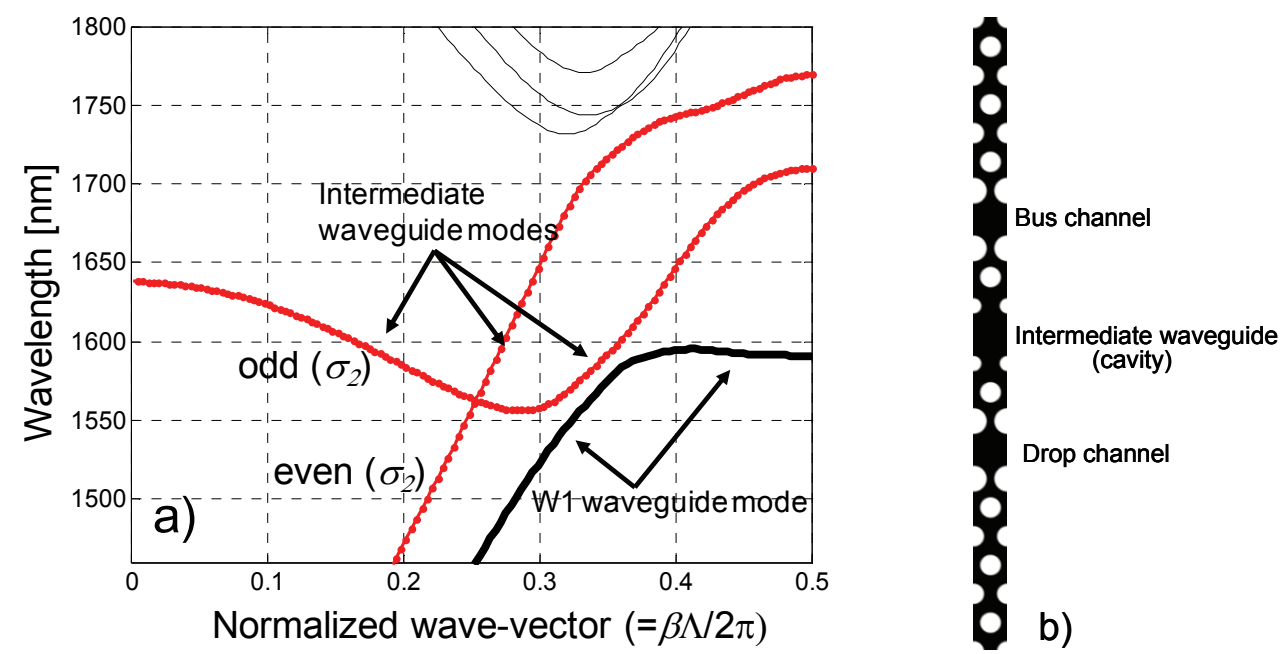

Fig. 8. a) The dispersion graph of the PhC channel drop device, calculated with MPB [5] using the supercell method. b) The supercell structure that is used to calculate the dispersion properties of the channel drop filter device in the cavity region.

The dispersion characteristics do not guarantee the operation in quasi degenerate regime, as depending on the effective cavity length $\left(L_{e f f}\right)$, different modes are supported by the cavity [15]. In order to find the correct cavity length, we performed 2D FDTD simulations by varying the cavity length by integer multiples of the lattice constant, as this changes the possible resonance wavelengths that are supported by the cavity, and hence its free spectral range.

The simulated transmission spectrum of a reference structure that has no cavity is shown in Fig 9a. Most of the light is transmitted to the out 1 port, while very little light is leaked to the out 2 and out 3 ports of the drop channel. The loss $(\sim 25 \%)$ is due to non optimal coupling of the waveguides to the PhC structure, see section 3.1.1.

Based on the graph shown in Fig. 8 and on the required wave-vector difference $\left((2 q+1) \pi / L_{\text {eff }}\right)$, we considered a cavity with a physical length $\left(L_{c a v}\right)$ of 10 holes as an initial starting point. Fig. $9 \mathrm{~b}$ shows the simulated transmission spectrum of this cavity. 
Two resonance modes can be seen around a wavelength of $1565 \mathrm{~nm}$. The two cavity modes are too far apart (ie. at different wavelengths) for achieving complete directional dropping. In other words, the degeneracy of these modes is poor, and approximately $20 \%$ of the input power is dropped to the out 2 and out 3 ports. Fig. 9c shows the transmission spectrum of the cavity with $L_{c a v}=11$ holes. The degeneracy is now better than for $L_{c a v}=$ 10 holes. However, two resonance modes are still too far apart; a broader one at $\lambda=1564$ $\mathrm{nm}$ and narrower at $\lambda=1566.5 \mathrm{~nm}$. The broader peak couples $\sim 20 \%$ of the input power to both out 2 and out 3 ports, while the narrower one couples $40 \%$ to the out 2 .

It turns out that $L_{c a v}=9$ holes gives the desired result, as shown in Fig. 9d. The quasidegeneracy of the modes occurs and a directional dropping with better than $95 \%$ efficiency to the out 2 port is obtained. Further fine tuning of the drop efficiency (by fine tuning the cavity length) can be done by either changing the hole diameters at the ends of the cavity or by shifting the hole positions slightly.

We have simulated the transmission spectra for cavity lengths down to $L_{c a v}=4$ holes. None of the cavities shorter than 9 holes showed complete channel dropping. It is likely that some cavity length longer than $L_{c a v}=11$ holes would show a quasi-degenerate resonance state. However, the $L_{c a v}=9$ holes provides the most compact design for the chosen parameters.
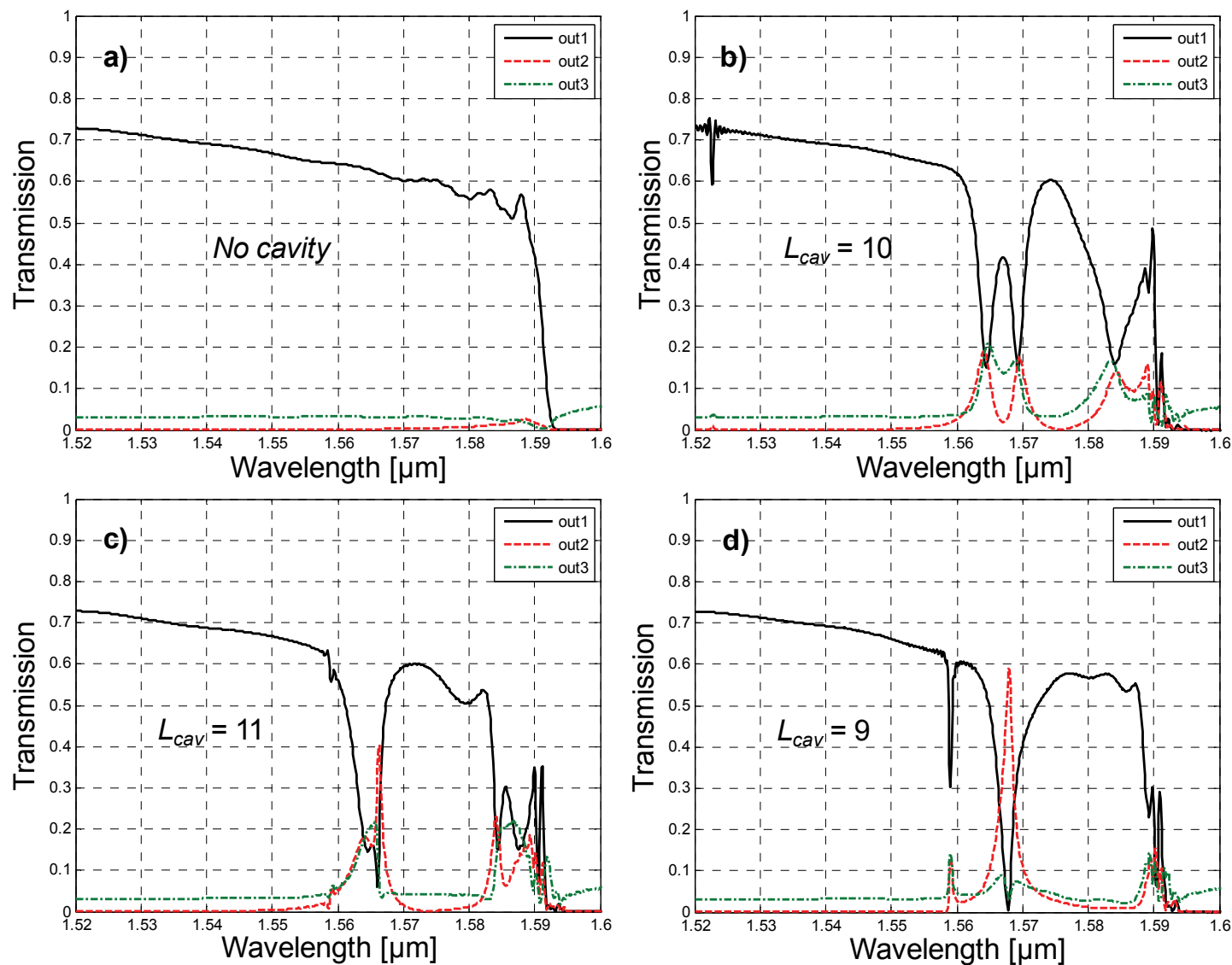

Fig. 9. Simulated (2D FDTD) transmission spectrum of the PhC channel drop filter (Fig. 2). a) Structure without cavity. b) Cavity length of 10 (omitted) holes. c) Cavity length of 11 holes. d) Cavity length of 9 holes. 


\subsubsection{The design and modeling of a W3-type cavity}

In the deep UV lithography process [18] feature size depends on the exposure dose. The features do not have the same optimal dose (the exposure dose required to get the desired size), and particularly for holes the optimal dose depends on the hole diameter. For this reason a PhC structure relying on a precise variation of hole diameters is a critical design from a fabrication point of view.

Taking this into account we have made a variation of the $\mathrm{PhC}$ structure discussed in the previous section. The period of the $\mathrm{PhC}$ remains at $440 \mathrm{~nm}$, while the slab hole diameter is reduced to $240 \mathrm{~nm}$ and the waveguide-boundary hole diameter to $330 \mathrm{~nm}$. The structural difference from the $\mathrm{PhC}$ device discussed in the previous section, is that the cavity is different; 3 rows of holes are omitted, and the cavity-boundary holes -which critically affect the cavity tuning- are the same as the slab holes. The length of the central row is 9 holes, while the two other rows are 10 holes long. The cavity is separated from the bus and drop channels by 2 rows of holes. A schematic picture of this $\mathrm{PhC}$ device is shown in Fig. 10.

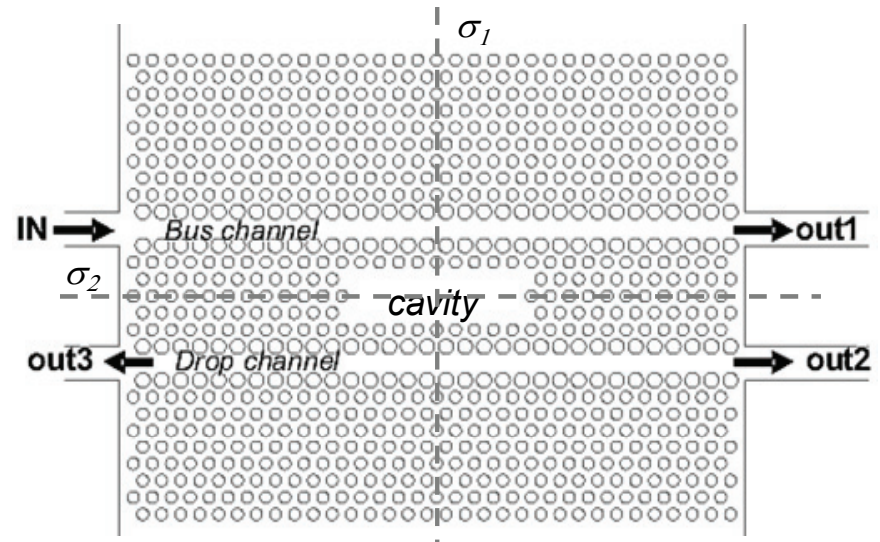

Fig. 10. A schematic picture of the PhC-cavity-based channel drop filter.

The dispersion properties of this device have been calculated in the same way as in the previous section. Fig.s $11 \mathrm{a}$ and $\mathrm{b}$ show the dispersion graph of the structure and the corresponding supercell for which the graph is calculated. We recognize several modes that are within the photonic band gap $(1430-1700 \mathrm{~nm})$. In this design a change in hole diameter changes the dispersion characteristics and therefore also the wave-vector distances between the modes are changed. For a certain hole diameter the difference is expected to be (an odd multiple of) $\pi$ for wave-vectors $\beta_{1}\left(\omega_{\text {res }}\right)$ and $\beta_{2}\left(\omega_{\text {res }}\right)$, where $\omega_{\text {res }}$ is the resonance frequency of the cavity and $\beta_{1,2}$ are the wave-vectors of two modes that are localized in the intermediate waveguide (and that will become degenerate cavity modes). 

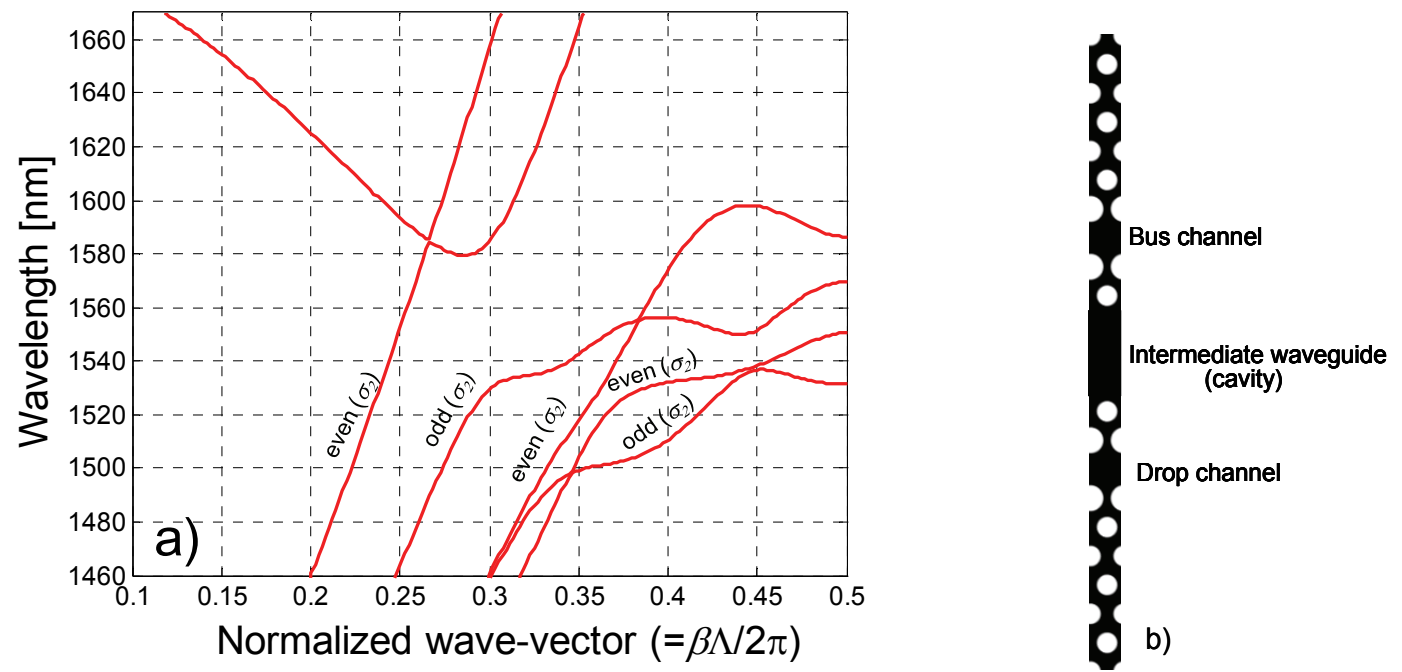

Fig. 11. a) The dispersion graph of the PhC channel drop device calculated with MPB using supercell method. b) The supercell structure that is used to calculate the dispersion properties of the channel drop filter device in the cavity region.

Based on the dispersion characteristics of the structure we made an initial guess for the hole diameters and fixed the cavity length (to 9 missing holes). Then a series of FDTD simulations have been performed by slightly changing the hole diameters around the initial guess values till the hole diameters are found that give the complete channel drop (for some wavelength) from input port to out 2 port.

Fig. 12a shows a result of such a simulation run. For the simulated structure a slab hole diameter of $240 \mathrm{~nm}$ and BHD of $330 \mathrm{~nm}$ were used. As a slab effective index we used 2.70 , as this index should better match the $2 \mathrm{D}$ simulation results with the full $3 \mathrm{D}$ results [7] than the index obtained with the effective index method. At a wavelength of $1507 \mathrm{~nm}$ the device clearly shows the desired channel drop operation; the light at this wavelength is fully coupled to the out 2 port. Unfortunately, the light at wavelengths larger than 1507 $\mathrm{nm}$ do not propagate undisturbed, but are affected by the cavity. Fig. 12b shows a snapshot of a continuous wave (CW) 2D FDTD simulation at a wavelength of $1507 \mathrm{~nm}$. The H-field distribution (Fig. 12b) clearly shows the complete channel drop operation. 

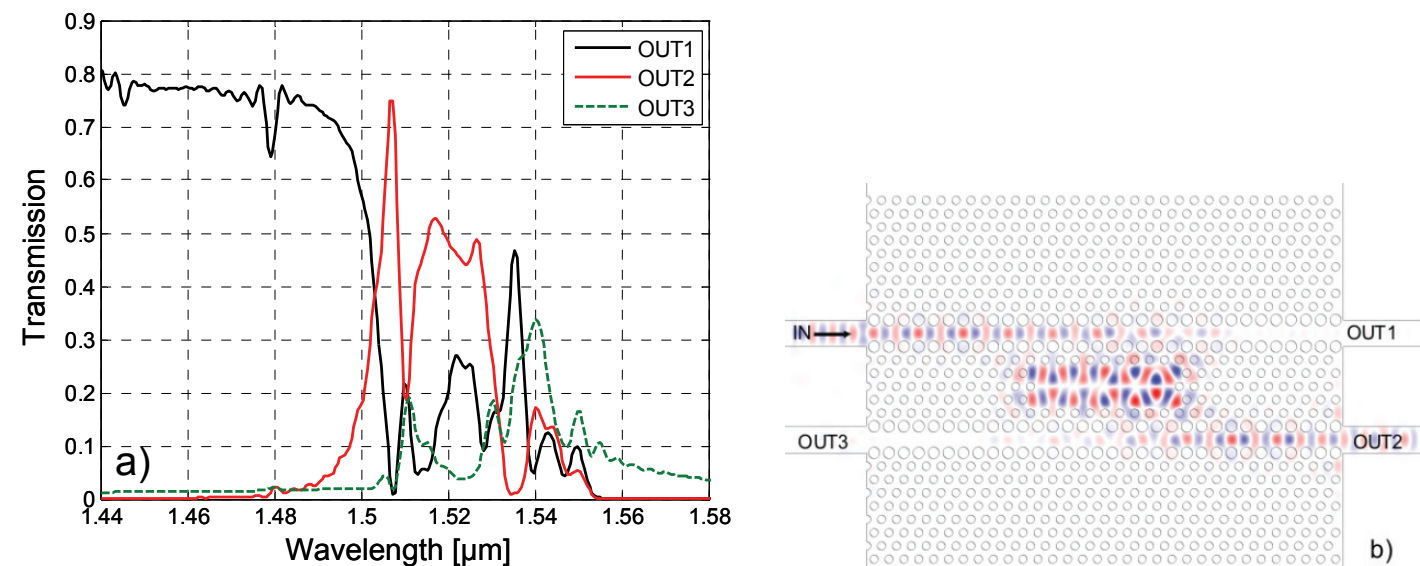

Fig. 12. 2D FDTD simulated transmission spectrum of the device. b) Simulated snapshot image of the Hfield profile at a wavelength of $1507 \mathrm{~nm}$.

Although this design provides a less ideal channel drop filter response than the design presented in previous section, it is recognized to be much easier to realize as it is less vulnerable to fabrication process variation than the design which includes the small holes around the cavity.

\subsubsection{Discussion}

The devices reported [15] and [19] are similar to our PhC channel drop filter device. In [15] 11 rows of holes separate the bus and drop channels, and there are two rows of small holes on both sides of a W1-type cavity. The optimum cavity length of the device is reported to be 20 holes. From the fabrication point of view, this design has some drawbacks. The diameter of the boundary holes adjacent to $\mathrm{W} 1$ waveguides is large in comparison to the period and the Si feature between the holes would be only $80 \mathrm{~nm}$. This is below the feature size limit of the $193 \mathrm{~nm}$ DUV. An additional challenge might come from the change in location of the small holes around the cavity. Due to the different relative locations of these holes, the target exposure dose to produce the desired hole diameter might be different for these rows.

A $\mathrm{PhC}$ channel drop device with a cavity length of 10 holes is reported in [19]. This device has 7 holes between the bus and drop channels, while the desired dispersion properties are obtained by narrowing the bus and drop channels to W0.65-type waveguide designs. While this design has the benefit of uniform hole diameter, the W0.65 waveguide design poses limitations as e.g. realizing bends in a W0.65 waveguide is difficult as they easily lead to discontinuities [7].

\subsubsection{Experimental: W3-type cavity}

Fig. 13 shows a SEM image of a DUV-fabricated PhC W3-type-cavity-based channel drop filter. Two versions of the device were fabricated; one with a waveguide boundary hole diameter (BHD) of $320 \mathrm{~nm}$ and the other with a BHD of $330 \mathrm{~nm}$. The slab hole diameter for both devices is approximately $240 \mathrm{~nm}$. Fig.s $14 \mathrm{a}$ and $14 \mathrm{~b}$ show the measured transmission spectra of the channel drop devices, normalized with respect to the 
transmission of a straight $\mathrm{W} 1$ waveguide of equal dimensions. Due to measurement noise, the transmission seems to be (unphysically) slightly above $0 \mathrm{~dB}$ in some regions.

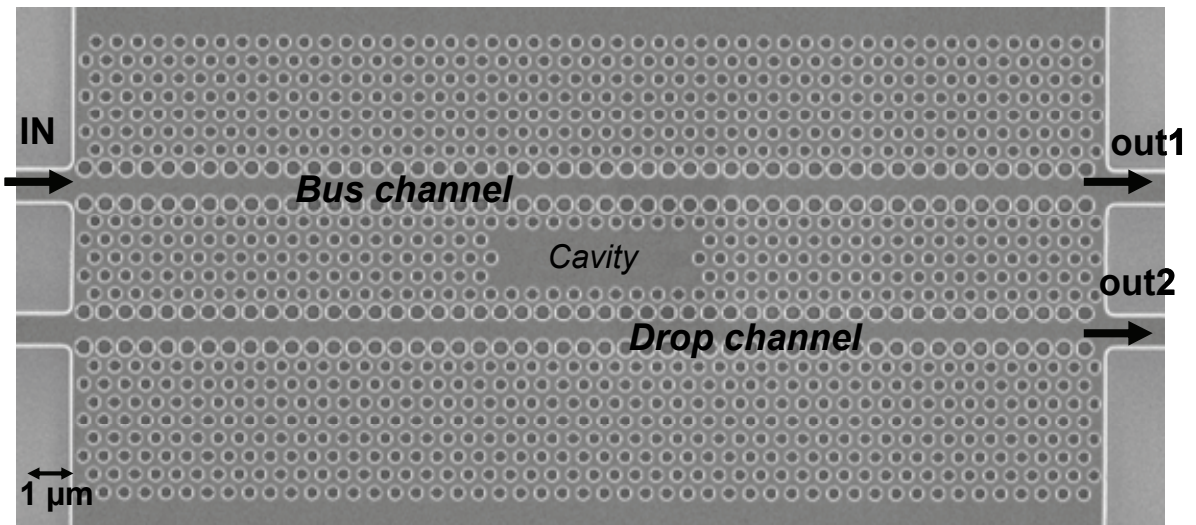

Fig. 13. SEM image (top view) of a PhC channel drop filter.

As seen from the measured transmission spectra, both channel drop filters have a drop wavelength of $1561 \mathrm{~nm}$. For a design with BHD of $330 \mathrm{~nm}$ the on/off ratio between ports out 1 and out 2 is higher; $16 \mathrm{~dB}$ in comparison to $10 \mathrm{~dB}$. The full width at half maximum of the drop band is approximately $2.5 \mathrm{~nm}$ for both designs. Fig. 14a also shows the transmission spectrum of a reference device without a cavity but otherwise similar. From this reference curve (OUT2 Ref.), it can be seen that the device suffers from considerable cross talk at wavelengths longer than $1570 \mathrm{~nm}$. Therefore, the device should not be operated at wavelengths larger than the drop wavelength. The device with $320 \mathrm{~nm}$ BHD appears to have less crosstalk outside the drop band and can be operated at slightly broader wavelength range. Based on the measurements, the cut-off wavelength of the bus channel waveguide for both devices is between $1600 \mathrm{~nm}$ and $1610 \mathrm{~nm}$.
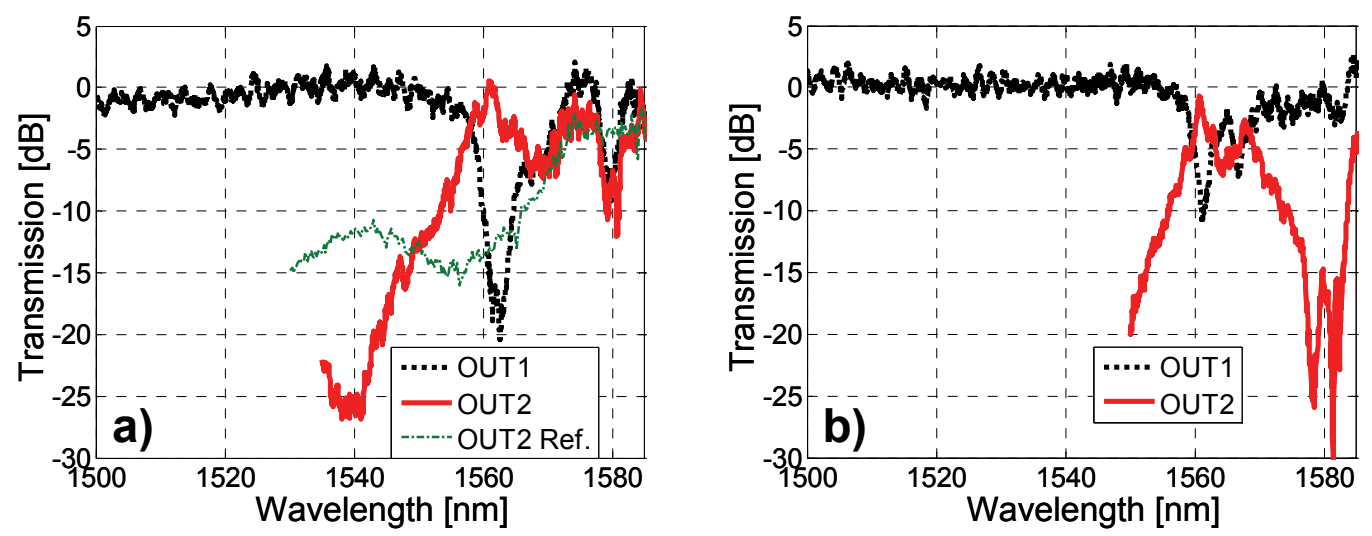

Fig. 14. Measured transmission spectra of channel drop filters from the in to the out 1 and out2 ports. a) device with $330 \mathrm{~nm}$ boundary hole diameter (BHD). Also, shown a reference curve (see text). b) device with $320 \mathrm{~nm}$ BHD. 


\subsubsection{Conclusions}

Based on computer simulations PhC cavity -based channel drop filters have been designed. These line defect devices require well chosen parameters for complete channel drop operation (100\% drop efficiency). Requirements for the fabrications process variables (such as exposure dose) are high. A design that requires only two different hole diameters turned out to be realizable, though with less ideal spectral properties as the design based three different hole diameters, that according to simulations provides nearly ideal channel drop filter response. These $\mathrm{PhC}$ devices suffer from high optical loss, particularly at wavelengths near the mode cut-off, where the loss is approximately $14 \mathrm{~dB}$, as discussed in section 3.1.1.2.

\subsubsection{Photonic-Crystal-Cavity-based Y Splitter}

We propose a Y splitter in a 2D photonic crystal with a triangular lattice of air holes suitable for mechano-optical switching. Full switching between the two output ports can be achieved at a certain wavelength owing to the presence of a resonant central cavity with well-chosen symmetry.

Experimental results confirm the operation of the device and its potential as an ultracompact mechanical switch.

\subsubsection{Introduction}

One of the basic components in optical networks is the Y splitter. Several ultracompact Y splitter designs in two-dimensional (2D) photonic crystals $(\mathrm{PhC})$ have been reported [2022]. PhC-based switches are particularly attractive due to their compactness and large $\mathrm{Y}$ splitting angle.

We propose a Y-splitter in a 2D photonic crystal slab with a triangular lattice of air holes, as shown in Fig. 15.

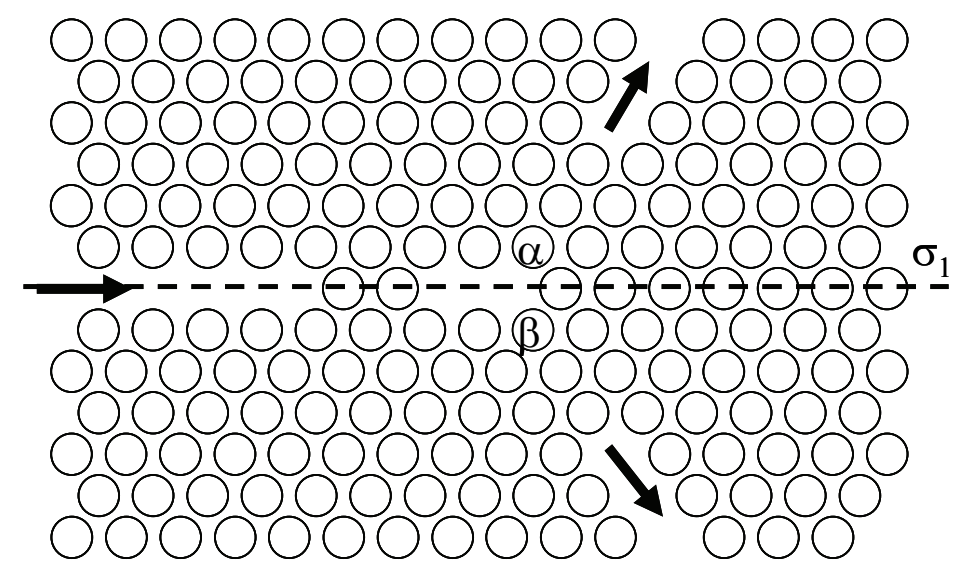

Fig. 15. The proposed PhC-cavity-based Y splitter. The holes marked with $\alpha$ and $\beta$ show the positions of the switching holes. By selectively filling the holes, different cavities (and therefore different coupling conditions) between the output ports are obtained. The symmetric cavity (both $\alpha$ and $\beta$ filled, or both empty) splits the light equally to the output ports, whereas the asymmetric cavity ( $\alpha$ or $\beta$ filled) couples light to only one of the ports.

This section has been published in: L.J. Kauppinen, T.J. Pinkert, H.J.W.M. Hoekstra, and R.M. de Ridder, Photonic Crystal Cavity-Based Y Splitter for Mechano-Optical Switching, IEEE Photonics technology letters, vol. 22, issue 13, pp. 966-968, 2010. 
By selectively filling the holes, labeled $\alpha$ and $\beta$ in Fig. 15, different cavity geometries are formed at the Y-junction. Filling both holes $\alpha$ and $\beta$ with the same material results in a symmetric cavity with respect to the $\sigma_{1}$ axis (see Fig. 15), whereas filling either hole $\alpha$ or $\beta$ an asymmetric cavity with respect to the $\sigma_{1}$ axis is obtained. As will be explained below, in case of such an asymmetric cavity and for a specific resonant wavelength, the light is coupled to only one of the output ports, whereas in the symmetric case the device acts as a Y splitter and the light is coupled equally to both output waveguides.

In the envisaged mechano-optical switch application, the signal output at the operating wavelength of the splitter device can be dynamically switched between the output ports by selectively filling holes $\alpha$ and $\beta$.

\subsubsection{Design and modeling}

The envisaged tuning of our device is based on selecting the drop port, via a nanoelectromechanical system, by changing the symmetry of the structure and hence the spatial field symmetries in the resonator. Such an operation can be achieved e.g. by perturbing the structure with an AFM tip [23-24]. Fig. 16 shows the PhC structure with its symmetry axes corresponding to the cases that both or none of the holes $\alpha$ and $\beta$ are filled $\left(\sigma_{1}\right)$, only hole $\alpha$ filled $\left(\sigma_{3}\right)$, and only hole $\beta$ filled $\left(\sigma_{2}\right)$. The operation of this kind of devices can be understood by group theoretical arguments [25-26], using that defects, in our case W1 waveguides and a multihole resonator, have the symmetry of a subgroup of the $\mathrm{PhC}$ point group (hereafter called group). Such a defect structure can support electromagnetic fields that have the properties of the irreducible representations of the group of the defect.

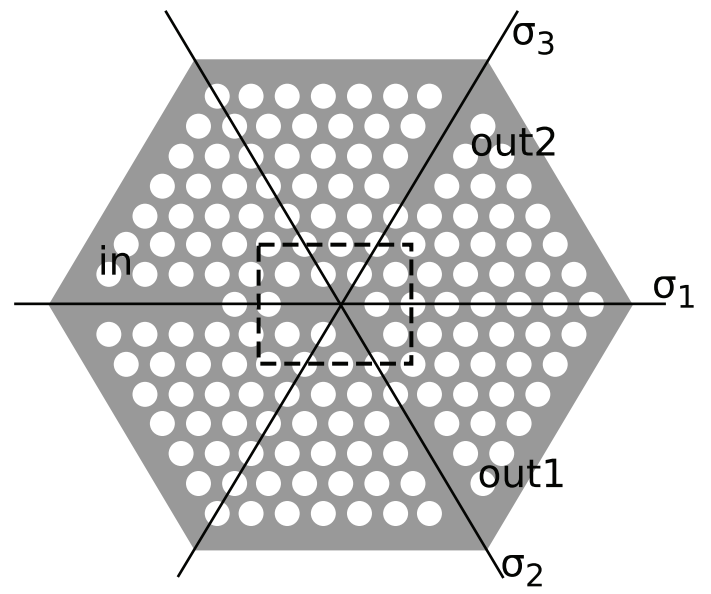

a

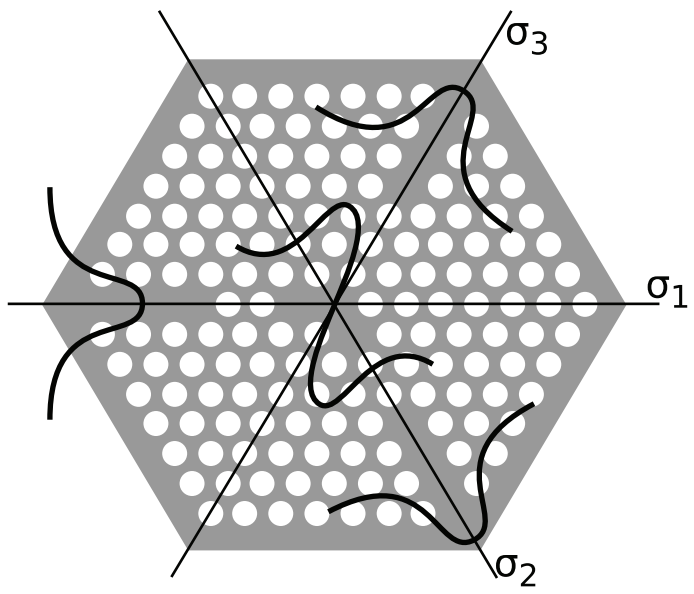

b

Fig. 16 Photonic crystal switch. a) The possible mirror symmetry axes of the design, the dashed rectangle indicates the resonator position; b) the $\chi=1$ fields of the photonic crystal waveguide and $\chi=-1$ field of the defect show intuitively that light cannot be coupled from the resonator into the out 2 waveguide.

The defects of the proposed switching device (in both switching states) have the symmetry of the $\mathrm{C} 2 \mathrm{v}$ group, which means that symmetric $(\chi=1)$ and anti-symmetric $(\chi=-1)$ field solutions (with respect to the concerned symmetry axes) do not interact. In Fig. 16b some possible field symmetries are sketched. If, at a certain frequency, only field solutions with the sketched symmetry are supported by the waveguides and resonator, a coupling may exist only between the in- and outl-waveguide and not 
between the in- and out2-waveguide. The projection of the resonator field (antisymmetric with respect to the $\sigma_{3}$ axis) onto the fields of the waveguides shows that the in- and outl-waveguide have a field overlap with the resonator field, but the resonator field has no overlap with the field of the out 2 waveguide owing to their symmetries. Therefore the transmission of light to this waveguide is prohibited.

The dispersion curves and symmetries of the modes that are supported by the waveguide and resonator have been calculated with 2D eigenstate simulations (using the plane wave expansion method [27]) for the case of either the $\alpha$ or $\beta$ hole filled. The results are shown in Fig. 17a. We see that there are two frequency ranges in which only a $\chi=1$ mode is available for the $\mathrm{W} 1$ waveguide, and in these regions two resonator states with a $\chi=-1$ symmetry are present, which can be used to obtain switching behavior. Fig. $17 \mathrm{~b}$ gives the results from 2D finite-difference time-domain (FDTD) simulations [28] of the transmission of light through the device for the frequency corresponding to the 'operation point' (see Fig. 3a), which shows that this type of device can in principle work as a (wavelength routing) switch. For the simulations of Figs. 3a and $3 \mathrm{~b}$ we have used lattice parameter $\Lambda=440 \mathrm{~nm}$, hole radius $r=135 \mathrm{~nm}$ and a slab-effective refractive index 2.9, corresponding to $240 \mathrm{~nm}$ thick silicon on silica at a wavelength of $1550 \mathrm{~nm}$. The results are applicable to other Si layer thicknesses near this value and shift with respect to frequency as the corresponding effective index changes.

By making the cavity symmetric ( $\alpha$ and $\beta$ filled), we can obtain $3 \mathrm{~dB}$ splitting of light from the input to both output channels. In order to test the optical design, we fabricated both symmetric and asymmetric devices, which differ only by the absence or presence, respectively, of an additional hole in the outl branch, so that device operation in both the symmetric and the asymmetric state could be studied without mechanical actuation.

It is known that using the effective index method for 2D calculations of the properties of photonic crystal slabs leads to results that are red-shifted in frequency with respect to the results from full three-dimensional calculations [3]. By estimating the error in the effective index, scaling the results from Fig. 17, and keeping the $\Lambda / r$-ratio approximately the same, we arrived at parameters $\Lambda=420 \mathrm{~nm}$ and $r=128 \mathrm{~nm}$, in order to obtain an operating point at $1550 \mathrm{~nm}$ wavelength. The actual hole diameter depends on the lithographic exposure dose, and as an exposure dose sweep is performed in the fabrication process, we obtained structures with varying hole diameters; ranging approximately + and $-20 \mathrm{~nm}$ around the target value.

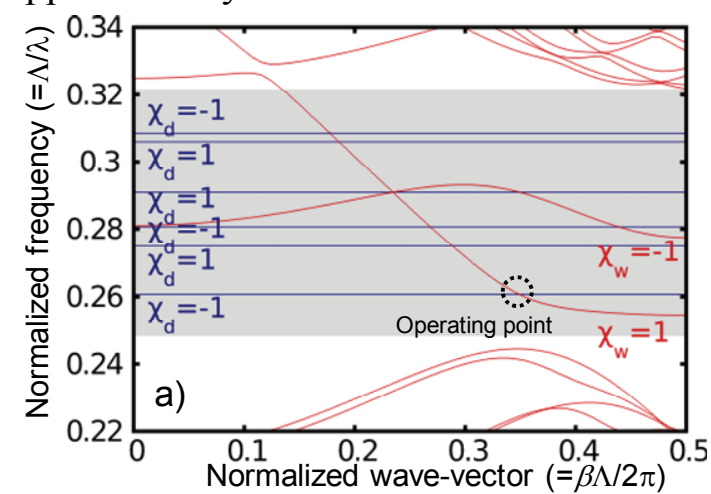

a)

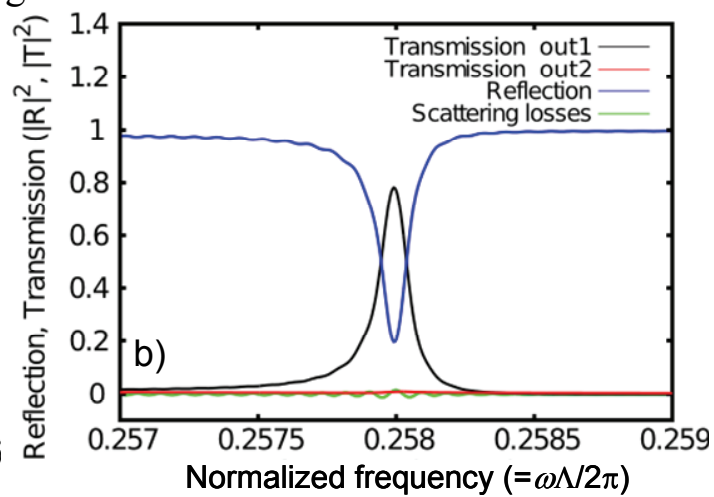

b)

Fig. 17. a) Dispersion relations of the photonic crystal waveguides. Also shown are the localized states of the cavity (horizontal lines) within the photonic band gap (grey band) of the bulk crystal. $\chi_{\mathrm{w}}$ and $\chi_{\mathrm{d}}$ give the 
Compact silicon photonic devices for MEMS-actuated optical switching

field symmetries for the waveguide fields and resonator fields, respectively. b) Reflection and transmission spectra of the device.

\subsubsection{Experimental}

The photonic crystal structures were fabricated in a 193-nm deep-UV lithography process by using the silicon photonics platform established at IMEC, Leuven. The device layer of the SOI wafer is a 220-nm thick silicon layer, with underneath it a 2- $\mu \mathrm{m}$ thick $\mathrm{SiO}_{2}$ layer. The PhC structure is formed by etching through the $\mathrm{Si}$ layer and stopped to the $\mathrm{SiO}_{2}$ layer. Fig. 18a shows a SEM image of one of the fabricated PhC structures. Light was coupled into the PhC device using a vertical fiber coupling method [29]. As a light source we used an Agilent 8164B tunable laser at an output power of $1 \mathrm{~mW}$. All the measured transmission spectra have been normalized with respect to a straight $\mathrm{Si}$ photonic wire waveguide, similar to the access waveguides (fiber-to-fiber). This removes the grating-coupler and access waveguide induced wavelength dependent loss $(16 \mathrm{~dB} \pm 2$ $\mathrm{dB}$ at the wavelength of maximum coupler transmission) from the data.

Fig. $18 \mathrm{~b}$ shows the measured transmission spectrum (hole $\beta$ filled) and the result of a corresponding 2D FDTD simulation. For the simulation we assumed a hole diameter of $250 \mathrm{~nm}$ (as estimated from the SEM image for the fabricated device) and an effective index of 2.82, while using a grid spacing of $10 \mathrm{~nm}$. The simulated curve has been fitted to the experimental data by shifting it in horizontal direction by $-23 \mathrm{~nm}$ and in vertical direction by $-7 \mathrm{~dB}$. The horizontal shift corresponds to effective index fitting, compensating the red shift caused by the effective index method. The vertical shift is due to out of plane losses, which the 2D simulation cannot account for. The typical loss of a W1 PhC waveguide of a corresponding length $(24 a)$, at a wavelength of $1560 \mathrm{~nm}, 20 \mathrm{~nm}$ below the mode cut-off (see the operating point indicated in Fig. 13a), is $14 \mathrm{~dB} \pm 2 \mathrm{~dB}$. Comparing this number to the maximum measured transmission, we conclude that the excess loss of the Y-junction is approximately $6 \mathrm{~dB}$, close to the 7-dB vertical shift mentioned above.

Further away from the mode cut-off, the $\mathrm{W} 1 \mathrm{PhC}$ waveguide loss is $5 \pm 1 \mathrm{~dB}$. Therefore a large part of the $\mathrm{Y}$-junction device loss at the operating point can be attributed to the slow light propagation in the $\mathrm{PhC}$ waveguide [9-10]. This can be seen from Fig. 13a: the slope of the $(\chi=1)$ dispersion curve of the $\mathrm{W} 1$ mode is decreasing from the operating point towards the mode cut-off. This in turn means that the group velocity of the light is moderately decreasing in this region, corresponding to increase of loss. 

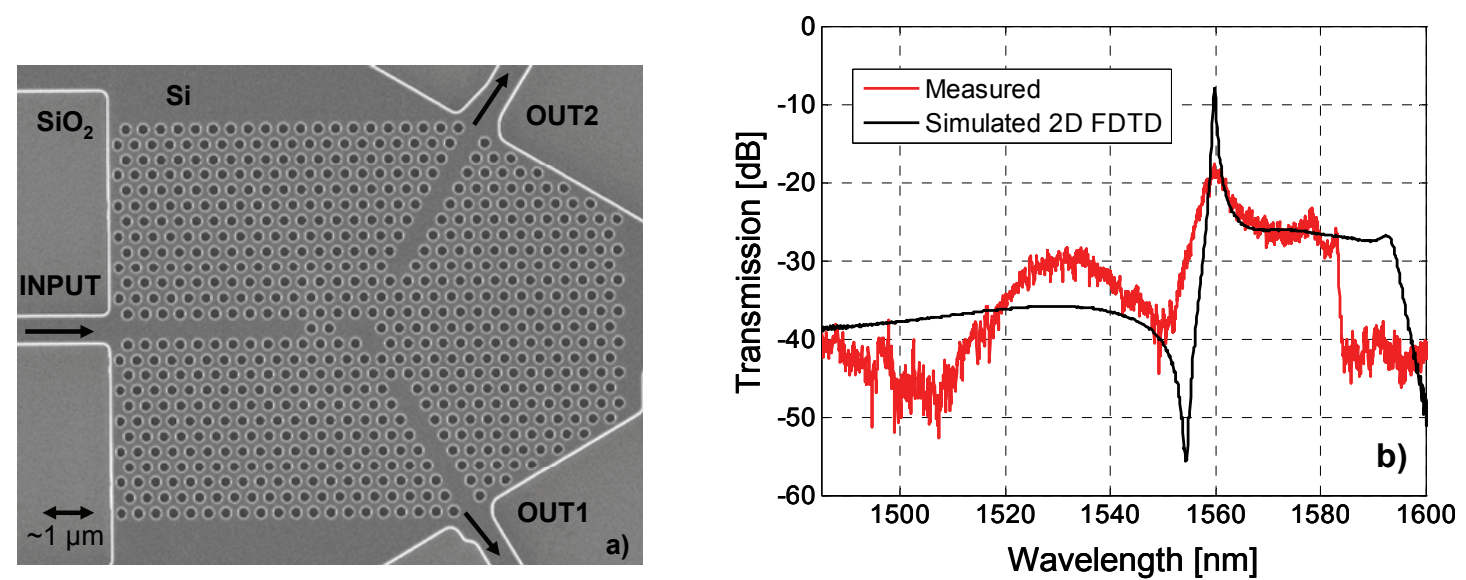

Fig. 18 a) SEM image (top view) of the PhC Y splitter. b) Measured and simulated transmission spectra for the asymmetric cavity design; transmission from the input to the outl waveguide branch, using the normalization procedure mentioned in the main text.

Fig. 19a shows a measured transmission spectrum of the asymmetric cavity (hole $\beta$ filled). It can be seen that at resonance most of the light is coupled through the cavity to the out1 waveguide branch, operating similar to a Fabry-Perot resonator. The on/off ratio at $1560 \mathrm{~nm}$ between ports outl and out2 is about $12 \mathrm{~dB}$. Fig. $19 \mathrm{~b}$ shows that the resonance wavelength is blue-shifted by approximately $13 \mathrm{~nm}$ for a $10 \mathrm{~nm}$ increase in hole diameter.

Fig.s 20a and 20b show the measured and simulated transmission spectra of the symmetric-cavity Y splitter in the optical C-band. In this case splitting of the optical power occurs in the cavity, and the light at a resonance wavelength is distributed between the output branches. We note that the design is very critical with respect to cavity symmetry, both experimentally and numerically. Even a slight deviation from the symmetric structure -which in our simulations was caused by a slightly misaligned FDTD calculation grid- was found to result in uneven coupling to the out 1 and out 2 ports.
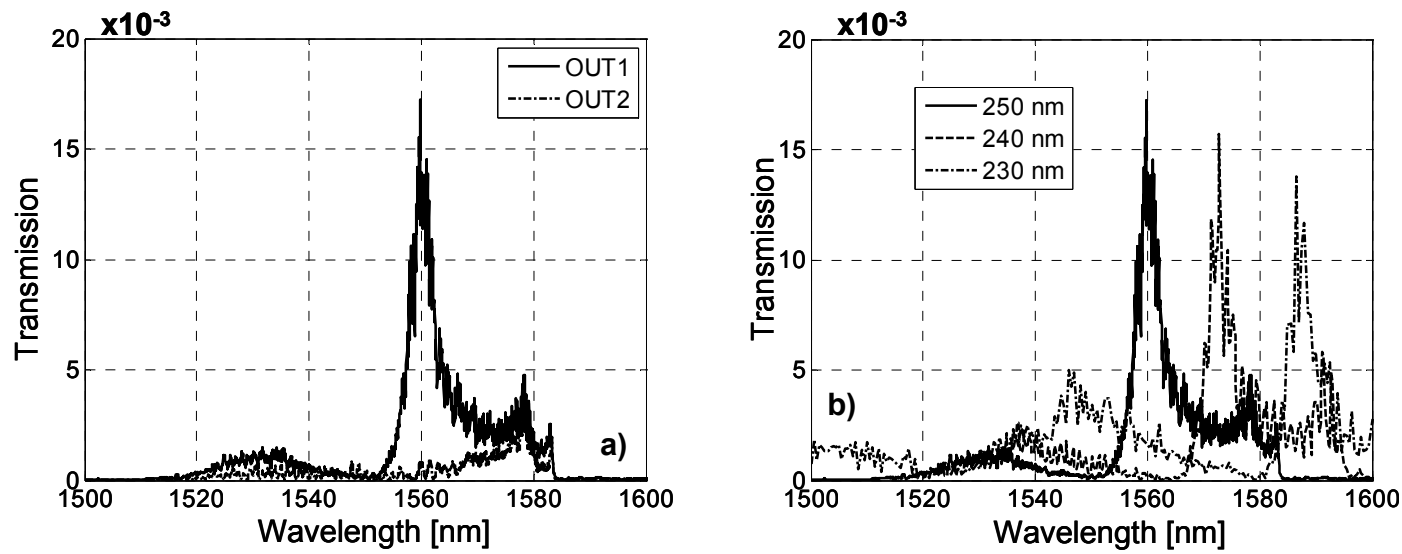

Fig. 19. Measured transmission spectra of the asymmetric cavity (with respect to the $\sigma_{1}$ axis). a) At out 1 and out 2 ports; b) at out 1 , for 3 different values of the $\mathrm{PhC}$ hole diameter. 

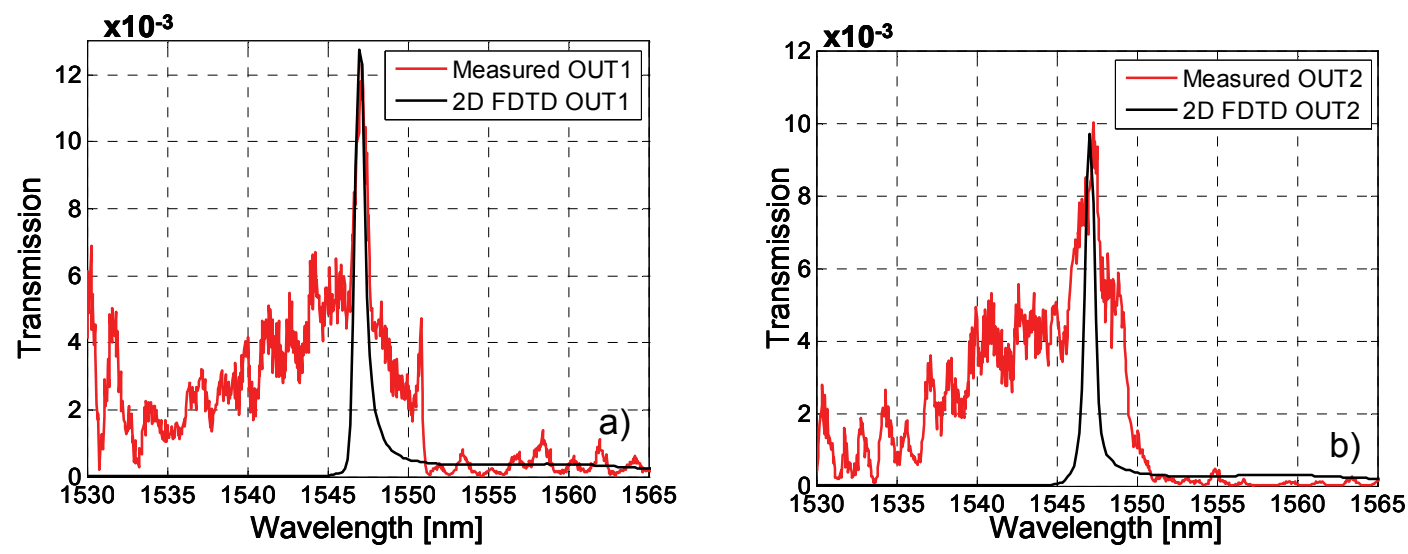

Fig. 20. Measured and simulated transmission spectra for the symmetric cavity (both holes filled). a) out1 port. b) out 2 port.

\subsubsection{Conclusions}

A photonic crystal cavity-based $\mathrm{Y}$ splitter has been designed for optical switching application. Its operation has been verified by simulations and experiment. By filling one of two specific holes with slab material, the symmetry of the device can be switched, thus selecting one of two output ports. A 12-dB on/off ratio between the output ports was measured. The optical losses of the device $(20 \mathrm{~dB})$, are assumed to originate mostly from the slow light effects, which cause increased out-of-plane scattering. It turns out to be challenging to achieve precisely symmetric light transmission between the output ports, since that requires the cavity to be perfectly symmetric. Therefore the device should preferably be operated as on/off switch in one of its asymmetric states.

\subsection{Waveguide grating and ring-resonator-based designs}

This section introduces the wavelength routing principles of waveguide grating and ringresonator-based designs. Both devices are based on directional coupling of light from one waveguide to the other one. A brief theoretical discussion on directional coupling is given. The schematic layouts of the devices are shown along with the measurement results.

\subsubsection{A directional coupler}

If the lateral separation between two waveguides is small, as in the middle section of Fig 21 , the light may couple between the waveguides. The coupling is based on the evanescent field tail that extends outside the waveguide boundaries and partially overlaps with the other waveguide. The energy can flow either way between the waveguides. The operation of the directional coupler can be theoretically described using coupled mode theory [30]; it is discussed in detail e.g. in [31]. The result of this analysis is that the following equation can be written for the modal field amplitudes of a symmetrical directional coupler: 


$$
\left[\begin{array}{l}
E_{o 1} \\
E_{o 2}
\end{array}\right]=e^{j \beta l_{c}}\left[\begin{array}{cc}
\cos \left(\kappa l_{c}\right) & j \sin \left(\kappa l_{c}\right) \\
j \sin \left(\kappa l_{c}\right) & \cos \left(\kappa l_{c}\right)
\end{array}\right]\left[\begin{array}{l}
E_{i 1} \\
E_{i 2}
\end{array}\right],
$$

in which $l_{c}$ is the (effective) length of the coupled section, and $\kappa$ is the coupling coefficient defined as:

$$
\kappa=\frac{\pi}{2 L_{c p}}
$$

in which $L_{c p}$ is the minimum coupling length needed for complete power transfer from one waveguide to the other, e.g., from INI to OUT2. The $l_{c}$ determines the power split ratio of a coupler, e.g. for a $3 \mathrm{~dB}$ coupler $l_{c}=0.5 L_{c p}$.

Another way to consider the above is as follows. Assuming again that the waveguides are single mode, a symmetric directional coupler supports two system modes, which are respectively symmetric mode and antisymmetric with respect to the central axis $\left(\sigma_{2}\right)$ of the coupler, see Fig. 21. In the coupling region, where the gap between the waveguides is small enough, the evanescent field tails of these modes overlap and due to this interaction the propagation constants of these modes are different.

When light is launched into the "upper" waveguide of the directional coupler from IN1, then in the coupling region the system modes with propagation constant $\beta_{s}$ (for symmetric mode) and $\beta_{a}$ (for antisymmetric mode) exist. After a propagation distance of $L_{\text {cp }}$, by definition, they have accumulated a phase difference of $\pi$, so that the sum of the system modal fields now concentrates in the "lower" waveguide, and all the power is coupled from IN1 to OUT2 (IN2 to OUT1). Therefore, the following equation for the coupling length holds:

$$
L_{c p}=\frac{\pi}{\beta_{s}-\beta_{a}}=\frac{\lambda}{2\left(n_{s}-n_{a}\right)},
$$

in which $n_{s}$ and $n_{a}$ are the modal indicies of the symmetric and antisymmetric mode, respectivly.

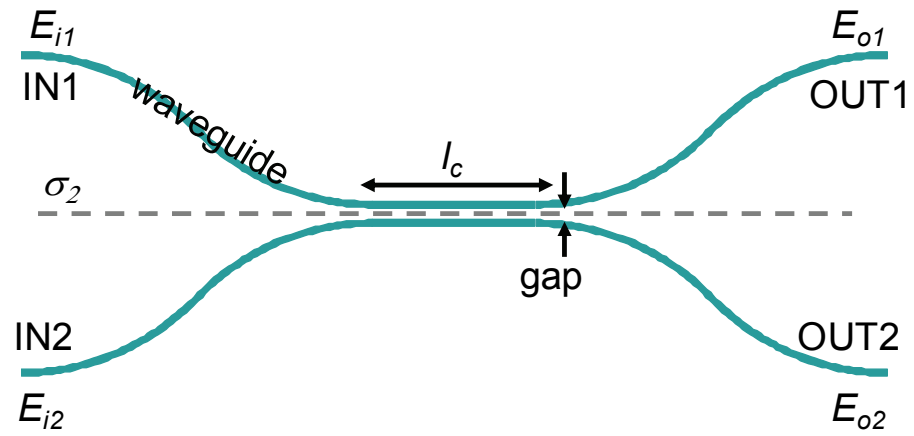

Fig. 21. A Schematic picture of directional coupler.

\subsubsection{Waveguide-grating-based add-drop multiplexer}

Fig. 22 shows a schematic picture of a grating-based optical add-drop multiplexer (OADM) [32]. In this design there are two 3-dB directional couplers. The first one splits the light from the $I N$ port equally to two waveguide branches. Identical waveguide gratings are present in each branch. If the wavelength of the light is in the photonic 
stopband of these gratings, the light is reflected from the gratings back to the first 3-dB coupler. As a 3-dB coupler introduces 90 degree phase difference -as can be seen from (1)- the reflected light from the grating is now coupled completely to the OUT2 port. The light with a wavelength outside the stopband goes to the second 3-dB coupler, where the coupling continues and all the light is eventually coupled to the OUT1 port.

The 3-dB coupler design is based on equation (3). The waveguides were chosen to be $500-\mathrm{nm}$ wide silicon photonic wires separated by a $200-\mathrm{nm}$ gap. The effective indices of the symmetric and antisymmetric modes for this geometry were calculated using a $2 \mathrm{D}$ modesolver. From the calculated indices a coupling length $L_{\mathrm{cp}}$ of $52.97 \mu \mathrm{m}$ at a wavelength of $1550 \mathrm{~nm}$ is obtained from (3). The 3-dB coupling length is conveniently exactly half of the $L_{\mathrm{cp}}$, and a length of $26.5 \mu \mathrm{m}$ was chosen for the 3 -dB coupler section. The gratings were designed following the same procedure as discussed in section 2.2.

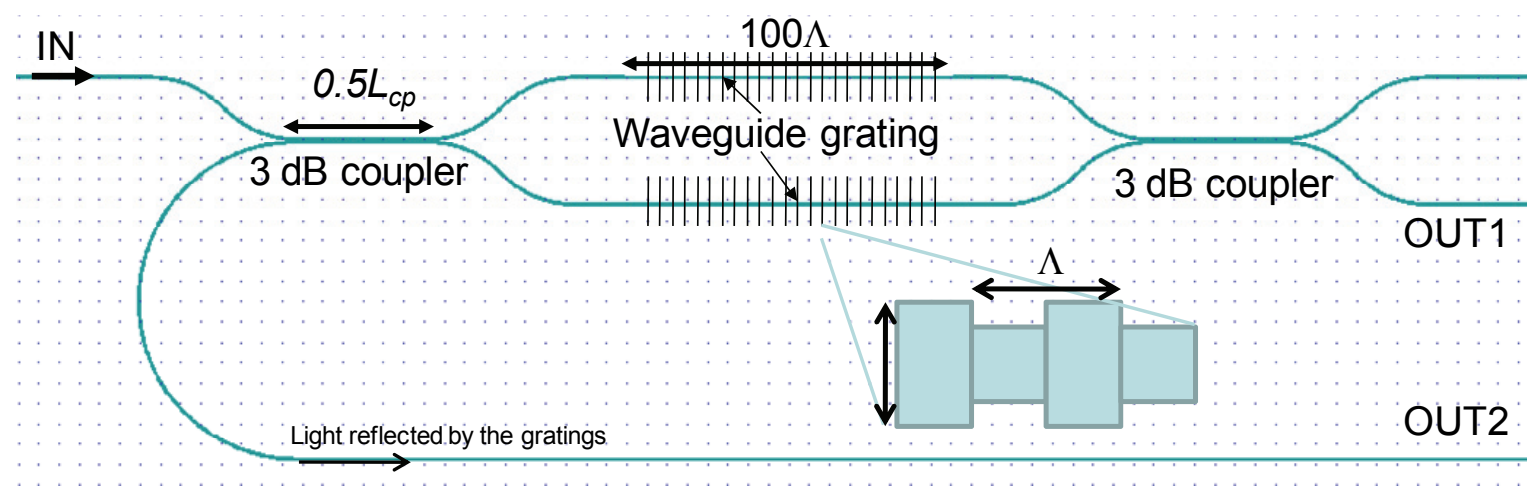

Fig. 22. A schematic picture of waveguide-grating-based wavelength router. Inset: schematic top view of the grating; see section 2.2 for details.

Fig. 23a shows measured transmission spectra of a grating-based wavelength selective router. The designed width of the grated waveguide is $320 \mathrm{~nm}$ and the grating is introduced by periodically varying the waveguide width by $20 \mathrm{~nm}$ in $450 \mathrm{~nm}$ periods (see section 2.2. for details). The length of the grated section is 100 periods $(45 \mu \mathrm{m})$.

A wavelength band with a width of approximately $8 \mathrm{~nm}$ with a center wavelength of $1506 \mathrm{~nm}$ is rejected from the OUT1 port by the grating and the light at these wavelengths propagates to the OUT2 port. The rejection ratio between the OUT1 and OUT2 ports is around $5 \mathrm{~dB}$ at wavelengths that are outside the stopband, while better than $10 \mathrm{~dB}$ on/off ratio is achieved at the stopband wavelengths.

Fig. $23 \mathrm{~b}$ shows measured transmission spectra of a similar grating structure in which the overall waveguide width is approximately $10 \mathrm{~nm}$ larger, which leads to stopband shift of $15 \mathrm{~nm}$. The waveguide width difference is caused by different photolithographic exposure dose, as discussed in 2.2.1. Due to this the waveguide width in the 3-dB coupler region, as well as the gap, are changed. This could change the properties of the directional coupler. However, the waveguide width change is compensated by the change in the gap: as the waveguide width increases and a mode becomes more confined in the waveguide, the gap between the waveguides decreases. According to our experience from experimental data; the waveguide width and the gap changes that result from the exposure dose difference cancel out one another's effect. This holds at least for values that are within reasonable range $( \pm 40 \mathrm{~nm})$ from the design target values. As a result, the 
operating wavelength range of the device (which is the stopband) can be conveniently tuned by the exposure dose during the fabrication.

Fig. 23c shows measured transmission spectrum of another variation of the device in which the waveguide width in the grating section is varied by $60 \mathrm{~nm}$. The index variation in the grating section is stronger than obtained with $20 \mathrm{~nm}$ width variation, and accordingly the stopband is wider and deeper. The optical C-band (1535-1560 nm) is almost completely rejected from the OUT1 port. For wavelengths from $1540 \mathrm{~nm}$ to 1555 $\mathrm{nm}$ better than $40 \mathrm{~dB}$ on/off ratio in transmitted power between OUT1 and OUT2 ports is achieved.

Efficient tuning of the whole grating spectrum requires a cantilever that entirely covers both gratings. As we have discussed in section 2.4.4, releasing a broad cantilever is challenging because a long release etching time is required. For this reason it is interesting to consider also the tuning of resonating Bloch modes. The transmission spectra that are measured at OUT2 port show clear dips at wavelengths that correspond to resonating Bloch modes of the gratings. At these dips the on/off ratio is better than $20 \mathrm{~dB}$. Due to the spatial distribution of these resonances [33] their properties can be tuned selectively with much narrower cantilevers, as discussed in section 2.1.4.
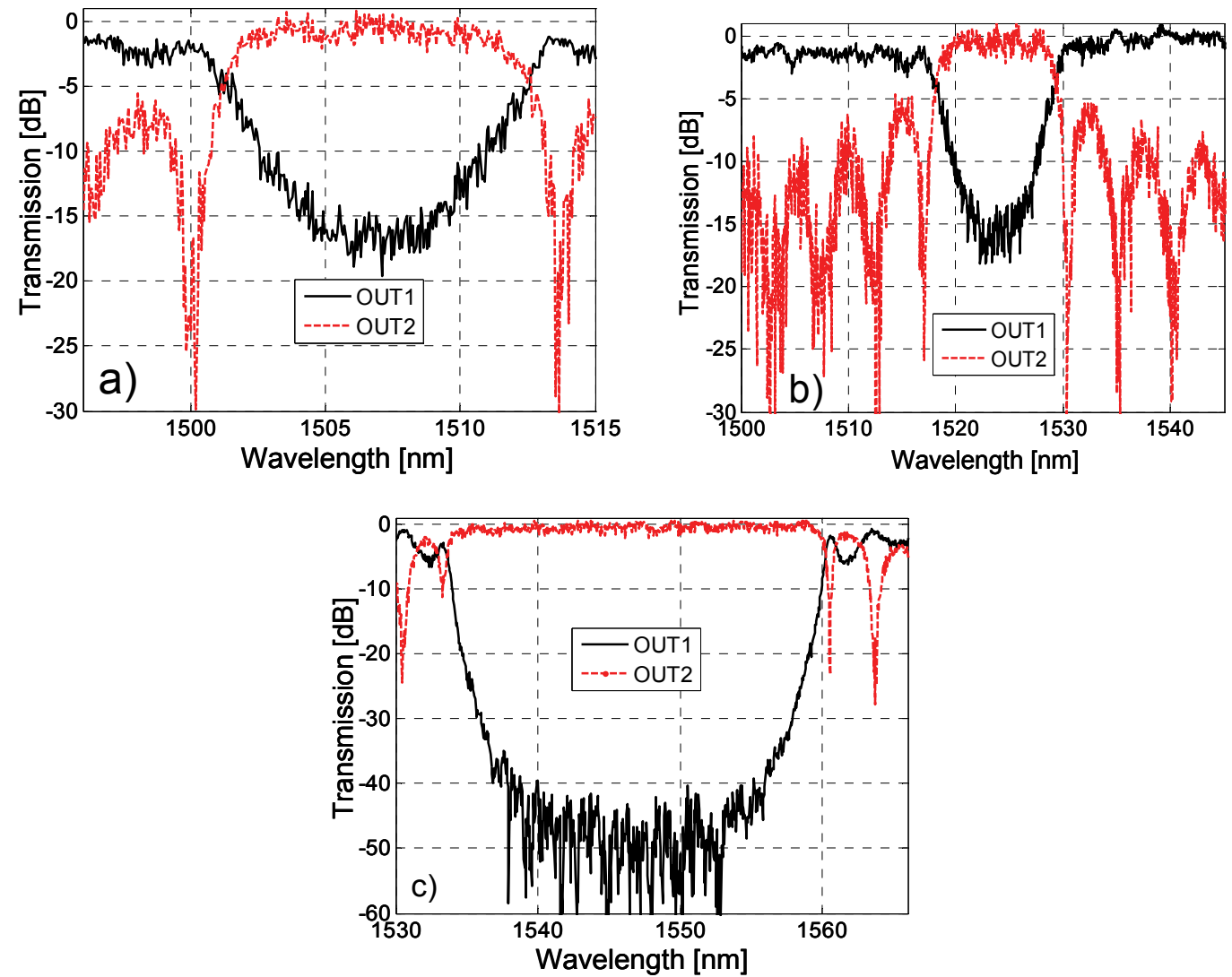

Fig. 23. Measured transmission spectra of different grating-based wavelength routers. a) Device that has a weak grating (width variation $20 \mathrm{~nm}$ ). b) Same as in a), but due to a different exposure dose the width of the waveguide structures is some $10 \mathrm{~nm}$ larger (see main text for details). c) Strong grating with a width variation of $60 \mathrm{~nm}$ (see section 2.2 for details of the gratings). 


\subsubsection{A ring resonator}

In a ring resonator, see Fig. 24a, a fraction of light is coupled from the input waveguide to the ring waveguide, using a directional coupler section, such as discussed in section 3.2.1. A part of the light that is coupled to the ring waveguide travels back to the point where it was coupled in. During this propagation the light accumulates a phase difference and if it arrives this point in phase match with respect to light in the input waveguide, due to constructive interference enhanced coupling of light to the ring occurs, leading to a resonance build up at the wavelengths that fulfill the phase match condition. In a steady state light power coupled to the ring at the resonance wavelength equals the power that the ring loses. If the ring is coupled symmetrically with two waveguides, as shown in Fig. $24 \mathrm{a}$, the light at the resonance wavelength is coupled from IN port through ring to OUT2 port. A detailed discussion about ring-resonators is given e.g. in [34].

The resonance wavelength of a ring resonator can be obtained from the following equation:

$$
\lambda_{\text {res }}=\frac{L_{\text {ring }}}{m} n_{\text {eff }},
$$

in which $L_{\text {ring }}$ is the round trip length of the ring and $m$ the cavity mode order ( $m=1$, $2 \ldots)$.

We have implemented racetrack-type ring resonators, coupled to adjacent waveguides, with varying coupling lengths $\left(l_{c}=12 \mu \mathrm{m}, 14 \mu \mathrm{m}\right.$ and $\left.16 \mu \mathrm{m}\right)$ and $\operatorname{ring}$ radii $(R=5 \mu \mathrm{m}$ and $10 \mu \mathrm{m})$. The waveguide is a single mode $\mathrm{Si}$ photonic wire waveguide and in the coupling region the gap between the waveguides is designed to be $200 \mathrm{~nm}$. A typical transmission spectrum measured for a 193-nm DUV-lithography-fabricated ringresonator is shown in Fig. 24b. The chosen coupling lengths and radii result is FSRs from 5 to $13 \mathrm{~nm}$, with Q-values better than 10000 and resonance dip depths of $>8 \mathrm{~dB}$.

The area required by the ring-resonator is approximately $40 \mu \mathrm{m} \times 30 \mu \mathrm{m}$, while for $\mathrm{PhC}$ cavity devices it is about $20 \mu \mathrm{m} \times 10 \mu \mathrm{m}$. The downside of the ring resonator is that it does not selectively drop only a single wavelength or wavelength range, but drops all the wavelengths that fulfill the resonance condition (4). The maximum free spectral range, i.e. the spectral distance between two adjacent resonances, for ring resonators fabricated in SOI technology and operating around wavelengths around $1500 \mathrm{~nm}$ is of the order of $10 \mathrm{~nm}$. The FSR increases for smaller rings, but unfortunately efficient coupling becomes more challenging the smaller the ring is. 

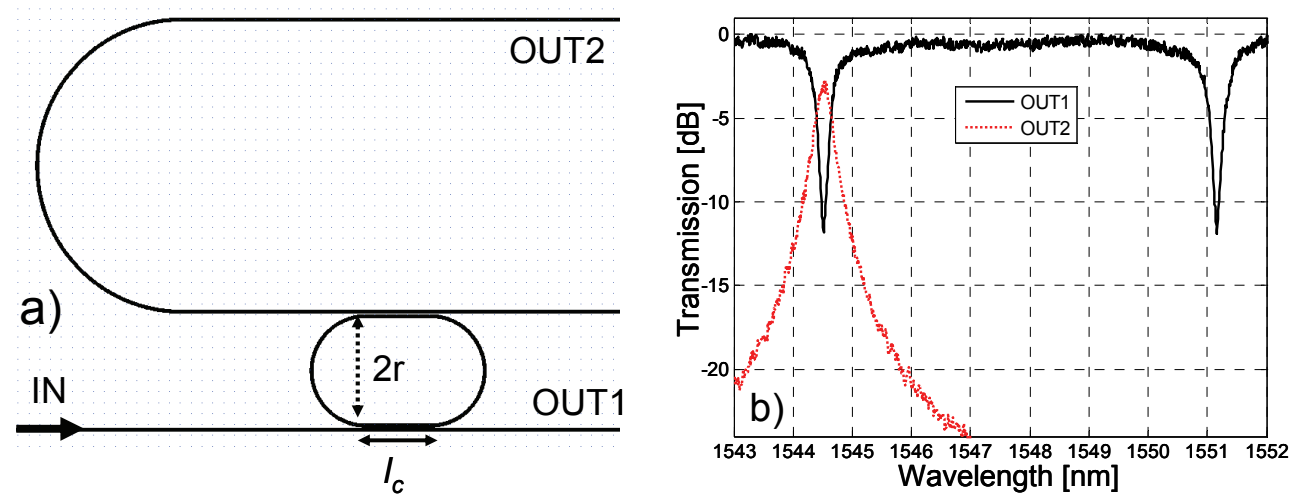

Fig. 24. a) A schematic top view of a ring-resonator-based wavelength router. In this so-called racetrack design, straight waveguide sections of length $l_{c}$ have been inserted in the ring in order to have a wellcontrolled and, if needed, strong coupling to the access waveguides. Light at the resonance wavelengths (4) is coupled through the ring to the OUT2 port. b) Measured transmission spectrum of a ring resonator with $r$ $=10 \mu \mathrm{m}, l_{c}=12 \mu \mathrm{m}$ and a coupler gap of $200 \mathrm{~nm}$. The transmission spectrum to the OUT2 port is measured for wavelengths $1543 \mathrm{~nm}$ to $1547 \mathrm{~nm}$.

\subsubsection{Conclusion}

Optical add-drop multiplexers, based on grated silicon photonic wires, have been designed and characterized. These devices are considerably larger in size than the $\mathrm{PhC}$ based devices discussed in the previous sections. However, the design less critical to process parameter variations and e.g. if the designed waveguide dimensions differ slightly the device still functions, yet at different wavelength (see Fig. 23a and b). In additional the optical loss is much smaller than in the $\mathrm{PhC}$ devices.

At the stopband wavelengths it is possible to get over $40 \mathrm{~dB}$ on/off ratio between the output ports over a wide wavelength range $(25 \mathrm{~nm})$. However, the on/off ratio is poor ( $5 \mathrm{~dB}$ ) outside the stopband region, except for the wavelengths that correspond to resonating Bloch modes. Therefore the spectral shift of the entire stopband does not provide a well defined output signal to OUT2 port. The spectral features of the resonating Bloch modes can be tuned with a narrow cantilever, that is easier to fabricate than a broad one that covers the whole grating (as discussed in chapter 2). Since this type of tuning of the Bloch modes also provides well defined output signals, it is preferred over tuning the entire stopband.

The ring resonator is robust and compact design, although for channel dropping it has the disadvantage that not only one specific wavelength is dropped. Large FSR would require small coupling length and small radii, resulting in shallow and low Q-value resonances. We observed a FSR of $\sim 13 \mathrm{~nm}$ by using a ring that has a radius of $5 \mu \mathrm{m}$ and coupling length of $12 \mu \mathrm{m}$. The loss of the ring resonators is less than $3 \mathrm{~dB}$.

\subsection{Discussion}

The performance requirements for a switch depend on the application. If for example, the switch is used to select a light path to one of several optical sensors, the performance requirements are somewhat different from those needed to perform high-speed packetswitching in an optical telecom network.

In Table 1 we compare the performance of our optical devices with respect to several parameters that are used to characterize the suitability of a switch for its indented 
applications. The PhC-cavity-based channel-drop filter (Sect. 3.1.2) is labeled PhC1, the $\mathrm{PhC}$-cavity-based Y splitter (Sect. 3.1.3) is named $\mathrm{PhC}$ 2, and waveguide grating (Sect. 3.2.2) and ring-resonator-based (Sect. 3.2.3) wavelength routers are shortened to WG and $\mathrm{RR}$, respectively.

The extinction ratio is the output power ratio of the output ports. Insertion loss is the loss that is caused by the optical switching element. The size of a device is its footprint on a chip. For waveguide grating and ring-resonator devices, also the area required by the access waveguides, that are essential part of the device, are taken into account. Bandwidth is the $3-\mathrm{dB}$ spectral width of the wavelength band that is routed by the device.

Table 1. Comparing of different devices designed for MEMS-actuated wavelength selective switching.

\begin{tabular}{|l|c|c|c|c|}
\hline & PhC1 & PhC2 & WG & RR \\
\hline Extinction ratio & $20 \mathrm{~dB}$ & $12 \mathrm{~dB}$ & $15-40 \mathrm{~dB}$ & $12-30 \mathrm{~dB}$ \\
\hline Insertion loss & $5-15 \mathrm{~dB}^{*}$ & $18 \mathrm{~dB}$ & $<2 \mathrm{~dB}$ & $<3 \mathrm{~dB}^{*}$ \\
\hline Size & $100 \mu \mathrm{m}^{2}$ & $100 \mu \mathrm{m}^{2}$ & $6000 \mu \mathrm{m}^{2}$ & $1200 \mu \mathrm{m}^{2}$ \\
\hline Bandwidth & $2-3 \mathrm{~nm}$ & $2-3 \mathrm{~nm}$ & $15-25 \mathrm{~nm}^{* *}$ & $<0.15 \mathrm{~nm}$ \\
\hline
\end{tabular}

*Depends on the wavelength, see section 5.2.4. **Depends on the grating.

Switching speed is one of the key properties of an optical switch. According to [35], the fastest commercially available switches are semiconductor-optical-amplifier-based on/off switches with a switching speed of $1 \mathrm{~ns}$ and lithium niobate electro-optical on/off switch with a switching speed of $10 \mathrm{ps}$. In [36], a switching rate of $8 \mathrm{kHz}$ is reported with an aluminum micro-bridge-actuated ring resonator. The speed of our MEMS-actuated optical switch depends on the micromechanical element; the resonance frequencies of our microcantilevers are in the $\mathrm{MHz}$ range [37], indicating a switching time of $\sim 1 \mathrm{~ns}$.

Another interesting property is the wavelength tuning range that can be achieved with the envisioned micromechanical tuning scheme; electrostatically actuated microcantilever that interacts with the evanescent field of the optical device. For a ring-resonator a wavelength tuning range of tens of nanometers is reported when actuated with an optical fiber tip [38]. The tuning range depends on how strongly the mechanical element interacts with the evanescent field. For waveguide devices this depends e.g. on the interaction length and on how close the mechanical element can be brought to the waveguide in controlled way.

In this thesis we also consider tuning in which the entire optical properties of the device are changed. In this case the microcantilever has submicrometre tips, which will enter the holes of a $\mathrm{PhC}$ and radically change its operation (section 4.6.). This type of tuning is a bit more difficult to realize due to require nanometre scale tips, however much stronger response, than with just evanescent field perturbation, is expected. Experimental tuning results are given in chapter 4 .

\subsection{Conclusions}

Several optical designs for mechano-optically tuned wavelength routing have been designed and realized. The PhC-based devices provide theoretically the most compact and ideal wavelength routing performance. However, the fabrication challenges to accurately produce these nanophotonical devices hamper the device performance. The result is non-ideal channel dropping. In addition, optical loss in $\mathrm{PhC}$ components is 
Compact silicon photonic devices for MEMS-actuated optical switching

relatively high, ranging from 5 to $20 \mathrm{~dB}$. The grating-based wavelength router is suitable to route wide wavelength bands and the optical loss of the device is within our measurement accuracy $\pm 2 \mathrm{~dB}$. Unfortunately, with $120 \mu \mathrm{m} \times 50 \mu \mathrm{m}$, the footprint of the device is quite large. The ring resonator performs well, and, according to our experimental data, is least vulnerable to fabrication process parameter variations. The device is also nearly as compact as the PhC-based routers. It has, however a limited free spectral range, which is disadvantageous for certain applications. 


\section{References}

[1] J. Singh, "Semiconductor optoelectronics: physics and technology", McGraw-Hill 1995.

[2] L.G. Ferreira and M.A.A. Pudenzi, "Waveguiding in a dielectric medium varying slowly in one transverse direction", Journal of the Optical Society of America, vol. 71, pp. 1377-1380, 1981.

[3] O.V. Ivanova, R. Stoffer, L. Kauppinen, and M. Hammer, "Variational effective index method for 3D vectorial scattering problems in photonics: TE Polarization", in Proc. PIERS 2009 Moscow pp. 10381042, 2009.

[4] J.D. Joannopoulos, S.G. Johnson, J.N. Winn, and R.D. Meade, "Photonic crystals: molding the flow of light", Princeton University Press, 2008.

[5] S.G. Johnson and J.D. Joannopoulos, "Block-iterative frequency-domain methods for Maxwell's equations in a planewave basis," Optics Express, vol. 8, pp. 173-190, 2001.

[6] C.G. Bostan, "Design and fabrication of quasi-2D photonic crystal components based on silicon-oninsulator technology", PhD thesis, University of Twente, Enschede, 2005.

[7] W. Bogaerts, "Nanophotonic Waveguides and photonic crystals in silicon-on-insulator", PhD thesis, Universiteit Gent 2004.

[8] S. Hughes, L. Ramunno, J.F. Young, and J.E. Sipe, "Extrinsic Optical Scattering Loss in Photonic Crystal Waveguides: Role of Fabrication Disorder and Photon Group Velocity," Phys. Rev. Lett. vol. 94, 033903, 2005.

[9] L.O'Faolain, T.P. White, D.O’Brien, X. Yuan, M.D. Settle, and T.F. Krauss, "Dependence of extrinsic loss on group velocity in photonic crystal waveguides," Opt. Exp., vol. 15, no. 20, pp. 13129-13138, 2007.

[10] Y. Akahane, T. Asano, B-S. Song, and S. Noda, "High-Q photonic nanocavity in a two-dimensional photonic crystal”, Nature, vol. 425, pp. 944-947, 2003.

[11] A. Yariv, Y. Xu, R.K. Lee, and A. Scherer, "Coupled-resonator optical waveguide: a proposal and analysis", Optics Letters, vol. 24, pp. 711-713, 1999.

[12] P. Sanchis, J. Marti, B. Luyssaert, P. Dumon, P. Bienstman, and R. Baets, "Analysis and design of efficient coupling in photonic crystal circuits", Optical and Quantum Electronics, vol. 37, pp. 133-147, 2005.

[13] P. Sanchis, P. Bienstman, B. Luyssaert, R. Baets, and J. Marti, "Analysis of butt coupling in photonic crystals", IEEE Journal of Quantum Electronics, vol. 40, pp. 541-550, 2004.

[14] S. Fan, P.R. Villeneuve, J.D. Joannopoulos M.J. Khan, C. Manolatou, and H.A. Haus, "Theoretical analysis of channel drop tunneling processes", Physical Review B, vol. 59, pp. 15882-15892, 1999.

[15] E. Drouard, H.T. Hattori, C. Grillet, A. Kazmierczak, X. Letartre, P. Rojo-Romeo, and P. Viktorovitch, "Directional channel-drop filter based on a slow Bloch mode photonic crystal waveguide section", Optics Express, vol. 13, pp. 3037-3048, 2005.

[16] S. Fan, P.R. Villeneuve, J.D. Joannopoulos, and H.A. Haus, "Channel drop filters in photonic crystals", Optics Express, vol. 3, pp. 4-11, 1998.

[17] A. Adibi, R.K. Lee, Y. Xu, A. Yariv, and A. Scherer, "Design of photonic crystal optical waveguides with singlemode propagation in the photonic bandgap", Electronics Letters, vol. 36, pp. 1376-1378, 2000 .

[18] Silicon Photonics Platform, http://www.epixfab.eu/

[19] M. Notomi, A. Shinya, S. Mitsugi, E. Kuramochi, and H-Y. Ryu, "Waveguides, resonators and their coupled elements in photonic crystal slabs", Optics Express, vol. 12, pp. 1551-1561, 2004.

[20] S.Y. Lin, E. Chow, J. Bur, S.G. Johnson, and J.D. Joannopoulos, "Low-loss, wide-angle Y splitter at $\sim 1.6-\mu \mathrm{m}$ wavelengths built with a two-dimensional photonic crystal," Opt. Lett., vol. 27, no. 16, pp. 1400-1402, 2002.

[21] H. Takeda and K. Yoshino, "Tunable light propagation in Y-shaped waveguides in two-dimensional photonic crystals utilizing liquid crystals as linear defects,” Phys. Rev. B, vol. 67, 073106, 2003.

[22] K. Inoue, Y. Sugimoto, N. Ikeda, Y. Tanaka, K. Asakawa, H. Sasaki, and K. Ishida, "Ultra-small photonic-crystal-waveguide-based Y-splitters useful in the near-infrared wavelength region,” Jpn. J. Appl. Phys., vol. 43, no. 4A, pp. L 446-L 448, 2004. 
[23] T. Takahata, K. Hoshino, K. Matsumoto, and I. Shimoyama, "Transmittance tuning of photonic crystal reflectors using an AFM cantilever,” Sens. Actuators A 128 , pp. 197-201, 2006.

[24] W.C.L. Hopman, K.O. van der Werf, A.J.F. Hollink, W. Bogaerts, V. Subramaniam, and R.M. de Ridder, "Modeling and experimental verification of the dynamic interaction of an AFM-tip with a photonic crystal microcavity," IEEE photon. Technol. Lett, vol. 20, pp. 57-59, 2008.

[25] M. Tinkham, Group theory and quantum mechanics. Dover Publications 2003.

[26] K. Sakoda. Optical properties of photonic crystals. Springer Series in Optical Sciences. Springer, 2nd edition, 2005.

[27] K.M. Leung and Y.F. Liu. "Full vector wave calculation of photonic band structures in face-centeredcubic dielectric media," Phys. Rev. Lett., vol. 65, pp. 2646-2649, 1990.

[28] A. Farjadpour, D. Roundy, A. Rodriguez, M. Ibanescu, P. Bermel, J.D. Joannopoulos, S.G. Johnson, and G. Burr, "Improving accuracy by subpixel smoothing in FDTD," Opt. Lett., vol. 31, pp. 29722974, 2006.

[29] D. Taillaert, F. van Laere, M. Ayre,W. Bogaerts, D. van Thourhout, P. Bienstman, R. Baets, "Grating couplers for coupling between optical fibers and nanophotonic waveguides," Jpn. J. Appl. Phys. vol. 45, pp. 6071-6077, 2006.

[30] A. Yariv, “Optical Electronics in modern communications”, Oxford University Press, 1997.

[31] C. Pollock and M. Lipson, "Integrated photonics", Kluwer Academic Publishers 2003.

[32] H. Yamada, T. Chu, S. Ishida, and Y. Arakawa, "Optical add-drop multiplexers based on Si-wire waveguides", Applied Physics Letters, vol. 86, 191107, 2005.

[33] W.C.L. Hopman, H.J.W.M. Hoekstra, R. Dekker, L. Zhuang, and R.M. de Ridder, "Far-field scattering microscopy applied to analysis of slow light, power enhancement, and delay times in uniform Bragg waveguide gratings", Optics Express, vol. 15, pp. 1851-1870, 2007.

[34] E.J. Klein, "Densely integrated microring-resonator based components for fiber-to-the-home applications", PhD thesis, University of Twente, 2007.

[35] R. Ramaswani and K.N. Sivarajan, "Optical networks: a practical perspective”, Morgan Kaufmann, 2002.

[36] G.N. Nielson, D. Seneviratne, F. Lopez-Royo, P.T. Rakich, Y. Avrahami, M.R. Watts, H.A. Haus, H.L. Tuller, and G. Barbastathis, "Integrated Wavelength-Selective Optical MEMS Switching Using Ring Resonator Filters", IEEE Photonics Technology Letters, vol. 17, pp. 1190-1192, 2006.

[37] S.M. Chakkalakkal Abdulla, J.W. Berenschot, M.J. de Boer, L.J. Kauppinen, R.M. de Ridder, and G.J.M. Krijnen, (2009) "Optimisation study of micro cantilevers for switching of photonic band gap crystals", In: Proceedings of the International Conference on Photonics in Switching 2009, pp. 15-19, 2009.

[38] P.T. Rakich, M. A. Popović, M.R. Watts, T. Barwicz, H.I. Smith, and E.P. Ippen, "Ultrawide tuning of photonic microcavities via evanescent field perturbation", Optics Letters, vol. 31, pp. 1241-1243, 2006. 


\section{Mechano-optical actuation}

In this chapter we present the experimental results of mechanically actuated integrated optical devices. The basics of electrostatic actuation of a cantilever are discussed followed by a section that briefly describes the fabrication process to monolithically integrate a microcantilever on an SOI photonics chip. The electrostatically actuated microcantilever was used to tune the optical properties of a ring-resonator and a photonic crystal waveguide. Experimental results from this tuning are given in sections 4.5 and 4.6. The chapter ends with conclusions.

\subsection{Introduction}

The mechanical actuation of integrated optical (IO) devices promises broad wavelength tuning range, low power consumption and rapid actuation cycles. These are particularly attractive properties when compared to commonly used thermo-optic (TO) based actuation [1]. Although this method provides a wide actuation range with moderate actuation speed $(700 \mathrm{kHz}$ in silicon [1]), the TO method is power hungry, making it less attractive for devices where many actuation elements are required.

Electro-optic modulation is another commonly used method to actuate IO devices. In silicon the Pockels or E/O Kerr effects are not practical and often the plasma dispersion method is used (see section 1.3.2.). In this method free carriers are injected to increase the conductivity that in turn causes the refractive index to change. Very fast modulation with this method can be achieved; signal rise time $<1 \mathrm{~ns}$ is reported in [2]. Although this method allows low voltage operation, the increased conductivity results in increased optical loss due to free carrier absorption. For this reason the method is not suitable to e.g. tune a resonance wavelength of a resonator.

In recent years the mechano-optical actuation of IO devices has been intensively investigated. Micromechanically actuated on/off switching of a ring resonator has been demonstrated with an aluminum microbridge that, when pulled close to the ring, kills the resonance due to increased optical loss [3]. Ultrawide tuning of a ring resonator has been demonstrated in [4] by using an optical fiber tip as a movable dielectric load that interacts with the evanescent field of the ring resonator. In addition there have been demonstrations of the interaction of the scanning tip of an atomic force microscope with nanophotonic structures $[5,6]$.

Such devices have potential applications in wavelength division multiplexed (WDM) telecom networks, where waveband switching is desired [7]. Potential future applications include modulation or switching in on-chip optical circuits, e.g. in optical interconnects between integrated electronic circuits. Particularly for on-chip applications, CMOScompatibility, low actuation voltage, and compact size are key properties.

In this chapter we demonstrate tuning of two different devices: a compact ring resonator and a photonic crystal waveguide, both fabricated on SOI. The tuning of the devices is obtained by perturbing the evanescent field with a monolithically integrated silicon nitride microcantilever. The actuation of the cantilever is done electrostatically, which has the advantage that the power required for actuation can be very low as the capacitance of the microcantilever is typically small, of the order of picofarads. 


\subsection{Electrostatic actuation of microcantilever}

In this section we briefly describe the physics of electrostatic actuation of a microcantilever. In a simplified approximation the mechanical properties of a cantilever can be modelled as a spring to which the electrostatic force is applied. For this modelling we consider a system as depicted in Fig. 1.
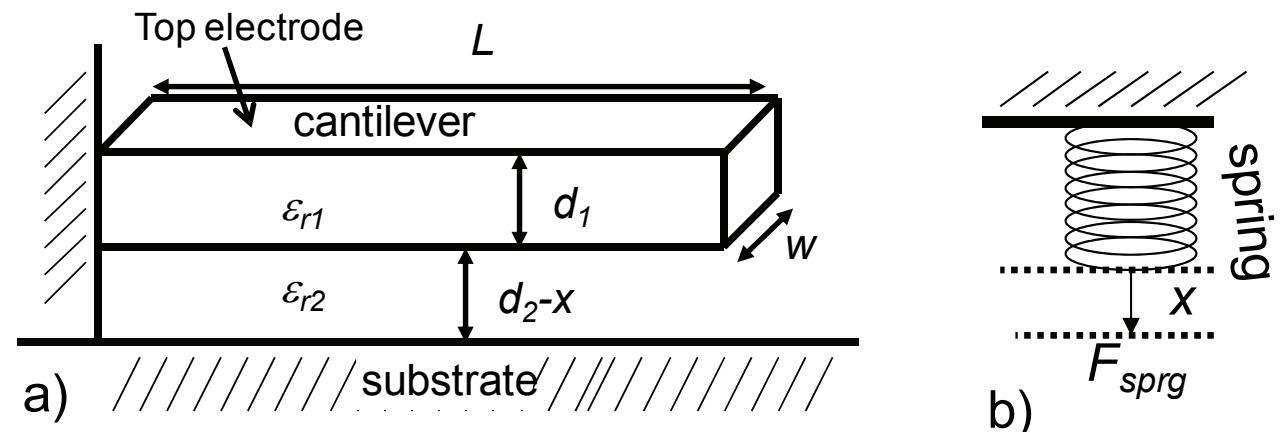

Fig. 1. a) Cantilever suspended above a substrate. The initial gap (without applied force) between cantilever and substrate is $d_{2}$. b) Spring model of the cantilever.

For this type of cantilever the spring constant can be calculated from the following equation [8]:

$$
k_{\text {sprg }}=\frac{Y w d_{1}^{3}}{4 L^{3}},
$$

in which $Y$ is Young's modulus, $w$ is the width, $d_{1}$ is the thickness and $L$ the is length of the cantilever. To stretch a spring distance $x$, see Fig. 1, a force of $F_{\text {sprg }}=k_{\text {sprg }} x$ is required.

To actuate the cantilever electrostatically, a voltage difference between the cantilever and the substrate is applied. From the electrical point of view the cantilever, shown in Fig. 1, can be seen as a capacitor to which the applied voltage $V$ causes a force $F_{c}$ according to:

$$
\begin{aligned}
& E_{c}=\frac{1}{2} C V^{2}=\frac{1}{2} A \frac{\varepsilon_{r 1} \varepsilon_{r 2} \varepsilon_{0}}{\left(d_{2}-x\right) \varepsilon_{r 1}+d_{1} \varepsilon_{r 2}} V^{2} \Rightarrow \\
& F_{c}=\frac{\partial E_{c}}{\partial x}=\frac{1}{2} A \varepsilon_{r 1} \frac{\varepsilon_{r 1} \varepsilon_{r 2} \varepsilon_{0}}{\left(\left(d_{2}-x\right) \varepsilon_{r 1}+d_{1} \varepsilon_{r 2}\right)^{2}} V^{2},
\end{aligned}
$$

in which $E_{c}$ is the energy stored in the capacitor, $C$ is the capacitance, $\varepsilon_{r 1}$ and $\varepsilon_{r 2}$ are the relative permittivities of the cantilever dielectric and the material in the gap, respectively, $\varepsilon_{0}$ is the vacuum permittivity, $d_{2}$ is the initial gap between the substrate and the cantilever, $A=w L$ is the surface area of the cantilever, and $x$ is the deflection caused by the applied voltage.

The spring force $F_{\text {sprg }}$ will be equal to the electric force $F_{c}$, arising from an applied voltage $V$ :

$$
\frac{1}{2} A \varepsilon_{r 1} \frac{\varepsilon_{r 1} \varepsilon_{r 2} \varepsilon_{0}}{\left(\left(d_{2}-x\right) \varepsilon_{r 1}+d_{1} \varepsilon_{r 2}\right)^{2}} V^{2}=k_{\text {sprg }} x
$$


Here we have assumed that the gravitational force can be neglected (due to the small mass of the cantilever). From (3) it is possible to calculate the required voltage to deflect the cantilever by an amount $x$ :

$$
V(x)=\sqrt{\frac{2 k x\left(\left(d_{2}-x\right) \varepsilon_{r 1}+d_{1} \varepsilon_{r 2}\right)^{2}}{\varepsilon_{r 1}^{2} \varepsilon_{r 2} \varepsilon_{0} A}} .
$$

It turns out that up to a critical voltage the deflection increases with voltage. However, the function $V(x)$ has a maximum $V_{p}$ where $\mathrm{d} V(x) / \mathrm{d} x=0$. At $V=V_{p}$, a pull-in phenomenon occurs: the cantilever becomes unstable and its deflection keeps increasing, even if the voltage is decreased. This typically results in catastrophic failure where the cantilever is pulled to the surface. As a rule of thumb, the deflection can be changed in a controlled way only over a range not exceeding $1 / 3$ of the total initial gap between the electrodes.

Fig. 2 shows the calculated voltage, from equation (4), as a function of the deflection $x$. For the cantilever we have used $k=3.24 \mathrm{~N} / \mathrm{m}$, which corresponds to the value that is found for a $1-\mu \mathrm{m}$ thick silicon nitride $\left(Y=280 \mathrm{GPa}, \varepsilon_{\mathrm{r} 1}=7.3\right)$ cantilever, having a length of $40 \mu \mathrm{m}$ and a width of $10 \mu \mathrm{m}$. The initial gap $\left(d_{2}\right)$ is chosen to be $400 \mathrm{~nm}$. It can be seen that a voltage of $6 \mathrm{~V}$ is required to defect the cantilever by $150 \mathrm{~nm}$, which is close to the maximum amount the cantilever can be deflected in controlled way.

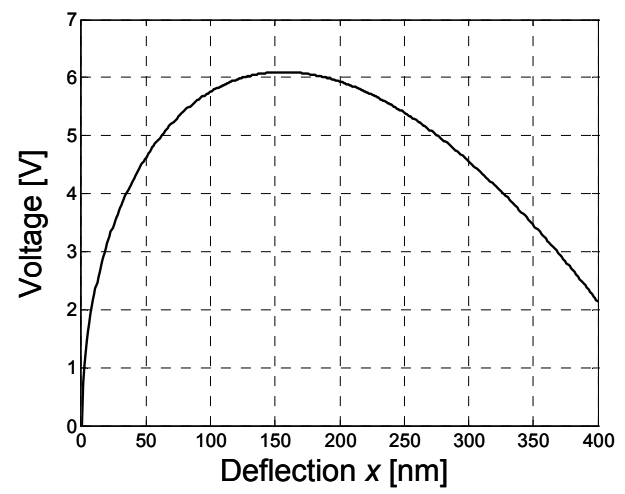

Fig. 2. Deflection response of cantilever (see text) to applied voltage.

\section{The actuation speed}

The speed of the cantilever can be estimated from its resonance frequency [8]:

$$
f=\frac{1}{2 \pi} \sqrt{\frac{k_{\text {sprg }}}{m_{0}}},
$$

in which the $m_{0}$ is the mass of the cantilever.

As a rule of thumb, the maximum actuation frequency is about half of this. However, there are factors that can slow down the speed. For our device the dominant limiting factor is the so-called squeeze force [9]. This force arises from the air trapped in the narrow gap between the cantilever and the substrate. This effect can be modelled as a damper-spring system. For a given cantilever it is possible to calculate damping ratio [10]: 


$$
\Gamma=\frac{\mu w^{2}}{4 \rho d_{2}^{3} d_{1} \pi f}
$$

in which $\mu$ is the air viscosity in the ambient atmosphere $\left(1.79 \cdot 10^{-5} \mathrm{Nsm}^{-2}\right)$ and $\rho$ is the density of the cantilever. From this damping ratio the time response of the system can be calculated. A highly damped system $(\Gamma>1)$ can be described in the Laplace domain by [10]:

$$
H(s)=\frac{K}{\left(1+s \tau_{1}\right)\left(1+s \tau_{2}\right)},
$$

in which $K$ is a constant, $s$ is a complex number and the time constants can be expressed as:

$$
\begin{gathered}
\tau_{1}=\frac{1}{\omega}\left(\Gamma+\sqrt{\Gamma^{2}-1}\right), \\
\tau_{2}=\frac{1}{\omega}\left(\Gamma-\sqrt{\Gamma^{2}-1}\right) .
\end{gathered}
$$

For the underdamped situation $(\Gamma<1)$, similar expressions can be found [11].

With the same cantilever parameters as used for calculating the results given in Fig. 2, and with $\rho=3440 \mathrm{~kg} / \mathrm{m}^{3}$ for silicon nitride, we calculate the step response of the system with two different gap values $d_{2}$. It can be seen from Fig. 3 that a smaller gap results in a slower response. From the graph we estimate switching frequencies of $25 \mathrm{kHz}$ (for 200 $\mathrm{nm}$ initial gap) and $225 \mathrm{kHz}$ (for $400 \mathrm{~nm}$ initial gap). The effect of the squeeze damping can be reduced by reducing the air pressure or designing the cantilever such that air can escape faster from the gap. This correspondingly increases the speed of the system. Based on the experimental data presented in [12-13] we expect orders of magnitude faster operation in low pressure conditions.

The other factor that can limit the speed of the actuation is the electrical RC delay that originates from charging and discharging of the capacitor through a contact resistance. As the capacitance of the microcantilever is typically small (of the order $1 \mathrm{pF}$ ), the effect of the contact resistance would need to be of the order $10 \mathrm{M} \Omega$ for the RC delay to become of the same order of magnitude as the squeeze film damping delay at atmospheric pressure.

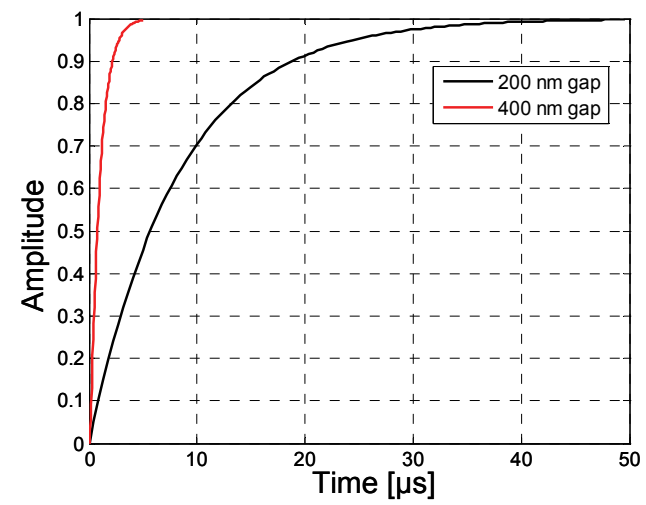

Fig. 3. The step response of a silicon nitride cantilever at two different initial gaps. 


\subsection{Monolithic integration of the microcantilever}

The optical devices, described in chapter 3, have been fabricated at the silicon photonics platform ePIXfab, established at IMEC, Leuven. The devices are fabricated on SOI wafers, containing a $220-\mathrm{nm}$ thick silicon device layer on top of a $2-\mu \mathrm{m}$ thick $\mathrm{SiO}_{2}$ layer. The ring resonator was formed by etching $200 \mathrm{~nm}$ into the Si device layer (leaving a thin Si slab in the etched areas); for photonic crystal devices, the device layer was completely etched through with a 220-nm etch depth. The smaller etch depth of the ring resonator was due to fabrication error.

A cross-section of the integrated mechano-optical structure is shown in Fig. 4. The microcantilevers were fabricated later on top of the optical structures by post-processing at the MESA+ labs. This post-processing starts with a deposition of 40-nm thick siliconrich silicon nitride ( $\mathrm{SiRN}$ ) layer, that functions as a protective layer during the etch steps that are performed later. For PhC devices, this layer is locally removed from the $\mathrm{PhC}$ holes, and at locations that will act as windows for underetching the $\mathrm{PhC}$ device, so that a membrane structure can be fabricated. Next follows the so-called sacrificial layer, for which we used $\mathrm{SiO}_{2}$. The thickness of this layer partially determines the initial gap between the optical device and the cantilever. A thickness of $200 \mathrm{~nm}$ was used for the ring-resonator, while $100 \mathrm{~nm}$ was selected for $\mathrm{PhC}$ device. The thinner layer for the $\mathrm{PhC}$ device is motivated by the desired formation of self-aligned tips that can enter the $\mathrm{PhC}$ holes (see section 4.6). The microcantilever is patterned into a $1-\mu \mathrm{m}$ thick SiRN layer that is deposited on top of the sacrificial layer. For electrostatic actuation a $58 \mathrm{~nm}$ thick chromium-gold layer is deposited on top of this $\mathrm{SiRN}$. The cantilever is patterned using photolithography, in which the pattern is transferred from a mask to the photoresist. The metal layers and the top SiRN layers are then etched according to these patterns (Fig. 4a). In a final step the cantilever is released by etching the sacrificial layer in buffered hydrofluoric acid (BHF) (Fig. 4b). The cantilevers that were used in the experiments are $10 \mu \mathrm{m}$ wide and $40 \mu \mathrm{m}$ long.

The main challenges in the cantilever fabrication are the control of the initial stress of the cantilever and of the etch processes that define and release the cantilever. A detailed discussion on the fabrication process and the mechanical properties of the cantilever will be published in [12-13].
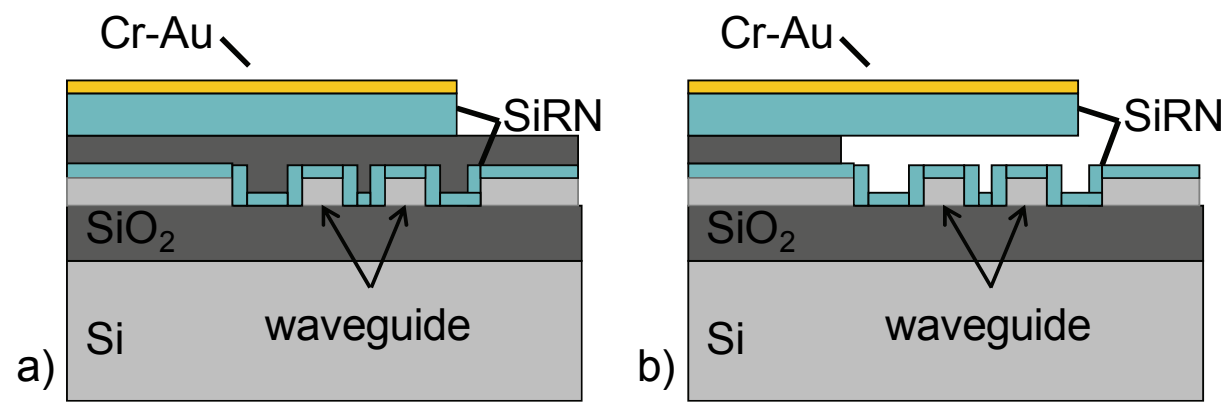

Fig. 4. Schematic cross-section of an integrated mechano-optical device. a) after cantilever patterning and before sacrificial etching. b) After sacrificial etching showing a cantilever suspended above an optical waveguide. 


\subsection{Measurement set-up}

Part of the measurement set-up is shown schematically in Fig. 5. The light is coupled to the device using grating couplers [14]. Transmission spectra were measured using a tunable laser and a photodetector module (Agilent 8164B and 81634B). The dynamic response was recorded by connecting the photodetector output to an oscilloscope. Contact probes, connected to a contact pad on the top surface of the cantilever and to the bulk silicon of the wafer, allowed electrical actuation of the cantilever. The contact resistance of the bulk silicon wafer was measured to be $\sim 10 \mathrm{k} \Omega$.

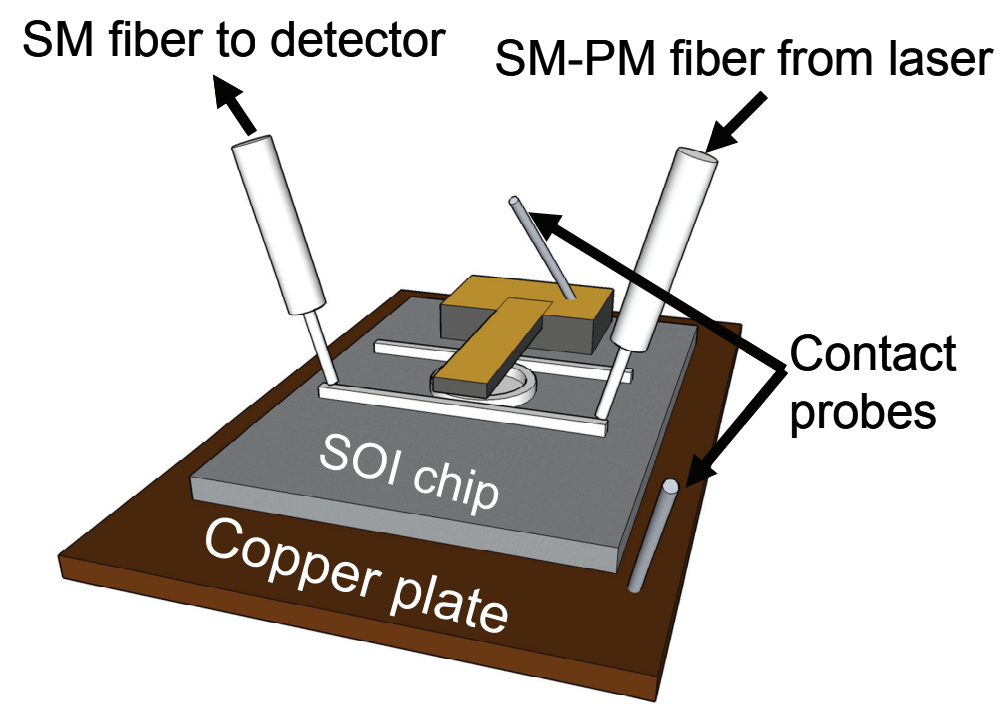

Fig. 5. Schematic of the setup used for optical transmission measurement and electrostatic actuation of the cantilever. Grating couplers, not shown in detail, couple light from a laser into the optical structure through single-mode (SM) polarization maintaining (PM) fiber, and from the structure to a photodetector.

\subsection{Micromechanically actuated ring resonator}

The resonance wavelength of the ring resonator, discussed in section 3.2.3, is changed by perturbation of the evanescent field of the ring waveguide, which increases the optical path length of the ring. If the interaction of the cantilever with the modal field causes a change $\Delta n_{\text {eff }}$ of the effective index over a perturbation length $L_{p e r t}$, this results in a change in the optical path length of $L_{\text {pert }} \Delta n_{\text {eff. }}$. For an optical ring resonance of order $m$, this leads to a shift of resonance wavelength $\Delta \lambda$, given by

$$
\Delta \lambda=\frac{L_{\text {pert }}}{m} \Delta n_{\text {eff }} .
$$

The magnitude of the effective index change depends on the overlap of the cantilever with the evanescent field. We have modelled the perturbation effect by using a twodimensional modesolver [15] to calculate $n_{\text {eff }}$ as a function of the air gap between the cantilever and the waveguide for TE-polarized light at a wavelength of $1550 \mathrm{~nm}$. As expected from the exponential tail of the evanescent field, the response is strongly nonlinear as shown in Fig. 6. It should be noted that due to pull-in phenomena the cantilever can be actuated in a controlled way only over $1 / 3$ of the initial distance between the electrodes (see section 4.2). This emphasizes the need to reduce the initial 
gap to obtain a large actuation range with a small cantilever deflection. If the initial gap between the cantilever and the waveguide is too large, the actuation effect is negligible (Fig. 6).

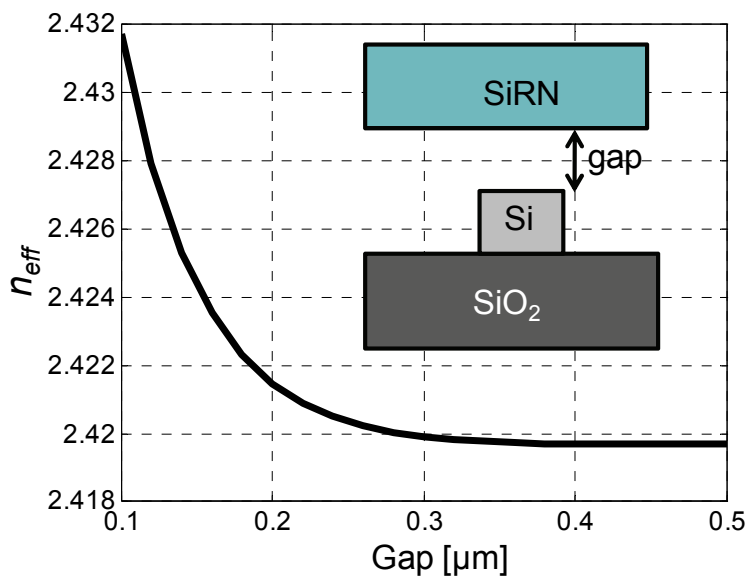

Fig. 6. Calculated effective index as a function of the air gap between the cantilever and the waveguide. Inset: cross-section used in the simulation.

We used 3D FDTD [16] to calculate the effect of the cantilever on directional coupling. In the calculation we considered a structure shown in Fig. 7. In this structure there is a pair of Si photonic wire waveguides (with a $200 \mathrm{~nm}$ gap between them), and a SiRN cantilever, modelled as a dielectric block above the waveguide pair. Wave propagation through the structure was simulated with and without the cantilever. We then compared the output powers of the ports out 1 and out2, at a wavelength of $1550 \mathrm{~nm}$. When the cantilever is present, with a $200 \mathrm{~nm}$ air gap between it and the waveguide pair, $2.435 \%$ of the input light is collected at out 2 port. Without the cantilever the number is $2.360 \%$. According to the FDTD results, $0.18 \%$ of the input light is lost due to the cantilever.

As a check for radiation loss induced by the cantilever, the extreme situation of the cantilever in direct contact with a single waveguide ( $0 \mathrm{~nm}$ gap) was analysed; $96.77 \%$ of the light was transmitted through the waveguide.
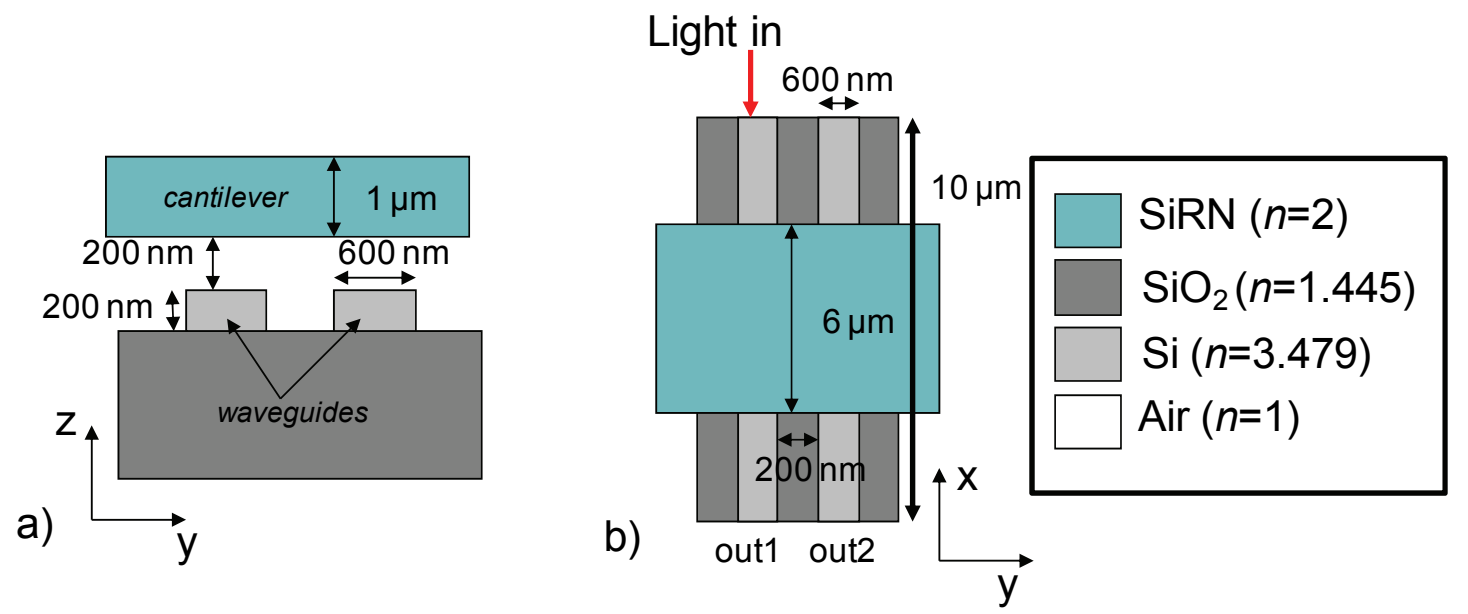

Fig. 7. Model of the structure used in 3D FDTD calculations to evaluate the effect of the cantilever on coupling. a) Cross-section; b) top view. 
The waveguide has been designed to be single mode for TE-polarized light at a wavelength of $1550 \mathrm{~nm}$. For single mode operation and efficient coupling, the waveguide width and the gap between the waveguides (in the coupling region) were designed to be $550 \mathrm{~nm}$ and $240 \mathrm{~nm}$, respectively. The ring resonator is a so-called racetrack design with a radius of $10 \mu \mathrm{m}$ and straight sections of $12 \mu \mathrm{m}$ length. The free spectral range of the ring is $6.6 \mathrm{~nm}$ (at $1550 \mathrm{~nm}$ ).

The microcantilever was monolithically integrated following the steps described in section 4.3. A scanning electron microscope (SEM) image of the integrated device is shown in Fig. 8.

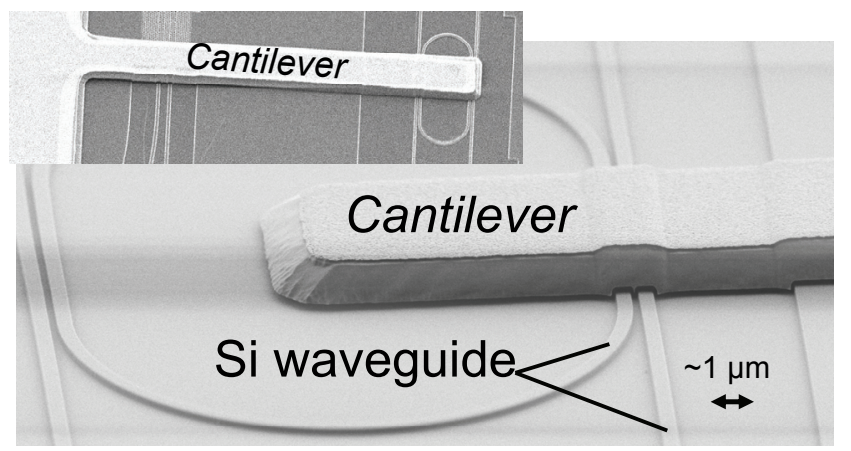

Fig. 8. a) SEM image of the fabricated device. Inset: top SEM image of another device variation.

Fig. 9a shows the measured transmission spectra for two different applied voltages, $0 \mathrm{~V}$ and $8.5 \mathrm{~V}$. It can be see that the resonance wavelength shifts as the cantilever moves closer to the waveguide. The quality factor of the resonance remains high; i.e. the presence of the cantilever does not appear to significantly increase loss in the resonator. However, as shown in Fig. 9b, when the cantilever is pulled in at $9.2 \mathrm{~V}$ (and touching the waveguide surface) a noticeable change of the quality factor; from 10000 to 5000 , was found.

Fig. 9c shows the measured resonance wavelength shift as a function of the actuation voltage. It can be seen that the response is strongly nonlinear with the $20 \mathrm{pm}$ wavelength shift corresponding to voltage change from $8 \mathrm{~V}$ to $8.5 \mathrm{~V}$. The maximum reversibly attainable wavelength shift was found to be $50 \mathrm{pm}$, at an applied voltage of $8.5 \mathrm{~V}$. The energy required for obtaining this shift is given by the energy required to charge the $(\sim 1$ $\mathrm{pF}$ ) capacitance of the cantilever to this voltage, which evaluates to $\sim 36 \mathrm{pJ}$. 


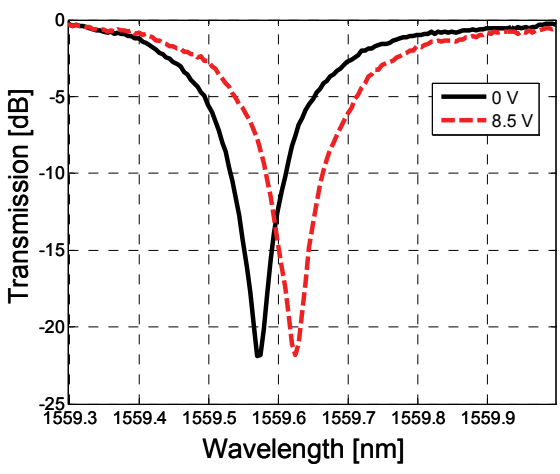

a)

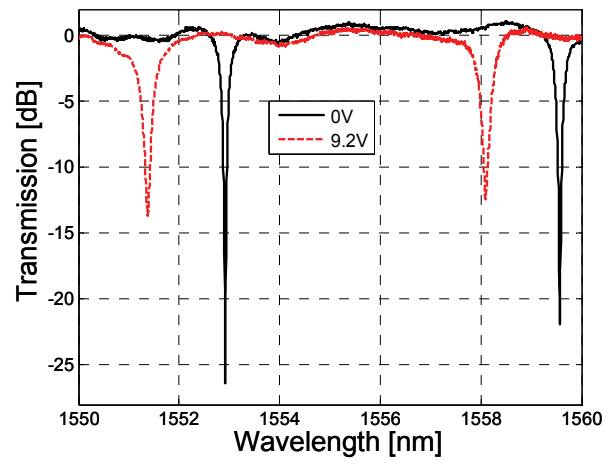

b)

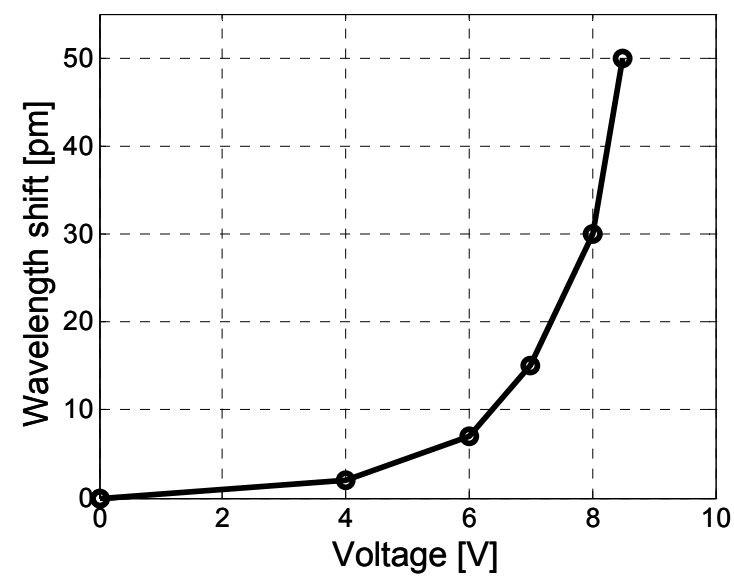

c)

Fig. 9. a) Transmission spectrum of a ring resonator measured for $0 \mathrm{~V}$ and for $8.5 \mathrm{~V}$ actuation voltages. b) Transmission spectrum of a ring resonator measured for $0 \mathrm{~V}$ and for $9.2 \mathrm{~V}$ actuation voltages. At $9.2 \mathrm{~V}$ the cantilever is pulled-in that results in large $(\sim 5 \mathrm{~nm})$ resonance wavelength shift and lowers the quality factor of the ring. Note that due to measurement noise and the used normalization procedure, the transmission is (unphysically) slightly above $0 \mathrm{~dB}$ in some regions. c) Measured resonance wavelength shift as a function of actuation voltage.

The required actuation voltage to obtain a certain resonance wavelength shift depends on the initial gap between the cantilever and the waveguide. We estimate the gap between the cantilever and the waveguide to be $305 \mathrm{~nm}$, resulting from $200 \mathrm{~nm}$ thick sacrificial layer, $40 \mathrm{~nm}$ protective SiRN layer and $65 \mathrm{~nm}$ upward bending of the cantilever due to tailored residual stress. Further on, based on the experimental data the cantilever can be tuned by $150 \mathrm{~nm}$ before pull-in occurs. As can be seen from Fig. 6, much stronger optical tuning could be achieved with a smaller initial gap. It should be noted that fabricating the cantilevers with a good yield becomes increasingly more challenging for smaller initial gaps. However, by optimizing the fabrication process, we expect to be able to reduce the initial gap to approximately $200 \mathrm{~nm}$ with acceptable yield, thus increasing the modulation strength by a factor of 2 or 3 .

To demonstrate dynamic operation of the switch, we have actuated the cantilever with a square wave signal (from 0 to $6 \mathrm{~V}$ ) while recording the optical transmittance through the device at a fixed wavelength. Fig. 10 shows the result for a $2 \mathrm{kHz}$ actuation signal. The 
signal rise time $(110 \mu \mathrm{s})$, shown in Fig. 10, is due to slow photodetector. From the preliminary results, obtained with a fast photodetector (see the appendix), we estimate a signal rise time of $16 \mu \mathrm{s}$, while the fall time is $4 \mu \mathrm{s}$. The maximum actuation frequency is thus limited to approximately $30 \mathrm{kHz}$.

Although higher frequencies can be used to modulate the optical signal, the peak value of the signal is cut due to rise time. This could be compensated by having an overshoot in the actuation signal, yet there would still be phase shift between the actuation and the optical signal. Based on the measured mechanical resonance frequency of the cantilever $(800 \mathrm{kHz})$, we expect rise times that are almost order of magnitude shorter than the measured values. We expect that the operation frequency is limited by the squeeze film damping of the cantilever $[9,10,17]$ (see also section 4.2 ).

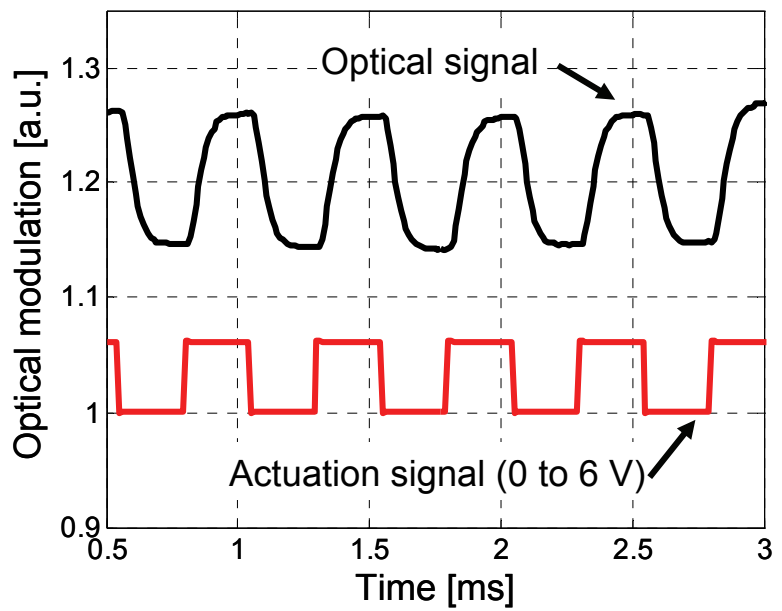

Fig. 10. Dynamic response of the electromechano-optically modulated ring resonator. The upper trace shows the transmission through the device at a fixed wavelength for a $2 \mathrm{kHz}$ square wave actuation signal (lower trace). Measured with a slow photo detector. Preliminary results with the fast detector; see the appendix.

\subsection{Micromechanically actuated photonic crystal waveguide}

Below preliminary results are presented aiming to demonstrate the tuning of a photonic crystal with a specially designed microcantilever. We consider a straight $\mathrm{PhC}$ waveguide that is formed by reducing the hole diameter of one $\mathrm{PhC}$ hole row (changed-hole $\mathrm{W} 1$ waveguide). The optical properties of this $\mathrm{PhC}$ device are actuated with a cantilever that covers the entire top surface of the photonic crystal, see Fig. 11a. The sacrificial $\mathrm{SiO}_{2}$ layer, that partially determines the gap between the $\mathrm{PhC}$ and the cantilever, had a thickness of $100 \mathrm{~nm}$ for this device. As mentioned in 4.3, such a thin layer allows the cantilever to have self-aligned tips that will enter the holes of the $\mathrm{PhC}$ as the cantilever approaches the PhC. A SEM image of the fabricated device is shown in Fig. 11a, the tips are clearly visible in Fig. 11b. 

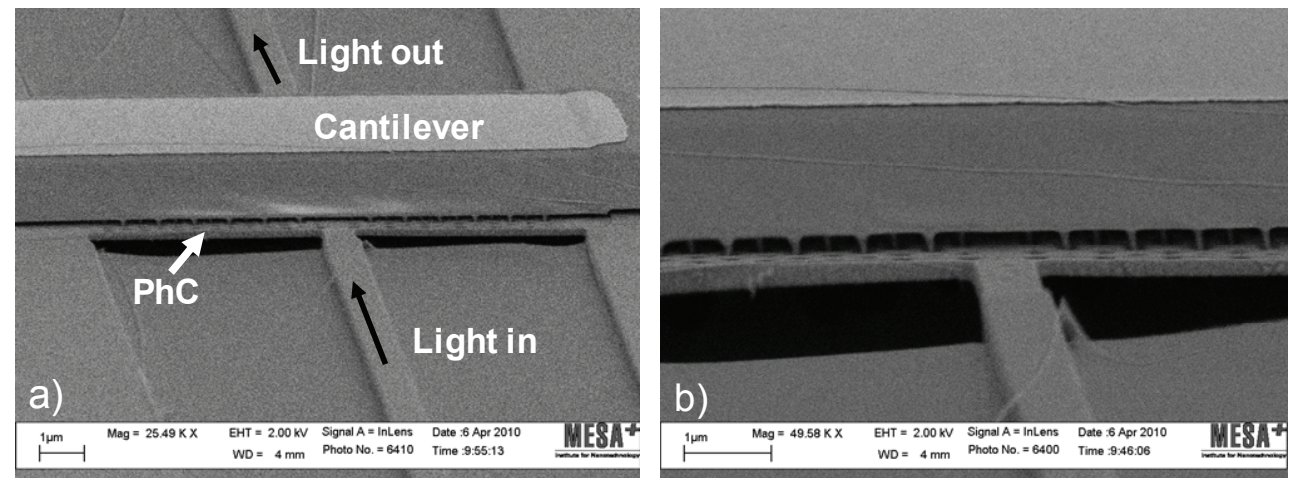

Fig. 11. a) A SEM image of the device. The cantilever covers the entire PhC. b) Detail of the same device. Small nitride tips, self-aligned with respect to the holes in the $\mathrm{PhC}$, protrude from the cantilever.

We have modelled the effect of the tips on the dispersion properties of the $\mathrm{PhC}$ waveguide with the MIT Photonic Bands (MPB) software [18]. Fig. 12a shows the dispersion graph of the $\mathrm{PhC}$ waveguide, as calculated from the structure (a so-called supercell, which is assumed to be repeated periodically) given in Fig. 12b. The slab hole diameter of the $\mathrm{PhC}$ is $270 \mathrm{~nm}$, the reduced hole diameter in the $\mathrm{PhC}$ waveguide is 140 $\mathrm{nm}$ and the period is $440 \mathrm{~nm}$. Fig. $12 \mathrm{~b}$ also shows the nitride tips with a diameter of 180 $\mathrm{nm}$ (grey disks) at the center of each large hole. In the two-dimensional calculations, the presence of the cantilever body was accounted for by changing the effective index of the slab material. The reference case (absence of the cantilever) was modelled with a slab effective index of 2.8495 , providing the reference curve. To model a cantilever without tips, we increased the slab effective index to 2.865. This corresponds to a situation where the nitride cantilever is $60 \mathrm{~nm}$ above the $220 \mathrm{~nm}$ thick Si slab. Finally we included tips into $\mathrm{PhC}$ holes and used the increased slab index (2.865) to model the presence of the cantilever body. From the graph it is clear that the tips have a large effect on the dispersion properties.
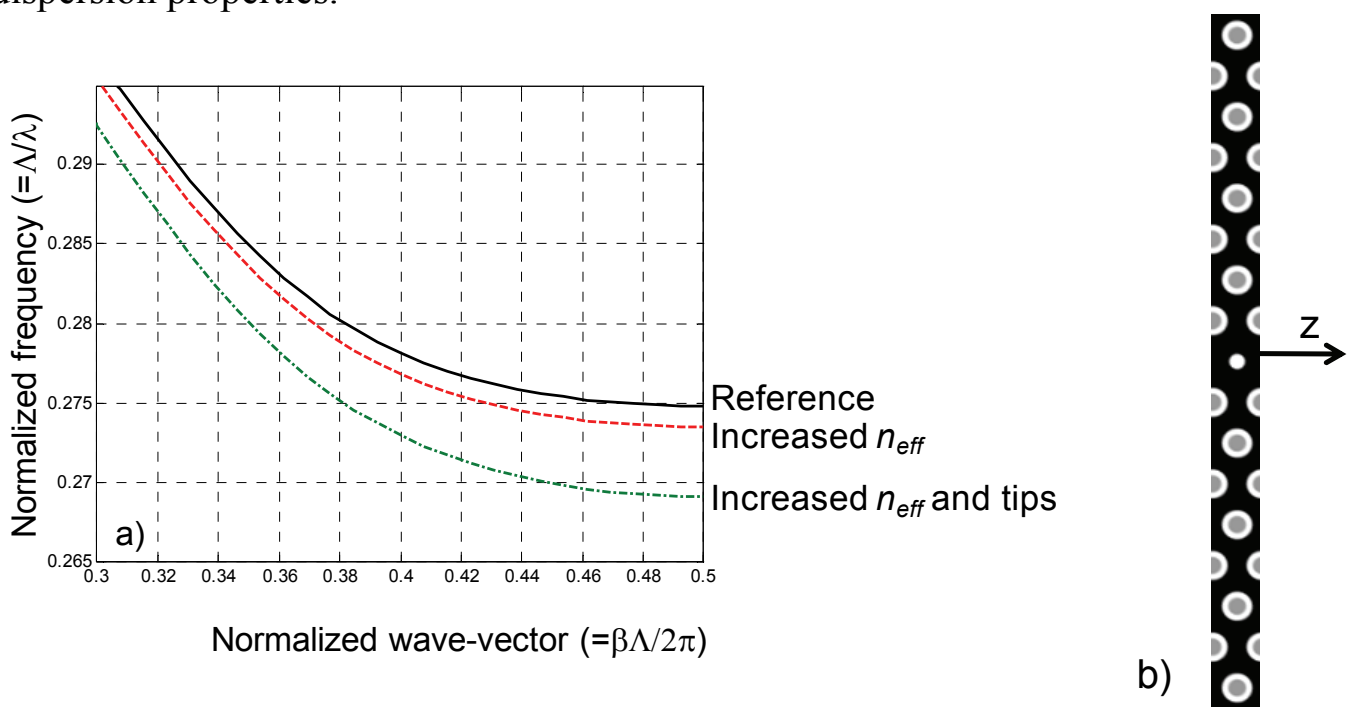

Fig. 12. a) The dispersion graph of the PhC waveguide calculated with MPB using supercell method. b) The supercell structure, to be repeated periodically along the z-axis to represent an infinitely long $\mathrm{PhC}$ waveguide running parallel to the $\mathrm{z}$-axis, that is used to calculate the dispersion properties. 
The optical response of the $\mathrm{PhC}$ waveguide, when actuated with a "tip-cantilever" was measured. The characterization procedure was similar to the one used for the ring resonator (section 4.5). However, here we do not measure a shift of a resonance wavelength, but the change in light transmission (at a constant wavelength) through the $\mathrm{PhC}$ waveguide. The slow-light properties of the $\mathrm{PhC}$ waveguide likely enhance the actuation effect.

Fig. 13 shows the optical modulation (at $1550 \mathrm{~nm}$ wavelength; normalized frequency 0.284 ) as a result of a $1-\mathrm{kHz}$ actuation signal. The modulation depth of the optical signal is approximately $7 \mathrm{~dB}$. The exact reason why the propagation loss increases when the cantilever is actuated closer to the $\mathrm{PhC}$ is not known. Mode cut-off of the $\mathrm{PhC}$ waveguide can be excluded as a potential mechanism, as it occurs at a wavelength longer than 1620 $\mathrm{nm}$ for all cantilever positions; also, as the cantilever moves closer to the waveguide, the cut-off wavelength is moved further away from the operating wavelength. There are two potential reasons: the dispersion properties change such that a part of the light is diffracted from the $\mathrm{PhC}$ waveguide (light line crossing), or the presence of the cantilever close to the $\mathrm{PhC}$ introduces such an abrupt index change that it leads to strong out-ofplane scattering.

When the cantilever is in the off state, the measured propagation loss of the $\mathrm{PhC}$ waveguide is less than $2 \mathrm{~dB}$, which is equal (within the measurement accuracy of $\pm 2 \mathrm{~dB}$ ) to that of a straight waveguide without any cantilever at all.

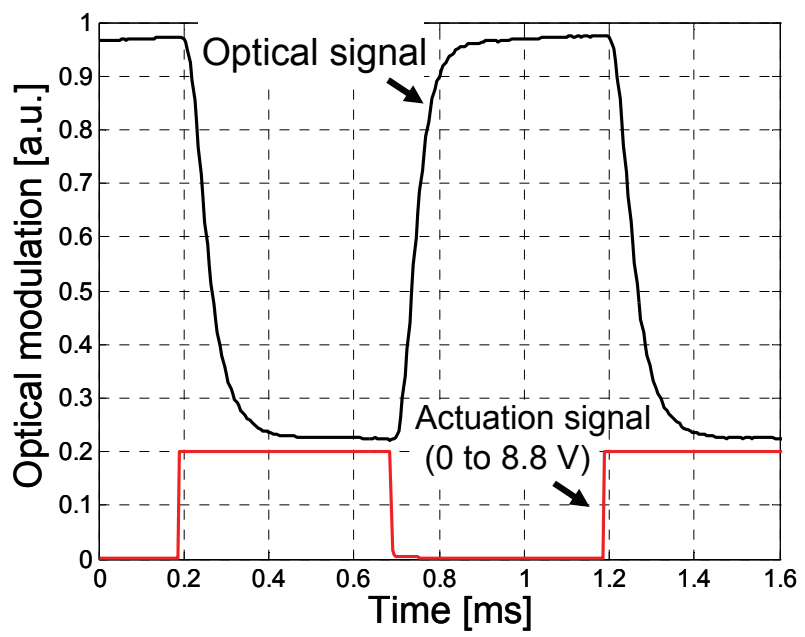

Fig. 13. Dynamic response. Transmission through the device at a fixed wavelength for a $1 \mathrm{kHz}$ square wave actuation signal. Rise (and fall) time of the optical signal is limited by the speed of the photodetector.

\subsection{Conclusions}

Electrostatically actuated microcantilevers were used to tune the optical properties of a racetrack ring-resonator and a photonic crystal waveguide. These cantilevers were monolithically integrated in wafer scale on SOI photonic chips.

The resonance wavelength of the ring-resonator was tuned by $50 \mathrm{pm}$ with an actuation voltage of $8.5 \mathrm{~V}$. The actuation of the resonance wavelength is obtained without notably increasing the optical loss. With a compact $(10 \mu \mathrm{m}$ by $10 \mu \mathrm{m})$ photonic crystal waveguide component a modulation depth of $7 \mathrm{~dB}$ was achieved with $8.8 \mathrm{~V}$ actuation voltage. For both devices the actuation frequency is limited to approximately $30 \mathrm{kHz}$. 
Mechano-optical actuation

Based on the measured mechanical resonance frequencies of the cantilevers $(\sim 800 \mathrm{kHz})$ in vacuum, we expect that the operation frequency is limited by the squeeze film damping of the cantilever. 


\section{References}

[1] G. Cocorullo, M. Iodice, I. Rendina, and P.M. Sarro, "Silicon Thermooptical Micromodulator with 700kHz - 3-dB Bandwidth", IEEE Photonics Technology Letters, vol. 7, pp. 363-365, 1995.

[2] Q. Xu, B. Schmidt, S. Pradhan, and M. Lipson, "Micrometre-scale silicon electro-optic modulator", Nature, vol. 435, pp. 325-327, 2005.

[3] G.N. Nielson, D. Seneviratne, F. Lopez-Royo, P.T. Rakich, Y. Avrahami, M.R. Watts, H.A. Haus, H.L. Tuller, and G. Barbastathis, "Integrated wavelength-selective optical MEMS switching using ring resonator filters", IEEE Photonics Technology Letters, vol. 17, pp. 1190-1192, 2005.

[4] P.T. Rakich, M. A. Popović, M.R. Watts, T. Barwicz, H.I. Smith, and E.P. Ippen, "Ultrawide tuning of photonic microcavities via evanescent field perturbation", Optics Letters, vol. 31, pp. 1241-1243, 2006.

[5] W.C.L. Hopman, K.O. van der Werf, A.J.F Hollink, W. Bogaerts, V. Subramaniam, and R.M. de Ridder, "Nano-mechanical tuning and imaging of a photonic crystal micro-cavity resonance," Optics Express, vol. 14, pp. 8745-8752, 2006.

[6] I. Märki, M. Salt, and H.P. Herzig, "Tuning the resonance of a photonic crystal microcavity with an AFM probe," Optics Express 14, pp. 2969-2978, 2006.

[7] M. Maier and M. Reisslein, "Trends in Optical Switching Techniques: A Short Survey," IEEE Network, vol. 22, pp. 42-47, 2008.

[8] N.V. Lavrik, M.J. Sepaniak, and P.G. Datskos, "Cantilever transducers as a platform for chemical and biological sensors", Review of Scientific Instruments, vol. 75, pp. 2229-2253, 2004.

[9] H. Hosaka, K. Itao, and S. Kuroda, "Damping characteristics of beam-shaped micro-oscillators", Sensors and Actuators A, vol. 49, pp. 87-95, 1995.

[10] G.J. Veldhuis, T. Nauta, C. Gui, J.W. Berenschot, and P.V. Lambeck, "Electrostatically Actuated Mechanooptical Waveguide ON-OFF Switch Showing High Extinction at a Low Actuation-Voltage", IEEE Journal of Selected Topics in Quantum Electronics, vol. 5, pp. 60-66, 1999.

[11] G.N. Nielson, "Micro-Opto-Mechanical Switching and Tuning for Integrated Optical Systems", Massachusetts Institute of Technology, PhD thesis, 2004.

[12] S.M. Chakkalakkal Abdulla, et al., "Tuning of Race-Track Ring Resonators by Integrated Bimorph Cantilevers", in preparation.

[13] S.M. Chakkalakkal Abdulla, PhD thesis, Faculty of Electrical Engineering, Mathematics and Computer Science, University of Twente, Enschede, The Netherlands, in preparation.

[14] D. Taillaert, F. van Laere, M. Ayre, W. Bogaerts, D. van Thourhout, P. Bienstman, and R. Baets, "Grating couplers for coupling between optical fibers and nanophotonic waveguides," Japanese Journal of Applied Physics, vol. 45, pp. 6071-6077, 2006.

[15] OlympIOs Integrated Optics Software. C2V, P.O. Box 318, 7500 AH Enschede, The Netherlands,(http://www.c2v.nl/software/).

[16] A. Farjadpour, D. Roundy, A. Rodriguez, M. Ibanescu, P. Bermel, J.D. Joannopoulos, S.G. Johnson, and G. Burr, "Improving accuracy by subpixel smoothing in FDTD," Optics Letters, vol. 31, pp. 29722974, 2006.

[17] H. Sumali, "Squeeze-film damping in the free molecular regime: model validation and measurement on a MEMS", Journal of Micromechanics and Microengineering, vol. 17, pp. 2231-2240, 2007.

[18] S.G. Johnson and J.D. Joannopoulos, "Block-iterative frequency-domain methods for Maxwell's equations in a planewave basis", Optics Express, vol. 8, pp. 173-190, 2001. 


\section{Summary and outlook}

This thesis describes the design, fabrication, and characterization of compact optical devices for sensing and switching applications. Our focus has been to realize the devices using CMOS-compatible fabrication processes. Particularly the silicon photonics fabrication platform, ePIXfab, has been used for this purpose. There are high hopes that silicon photonics, by utilizing the CMOS processing tools and standardized fabrication processes, can reduce the cost of photonic integrated circuits and open new markets to this technology-driven field of research.

Silicon photonics is currently in a state where separate components and fabrication technologies are actively developed, like the ones described in this thesis. Unlike for electronic CMOS ICs, no global standardized fabrication process exists for photonic integrated circuits, despite great efforts made by the ePIXnet and ePIXfab communities. The logical next step ahead would be standardization of the components and technologies, such that they could be effectively used. Due to the multidisciplinarity and complexity of these technologies it is unlikely that all fabrication will be done in one place. Instead, a distributed fabrication network providing different process options seems to be more feasible.

\section{Sensor work}

In chapter 2 we exploited waveguide-grating-based sensors. The waveguide grating is a simple building block for sensors; essentially it is just a straight waveguide requiring laser source and photodetector for sensor operation. The sensitivity of the grating based can be tailored (taking into account the process limitations) by changing grating parameters like length and the strength of the grating.

Grated silicon photonic wires (GSPWs) were designed and fabricated using the ePIXfab fabrication platform to realize cost effective, compact and sensitive biosensor.

According to our experimental results, cladding index changes as small as $10^{-5}$ RIU can be detected with the GSPWs. The length of the device is smaller than $180 \mu \mathrm{m}$, allowing dense integration of the sensor elements on an optical chip. As a demonstration the sensor was used to monitor the evaporation process of isopropanol from an aqueous solution. Label-free enzyme sensing was also demonstrated with the sensor, after depositing an immobilized enzyme-specific anti-body layer on the sensing element. Such sensors are expected to have applications in, e.g., medical diagnostics and detection of contaminants in the food and feed industry.

The GSPW that was developed and characterized in this thesis is just one part of a sensor. The other important parts are the biochemicals (that enable selectivity), (micro)fluidics, light source, photodetector and electrical read-out circuitry. Particularly for label-free sensing it would be of interested to pattern and deposit the biochemicals (receptor layers) on wafer scale in mass production way. In general it is debatable what the level of integration of these components should be. If all the functions are integrated on one chip (so called lab-on-chip approach), compact devices can be obtained. However, if the integration technology is complex, the cost of the device might be high, especially if the sensor is not reusable. On the other hand, set-ups based on devices with a low level of integration, such as commercial surface plasmon resonance based systems, are neither cheap nor compact. 
A new integrated optical read-out method for microcantilever displacements was developed. The optical read-out of cantilever deflection can for example be used in microcantilever sensor applications. These sensors are based on deflection of a cantilever when exposed to an analyte, such as hydrogen gas. The optical detection of cantilever deflection is a particularly attractive property for measurements in hazardous environments where electric contacts cannot be used.

Our optical cantilever read-out sensor is based on the spatial localization of a resonance in waveguide gratings. High displacement sensitivity is obtained by placing the microcantilever such that it interacts with the evanescent field of this resonance. The properties of the sensors were theoretically evaluated with computer simulations. Based on these results, a sub-nanometer detection limit was found for the deflection of silicon oxide cantilevers. Fabrication aspects of this sensor were studied. For the optical part, a well-established silicon nitride waveguide grating technology was chosen. The emphasis of the technological work was on the integration of the fabrication processes for optical devices and microcantilevers. After selecting a material that provides a high etch selectivity between different layers, a test fabrication was done. We tested isotropic reactive ion etching for releasing the cantilevers, as opposed to wet etching that requires a time-consuming and cumbersome freeze-drying step. The dry-etching method was found to result in high surface roughness of silicon nitride layers, likely ruining the optical properties of the waveguides in this layer. Based on the first fabrication test, the initial bending of the cantilever was indentified as an important factor for further study. This work was continued in a new $\mathrm{PhD}$ project.

\section{Micromechano-optical actuation}

The aim of the research was to implement an electromechanically actuated compact optical switch. The micromechanical actuation could be an attractive alternative to thermo-optical actuation due to its much smaller power consumption, yet providing similar tuning range and speed. In earlier work done in this field use was made of components that are not particularly compact and/or are assembled using bonding techniques; the mechanical part is attached to the optics chip. Our approach was to use a standardized silicon photonics fabrication platform, to design compact optical devices, and then do further processing with CMOS-compatible technology to monolithically integrate microcantilevers on these optics chips.

In chapter 3 we presented several optical devices that were designed to realize a compact mechanically actuated optical switch. Considering the available cantilever technology, the main focus in the design was on devices that could be actuated with a $10-\mu \mathrm{m}$ wide cantilever. For typically sized photonic crystal devices this implies that the cantilever covers the whole PhC. However, we also designed a PhC component that could be actuated by just manipulating one of its holes (with a diameter of only $250 \mathrm{~nm}$ ). Since photonic-crystal components are known to be challenging to design, and are infamous for their high optical loss, we also implemented more conventional, yet less compact, designs based on waveguide gratings and ring-resonators.

In chapter 4 , the micromechanical actuation of a ring resonator and a photonic crystal were demonstrated. We also discussed the basic mechanical properties of an electrostatically actuated microcantilever. From this theoretical discussion we predicted a low actuation voltage $(<6 \mathrm{~V})$, and an actuation frequency of better than $25 \mathrm{kHz}$ (limited 
by squeeze film damping). Actuating a cantilever in close proximity to a ring resonator resulted in a shift of the resonance wavelength by $50 \mathrm{pm}$. The required actuation voltage was $8.5 \mathrm{~V}$. The modulation frequency was limited to $30 \mathrm{kHz}$, likely due to squeeze film damping.

A similar cantilever was used to modulate the intensity of light that propagates in a compact $(10 \mu \mathrm{m}$ by $10 \mu \mathrm{m})$ photonic crystal $(\mathrm{PhC})$ device. For the $\mathrm{PhC}$ actuation the cantilever was designed such that it has small tips that can enter the holes of the PhC. A computer simulation showed that this significantly changes the dispersion properties of the $\mathrm{PhC}$ device. A modulation depth of $7 \mathrm{~dB}$ was measured, with potential actuation frequencies up to $30 \mathrm{kHz}$.

The speed of mechanically actuated optical devices is poor compared to the nearly 1 $\mathrm{MHz}$ actuation frequency that is possible with the thermo-optic method in silicon. Even if the power consumption of the cantilever-tuned device is much smaller, the speed likely limits the possible applications. Assuming that the speed is limited by the squeeze damping, it could be increased by designing the cantilever such that air can escape faster from the small gap that is between the cantilever and the optics chips. This could be achieved e.g. by reducing the width of the cantilever. The narrow cantilever in turn requires optical devices where only very local actuation results in a strong optical response; this can be achieved e.g. with the aforementioned photonic crystal devices. Another straightforward approach is to operate the device at low pressure conditions. 


\section{Samenvatting}

Dit proefschrift beschrijft ontwerp, vervaardiging en karakterisering van compacte optische componenten voor toepassing in sensoren en optische schakelaars. Het werk was gericht op realisatie van de componenten in een fabricageproces dat compatibel is met de processen die gebruikt worden voor de vervaardiging van standaard (CMOS) elektronica. Hiervoor is in het bijzonder gebruik gemaakt van een Europees "fabricageplatform" voor fotonische componenten in silicium, genaamd "ePIXfab". Men verwacht dat het gebruik van de gestandaardiseerde gereedschappen en fabricageprocessen voor CMOS de kosten van silicium fotonica kan reduceren en nieuwe markten kan openen.

Ten tijde van het schrijven van dit proefschrift (2010) wordt actief gewerkt aan de ontwikkeling van losse componenten en de benodigde technologie, zoals die bijvoorbeeld beschreven worden in dit proefschrift. Anders dan het geval is voor CMOS elektronica, bestaat er nog geen algemeen gestandaardiseerd fabricageproces voor geïntegreerde fotonische schakelingen, ondanks de krachtige inspanningen die verricht zijn door de onderzoekers die samenwerkten in het Europese netwerk "ePIXnet" en die worden voortgezet door middel van ePIXfab. De logische volgende stap is de standaardisatie van componenten en technologieën, zodat deze efficiënt kunnen worden ingezet. Wegens de multidisciplinaire aard en de complexiteit van deze technologieën is het onwaarschijnlijk dat alle fabricagestappen op een enkele locatie zullen worden uitgevoerd. Een ruimtelijk gespreid fabricagenetwerk dat een variatie aan procesopties kan bieden lijkt een realistischer vooruitzicht.

\section{Sensorwerk}

In hoofdstuk 2 zijn sensoren besproken die gebaseerd zijn op optische golfgeleiders waarin een traliestructuur is aangebracht. Een dergelijk "golfgeleidertralie" is een relatief eenvoudige bouwsteen voor sensoren, die slechts bestaat uit een recht golfgeleidend kanaal waarin een periodieke geometrische verstoring is aangebracht. Voor toepassing als sensor moeten nog een laserlichtbron en een fotodetector worden toegevoegd. De gevoeligheid van de sensor kan -binnen de grenzen die door het fabricageproces worden gesteld- worden aangepast aan de specifieke toepassing, door tralieparameters zoals lengte en sterkte van de periodieke verstoring te kiezen.

De golfgeleidertralies in dit werk, die zijn gebaseerd op optische kanalen met dwarsafmetingen kleiner dan $1 \mu \mathrm{m}$, zogenaamde "fotonische draden" (photonic wires), zijn ontworpen en vervaardigd op het ePIXfab platform. Hiermee zijn relatief goedkope, compacte en gevoelige biosensoren gerealiseerd.

Onze experimentele resultaten laten zien dat veranderingen van de brekingsindex in het medium grenzend aan het golfgeleidertralie van $10^{-5}$ brekingsindexeenheid kunnen worden gedetecteerd met deze golfgeleidertralies. Het actieve deel van de optische component heeft een lengte kleiner dan $180 \mu \mathrm{m}$, waardoor in principe grote aantallen sensorelementen in grote dichtheid op een enkele optische "chip" kunnen worden geïntegreerd.

De werking van de sensor is gedemonstreerd door het verdampingsproces van isopropanol uit een waterige oplossing in de tijd te volgen. Ook is de concentratie van een enzym gemeten zonder dat dit met van een extra chemisch "label" werd voorzien (het zogenaamde "label-free sensing"). Hiertoe werd het optisch gevoelige element voorzien 
van een dun laagje biologische antilichamen met een specifieke affiniteit voor het te detecteren enzym. Verwacht wordt dat dergelijke sensoren toepassing zullen vinden in bijvoorbeeld de medische diagnostiek en voor de detectie van verontreinigingen in de voedsel- en voederindustrie.

Het silicium golfgeleidertralie dat in dit werk werd ontwikkeld en gekarakteriseerd is slechts een onderdeel van een complete sensor. De andere belangrijke onderdelen zijn de biochemicaliën die de selectiviteit van de sensor bepalen (de zogenaamde "receptorlagen"), het microfluïdische systeem dat de te analyseren materialen aan het gevoelige element toevoert, de lichtbron, de fotodetector en de elektronica voor signaalverwerking en presentatie van de meetresultaten. In het bijzonder voor de massafabricage van elementen voor label-free sensing is het interessant om diverse receptorlagen selectief te kunnen aanbrengen en structureren op de schaal van complete siliciumplakken (de zogenaamde wafers die als basis dienen voor de fabricage van zowel elektronische als optische schakelingen). De meest wenselijke schaal van integratie van optische componenten is niet onomstreden. Zeer compacte microsystemen kunnen worden verkregen door integratie van alle functies op een enkele chip (de zogenaamde "lab-on-a-chip"-aanpak). Als echter de integratietechnologie complex is, wordt de prijs van de microsystemen te hoog, in het bijzonder voor sensoren die niet herbruikbaar zijn. Anderzijds zijn systemen met een laag integratieniveau goedkoop noch compact, zoals de huidige systemen gebaseerd op resonantie van oppervlakteplasmonen.

Een geheel ander type sensoren maakt gebruik van microbalkjes die verbuigen onder invloed van een te meten grootheid, bijvoorbeeld de concentratie van waterstofgas. Optische detectie van de verplaatsing van zo'n balkje maakt het mogelijk sensoren te ontwikkelen die geen rechtstreekse elektrische verbindingen nodig hebben en die daardoor bijzonder geschikt zijn voor toepassingen in ruimten met explosiegevaar.

Een nieuwe optische uitleesmethode voor de positie van een microbalkje is gebaseerd op de ruimtelijke lokalisatie van een resonantie in een golfgeleidertralie. Een grote verplaatsingsgevoeligheid is verkregen door het microbalkje in het evanescente optische veld van de tralieresonantie te plaatsen. De eigenschappen van de sensoren zijn theoretisch onderzocht door middel van computersimulaties. Op basis van die resultaten is gevonden dat de detectielimiet voor verplaatsingen van een siliciumoxide-balkje kleiner is dan $1 \mathrm{~nm}$. Op grond van een studie naar de vervaardigingsmogelijkheden van een dergelijke sensor is ervoor gekozen om het golfgeleidertralie te realiseren in een beproefde siliciumnitride-technologie. Het technologische werk concentreerde zich op de integratie van de optische structuren met de microbalkjes. Hierbij was de keuze van materialen belangrijk die een hoge mate van selectiviteit bieden bij de noodzakelijke etsprocessen. Vergelijkende tests zijn uitgevoerd van een isotroop reactief plasmaetsproces en een nat chemisch proces. De plasma-etsmethode bleek een grote ruwheid van de optische siliciumnitridelaag te veroorzaken, leidend tot onacceptabele optische verliezen. Daarom werd de voorkeur gegeven aan het natte proces, ondanks het daarvoor vereiste omslachtige en tijdrovende vriesdroogproces. Een eerste testfabricage leidde tot de conclusie dat meer onderzoek nodig is naar de initiële kromming van de microbalkjes t.g.v. interne spanningen die door het fabricageproces veroorzaakt worden. Dit werk is afgesplitst en voortgezet in een nieuw promotieproject. 


\section{Samenvatting}

\section{Micromechano-optische actuatie}

Het doel van dit onderzoek was de realisatie van een elektromechanisch bekrachtigde compacte optische schakelaar. Micromechanische actuatie kan een aantrekkelijk alternatief zijn voor thermo-optische actuatie wegens het veel geringere energieverbruik bij een modulatiebereik en -snelheid die van gelijke grootteorde zijn. Eerder werk in dit veld heeft componenten opgeleverd die niet bijzonder compact zijn en/of gebruik maken van een hybride techniek waarin optische en mechanische onderdelen van de component op aparte substraten worden vervaardigd, die later op elkaar worden bevestigd. In onze benadering daarentegen wordt eerst het optische deel van de component vervaardigd op het ePIXfab platform, waarna de elektromechanisch te bekrachtigen microbalkjes hier in een CMOS-compatibel proces monolithisch mee worden geïntegreerd.

Hoofdstuk 3 beschrijft verschillende optische componenten die ontworpen zijn voor toepassing in een compacte mechanisch bekrachtigde optische schakelaar. Op basis van fotonische kristallen kunnen uiterst compacte optische structuren ontworpen worden. Op grond van de beschikbare vervaardigingstechnologie voor microbalkjes werd het optisch ontwerp hoofdzakelijk gericht op structuren die bestuurd kunnen worden met $10 \mu \mathrm{m}$ brede balkjes. Gezien de typische afmetingen van fotonische kristalstructuren die werken bij infrarode golflengten, zal een balkje het fotonisch kristal nagenoeg volledig bedekken. We hebben echter ook een optische structuur ontworpen waarmee het schakeleffect wordt bereikt door selectief de vulling van een specifiek gat (met een diameter van ca. $250 \mathrm{~nm}$ ) in een fotonisch kristal te manipuleren. Aangezien ontwerp en voldoende nauwkeurige fabricage van fotonische een aanzienlijke uitdaging vormen en dit type structuren relatief hoge optische verliezen vertonen, zijn ook conventionelere doch minder compacte ontwerpen gemaakt, gebaseerd op golfgeleidertralies en ringresonatoren.

Hoofdstuk 4 beschrijft de experimentele resultaten van elektromechanisch bekrachtigde ringresonatoren en fotonische kristalstructuren. Daarbij worden tevens de elementaire mechanische eigenschappen van elektrostatisch bekrachtigd microbalkjes besproken. Op grond van deze theoretische beschouwing konden een actuatiespanning van minder dan 6 $\mathrm{V}$ en een actuatiefrequentie van meer dan $25 \mathrm{kHz}$ worden voorspeld. De actuatiefrequentie wordt theoretisch begrensd door het dempende effect van een dun luchtlaagje tussen het balkje en de onderliggende optische structuur (de zogenaamde "squeeze-film"-demping). Experimenteel werden een actuatiespanning van $8.5 \mathrm{~V}$ en een maximale actuatiefrequentie van $30 \mathrm{kHz}$ gevonden.

Met een balkje van overeenkomstige afmetingen is de lichtintensiteit gemoduleerd die wordt doorgelaten door een fotonische kristalstructuur met afmetingen van $10 \mu \mathrm{m} \times 10$ $\mu \mathrm{m}$. Het balkje is zodanig ontworpen dat het kleine uitsteeksels bezit die precies in de gaatjes van het fotonisch kristal vallen. Uit computersimulaties is gebleken dat hiermee de dispersie-eigenschappen van het fotonisch kristal sterk beïnvloed kunnen worden. Een modulatiediepte van $7 \mathrm{~dB}$ is gemeten.

De snelheid van mechanisch bekrachtigde optische componenten blijkt aanzienlijk lager te zijn dan de bijna $1 \mathrm{MHz}$ die mogelijk is met thermo-optische actuatie in silicium. Ook al is het energieverbruik van de mechano-optische actuatoren veel geringer, toch zal de geringere snelheid de mogelijke toepassingen beperken. Indien de snelheid door squeezefilm-demping wordt beperkt, kan het helpen om het balkje zo te ontwerpen dat de lucht gemakkelijker kan ontsnappen, bijvoorbeeld door het balkje smaller te maken. Een optische structuur die ook met zeer smalle balkjes een sterk schakeleffect vertoont is 


\section{Samenvatting}

reeds ontworpen. Een andere voor de hand liggende, doch wellicht minder praktische, oplossing is het bedrijven van de schakelaar in vacuüm. 
Appendix: Dynamic response of cantilever

\section{Appendix: Dynamic response of cantilever}

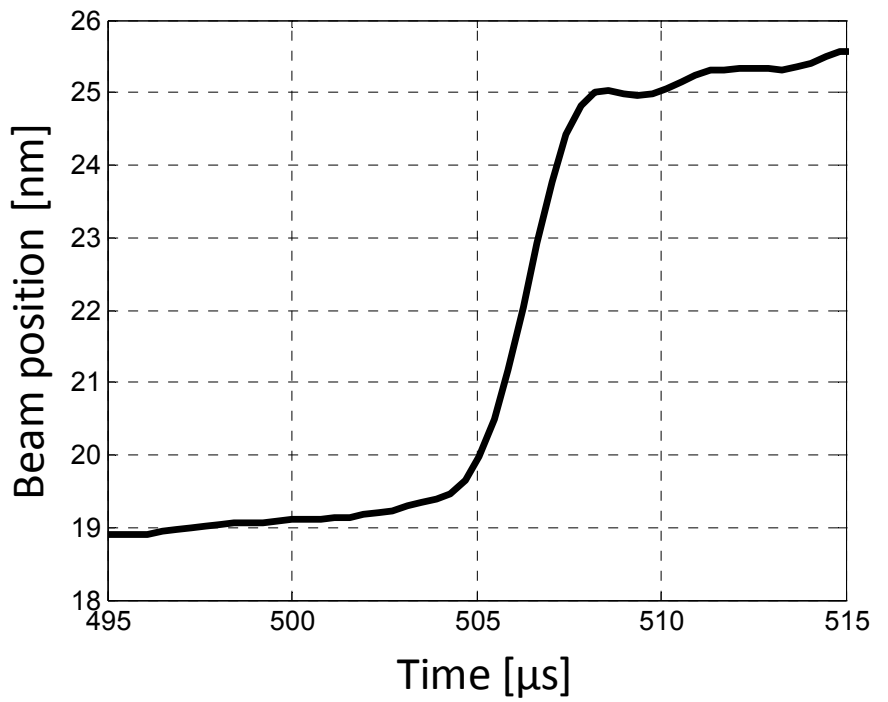

Fig. 1. The rise time response of a $40 \mu \mathrm{m}$ long cantilever as measured with a vibrometer. The actuation signal (not shown) is a $2 \mathrm{~V}$ square wave.

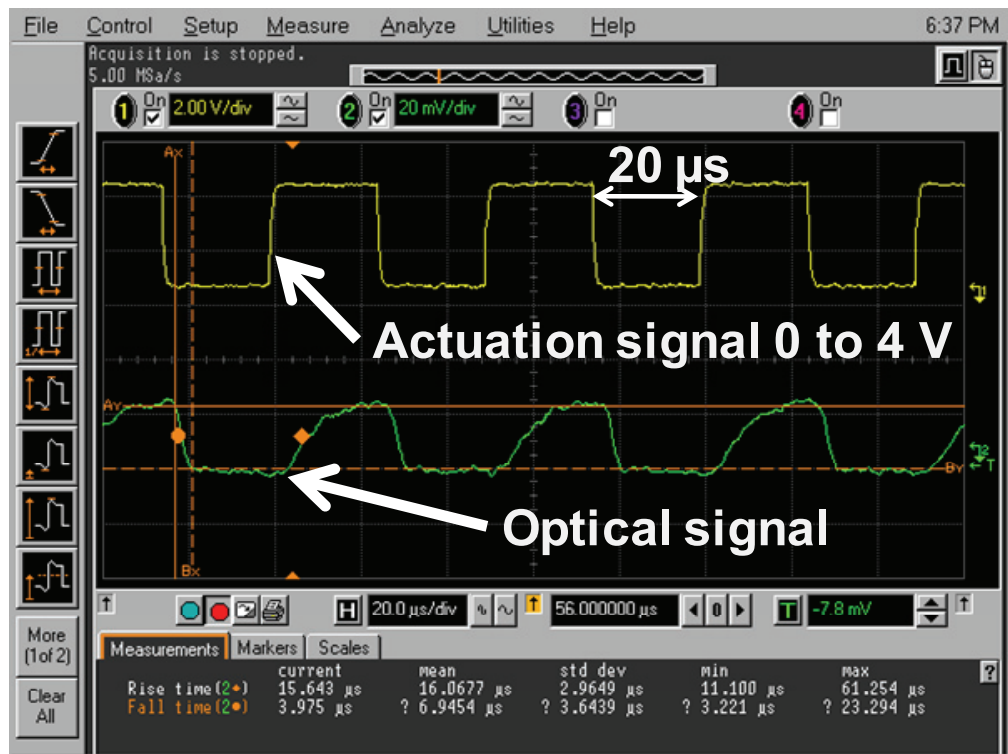

Fig. 2. Dynamic response of the electromechano-optically modulated ring resonator (with a $100 \mu \mathrm{m}$ long cantilever). The lower trace shows the transmission through the device (see section 4.5) at a fixed wavelength for a $25 \mathrm{kHz}$ square wave actuation signal (upper trace). 


\section{List of Publications}

\section{Peer-Reviewed Journal Articles}

1. L.J. Kauppinen, T.J. Pinkert, H.J.W.M. Hoekstra and R.M. de Ridder, "Photonic crystal cavity-based Y splitter for mechano-optical switching", IEEE photonics technology letters, vol. 22, pp. 966-968, 2010.

2. L.J. Kauppinen, H.J.W.M. Hoekstra and R.M. de Ridder, "A compact refractometric sensor based on grated silicon photonic wires", Sensors and actuators B (Chemical), vol. 139, pp. 194-198 2009.

\section{Conferences \\ Oral presentations}

3. L.J. Kauppinen, T.J. Pinkert, H.J.W.M. Hoekstra and R.M. de Ridder, "Photonic Crystal Cavity-Based Wavelength Routers", In: Proceedings of the 15th European Conference on Integrated Optics (ECIO), 7-9 April 2010, Cambridge, United Kingdom. ThG6, London: IET.

4. L.J. Kauppinen, M. Dijkstra, H.J.W.M. Hoekstra and R.M. de Ridder, "Label-free Enzyme Sensing with Grated Silicon Photonic Wire", In: Proceedings of the 2009 Annual Symposium of the IEEE Photonics Benelux Chapter, 05-06 Nov 2009, Brussels, Belgium. pp. 41-44. VUBPress Brussels University Press.

5. L.J. Kauppinen, H.J.W.M. Hoekstra and R.M. de Ridder, "Refractometric sensor based on silicon photonic wires", In: 3rd EOS Topical Meeting on Optical Microsystems (OMS09), pp. 27-30 Sep 2009, Capri, Italy. 2118. European Optical Society (EOS).

6. L.J. Kauppinen, H.J.W.M. Hoekstra, M. Dijkstra, R.M. de Ridder and G.J.M. Krijnen, "Grated waveguide optical cavity as a compact sensor for sub-nanometre cantilever deflections", In: Proceedings of the 14th European Conference on Integrated Optics (ECIO), 11-13 June 2008, Eindhoven, The Netherlands. pp. 111-114. Eindhoven University of Technology.

\section{Poster presentations}

7. L.J. Kauppinen, H.J.W.M. Hoekstra, M. Dijkstra, R.M. de Ridder and G.J.M. Krijnen, "Grated waveguide-based optical cavities as compact sensors for subnanometre cantilever deflections, and small refractive-index changes", In: Book of Abstracts 9th European Conference on Optical Chemical Sensors and Biosensors (Europtrode IX), 30-3-2008-02-04-2008, Dublin, Ireland. P084. Dublin City University. 


\section{Contributed}

8. F. Ay, L.J. Kauppinen, J.D.B. Bradley, K. Wörhoff, R.M. de Ridder and M. Pollnau, "Focused ion beam milled on-chip resonator nanostructures for applications in rare-earth-ion-doped A12O3 active waveguides", In: LEOS Annual Meeting Conference Proceedings, 2009. LEOS '09, IEEE, 4-8 Oct. 2009, BelekAntalya, Turkey. pp. 379-380. IEEE Photonics Society.

9. S.M. Chakkalakkal Abdulla, J.W. Berenschot, M.J. de Boer, L.J. Kauppinen, R.M. de Ridder and G.J.M. Krijnen, "Optimisation study of micro cantilevers for switching of photonic band gap crystals", In: Proceedings of the International Conference on Photonics in Switching 2009, 15-19 Sep 2009, Pisa, Italy. pp. 1-2. IEEE Photonics in Switching.

10. O.V. Ivanova, R. Stoffer, L.J. Kauppinen and M. Hammer, "Variational Effective Index Method for 3D Vectorial Scattering Problems in Photonics: TE Polarization", In: PIERS 2009 Moscow Proceedings, 18-21 Aug 2009, Moscow. pp. 1038-1042. The Electromagnetics Academy.

11. V.S. Pham, L.J. Kauppinen, M. Dijkstra, H.A.G.M. van Wolferen, R.M. de Ridder and H.J.W.M. Hoekstra, "Photonic crystal waveguides integrated with microcantilevers as a novel platform for gas sensors", In: Proceedings of the 7th International Symposium on Modern Optics and Its Applications (ISMOA), 10-14 August 2009, Bandung, Indonesia. pp. 68-68. Bandung Institute of Technology.

12. V.S. Pham, L.J. Kauppinen, M. Dijkstra, H.A.G.M. van Wolferen, R.M. de Ridder and H.J.W.M. Hoekstra, "Fabrication of mechano-optical sensors for hydrogen gas", In: Annual Workshop on Semiconductor Advances for Future Electronics and Sensors (SAFE), 26-27 Nov 2009, Veldhoven, the Netherlands. pp. 44-47. Technology Foundation STW.

13. V.S. Pham, L.J. Kauppinen, M. Dijkstra, H.A.G.M. van Wolferen, R.M. de Ridder and H.J.W.M. Hoekstra, "Simulation and fabrication of a mechano-optical sensor for nano-displacements", In: 1st Nano Today Conference, 2-5 Aug 2009, Biopolis, Singapore. pp. 33-33. Institute of Bioengineering and Nanotechnology, Singapore.

14. V.S. Pham, L.J. Kauppinen, H.J.W.M. Hoekstra, M. Dijkstra, H.A.G.M. van Wolferen, G.J.M. Krijnen and R.M. de Ridder, "Fabrication of microcantileverbased IO grated waveguide sensors for detection of nano-displacements", In: Proceedings of the 13th Annual Symposium of the IEEE LEOS Benelux Chapter, 27,28 November 2008, Enschede, The Netherlands. pp. 155-158. IEEE LEOS Benelux Chapter.

15. H.J.W.M. Hoekstra, R. Dekker, M. Dijkstra, C. Dongre, M. Hoekman, L.J. Kauppinen, P.V. Lambeck, M. Pollnau, H.P. Uranus, "Integrated optics sensors for multi-sensing platforms", In: Proceedings "The Sense of Contact", 4 April 2007, Zeist, The Netherlands. 5. Technology Foundation STW. 


\section{Acknowledgements}

I am grateful for Dutch Technology Foundation (STW) and the nanotechnology investment program NanoNed, by the Dutch Ministry of Economic Affairs, for financing the research work reported in this thesis.

Many people have contributed to this thesis and I would like to take the opportunity to thank all those people.

I thank my promoter Markus Pollnau and my supervisors Hugo Hoekstra and René de Ridder for the opportunity complete my Ph.D in the IOMS group.

As my daily supervisors Hugo and René deserve big thanks for their kind and supportive guidance during the work. I particularly appreciated the patience in reading my paper manuscripts and providing valuable feedback.

I would like to express my appreciation to the rest of the IOMS scientific staff, including Alfred Driessen, Kerstin Wörhoff and Paul Lambeck.

I thank IOMS group sectaries Rita ter Weele-Stokkers and Annitta David for their patience and good administrative support.

I like to thank all the members of the graduation committee: prof. dr. M. Pollnau, dr. ir. R.M. de Ridder, prof. dr. K.J. Boller, dr. H.J.W.M. Hoekstra, prof. dr. G.J.M. Krijnen and prof. dr. H.W.M. Salemink for their time and efforts in reviewing this thesis.

During the first 18 months I had the privilege to participate in many fruitful technical and scientific discussions in biweekly "multi-sensing" meetings with Hugo Hoekstra, Gjis Krijnen, Marcel Hoekman, Henri Uranus and Meindert Dijkstra. I truly appreciate these meetings in helping me to build my expertise in integrated optics.

I also want to acknowledge the optics theory meetings with Manfred Hammer, Remco Stoffer, Milan Maksimović, René de Ridder, Hugo Hoekstra and Alyona Ivanova as very useful insight into theoretical and modelling aspects. I am grateful for all the tips and advices I got from you during these meetings.

I am grateful to Anton Hollink for his technical support and his phenomenal talent in keeping the lab up \& running.

Meindert Dijkstra deserves big thanks for all the cleanroom work and all the technical advices he provided.

I also want to acknowledge the technical help of Meint de Boer and Erwin Berenschot, particularly in cantilever process integration.

I thank Silicon photonics platform staff Pieter Dumon and Jin Guo for advices and help in platform use and mask design. 
I wish to thank Shahina Mumthaz for co-operation in the "optical switch" project. I appreciate her willingness to spend countless hours in the cleanroom to get the devices, only to join me in the lab for more countless hours in measuring the devices :-)

During his M.Sc. thesis work Tjeerd Pinkert initiated the photonic crystal Y-splitter work, reported in chapter 3. I thank Tjeerd for all the nice scientific and non-scientific discussions we had, as well as for his active participation in publishing the results of the Y-splitter device.

Special thanks for Jing Yang and Chaitanya Dongre for being in a same situation; that is being a final year $\mathrm{PhD}$. I appreciate all the conversations we had about finishing the $\mathrm{PhD}$, job hunting and life after the book is done.

I highly appreciate the efforts of Dimitri Geskus for being the spirit and organizer of the (P)IOMS group.

I thank Muralidharan Balakrishnam for accompanying me for *real* warm lunch every day during his stay in UT.

I thank René de Ridder for translating the summary into Dutch.

Saara-Maarit Reijn, kiitokset monista suomen kielisistä keskusteluista!

I also wish to extend my thanks to the other current and former members of the IOMS group. Fei Sun, Nur Ismail, Edward Bernhardi, So Van Pham, Fehmi Civitci, Imran Akca, Edwin Klein, Ronald Dekker, Gamar Hussein, Wico Hopman, Laura Agazzi, Jonathan Bradley, Abu Aravazhi, Marko van Dalfsen, Henry Kelderman, Feridun Ay, Gabriël Sengo and Henk van Wolferen. You made my stay enjoyable and memorable, my sincere thanks!

I wish to thank my roommate Irene for shearing a flat with me and all those fun times we had.

Last but not least I wish to thank my family for giving me the strength to go on during the hard times. I thank my brother Juha, my sister in law Anu and my niece Sara for many nice moments that we have had. I want to thank my parents Veikko and Pirkko for the love, support and encouragement. 

Compact integrated optical devices for optical sensor and switching applications 
Graduation committee:

Chairman and Secretary:

Prof. Dr. Ir. A.J. Mouthaan

Promoter:

Prof. Dr. M. Pollnau

Assistant Promoter:

Dr. Ir. R.M. de Ridder

\section{Members:}

Prof. Dr. K.J. Boller

Dr. H.J.W.M. Hoekstra

Prof. Dr. G.J.M. Krijnen

Prof. Dr. H.W.M. Salemink

\section{University of Twente}

University of Twente

University of Twente

University of Twente

University of Twente

University of Twente

TU Delft

The research described in this thesis was carried out at the Integrated Optical MicroSystems (IOMS) Group, Faculty of Electrical Engineering, Mathematics and Computer Science, MESA+ Institute for Nanotechnology, University of Twente, P.O. Box 217, 7500 AE Enschede, The Netherlands.

This work was financially supported by the Dutch Technology Foundation (STW) for the first 18 months and the last 30 months by the nanotechnology investment program NanoNed, by the Dutch Ministry of Economic Affairs.

Cover design:

Front: Vertical fiber coupling to a silicon photonics chip.

Reverse: Scanning electron microscope (SEM) image of a microcantilever on top of silicon waveguides (top), SEM image of a photonic crystal membrane (bottom).

Printed by Wöhrmann Print Service, Zutphen, The Netherlands.

ISBN: 978-90-365-3088-0 


\title{
COMPACT INTEGRATED OPTICAL DEVICES FOR OPTICAL SENSOR AND SWITCHING APPLICATIONS
}

\section{DISSERTATION}

\author{
to obtain \\ the degree of doctor at the University of Twente, \\ on the authority of the rector magnificus, \\ prof. dr. H. Brinksma, \\ on the account of the decision of the graduation committee, \\ to be publicly defended \\ on Thursday the $7^{\text {th }}$ of October 2010 at 16:45
}

\section{Lasse Juhana Kauppinen}

Born on the $2^{\text {nd }}$ of March 1978

in Anjalankoski, Finland 
This dissertation is approved by:

the promoter: Prof. Dr. M. Pollnau

the assistant promoter: Dr. Ir. R.M. de Ridder 




\section{Contents}

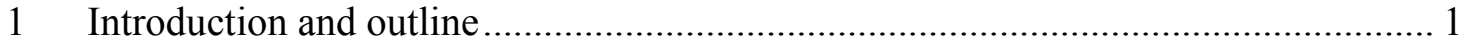

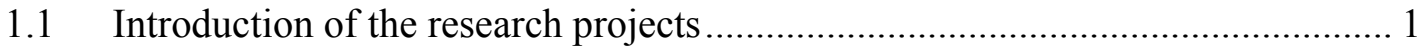

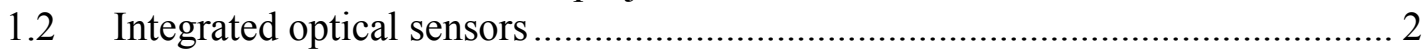

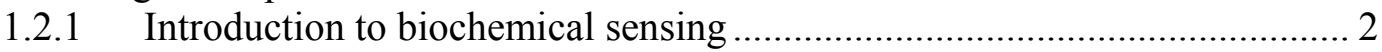

1.2.1.1 Label-free integrated optical sensors ............................................. 3

1.2.2 Integrated optical read-out of microcantilever sensors ............................... 8

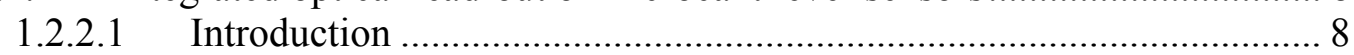

1.2.2.2 Optical read-out methods.................................................... 8

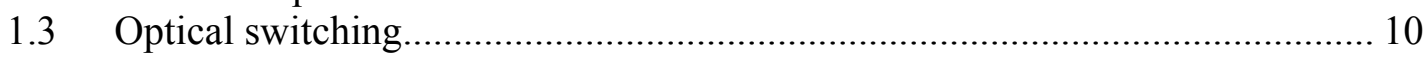

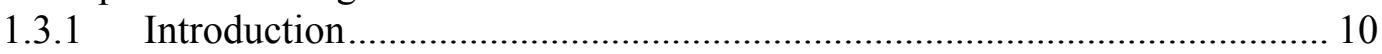

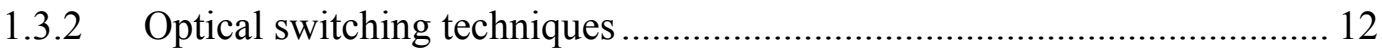

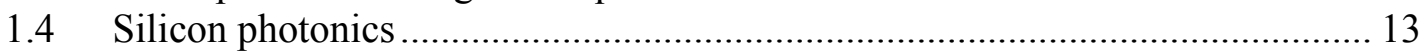

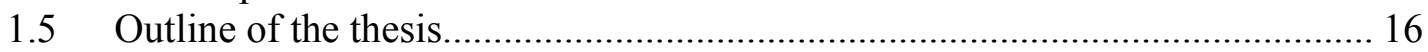

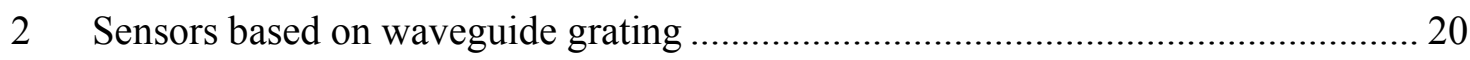

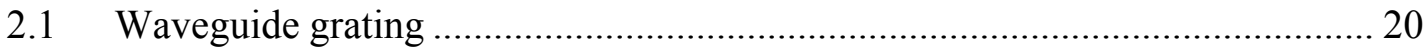

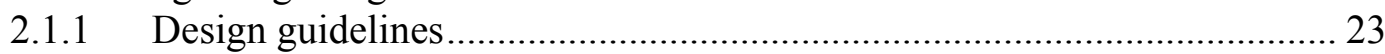

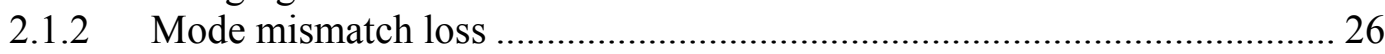

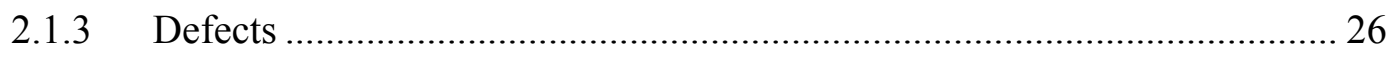

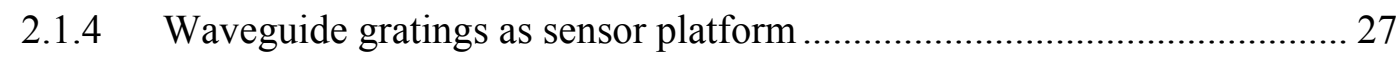

2.2 Design and characterization of the grated silicon photonic wire sensor ............ 31

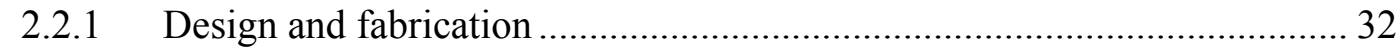

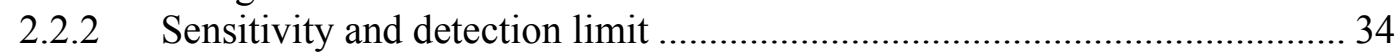

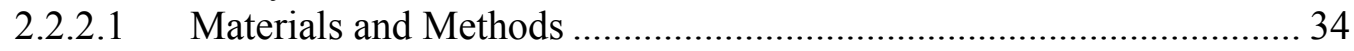

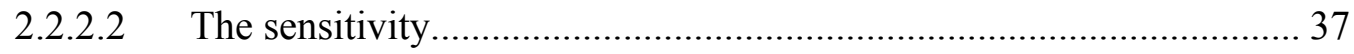

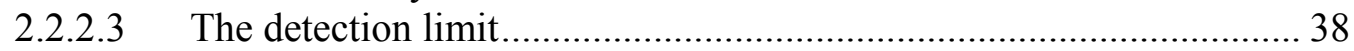

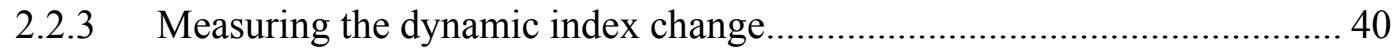

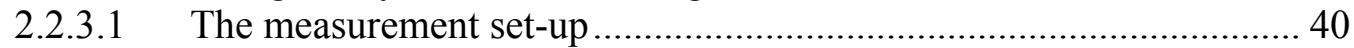

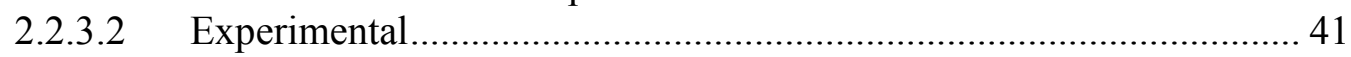

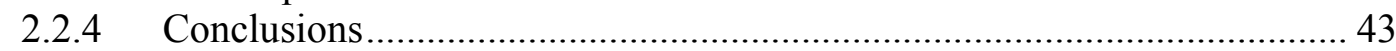

2.3 Label-free enzyme sensing with grated silicon photonic wire ......................... 43

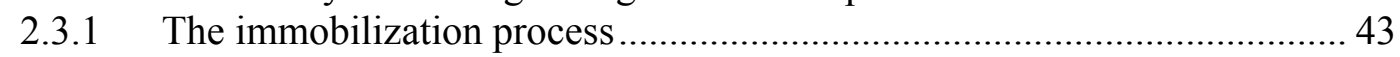

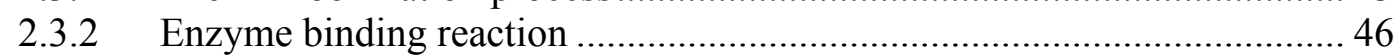

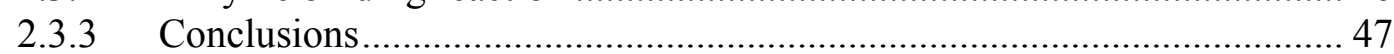

2.4 Grated Waveguide Optical Cavity as a Compact Sensor for Sub-nanometre

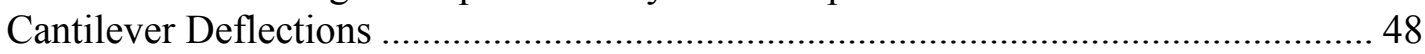

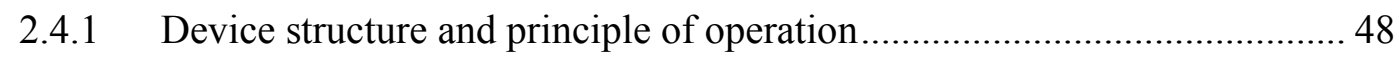

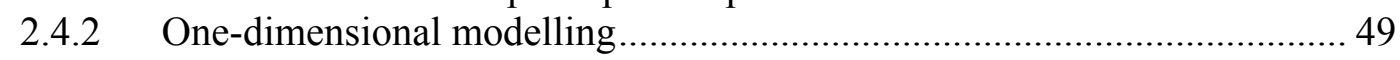

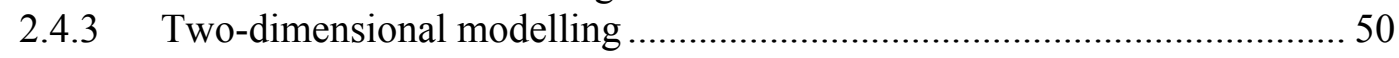

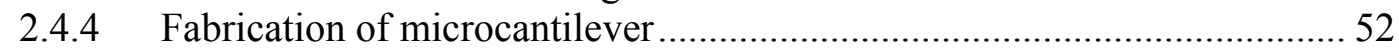

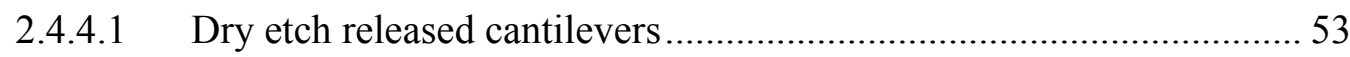

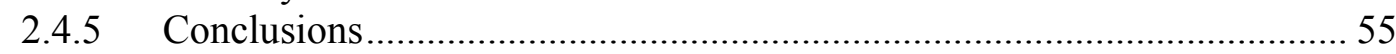

3 Compact silicon photonic devices for MEMS-actuated optical switching .............. 59

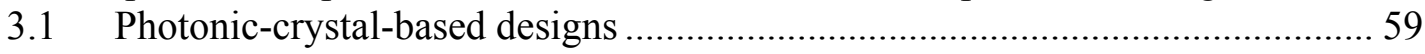




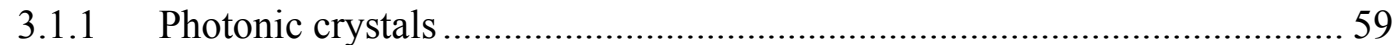

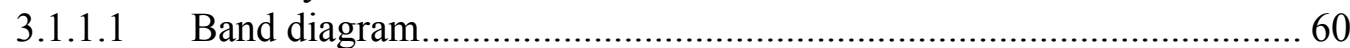

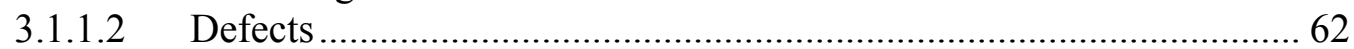

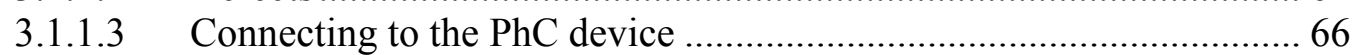

3.1.2 Photonic-crystal-cavity-based channel drop filter ..................................... 66

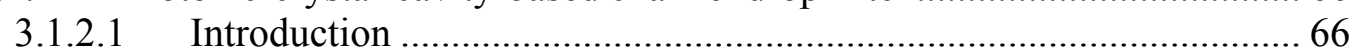

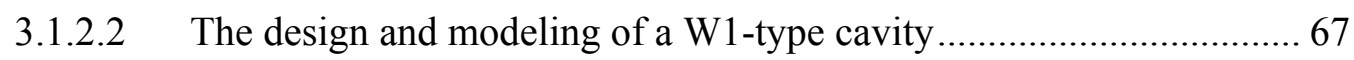

3.1.2.3 The design and modeling of a W3-type cavity ................................. 70

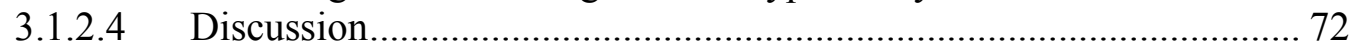

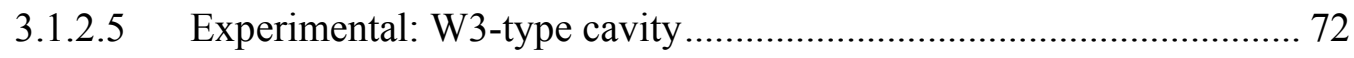

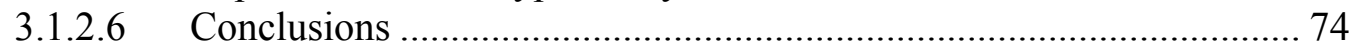

3.1.3 Photonic-Crystal-Cavity-based Y Splitter ........................................... 74

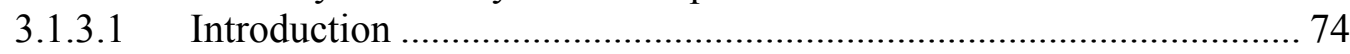

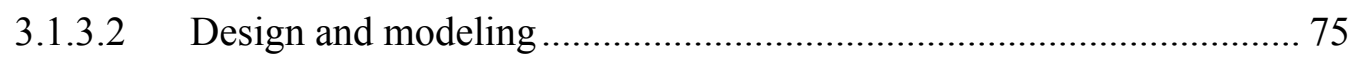

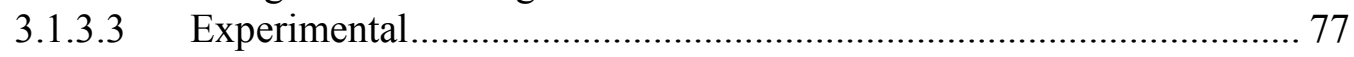

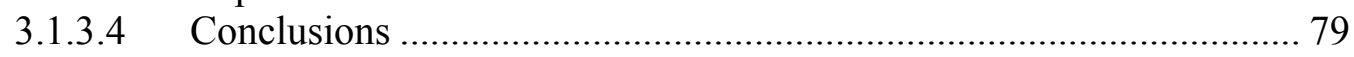

3.2 Waveguide grating and ring-resonator-based designs .................................. 79

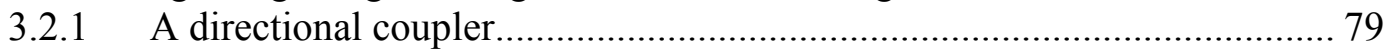

3.2.2 Waveguide-grating-based add-drop multiplexer ...................................... 80

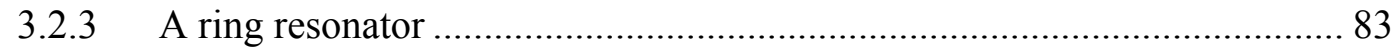

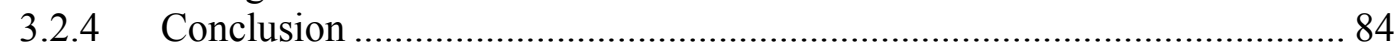

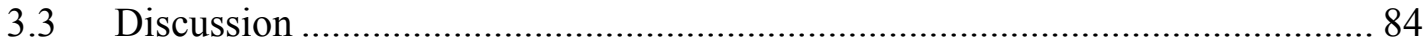

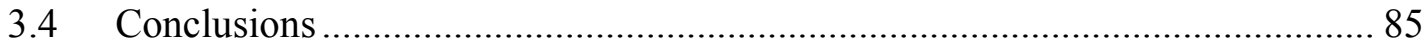

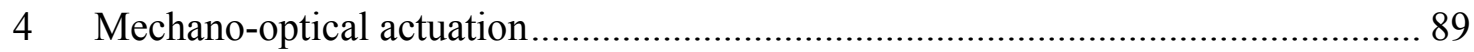

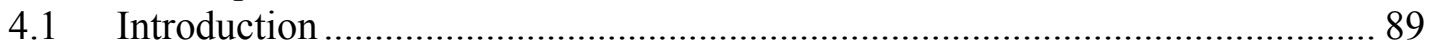

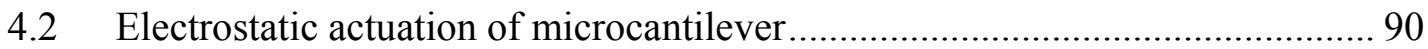

4.3 Monolithic integration of the microcantilever ................................................. 93

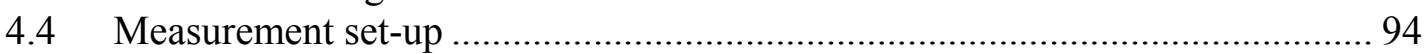

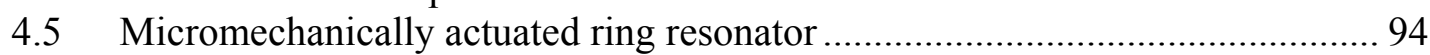

4.6 Micromechanically actuated photonic crystal waveguide ............................... 98

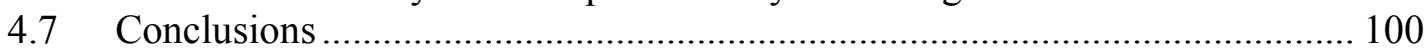

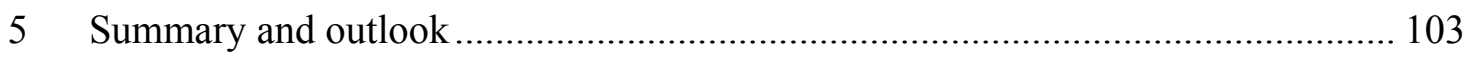

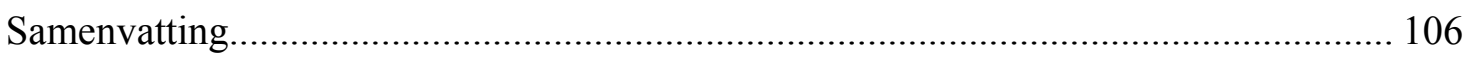

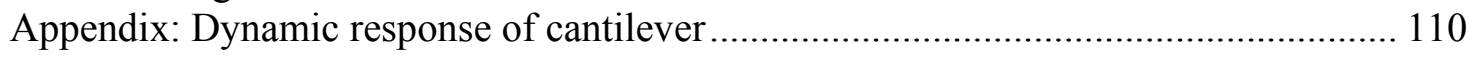

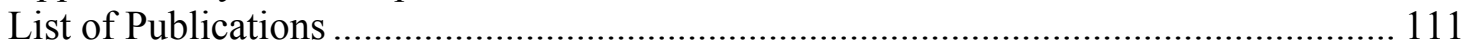

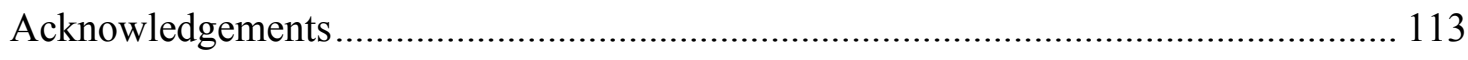






\section{Introduction and outline}

This chapter presents a general introduction of the thesis. An introduction of the research projects is first given as background information. The motivation for the development of the sensor and switching devices is given in the introduction sections. Also a brief introduction to optical switching and sensing is provided. Furthermore, an introduction of the silicon photonics fabrication platform, that was used to fabricate most of the devices reported in this thesis, is given, as well as the motivation for the use of silicon photonics. The chapter ends with the outline of the thesis.

\subsection{Introduction of the research projects}

The work reported in this thesis focuses on two topics; optical sensing and optical switching. The first 18 months of my $\mathrm{PhD}$ period were funded by the STW project called "Multi-sensing arrays of separately accessible optics sensors". The remaining 30 months I worked in the project titled "Optical switching by NEMS-actuated resonator arrays", funded by the nanotechnology investment program NanoNed, by the Dutch Ministry of Economic Affairs.

The aim of the multi-sensing project was to realize a compact and highly sensitive sensor unit that could be used for multi-analyte sensing. The focus was on utilizing waveguide grating based sensors. The work can be divided into two sub-projects; in the cantilever sensor project a novel optical detection method to measure nanodisplacements of microcantilevers was developed while in the biosensing project the sensing properties of deep UV lithography fabricated grated silicon photonic wires were studied. The cantilever project was later split off as a new $\mathrm{PhD}$ project funded by the Dutch Ministry of Economic Affairs through the Point-One MEMSland project, and is continued by So Van Pham in the IOMS group.

The aim of the optical switching project was to realize a compact mechano-optically actuated optical switching device. The project was a joint research effort by the AAMP, IOMS and TST groups of the University of Twente. In the project I continued the work initiated by my predecessor Wico Hopman, whose contribution is reported in his $\mathrm{PhD}$ thesis [1]. My contribution was to provide the optical devices that could be mechanooptically tuned with a monolithically integrated microcantilever. The project was conducted in close co-operation with the TST group, particularly with Shahina Chakkalakkal Abdulla who was responsible for the microcantilever design and fabrication.

In the following sections the introductions to the research topics covered in this thesis are given. Following the division that originates from two separate projects, the introduction and the remaining part of the thesis are each divided in two sections. In chapter 2 the sensor work is reported; chapters 3 and 4 deal with the optical switching work, where chapter 3 discusses the optical designs implemented for the mechano-optical switching and chapter 4 reports the experimental results obtained from the monolithically integrated switching devices. 


\subsection{Integrated optical sensors}

In this section an introduction to integrated optical (IO) sensors is given. As the sensor work in this thesis focuses on implementing a label-free biochemical sensor and nanodisplacement sensor, an introduction of both topics is given.

First the optical biochemical sensors are discussed. A brief introduction of biochemical sensing is given, followed by section that introduces some of the most common IO labelfree biosensor types. The other sensor section (1.2.2) focuses on IO devices that are used to measure small displacements of microcantilevers. These types of devices can be used as an optical read-out method for cantilever sensors that are based on observing reactions that cause the cantilever to deflect.

\subsubsection{Introduction to biochemical sensing}

Biosensing aims at recognizing some specific biomolecules (analytes) from a sample. For this purpose a biosensor consists of two basic components: a biological recognition system (bioreceptor) and a transducer.

An example of a bioreceptor is an antibody. It is a complex biomolecule that immune system cells produce when exposed to molecules called antigens. The specific structure of the antibody targets only an antigen that matches to its structure. As a result of this, the antibody and the antigen can bind together. For more detailed discussion about bioreceptors and they properties we refer to [2].

A transducer is needed to convert the biorecognition event (e.g. binding of an antibody and an antigen) into a measurable effect. Typical transducer types used in biosensors rely on optical, electrochemical or mass-based methods. In electrochemical detection, see e.g. [3], the transduction principle is the detection of an electric current caused by oxidation or reduction of species at the electrode. Mass-based detection methods exploit the fact that a mechanical resonance frequency of a piezoelectric element changes as its mass changes. A possible implementation of this detection scheme involves the use of microcantilevers, see e.g. [4]. Optical transduction methods can be roughly divided in two categories; labeled and label-free methods. Next we will briefly discuss the general properties of both of these optical methods and motivate our focus on label-free sensors.

In the labeled detection scheme either the analytes to be detected or the bioreceptors are labeled with e.g. fluorescent tags, such as dyes. Upon excitation with light, the fluorescent dye will emit photons at a certain wavelength that is specific to the dye. The emitted light can be detected with a sensitive photodetector, and based on the intensity of the emitted light the concentration of the analyte is obtained.

In the label-free sensing scheme the analytes to be measured are not labeled, but typically the receptors are immobilized on the sensor's surface. The surface layer containing the receptors changes thickness upon capturing an analyte. The sensor's transduction mechanism translates the thickness change to a measurable signal.

In label-free detection the target molecules are detected in their natural form, allowing quantitative and kinetic analysis, which is typically not possible by using analytes that are labeled with fluorescent tags. This is due to two main effects: first, it is difficult to control the number of fluorescent tags attached to each molecule, and second, the labeling procedure changes the properties of the analyte. Although the fluorescence-based detection is highly sensitive, the fluorescence light signal scales with the sample volume 
and is typically very weak, requiring photon counting methods. In label-free methods the signal is related to sample concentration and good sensitivity can be obtained with ultrasmall sample volumes.

In label-free sensing much attention has been given to sensors that are capable of multianalyte detection. A straightforward way to implement this type of sensor is to arrange the sensing elements into an array layout, in which each element is functionalized to detect a specific molecule. The following medical diagnostic case illustrates the attractiveness of using a label-free sensor array, capable of multi-analyte detection.

A patient has a virus infection in his central nervous system. In most of the cases one of 15 possible viruses is causing the infection. The infection can be analyzed from cerebrospinal fluid, which is taken with a Lumbar puncture method. Due to the nature of this method it cannot be repeated for some time, which implies that this diagnostic sample should be used as efficiently as possible. The sample contains a very small amount of viruses, and in order to identify them, a real-time polymerase chain reaction (PCR) is used to multiply the genome of the virus. In a real-time PCR procedure, often a dye is used that fluoresces strongly when bound to the multiplied genome, allowing the detection of the multiplication. Typically only one (or in some cases a few) virus(es) can be indentified with one PCR based test.

It would clearly be advantageous to be able to cover all 15 viruses simultaneously in a single diagnostic test. Also, considering the good sensitivity provided by detection with immobilized receptors, the virus could be indentified directly from the diagnostic sample without the PCR step. The main aim of the development of label-free integrated optical sensors for this kind of tests is to add value compared to conventional methods (such as the real-time PCR), as measured by factors such as the time and workforce required to perform the test, the cost of test equipment, and the cost of a single test. The portability of the test equipment may also play an important role.

\subsubsection{Label-free integrated optical sensors}

In this section we introduce integrated optical sensor structures, used for label-free refractive-index-based sensing, that have received most attention in recent research work reported in the literature. These sensor structures can be divided in three categories: surface-plasmon-based, interferometric and resonance-based sensors.

For a comprehensive review of optical biochemical sensors we refer to review papers [57].

In order to evaluate and compare the performance of the optical sensors we introduce two important parameters that are often used for this purpose: sensitivity $\left(S_{n}\right)$ and detection limit $(D L)$. The sensitivity is an intrinsic property of a sensor, defined as the change of the transduction signal $\left(\Delta T_{t s}\right)$ per unit change of analyte concentration $(\Delta A)$.

$$
S_{n}=\frac{\Delta T_{t s}}{\Delta A} \text {. }
$$


In many cases, the concentration of the analyte is deduced from a change in refractive index (either directly caused by the analyte, or through its interaction with a receptor). For optical sensors that respond to a change in refractive index by wavelength-shifting some spectral feature, the sensitivity is specified as wavelength shift per refractive index unit (RIU) change caused by the analyte. For example, addition of sugar to water causes the refractive index to change and this change can then be measured with an optical sensor that has a certain sensitivity to the index change. The detection limit depends on the signal-to-noise ratio (SNR) of the transduction signal and is defined as:

$$
D L=\frac{S N R}{S_{n}} .
$$

Often used transduction mechanism is to observe analyte caused changes in the effective modal index $\left(n_{e f f}\right)$ of the waveguide. This effective index is defined as:

$$
n_{\text {eff }}=\frac{\beta \lambda_{0}}{2 \pi},
$$

in which $\beta$ is the propagation constant and $\lambda_{0}$ is the vacuum wavelength. The effective index depends, among many things, on the refractive index of the materials that form the waveguide structure.

\section{$\underline{\text { Surface plasmon }}$}

Probably the most well-known label-free optical transduction mechanism is surface plasmon resonance (SPR) [8]. The surface plasmon originates from the interaction between photons and the free electron cloud of the metal. Integrated optical waveguides can be used to excite the SPR wave (SPW), as shown in Fig. 1. At a certain wavelength the propagation constant of the waveguide mode matches that of the SPW; this is known as the phase-matching condition. As power transfer from the waveguide mode to the lossy SPW mode is maximum at this phase-matched condition, the light transmission through the waveguide has a dip at this wavelength.

The wavelength for phase matching depends on the dielectric material that is adjacent to the metal, and therefore index changes in this dielectric can be monitored. Moderate to good detection limits have been reported for waveguide based SPR devices; e.g. $1 \cdot 10^{-4}$ RIU [9] and $1.2 \cdot 10^{-6}$ RIU [10], although the interaction lengths are relatively long; 0.7 $\mathrm{mm}$ and $1.8 \mathrm{~mm}$, respectively.

The SPR imaging type sensor, see e.g. [11], is an array type sensor responding to the need of simultaneous multi-analyte detection. Arrays of SPR strips are illuminated and the intensity reflected by each element is measured with a 2D array detector (e.g. CCD image sensor). Detection limits of $3 \cdot 10^{-5}$ [12] and $3 \cdot 10^{-6}$ [13] RIU have been reported. Although this type of sensor is capable of multi-analyte detection it uses bulky optics and the SPR element is nothing more than an array of microfabricated metal strips. Today commercially available SPR sensors are far from being handheld multi-analyte devices. 


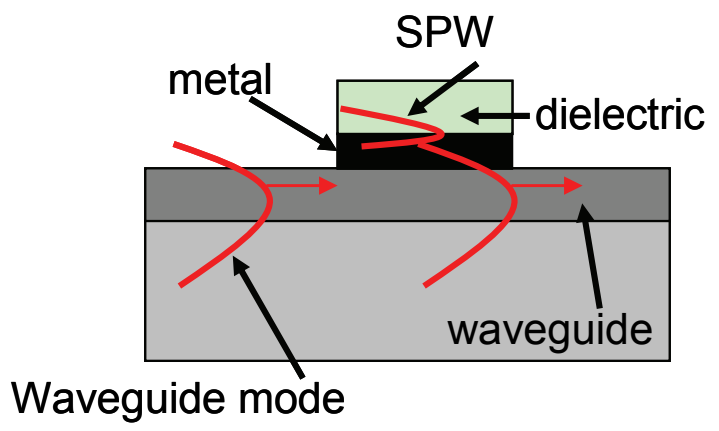

Fig. 1. A schematic cross-section of a waveguide coupled surface plasmon device.

\section{$\underline{\text { Interferometer based }}$}

Interferometer-based sensors measure the optical path length changes caused by the analyte. Probably the most well-known type in this class of sensors is the Mach-Zehnder interferometer (MZI), see Fig 2. Light from an input waveguide is distributed, usually with equal power, over two waveguide branches, often using a so-called 3-dB coupler. In a sensing branch, the analyte causes a certain effective index change (e.g. by binding to the immobilized receptor layer on the waveguide surface) that in turn introduces an optical path length difference between the branches. The light from the two waveguide branches is recombined by a $3 \mathrm{~dB}$ coupler. The distribution of light over the output waveguides depends on the path length difference. By introducing phase modulation to increase sensitivity, a detection limit of the order of $5 \cdot 10^{-8}$ RIU has been achieved [14]. However, in order to introduce sufficient optical path length difference to detect a very small index change, the required length of the MZI device is typically quite long; ranging from a few millimetres to several centimetres. As the device dimensions increase, the undesirable sensitivity to temperature gradients increases as well. A spiral layout has been explored allowing a design that is insensitive to temperature gradients, and that reduces the footprint of the MZI, enabling a compact array configuration for multisensing [15-16].

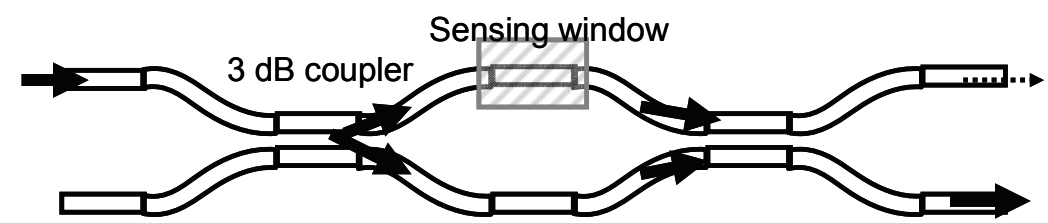

Fig. 2. Top view of Mach-Zehnder interferometer. The input light is split over two waveguide branches. The sensing window defines the region in which the analyte solution is applied. The index change in the sensing branch causes a change in the optical path length difference between the two branches.

\section{Resonance based}

The resonance wavelength of integrated guided-wave optical resonators depends on the effective refractive index of the waveguiding medium. Since in the refractive index-based label-free sensing scheme the analyte causes the effective index to change, the presence of the analyte can be detected by monitoring the change of the resonance wavelength. The crucial feature in achieving a small detection limit is the spectral sharpness of this 
resonance, which is related to the so-called quality factor $Q$. The higher the quality factor is, the smaller index change can be detected [17].

In ring-resonator-based sensors, the sample is applied to a sensing window covering the ring waveguide (Fig. 3a). The effective-index change of the ring waveguide causes the resonance wavelength to change, which can be observed by measuring the transmission spectrum, see e.g. [18-19]. A detection limit of $10^{-5}$ RIU has been reported [18]. The footprint of a ring resonator is typically considerably smaller than that of an MZI (e.g. $500 \mu \mathrm{m}^{2}$ vs. $2 \mathrm{~mm}^{2}$ ), and the integration into an array layout is easier. Unfortunately the quality factor of ring resonators is typically limited to $\sim 10^{4}[18]$, due to optical loss of the ring waveguide, which is mostly caused by surface roughness that results in out-of-plane scattering. This imposes a lower bound on the achievable detection limit $[5,17]$.

Sensors based on resonators in a photonic crystal $(\mathrm{PhC})$ (see Fig. 3b) operate in a similar way as ring-resonator sensors: the resonance frequency changes according to index change. An impressive set-up involving an array of $\mathrm{PhC}$ resonators for multianalyte sensing is reported in [20]. The array element is a 1D PhC structure with a defect, to which the light is coupled from the access waveguide, see Fig. 3b. Each array element has a unique resonance wavelength and by monitoring the transmission spectrum of the access waveguide the response of the individual sensor element can be extracted. Multi channel sensing with a detection limit of $7 \cdot 10^{-5}$ RIU is reported in [20].
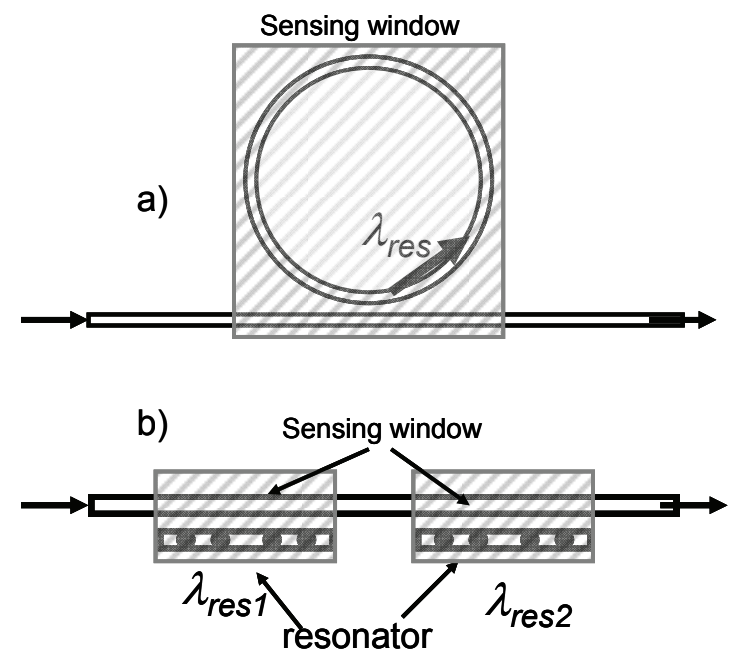

Fig. 3. a) Top view of a ring resonator. Light at a resonance wavelength is coupled from the access waveguide to the ring. The resonance wavelength depends on the effective index of the ring waveguide. $b$ ) A top view of a sensor based on a 1D photonic crystal resonator. The resonance wavelength depends on the effective index of the resonator.

The waveguide gratings and photonic crystal waveguides are resonator-based devices as well. However, these devices do not have a separate resonance cavity to which the light is coupled from the waveguide. For this reason these devices are less suitable for array layout, e.g. due to possible reflections between cavities. Nevertheless, the spectral features of e.g. waveguide gratings typically have a larger dynamic range (stopband attenuation) than e.g. ring resonators.

In a waveguide grating, see Fig. $4 \mathrm{a}$, the spectral position of the photonic stopband depends on the effective index, and by monitoring the changes of the stopband position the index change of the cladding medium can be measured. Typically, waveguide 
gratings are somewhat larger in size than ring resonators. Detection limits of $4 \cdot 10^{-4} \mathrm{RIU}$ [21] and $2.8 \cdot 10^{-5} \mathrm{RIU}$ (this thesis, section 2.2.) have been reported for waveguide-grating based-sensors. The detection limit of a waveguide grating can be increased by tuning the grating parameters, although at some point optical losses and non uniformities of the grating structure due to fabrication technology will set a lower limit.

The operating principle of a photonic crystal waveguide sensor, see Fig. $4 \mathrm{~b}$, is based on the fact that the cut-off wavelength of the waveguide depends on the effective index. The index changes are therefore measured by observing the spectral shift of the cut-off wavelength. Additional sensitivity is expected from the field profile of the waveguide mode near the surface and from liquid penetrating into the holes of the $\mathrm{PhC}$ [22]. By assuming a spectral resolution of $5 \mathrm{pm}$, a detection limit of $8 \cdot 10^{-5}$ RIU can be estimated for the device reported in [22].

a)

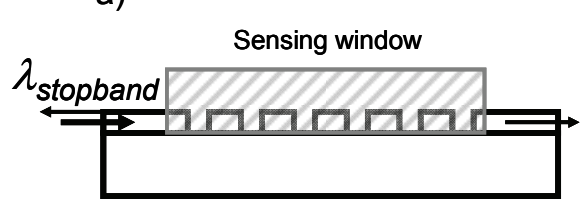

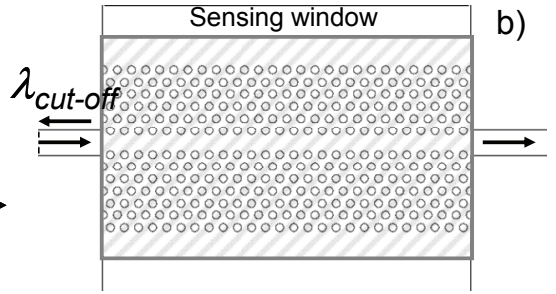

Fig. 4. a) Side view of a grated waveguide. Spectral position of the photonic stopband of a grating depends on the index of the cladding material. b) Top view of a line-defect waveguide in a 2D photonic crystal. The cut-off wavelength of the waveguide depends on the effective index of the environment.

Resonance-based sensor devices, such as ring resonators and $1 \mathrm{D} \mathrm{PhC-cavities,} \mathrm{have} \mathrm{the}$ advantage that they can be easily arranged into array layout for multianalyte sensing. However, on-chip integration of a spectrometer that can resolve a wavelength e.g. at a resolution of $5 \mathrm{pm}$ is challenging. Also, as the spectral sharpness of e.g. the ring resonator depends on the coupling condition between the access waveguide and the ring waveguide, it might be altered by the presence of the molecules in this region.

\section{Methods to increase sensitivity}

Apart from obvious parameter optimization, there are some ways to improve the performance of the sensor.

A slot waveguide based designs attempt to increase the sensitivity with a special waveguide design, see Fig 5. In a slot waveguide the highest field intensity is between two high-index strips, where a sensing medium can be, and hence a high sensitivity can be expected. Indeed, impressive sensitivity of $212 \mathrm{~nm} / \mathrm{RIU}$ has been reported for a slot waveguide ring-resonator, although the detection limit of $2.3 \cdot 10^{-4}$ is only moderate [23]. Porous silicon can be used as a waveguiding material. Due to porosity the surface area of the sensor is considerably increased and improved sensitivity is expected [24].

Gold nanoparticles can be used to greatly increase the SPR reflectivity shift caused by the binding of DNA. 1000-fold increase in sensitivity is reported in [25]. 


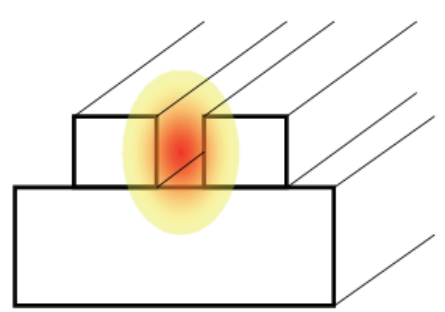

Fig. 5. Slot waveguide geometry. In a slot waveguide the highest field intensity is in the cladding between two high index strips. Therefore the effective index of this waveguide depends strongly on the refractive index of the material that is between the strips.

\subsubsection{Integrated optical read-out of microcantilever sensors}

This section discusses microcantilever-based sensors, in particular integrated optical read-out methods suitable for detection of the deflection of a microcantilever. The physical principles of cantilever sensors are briefly described. A brief summary is given of the integrated optical read-out methods that are reported in the recent literature. Our proposed read-out method, which involves measuring the wavelength shift of an optical resonance of a grated waveguide, is discussed in detail in chapter 2.

\subsubsection{Introduction}

Microcantilever-based sensors can be used to detect the effect of adsorption or absorption of a species by a sensitive layer that is deposited on a micromachined cantilever. These effects cause changes in the surface stress [26], leading to deflection of the cantilever.

A large number of microcantilever-based sensors are reported in the literature. Fritz et al. functionalized Si cantilevers on one side with different oligonucleotide base sequences [27]. DNA hybridization depending on the base sequence was demonstrated. Baller et al. [28] report a cantilever-array-based artificial nose. Different polymer coated cantilevers respond to gaseous analytes due to the swelling effect as the gas is absorbed by the polymer. Artificial flavours, such as lemon, cherry, rum, vanilla, orange and bitter almond, were successfully detected with the sensor.

An interesting type of cantilever-based sensor is the use of cantilevers as pixel elements in an uncooled infrared (IR) imaging system [29-30]. In this case the cantilever response is based on thermal expansion which depends on the amount of IR light absorbed.

Hydrogen gas detection with palladium-coated cantilevers is a widely studied topic, e.g. [31-32].

Several electric methods exist for measuring the deflection of a microcantilever, e.g. piezoelectric, piezoresistive and capacitive. Here we will focus on a comparison of integrated optical read-out methods that have the advantage that no electric connection to the cantilever is required.

\subsubsection{Optical read-out methods}

Often, the displacement of a free-space optical beam is detected as a measure of the cantilever deflection [29-30, 33]. Although the method is simple and accurate, it is bulky, and therefore dense and compact monolithic integration of cantilever sensors is not possible with this method. In addition, the slow response of the large position-sensitive 
photodetectors that are needed for measuring optical beam displacement limits the bandwidth of this method.

Different designs for integrated optical read-out of microcantilever deflection have been proposed and demonstrated. These read-out methods can be roughly divided into two categories. The first one involves exploiting a structural change of the optical device, e.g. displacement of a waveguide attached to the cantilever, which changes the amount of light that can propagate through the device. The second one uses the effect caused by perturbation of the evanescent field of a waveguiding structure. The cantilever, moving in close proximity to the optical device changes its effective refractive index, which, in turn, modulates the light transmission through the device. Table 1 summarizes some of the reported integrated read-out methods and their deflection sensitivities (change of power transmission coefficient $\Delta T$ per unit cantilever deflection $\Delta z$ ).

Table 1.

Different integrated optical designs to detect cantilever deflection and their performance.

\begin{tabular}{|l|l|}
\hline Optical read-out device & Sensitivity $\Delta \mathbf{T} / \Delta \mathbf{z}$ \\
\hline Waveguiding cantilever \#1 & $1 \mu \mathrm{m}^{-1}[34]$ \\
\hline Waveguiding cantilever \#2 & $0.24 \mu \mathrm{m}^{-1}[35]$ \\
\hline Photonic crystal cavity & $0.1 \mu \mathrm{m}^{-1}[37]$ \\
\hline Multi-mode interference (MMI) couplers & $5 \mu \mathrm{m}^{-1}[36]$ \\
\hline Waveguide grating & $60 \mu \mathrm{m}^{-1}[38]$ \\
\hline Slot waveguide resonator & $33000 \mu \mathrm{m}^{-1}[39]$ \\
\hline Ring resonator & $500 \mu \mathrm{m}^{-1}[40]$ \\
\hline
\end{tabular}

The principle of waveguiding-cantilever devices $[34,35]$ is shown in Fig. 6. A segment of an optical waveguide is under-etched to form the cantilever. A small gap between the cantilever waveguide and the non-under-etched (fixed) part of the waveguide allows the cantilever to move. Deflection of the cantilever changes the coupling between the cantilever waveguide and the fixed waveguide.

The read-out methods reported in [36] and [37] are variations of this type of design. In [36] 10 cantilever waveguides couple the outputs of a $1 \times 5$ MMI splitter to the inputs of a $5 \times 1$ MMI combiner. In [37], a 2D photonic crystal, containing a resonance cavity, is patterned on the top surface of the cantilever. Bending of the cantilever causes deformation of the cavity, which results in a shift of resonance wavelength.

Although the read-out methods based on structural change are relatively simple, sensitive and pose no serious challenges in fabrication, it is a disadvantage that the light propagates in a cantilever structure, which severely limits the options for cantilever design and choice of the receptor layer, in particular in case of absorbing receptor layers. Also the deflection sensitivities are not as high as the ones obtained with methods using perturbation of the evanescent field. 


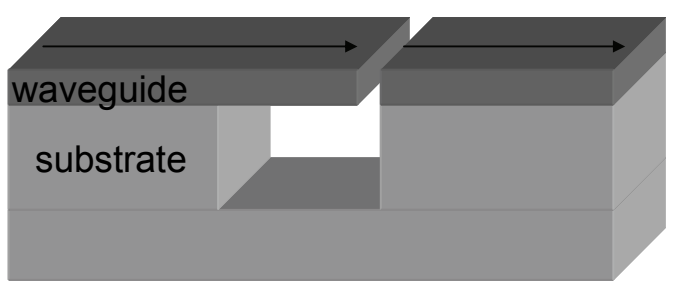

Fig 6. Schematic picture of the waveguiding-cantilever type of sensor. The deflection of the cantilever changes the transmission through the small air gap.

A sensor, in which a microbridge is bonded to an optical chip and located above a waveguide grating, is reported in [38]. This type of read-out exploits the wavelength shift of the photonic stopband of the waveguide grating as the air gap between the grating and the mechanical element changes. Therefore, the size of the mechanical element should at least be equal to the size of the grating. Barrios [39] presents a theoretical study on a nanomechanical sensor employing a disk resonator in a waveguide structure where a slot is present parallel to the disk surface. A deflection of the disk changes the width of the slot, which, in turn, changes the resonance wavelength. Although an extremely high deflection sensitivity is predicted, it poses serious fabrication challenges, e.g. regarding the required etch selectivity between layers.

It is also possible to use an optical ring resonator as a sensor platform for a cantilever deflection read-out [40-41]. In this case the cantilever deflection causes a shift of the resonance wavelength as the presence of the cantilever increases the optical path length of the ring resonator. The biggest challenge for fabricating sensors that are based on perturbation of the evanescent field, is the monolithic integration of the cantilevers and the optical readout circuit, as will be discussed in section 2.4.4.1.

\subsection{Optical switching}

In this section we will introduce applications for optical switches and then briefly discuss some of the most common switching principles.

\subsubsection{Introduction}

Switching functions for optical signal can be realized either in the optical or the electrical domain. In electrical-domain switching, the optical signal is first converted to an electronic signal to which the switching function is applied. Then, the switched electrical signal is converted back to an optical signal. In optical-domain switching, the signal remains optical, although electronic circuits are often used to drive such an optical switch.

At present, it depends on the switching application which one of these two switching domains (electrical or optical) should be used. It is argued that e.g. in optical telecom networks the optical switches are useful in circuit switching applications while for packet switching the technology is not mature enough, due to the fact that an optical random access memory does not exist and all optical packet header processing techniques are primitive, giving advantage to the electrical switches [42]. In general the conversion from optical to electrical signal should be avoided for trivial signal functions due to the high cost of signal conversions (optical-to-electrical-to-optical) [43].

The main application areas for optical switches in the networks are provisioning (e.g. reconfiguring lightpaths), and protection switching. The required switching speeds for these 
applications are in the range of milliseconds, which is well within reach of optical switching technologies [43]. The total number of ports required for provisioning and protection switching can be higher than 1000 [43]. The high number of ports requires a huge amount of switches. This favors optical switching, since the power consumption of a typical $64 \times 64$ electronic integrated circuit switch is $25 \mathrm{~W}$, whereas an optical MEMS based switch would need only a fraction of this [43].

The other main advantage of optical switching is that it allows waveband switching, i.e. selectively switching certain wavelength ranges that are contained in a broadband multiwavelength signal. This is a particularly attractive property for switching applications in optical networks that use wavelength division multiplexing (WDM) to achieve high data bandwidth. WDM systems replace a single carrier wavelength, possibly modulated with an ultrahigh bandwidth signal, by several carrier wavelengths, called channels, each modulated at a much lower rate. There can be tens of channels in a single fiber, each operating at a data rate of e.g. $40 \mathrm{~Gb} / \mathrm{s}$. At a network node there may be a need to switch some of these channels to a different light path. If this is done electronically, all the channels are first demultiplexed and converted to electrical signals for switching. Apparently this causes unnecessary processing for the channels that are not switched, and due to high cost of electrical switches, optical switching is preferred [44].

Apart from their use in optical networks, optical switches could be used for on-chip switching and modulation. A possible application is to access individual optical sensor elements on an integrated sensor array chip, see Fig 7. Other potential applications are in optical instruments (e.g. in an optical spectrum analyzer).

An important future application area is in on-chip optical networks that may replace some of the electrical interconnections, for example for distributing high-speed clock signals and handling massive data streams in processors.

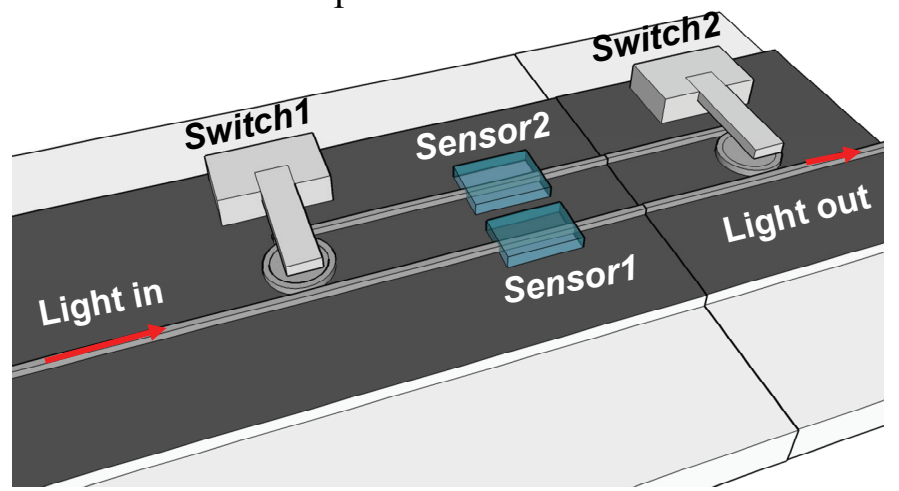

Fig. 7. A conceptual picture; an optical switch is used to access a sensor element. The switch1 controls to which sensor the light is coupled while the switch2 can be used to control from which sensor the signal is read-out. 


\subsubsection{Optical switching techniques}

Several transduction principles can be used for obtaining optical switching, some of which will be briefly discussed below.

\section{Thermo-optic (TO) switching:}

The refractive index of a material depends on its temperature. Therefore it is possible to change the effective index of a waveguide by heating or cooling it, which can be exploited for obtaining a temperature-induced optical switching function in properly designed optical devices. Thermo-optical IO switches are typically designed very similar to the ones used for sensor applications, presented in section 1.2.1.2. The thermo-optic coefficient of the material determines how large an index change is caused per unit temperature change. Compared to other commonly used waveguide materials silicon has a high thermo-optic coefficient of the order of $10^{-4} /{ }^{\circ} \mathrm{C}$. This enables the achievement of relatively large index changes, compared to those attainable with other relevant effects, such as the electro-optic effect. However, the thermal conductivity limits the switching time, typical values in silicon-nitride-based waveguide devices being around $0.1 \mathrm{~ms}$ [45], whereas in silicon, having a much larger thermal conductivity, switching times down to 1 $\mu \mathrm{s}$ have been demonstrated [46]. The biggest disadvantage of TO devices is the power consumption, which is needed even in the static case, as at least one of the on or off states of a TO switch requires continuous local heating. Additionally heat dissipation from a switch element to another may cause cross-talk problems in a TO switch array.

\section{Electro-optic (EO) switching:}

In some materials the (linear) Pockels effect or the (quadratic) electro-optic Kerr effect causes the refractive index to change upon the application of an electric field [47-48]. As the Pockels effect is stronger, thus requiring smaller operation voltages, it is preferred, although it occurs only in non-centrosymmetric crystalline materials. Lithium niobate is a commonly used material for EO switches.

The electro-optic effect is fast, allowing sub-nanosecond switching times [43] with small power consumption; yet the attainable index changes are much smaller than with the TO effect.

\section{Plasma dispersion:}

The presence of free charge carriers in a material causes optical absorption loss, represented by the imaginary part of the refractive index [49]. According to the KramersKronig relation, the real part of the refractive index will change as well. Hence, by injecting electrons (e.g. with an electric current) into some specific region, it is possible to locally change the refractive index. In this way very fast $(>1 \mathrm{~ns})$ and low power $(\sim 10$ $\mu \mathrm{W})$ switching can be achieved [50]. The trade-off is that the phenomena inherently include optical absorption loss, which becomes especially noticeable if a large tuning range is to be realized. 


\section{Mechanical switching:}

As already mentioned in 1.2.2.2, the mechano-optical interaction, which can be used for optical switching, can be roughly divided into two categories: the ones where the mechanical elements are introduced into the optical path and the other ones where the mechanical element is interacting with the evanescent field of the guided light.

This type of switching is not yet as established as the other techniques discussed above, however some related work on the topic has been published. In the late 90's these types of devices were implemented with bonding techniques, i.e. the mechanical part was attached to the optics chips [38, 51]. Later there have been few demonstrations with monolithically integrated cantilevers. In [52] MEMS-actuated switching of a ring resonators is demonstrated with an aluminum microbridge that, when pulled close to the ring, dispatches the resonance due to increased optical loss. Modulation of a photonic crystal waveguide with a polysilicon cantilever is demonstrated in [53]. Also in this case the cantilever causes absorption of light when pulled close to the waveguide. In addition, there have been demonstrations of the AFM cantilever interaction with nanophotonic devices [54-55]. These types of integrated devices aim at providing low power consumption, wide actuation range, and a switching speed at least comparable to thermooptic devices. The micromechanical actuation of an IO device is a major topic of this thesis, which is discussed in chapter 4.

The devices that rely on introducing a mechanical element into the optical path can be seen as miniaturized versions of the bulk mechano-optical switches. An example of such a device is a MEMS mirror that is typically a movable thin film fabricated on a siliconon-insulator wafer. It is possible to fabricate a compact array of thousands of MEMS mirror switch elements [43]. As the required power consumption of an electrostatically actuated mechanical switch is low, this switching technique is attractive for applications requiring a high number of ports. The switching speed of mirror-based devices is on the order of $10 \mathrm{~ms}$ [43].

\subsection{Silicon photonics}

Although very expensive processing equipment is needed to fabricate state-of-the-art silicon complementary metal-oxide-semiconductor (CMOS) devices, the investments have been justified by the economy of mass production which has proven to bring down the price per device to a sufficiently low level so as to open markets to the devices. The ability to use the same standardized process to produce a wide variety of devices can be claimed to be at least partly the reason behind the CMOS glory path. Silicon can be seen as an ideal material for optical-electrical integration, due to the maturity of Si based technology. It has already been demonstrated that the tools that are used for the modern CMOS technology can be used to fabricate micro- and nanophotonic devices as well [6]. The research described in this thesis is partly motivated by the desire to explore the interesting possibility to fabricate state-of-the-art photonic devices with potentially low cost by sharing the equipment with electronics industry and research, and by applying the same standardized processes. 


\section{Silicon photonics fabrication platform, ePIXfab}

Most of the optical devices that are presented in this thesis were fabricated using the silicon photonics platform, known as ePIXfab, established at IMEC, Leuven. This fabrication platform allows the realization of submicrometre features on silicon by using the same state-of-the-art lithographic tools that are used to make modern CMOS transistors. We used two different deep-UV lithographic processes to realize our silicon photonic devices, viz. an older one, employing a 248-nm light source, and a newer one, operating at $193 \mathrm{~nm}$. The specified minimum feature sizes of these processes are given in table 2.

Table 2. Minimum feature sizes of ePIXfab deep UV lithography processes

\begin{tabular}{|l|l|l|}
\hline \multirow{2}{*}{ Feature } & \multicolumn{2}{|c|}{ Minimum size } \\
\cline { 2 - 3 } & $\mathbf{1 9 3} \mathbf{~ n m}$ DUV & $\mathbf{2 4 8} \mathbf{~ n m}$ DUV \\
\hline Periodic structure, pitch & $300 \mathrm{~nm}$ & $400 \mathrm{~nm}$ \\
\hline Holes, diameter & $100 \mathrm{~nm}$ & $200 \mathrm{~nm}$ \\
\hline Holes, spacing between & $90 \mathrm{~nm}$ & $120 \mathrm{~nm}$ \\
\hline Lines, width & $120 \mathrm{~nm}$ & $200 \mathrm{~nm}$ \\
\hline Lines, spacing between & $100 \mathrm{~nm}$ & $150 \mathrm{~nm}$ \\
\hline Trenches, width & $100 \mathrm{~nm}$ & $200 \mathrm{~nm}$ \\
\hline
\end{tabular}

The processing is done on 200-mm SOI wafers, supplied by SOITEC [56], having a 220$\mathrm{nm}$ thick silicon device layer on top of a $2-\mu \mathrm{m}$ thick silicon dioxide layer, all on a $\sim 700$ $\mu \mathrm{m}$ thick silicon handle wafer. The process provides two possible etching depths: $70 \mathrm{~nm}$ and $220 \mathrm{~nm}$. The shallow etch is mainly used to define grating couplers at the ends of the access waveguides. These couplers allow efficient coupling of light to and from the outside world without need for dicing and polishing waveguide end facets. The $220-\mathrm{nm}$ etch depth provides high index contrast, which allows the realization of compact devices. To use this fabrication platform the designer reserves a certain die area, called a mask allocation block, for his/her devices. The size of these blocks is standardized in the platform. The typical size of such a block is $6 \mathrm{~mm}$ by $2.5 \mathrm{~mm}$. The blocks from several designers are combined into an area of roughly $12 \mathrm{~mm}$ by $7.5 \mathrm{~mm}$ to form a master die, as depicted in Fig. 8. A photograph of a fabricated SOI wafer is shown in Fig. 9. Many copies of this master die are then replicated on the wafer with stepper lithography. This allows one to vary the lithographic exposure dose for these dies, which, in turn, means that size variations are obtained. Thus, it is possible to have different waveguide widths in each die, although the designed structures are identical for each die.

The so-called target exposure dose should give exactly the same dimensions on a wafer as designed on the mask. This target exposure dose depends on the structure and its dimensions, and the designer should carefully take this into account.

For a more detailed discussion about the process we refer to [57-58]. 


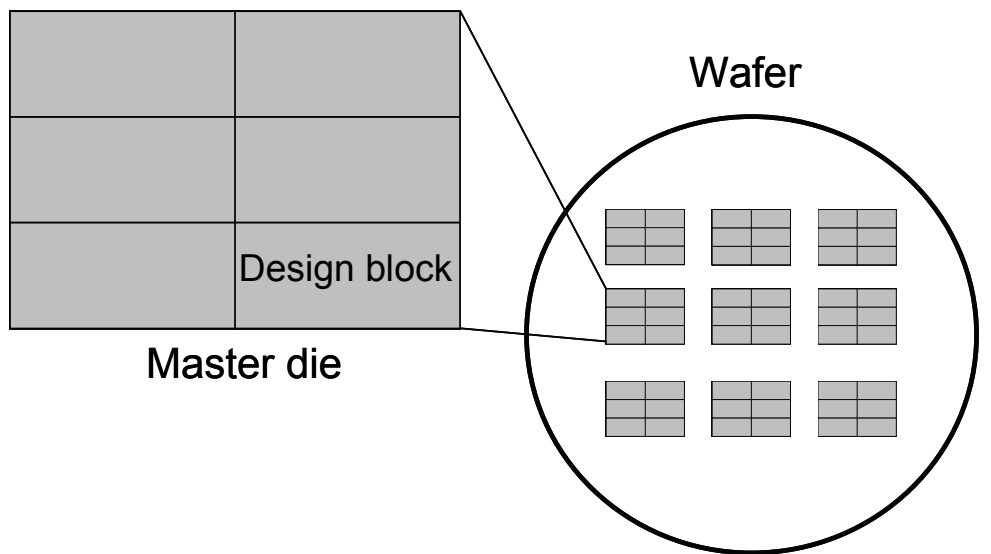

Fig. 8. A Schematic picture of a wafer. A master die that is repeated many times on a wafer is composed of design blocks.

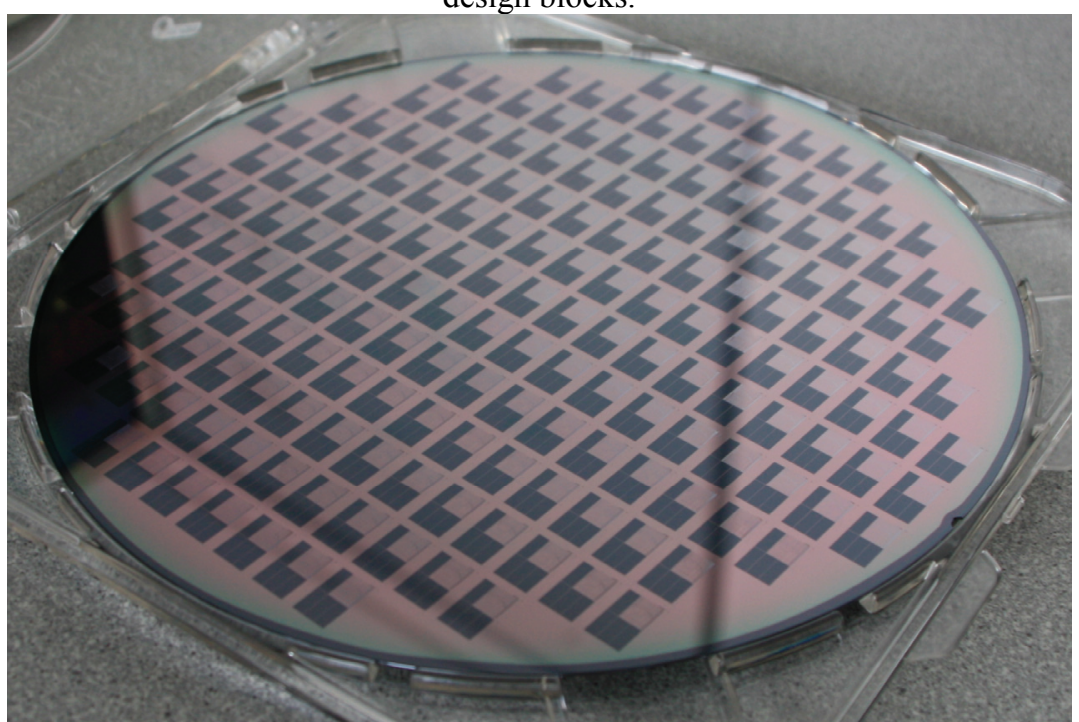

Fig. 9. A photograph of a processed 8 inch SOI wafer. In total there are 186 dies (of size $12.1 \mathrm{~mm}$ by 8.6 $\mathrm{mm})$ on the wafer. 


\subsection{Outline of the thesis}

The current chapter, 1, gives a general introduction to research topics covered in this thesis. These are biosensing, integrated read-out of microcantilever deflection, and optical switching.

Chapter 2 presents the sensor work. The chapter focuses on sensors based on waveguide gratings. An introduction of the optical properties and design issues of waveguide gratings is given. The experimental results of grated silicon photonic wire sensors, applied to bulk refractive-index sensing and label-free enzyme sensing, are given. A novel optical detection method to monitor nanodisplacements of a microcantilever is presented, and the method's potential use as a sensor is discussed.

Chapter 3 discusses the optical designs that were made for MEMS-actuated optical switching. These include cavities in photonic crystals, as well as waveguide gratings and ring resonators. The design procedure for each device is given, and the results of optical characterization are shown.

Chapter 4 gives the experimental results of MEMS-actuated devices. Mechano-optical tuning of a ring-resonator and a photonic crystal waveguide is demonstrated with an electrostatically actuated integrated microcantilever.

Chapter 5 presents an outlook on possible future developments and applications. 


\section{References}

[1] W.C.L. Hopman, "Light-flow characterization and manipulation in 1 and 2 dimensional photonic crystals", PhD thesis, University of Twente, 2007.

[2] T. Vo-Dinh, "Biomedical photonics handbook", CRC Press 2003.

[3] R.E. Gyurcsanyi, Z. Vagfoldi, K. Toth, and G. Nagy, "Fast response potentiometric acetylcholine biosensor", Electroanalysis, vol. 11, pp. 712-718, 1999.

[4] G.A. Campbell, D. Delesdernier, and R. Mutharasan, "Detection of airborne Bacillus anthracis spores by an integrated system of an air sampler and a cantilever immunosensor", Sensors and Actuators BChemical, Vol. 127, pp. 376-382, 2007.

[5] X. Fan, I.M. White, S.I. Shopova, H. Zhu, J.D. Suter, and Y. Sun, "Sensitive optical biosensors for unlabeled targets: A review", Analytica Chimica Acta, vol. 620, pp. 8-26, 2008.

[6] V.M.N. Passaro, F. Dell'Olio, B. Casamassima, and F. De Leonardis, "Guided-Wave Optical Biosensors", Sensors, vol. 7, pp. 508-536, 2007.

[7] P.V. Lambeck, "Integrated optical sensors for the chemical domain," Measurement Science \& Technology, vol. 17, pp. 93-116, 2006.

[8] B. Liedberg, C. Nylander, and I. Lunström, "Surface plasmon resonance for gas detection and biosensing", Sensors and Actuators, vol. 4, pp. 299-304, 1983.

[9] R.D. Harris, B.J. Luff, J.S. Wilkinson, J. Piehler, A. Brecht, G. Gauglitz, and R.A. Abuknesha, "Integrated optical surface plasmon resonance immunoprobe for simazine detection", Biosensors \& Bioelectronics, vol. 14, pp. 377-386, 1999.

[10] J. Dostálek, J. Čtyroký, J. Homola, E. Brynda, M. Skalský, P. Nekvindová, J. Špirková, J. Škvor, and J. Schröfel, "Surface plasmon resonance biosensor based on integrated optical waveguide", Sensors and Actuators B: Chemical, vol. 76, pp. 8-12, 2001.

[11] C.E.H. Berger, T.A.M. Beumer, R.P.H. Kooyman, and J. Greve, "Surface Plasmon Resonance Multisensing", Analytical Chemistry, vol. 70, pp. 703-706, 1998.

[12] E. Fu, T. Chinowsky, J. Foley, J. Weinstein, and P. Yager, "Characterization of a wavelength-tunable surface plasmon resonance microscope", Review of Scientific Instruments, vol. 75, pp. 2300-2304, 2004.

[13] M. Piliarik, H. Vaisocherová, and J. Homola, "A new surface plasmon resonance sensor for highthroughput screening applications", Biosensors and Bioelectronics, vol. 20, pp. 2104-2110, 2005.

[14] R.G. Heideman and P.V. Lambeck, "Remote opto-chemical sensing with extreme sensitivity: design, fabrication and performance of a pigtailed integrated optical phase-modulated Mach-Zehnder interferometer system", Sensors and Actuators B-Chemical, vol. 61, pp. 100-127, 1999.

[15] M. Hoekman, M. Dijkstra, and H.J.W.M. Hoekstra, "Design of a Spiral-Shaped Mach-Zehnder Interferometric Sensor for Refractive Index Sensing of Watery Solutions", IEEE/LEOS Benelux Annual Workshop, Eindhoven, The Netherlands, 25. May 2007.

[16] A. Densmore, D.-X. Xu, S. Janz, P. Waldron, T. Mischki, G. Lopinski, A. Delâge, J. Lapointe, P. Cheben, B. Lamontagne, and J. H. Schmid, "Spiral-path high-sensitivity silicon photonic wire molecular sensor with temperature-independent response", Optics Letters, vol. 33, pp. 596-598, 2008.

[17] I.M. White and X. Fan, "On the performance quantification of resonant refractive index sensors", Optics Express, vol. 16, pp. 1020-1028, 2008.

[18] K. De Vos, I. Bartolozzi, E. Schacht, P. Bienstman, and R. Baets, "Silicon-on-Insulator microring resonator for sensitive and label-free biosensing", Opt. Express, vol. 15, pp. 7610-7615, 2007.

[19] C. Chao, W. Fung, and L.J. Guo, "Polymer microring resonators for biochemical sensing applications", IEEE Journal of Selected Topics in Quantum electronics, vol. 12, pp. 134-142, 2006.

[20] S. Mandal and D. Erickson, "Nanoscale optofluidic sensor arrays", Optics Express, vol. 16, pp. $1623-$ $1631,2008$.

[21] W.C.L. Hopman, P. Pottier, D.Yudistira, J. van Lith, P.V. Lambeck, R.M. De LaRue, A. Driessen, H.J.W.M. Hoekstra, and R.M. de Ridder, "Quasi-one-dimensional photonic crystal as a compact building-block for refractometric optical sensors”, IEEE J. Select. Topics Quantum Electron, vol. 11, pp. 11-16, 2005. 
[22] N. Skivesen, A. Têtu, M. Kristensen, J. Kjems, L.H. Frandsen, and P.I. Borel, "Photonic-crystal waveguide biosensor", Optics Express, vol. 15, pp. 3169-3176, 2007.

[23] C.A. Barrios, K.B. Gylfason, B. Sánchez, A. Griol, H. Sohlström, M. Holgado, and R. Casquel, "Slotwaveguide biochemical sensor", Optics Letters, vol. 32, pp. 3080-3082, 2007.

[24] G. Rong, A. Najmaie, J.E. Sipe, and S.M. Weiss, "Nanoscale porous silicon waveguide for labelfree DNA sensing", Biosensors \& Bioelectronics, vol. 23, pp. 1572-1576, 2008.

[25] L. He, M.D. Musick, S.R. Nicewarner, F.G. Salinas, S.J. Benkovic, M.J. Natan, and C.D. Keating, "Colloidal Au-enhanced surface plasmon resonance for ultrasensitive detection of DNA hybridization", Journal of the American Chemical Society, vol. 122, pp. 9071-9077, 2000.

[26 ] C. Ziegler, "Cantilever-based biosensors", Analytical and Bioanalytical Chemistry, vol. 379, pp. 946959, 2004.

[27] J. Fritz, M. K. Baller, H.P. Lang, H. Rothuizen, P. Vettiger, E. Meyer, H.-J. Güntherodt, Ch. Gerber, and J.K. Gimzewski, "Translating biomolecular recognition into nanomechanics", Science, vol. 288, pp. 316-318, 2000.

[28] M.K. Baller, H.P. Lang, J. Fritz, Ch. Gerber, J.K. Gimzewski, U. Drechsler, H. Rothuizen, M. Despont, P. Vettiger, F.M. Battiston, J.P. Ramseyer, P. Fornaro, E. Meyer, and H.-J. Güntherodt, "A cantilever array-based artificial nose", Ultramicroscopy, vol. 82, pp. 1-9, 2000.

[29] Y. Zhao, M. Mao, R. Horowitz, A. Majumdar, J. Varesi, P. Norton, and J. Kitching, "Optomechanical uncooled infrared imaging system: design, microfabrication, and performance", Journal of Microelectromechanical systems vol. 11, pp. 136-146, 2002.

[30] F. Dong, Q. Zhang, D. Chen, Z. Miao, Z. Xiong, Z. Guo, C. Li, B. Jiao, and X. Wu, "Uncooled infrared imaging device based on optimized optomechanical micro-cantilever array", Ultramicroscopy, vol. 108, pp. 579-588, 2008.

[31] S. Okuyama, Y. Mitobe, K. Okuyama, and K. Matsushita, "Hydrogen gas sensing using a Pd-coated cantilever" Japanese Journal of Applied Physics, vol 39, pp. 3584-3590, 2000.

[32] D. Iannuzzi, M. Slaman, J.H. Rector, H. Schreuders, S. Deladi, and M.C. Elwenspoek, "A fibertop cantilever for hydrogen detection", Sensors and Actuators B, vol. 121, pp. 706-708, 2007.

[33] N.V. Lavrik, M.J. Sepaniak, and P.G. Datskos, "Cantilever transducers as a platform for chemical and biological sensors", Review of Scientific Instruments, vol. 75, pp. 2229-2253, 2004.

[34] K. Zinoviev, C. Dominguez, J.A. Plaza, V.J.C. Busto, and L.M. Lechuga, "A novel optical waveguide microcantilever sensor for the detection of nanomechanical forces", Journal of Lightwave Technology, vol. 24, pp. 2132-2138, 2006.

[35] M. Nordström, D.A. Zauner, M. Calleja, J. Hübner, and A. Boisen, "Integrated optical readout for miniaturization of cantilever-based sensor system", Applied Physics Letters, vol. 91, p. 103512, 2007.

[36] M. Li, W.H.P. Pernice, and H. X. Tang, "Broadband all-photonic transduction of nanocantilevers", Nature Nanotechnology, vol. 4, pp. 377-382, 2009.

[37] C. Lee and J. Thillaigovindan, "Optical nanomechanical sensor using a silicon photonic crystal cantilever embedded with a nanocavity resonator", Applied Optics, vol. 48, pp. 1797-1803, 2009.

[38] W. Gabathuler and W. Lukosz, "Electro-nanomechanically wavelength-tunable integrated-optical Bragg reflectors Part II: Stable device operation”, Optics Communications, vol. 145, pp. 258-264, 1998.

[39] C.A. Barrios, "Ultrasensitive nanomechanical photonic sensor based on horizontal slot-waveguide resonator", IEEE Photonics Technology Letters, vol. 18, pp. 2419-2421, 2006.

[40] P.T. Rakich, M. A. Popovic', M.R. Watts, T. Barwicz, H.I. Smith, and E.P. Ippen, "Ultrawide tuning of photonic microcavities via evanescent field perturbation", Optics Letters, vol. 31, 2006.

[41] G.N. Nielson, D. Seneviratne, F. Lopez-Royo, P.T. Rakich, Y. Avrahami, M.R. Watts, H.A. Haus, H.L. Tuller, and G. Barbastathis, "Integrated wavelength-selective optical MEMS switching using ring resonator filters”, IEEE Photonics Technology Letters, vol. 17, pp. 1190-1192, 2005.

[42] R. Ramaswami, "Optical Networking Technologies: What Worked and What Didn't" IEEE Communications Magazine, pp. 132-139, September 2006.

[43] R. Ramaswami and K.N. Sivarajan, "Optical networks: a practical perspective", second edition, Morgan Kaufmann 2002. 
[44] M. Maier and M. Reisslein, "Trends in Optical Switching Techniques: A Short Survey," IEEE Network, vol. 22, pp. 42-47, 2008.

[45] E.J. Klein, "Densely integrated microring-resonator based components for fiber-to-the-home applications", PhD thesis, University of Twente, 2007.

[46] G. Cocorullo, M. Iodice, I. Rendina, and P.M. Sarro, "Silicon Thermooptical Micromodulator with 700-kHz - 3-dB Bandwidth", IEEE Photonics Technology Letters, vol. 7, pp. 363-365, 1995.

[47] A. Schneider, I. Biaggio, and P. Günther, "Teraherz-induced lensing and its use for the detection of teraherz pulses in a birefringent crystal," Applied Physics Letters, Vol. 84, pp. 2229-2231, 2004.

[48] R. Dekker, A. Driessen, T. Wahlbrink, C. Moormann, J. Niehusmann, and M. Först, "Ultrafast Kerrinduced all-optical wavelength conversion in silicon waveguides using $1.55 \mu \mathrm{m}$ femtosecond pulses," Optics Express, Vol. 14, pp. 8336-8346, 2006.

[49] J. Singh, "Semiconductor optoelectronics: physics and technology", McGraw-Hill 1995.

[50] Q. Xu, B. Schmidt, S. Pradhan, and M. Lipson, "Micrometre-scale silicon electro-optic modulator", Nature, vol. 435, pp. 325-327, 2005.

[51] G.J. Veldhuis, T. Nauta, C. Gui, J.W. Berenschot, and P.V. Lambeck, "Electrostatically Actuated Mechanooptical Waveguide ON-OFF Switch Showing High Extinction at a Low Actuation-Voltage", IEEE Journal of Selected Topics in Quantum Electronics, vol. 5, pp. 60 - 66, 1999.

[52] G.N. Nielson, D. Seneviratne, F. Lopez-Royo, P.T. Rakich, Y. Avrahami, M.R. Watts, H.A. Haus, H.L. Tuller, and G. Barbastathis, "Integrated wavelength-selective optical MEMS switching using ring resonator filters", IEEE Photonics Technology Letters, vol. 17, pp. 1190-1192, 2005.

[53] P.T. Rakich, M. A. Popović, M.R. Watts, T. Barwicz, H.I. Smith, and E.P. Ippen, "Ultrawide tuning of photonic microcavities via evanescent field perturbation", Optics Letters, vol. 31, pp. 1241-1243, 2006.

[54] W.C.L. Hopman, K.O. van der Werf, A.J.F Hollink, W. Bogaerts, V. Subramaniam, and R.M. de Ridder, "Nano-mechanical tuning and imaging of a photonic crystal micro-cavity resonance," Optics Express, vol. 14, pp. 8745-8752, 2006.

[55] I. Märki, M. Salt, and H.P. Herzig, "Tuning the resonance of a photonic crystal microcavity with an AFM probe," Optics Express 14, pp. 2969-2978, 2006.

[56] Soitec, S.A.Parc Technologique des Fontaines, 38190, Bernin, France. (www.soitec.com)

[57] W. Bogaerts, "Nanophotonic Waveguides and photonic crystals in silicon-on-insulator", PhD thesis, Universiteit Gent 2004.

[58] P. Dumon,W. Bogaerts, R. Baets, J.-M. Fedeli, and L. Fulbert, "Towards foundry approach for silicon photonics: silicon photonics platform ePIXfab", IEEE Electronics Letters, vol. 45, no. 12, 2009. 


\section{Sensors based on waveguide grating}

In this chapter waveguide grating based sensors for bio- and nanodisplacement sensing are presented. First the optical properties of waveguide gratings are discussed with a focus on modeling and design issues. The use of a waveguide grating as a sensor platform for refractometric sensing and for sensing of nanodisplacements is discussed and important sensor parameters are introduced. The properties of a grated silicon photonic wire for label- free biochemical sensing applications are discussed and sensitivity, as well as detection limit, of this device is experimentally determined. A new integrated optical detection method to observe the displacement of a microcantilever is presented and options to fabricate this device are discussed.

\subsection{Waveguide grating}

In this section some properties of waveguide gratings are discussed. The focus is on modeling and design issues related to optical losses that are specific to waveguide gratings. Design boundaries to obtain low loss operation are presented. A grating defect and some of its potential applications are shortly described. The use of waveguide gratings as sensors to detect refractive index changes in the proximity of the grating is also discussed.

If the properties of periodic structures are to be exploited in integrated optics, the $2 \mathrm{D}$ lateral confinement provided by channel waveguides needs to be combined with a periodic variation in the third dimension. The most common way to do this is to periodically vary the thickness or width of the waveguide. An example of such a "grated waveguide" or "waveguide grating" is schematically depicted in Fig. 1a.

Exact modelling of such essentially 3-dimensional structures is rather involved, requiring huge computing resources. Depending on the waveguide geometry the model can be reduced to $2 \mathrm{D}$, see Fig. 1b, without losing much information. However, this is not always the case. In particular, the strong modal dispersion of strip waveguides in high-indexcontrast material systems causes a large uncertainty in the parameters of a 2D model [1], see also section 2.2.1.

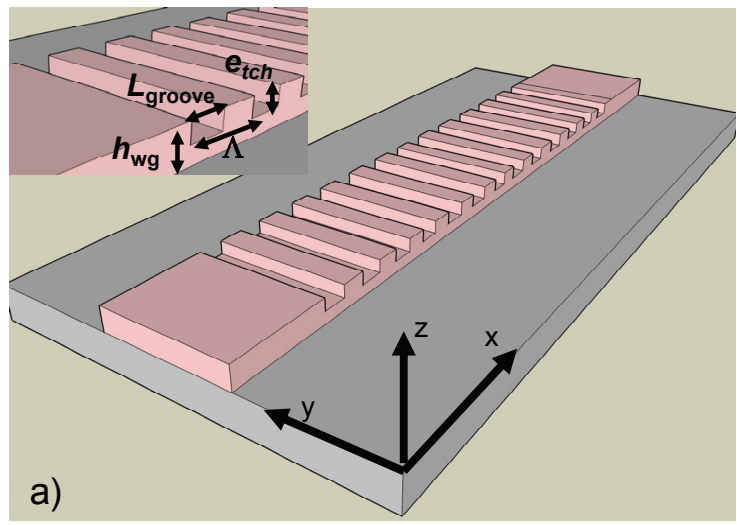



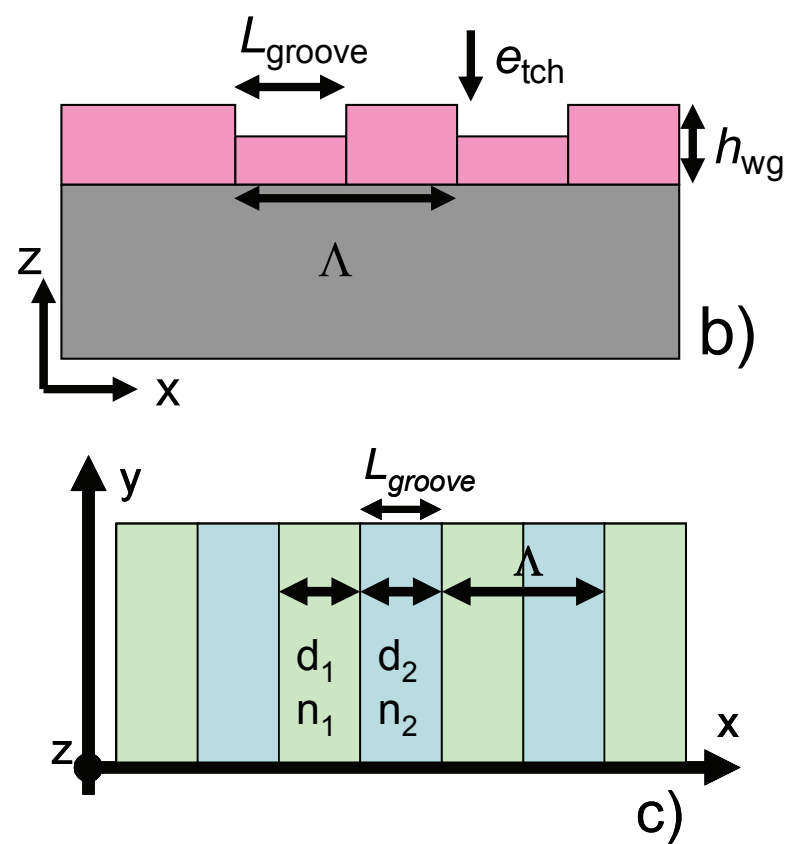

Fig. 1. A schematic 3D picture of a waveguide grating. The grating is formed by periodically altering the thickness of the waveguide. The depth of the groove depends on the etch depth $\left(\mathrm{e}_{\mathrm{tch}}\right)$. b) Schematic picture of waveguide grating reduced to $2 \mathrm{D}$. c) A schematic picture of waveguide grating reduced to $1 \mathrm{D}$. The equivalent $1 \mathrm{D}$ indices $\left(n_{1}\right.$ and $\left.n_{2}\right)$ are calculated using e.g. a mode solver. The layer thicknesses $d_{1}$ and $d_{2}$ correspond to groove length $\left(L_{\text {groove }}\right)$ and ridge length $\left(\Lambda-L_{\text {groove }}\right)$.

The 2D structure can be reduced further to $1 \mathrm{D}$, see Fig. 1c, for efficient modeling. However, in this case some crucial information is lost. In 1D modeling, out-of-plane losses are ignored and the effect of the waveguide geometry is not fully taken into account.

We will use the reduced $1 \mathrm{D}$ and 2D models to illustrate some properties of light propagation in a waveguide grating. Wave propagation in a periodic structure, such as a waveguide grating, can be formulated according to Bloch's theorem [2]. According to it, the wave function in a $1 \mathrm{D}$ periodic structure can be expressed as:

$$
\psi(x, t)=e^{j(\omega t-\beta x)} u(x) .
$$

In which $u$ is the amplitude of the wave, $\beta$ is the wave-vector (also known as propagation constant), $\omega$ is the angular frequency and $t$ is the time. From the periodicity of the structure it follows that the wave-vectors that differ by integer multiples of $2 \pi / \Lambda$ (where $\Lambda$ is the period) are equal; i.e. the wave-vector repeats itself [3]. This implies that the wave-vector of this Bloch state can be reduced to the so-called Brillouin zone $[-\pi / \Lambda$, $\pi / \Lambda]$. A dispersion graph of a grating is obtained by solving the wave-vector values as function of angular frequency in the Brillouin zone. The dispersion graph is useful in analyzing the properties of gratings (and other periodic structures).

As an example, the dispersion properties of a waveguide grating are calculated. We consider a shallow ridge waveguide with a ridge height $\left(h_{\mathrm{wg}}\right)$ of $5 \mathrm{~nm}$ defined in a $275 \mathrm{~nm}$ thick layer of $\mathrm{Si}_{3} \mathrm{~N}_{4}(n=1.981)$ that is on top of a $\mathrm{SiO}_{2}$ cladding $(n=1.445)$. If a grating is defined to this waveguide by etching a periodic pattern of $55 \mathrm{~nm}$ deep grooves (in the 
z-direction) having a length ( $\left.L_{\text {groove }}\right)$ of $240 \mathrm{~nm}$ and a period of $490 \mathrm{~nm}$, the equivalent 1D layer stack indices (as calculated with a 2D mode solver) are $n_{1}=1.59289$ and $n_{2}=$ 1.53209. Figs $2 \mathrm{a}$ and $2 \mathrm{~b}$ show the dispersion diagrams for the infinite layer stack, obtained by computing definite frequency eigenstates of Maxwell's equations using the MIT Photonics Band (MPB) package [4]. As seen from Fig. 2b, for some wavelengths there is no propagating mode in a grating as there is no wave-vector for these wavelengths. This wavelength range is the so-called photonic stopband. In a specific case the energy of the propagating wave is coupled from the forward propagating wave to the backward propagating wave. In terms of wave-vectors phase matching is required; $\beta_{f}-\beta_{b}$ $=m 2 \pi / \Lambda$, in which $m$ is an integer and subscripts $f$ and $b$ refer to backward and forward directions, respectively. In this case the light is reflected by the grating. In the stopband the Bloch mode is named as evanescent Bloch mode [5].

The dispersion graph also shows the so-called lightlines, which are the dispersion curves of plane waves travelling parallel to the waveguide in the corresponding cladding material, given by $\omega / \beta=c / n_{\text {clad }}$.

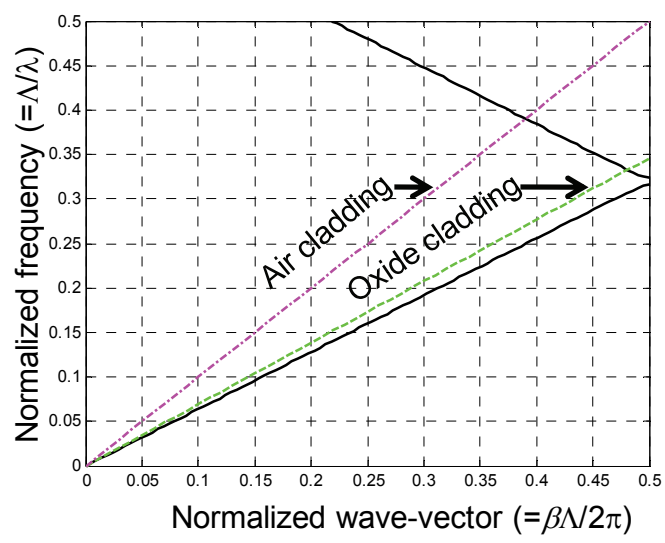

a)

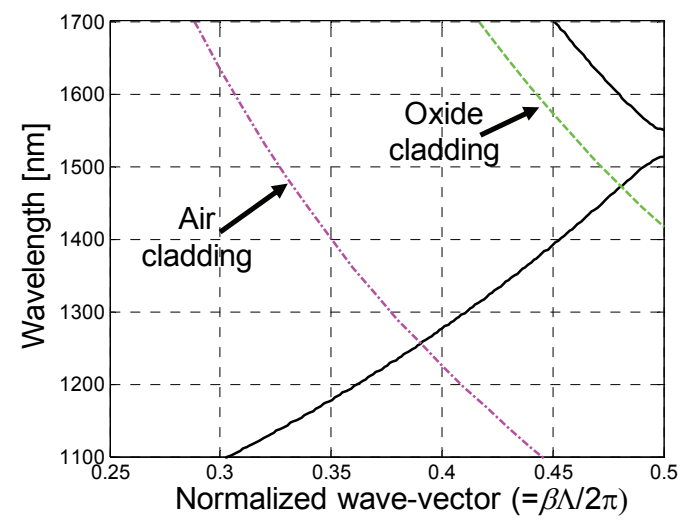

b)

Fig. 2. Dispersion graph of a 1D grating, also showing the lightlines for air and the oxide cladding (coloured dashed lines). a) Normalized dispersion graph. b) Magnified part of Fig. 2a, around the lowestorder stopband, with a real wavelength scale instead of normalized frequency.

If, for a given propagation constant of the grating mode, the frequency is above the lightline, part of the light radiates from the grating. This is because in that case the grating mode $(\beta)$ can always phase-match to a plane wave propagating at an angle $\theta$ to the surface normal in the cladding $\left(\beta_{\text {clad }} \sin \theta\right)$, according to [6]:

$$
\beta_{\text {clad }} \sin \theta=\beta-\frac{m 2 \pi}{\Lambda} \text {. }
$$

For our grating below $1470 \mathrm{~nm}$ the loss starts to increase as the lower dispersion curve crosses the lightline of the oxide cladding at this point (see Fig. 2b).

To obtain more quantitative data of the properties of the grating, we have performed 2D calculations. Fig. 3 shows a simulated transmission spectrum, obtained using the 2D finite difference time domain (FDTD) method [7], of the grating structure that is shown in the inset of Fig. 3. This is the 2D reduced model of the grating and the parameters are the same as the ones that were used for the 1D calculations to obtain the data shown in Fig. 2. For the 2D simulation a grating length of 100 periods was chosen. 
From the transmission spectrum we can see the effect of crossing the lightline for this grating. For wavelengths below $1470 \mathrm{~nm}$ the loss increases with the wavelength difference from this lightline crossing point, as predicted from the dispersion graph. In the simulated transmission spectrum (see Fig. 3) there are peaks in the transmission band. These resonance peaks are explained next.

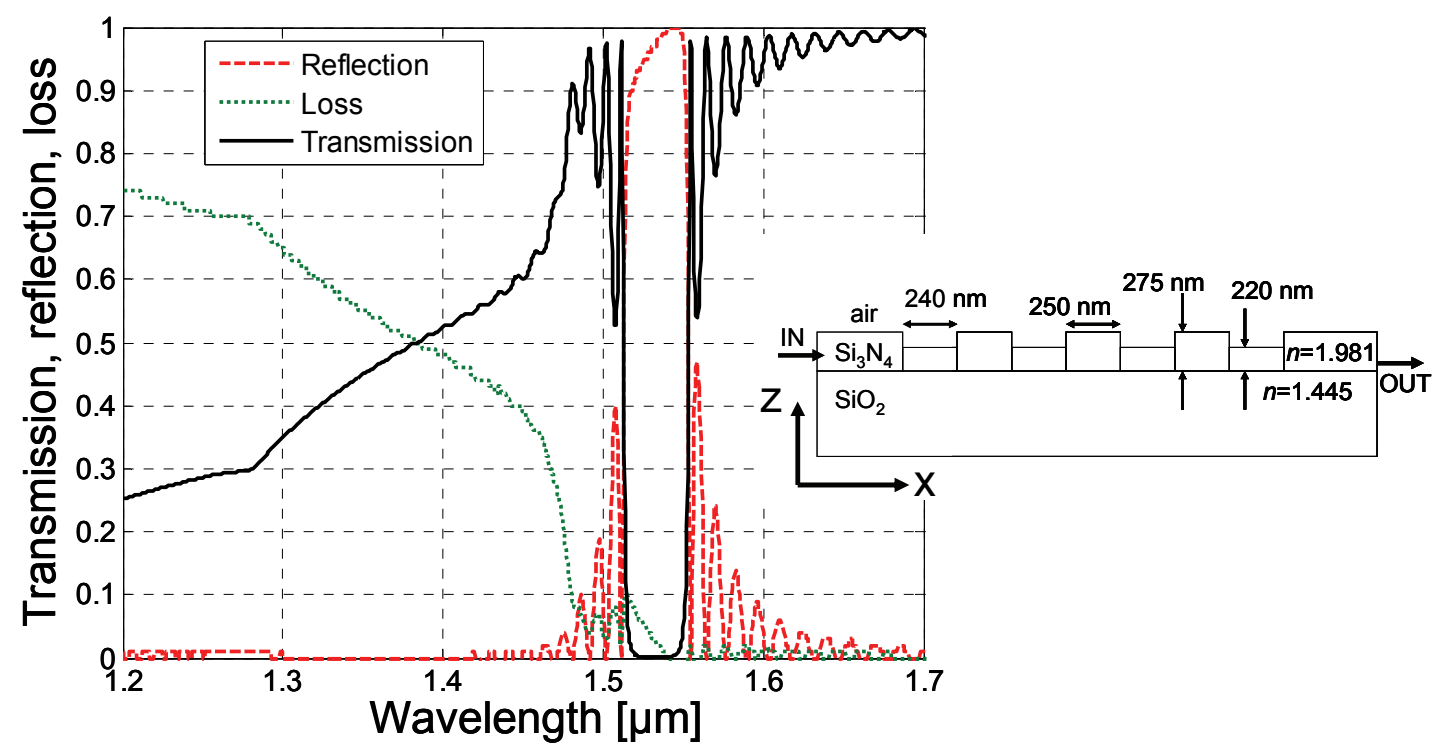

Fig. 3. Simulated transmission spectrum using the same grating parameters as for Fig. 2. The loss increases as the wavelength becomes shorter than the wavelength of $1470 \mathrm{~nm}$, where the dispersion curves of the cladding and the grating modes cross (see Fig. 2b). Inset: schematic picture of the grating cross-section used in the calculation.

If the grating is finite in the $\mathrm{x}$-direction, resonances can be observed in the transmission band. They are similar to Fabry-Pérot cavity resonances of the Bloch modes [8]. Propagation constants of these (forward and backward propagating) modes are given by [8-9];

$$
\pm \beta=\frac{\pi}{\Lambda}-\frac{\pi q}{N \Lambda}, q=[1, N],
$$

in which $N$ is the number of periods in the grating.

When constructive interference occurs, these modes create a standing (electromagnetic) wave, and the nodes of these standing waves are located at $x=N \Lambda s / q$, with $s=[0, q]$.

In sensor applications, discussed in this book, we have exploited the sharp spectral features of the resonating Bloch modes in the design of a compact and highly sensitive sensors for the detection of nanodisplacements (section 2.4) and for refractometric sensing (sections 2.2 and 2.3).

\subsubsection{Design guidelines}

In this section some design guidelines are given to avoid the losses originating from the lightline crossing. 
Based on the fact that the normalized frequency $(\Lambda / \lambda)$ of the cladding lightline is $1 / 2 n_{\text {clad }}$ at the Brillouin zone edge, it is possible to write a simple equation for maximum period $\Lambda_{\max }$, in order to have the stopband at the lossless side of the lightline:

$$
\Lambda_{\text {max }}=\frac{\lambda_{\text {edge }}}{2 n_{\text {clad }}},
$$

where $\lambda_{\text {edge }}$ is the wavelength at the lower wavelength stopband edge. For a silicon oxide cladding $n_{\text {clad }}=1.445$ (at infrared wavelengths), and assuming the band edge of $\lambda_{\text {edge }}=1500 \mathrm{~nm}$, a maximum period of $520 \mathrm{~nm}$ is obtained from equation (17).

An estimate can be given of the minimum operating wavelength $\lambda_{\text {Omin }}$ that is the shortest wavelength at which the grating is still on the lossless side of the lightline, by just considering the properties of a 1D periodic structure (which of course cannot have radiation loss). It is the wavelength corresponding to the crossing of the light line and the dispersion curve of the grating mode of an equivalent 1D structure (see Fig. 2a). The crossing point of two linear curves can be calculated, if the slope and the value of the curves are known in one point. By assuming that the slope of the dispersion curve of the grating mode can be approximated to be $-1 / n_{a v}$, where $n_{a v}$ is the average index of the grating, and the slope of the cladding mode (lightline) is $1 / n_{\text {clad }}$, an equation that gives an approximate value for $\lambda_{O \min }$ is obtained:

$$
\lambda_{\text {Omin }}=\frac{2 \lambda_{\text {edge }} \Lambda\left(n_{a v}+n_{\text {clad }}\right)}{2 n_{a v} \Lambda+\lambda_{\text {edge }}}
$$

Equation (5) gives a good approximation only for weak gratings. Fig. 4 shows a comparison of the minimum lossless operating wavelength as calculated from equation (5) and as obtained using the MPB [3]. The following grating parameters were used: $\Lambda=490 \mathrm{~nm}, d_{1}=250 \mathrm{~nm}, n_{1}=1.55 \ldots 1.98, d_{2}=240 \mathrm{~nm}$ and $n_{2}=1.53$.

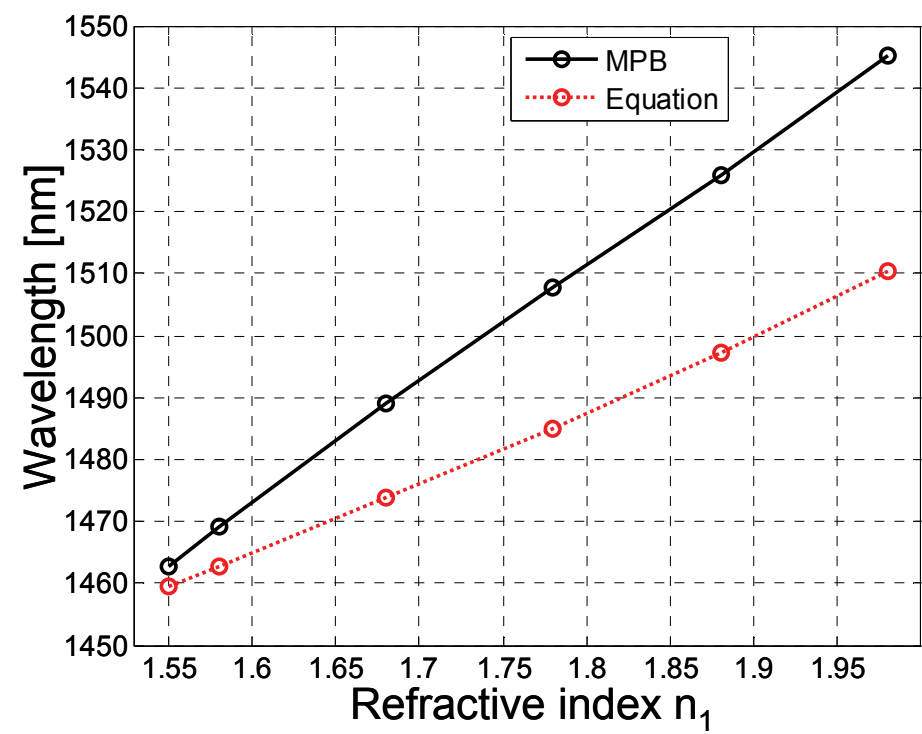

Fig. 4. Minimum operating lossless wavelength for a waveguide grating. Comparison between the data obtained from equation 5 and from MPB calculations. 
It can be seen that the equation gives the minimum operating wavelength $\lambda_{O \min }$ at better than $15 \mathrm{~nm}$ accuracy if $n_{1}-n_{2}<0.1$. For strong gratings the simple approximation is no longer valid as the nonlinearity of the dispersion curve increases. For these cases, the minimum operating wavelengths obtained using the MPB are considerable larger. Nevertheless equation (5) is a useful design tool by providing a quick and rough estimate of the minimum wavelength for lossless operation.

As an example, let us assume that a grating is designed to have $\lambda_{\text {edge }}=1500 \mathrm{~nm}$, and that it is to be fabricated on a waveguide having $n_{\text {eff }}=1.6$ and $n_{\text {clad }}=1.445$. We wish to find a design boundary for the grating period and see what is the minimum wavelength for lossless operation. By inserting the parameters into equation (5) (using $n_{a v}=n_{\text {eff }}$ ), a minimum operating point as a function of period is obtained as shown in Fig. 5. The maximum period can be calculated from equation (4) as well as the minimum period by placing $n_{\text {clad }} \rightarrow n_{a v}$. For the calculation of minimum period it is assumed that maximum $n_{a v}$ is used $\left(n_{e f f}\right)$ and keeping in mind that we set $\lambda_{\text {edge }}=1500 \mathrm{~nm}$. It can be seen from Fig. 5 that it is not possible to design a grating that would be lossless for wavelengths below $\lambda_{\text {Omin }}=1425 \mathrm{~nm}$.

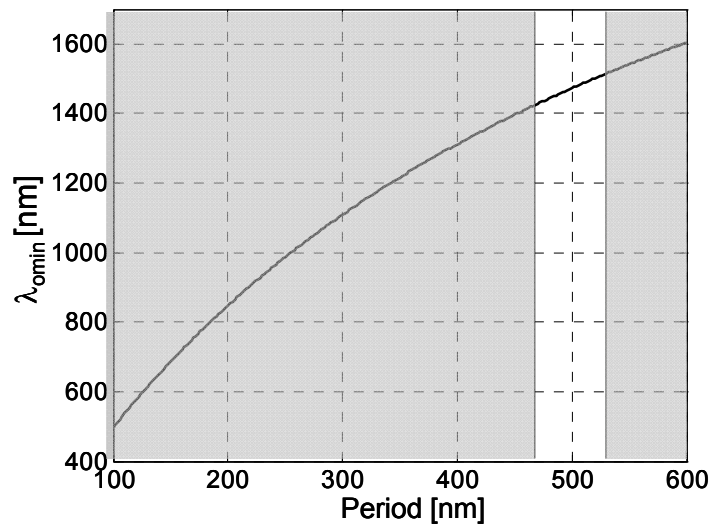

Fig. 5. Minimum lossless operating point as a function of the grating period. The shaded regions correspond to periods that are outside the boundaries set by equation (4), see text.

This type of design rule is useful, e.g. in the design of a waveguide laser that uses grating(s) as mirror(s). The grating operates as a mirror for the wavelengths that are in the photonic stopband of the grating. The wavelength of the pump light, used to excite the laser operation, is often much shorter than the laser wavelength and may therefore operate the grating at the lossy side of the lightline. For sensor applications low loss operation is important to get the best possible signal-to-noise ratio. Also to realize a compact sensor, the period should be small, which favors high index materials $(n>2)$.

It should be noted that in some cases it is not possible to have the periodic devices operating at the lossless side of the lightline, e.g. for some $\mathrm{PhCs}$ defined in III-V group materials [10]. However, if the device length is kept short, as can be the case for the $\mathrm{PhC}$ based devices, the absolute loss may still be acceptable.

The phenomenon of light radiating from a grating operating above the lightline is exploited in grating couplers [11], which are waveguide gratings that are intentionally 
designed to operate in this regime, such that the light in a given wavelength range is coupled into or out of a waveguide at a coupling angle $\theta$, given by (2).

\subsubsection{Mode mismatch loss}

Apart from radiation loss caused by crossing the lightline, there can be additional loss, depending on the geometry of the grating structure. For example, if the low-effectiveindex parts of the grating period are defined by etching as shown in Fig. 1, increasing the etch depth increases the loss. The origin of this loss is modal field mismatch between the incoming waveguide mode and the Bloch mode of the grating [12]. This type of loss affects a wide spectral band, including the stopband. The loss in the stopband is seen e.g. in a simulated transmission spectrum of Fig. 3: the loss is smaller at the longest wavelength.

The loss for the Bloch modes near the band edge is also due to mode mismatch between the Bloch mode and the waveguide mode, which leads to coupling to radiation modes. In order to minimize the mode mismatch loss the grating should be introduced gradually in tapered manner [12-13].

It should be noted that in the ideal grating the Bloch modes are lossless. In real gratings fabrication imperfections cause loss, which is particularly large for the slow Bloch modes due to increased life time of the resonating photons.

\subsubsection{Defects}

A defect is a structural imperfection in a periodic structure. In a waveguide grating a defect can be e.g. a waveguide segment or a grating section that has a different period than the surrounding grating. In optics such defects are often called cavities. Fig. 6a shows a defect in a waveguide grating. Light can be trapped in a cavity as a localized state if the frequency is in the photonic stopband of the surrounding grating. Light can be coupled from the input waveguide to the defect mode e.g. via an evanescent Bloch mode. If the cavity length is well chosen, a Fabry-Pérot resonator type operation is achieved; the surrounding grating sections function as mirrors for the cavity. Due to phase matching at resonance, energy is fed from the input side to the cavity while at the output side a fraction of energy escapes from the cavity via an evanescent Bloch mode. In the steady state, for a lossless structure, the power coupled to the cavity equals to the power transferred to the output. In a grating the reflection comes from distributed Bragg reflections. Therefore, in order to minimize the losses one should take into account the mode mismatch loss discussed above. Fig. 6b shows the transmission spectrum of a grated waveguide that has a waveguide segment with length $0.9 \Lambda$ in the middle of the grating. In this case the cavity loss is about $5 \mathrm{~dB}$.

At resonance, the field intensity is enhanced in a cavity. This phenomenon can be exploited in opto-optical devices. An intense light pulse at a wavelength corresponding to a defect mode resonance can be used to create an index change in a material whose index depends strongly on light intensity. This index change in turn changes the resonance peak position by some amount $\Delta \lambda$. Thanks to the cavity enhanced intensity, a required index change $\Delta n$ can be induced with a less intense pulse than would be needed without exploiting the resonant field enhancement. This kind of design can be used e.g. as an optical switch [14], in which the switching is done with intense optical pulses. Other 
grating cavity applications are e.g. optical delay lines [15], sensors [16 and 17] and waveguide lasers [18].
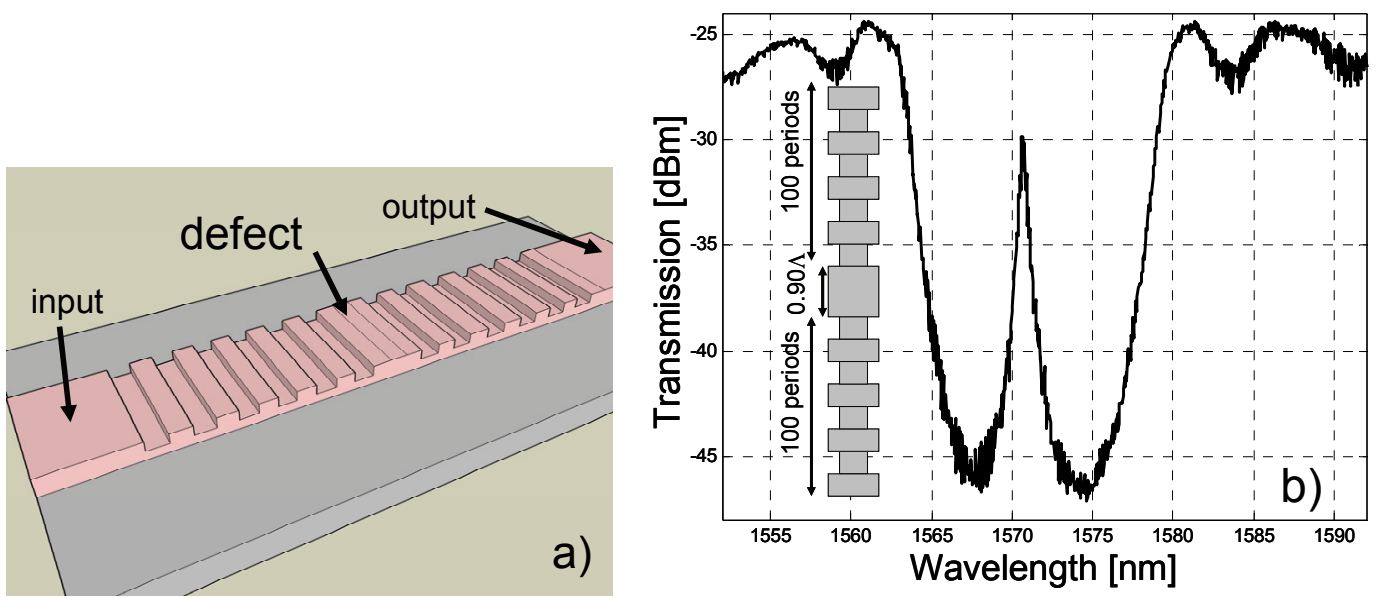

Fig. 6. a) Schematic picture of a defect in a waveguide grating b) An example of a measured transmission spectrum of a waveguide grating that has a defect cavity in the center. The peak in the middle of the stopband originates from the defect cavity. Inset: Schematic top view picture of the measured defective grating.

\subsubsection{Waveguide gratings as sensor platform}

In this section we discuss the use of waveguide gratings as sensors to detect refractive index changes in the proximity of the grating. The sensitivity of the grating to the index changes can be exploited to make sensors for biosensing and nanodisplacement.

\section{Refractometric sensing}

The effective index of the grated waveguide depends on the cladding material index. Therefore, if it is possible to observe the effective index change, it is also possible to measure the change in the cladding material index. For a waveguide grating the effective index change causes the spectral position of the stopband to shift, and this shift is often used to measure the cladding index change. A schematic picture of a waveguide grating sensor is shown in Fig. 7a.

The sharp spectral phenomena of a grated waveguide near the stopband edge exhibit a strong sensitivity to changes in the ambient refractive index. A small cladding-index change $\Delta n_{\text {clad }}$ causes the first-order Bragg wavelength $\lambda_{B}[6]$ of a grating to shift in accordance with:

$$
\Delta \lambda_{B} \approx 2 \eta \Delta n_{\text {clad }} \Lambda
$$

in which $\eta$ is the fraction of energy density in the cladding. 

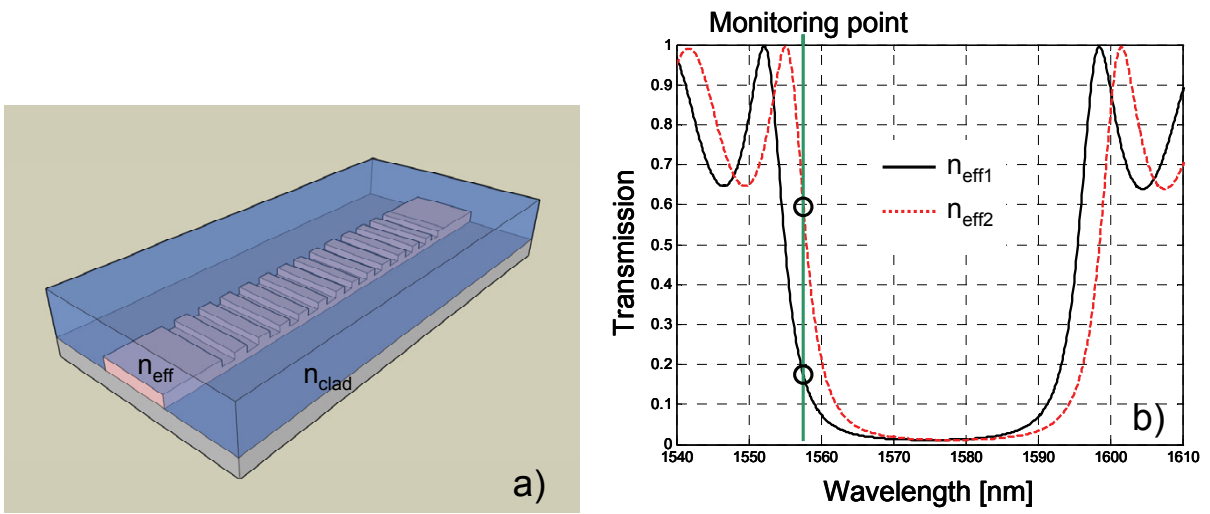

Fig. 7. a) Waveguide grating as a sensor; the index change in the cladding index $n_{\text {clad }}$ causes a change in the effective index of the grating $n_{\text {eff }}$ that in turn shifts the stopband. B) The stopband shift due to index change; $n_{\text {eff } 2}>n_{\text {eff } 1}$. By measuring at a fixed wavelength (monitoring point) at the band edge, a large change in the transmission, corresponding to a small index change, can be observed.

Hence by detecting a wavelength shift, a change in the cladding index can be observed. To fully exploit the sharp spectral features of such a waveguide grating, we monitor the change in transmitted power at a stopband edge, see Fig. 7b. As the cladding index changes, the spectral position of this edge shifts according to (6). A change in transmitted power $\Delta T$ depends on both the slope of the stopband edge and the cladding-index change according to:

$$
\Delta T \approx 2 \eta \Lambda \frac{\partial T}{\partial \lambda} \Delta n_{c l a d}
$$

Therefore, a steep stopband edge and a large energy density fraction in the cladding $(\eta)$ will result in high sensitivity to cladding-index changes. The energy density fraction $(\eta)$ depends on the geometry of the structure and the material properties. The slope of the stopband edge depends on the length and strength (i.e. index contrast between high- and low-index regions) of the grating [20].

The detection limit of the sensor depends on how accurately the small power change $\Delta T$ can be measured, which depends on, among many things, laser power fluctuation and dark current noise of the photodetector.

The accuracy, at which the small power change $\Delta T$ can be measured, sets the detection limit of the sensor. Therefore many things such as laser power fluctuation and dark current noise of the photodetector contribute to detection limit.

\section{Nanodisplacement sensing}

The waveguide grating's response to an effective index change can be exploited to observe nanodisplacements of a micromechanical element (ME) that is in the proximity of the grating [21]. In [21] the ME is attached to the optical chip by bonding. From the fabrication point of view monolithic integration of a micromechanical structure that covers the entire grating is very challenging (see sections 2.4.4.1 and 4.3). However, recalling the spatial positions of the resonating Bloch modes and taking into account that the electromagnetic field strength is enhanced at the resonance wavelength (leading to the situation where also light matter interaction is enhanced) one can exploit these resonances for nanodisplacement sensing. Due to localization of these resonances much smaller ME 
can be used, allowing monolithic integration. Although this comes at the expense of sensitivity, a sub-nanometre deflection sensitivity is achievable as will be discussed in section 2.4. Spatial distributions of some resonance modes are shown in Fig. 8. If a perturbation, say a dielectric microcantilever, is placed such that it is in the evanescent field region of a resonance mode, a shift in the resonance wavelength is observed.
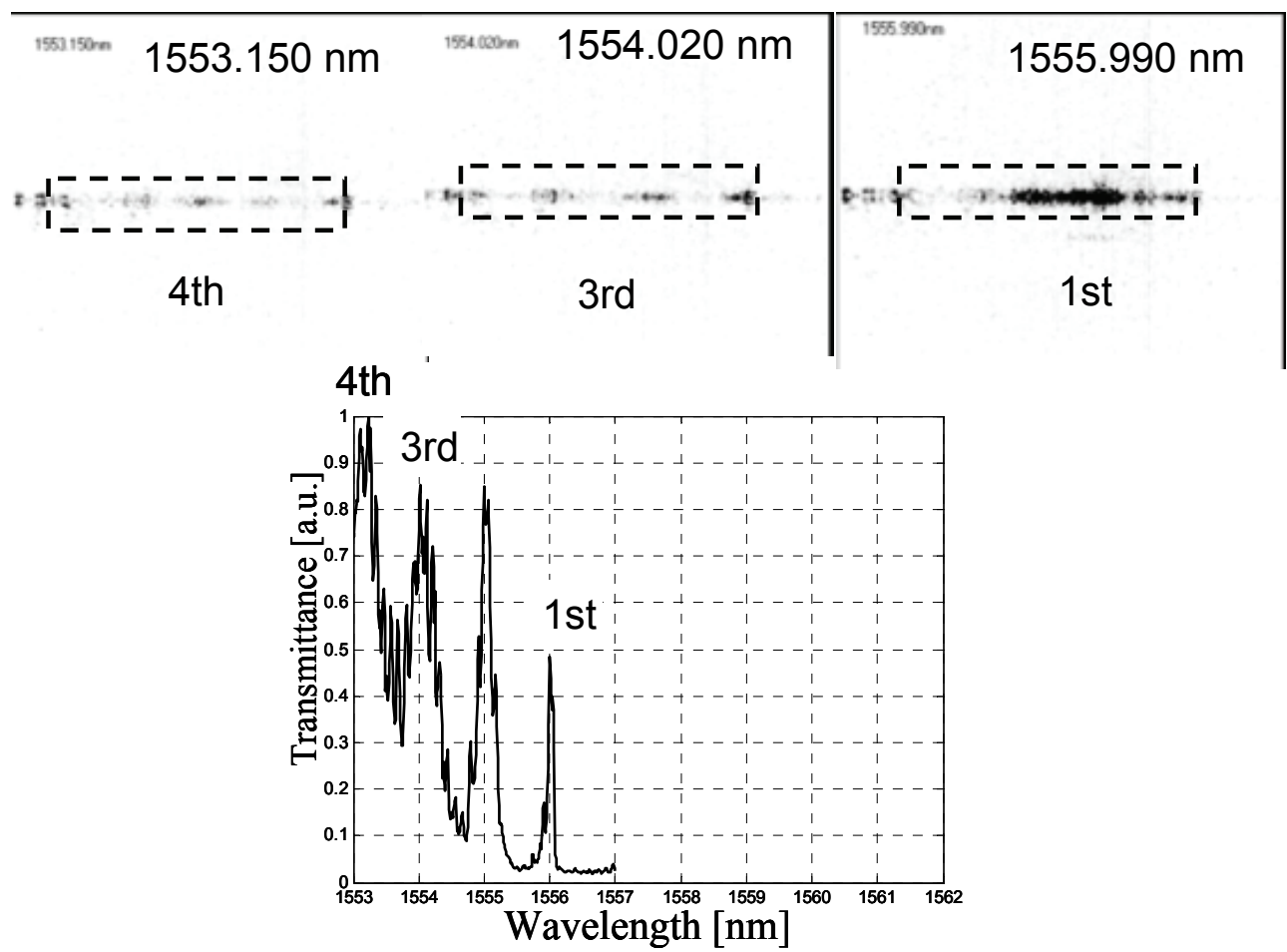

Fig. 8. Transmission spectrum of the grated waveguide, showing the four near band edge resonances. Also shown are the (top views of) spatial distributions of these resonances as captured with the infrared camera. The dashed line shows the position of the grated waveguide, light propagates from left to right.

By placing a cantilever above a waveguide grating, as shown in Fig. 9, the deflection of the cantilever by $\Delta z$ causes a change in the resonance wavelength. By monitoring the transmittance of the grating at the edge of the resonance wavelength, a small deflection can be deducted from a small transmission power change $\Delta T$. Optical deflection sensitivity is defined as:

$$
S_{o d}=\frac{\Delta T}{\Delta z}
$$

The deflection sensitivity, in case of waveguide grating device, depends on many factors, such as the spectral sharpness of the resonance, waveguide geometry and the cantilever parameters. We will next briefly discuss how the deflection $\Delta z$ relates to other measurable quantities like gas concentration. The factors related to $S_{o d}$ are discussed in section 2.4 via a modeling study of a sensor device.

As discussed in chapter 1, the integrated optical read-out methods for nanodisplacements have applications in microcantilever based sensors. These types of sensors, see e.g. Fig. 9 , can be used to detect the effect of adsorption or absorption. The adsorption causes 
changes in the surface stress [22], leading to deflection of the cantilever. In such scheme one side of the cantilever is coated with a material to which some specific molecule binds. The adsorption induces a differential surface stress between the top and the bottom side of the cantilever, and the cantilever will deflect according to Stoney's equation [23];

$$
\Delta z=3 \Delta \sigma L^{2}(1-v) /\left(Y h^{2}\right),
$$

in which $\Delta z$ is the tip displacement of a cantilever having length $L$, thickness $h$, Poisson's ratio $v$ and Young's modulus $Y$, and $\Delta \sigma$ is the differential surface stress.

Apart from adsorption also absorption can cause cantilever to bend. The absorption causes volume expansion, which in turn causes a bimaterial cantilever to deflect according to [24]:

$$
\Delta z=3\left(\alpha_{1}-\alpha_{2}\right)\left(\frac{h_{1}+h_{2}}{h_{2}^{2} K}\right) C L^{2},
$$

where

$$
K=4+6\left(\frac{h_{1}}{h_{2}}\right)+4\left(\frac{h_{1}}{h_{2}}\right)^{2}+\left(\frac{Y_{1}}{Y_{2}}\right)\left(\frac{h_{1}}{h_{2}}\right)^{3}+\left(\frac{Y_{2}}{Y_{1}}\right)\left(\frac{h_{2}}{h_{1}}\right),
$$

$C$ is the ambient parameter change that causes the volume expansion and $\alpha_{\mathrm{i}=1,2}$, are the expansion coefficients with the subscripts referring to the layers of the structure.

In case of for example hydrogen gas absorption the $C$ is the gas concentration, which is, according to Sieverts' law, directly proportional to the square root of the external pressure of the gas molecules [24]. Also temperature difference between the cantilever top and bottom side, or an ambient temperature change (in case the thermal expansion coefficients are different) can cause cantilever to deflect, according to equation (2).

From the equations (9) and (10), it can seen that the mechanical sensitivity of the cantilever to adsorption or absorption induced deflection can be increased by increasing the length of the cantilever, reducing its thickness or by selecting the cantilever materials such that the Young's modulus is small.

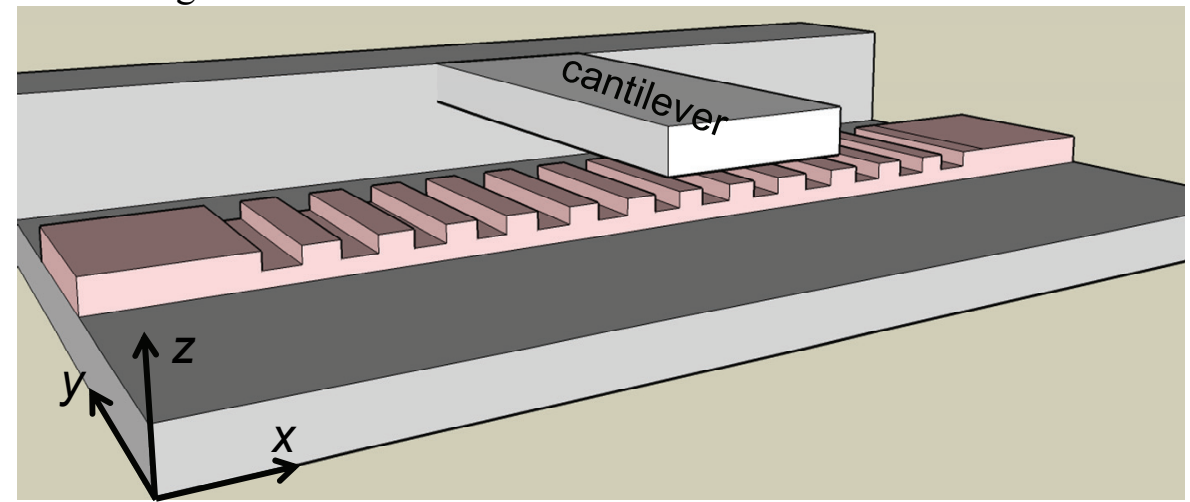

Fig. 9. The grated waveguide-cantilever sensor device. The (up and down) movement of the cantilever changes the transmission of light through the grating.

In the next sections we will discuss in detail the sensor devices that we have implemented based on waveguide gratings. In section 2.4 we present $1 \mathrm{D}$ and 2D modelling results of 
our proposed waveguide grating-cantilever sensor. Also the fabrication issues are shortly discussed based on preliminary test fabrication. Sections 2.2 and 2.3 focus on refractometric sensing by evaluating the sensing performance of the silicon waveguide gratings fabricated in SOI technology.

\subsection{Design and characterization of the grated silicon photonic wire sensor}

In this section the design issues related to a deep UV lithography fabricated grated silicon waveguide are discussed. The sensor's properties are characterized with waterisopropanol and sucrose solutions. The detection limit and the sensitivity of the grated waveguide based sensor to bulk index changes are measured, and the factors that affect on these sensor parameters are discussed. We also explore the properties of the sensor to monitor on-chip chemical reaction by monitoring the evaporation of isopropanol from a watery solution. This evaporation is a dynamic process owning to the fact that the refractive index of the liquid is in a state of continuous change and it therefore mimics the label-free biosensing which will be discussed in section 2.3 .

Various types of silicon-based integrated optical (IO) sensors for label-free sensing have recently been reported, including designs based on the Mach-Zehnder interferometer [25], the ring-resonator [26], waveguide gratings [27-28], porous silicon waveguides [29] and photonic crystals [30-31]. These sensors are based on a change in transmission due to change of refractive index in the proximity of the sensor. To meet the requirements of a small sample volume and to enable dense integration of sensor elements, these sensor designs can be made more compact than in (for example) silicon nitride based devices, because of the high refractive index of silicon. The key advantage of the silicon-based IO sensors can be claimed to be the huge potential to achieve high levels of integration in a cost-effective way by utilizing the CMOS infrastructure. Recent advances in silicon photonics, such as the waveguide photodetectors [32] and the hybrid light sources [33] are examples of building blocks that will allow further miniaturization of these sensors. Apart from pure bulk index sensing, Si photonic wires can be functionalized to versatile biosensor by immobilizing biointerface on the silicon surface [26]. In such a sensor, the sensitivity on the upper cladding index change and compact size are key factors.

It is well-known that a partly grated waveguide defines a cavity in the grated region for wavelengths outside the photonic stopband. For wavelengths near the stopband edge, particularly sharp spectral features can be observed, which are related to the strong modal dispersion and to the increased modal reflectivity in that wavelength region [34], (see section 2.1). These sharp spectral phenomena exhibit a strong sensitivity to changes of the ambient refractive index, which can be exploited in sensor applications.

This section has been published in: Kauppinen, L.J. and Hoekstra, H.J.W.M. and de Ridder, R.M. (2009) A compact refractometric sensor based on grated silicon photonic wires. Sensors and actuators B (Chemical), 139 (1). pp. 194-198. 


\subsubsection{Design and fabrication}

The grated silicon photonic wires (GSPW) have been fabricated using the standard 248$\mathrm{nm}$ deep-UV lithography process for photonic crystals and photonic wires established at IMEC, Leuven [35]. In this process the photonic devices are patterned in a $220 \mathrm{~nm}$ thick top layer of a silicon on insulator (SOI) wafer, using inductively coupled plasma (ICP) etching. The design of the optical structures is constrained mainly by the used waveguide material (silicon) which is transparent at wavelengths $>1100 \mathrm{~nm}$ with a refractive index of 3.45 in the near infrared, as well as by the process limitations requiring a grating period $>400 \mathrm{~nm}$, silicon feature size $>200 \mathrm{~nm}$, and air feature size (gap between two Si features) $>150 \mathrm{~nm}$. The grid size used in a mask design is $5 \mathrm{~nm}$, which determines the minimum feature resolution.

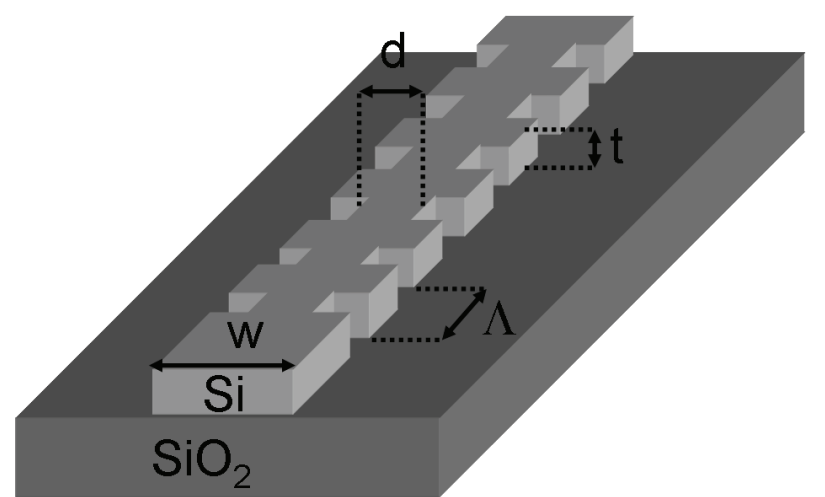

Fig. 10. Lay-out of a grated silicon photonic wire. Waveguide widths $w$ and $d$ are of the order of 400-300 $\mathrm{nm}, \Lambda=450 \mathrm{~nm}$ and the waveguide thickness $t$ is $220 \mathrm{~nm}$.

We have realized the grating structure by periodically varying the waveguide width as shown in Fig. 10. In order to have the first order photonic stopband in the $1.5 \mu \mathrm{m}$ wavelength region of the available equipment, the dimensions for the wide $(w)$ and narrow $(d)$ segments need to be carefully chosen. Although only time-consuming 3dimensional calculations can provide full and accurate information on the transmission properties of the structure [36], we have chosen to use a highly efficient 2-dimensional method. The central wavelength of the stopband is given by the well-known equation:

$$
\lambda_{\mathrm{B}}=2 n_{\mathrm{av}} \Lambda,
$$

where $\lambda_{\mathrm{B}}$ is the Bragg wavelength, $\Lambda$ the period of the grating, and $n_{\mathrm{av}}$ the average modal index of the grating, found by averaging modal indices of the narrow $\left(n_{\mathrm{d}}\right)$ and wide $\left(n_{\mathrm{w}}\right)$ waveguide section over one grating period. If the length ratio of the narrow to the wide waveguide sections is $\xi$ (duty cycle), the average index can be calculated as $n_{\mathrm{av}}=$ $\xi n_{\mathrm{d}}+(1-\xi) n_{\mathrm{w}}$. A 2D modesolver using multi grid finite difference method [37] was used to calculate the allowable range for $w$ and $d$, such that the waveguiding sections are single mode for a TE mode at wavelength of $1520 \mathrm{~nm}$. The same mode solver also produced the corresponding effective refractive indices $n_{w}$ and $n_{d}$. 


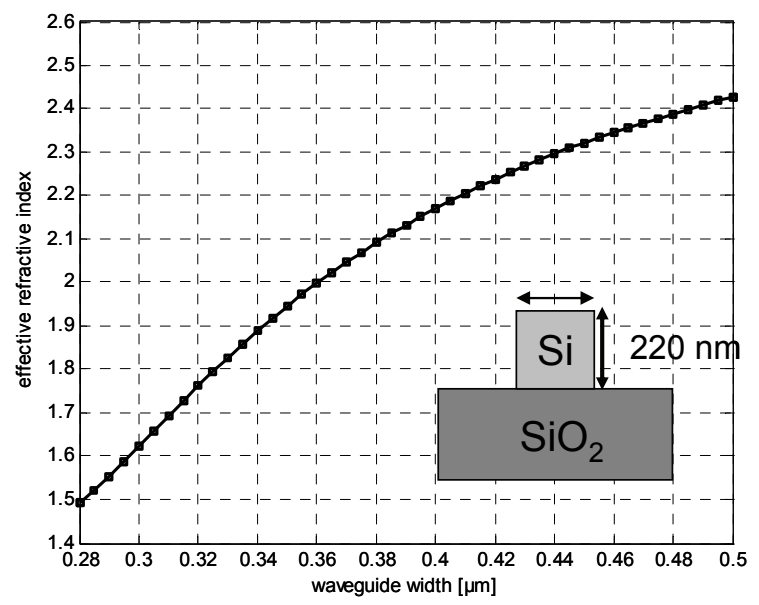

Fig. 11. The effective index versus waveguide width at $1520 \mathrm{~nm}$ wavelength. The height of the waveguide is $220 \mathrm{~nm}$.

The effective refractive index as a function of waveguide width (at wavelength of 1520 $\mathrm{nm})$ is shown in Fig. 11. The graph is used to choose the waveguide widths $d$ and $w$ leading to the desired $\lambda_{B}$. The period $\Lambda$ and the duty cycle are restricted by the process limitations. Because of the high index material (Si) and a minimum period of $400 \mathrm{~nm}$, relatively narrow waveguides are needed.

Since the effective index of the waveguide also depends on the wavelength, see Fig 12, the $\lambda_{B}$ values (in general) cannot be accurately calculated from (12) by just using the indices of Fig.11. However, we can use it to choose $w$ and $d$ for $\lambda_{B}=1520 \mathrm{~nm}$. By keeping in mind the minimum resolution $(5 \mathrm{~nm})$, we choose $w=320 \mathrm{~nm}$ and $d=300$ $\mathrm{nm}$. To obtain design variations, we have chosen the structural dimensions $d$ and $w$ to be between 300 and $400 \mathrm{~nm}$, while the grating period $\Lambda$ is fixed to $450 \mathrm{~nm}$, and the duty cycle $\xi$ to 0.45 . The difference $w-d$ is varied from $20 \mathrm{~nm}$ to $90 \mathrm{~nm}$.

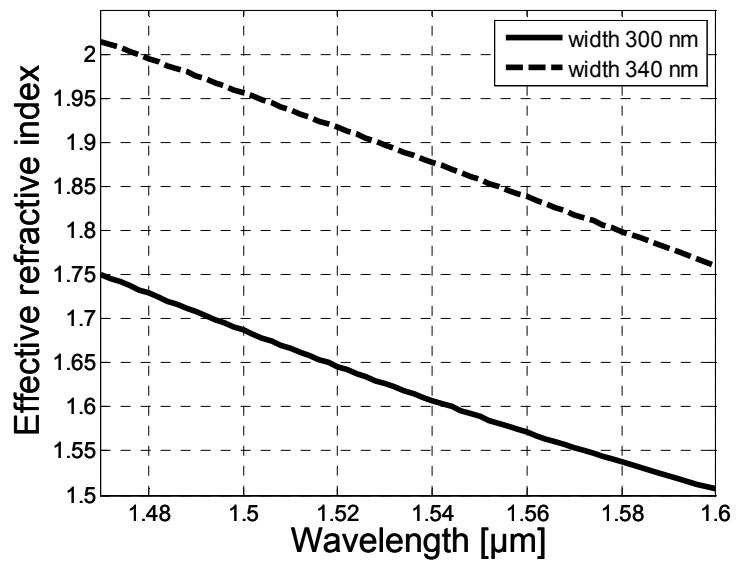

Fig. 12. Effective index as a function of wavelength for two waveguide widths.

In the fabrication process an exposure dose sweep is performed across the wafer. The sweep will give additional variety of the fabricated gratings, as the size of small features on a wafer depends on the exposure dose, shown in Fig. 13. 

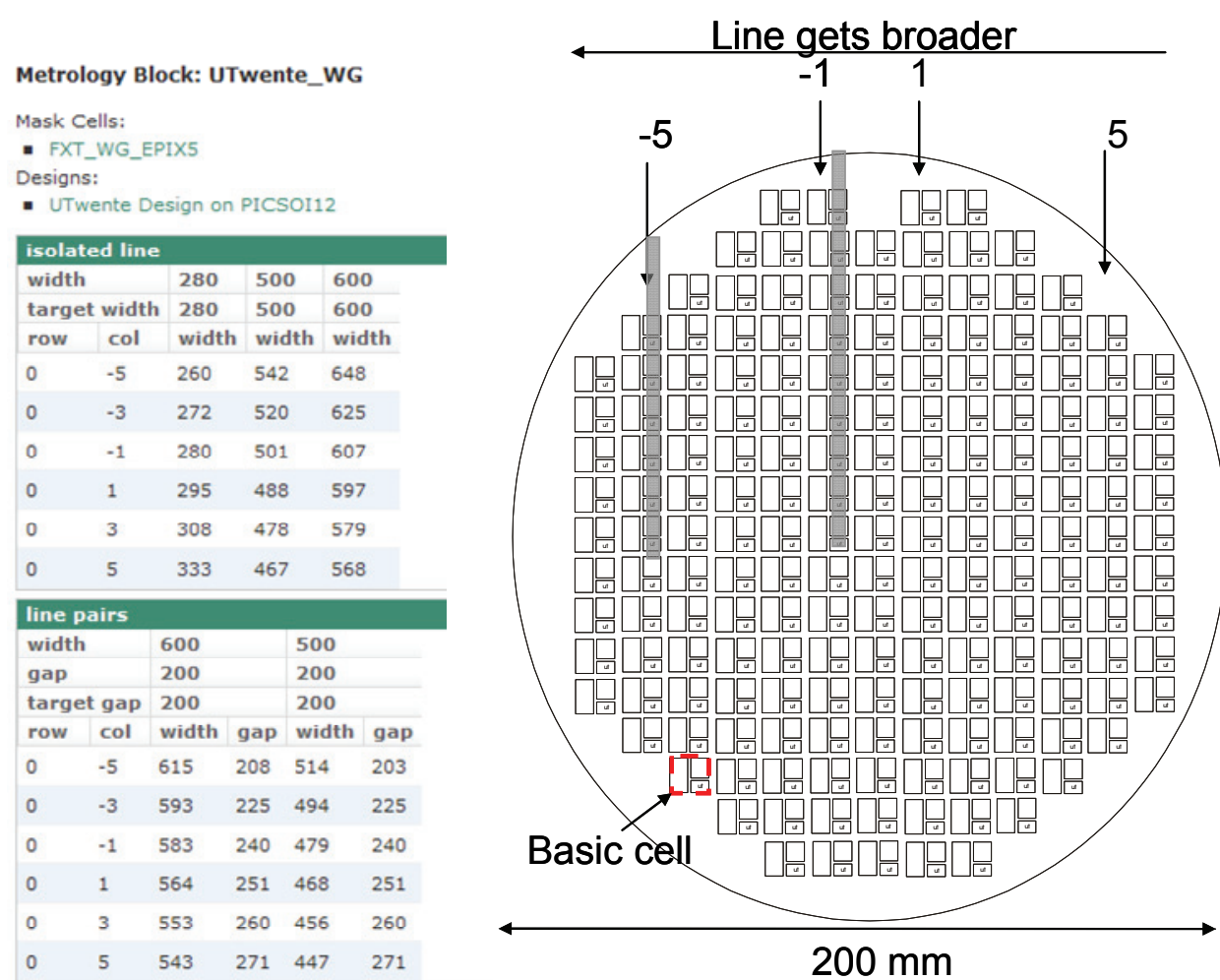

Fig. 13. The effect of exposure dose sweep on feature size. On the left the tables show the feature sizes as measured in IMEC [35]. On the right is a schematic picture of the wafer. A basic cell, of size $12 \mathrm{~mm} \cdot 7$ $\mathrm{mm}$, containing the photonic structures, is repeated across the wafer using stepper lithography. Due to exposure dose sweep, different cell columns get different feature sizes. The columns named as $-5,-1,1$ and 5 are pointed with arrows. The gray shaded areas are the columns that were chosen for first

characterization. The gratings in column -1 have stopbands in the desired wavelength range (1500-1600 $\mathrm{nm}$ ) when the top cladding index is 1.32 (e.g. water) while the gratings in column -5 have the stopbands in same the range for top cladding index of 1 (air).

\subsubsection{Sensitivity and detection limit}

To evaluate the sensitivity and bulk index detection limit of the GSPW, we have implemented a flow cuvette system that allows an easy way to apply different solutions for sensing. It also eliminates the evaporation of liquid hence the index of the solution remains constant during the measurement. We have aimed to get as good signal-to-noiseratio (SNR) as possible, while keeping the signal integration time short, to not limit the measurement scheme for slow reactions only. For this purpose we have used signal modulation. The purpose of the experiment is to determine the achievable detection limit of GSPW sensor in a laboratory conditions and indentify the factors that set the detection limit.

\subsubsection{Materials and Methods}

The detection limit of the GSPW sensor on bulk refractive index changes is evaluated by using water at various sugar concentrations. The refractive index of the water-sugar solution depends linearly on the sugar concentration with a ratio of $1.4 \cdot 10^{-3} \mathrm{RIU} / \mathrm{wt} . \%$ (percent by weight) [38]. For the experiment, a solution with a sugar concentration of 1 $\mathrm{mg} / \mathrm{ml}$ was produced by adding $200 \pm 5 \mathrm{mg}$ of sugar to $200 \pm 2 \mathrm{ml}$ of water. In this way, a 
concentration accuracy of $\pm 0.05 \mathrm{mg} / \mathrm{ml}$ was obtained. Solutions of lower concentrations were produced by diluting from a $1 \mathrm{mg} / \mathrm{ml}$ solution to the desired sugar concentration, resulting in good accuracy, e.g. $0.2 \pm 0.015 \mathrm{mg} / \mathrm{ml}\left(\Delta n=2.8 \pm 0.2 \cdot 10^{-5} \mathrm{RIU}\right)$. All the solutions were produced from same water bottle in order to avoid a case where water obtained on a different day might have a slightly different refractive index.

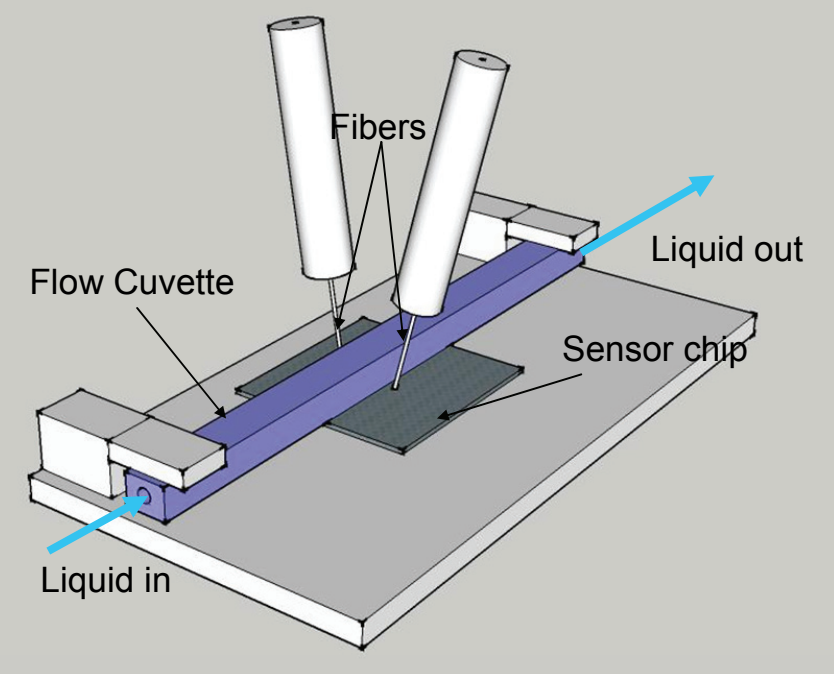

Fig. 14. Schematic picture of the measurement setup. Small opening in a cuvette allows liquid to touch the surface of the sensor chip.

A schematic of our measurement setup is shown in Fig. 14. We used grating couplers at the ends of the waveguides to couple light in and out from the sensor. With this coupling method the losses are 15-25 dB (depending on the wavelength), which is good enough for characterization. However, for a packaged sensor array chip different coupling scheme should be used to ensure that each sensor element receives enough power for high detection limit.

As a light source, we used a tunable infrared laser (Agilent 8164B) and as a photodiode Agilent's 81634B photodiode module. The flow cuvette was clamped on top of the sensor chip. The cuvette has a small opening of $1 \mathrm{~mm} \cdot 3 \mathrm{~mm}$ that allows liquid to contact the surface of the sensor chip. Attention was paid to cuvette design to ensure that the liquid refreshment at the cuvette opening is sufficient. The entire liquid volume of the cuvette system, including the input and output pipes, is approximately $2 \mathrm{ml}$. However, to ensure refreshment of the liquid during the experiments, several millilitres of solution is slowly passed through the cuvette.

If high detection limit is required, the effect arising from the high thermo-optic coefficient of silicon $\left(16-18.5 \cdot 10^{-5} /{ }^{\circ} \mathrm{C}\right)$ should be taken into account. We used active temperature control with a thermo-electric cooling unit that was mounted under the sensor chip. Considering the high thermal conductivity of silicon and the presence of a metallic heat sink under the chip, the temperature of the sensor chip was expected to remain constant. Liquids passing the sensor might cause temperature shifts if the liquid has a temperature that is different to that of the sensor. To reduce this effect, we kept the liquids in a temperature controlled laboratory for 1 day immediately before the experiment. During the experiment, the whole set-up was enclosed in a box which 
reduced the vibration of the coupling fibers due to air flow. This box also reduced possible background light that might reach the output fiber, although in our experience such an effect was shown to be small.

The refractive index sensing properties of the sensor were evaluated by flowing sucrose solutions at various concentrations through the sensor. We first determined an operating point at which the sensitivity of the sensor is high for the index changes. This operating point is near the stop-band edge of the grating. Once the operating point is set, the wavelength of the laser source is kept fixed and the changes in transmission power can be measured according to equation (7).

To improve the SNR and better detect small transmission power changes, the laser signal was modulated with a sine wave at a frequency of $3 \mathrm{kHz}$. The sampling frequency was chosen to be $10 \mathrm{kHz}$ and a total of 32760 samples were recorded, which is the maximum number of samples that our equipment can store. Since the laser is modulated at a specific frequency, we can focus on just this frequency and consider all the other frequency components as noise. The amplitude of the $3 \mathrm{kHz}$ frequency component is proportional to the transmission power going through the sensor.

The slope of the stopband edge is one of the factors that determine the detection limit of the GSPW to refractive index changes, as discussed in section 2.1.4. As the difference $w$ $d$ increases, the index difference $n_{w}-n_{d}$ increases as well. This leads, next to a wider stopband, to a higher modal reflectance at the onsets of the grating, but also to higher losses on reflection at these onsets. The higher reflectance leads to sharper spectral features on the one hand, which are obscured on the other hand by the increased losses [36]. To choose the optimum GSPW for the envisioned sensor experiment an experimental study of the stopband spectral features has been performed.

Fig. 15a shows measured transmission spectra of three different gratings that are all 200 periods long while the designed $w-d$ is varied by increasing the $w$ in $20 \mathrm{~nm}$ steps. The measured spectrum is normalized with respect to a straight photonic wire waveguide including the coupling loss. The uncertainty in coupling loss, due to small changes in coupling conditions and measurement noise, is assumed to be $\pm 2 \mathrm{~dB}$. The effect of the increased grating strength can be seen from the spectra; the stopband edges get steeper and the stopband gets deeper for larger values of $w$. As the average index of the grating is also increased, the stopband shifts to a longer wavelength.

Fig. 15b shows the effect of the grating length; as the length is increased the edges get steeper and the stopband gets deeper. According to our measurements, the stopband edge slope of a grated silicon photonic wire with $w-d=120 \mathrm{~nm}$ and a length of 200 periods equal to the slope of the 800 periods long grating with $w-d=20 \mathrm{~nm}$. The period of the strong grating has to be reduced to $400 \mathrm{~nm}$ to avoid a too narrow waveguide width and to have the stopband at the same wavelength range as the 800 periods long grating. The benefit of strong grating in reducing the size of the sensors, and still maintaining high sensitivity, is obvious.

The grating parameters for sensing should be chosen depending on the application. If small index changes are measured, then the slope of the stopband edge should be as steep as possible. For large index changes less steep stopband edge is beneficial as it provides larger measurement rage (at the cost of sensitivity). 

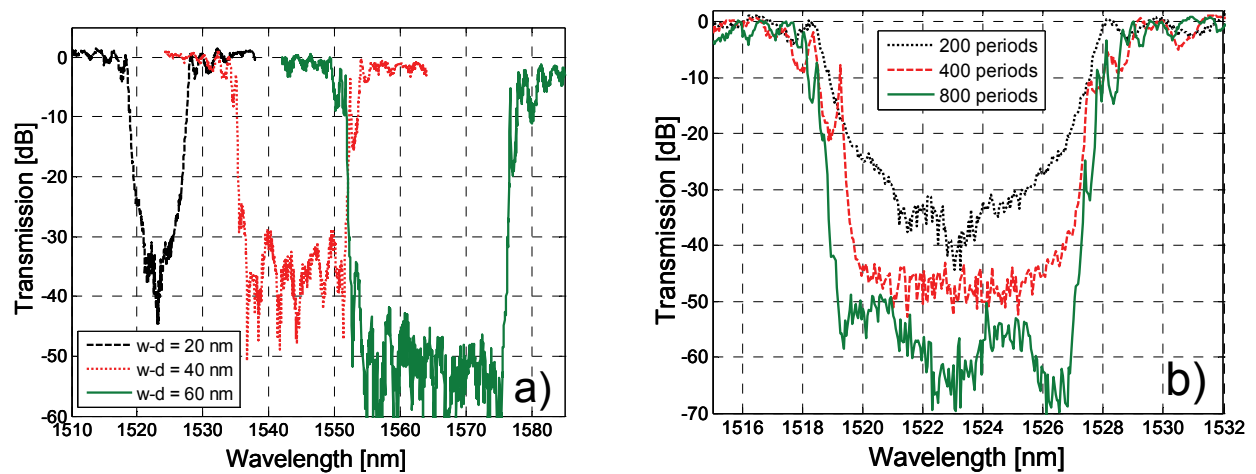

Fig. 15. a) the effect of grating strength on transmission spectrum. The dimension $w$ is changed in steps of $20 \mathrm{~nm}$, while $d$ is fixed. b) The effect of the grating length on transmission spectrum. The grating length varied from 200 periods to 800 periods, for a grating with $\Lambda=450 \mathrm{~nm}$ and $w-d=20 \mathrm{~nm}$.

Due to the fundamental limitations of the photolithographic process, sharp corners will not be faithfully reproduced, resulting in smoothened corners, in particular in narrow structures. This smoothening effect is clearly visible in Fig. 16, showing that the grating shape, which had been designed to consist of rectangular segments, has deteriorated to a sinusoidal-like one. The corner smoothening effect reduces the index contrast between high- and low-index segments. This effect likely reduces the obtainable detection limit as a higher effective index contrast of a segment would provide a steeper band edge slope, although the effect of reduced losses due to lower contrast should be considered [36].

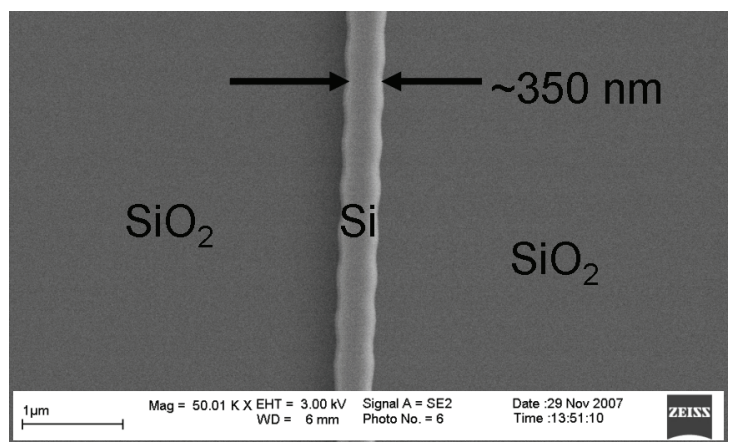

Fig. 16. SEM picture of $350 \mathrm{~nm}$ wide grated photonic wire. Lithography smoothens the rectangular shape and a sinusoidal-like grating is obtained.

\subsubsection{The sensitivity}

To get the estimates of the sensitivity and the index resolution, the stopband position shift and the band edge slope were measured. The stopband shift of a 400 period grating was determined by dropping a water droplet on the sample area $(10 \mathrm{~mm} \times 5 \mathrm{~mm})$. The water droplet $\left(n_{\text {clad }}=1.32\right)$ provides an index shift of 0.32 in the upper cladding. The measured stopband positions before and after depositing the water droplet are shown in Fig. 17. 


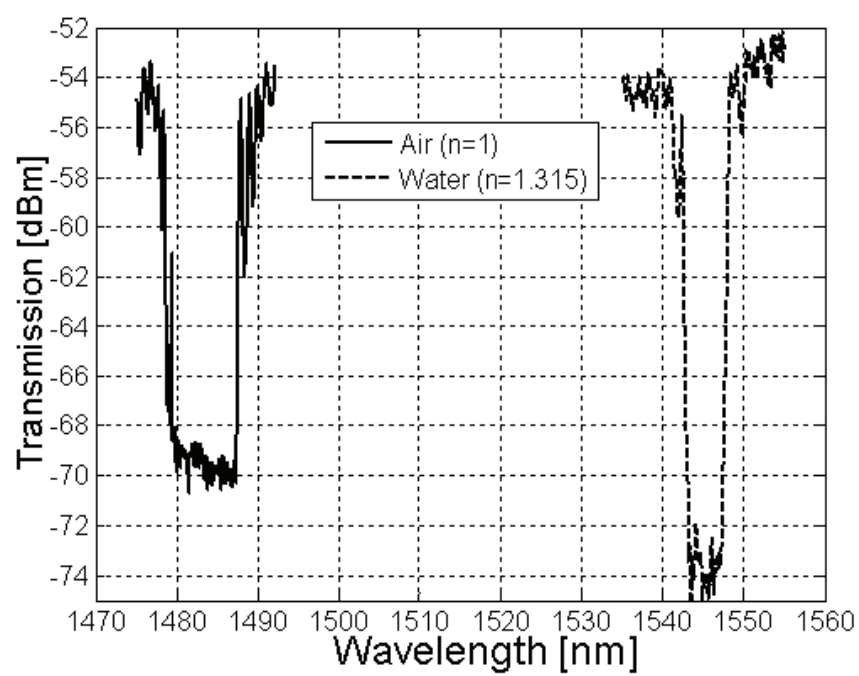

Fig. 17. Stopband positions with air (left) and water (right) claddings.

It can be seen that the shift is roughly $60 \mathrm{~nm}$, which gives a sensitivity of $188 \mathrm{~nm} / \mathrm{RIU}$ according to (6). The slope of the stopband edge at a measured power level (1 nW) is 20 $\mathrm{nW} / \mathrm{nm}$. Assuming that the noise level enables a measurement resolution $\Delta T$ of $10^{-2}$, a resolution of $\Delta \mathrm{n}_{\text {clad }}=10^{-6}$ can be obtained. However, better estimate of the index resolution and limiting factors are obtained by directly measuring it, as will be discussed in the next section.

\subsubsection{The detection limit}

For the determination of the bulk index detection limit, experiments were performed using sucrose solutions, at varying concentrations from $1 \mathrm{mg} / \mathrm{ml}$ to $0 \mathrm{mg} / \mathrm{ml}$ in steps of $0.2 \mathrm{mg} / \mathrm{ml}$. We recorded the transmission power as a function of time as we pumped sucrose solutions through the sensor. From the results, as shown in Fig 18, it is clear that a concentration of $0.2 \mathrm{mg} / \mathrm{ml}$ can be detected. Also, concentration changes in steps of 0.2 $\mathrm{mg} / \mathrm{ml}$ are clearly resolvable. 

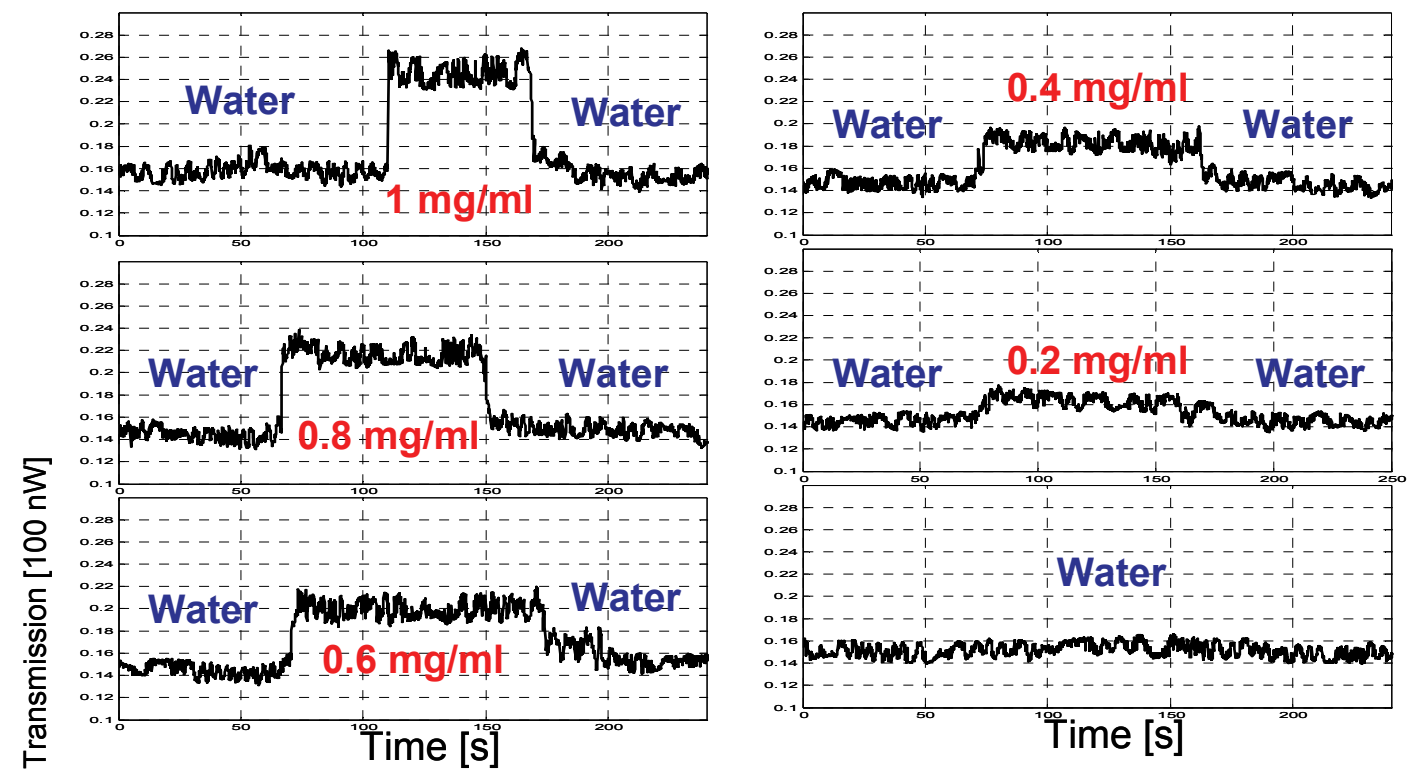

Fig. 18. Sensors response on various sugar concentrations.

The short-term stability of the signal is low. From the results, it can be seen that noise accounts for approximately $10 \%$ of the signal. However, the medium term stability is good, i.e. signal level does not change much over a time periods ranging from one minute to ten minutes. Therefore an integration time of few minutes could be set to improve the $\mathrm{SNR}$, if slow reactions are measured.

As mentioned above to achieve an improved SNR, we repeated the experiment by modulating the laser at a frequency of $3 \mathrm{kHz}$. With this method, the short-term stability of the signal does not play a role as only a single frequency component is considered. However, due to the limited number of samples that can be stored with our equipment, the signal integration time remained short, at $\sim 3.3$ seconds. From the recorded $3 \mathrm{kHz}$ frequency components, shown in Fig.19a, we can clearly observe the concentration changes at a resolution of $0.2 \mathrm{mg} / \mathrm{ml}$. However, the amplitudes of the frequency components corresponding to water appear to vary slightly due to signal variations in time period that is longer than our integration time (i.e. low frequency noise is still present).

The experiment was repeated again about 30 minutes later. This time, the order at which the solutions were applied was changed. When we compare the results, see Fig 19b, there appears to be a systematic difference between the results. We believe that this difference is due to temperature change. Based on the thermo-optic coefficient of silicon, this systematic difference corresponds to a temperature change of $\sim 0.1{ }^{\circ} \mathrm{C}$.

By using the modulation the SNR is better than $20 \mathrm{~dB}$ and the sensors' index resolution is essentially limited only by thermal stability. Sugar concentrations of $0.2 \mathrm{mg} / \mathrm{ml}$ can be detected. This concentration corresponds to refractive index change of $2.8 \cdot 10^{-5}$. To the author's best knowledge, detection limit beyond $10^{-4}$ in bulk refractive index change has not yet been reported with a grated waveguide type sensor.

The results clearly indicate the need for a temperature control element and the necessity of a reference GSPW sensor, if a detection limit better than $10^{-5}$ on refractive index change is required. A reference sensor can be used to compensate the effect of 
temperature fluctuation on refractive index and geometry of the sensor structure, as well as the refractive index of the main solvent for the species to be measured.
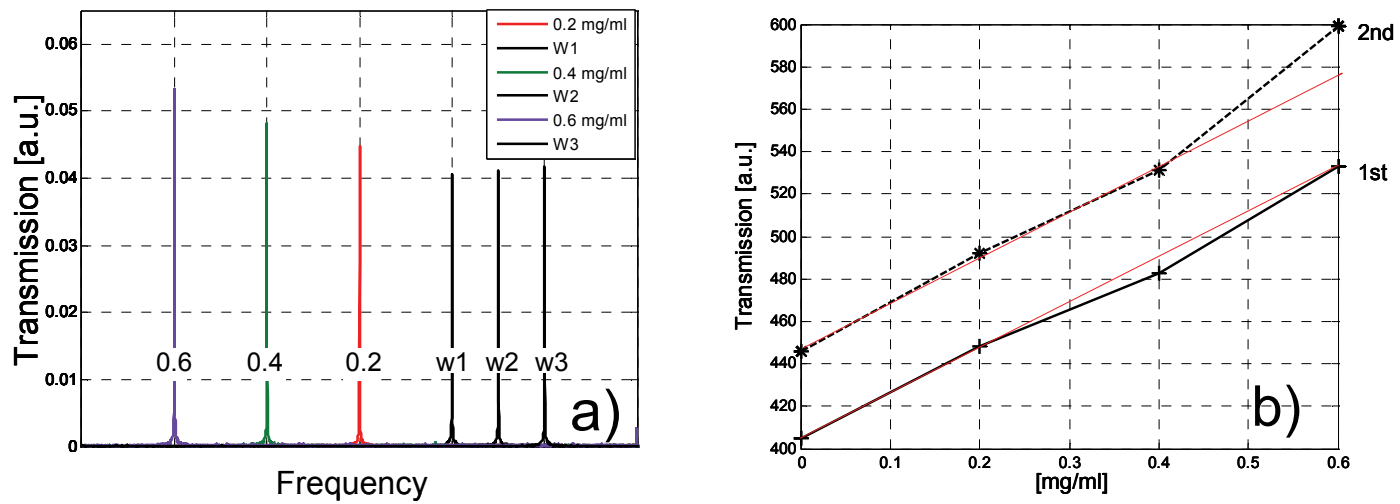

Fig. 19. a) Measured $3 \mathrm{kHz}$ frequency components (see text). Components have been shifted in frequency axis for better visualization. Order of solutions: $0.2 \mathrm{mg} / \mathrm{ml} \rightarrow>$ water $1->0.4 \mathrm{mg} / \mathrm{ml}->$ water2 -> $0.6 \mathrm{mg} / \mathrm{ml}$ $>$ water3. b) Sensors response on different sugar concentrations. The $2^{\text {nd }}$ curve was measured approximately 30 minutes after the first one. Order of solutions for the $2^{\text {nd }}$ curve: $0.4 \mathrm{mg} / \mathrm{ml}->$ water $->0.2$ $\mathrm{mg} / \mathrm{ml}->$ water $->0.6 \mathrm{mg} / \mathrm{ml}->$ water. The linear curves are fitted lines with slope $0.1 \mathrm{mg} / \mathrm{ml}$ per 20 transmission units.

\subsubsection{Measuring the dynamic index change}

As discussed earlier (see e.g. section 1.2.1), the main application area for the GSPW sensor is to follow the progress of on-chip chemical or physical processes, provided that these processes cause a change of the refractive index. As a prelude towards label-free biosensing we performed an experiment in which we followed evaporation of isopropanol from a watery solution.

\subsubsection{The measurement set-up}

A schematic picture of the measurement set-up is shown in Fig. 20. A tunable laser is used as a light source from which the light is coupled with the help of microscope objectives to the waveguide. The objectives and the sample are on 3D translation stages that can be aligned for optimum light coupling. Depending on which objectives are used, different input spot sizes are obtained. The selection of the objectives has been done by experimentally trying different objectives to obtain optimum coupling to the silicon photonic wires. For optimal coupling the spot size (and shape) should match the mode profile of the waveguide.

The typical coupling loss for our end fire setup is about $25 \mathrm{~dB}$. However, when the sample is diced for the end-fire coupling the waveguide end facets may get damaged and the coupling loss can accordingly be much higher. 


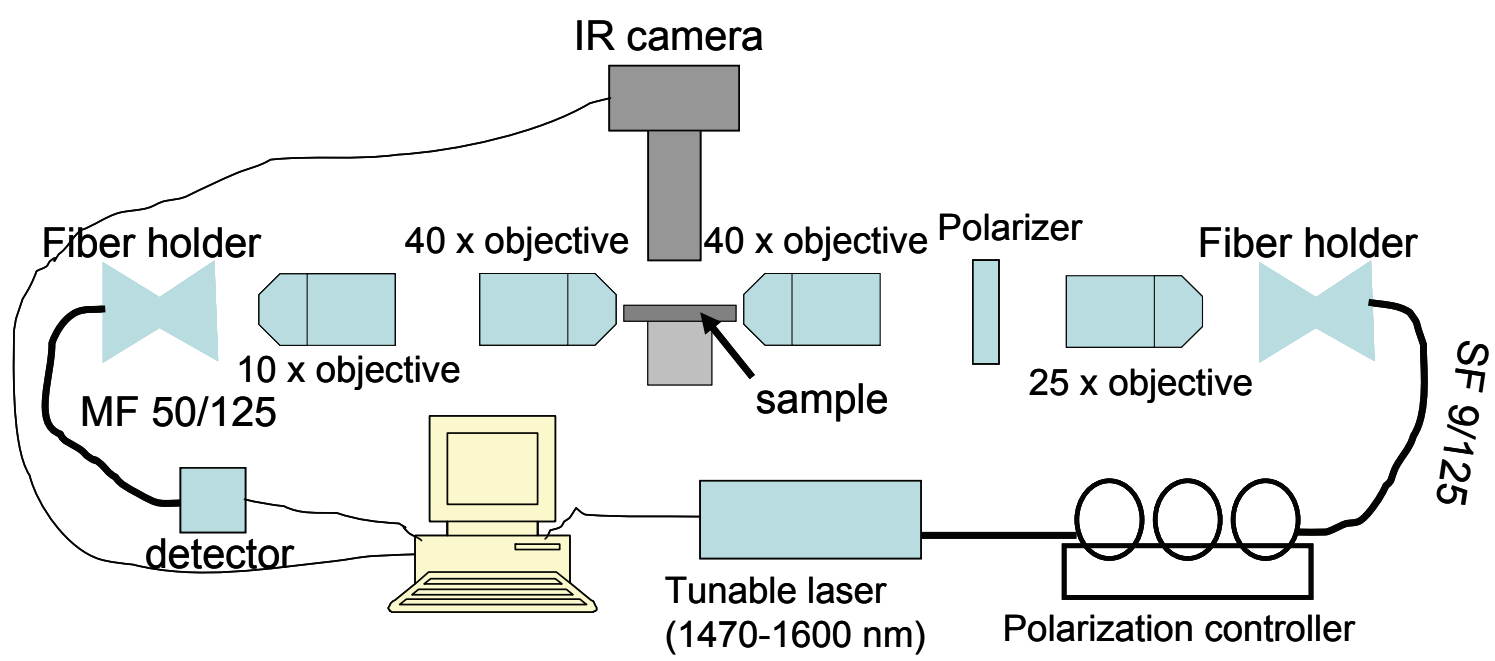

Fig. 20. A schematic picture of the end-fire set-up used to characterize the devices. Light from the laser is guided with a single mode fiber (SF) to the fiber holder from which it propagates in free space and is focused to the end faced of the waveguide by using microscope objectives. At the output side multi mode fiber (MF) is used to collect the light and guide it to the photodetector. The infrared (IR) camera helps in coupling procedure by visualizing the infrared light.

\subsubsection{Experimental}

The suitability to use the GSPW to measure small refractive index changes at a fixed wavelength, and thereby exploiting the spectral sharpness of the stopband edge, was evaluated by using a water-isopropanol solution. Isopropanol evaporates faster than water in ambient temperature and pressure conditions (air at $20^{\circ} \mathrm{C}$ and 1 atm pressure). Therefore the refractive index of a water-isopropanol droplet will decrease with time at some rate. Consequently, when a droplet of water-isopropanol solution is applied to a grating, a gradual stopband shift to lower wavelengths can be observed.

To demonstrate this effect, the droplet of approximately 9 wt. \% isopropanol solution was applied to the grating, and transmitted power at the stopband edge was recorded as a function of time, as shown in Fig. 21. The wavelength was fixed at $1550 \mathrm{~nm}$. The data gap between 400 and 500 seconds was caused by a limitation of the measurement set-up. Fig. 22 shows two transmission spectra, one taken immediately after applying the solution and the other after 14 minutes had passed. The time required for a spectral scan is about 2 minutes. 


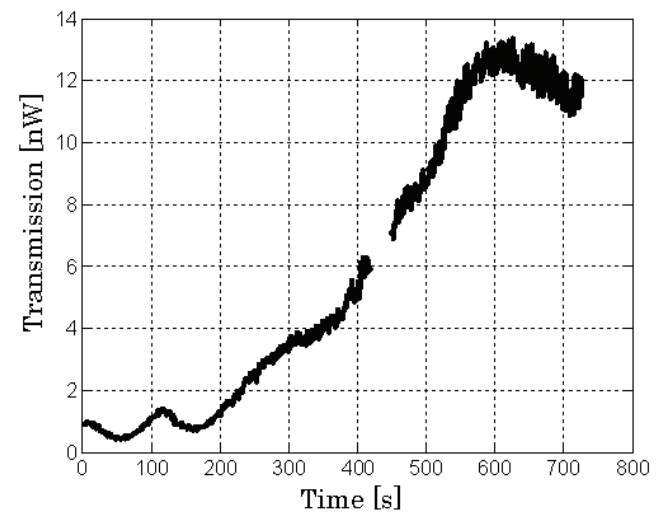

Fig. 21. Transmitted power measured at the stopband edge $(1550 \mathrm{~nm})$ as a function of time during the evaporation of isopropanol from solution.

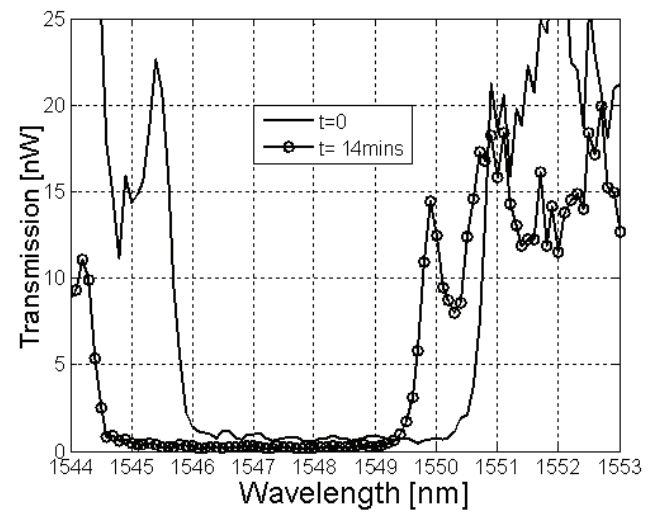

Fig. 22. Transmission spectra, measured at the beginning and at the end of the experiment.

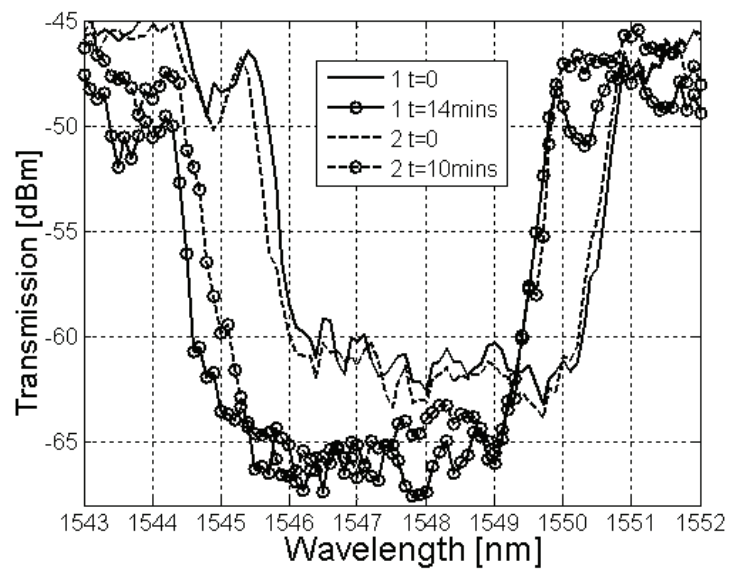

Fig. 23. Repeated measurement of the stopband position shift due to the evaporation of isopropanol. A fresh droplet of solution was used in the second experiment. Solid lines correspond to the first experiment.

The refractive index of the droplet was measured with an Abbe refractometer before and after the experiment. The index of the solution had changed by $5 \cdot 10^{-3}$ over the 16 minutes duration of the entire experiment. The measured index change agrees with the index change that can be calculated from the stopband shift: the stopband shift shown in Fig. 22 is roughly $1 \mathrm{~nm}$, while from Fig. 9 it can be deduced that a shift of $1 \mathrm{~nm}$ corresponds to a refractive index change of $5.3 \cdot 10^{-3}$ in the upper cladding material.

The experiment was repeated by dropping a new droplet of the same solution. The initial and the final stopband positions of the first and the second experiment are nearly equal, as shown in Fig. 23.

The power levels of the transmission spectra measured at the beginning and at the end of the isopropanol evaporation experiment are different; see Figs. 22 and 23. This is due to alignment drift of the measurement set-up. A stabilization period before the experiment can be used to reduce the drift. However, in our end-fire set-up, the coupling conditions change when the droplet is applied to the sample, and due to evaporation of the isopropanol such a stabilization period cannot be applied in this experiment. 


\subsubsection{Conclusions}

It was experimentally shown that using the near stopband edge phenomena of the GSPW, a resolution of the order of $10^{-6}$ to bulk index change is feasible assuming $1 \%$ noise level. It was also demonstrated that the device is suitable to measure the index change of a liquid cladding in real time, which can be used to monitor on chip chemical reactions. Signal modulation -- in combination with a typical photodiode -- is sufficient to achieve a signal-to-noise ratio at which the detection limit of a GSPW sensor is limited only by thermal stability. A detection limit of $2.8 \cdot 10^{-5}$ on bulk index changes has been measured. Lower detection limits call for thermal stability of better than $0.1{ }^{\circ} \mathrm{C}$ or a reference GSPW sensor to compensate for the thermal effects.

Finally we note that low-loss light coupling into silicon waveguides needs careful attention. Especially when considering a packaged chip that houses arrays of sensor waveguides, the optical power reaching the detector should remain well above the noise level of the detector. Robust and efficient fiber-chip coupling solutions exist, for example involving an intermediate waveguide [39].

\subsection{Label-free enzyme sensing with grated silicon photonic wire}

An interesting application field for IO sensors is label-free bio-sensing. In this sensing scheme, the target bio-molecules are attracted to the surface of the IO sensor. Due to exponential nature of the evanescent field, the sensitivity is high near the surface and much smaller concentrations, than with the pure bulk index sensing, can be detected. The surface sensing scheme using immobilized receptors also enables selectivity; the sensor is practically only sensitive to the specific molecules that attach to the surface.

As a demonstration of label-free bio-sensing, we have monitored the binding process of enzymes on an immobilized antibody layer that we applied to a surface of a GSPW sensor (see section 2.2). Also we have used the sensor to monitor the antibody immobilization process.

\subsubsection{The immobilization process}

To obtain an antibody layer for the bio-sensing experiment, an immobilization process is required. We followed the standard immobilization process developed by Imenz b.v. [40]. This process consists of five main steps as shown in Fig 24. The first two steps were performed at chip-scale in a cleanroom. The remaining three steps were done at sensor scale by using the flow cuvette (see Fig. 24). This allowed us to use the sensor to monitor the immobilization process.

This section has been published in: Kauppinen, L.J. and Dijkstra, M. and Hoekstra, H.J.W.M. and de Ridder, R.M. (2009) Label-free Enzyme Sensing with Grated Silicon Photonic Wire. In: Proceedings of the 2009 Annual Symposium of the IEEE Photonics Benelux Chapter, 05-06 Nov 2009, Brussels, Belgium. pp. 41-44. VUBPress Brussels University Press. 


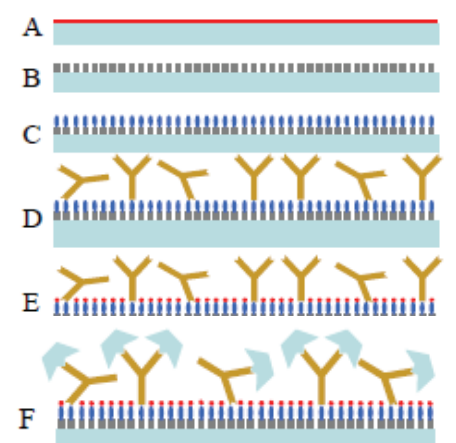

Fig. 24. Immobilization process (A-E) and enzyme binding (F). (A) Surface cleaning and activation, (B) Silanization, (C) Glutaraldehyde, (D) Antibody immobilization and (E) Blocking of reactive carbonyl groups.

The immobilization procedure begins by cleaning the chip with acetic acid and $\mathrm{H}_{2} \mathrm{O}_{2}$ (ratio 4:1). Silanization is performed using a silane solution that will result in a monolayer of silane on the waveguide surface. After silanization, the sensor chip is washed eight times with ethanol and once with phosphate buffered saline (PBS). Silane forms a surface to which glutaraldehyde molecules attach. Glutaraldehyde is used as a surface for anti-bodies selective to an enzyme known as PepN. Blocking solution is required to block non-specific binding on carbonyl groups in glutaraldehyde interface.

Initially, we measured the stop-band position of the GSPW after the silization. In this case, the GSPW sensor is covered by a monolayer of silane, and then air. Next, a PBS solution was flowed into the cuvette. This solution replaces air as top cladding, and the stopband shift of approximately $45 \mathrm{~nm}$ was observed, as shown in Fig. 25. We measured the refractive index of the PBS solution using an Abbe refractometer $(n=1.3348)$. The stopband shift was roughly $10 \mathrm{~nm}$ smaller than we expected from a bulk index sensing experiment (reported in the previous section) for this type of grating. This indicates that the sensor had become less sensitive to bulk index changes after silanization. This, in turn, could mean that silanization had formed a thicker silane layer than just the desired monolayer.

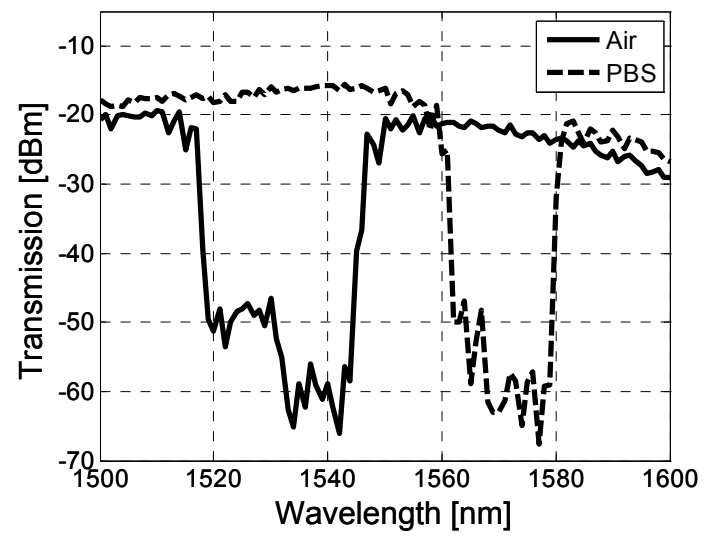

Fig. 25. Stopband positions after silanization in air (solid line) and in PBS solution (dashed line)

After the PBS solution, a glutaraldehyde solution is flowed into the cuvette to form a surface for the anti-bodies to attach to. As the glutaraldehyde solution has a higher 
refractive index $(n=1.3480)$ than the PBS, we expected the shift in the stop-band position to continue. Surprisingly, the shift was in the "wrong" direction: into a lower wavelength region. We have measured the same unexpected stopband shift several times. The reaction time for the glutaraldehyde process step is 1 hour. During this time, we noticed a stopband shift, of few nanometres, back to higher wavelengths, see Fig. 26a. Fig. 26b shows the measured reaction curve of glutaraldehyde binding, as recorded in the monitoring point, shown in Fig 26a, after 240 seconds from injecting the solution into the cuvette. The reaction has saturated after 60 minutes, which corresponds to specified reaction time. PBST $(n=1.3345)$ was flowed in as an intermediate step; however the stopband position remained at lower wavelengths than before the glutaraldehyde step.

Monitoring point
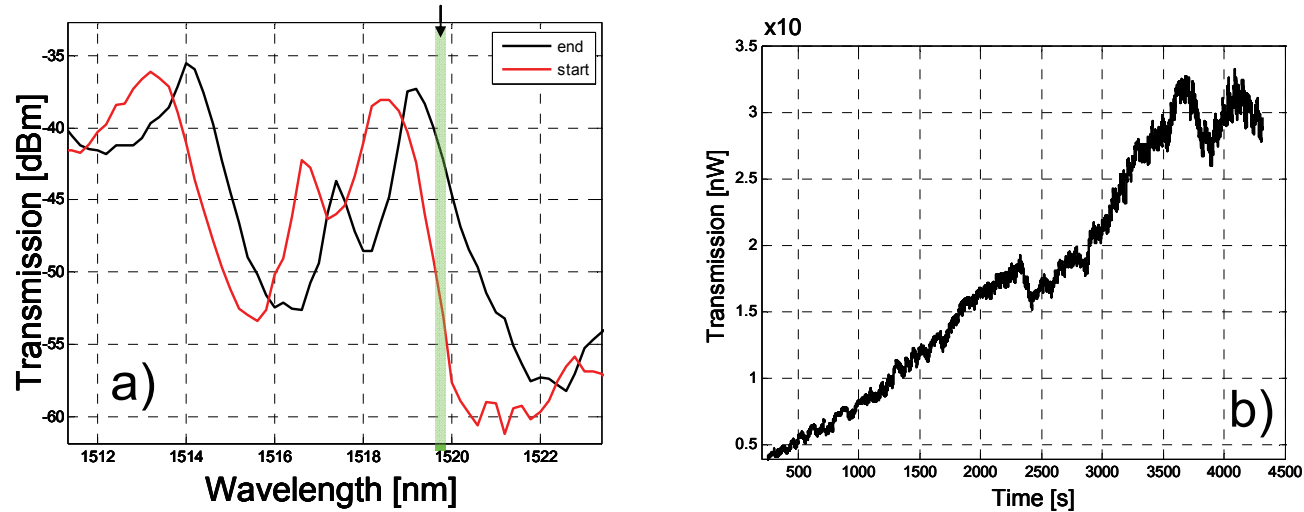

Fig. 26. a) Glutaraldehyde reaction. a) Transmission spectrum at the band edge showing the stopband edge position initially (start) and after $1 \mathrm{~h}$ (end). b) The reaction curve as measured after 4 minutes from injecting the Glutaraldehyde solution into cuvette. The reaction has saturated after 60 minutes.

The PepN antibodies were immobilized on the glutaraldehyde surface by flowing an antibody solution into the cuvette. The reaction time for this step is specified as one hour at $37{ }^{\circ} \mathrm{C}$; however, to avoid complications due to the thermo-optic effect, we performed the step at room temperature $\left(19^{0} \mathrm{C}\right)$. To allow more time for antibodies to bind, we kept the solution in cuvette for 1.5 hours. We measured deformed stopband shapes while the antibody solution was in a cuvette; see Fig 27 . One possible reason for this is that clustering of the antibody molecules near the surface may destroy the uniformity of the grating.

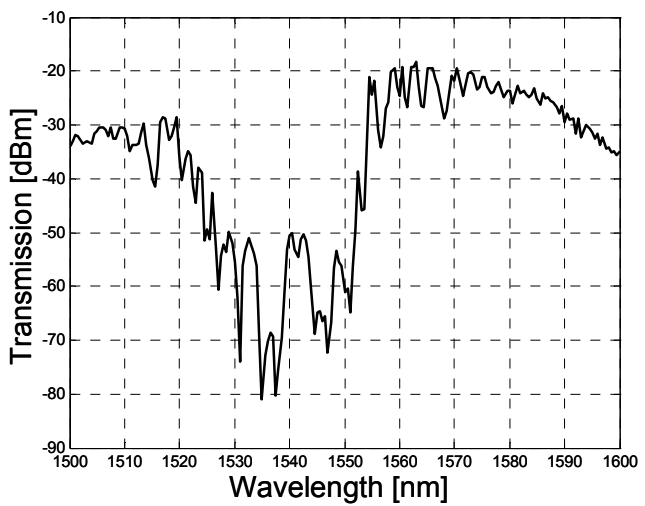

Fig. 27. Deformed stopband stop-band shape due to antibody solution. 
Adding the blocking solution restored the shape of the spectrum, see Fig. 28a. Also, a slight shift to the higher wavelengths was observed, indicating an index increase due to both immobilized antibodies and the attached blocking molecules. The reaction of blocking solution is rapid and strong, as shown in Fig.s 28b. The stopband edge passes the monitoring point in few minutes. After 30 minutes the reaction appears to be saturated as no further stopband shift is measured.
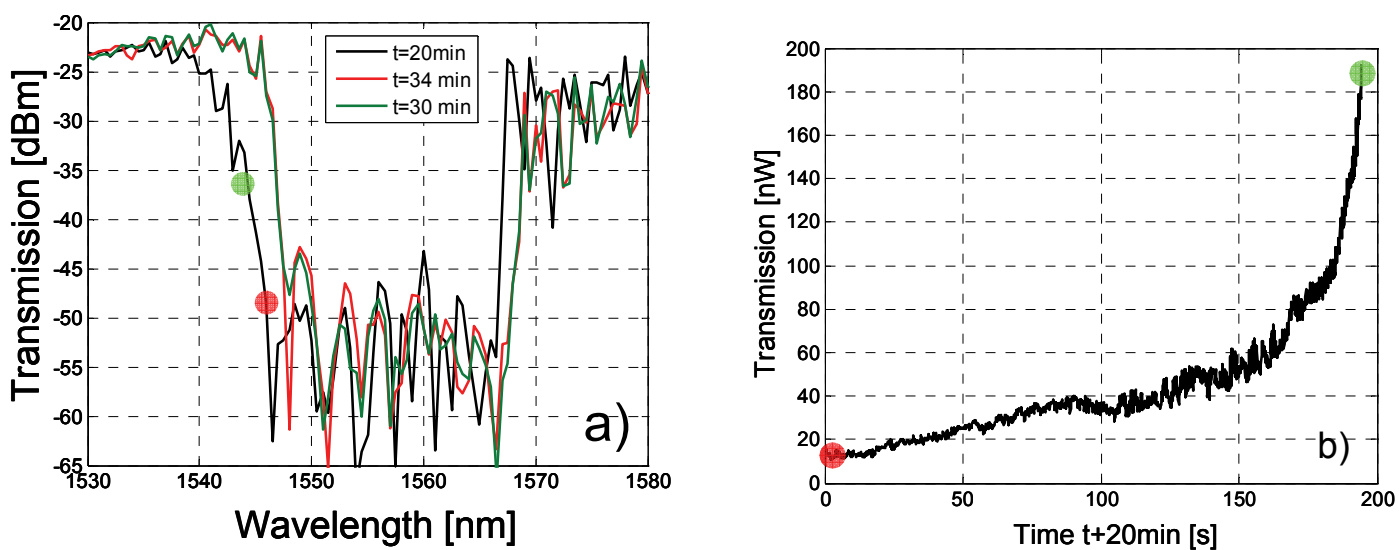

Fig. 28. a) Transmission spectrum with blocking solution. After 30 mins the reactions appears to have saturated. b) The reaction curve of carbonyl group blocking. The reaction is rapid and the stopband edge slides quickly through the monitoring point $(\lambda=1546.1 \mathrm{~nm})$. The corresponding points indentified in the transmission spectrum (Fig. 21a)

\subsubsection{Enzyme binding reaction}

The PepN enzyme binding on the antibody interface was monitored by measuring the transmittance using a couple of operation points near the stopband edge. The reaction time for enzyme binding is one hour. Based on measurement results, the enzyme binding appeared to be active during the first 30 minutes after which the activity fell to a very low level; see Fig 29. The response of the enzymes depends on the number of antibody sites. If there are fewer antibody sites than enzymes, the response saturates more quickly and the total signal change is smaller compared to the situation where there is excess amount of antibody sites.

The total wavelength shift caused by the enzyme binding is estimated to be $230 \mathrm{pm}$, which corresponds to bulk index change of $\sim 10^{-3}$. This index change is high, and given the sensor's detection limit of $10^{-5}, 100$ times smaller enzyme concentration than in our sample, can potentially be detected. Due to the compact size of the GSPW sensor, the sample volume can be very small, down to the picolitre level. 


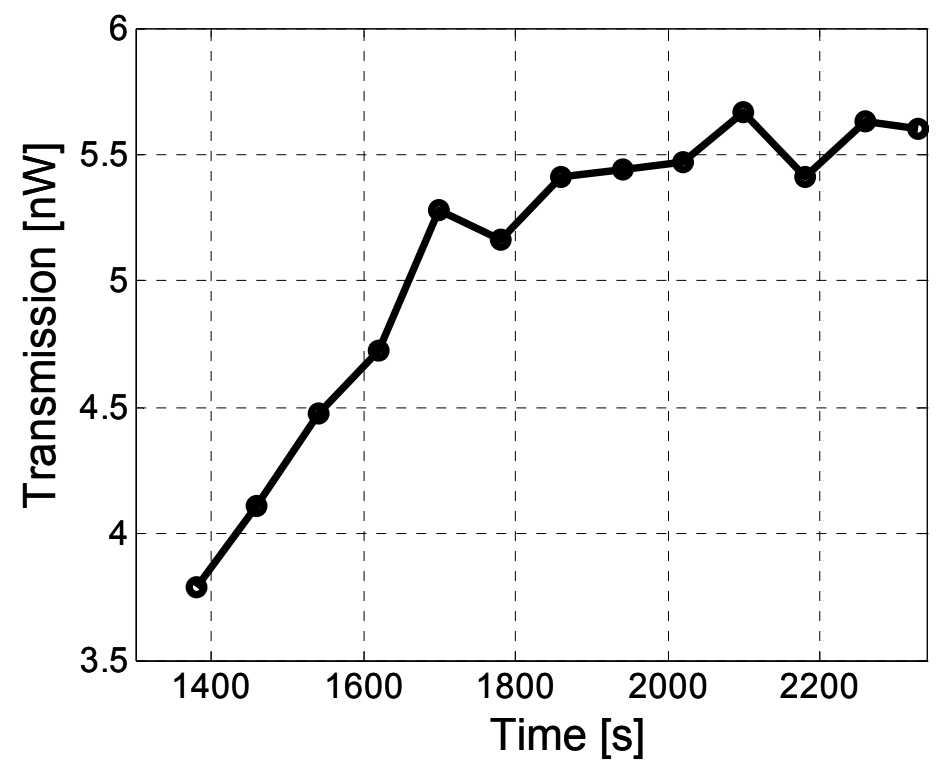

Fig. 29. Reaction curve of enzyme binding process. The saturation in enzyme binding happens after about 30 minutes from injecting the solution.

\subsubsection{Conclusions}

Label-free enzyme sensing, using a GSPW sensor, was demonstrated by immobilizing the enzyme antibody interface on a surface of the sensor. Enzyme binding on this interface was measured. Also the immobilization process was monitored with the sensor. This can deliver calibration data that may be crucial considering variations in different immobilization processes. The compact size of the device enables the use of $<1 \mathrm{pl}$ sample volumes. The CMOS compatible fabrication process, mass production potential and compact size make these devices a very attractive sensor platform. 


\subsection{Grated Waveguide Optical Cavity as a Compact Sensor for Sub-nanometre Cantilever Deflections}

We propose a compact, novel and highly sensitive integrated read-out scheme to detect small deflections of a cantilever in close proximity to a grated waveguide (GWG) structure. In optical resonator (such as $G W G$ ) the electromagnetic field strength is enhanced at the resonance wavelength. This leads to the situation where also light matter interaction is enhanced.

We discuss modelling results for an $\mathrm{SiO}_{2}$ cantilever to be integrated with an optical cavity defined by a grated $\mathrm{Si}_{3} \mathrm{~N}_{4}$ waveguide. The aim is not to provide theoretically optimized design, but to provide discussion of relevant topics and provide information how different parameters affect on sensor performance. Motivation for the work is to get parameter range to fabricate $1^{\text {st }}$ batch of sensors using a safe design.

\subsubsection{Device structure and principle of operation}

We consider a grating defined in a shallow ridge silicon nitride $\left(\mathrm{Si}_{3} \mathrm{~N}_{4}\right)$ waveguide $(\mathrm{WG})$, as shown in the inset of Fig. 30. A waveguide width of $5 \mu \mathrm{m}$, a ridge height of $5 \mathrm{~nm}$ and a core height of $275 \mathrm{~nm}$ were chosen, leading to single mode operation. The grating can be realized on the WG e.g. with laser interference lithography [41].

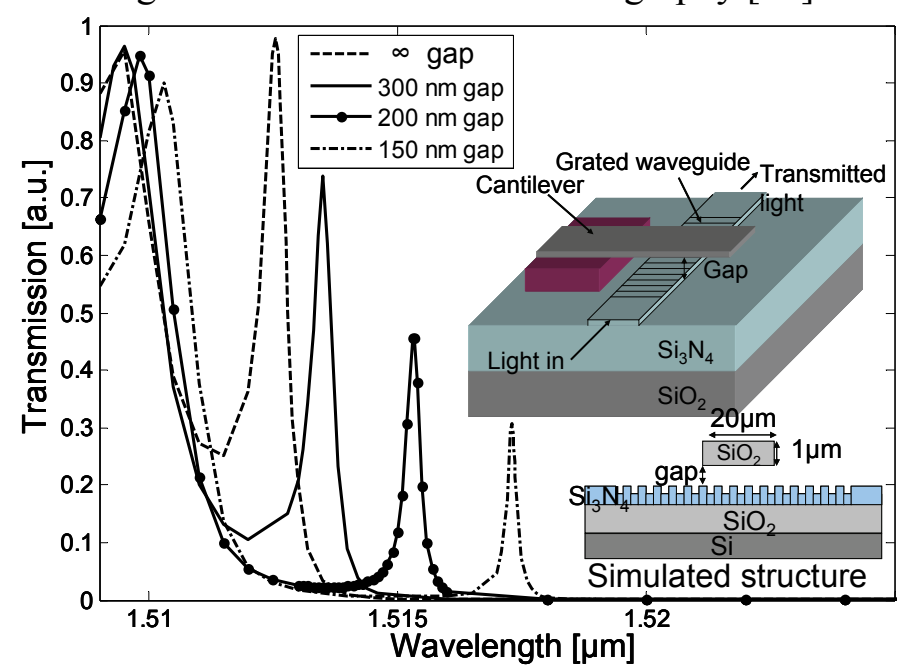

Fig. 30. Simulated transmission spectra of a 200-period grating, with the cantilever position as parameter, using a 2D bidirectional eigenmode propagation method [42]. Insets: device structure and its 2D model.

A very compact and stable sensor element can be realized by monolithically integrating a microcantilever structure with the GWG, using conventional layer deposition and sacrificial layer etching techniques. The device can be functionalized by depositing a sensitive layer on top of the cantilever, e.g. palladium $(\mathrm{Pd})$ for hydrogen $\left(\mathrm{H}_{2}\right)$ sensing. Absorption of $\mathrm{H}_{2}$ into $\mathrm{Pd}$ will cause the cantilever to bend [43]. This bending of the cantilever can then be optically detected by exploiting the properties of the GWG.

This section has been published in: Kauppinen, L.J. and Hoekstra, H.J.W.M. and Dijkstra, M.

and de Ridder, R.M. and Krijnen, G.J.M. (2008) Grated waveguide optical cavity as a

compact sensor for sub-nanometre cantilever deflections. In: Proceedings of the 14th

European Conference on Integrated Optics (ECIO), 11-13 June 2008, Eindhoven, The

Netherlands. pp. 111-114. Eindhoven University of Technology. 
The presence of a dielectric object, in this case a cantilever, in the evanescent-field region of the GWG may lead to the occurrence of propagating modes for wavelengths inside the stopband of the unloaded grating, and so to resonances (defect modes) inside the stopband, as shown in Fig. 30. The first near band edge resonance of a grated waveguide has its field maxima at a center of the grating [44]. If perturbation, say dielectric cantilever, is placed such that it is in the evanescent field region of this resonance mode, a shift in resonance frequency will occur. As the cantilever approaches the grating, the first near band-edge resonance peak is pulled inside the stopband and its spectral width decreases. This effect can be used for the detection of cantilever displacements.

\subsubsection{One-dimensional modelling}

The optical deflection sensitivity (dT/dgap) depends strongly on the maximum slope of the transmission peak of the mode that the cantilever pulls into the stopband. To analyze the effect of cantilever width on the slope of the transmission peak, 1D calculations were performed applying the transfer matrix method to the cantilever-loaded grating structure. The cantilever-induced effective-index change was calculated with a $2 \mathrm{D}$ mode solver, and the obtained values were used in the 1D calculations. The modelled 1D grating is composed of layers arranged as HLHL...H'L'H'L'...HLHL, where H and L represent high- and low-index layers, respectively, and $\mathrm{H}^{\prime}$ and $\mathrm{L}$ ' are the corresponding indices in the cantilever induced defect region. The period of the modelled grating is $490 \mathrm{~nm}$ and the refractive indices of the layers $H$ and $L$ are 1.5928 and 1.53211 , respectively. The proximity of the cantilever increases the indices below it by $\sim 0.5 \%$. A cantilever induced defect region width of 20 to 30 periods in a 100-period grating produces the steepest slope, as shown in Fig. 31. The slope also depends strongly on the grating length. Using the same method as above, the slope was calculated as a function of grating length. Two cases were studied: (a) the cantilever width is fixed at 20 periods, and (b) the relative cantilever width is constant, in this case at $20 \%$ of the total grating length. Doubling the grating length provides more than one order of magnitude slope improvement, as shown in Fig. 32. A large spectral slope means a high quality factor $Q$ of the defect mode. However, the 1D method does not account for scattering loss that is often large for a high Q resonance [44]. Because of noise considerations, the detection limit for cantilever deflection depends on the peak amplitude. 


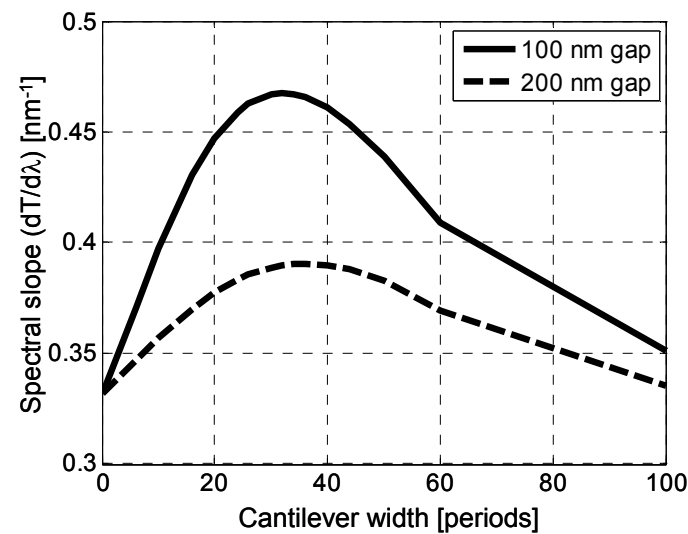

Fig. 31. Spectral slope of defect-mode transmission peak in a 100 -period grating versus of cantilever width.

\subsubsection{Two-dimensional modelling}

A 2D bidirectional eigenmode propagation (BEP) method [42] was applied to the model shown in Fig. 33, to analyze the effect of cantilever thickness on the deflection sensitivity and to account out of plane losses that affect on the detection limit. The defect-mode spectra corresponding to various cantilever thicknesses are shown in Fig. 34. Thinner cantilevers induce defect modes closer to the stopband edge and with higher transmittance than thicker ones. The difference in slope is small and thus the difference in deflection sensitivity comes mainly from the spectral shift. Since the defect modes of the thicker cantilevers are deeper in the stopband, they experience a larger spectral shift when the gap decreases from infinity to $200 \mathrm{~nm}$. This suggests that the optical sensitivity is higher for the thicker cantilevers at this gap range ( $\infty$ to $200 \mathrm{~nm}$ ).

The optical deflection sensitivity of the grating was calculated with two different cantilever thicknesses, $200 \mathrm{~nm}$ and $1 \mu \mathrm{m}$. Fig. 35 shows the transmitted power versus the cantilever deflection for 2 different initial gaps, $200 \mathrm{~nm}$ and $300 \mathrm{~nm}$ where the wavelength is fixed at the corresponding resonance peak of the defect mode. From Fig. 35 it can be seen that the deflection sensitivity at an initial $300 \mathrm{~nm}$ gap is higher for the thick cantilever, although the difference is not large. However, at $200 \mathrm{~nm}$ gap the thin cantilever is preferred due to a higher transmission power and a slightly higher sensitivity.

The theoretical deflection sensitivity can be estimated from the graphs in Fig. 35. The sensitivity slope of the $200 \mathrm{~nm}$ thick cantilever at $200 \mathrm{~nm}$ initial gap is $0.058 / \mathrm{nm}$. By assuming that the noise level allows power detection at an accuracy of $10^{-2}$ (e.g. transmission unit is in $\mu \mathrm{W}$ and noise level is $<10 \mathrm{nW}$ ), the deflection can be detected with a resolution of $0.17 \mathrm{~nm}$. Higher sensitivity is possible, e.g. with a longer grating, provided that the losses remain low. 


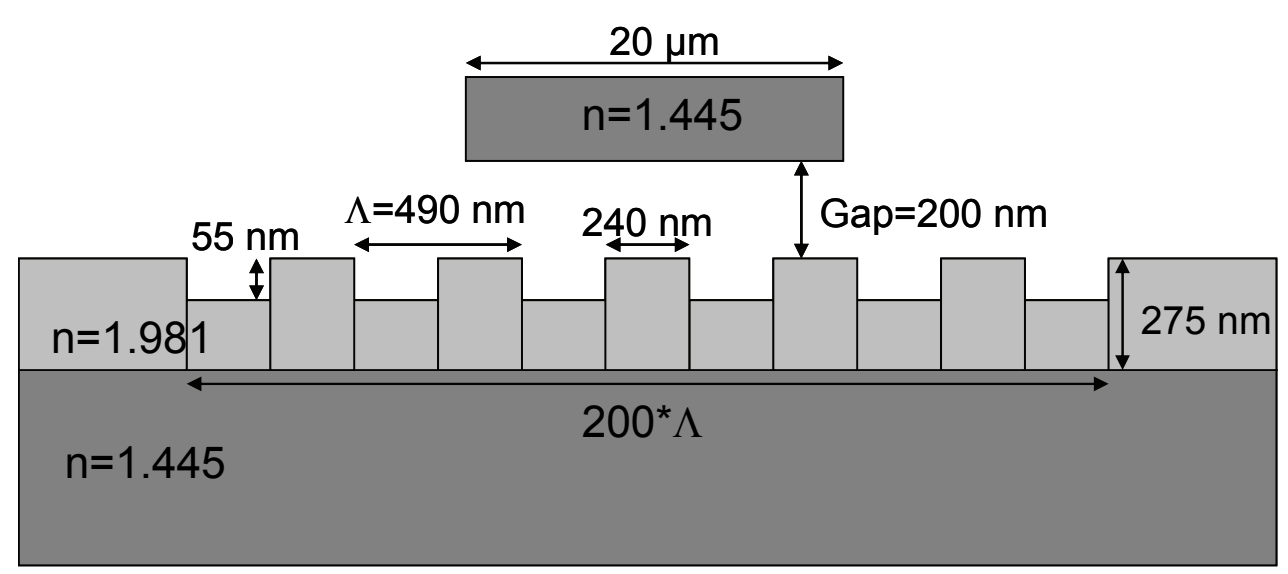

Fig. 33. 2D cross-sectional model of the device used in simulations.

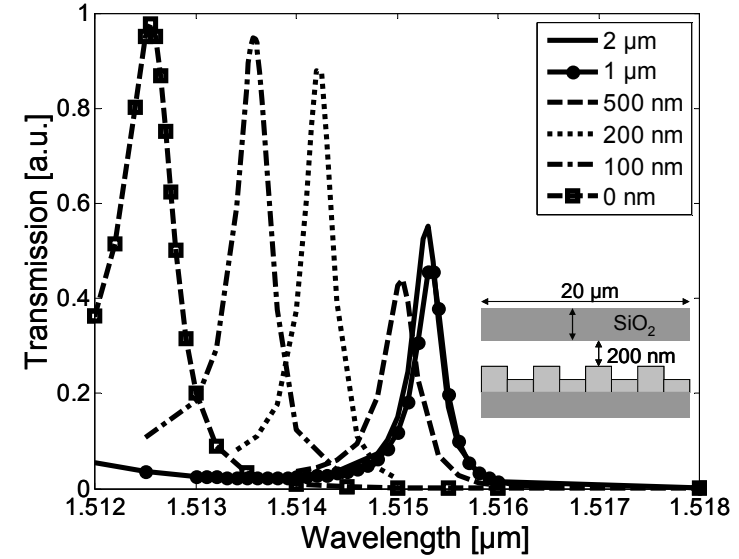

Fig. 34. Defect mode positions obtained with different cantilever thicknesses. Thinner cantilevers induce defect modes that are closer to the stopband edge.

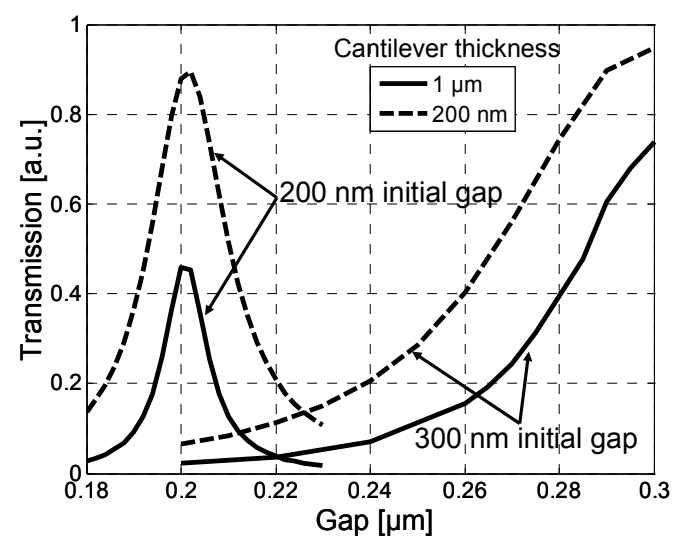

Fig. 35. Simulated transmitted power versus cantilever deflection, calculated for two cantilever thicknesses and two fixed wavelengths corresponding to resonances at 200 and $300 \mathrm{~nm}$ initial gap values.

The choice of cantilever thickness should be carefully considered to obtain maximum sensitivity and stable operation. Thinner cantilevers are more sensitive to mechanical bending that arises from differential surface stress, as follows from Stoney's model [23], see equation (1). However, the thermal and mechanical stability of thin cantilevers is low due to this high sensitivity. Also, if a thin cantilever is coated with a metal layer, the evanescent field of the WG may reach the metal layer through the thin silicon dioxide layer, increasing optical loss. Partial metal coating can be used to avoid such a loss, but at the expense of smaller sensitive area and therefore smaller surface stress.

From the fabrication point of view, it is convenient to have the initial gap between the WG and the cantilever as large as possible. For the considered WG design, the maximum gap for getting an optical response on downward deflection is around $400 \mathrm{~nm}$. For this initial gap there is not a large difference in optical sensitivity between thin $(200 \mathrm{~nm})$ and thick $(1 \mu \mathrm{m})$ cantilevers. 
For maximum sensitivity, a thin cantilever with a small initial gap should be selected, whereas a safer design calls for a thick cantilever with a large initial gap.

\subsubsection{Fabrication of microcantilever}

Below we will briefly describe in general a typical fabrication procedure of surface micromechanical cantilever. The main issues to fabricate the GWG cantilever sensor are discussed as well as the properties of dry etch method to release the cantilevers.

On substrate (which in our case has the waveguiding structure) so-called sacrificial layer is deposited and on top of this is deposited a layer to which the cantilever is patterned, e.g. using photolithography. After the cantilever structure is patterned, it is released by etching the sacrificial layer. The cantilever can be anchored to the substrate simply by defining it's geometry such that the release etch will not release the whole structure, as shown in Fig. 36. It is also possible to etch an anchor hole into the sacrificial layer prior to depositing the cantilever layer. The later anchoring option requires additional mask and photolithography steps, but results in well defined cantilever length.

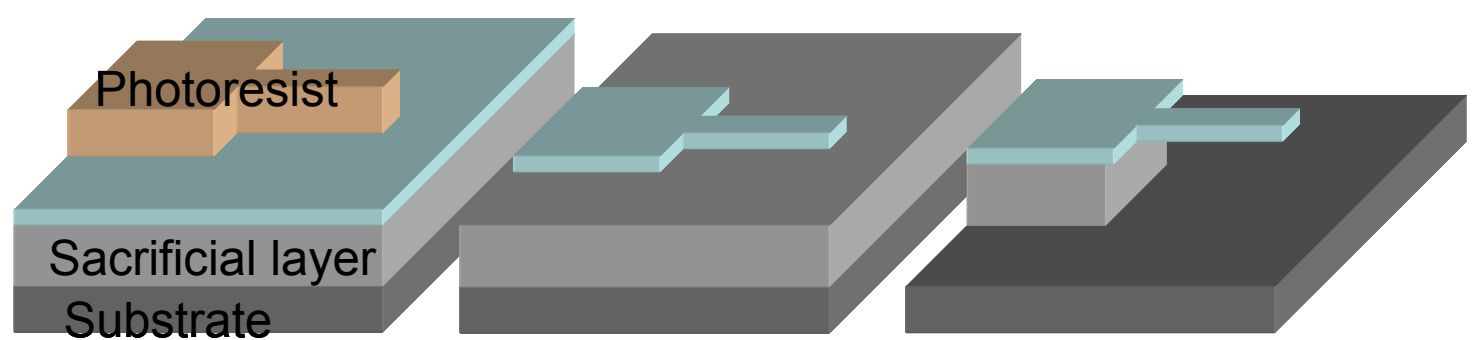

Fig. 36. Cantilever fabrication steps; definition of cantilever structure and release by etching the sacrificial layer.

The sacrificial material needs to be selected such that high selectivity in etching can be achieved between the surround layers. Commonly the sacrificial layer is either $\mathrm{Si}$ or $\mathrm{SiO}_{2}$. Several etchants have high selectivity between $\mathrm{Si}$ and $\mathrm{SiO}_{2}$. Silicon can be etched with high selectivity e.g. using wet etchants $\mathrm{KOH}, \mathrm{TMAH}, \mathrm{EDP}$. The $\mathrm{SiO}_{2}$ can be etched e.g. with BHF or by dry etching with gaseous HF.

If wet etching is used to release the cantilever, one needs to take stiction into account. Stiction occurs after wet etching due to capillary forces and it limits the cantilever length, $L$, according to equation 4 [45];

$$
L<L_{\text {crit }}=\sqrt[4]{\frac{3}{8} \frac{Y h^{3} g^{2}}{\gamma_{s}}},
$$

in which $Y$ is the Young's modulus, $h$ the thickness of the cantilever, $g$ the gap between the cantilever and the substrate and $\gamma_{s}$ is the adhesion energy per unit area. If the cantilever length exceeds the critical length $L_{c r i t}$ stiction will occur.

For integrated optics applications, in which the evanescent field perturbation is exploited, the cantilever should be within reach of this field. Typically this means that a distance of smaller than $400 \mathrm{~nm}$ is required. To avoid stiction after wet etching special drying methods can be used, e.g. freeze drying [46] or critical point drying [47]. Since the 
reduction of adhesion energy $\gamma_{s}$ allows longer cantilever length, a liquid that has a lower surface tension than normally used water, can be selected for rinsing. Also stiction (surface) area can be reduced by introducing bumps or by increasing the surface roughness. Dry etching has a clear benefit, since no liquid is involved and the stiction risk is negligible. However, depending on material selection, dry etching may not be possible or wet etching may provide much higher selectivity between different layers.

Initial stress in the layer(s), that form(s) the cantilever, may cause significant initial bending after the cantilever is released. This is unwanted effect, if the initial gap increases such that the evanescent field is not affected by the cantilever. Intrinsic stress inside the thin film originates from growth mechanism that has resulted in density or compositional gradients. In a cantilever that is composed of several layers the stress may additionally originate due to mismatch between the thermal expansion coefficients of the layers [48]. However, it is also possible to use layer stack such a way that different film stresses compensate one another, effectively reducing the stress and initial bending [49].

\subsubsection{Dry etch released cantilevers}

Dry etching is attractive option to release cantilevers since no additional steps are required to minimize the stiction risk during the release etch, as no liquid is involved. This etch method is versatile as it can be used to etch either anisotropic or isotropic etch profile by just changing the etch parameters, see e.g. [49]. In order to study the suitability of this etch technique as an etch step to release the microcantilevers, we performed an etch test by using the isotropic etch properties of an inductively coupled plasma (ICP) etch.

The layer stack required for the sensor that is presented in section 2.2. is: $\mathrm{Si}_{3} \mathrm{~N}_{4}$, poly-Si and $\mathrm{SiO}_{2}$. The poly-Si was selected as sacrificial layer as $\mathrm{Si}$ is the main target for the etchant gas used in the ICP, and therefore provides the best possible etch selectivity between the layers. The $200 \mathrm{~nm}$ thick stochiometric silicon nitride layer was deposited using low pressure chemical vapor deposition (LPCVD) method. This layer is the layer in which the optical devices for the cantilever deflection read-out can be patterned. The sacrificial layer, $400 \mathrm{~nm}$ of polysilicon was deposited as well by using the LPCVD method. The device layer for the microcantilevers is a $1 \mu \mathrm{m}$ thick plasma enhanced chemical vapor deposited (PECVD) silicon dioxide layer. The cantilevers were patterned by means of photolithography on the $\mathrm{SiO}_{2}$ layer and etched in $\mathrm{BHF}$. The $\mathrm{SiO}_{2}$ is etched over 600 times faster than the sacrificial poly-Si layer, meaning that the sacrificial layer is an excellent etch stop layer in this step. Finally the microstructures were released using the isotropic plasma etch using Sulfur hexafluoride $\left(\mathrm{SF}_{6}\right)$ gas as an etchant.

In Fig. 37a microscope image is shown of the etch hole after etching and resist strip off. The total under etch is approximately $20 \mu \mathrm{m}$. From the gradient it can be concluded that the silicon nitride is attacked by the etchant (as expected). 


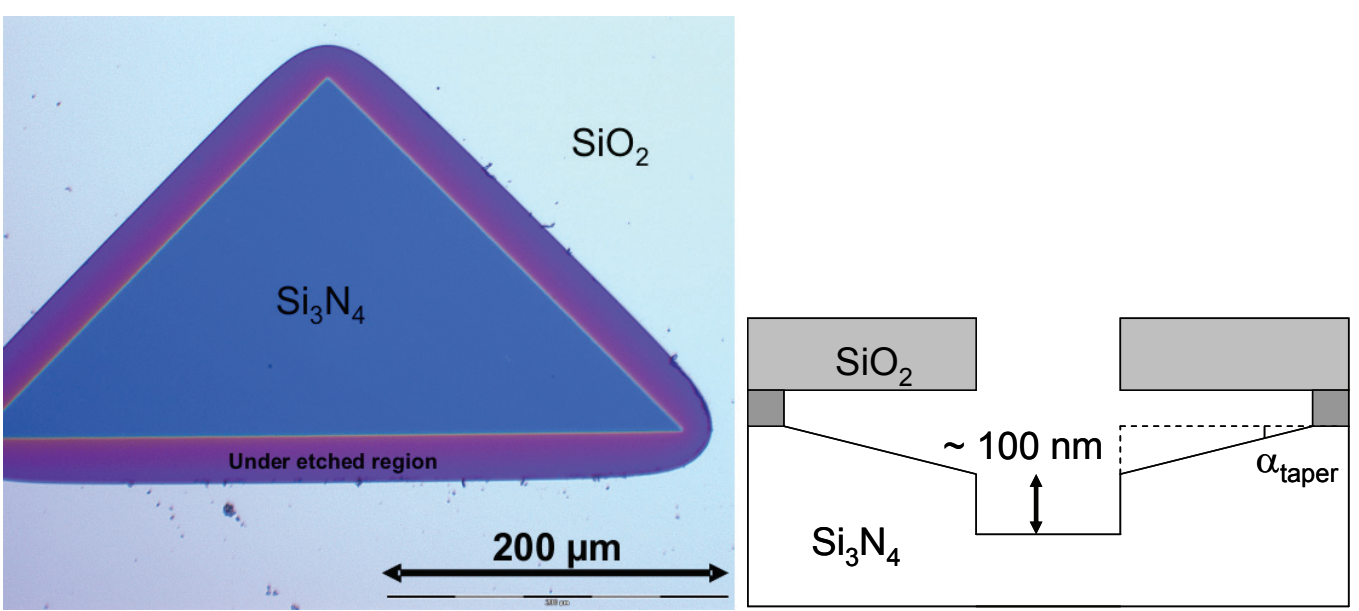

Fig. 37. a) Microscope image of the etch hole. An under etch of approximately $22 \mu \mathrm{m}$ is seen from the image. Color changes indicate tapered etch profile in $\mathrm{SiN}$ layer. b) schematic of the etch profile in a $\mathrm{Si}_{3} \mathrm{~N}_{4}$ layer. Under the etch hole the nitride is etched much faster than under the $\mathrm{SiO}_{2}$ roof, where $\alpha_{\text {taper }}=0.05^{\circ}$.

Directly under the etch hole, the nitride was etch by $\sim 100 \mathrm{~nm}$, whereas under the $\mathrm{SiO}_{2}$ roof the etch profile is tapered, as shown in the schematic picture in Fig 37b. Based on the Dektak surface profile measurement, the horizontal etch speed of the poly-Si is 1200 times faster than the vertical etch rate for the $\mathrm{Si}_{3} \mathrm{~N}_{4}$ under the $\mathrm{SiO}_{2}$ roof. For the integrated device this means that the waveguide should be under a protective (photoresist) roof during this etch step. The sacrificial material could be removed through such an etch windows as shown in Fig 38.

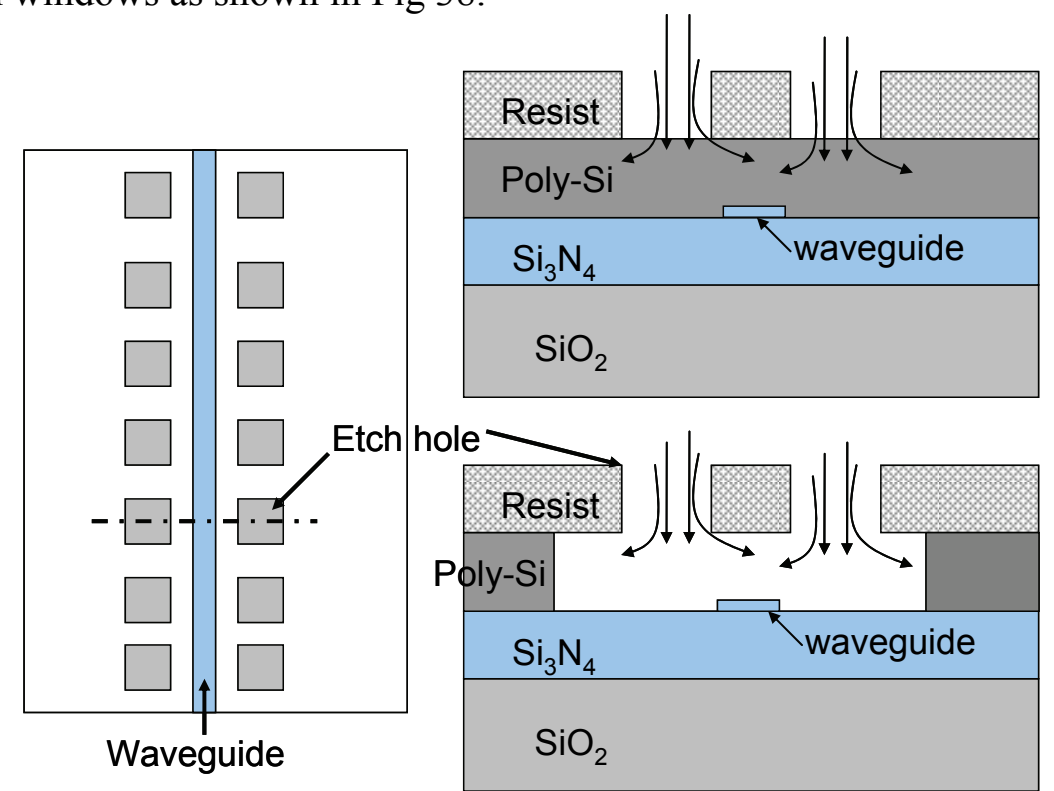

Fig. 38. Etch holes to remove the sacrificial material; on left schematic top view, on right schematic crosssection.

The unavoidable result is that the tapered etch profile (see Fig. 37b) would most likely affect the optical properties of the waveguide grating, although the taper angle $\alpha_{\text {taper }}$ is very small; 0.05 degrees. The SEM image in Fig. 39 gives a hint about the biggest disadvantage of this etch method; high surface roughness of the $\mathrm{Si}_{3} \mathrm{~N}_{4}$ layer after the dry 
etch. Later AFM study revealed that the surface roughness is of the order of the waveguide ridge $(\sim 5 \mathrm{~nm})$ [50]. Given the disadvantages of this release etch method, a wet etchant (TMAH) in combination with a freeze drying method was chosen; with successful results [50]. However, it should be noted that the isotropic dry etching with another etchant gas, Xenon difluoride $\left(\mathrm{XeF}_{2}\right)$, might yield better results than $\mathrm{SF}_{6}$, as etch selectivity between $\mathrm{Si}$ and $\mathrm{Si}_{3} \mathrm{~N}_{4}$ is much higher than with the $\mathrm{SF}_{6}$. Unfortunately this etchant was not available at time being.

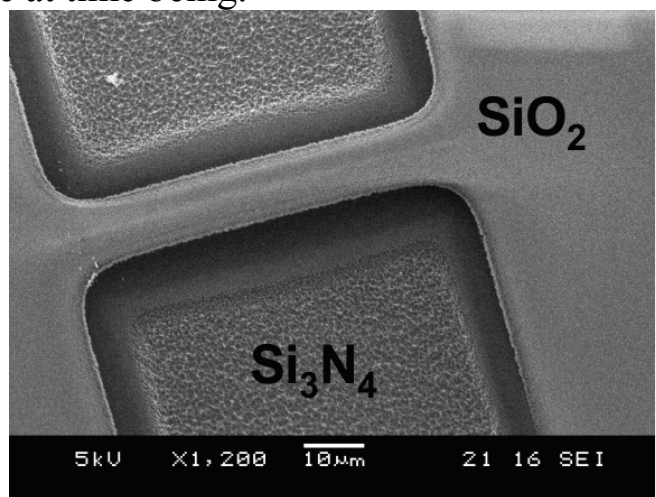

Fig. 39. SEM image of the released microbridge. The isotropic etching with $\mathrm{SF}_{6}$ results in high surface roughness in $\mathrm{Si}_{3} \mathrm{~N}_{4}$ layer. Although the roughness is smaller under the microbridge, it is still in the order of the planned waveguide ridge $(5 \mathrm{~nm})$.

\subsubsection{Conclusions}

Based on our simulations we have shown that GWG can be used to sense deflections of micromechanical cantilevers at sub-nanometre resolution. The presented read-out scheme is a good candidate to enable dense integration of cantilever sensors, providing an accurate and stable optical detection of cantilever bending. Issues related to cantilever fabrication and integration with the optical design have been discussed. The initial bending of the cantilever and the selection of the proper etch process have been identified as key issues for further process integration study. 


\section{References}

[1] M. Gnan, G. Bellanca, H.M.H. Chong, P. Bassi, and R.M. de la Rue, "Modelling of photonic wire Bragg gratings", Optical and Quantum Electronics, vol. 38, pp. 133-148, 2006.

[2] J. Singh, "Semiconductor optoelectronics: physics and technology", McGraw-Hill 1995.

[3] J.D. Joannopoulos, S.G. Johnson, J.N. Winn, and R.D. Meade, "Photonic crystals: molding the flow of light", Princeton University Press, 2008.

[4] S.G. Johnson and J.D. Joannopoulos, "Block-iterative frequency-domain methods for Maxwell's equations in a planewave basis," Optics Express, vol. 8, pp. 173-190, 2001.

[5] P. Yeh, "Optical waves in layered media", Wiley, 1988.

[6] C. Pollock and M. Lipson, "Integrated photonics", Kluwer Academic Publishers 2003.

[7] A. Farjadpour, D. Roundy, A. Rodriguez, M. Ibanescu, P. Bermel, J.D. Joannopoulos, S.G. Johnson, and G. Burr, "Improving accuracy by subpixel smoothing in FDTD," Optics Letters, vol. 31, pp. 29722974, 2006.

[8] H.J.W.M. Hoekstra, W.C.L. Hopman, J. Kautz, R. Dekker, and R.M. de Ridder, "A simple coupled mode model for near band-edge phenomena in grated waveguides", Optical and Quantum Electronics, vol. 38, pp. 799-813, 2006.

[9] Alex Figotin and Ilya Vitebskiy, "Slow light in photonic crystals", Waves in Random and Complex Media, vol. 16, No. 3, pp. 293-382, 2006.

[10] A.A.M. Kok, Jos J. G. M. van der Tol, Roel Baets, and Meint K. Smit, "Reduction of propagation loss in pillar-based photonic crystal waveguides", Journal of Lightwave Technology, vol. 27, pp. $3904-$ 3911, 2009.

[11] D. Taillaert, F. van Laere, M. Ayre, W. Bogaerts, D. van Thourhout, P. Bienstman, and R. Baets, "Grating couplers for coupling between optical fibers and nanophotonic waveguides", Japanese Journal of Applied Physics, vol. 45, pp. 6071-6077, 2006.

[12] M. Palamaru and P. Lalanne, "Photonic crystal waveguides: Out-of-plane losses and adiabatic modal conversion", Applied Physics Letters, vol. 78, pp. 1466-1468, 2001.

[13] C. Sauvan, G. Lecamp, P. Lalanne, and J.P. Hugonin, "Modal-reflectivity enhancement by geometry tuning in photonic crystal microcavities", Optics Express, vol. 13, pp. 245-255, 2005.

[14] G.M. Jie Shen, Z. Zhang, Z. Hua, and S.H. Tang, "Ultrafast all-optical switching in onedimensional photonic crystal with two defects", Optics Express, vol. 14, pp. 858-865, 2006.

[15] S. Zhu, N. Liu, H. Zheng, and H. Chen, "Time delay of light propagation through defect modes of onedimensional photonic band-gap structures", Optics Communications, vol. 174, pp. 139-144, 2000.

[16] H. Alatas, H. Mayditia, H. Hardhienata, A.A. Iskander, and M.O. Tjia, "Single-frequency refractive index sensor based on a finite one-dimensional photonic crystals with two defects", Japanese Journal of Applied Physics, vol. 45, pp. 6754-6758, 2006.

[17] P. Prabhathan, V.M. Murukeshan, Z.Jing, and P.V. Ramana, "Compact SOI nanowire refractive index sensor using phase shifted Bragg grating", Optics Express, vol. 17, pp. 15330-15341, 2009.

[18] F. Ay, L.J. Kauppinen, J.D.B. Bradley, K. Wörhoff, R.M. de Ridder, and M. Pollnau, "Focused ion beam milled on-chip resonator nanostructures for applications in rare Earth-ion-doped $\mathrm{Al}_{2} \mathrm{O}_{3}$ active waveguides", IEEE Photonics Society Annual Meeting, Antalya, Turkey, 2009.

[19] W.C.L. Hopman, H.J.W.M. Hoekstra, R. Dekker, L. Zhuang, and R.M. de Ridder, "Far-field scattering microscopy applied to analysis of slow light, power enhancement, and delay times in uniform Bragg waveguide gratings", Optics Express, vol. 15, pp. 1851-1870, 2007.

[20] W.C.L. Hopman, P. Pottier, D.Yudistira, J. van Lith, P.V. Lambeck, R.M. De LaRue, A. Driessen, H.J.W.M. Hoekstra, and R.M. de Ridder, "Quasi-one-dimensional photonic crystal as a compact building-block for refractometric optical sensors”, IEEE J. Select. Topics Quantum Electron, vol. 11, pp. 11-16, 2005.

[21] W. Gabathuler and W. Lukosz, "Electro-nanomechanically wavelength-tunable integrated-optical Bragg reflectors - Part II: Stable device operation", Optics Communications, vol. 145, pp. 258-264, 1998. 


\section{Sensors based on waveguide grating}

[22] C. Ziegler, "Cantilever-based biosensors", Analytical and Bioanalytical Chemistry, vol. 379, pp. 946959, 2004.

[23] N.V. Lavrik, M.J. Sepaniak, and P.G. Datskos, "Cantilever transducers as a platform for chemical and biological sensors", Review of Scientific Instruments, vol. 75, pp. 2229-2253, 2004.

[24] Z. Hu, T. Thundat, and R.J. Warmack, "Investigation of adsorption and absorption-induced stresses using microcantilever sensors", Journal of Applied Physics, Vol. 90, pp. 427-431, 2001.

[25] A. Densmore, D.-X. Xu, S. Janz, P. Waldron, T. Mischki, G. Lopinski, A. Delâge, J. Lapointe, P. Cheben, B. Lamontagne, and J. H. Schmid, "Spiral-path high-sensitivity silicon photonic wire molecular sensor with temperature-independent response", Optics Letters, vol. 33, pp. 596-598, 2008.

[26] K. De Vos, I. Bartolozzi, E. Schacht, P. Bienstman, and R. Baets, "Silicon-on-Insulator microring resonator for sensitive and label-free biosensing", Opt. Express, vol. 15, pp. 7610-7615, 2007.

[27] L.J. Kauppinen, H.J.W.M. Hoekstra, and R.M. de Ridder, "A compact refractometric sensor based on grated silicon photonic wires", Sensors and Actuators B: Chemical, vol. 139, pp. 194-198, 2009.

[28] A.S. Jugessur, J. Dou, J.S. Aitchison, R.M. De La Rue, and M. Gnan, "A photonic nano-Bragg grating device integrated with microfluidic channels for bio-sensing applications", Microelectronic Engineering, vol. 86, pp. 1488-1490, 2009.

[29] G. Rong, A. Najmaie, J.E. Sipe, and S.M. Weiss, "Nanoscale porous silicon waveguide for labelfree DNA sensing”, Biosensors \& Bioelectronics, vol. 23, pp. 1572-1576, 2008.

[30] S. Mandal and D. Erickson, "Nanoscale optofluidic sensor arrays", Optics Express, vol. 16, pp. 1623 $1631,2008$.

[31] N. Skivesen, A. Têtu, M. Kristensen, J. Kjems, L.H. Frandsen, and P.I. Borel, "Photonic-crystal waveguide biosensor", Optics Express, vol. 15, pp. 3169-3176, 2007.

[32] V. Sorianello, M.Balbi, L.Colace, G.Assanto, L.Socci, L.Bolla, G.Mutinati, and M.Romagnoli, "Guided-wave photodetectors in germanium on SOI optical chips", Physica E, vol. 41, pp. 1090-1093, 2009.

[33] A.W. Fang, R. Jones, H. Park, O. Cohen, O. Raday, M.J. Paniccia and J.E. Bowers, "Integrated AlGaInAs-silicon evanescent racetrack laser and photodetector", Optics Express, vol. 15, pp. 23152322, 2007.

[34] H.J.W.M. Hoekstra, W.C.L. Hopman, J. Kautz, R. Dekker, and R.M. de Ridder, “A simple coupled mode model for near band-edge phenomena in grated waveguides", Optical and quantum electronics, vol. 38, pp. 799-813, 2006.

[35] Silicon Photonics Platform, http://www.epixfab.eu/

[36] M. Gnan, G. Bellanca, H.M.H. Chong, P. Bassi, and R.M. de la Rue, "Modelling of photonic wire Bragg gratings", Opt. Quantum Electron., vol. 38, pp. 133-148, 2006.

[37] OlympIOs Integrated Optics Software. C2V, P.O. Box 318, 7500 AH Enschede, The Netherlands,(http://www.c2v.nl/software/)

[38] M-K. Kwon and W.H. Steier, "Microring-resonator-based sensor measuring both the concentration and temperature of asolution", Optics Express, vol. 16, pp. 9372-9377, 2008.

[39] R. Costa, G. Cusmai, A. Melloni, and M. Martinelli, "TE-TM coupling of a standard fiber to a Si-wire waveguide", in: Proceedings of the 13th European Conference on Integrated Optics (ECIO'07), Copenhagen, Denmark, 2007, ThC3.

[40] IMEnz Bioengineering BV, L.J. Zielstraweg 1, 9713 GX Groningen, The Netherlands.

[41] W.C.L. Hopman, R. Dekker, D. Yudistira, W.F.A. Engbers, H.J.W.M. Hoekstra, and R.M. de Ridder, "Fabrication and characterization of high-quality uniform and apodized $\mathrm{Si}_{3} \mathrm{~N}_{4}$ waveguide gratings using laser interference lithography”, IEEE Photonics Technology Letters, vol. 18, pp. 1855-1857, 2006.

[42] OlympIOs Integrated Optics Software. C2V, P.O. Box 318, 7500 AH Enschede, The Netherlands, (http://www.c2v.nl/software/)

[43] Y.I. Chou, H.C. Chiang, and C.C. Wang, "Study on Pd functionalization of microcantilever for hydrogen detection promotion", Sensors and Actuators B, vol. 129, pp. 72-78, 2008. 


\section{Sensors based on waveguide grating}

[44] W.C.L. Hopman, H.J.W.M. Hoekstra, R. Dekker, L. Zhuang, and R.M. de Ridder, "Far-field scattering microscopy applied to analysis of slow light, power enhancement, and delay times in uniform Bragg waveguide gratings", Optics Express, vol. 15, pp. 1851-1870, 2007.

[45] N. Tas, T. Sonnenberg, H. Jansen, R. Legtenberg, and M. Elwenspoek, "Stiction in surface micromachining", Journal of Micromechanics and Microengineering, 6, pp. 385-397, 1996.

[46] H. Guckel, J.J Sniegowski, T.R. Christenson, S. Mohney, and T.F Kelly, "Fabrication of micromechanical devices from polysilicon films with smooth surfaces", Sensors Actuators, vol. 20 pp. $117-22,1989$.

[47] G.T. Mulhern, D.S. Soane, and R.T. Howe, “carbon dioxide drying of microstructures", Proc. 7th Int. Conf. on Solid-State Sensors and Actuators (Transducers'93) (Yokohama, 1993) pp. 296-9, 1993.

[48] D. Resnik, U. Aljanc ic $^{`}$, D. Vrtačnik, M. Možek and S. Amon, "Mechanical stress in thin film microstructures on silicon substrate", Vacuum, vol. 80, pp. 236-240, 2005.

[49] P. Griss and G. Stemme, "Side-opened out-of-plane microneedles for microfluidic transdermal liquid transfer", Journal of Microelectromechanical systems, vol. 12, pp. 296-301, 2003.

[50] Pham Van So, L.J. Kauppinen, H.J.W.M. Hoekstra, M. Dijkstra, H.A.G.M. van Wolferen, G.J.M. Krijnen, and R.M. de Ridder, "Fabrication of microcantilever-based IO grated waveguide sensors for detection of nano-displacements", In: Proceedings of the 13th Annual Symposium of the IEEE LEOS Benelux Chapter, pp. 155-158, 2008. 


\section{Compact silicon photonic devices for MEMS-actuated optical switching}

In this chapter we present the optical structures that were designed and fabricated for MEMS-actuated switching. Also the results of optical characterization of these devices are presented and discussed.

The design work aimed at realizing compact devices that can be actuated with a cantilever-type MEMS. In the design work we have applied and used the existing body of knowledge on design and theory that is available in textbooks and journal publications. By using computer simulations the designs have been tailored such that they can be realized in ePIXfab fabrication platform.

All studied devices were designed to perform wavelength routing; light of a certain wavelength is routed through the device to a wavelength-specific output port. By perturbing the device with the MEMS actuator, either the wavelength for a given route is tuned, or this routing is cancelled (on/off switch). The perturbation effect of the cantilever on the optical structure is similar to the one discussed in section 2.4; it changes the effective index in a section of the optical device.

We have focused on four different designs. Judging by its theoretical specifications, a photonic-crystal-cavity-based channel drop filter (4-port) is an ideal device; as a PhCbased device it is compact and the cavity provides a mechanism for strongly wavelength selective routing. Another PhC-cavity-based device is a Y-splitter-type device (3-port) in which a certain wavelength is routed to one of the two output waveguides via cavity. A waveguide-grating-based device represents another 3-port device that can route a broad wavelength band from the input to the desired output port. The last design is the wellknown ring resonator, a 4-port device that has been widely studied for wavelength routing. However, very little effort has so far been made to realize a mechanically tuned ring resonator. We have implemented compact racetrack-type ring resonators where the resonance, and hence the drop wavelength, can be tuned with MEMS.

The discussion and conclusion sections at the end of the chapter summarize the performance of the devices.

\subsection{Photonic-crystal-based designs}

In this section we present photonic-crystal-based devices for optical switching. First the general properties of PhCs are discussed, followed by sections that present the switching structures in detail.

\subsubsection{Photonic crystals}

In this section we will discuss the basic properties of photonic crystals (PhCs). We focus on the most common photonic crystal structure: the two-dimensional (2D) slab-type photonic crystal, usually called "photonic crystal slabs" (PCSs), with a triangular lattice of air holes. The energy band diagram -also known as the photonic band structure-, the photonic bandgap, and guiding and trapping of light with crystal defects will be briefly explained. 
The propagation of light in $\mathrm{PhCs}$ has some mathematical similarities to the electron transport in atomic crystals. From intuition one would think that since in the atomic crystal there are so many atoms, at least a few of them would be on the electron's way and the electron would lose some of its kinetic energy in a series of collisions with the crystal atoms. However, the electron can go through such a perfect crystal without scattering at all. The explanation of this phenomenon is that for certain energy ranges, called bands, the electrons can move from unit cell to unit cell as described by a so-called Bloch wave, according to Bloch's theorem [1]. Note that in real atomic crystals the vibrations disturb the crystal structure and electrons do scatter.

As photons do not carry electric charge, like electrons do, they cannot be guided or "trapped" in the crystal by electric potential distributions. Photons can be guided and trapped by crystal defects, as will be discussed in more detail in section 3.1.1.2.

As the periodicity of structures supporting Bloch waves is typically of the order of the wavelength, and the wavelength of light is larger than that of an electron, photonic crystal structures are considerably larger than, e.g., the atomic crystals of semiconductor materials. Nevertheless, for integrated optics (IO), photonic crystals provide a way to realize ultracompact optical devices due to light confinement by structures in the crystal. Ideally the photons would propagate without scattering in a photonic crystal as the electrons do in an atomic crystal. Unfortunately the available fabrication technologies are not mature enough to produce such high-quality $\mathrm{PhCs}$.

The fabrication of 3D photonic crystal structures is challenging, as well as their integration with 'conventional' IO devices that rely on planar fabrication techniques. For this reason the most popular IO photonic crystals are the slab-type photonic crystals in which the crystal is formed by introducing a 2D-periodic refractive index pattern in the slab plane, as shown in Fig. 1.

The propagating modes in photonic crystals are characterized by dispersion curves, sometimes called photonic bands or energy bands, which correspond to propagating modes in the crystal. In contrast to the dispersion curves of waveguide gratings, in $\mathrm{PhCs}$ all possible propagation directions in the slab plane need to be considered. Due to the periodicity PhCs may also have energy (wavelength) ranges at which no (running) wave propagation is possible in the crystal.

\subsubsection{Band diagram}

Structures such as shown in Fig. 1, having two-dimensional periodicity, also have structural variation in the third dimension, thus requiring three-dimensional optical modelling for finding accurate solutions of Maxwell's equations. Since full 3D modelling of such structures requires huge computing resources, several approaches have been taken to reduce the problem to 2D. The first one is simply ignoring the third $(z)$ dimension, which is equivalent to extending the structure infinitely into the $z$ direction to a purely $2 \mathrm{D} \mathrm{PhC}$, and considering only waves that travel parallel to the $x-y$ plane. A second approach is to include one effect of the finite $z$-dimension, by replacing the refractive index of the "background" material by an effective index of the (unperturbed) slab, often simply calculated using the effective index method (EIM) [2]. This type of 2D modeling in general produces similar dispersion diagrams as full 3D methods. However, out-off-plane losses are neglected; in addition, the 2D method introduces a considerable red shift to the spectral phenomena [3]. 


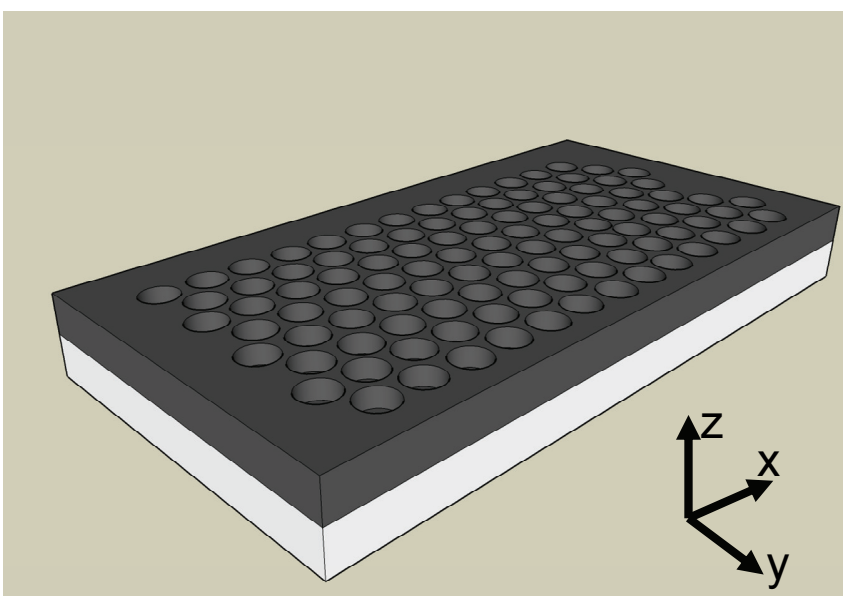

Fig. 1. Schematic picture of a photonic crystal slab formed by a triangular lattice of air holes in the slab.

Fig. 2 shows the band diagram of a PCS with parameters: $n_{\text {slab }}=3.479$, Si layer thickness $220 \mathrm{~nm}$, semi-infinite oxide layer, lattice period $\Lambda=440 \mathrm{~nm}$ and hole diameter $270 \mathrm{~nm}$. The inset of Fig. 2 shows the irreducible Brillouin zone of the triangular lattice and the high symmetry points of the zone. The irreducible Brillouin zone is the smallest region within the Brillouin zone for which the normalized frequency values as a function of wave-vector are not related by symmetry [4]. The Brillouin zone (BZ) can be constructed from the irreducible zone. Also shown in Fig. 2 is the band diagram of the corresponding $2 \mathrm{D}$ PhC obtained by using the effective index method $\left(n_{\text {eff }}=2.85\right)$. It can be seen that the bands calculated with the 3D method are at higher frequencies than the ones obtained with the 2D method. Although the finite thickness of the Si layer is taken into account by the effective index, the calculation of the field distribution in the 2D method assumes infinite thickness. The frequency shift can be understood by considering the fact that the energy of a mode is concentrated in a high-index material (being orthogonal to lower modes) in order to minimize the frequency [4]. In the actual 3D structure the mode extends into low index material in the $z$-direction (which, in the 2D approximation, is assumed uniform), which tends to increase the frequency of the mode(s). 


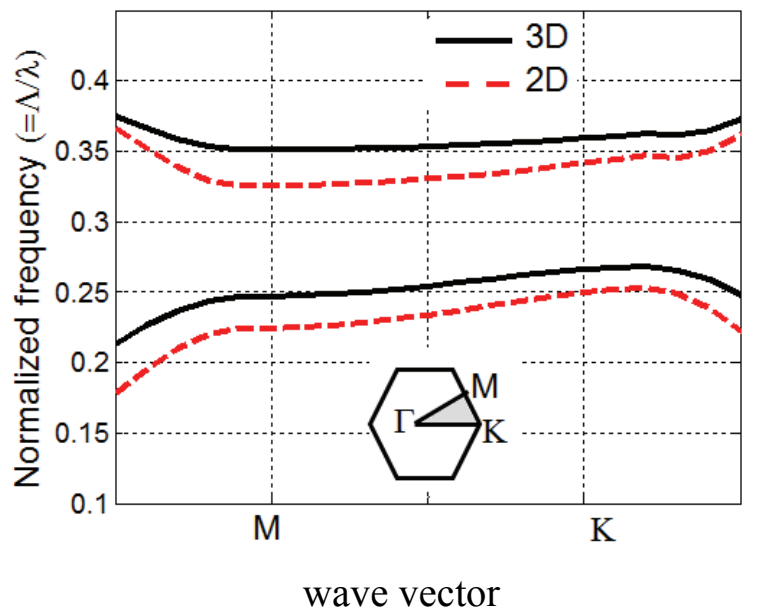

Fig. 2. Band diagram of a photonic crystal slab with $n_{\text {slab }}=3.479$, Si layer thickness $220 \mathrm{~nm}$, oxide layer thickness of $2 \mu \mathrm{m}$, triangular lattice of holes with period $\Lambda=440 \mathrm{~nm}$ and hole diameter $270 \mathrm{~nm}$.

Calculations have been done for TE polarized light with MIT Photonic-Bands (MPB) [5] for a full 3D

structure (solid lines) and for the 2D-reduced structure (dashed line). Inset: The Brillouin zone of the triangular lattice, the irreducible zone gray-shaded. Also shown are the high symmetry points $(\Gamma, \mathrm{K}$ and $\mathrm{M})$.

Dispersion curves, and hence photonic stopbands are polarization dependent. Only when the stopbands for both polarizations overlap for all possible propagation directions, one can speak of a (full) photonic bandgap (PBG). This overlap of stopbands for TE and TM polarization depends for 2D PhCs with a triangular lattice of air holes on the ratio of the lattice period to the hole radius. Unfortunately this PBG occurs typically (depending on the slab index) at normalized frequencies around 0.4. From Fig. 2a of chapter 2, it can be seen that the lightlines of the oxide cladding and the air cladding are at the normalized frequencies below 0.35 and 0.5 , respectively. Note that the same wave-vector range $(0-$ $\pi / \Lambda$ ) holds for a reduced $\mathrm{BZ}$ of a typical $\mathrm{PhC}$ waveguide, as will be discussed in the next section. To avoid out-of-plane diffraction losses that may arise for modes above the lightline the $\mathrm{PhC}$ should be designed such that the bandgap is at the lossless side of the lightlines. Based on our discussion above, this is more difficult to realize if operation at both polarization states is required.

\subsubsection{Defects}

The presence of defects in a $\mathrm{PhC}$ structure may introduce additional energy levels within the original bandgap. Light can be confined to these defects, provided that the energy of the photons matches the energy level of the defect.

\section{Line defects, acting as waveguides}

A particularly useful type of defect is created if an entire row of holes is removed. In this case a PhC waveguide called W1 is formed. Light in such a waveguide is confined in the z-direction by total internal reflection (TIR) and in other transverse direction by the photonic crystal due to the presence of a bandgap (gap guided). If a PCS is formed with 
air holes in a high-index material, such as silicon $(n \cong 3.5)$, only a few rows of holes around the W1 waveguide are sufficient to virtually confine the light to the waveguide.
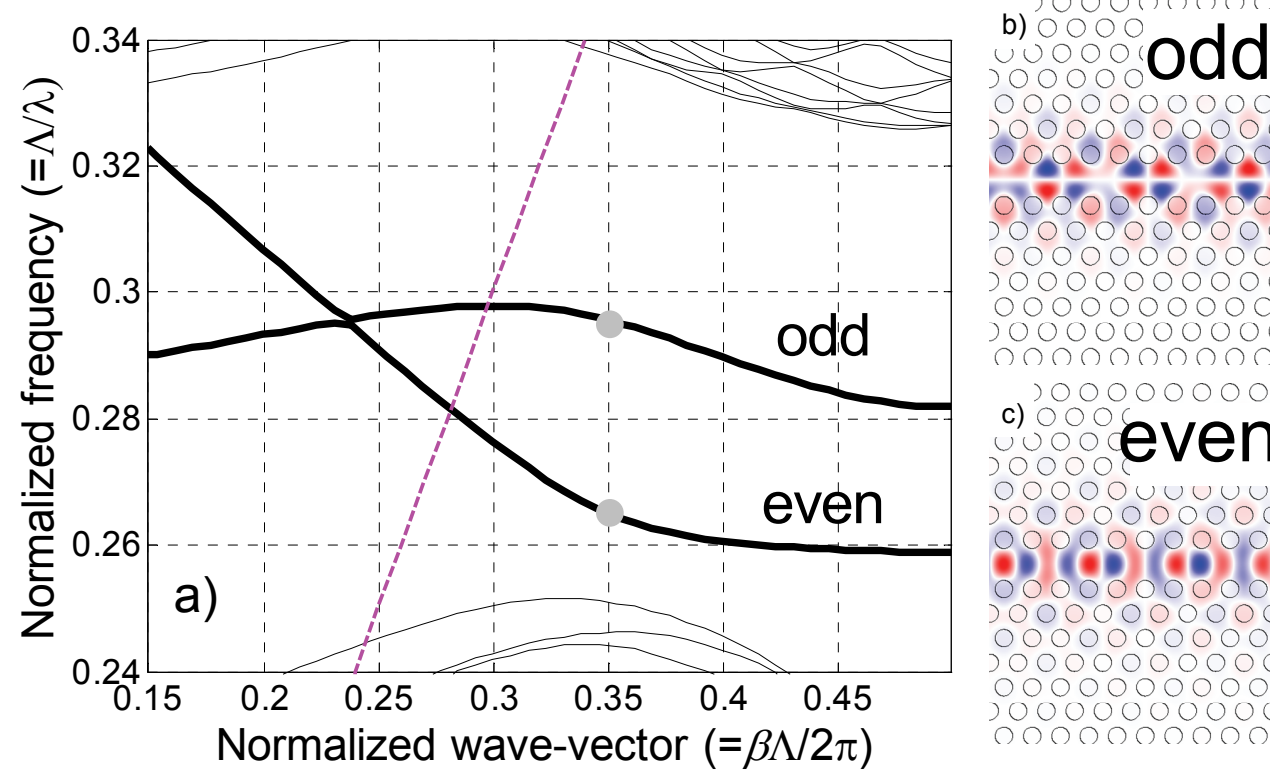

Fig. 3. a) Calculated dispersion diagram of a photonic crystal W1 waveguide in the $\Gamma$-K-direction, showing two H-like (TE-modes), I and II. The dashed line is the light line of the air cladding. b) and c) The H-field profiles of the W1 modes at a normalized wave-vector value of 0.35 .

A calculated dispersion graph of a W1 waveguide in a 2D photonic crystal with a period / hole radius ratio of $440 / 135$ and $n_{\text {eff }}=2.85$ is shown in Fig. 3a. This type of graph is also called a projected band diagram as the $k$-vectors belonging to the $\mathrm{BZ}$ of the $\mathrm{PhC}$ are projected to the reduced $\mathrm{BZ}$ of the waveguide. The mathematical details of this projection procedure are discussed e.g. in [6]. From Fig. 3 it can be seen that the waveguide has two H-like modes, i.e., modes with the only H-component along $z$ [6], that are in the photonic bandgap. Figs. $3 \mathrm{~b}$ and $3 \mathrm{c}$ show the corresponding $\mathrm{H}$-fields of these localized Bloch states (i.e. the guided modes) at the normalized wave-vector value of 0.35. Based on these field plots, the modes are identified as odd (antisymmetric) and even (symmetric), with respect to the centre-line of the waveguide. For practical reasons, related to coupling light into a $\mathrm{PhC}$ structure using single-mode channel waveguides, the even mode is preferred. As seen from Fig. 3a, single mode operation at telecom wavelengths (e.g. 1530-1565 nm) requires a small period (of the order of $400 \mathrm{~nm}$ ). From the fabrication point of view, the small period is a challenge and a number of ways to make the W1-type waveguide single mode and/or with improved dispersion profile have been proposed. Some of these designs and their properties are discussed in detail in e.g. [7]. In general the improved designs are based on reducing the effective index of the $\mathrm{PhC}$ waveguide by reducing the width or the index of the core region.

\section{Losses in $\mathrm{PhC}$ waveguides}

Fig. 4 shows the transmission spectra of three different photonic crystal waveguides. The spectra have been normalized with respect to the measured transmission spectrum of a 
photonic wire waveguide, with a cross-section of $500 \mathrm{~nm}$ (width) by $220 \mathrm{~nm}$ (height). Typical propagation loss of such a waveguide is $0.24 \pm 0.16 \mathrm{~dB} / \mathrm{mm}$ [7].

The PhC WG1 is a W1-type waveguide with a slab hole diameter of $270 \mathrm{~nm}$. The $\mathrm{PhC}$ WG2 and WG3 are W1-type waveguides with increased boundary hole diameter (BHD), see section 3.1.2.2. The PhC WG2 has a slab hole diameter of $250 \mathrm{~nm}$ and BHD of 330 $\mathrm{nm}$ and the PhC WG3 has a slab hole diameter of $255 \mathrm{~nm}$ and BHD of $340 \mathrm{~nm}$. All three $\mathrm{PhCs}$ have the same period, $440 \mathrm{~nm}$, and the same length, 24 periods.

It can be seen that the additional loss of these straight $\mathrm{PhC}$ waveguides compared to a conventional waveguide is about $5 \mathrm{~dB}$ with increasing trend near the mode cut-off wavelength. At a wavelength of $\sim 20 \mathrm{~nm}$ below the mode cut-off the loss is $14 \mathrm{~dB} \pm 2 \mathrm{~dB}$. The loss near the mode cut-off is high due to slow light propagation [8-9].

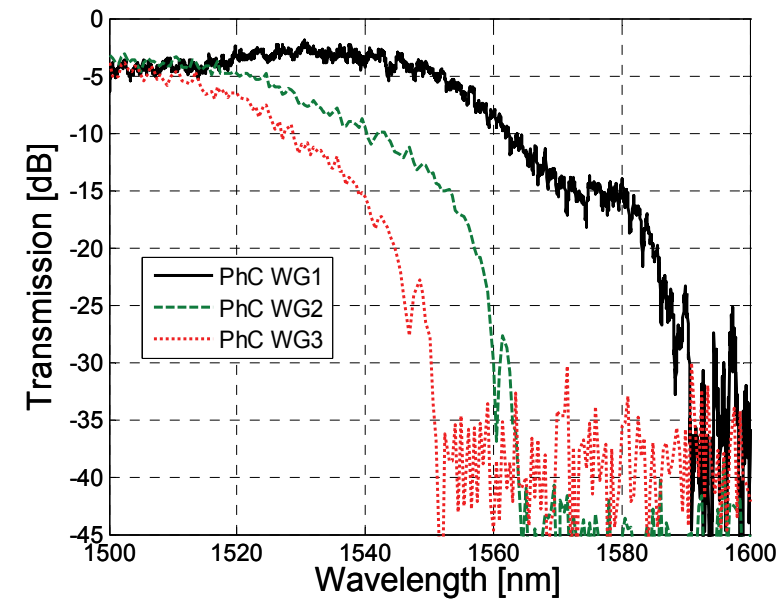

Fig. 4. The transmission spectra of three different photonic crystal waveguides (see text).

\section{Photonic crystal cavity}

An example of another interesting defect-type is shown in Fig. 5. It is an isolated defect that can act as a resonant optical cavity. Photons having a wavelength inside the PBG of the crystal and matching a resonant mode of the defect, are trapped inside this cavity in the $x-y$-plane by the surrounding crystal structure and in the $z$-direction due to TIR. Unlike the case of $\mathrm{W} 1$ defects, where a continuity of modes exists over a certain wavelength range, a cavity only supports certain discrete modes. Light can be coupled from a waveguide (e.g. W1) to the cavity, by locating the waveguide close to the cavity, separated by just a few holes, so that there is some (generally small) overlap between the evanescent fields of waveguide and cavity modes. The resonating cavity mode has to interfere constructively with the input light that feeds the energy to the cavity. As the cavity loss and out-coupling increase with the stored energy, at some point a steady state is achieved where the power fed into the cavity equals the lost power. In the steady state for a given input power a constant amount of electromagnetic energy is stored in the cavity. This amount can be calculated from the so-called Q-factor, which is defined as the ratio of the time-average energy stored at a resonance frequency to the energy dissipated in one period of this frequency. 


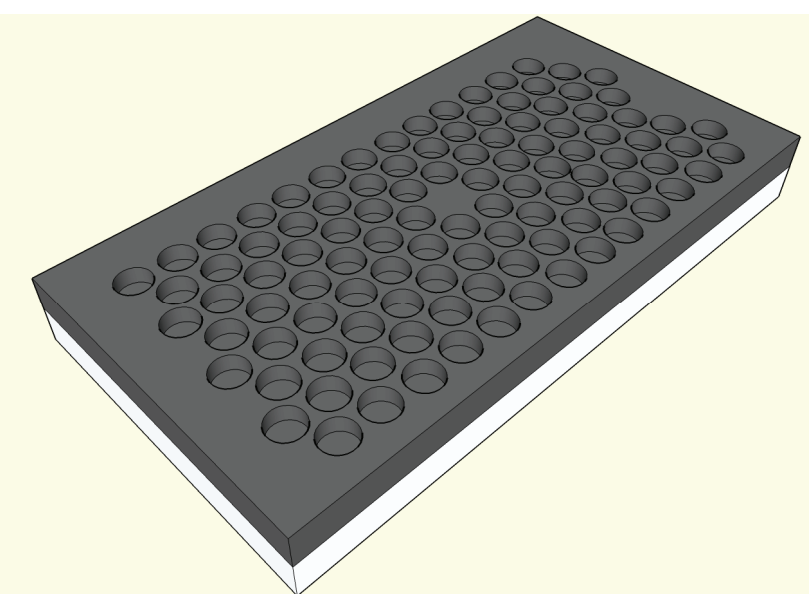

Fig. 5. Point defect in a 2D slab photonic crystal.

One of the key advantages of cavity resonators in a $\mathrm{PhC}$ is that they can be made compact. However, as the cavity size gets smaller, the losses increase. This can be explained by decomposing the resonant mode into its spatial Fourier components (i.e. plane waves). A large number of such plane wave components are needed for a strongly confined mode in a small cavity (e.g. below the wavelength). Losses arise when such components can couple to freely propagating plane waves in the cladding regions above and below the slab. In order to prevent this coupling, all the plane wave components should fulfill the TIR condition. If the absolute value of the tangential component of the $k$-vector of the plane wave is between 0 and $2 \pi / \lambda$, the light leaks from the surface of the microcavity to radiation modes. In order to obtain a low-loss, high-Q cavity, the amount of light radiating from the surface should be minimized. This can be accomplished by designing the cavity structure in such a way that the number of plane wave components is minimized and that there are no tangential components in the range from 0 to $2 \pi / \lambda[10]$. The shape of the cavity can be tuned, e.g. by shifting the position of the holes around the cavity. Shifting the position of the holes (in a $\mathrm{PhC}$ ) requires special accuracy in the fabrication process, as the typical shift is only a few tens of nanometers. As the shape of the cavity is tuned to obtain the maximum Q-factor, also the resonance wavelength is tuned. For these reasons it is challenging to fabricate a high-Q cavity operating at a specific wavelength for a given PCS; both the Q-factor and the resonance wavelength of such cavities are extremely sensitive to the process variations, which may cause serious problems with reproducibility.

Microcavities can be used in several applications. The development of cavities in compound semiconductor materials (such as InGaAsP) is motivated by their application for low-threshold (nano)lasers. The wavelength-selectivity of microcavities makes them interesting for application in ultracompact wavelength drop filters in optical network applications. In this configuration the cavity is placed in the proximity of the $\mathrm{PhC}$ waveguide. Light at a resonant wavelength is coupled to the cavity and can then be further coupled to the drop (output) channel.

Several coupled microcavities, arranged in line, form a special type of waveguide, where light will propagate by tunneling from cavity to cavity at low group velocity propagation. By exploiting the rotational symmetry of the field inside each cavity, very sharp bends 
can be achieved with this kind of waveguide [11]. The disadvantage is that this type of waveguide operates only for a narrow wavelength band.

\subsubsection{Connecting to the $\mathrm{PhC}$ device}

The PhC structure has to be connected to the outside world somehow. In the most common case waveguides are used. Due to the fundamental difference in light guiding properties, the coupling of a conventional waveguide mode to a $\mathrm{PhC}$ waveguide mode introduces coupling loss. Efficient coupling schemes (discussed e.g. in [12-13]) have been developed that considerably reduce the coupling loss.

\subsubsection{Photonic-crystal-cavity-based channel drop filter}

This section introduces design principles for PhC-cavity-based channel drop filters. Computer simulations have been performed in order to obtain device parameters that on the one hand would give the wavelength selective channel drop performance and on the other hand would result in a device that can be realized on the selected fabrication platform. Two variations of the device have been designed; one optimized for performance and one with better tolerance to fabrication process variations. In a discussion section our design is compared to similar designs reported in the literature. The section ends with a presentation of the experimental results of the $\mathrm{PhC}$ channel drop device.

\subsubsection{Introduction}

A schematic picture of the cavity-based channel drop filter is shown in Fig 6. In the ideal case, the light of a specific wavelength, propagating along the bus channel is transmitted to the drop port (out2) while the light at the other wavelengths continues traveling through the bus channel to output port out 1 . The cavity should be designed such that the power at the drop wavelength is fully coupled to the drop port. A fundamental challenge is that if the cavity supports only one mode, it decays the power to all the possible output ports. However, with an appropriate design $100 \%$ of the input power, at a resonance wavelength of the cavity, can be dropped from the bus channel to the drop port [14-15].

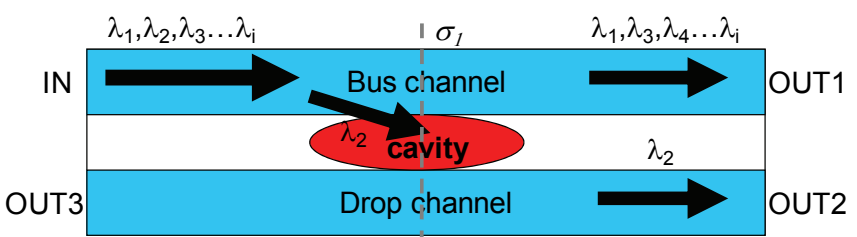

Fig. 6. A Schematic picture of a cavity-based wavelength selective channel drop filter.

This kind of performance requires (quasi)degenerate resonant modes with different field symmetries [14-16] at the operation wavelength. These resonant modes should have opposite symmetry with respect to the mirror plane centered on the cavity and perpendicular to the waveguides ( $\sigma_{l}$ in Fig. 6). The odd resonant mode excites forward and backward traveling guided modes in the $\mathrm{PhC}$ waveguides with $\pi$ phase shift, whereas the even resonant mode excites them in phase, thus causing destructive interference for the backward traveling waves [15-16]. 
It is possible to achieve such a complete directional channel dropping by applying a design of two coupled cavities or a design based on single cavity. The two cavity approach requires careful design work to balance the direct and indirect coupling rates between the cavities [14]. As pointed out in [15] the single-cavity-type of design has a clear benefit as the requirements to fabricate two cavities with exactly tailored structure is more challenging. For this reason, we have chosen to focus on channel drop filter designs that are based on a single cavity. In our design work, we have followed the principles described in [15] and [16] to design photonic-crystal-cavity-based channel drop filters.

\subsubsection{The design and modeling of a W1-type cavity}

The PhC structure under study, shown in Fig. 7, consists of two symmetrically arranged modified W1-type waveguides, separated by 7 rows of holes, with a cavity in between. The diameters of the boundary holes adjacent to the waveguides are made larger than the rest of the slab holes, in order to obtain single mode operation [17] and to adjust the dispersion curves for the cavity cross-section such that the desired wavelength drop performance is achieved. The cavity is formed by omitting a row of holes at the center of the structure between the waveguides, as shown in Fig. 7. In addition the boundary holes around the cavity are made smaller than the slab holes in order to adjust the dispersion properties of the structure as will be discussed below.

As suggested in [15] the wavelength dropping properties of this type of $\mathrm{PhC}$ structure can be analyzed by calculating the dispersion characteristics of the structure. They can be obtained by computing definite frequency eigenstates of Maxwell's equations using the MIT Photonics Band (MPB) package [5]. For computational efficiency, a so-called supercell approach is used, which assumes that a sub-structure (the supercell, shown as a slice in Fig. 8b) is repeated periodically. In this calculation, which cannot directly model the finite-length resonator, the cavity defect is treated as an intermediate waveguide.

The diameter of the holes surrounding the cavity can be chosen such that the cavity supports the quasi-degenerate modes needed for directional channel dropping. Based on MPB calculations we have chosen a 440-nm lattice period, a 200-nm diameter for the cavity boundary holes, and a 340-nm diameter for the boundary holes around the waveguides. The remaining "interior" holes, further denoted as "slab holes" have a diameter of $270 \mathrm{~nm}$. The calculated dispersion graphs of the structure are shown in Fig. 8a. The slow-light-regime of the intermediate waveguide section (that forms the cavity) provides the desired dispersion characteristics, showing bimodal behavior over an extended frequency range. The requirement for opposite symmetry with respect to the plane perpendicular to the propagation direction $\left(\sigma_{1}\right)$, implies that the propagation constants of the modes should differ by $(2 q+1) \pi / L_{\text {eff }}[15]$, in which $q$ is an integer and $L_{\text {eff }}$ is the effective length of the cavity. The symmetry with respect to the mirror plane parallel to the propagation direction $\left(\sigma_{2}\right)$, determines whether the dropped power propagates to the forward or backward direction [15]. 


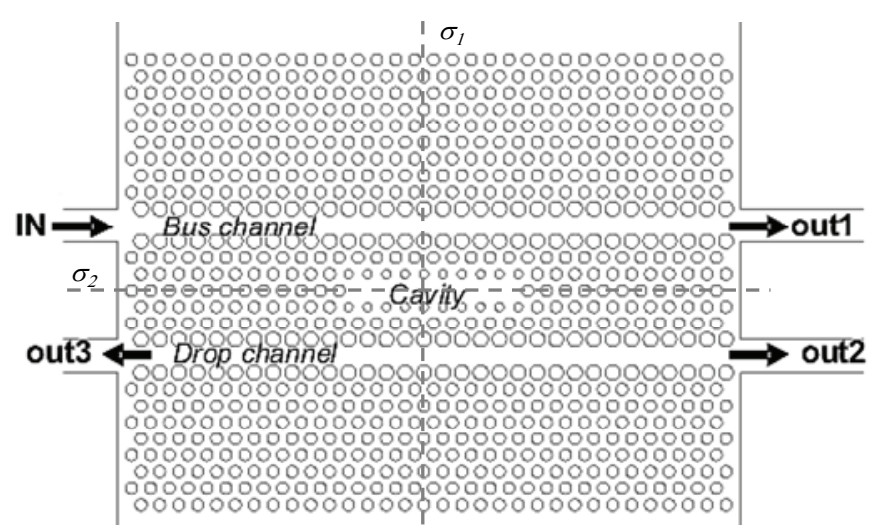

Fig. 7. A schematic picture of the PhC-cavity-based channel drop filter. Mirror plane perpendicular to propagation direction $\sigma_{l}$, mirror plane parallel to propagation direction

$\sigma_{2}$.
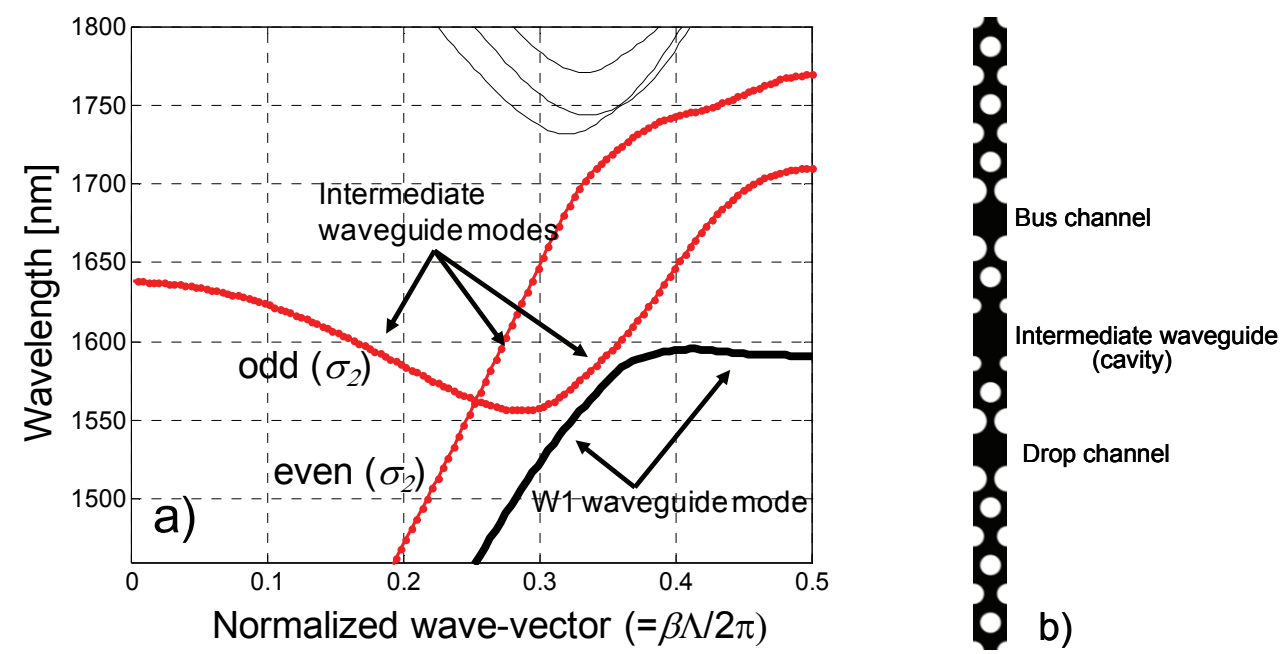

Fig. 8. a) The dispersion graph of the PhC channel drop device, calculated with MPB [5] using the supercell method. b) The supercell structure that is used to calculate the dispersion properties of the channel drop filter device in the cavity region.

The dispersion characteristics do not guarantee the operation in quasi degenerate regime, as depending on the effective cavity length $\left(L_{e f f}\right)$, different modes are supported by the cavity [15]. In order to find the correct cavity length, we performed 2D FDTD simulations by varying the cavity length by integer multiples of the lattice constant, as this changes the possible resonance wavelengths that are supported by the cavity, and hence its free spectral range.

The simulated transmission spectrum of a reference structure that has no cavity is shown in Fig 9a. Most of the light is transmitted to the out 1 port, while very little light is leaked to the out 2 and out 3 ports of the drop channel. The loss $(\sim 25 \%)$ is due to non optimal coupling of the waveguides to the PhC structure, see section 3.1.1.

Based on the graph shown in Fig. 8 and on the required wave-vector difference $\left((2 q+1) \pi / L_{\text {eff }}\right)$, we considered a cavity with a physical length $\left(L_{c a v}\right)$ of 10 holes as an initial starting point. Fig. $9 \mathrm{~b}$ shows the simulated transmission spectrum of this cavity. 
Two resonance modes can be seen around a wavelength of $1565 \mathrm{~nm}$. The two cavity modes are too far apart (ie. at different wavelengths) for achieving complete directional dropping. In other words, the degeneracy of these modes is poor, and approximately $20 \%$ of the input power is dropped to the out 2 and out 3 ports. Fig. 9c shows the transmission spectrum of the cavity with $L_{c a v}=11$ holes. The degeneracy is now better than for $L_{c a v}=$ 10 holes. However, two resonance modes are still too far apart; a broader one at $\lambda=1564$ $\mathrm{nm}$ and narrower at $\lambda=1566.5 \mathrm{~nm}$. The broader peak couples $\sim 20 \%$ of the input power to both out 2 and out 3 ports, while the narrower one couples $40 \%$ to the out 2 .

It turns out that $L_{c a v}=9$ holes gives the desired result, as shown in Fig. 9d. The quasidegeneracy of the modes occurs and a directional dropping with better than $95 \%$ efficiency to the out 2 port is obtained. Further fine tuning of the drop efficiency (by fine tuning the cavity length) can be done by either changing the hole diameters at the ends of the cavity or by shifting the hole positions slightly.

We have simulated the transmission spectra for cavity lengths down to $L_{c a v}=4$ holes. None of the cavities shorter than 9 holes showed complete channel dropping. It is likely that some cavity length longer than $L_{c a v}=11$ holes would show a quasi-degenerate resonance state. However, the $L_{c a v}=9$ holes provides the most compact design for the chosen parameters.
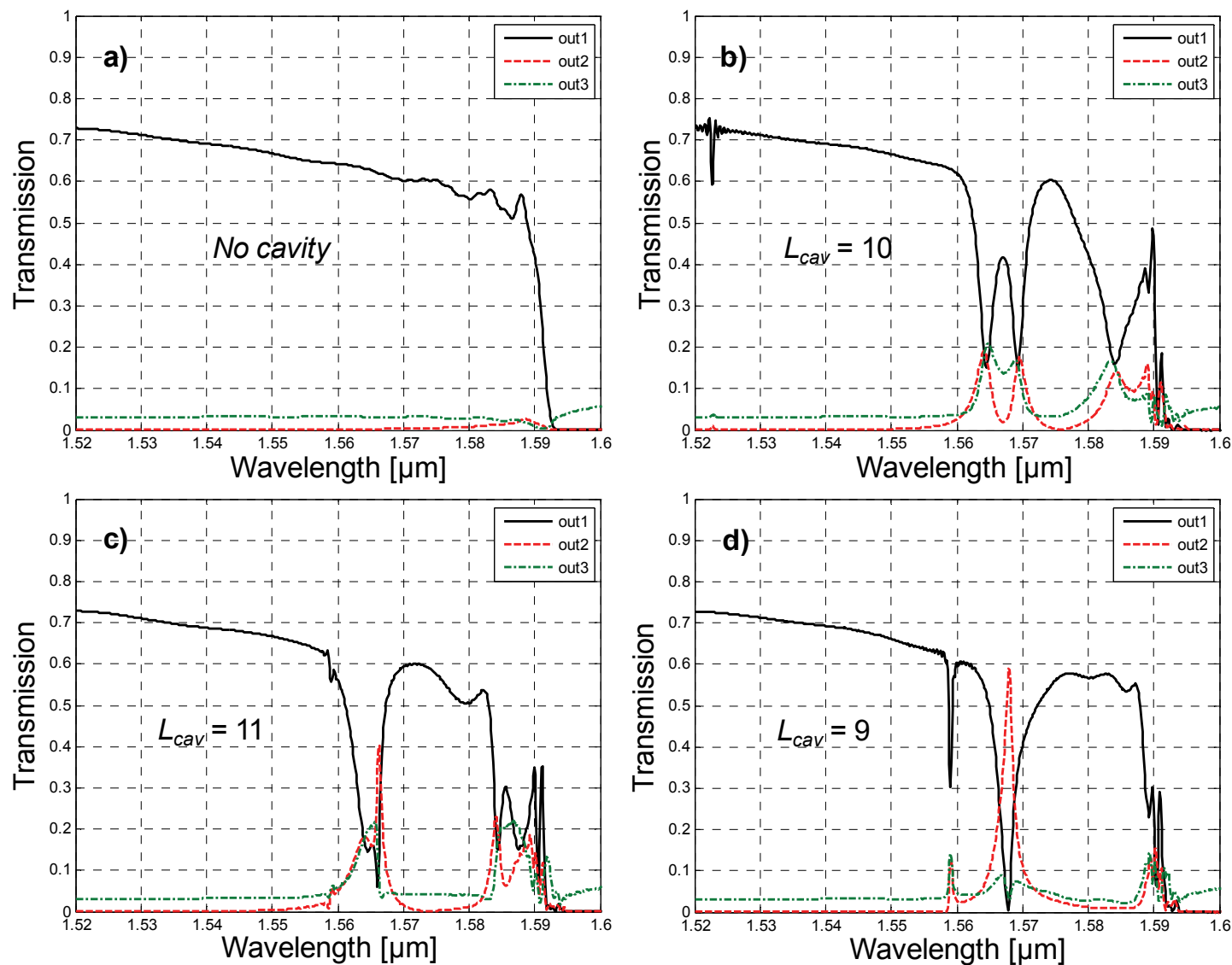

Fig. 9. Simulated (2D FDTD) transmission spectrum of the PhC channel drop filter (Fig. 2). a) Structure without cavity. b) Cavity length of 10 (omitted) holes. c) Cavity length of 11 holes. d) Cavity length of 9 holes. 


\subsubsection{The design and modeling of a W3-type cavity}

In the deep UV lithography process [18] feature size depends on the exposure dose. The features do not have the same optimal dose (the exposure dose required to get the desired size), and particularly for holes the optimal dose depends on the hole diameter. For this reason a PhC structure relying on a precise variation of hole diameters is a critical design from a fabrication point of view.

Taking this into account we have made a variation of the $\mathrm{PhC}$ structure discussed in the previous section. The period of the $\mathrm{PhC}$ remains at $440 \mathrm{~nm}$, while the slab hole diameter is reduced to $240 \mathrm{~nm}$ and the waveguide-boundary hole diameter to $330 \mathrm{~nm}$. The structural difference from the $\mathrm{PhC}$ device discussed in the previous section, is that the cavity is different; 3 rows of holes are omitted, and the cavity-boundary holes -which critically affect the cavity tuning- are the same as the slab holes. The length of the central row is 9 holes, while the two other rows are 10 holes long. The cavity is separated from the bus and drop channels by 2 rows of holes. A schematic picture of this $\mathrm{PhC}$ device is shown in Fig. 10.

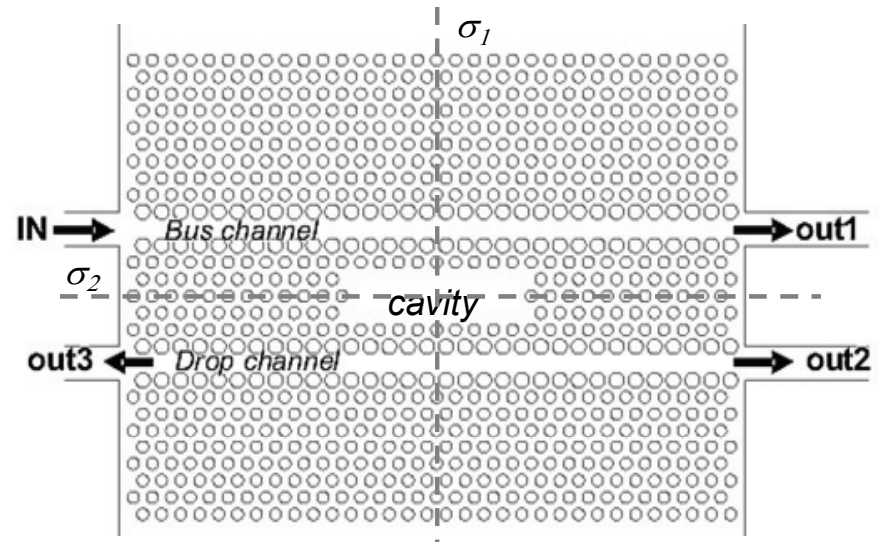

Fig. 10. A schematic picture of the PhC-cavity-based channel drop filter.

The dispersion properties of this device have been calculated in the same way as in the previous section. Fig.s $11 \mathrm{a}$ and $\mathrm{b}$ show the dispersion graph of the structure and the corresponding supercell for which the graph is calculated. We recognize several modes that are within the photonic band gap $(1430-1700 \mathrm{~nm})$. In this design a change in hole diameter changes the dispersion characteristics and therefore also the wave-vector distances between the modes are changed. For a certain hole diameter the difference is expected to be (an odd multiple of) $\pi$ for wave-vectors $\beta_{1}\left(\omega_{\text {res }}\right)$ and $\beta_{2}\left(\omega_{\text {res }}\right)$, where $\omega_{\text {res }}$ is the resonance frequency of the cavity and $\beta_{1,2}$ are the wave-vectors of two modes that are localized in the intermediate waveguide (and that will become degenerate cavity modes). 

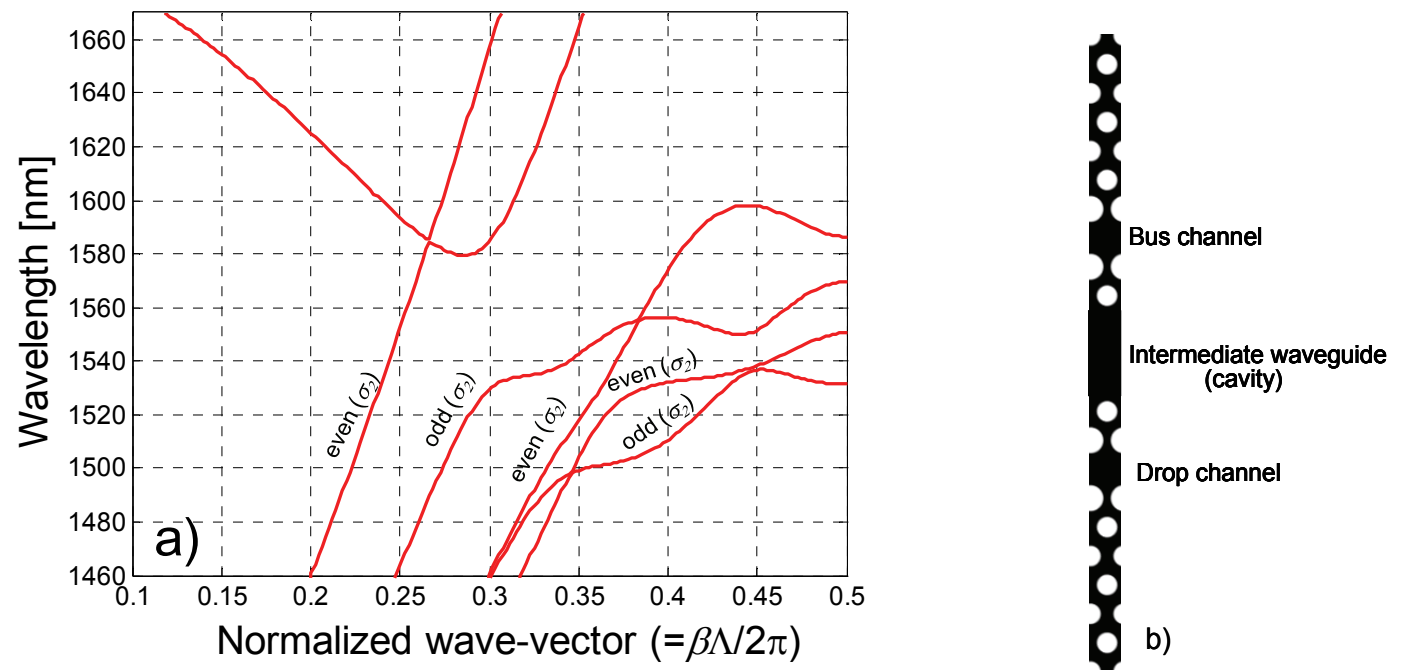

Fig. 11. a) The dispersion graph of the PhC channel drop device calculated with MPB using supercell method. b) The supercell structure that is used to calculate the dispersion properties of the channel drop filter device in the cavity region.

Based on the dispersion characteristics of the structure we made an initial guess for the hole diameters and fixed the cavity length (to 9 missing holes). Then a series of FDTD simulations have been performed by slightly changing the hole diameters around the initial guess values till the hole diameters are found that give the complete channel drop (for some wavelength) from input port to out 2 port.

Fig. 12a shows a result of such a simulation run. For the simulated structure a slab hole diameter of $240 \mathrm{~nm}$ and BHD of $330 \mathrm{~nm}$ were used. As a slab effective index we used 2.70 , as this index should better match the $2 \mathrm{D}$ simulation results with the full $3 \mathrm{D}$ results [7] than the index obtained with the effective index method. At a wavelength of $1507 \mathrm{~nm}$ the device clearly shows the desired channel drop operation; the light at this wavelength is fully coupled to the out 2 port. Unfortunately, the light at wavelengths larger than 1507 $\mathrm{nm}$ do not propagate undisturbed, but are affected by the cavity. Fig. 12b shows a snapshot of a continuous wave (CW) 2D FDTD simulation at a wavelength of $1507 \mathrm{~nm}$. The H-field distribution (Fig. 12b) clearly shows the complete channel drop operation. 

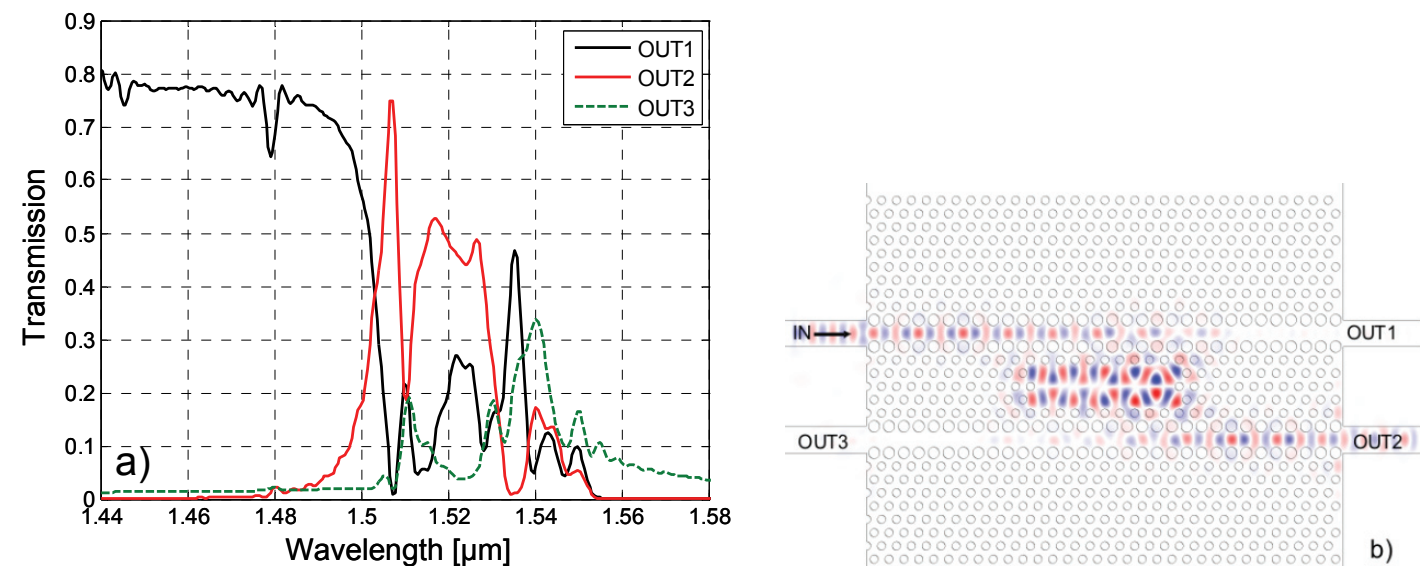

Fig. 12. 2D FDTD simulated transmission spectrum of the device. b) Simulated snapshot image of the Hfield profile at a wavelength of $1507 \mathrm{~nm}$.

Although this design provides a less ideal channel drop filter response than the design presented in previous section, it is recognized to be much easier to realize as it is less vulnerable to fabrication process variation than the design which includes the small holes around the cavity.

\subsubsection{Discussion}

The devices reported [15] and [19] are similar to our PhC channel drop filter device. In [15] 11 rows of holes separate the bus and drop channels, and there are two rows of small holes on both sides of a W1-type cavity. The optimum cavity length of the device is reported to be 20 holes. From the fabrication point of view, this design has some drawbacks. The diameter of the boundary holes adjacent to $\mathrm{W} 1$ waveguides is large in comparison to the period and the Si feature between the holes would be only $80 \mathrm{~nm}$. This is below the feature size limit of the $193 \mathrm{~nm}$ DUV. An additional challenge might come from the change in location of the small holes around the cavity. Due to the different relative locations of these holes, the target exposure dose to produce the desired hole diameter might be different for these rows.

A $\mathrm{PhC}$ channel drop device with a cavity length of 10 holes is reported in [19]. This device has 7 holes between the bus and drop channels, while the desired dispersion properties are obtained by narrowing the bus and drop channels to W0.65-type waveguide designs. While this design has the benefit of uniform hole diameter, the W0.65 waveguide design poses limitations as e.g. realizing bends in a W0.65 waveguide is difficult as they easily lead to discontinuities [7].

\subsubsection{Experimental: W3-type cavity}

Fig. 13 shows a SEM image of a DUV-fabricated PhC W3-type-cavity-based channel drop filter. Two versions of the device were fabricated; one with a waveguide boundary hole diameter (BHD) of $320 \mathrm{~nm}$ and the other with a BHD of $330 \mathrm{~nm}$. The slab hole diameter for both devices is approximately $240 \mathrm{~nm}$. Fig.s $14 \mathrm{a}$ and $14 \mathrm{~b}$ show the measured transmission spectra of the channel drop devices, normalized with respect to the 
transmission of a straight $\mathrm{W} 1$ waveguide of equal dimensions. Due to measurement noise, the transmission seems to be (unphysically) slightly above $0 \mathrm{~dB}$ in some regions.

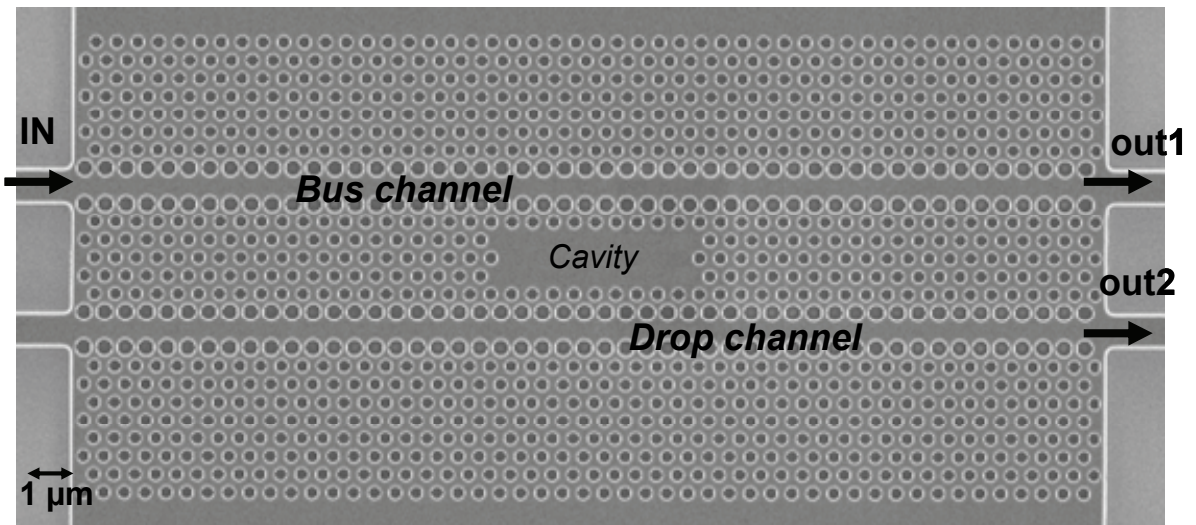

Fig. 13. SEM image (top view) of a PhC channel drop filter.

As seen from the measured transmission spectra, both channel drop filters have a drop wavelength of $1561 \mathrm{~nm}$. For a design with BHD of $330 \mathrm{~nm}$ the on/off ratio between ports out 1 and out 2 is higher; $16 \mathrm{~dB}$ in comparison to $10 \mathrm{~dB}$. The full width at half maximum of the drop band is approximately $2.5 \mathrm{~nm}$ for both designs. Fig. 14a also shows the transmission spectrum of a reference device without a cavity but otherwise similar. From this reference curve (OUT2 Ref.), it can be seen that the device suffers from considerable cross talk at wavelengths longer than $1570 \mathrm{~nm}$. Therefore, the device should not be operated at wavelengths larger than the drop wavelength. The device with $320 \mathrm{~nm}$ BHD appears to have less crosstalk outside the drop band and can be operated at slightly broader wavelength range. Based on the measurements, the cut-off wavelength of the bus channel waveguide for both devices is between $1600 \mathrm{~nm}$ and $1610 \mathrm{~nm}$.
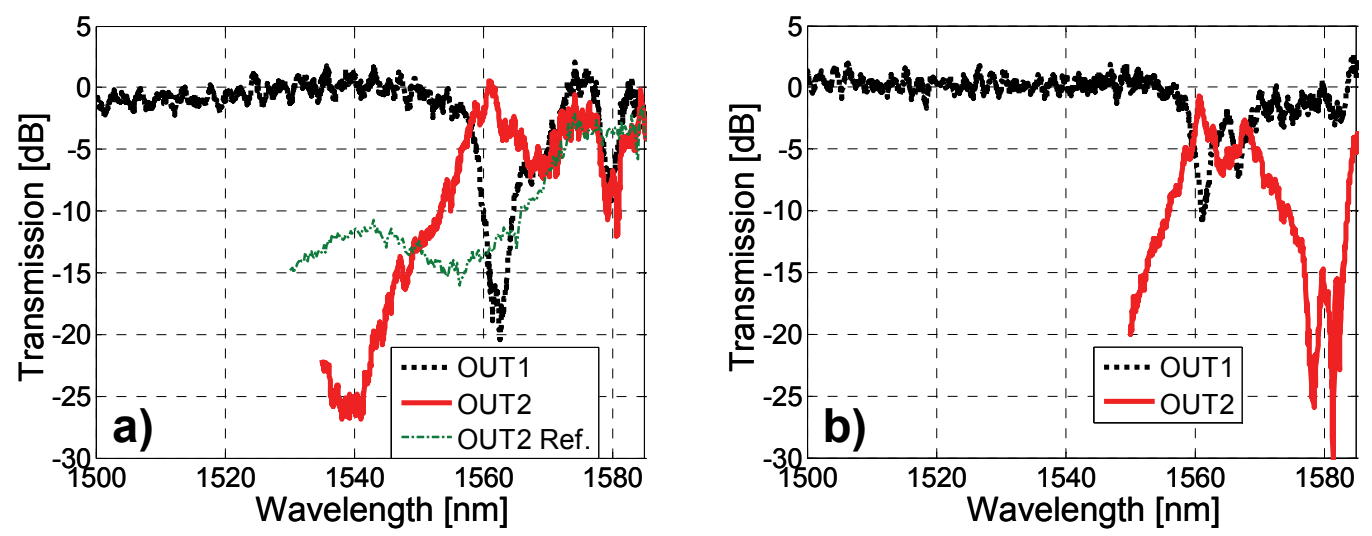

Fig. 14. Measured transmission spectra of channel drop filters from the in to the out 1 and out2 ports. a) device with $330 \mathrm{~nm}$ boundary hole diameter (BHD). Also, shown a reference curve (see text). b) device with $320 \mathrm{~nm}$ BHD. 


\subsubsection{Conclusions}

Based on computer simulations PhC cavity -based channel drop filters have been designed. These line defect devices require well chosen parameters for complete channel drop operation (100\% drop efficiency). Requirements for the fabrications process variables (such as exposure dose) are high. A design that requires only two different hole diameters turned out to be realizable, though with less ideal spectral properties as the design based three different hole diameters, that according to simulations provides nearly ideal channel drop filter response. These $\mathrm{PhC}$ devices suffer from high optical loss, particularly at wavelengths near the mode cut-off, where the loss is approximately $14 \mathrm{~dB}$, as discussed in section 3.1.1.2.

\subsubsection{Photonic-Crystal-Cavity-based Y Splitter}

We propose a Y splitter in a 2D photonic crystal with a triangular lattice of air holes suitable for mechano-optical switching. Full switching between the two output ports can be achieved at a certain wavelength owing to the presence of a resonant central cavity with well-chosen symmetry.

Experimental results confirm the operation of the device and its potential as an ultracompact mechanical switch.

\subsubsection{Introduction}

One of the basic components in optical networks is the Y splitter. Several ultracompact Y splitter designs in two-dimensional (2D) photonic crystals $(\mathrm{PhC})$ have been reported [2022]. PhC-based switches are particularly attractive due to their compactness and large $\mathrm{Y}$ splitting angle.

We propose a Y-splitter in a 2D photonic crystal slab with a triangular lattice of air holes, as shown in Fig. 15.

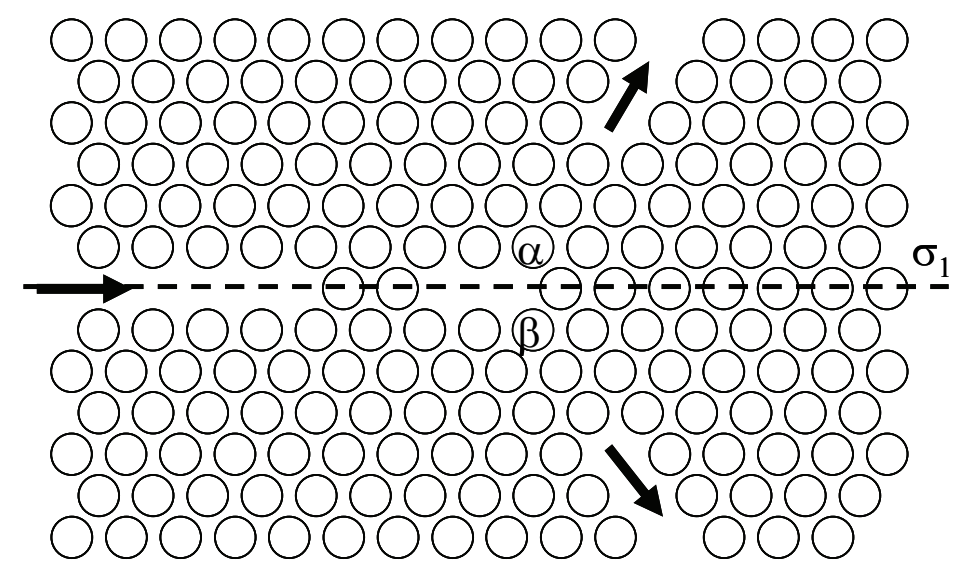

Fig. 15. The proposed PhC-cavity-based Y splitter. The holes marked with $\alpha$ and $\beta$ show the positions of the switching holes. By selectively filling the holes, different cavities (and therefore different coupling conditions) between the output ports are obtained. The symmetric cavity (both $\alpha$ and $\beta$ filled, or both empty) splits the light equally to the output ports, whereas the asymmetric cavity ( $\alpha$ or $\beta$ filled) couples light to only one of the ports.

This section has been published in: L.J. Kauppinen, T.J. Pinkert, H.J.W.M. Hoekstra, and R.M. de Ridder, Photonic Crystal Cavity-Based Y Splitter for Mechano-Optical Switching, IEEE Photonics technology letters, vol. 22, issue 13, pp. 966-968, 2010. 
By selectively filling the holes, labeled $\alpha$ and $\beta$ in Fig. 15, different cavity geometries are formed at the Y-junction. Filling both holes $\alpha$ and $\beta$ with the same material results in a symmetric cavity with respect to the $\sigma_{1}$ axis (see Fig. 15), whereas filling either hole $\alpha$ or $\beta$ an asymmetric cavity with respect to the $\sigma_{1}$ axis is obtained. As will be explained below, in case of such an asymmetric cavity and for a specific resonant wavelength, the light is coupled to only one of the output ports, whereas in the symmetric case the device acts as a Y splitter and the light is coupled equally to both output waveguides.

In the envisaged mechano-optical switch application, the signal output at the operating wavelength of the splitter device can be dynamically switched between the output ports by selectively filling holes $\alpha$ and $\beta$.

\subsubsection{Design and modeling}

The envisaged tuning of our device is based on selecting the drop port, via a nanoelectromechanical system, by changing the symmetry of the structure and hence the spatial field symmetries in the resonator. Such an operation can be achieved e.g. by perturbing the structure with an AFM tip [23-24]. Fig. 16 shows the PhC structure with its symmetry axes corresponding to the cases that both or none of the holes $\alpha$ and $\beta$ are filled $\left(\sigma_{1}\right)$, only hole $\alpha$ filled $\left(\sigma_{3}\right)$, and only hole $\beta$ filled $\left(\sigma_{2}\right)$. The operation of this kind of devices can be understood by group theoretical arguments [25-26], using that defects, in our case W1 waveguides and a multihole resonator, have the symmetry of a subgroup of the $\mathrm{PhC}$ point group (hereafter called group). Such a defect structure can support electromagnetic fields that have the properties of the irreducible representations of the group of the defect.

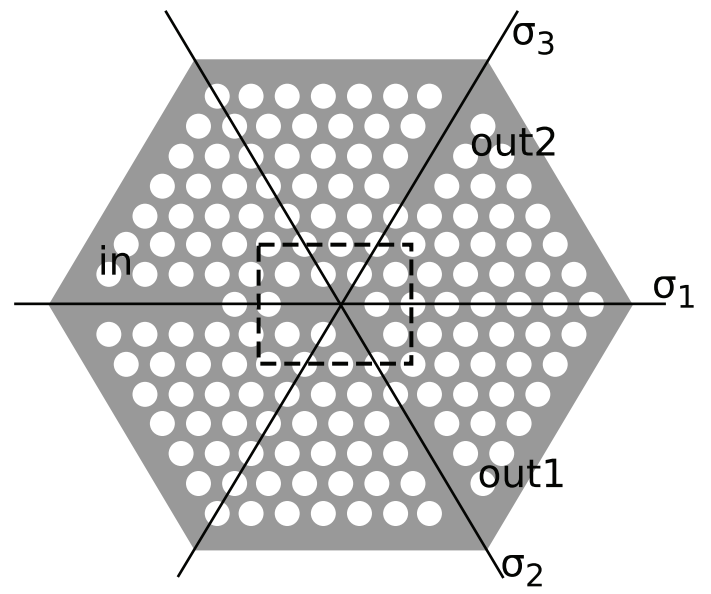

a

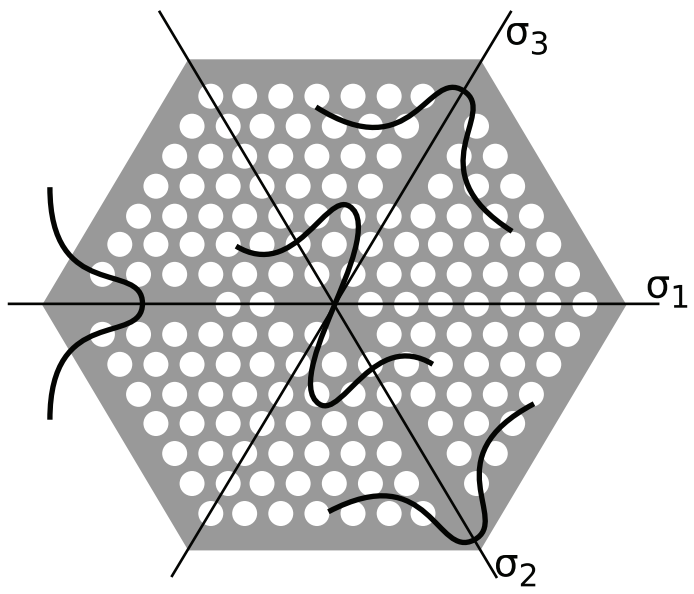

b

Fig. 16 Photonic crystal switch. a) The possible mirror symmetry axes of the design, the dashed rectangle indicates the resonator position; b) the $\chi=1$ fields of the photonic crystal waveguide and $\chi=-1$ field of the defect show intuitively that light cannot be coupled from the resonator into the out 2 waveguide.

The defects of the proposed switching device (in both switching states) have the symmetry of the $\mathrm{C} 2 \mathrm{v}$ group, which means that symmetric $(\chi=1)$ and anti-symmetric $(\chi=-1)$ field solutions (with respect to the concerned symmetry axes) do not interact. In Fig. 16b some possible field symmetries are sketched. If, at a certain frequency, only field solutions with the sketched symmetry are supported by the waveguides and resonator, a coupling may exist only between the in- and outl-waveguide and not 
between the in- and out2-waveguide. The projection of the resonator field (antisymmetric with respect to the $\sigma_{3}$ axis) onto the fields of the waveguides shows that the in- and outl-waveguide have a field overlap with the resonator field, but the resonator field has no overlap with the field of the out 2 waveguide owing to their symmetries. Therefore the transmission of light to this waveguide is prohibited.

The dispersion curves and symmetries of the modes that are supported by the waveguide and resonator have been calculated with 2D eigenstate simulations (using the plane wave expansion method [27]) for the case of either the $\alpha$ or $\beta$ hole filled. The results are shown in Fig. 17a. We see that there are two frequency ranges in which only a $\chi=1$ mode is available for the $\mathrm{W} 1$ waveguide, and in these regions two resonator states with a $\chi=-1$ symmetry are present, which can be used to obtain switching behavior. Fig. $17 \mathrm{~b}$ gives the results from 2D finite-difference time-domain (FDTD) simulations [28] of the transmission of light through the device for the frequency corresponding to the 'operation point' (see Fig. 3a), which shows that this type of device can in principle work as a (wavelength routing) switch. For the simulations of Figs. 3a and $3 \mathrm{~b}$ we have used lattice parameter $\Lambda=440 \mathrm{~nm}$, hole radius $r=135 \mathrm{~nm}$ and a slab-effective refractive index 2.9, corresponding to $240 \mathrm{~nm}$ thick silicon on silica at a wavelength of $1550 \mathrm{~nm}$. The results are applicable to other Si layer thicknesses near this value and shift with respect to frequency as the corresponding effective index changes.

By making the cavity symmetric ( $\alpha$ and $\beta$ filled), we can obtain $3 \mathrm{~dB}$ splitting of light from the input to both output channels. In order to test the optical design, we fabricated both symmetric and asymmetric devices, which differ only by the absence or presence, respectively, of an additional hole in the outl branch, so that device operation in both the symmetric and the asymmetric state could be studied without mechanical actuation.

It is known that using the effective index method for 2D calculations of the properties of photonic crystal slabs leads to results that are red-shifted in frequency with respect to the results from full three-dimensional calculations [3]. By estimating the error in the effective index, scaling the results from Fig. 17, and keeping the $\Lambda / r$-ratio approximately the same, we arrived at parameters $\Lambda=420 \mathrm{~nm}$ and $r=128 \mathrm{~nm}$, in order to obtain an operating point at $1550 \mathrm{~nm}$ wavelength. The actual hole diameter depends on the lithographic exposure dose, and as an exposure dose sweep is performed in the fabrication process, we obtained structures with varying hole diameters; ranging approximately + and $-20 \mathrm{~nm}$ around the target value.

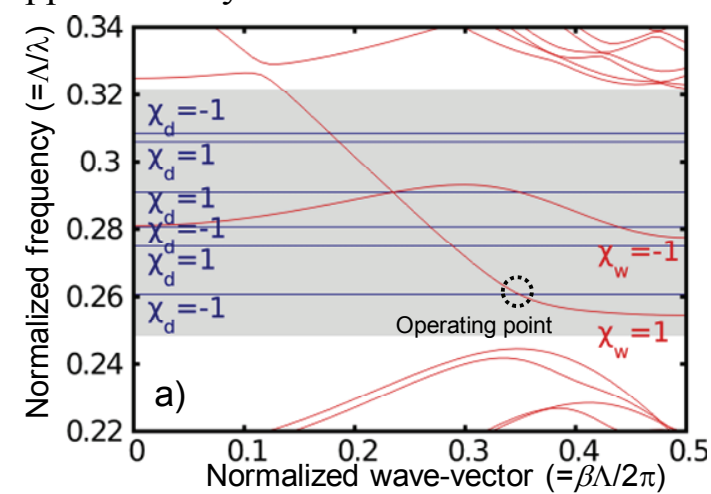

a)

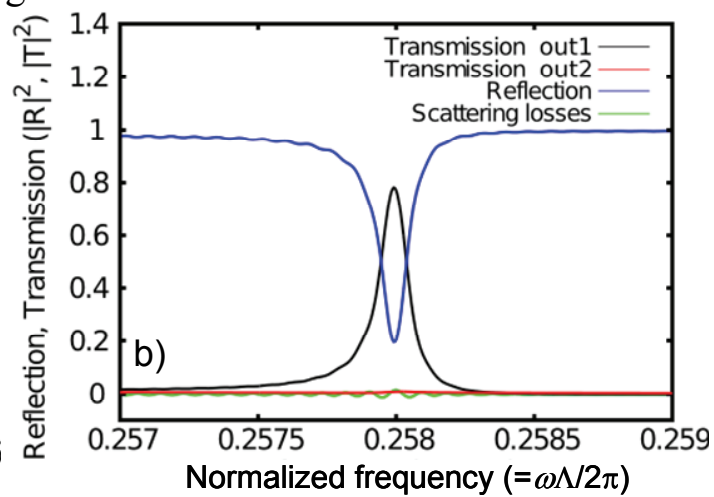

b)

Fig. 17. a) Dispersion relations of the photonic crystal waveguides. Also shown are the localized states of the cavity (horizontal lines) within the photonic band gap (grey band) of the bulk crystal. $\chi_{\mathrm{w}}$ and $\chi_{\mathrm{d}}$ give the 
Compact silicon photonic devices for MEMS-actuated optical switching

field symmetries for the waveguide fields and resonator fields, respectively. b) Reflection and transmission spectra of the device.

\subsubsection{Experimental}

The photonic crystal structures were fabricated in a 193-nm deep-UV lithography process by using the silicon photonics platform established at IMEC, Leuven. The device layer of the SOI wafer is a 220-nm thick silicon layer, with underneath it a 2- $\mu \mathrm{m}$ thick $\mathrm{SiO}_{2}$ layer. The PhC structure is formed by etching through the $\mathrm{Si}$ layer and stopped to the $\mathrm{SiO}_{2}$ layer. Fig. 18a shows a SEM image of one of the fabricated PhC structures. Light was coupled into the PhC device using a vertical fiber coupling method [29]. As a light source we used an Agilent 8164B tunable laser at an output power of $1 \mathrm{~mW}$. All the measured transmission spectra have been normalized with respect to a straight $\mathrm{Si}$ photonic wire waveguide, similar to the access waveguides (fiber-to-fiber). This removes the grating-coupler and access waveguide induced wavelength dependent loss $(16 \mathrm{~dB} \pm 2$ $\mathrm{dB}$ at the wavelength of maximum coupler transmission) from the data.

Fig. $18 \mathrm{~b}$ shows the measured transmission spectrum (hole $\beta$ filled) and the result of a corresponding 2D FDTD simulation. For the simulation we assumed a hole diameter of $250 \mathrm{~nm}$ (as estimated from the SEM image for the fabricated device) and an effective index of 2.82, while using a grid spacing of $10 \mathrm{~nm}$. The simulated curve has been fitted to the experimental data by shifting it in horizontal direction by $-23 \mathrm{~nm}$ and in vertical direction by $-7 \mathrm{~dB}$. The horizontal shift corresponds to effective index fitting, compensating the red shift caused by the effective index method. The vertical shift is due to out of plane losses, which the 2D simulation cannot account for. The typical loss of a W1 PhC waveguide of a corresponding length $(24 a)$, at a wavelength of $1560 \mathrm{~nm}, 20 \mathrm{~nm}$ below the mode cut-off (see the operating point indicated in Fig. 13a), is $14 \mathrm{~dB} \pm 2 \mathrm{~dB}$. Comparing this number to the maximum measured transmission, we conclude that the excess loss of the Y-junction is approximately $6 \mathrm{~dB}$, close to the 7-dB vertical shift mentioned above.

Further away from the mode cut-off, the $\mathrm{W} 1 \mathrm{PhC}$ waveguide loss is $5 \pm 1 \mathrm{~dB}$. Therefore a large part of the $\mathrm{Y}$-junction device loss at the operating point can be attributed to the slow light propagation in the $\mathrm{PhC}$ waveguide [9-10]. This can be seen from Fig. 13a: the slope of the $(\chi=1)$ dispersion curve of the $\mathrm{W} 1$ mode is decreasing from the operating point towards the mode cut-off. This in turn means that the group velocity of the light is moderately decreasing in this region, corresponding to increase of loss. 

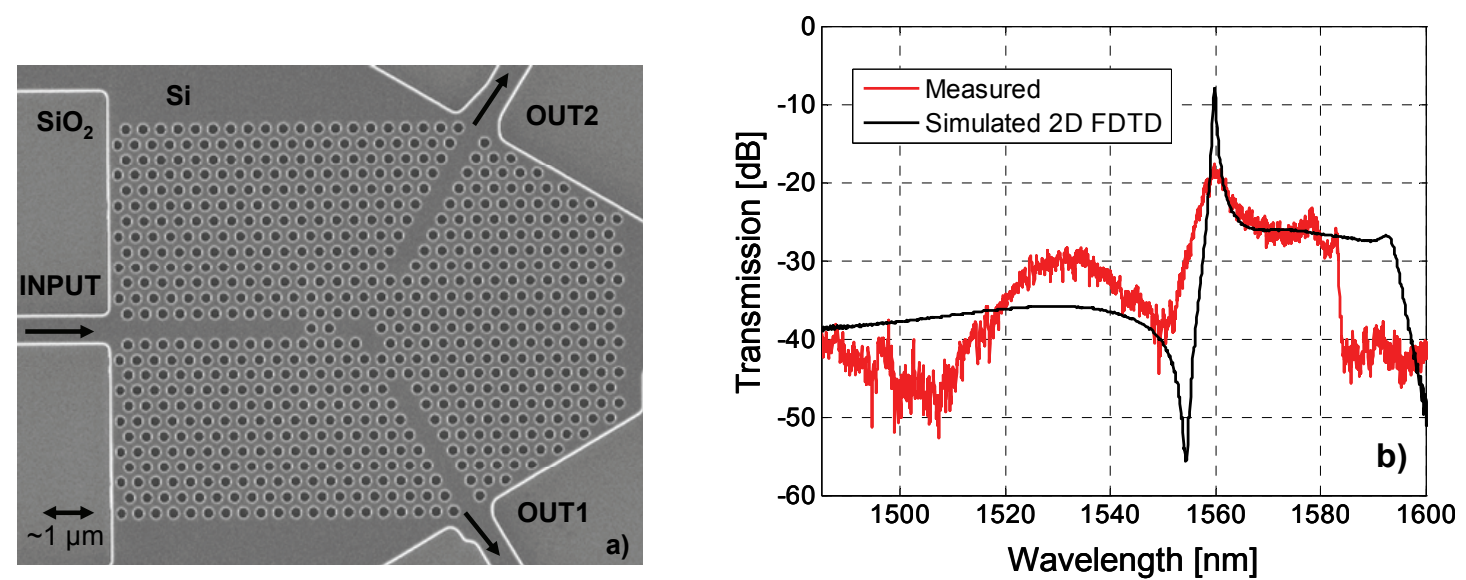

Fig. 18 a) SEM image (top view) of the PhC Y splitter. b) Measured and simulated transmission spectra for the asymmetric cavity design; transmission from the input to the outl waveguide branch, using the normalization procedure mentioned in the main text.

Fig. 19a shows a measured transmission spectrum of the asymmetric cavity (hole $\beta$ filled). It can be seen that at resonance most of the light is coupled through the cavity to the out1 waveguide branch, operating similar to a Fabry-Perot resonator. The on/off ratio at $1560 \mathrm{~nm}$ between ports outl and out2 is about $12 \mathrm{~dB}$. Fig. $19 \mathrm{~b}$ shows that the resonance wavelength is blue-shifted by approximately $13 \mathrm{~nm}$ for a $10 \mathrm{~nm}$ increase in hole diameter.

Fig.s 20a and 20b show the measured and simulated transmission spectra of the symmetric-cavity Y splitter in the optical C-band. In this case splitting of the optical power occurs in the cavity, and the light at a resonance wavelength is distributed between the output branches. We note that the design is very critical with respect to cavity symmetry, both experimentally and numerically. Even a slight deviation from the symmetric structure -which in our simulations was caused by a slightly misaligned FDTD calculation grid- was found to result in uneven coupling to the out 1 and out 2 ports.
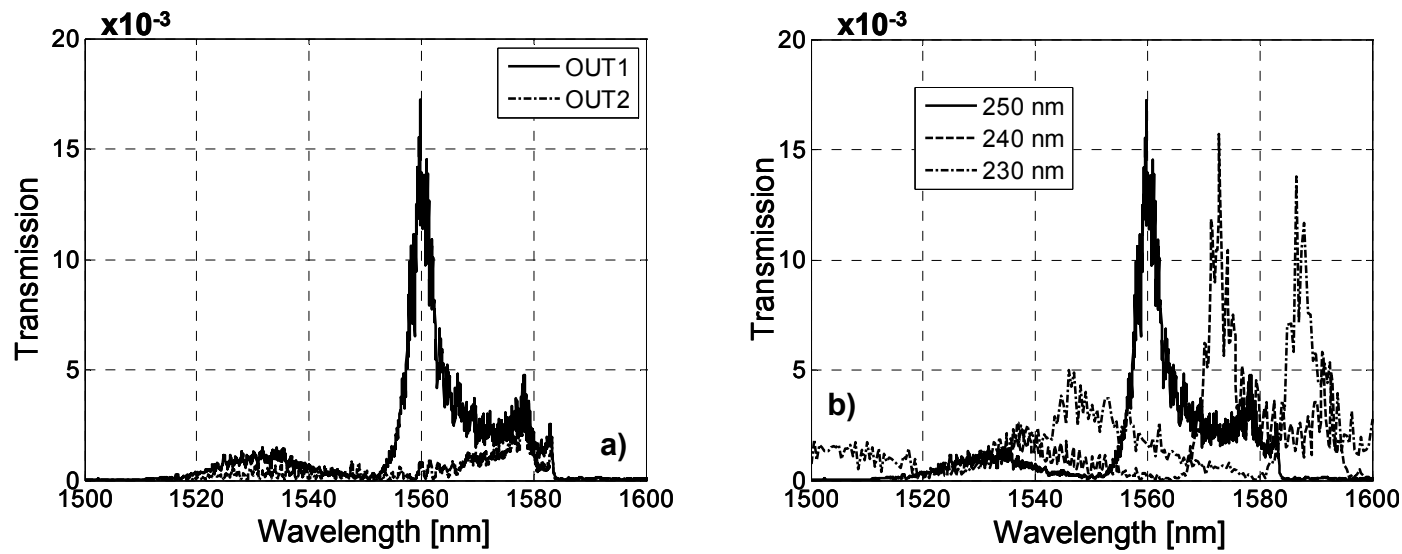

Fig. 19. Measured transmission spectra of the asymmetric cavity (with respect to the $\sigma_{1}$ axis). a) At out 1 and out 2 ports; b) at out 1 , for 3 different values of the $\mathrm{PhC}$ hole diameter. 

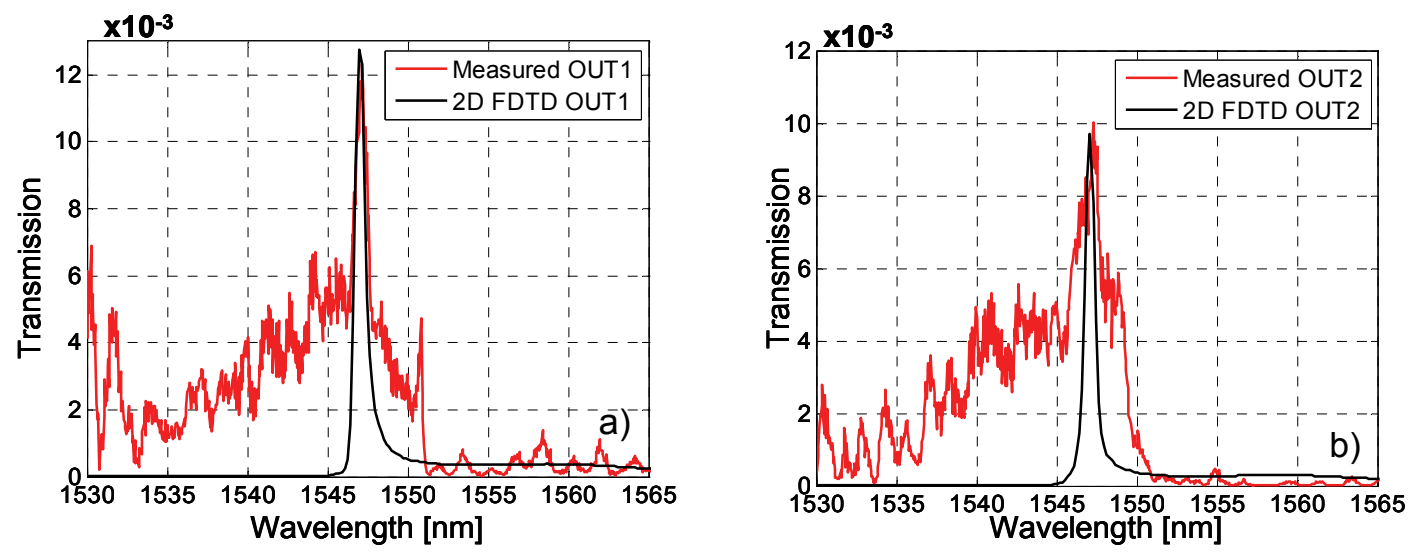

Fig. 20. Measured and simulated transmission spectra for the symmetric cavity (both holes filled). a) out1 port. b) out 2 port.

\subsubsection{Conclusions}

A photonic crystal cavity-based $\mathrm{Y}$ splitter has been designed for optical switching application. Its operation has been verified by simulations and experiment. By filling one of two specific holes with slab material, the symmetry of the device can be switched, thus selecting one of two output ports. A 12-dB on/off ratio between the output ports was measured. The optical losses of the device $(20 \mathrm{~dB})$, are assumed to originate mostly from the slow light effects, which cause increased out-of-plane scattering. It turns out to be challenging to achieve precisely symmetric light transmission between the output ports, since that requires the cavity to be perfectly symmetric. Therefore the device should preferably be operated as on/off switch in one of its asymmetric states.

\subsection{Waveguide grating and ring-resonator-based designs}

This section introduces the wavelength routing principles of waveguide grating and ringresonator-based designs. Both devices are based on directional coupling of light from one waveguide to the other one. A brief theoretical discussion on directional coupling is given. The schematic layouts of the devices are shown along with the measurement results.

\subsubsection{A directional coupler}

If the lateral separation between two waveguides is small, as in the middle section of Fig 21 , the light may couple between the waveguides. The coupling is based on the evanescent field tail that extends outside the waveguide boundaries and partially overlaps with the other waveguide. The energy can flow either way between the waveguides. The operation of the directional coupler can be theoretically described using coupled mode theory [30]; it is discussed in detail e.g. in [31]. The result of this analysis is that the following equation can be written for the modal field amplitudes of a symmetrical directional coupler: 


$$
\left[\begin{array}{l}
E_{o 1} \\
E_{o 2}
\end{array}\right]=e^{j \beta l_{c}}\left[\begin{array}{cc}
\cos \left(\kappa l_{c}\right) & j \sin \left(\kappa l_{c}\right) \\
j \sin \left(\kappa l_{c}\right) & \cos \left(\kappa l_{c}\right)
\end{array}\right]\left[\begin{array}{l}
E_{i 1} \\
E_{i 2}
\end{array}\right],
$$

in which $l_{c}$ is the (effective) length of the coupled section, and $\kappa$ is the coupling coefficient defined as:

$$
\kappa=\frac{\pi}{2 L_{c p}}
$$

in which $L_{c p}$ is the minimum coupling length needed for complete power transfer from one waveguide to the other, e.g., from INI to OUT2. The $l_{c}$ determines the power split ratio of a coupler, e.g. for a $3 \mathrm{~dB}$ coupler $l_{c}=0.5 L_{c p}$.

Another way to consider the above is as follows. Assuming again that the waveguides are single mode, a symmetric directional coupler supports two system modes, which are respectively symmetric mode and antisymmetric with respect to the central axis $\left(\sigma_{2}\right)$ of the coupler, see Fig. 21. In the coupling region, where the gap between the waveguides is small enough, the evanescent field tails of these modes overlap and due to this interaction the propagation constants of these modes are different.

When light is launched into the "upper" waveguide of the directional coupler from IN1, then in the coupling region the system modes with propagation constant $\beta_{s}$ (for symmetric mode) and $\beta_{a}$ (for antisymmetric mode) exist. After a propagation distance of $L_{\text {cp }}$, by definition, they have accumulated a phase difference of $\pi$, so that the sum of the system modal fields now concentrates in the "lower" waveguide, and all the power is coupled from IN1 to OUT2 (IN2 to OUT1). Therefore, the following equation for the coupling length holds:

$$
L_{c p}=\frac{\pi}{\beta_{s}-\beta_{a}}=\frac{\lambda}{2\left(n_{s}-n_{a}\right)},
$$

in which $n_{s}$ and $n_{a}$ are the modal indicies of the symmetric and antisymmetric mode, respectivly.

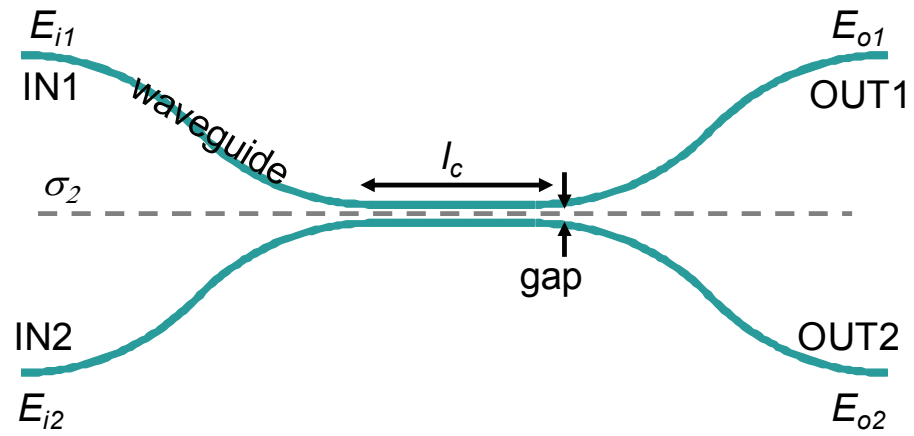

Fig. 21. A Schematic picture of directional coupler.

\subsubsection{Waveguide-grating-based add-drop multiplexer}

Fig. 22 shows a schematic picture of a grating-based optical add-drop multiplexer (OADM) [32]. In this design there are two 3-dB directional couplers. The first one splits the light from the $I N$ port equally to two waveguide branches. Identical waveguide gratings are present in each branch. If the wavelength of the light is in the photonic 
stopband of these gratings, the light is reflected from the gratings back to the first 3-dB coupler. As a 3-dB coupler introduces 90 degree phase difference -as can be seen from (1)- the reflected light from the grating is now coupled completely to the OUT2 port. The light with a wavelength outside the stopband goes to the second 3-dB coupler, where the coupling continues and all the light is eventually coupled to the OUT1 port.

The 3-dB coupler design is based on equation (3). The waveguides were chosen to be $500-\mathrm{nm}$ wide silicon photonic wires separated by a $200-\mathrm{nm}$ gap. The effective indices of the symmetric and antisymmetric modes for this geometry were calculated using a $2 \mathrm{D}$ modesolver. From the calculated indices a coupling length $L_{\mathrm{cp}}$ of $52.97 \mu \mathrm{m}$ at a wavelength of $1550 \mathrm{~nm}$ is obtained from (3). The 3-dB coupling length is conveniently exactly half of the $L_{\mathrm{cp}}$, and a length of $26.5 \mu \mathrm{m}$ was chosen for the 3 -dB coupler section. The gratings were designed following the same procedure as discussed in section 2.2.

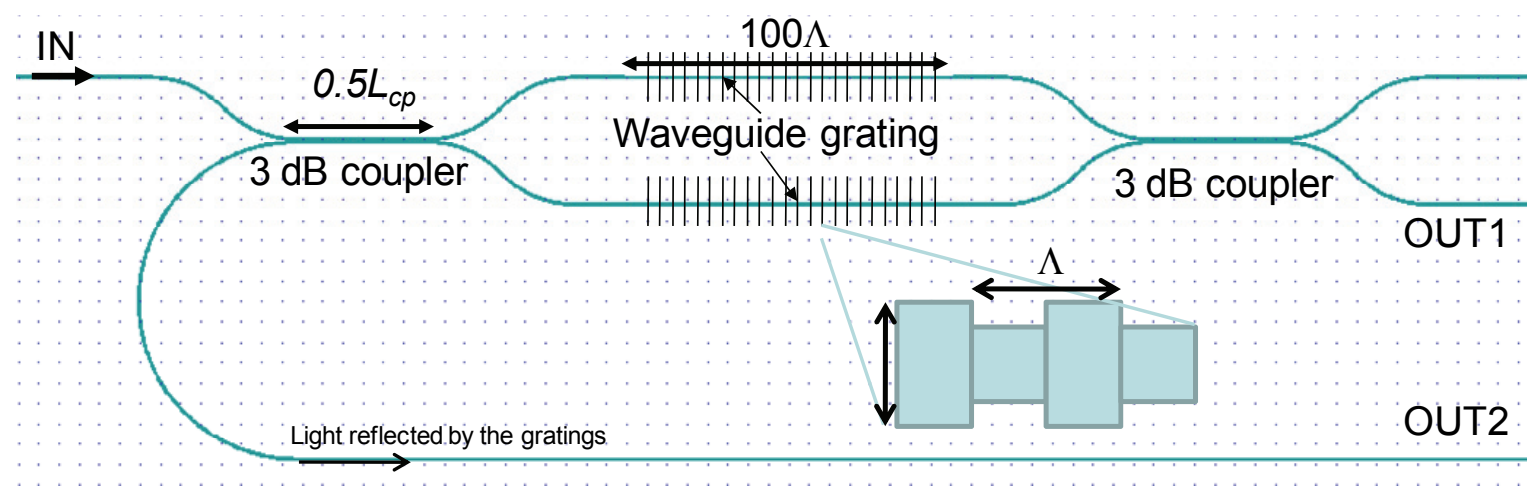

Fig. 22. A schematic picture of waveguide-grating-based wavelength router. Inset: schematic top view of the grating; see section 2.2 for details.

Fig. 23a shows measured transmission spectra of a grating-based wavelength selective router. The designed width of the grated waveguide is $320 \mathrm{~nm}$ and the grating is introduced by periodically varying the waveguide width by $20 \mathrm{~nm}$ in $450 \mathrm{~nm}$ periods (see section 2.2. for details). The length of the grated section is 100 periods $(45 \mu \mathrm{m})$.

A wavelength band with a width of approximately $8 \mathrm{~nm}$ with a center wavelength of $1506 \mathrm{~nm}$ is rejected from the OUT1 port by the grating and the light at these wavelengths propagates to the OUT2 port. The rejection ratio between the OUT1 and OUT2 ports is around $5 \mathrm{~dB}$ at wavelengths that are outside the stopband, while better than $10 \mathrm{~dB}$ on/off ratio is achieved at the stopband wavelengths.

Fig. $23 \mathrm{~b}$ shows measured transmission spectra of a similar grating structure in which the overall waveguide width is approximately $10 \mathrm{~nm}$ larger, which leads to stopband shift of $15 \mathrm{~nm}$. The waveguide width difference is caused by different photolithographic exposure dose, as discussed in 2.2.1. Due to this the waveguide width in the 3-dB coupler region, as well as the gap, are changed. This could change the properties of the directional coupler. However, the waveguide width change is compensated by the change in the gap: as the waveguide width increases and a mode becomes more confined in the waveguide, the gap between the waveguides decreases. According to our experience from experimental data; the waveguide width and the gap changes that result from the exposure dose difference cancel out one another's effect. This holds at least for values that are within reasonable range $( \pm 40 \mathrm{~nm})$ from the design target values. As a result, the 
operating wavelength range of the device (which is the stopband) can be conveniently tuned by the exposure dose during the fabrication.

Fig. 23c shows measured transmission spectrum of another variation of the device in which the waveguide width in the grating section is varied by $60 \mathrm{~nm}$. The index variation in the grating section is stronger than obtained with $20 \mathrm{~nm}$ width variation, and accordingly the stopband is wider and deeper. The optical C-band (1535-1560 nm) is almost completely rejected from the OUT1 port. For wavelengths from $1540 \mathrm{~nm}$ to 1555 $\mathrm{nm}$ better than $40 \mathrm{~dB}$ on/off ratio in transmitted power between OUT1 and OUT2 ports is achieved.

Efficient tuning of the whole grating spectrum requires a cantilever that entirely covers both gratings. As we have discussed in section 2.4.4, releasing a broad cantilever is challenging because a long release etching time is required. For this reason it is interesting to consider also the tuning of resonating Bloch modes. The transmission spectra that are measured at OUT2 port show clear dips at wavelengths that correspond to resonating Bloch modes of the gratings. At these dips the on/off ratio is better than $20 \mathrm{~dB}$. Due to the spatial distribution of these resonances [33] their properties can be tuned selectively with much narrower cantilevers, as discussed in section 2.1.4.
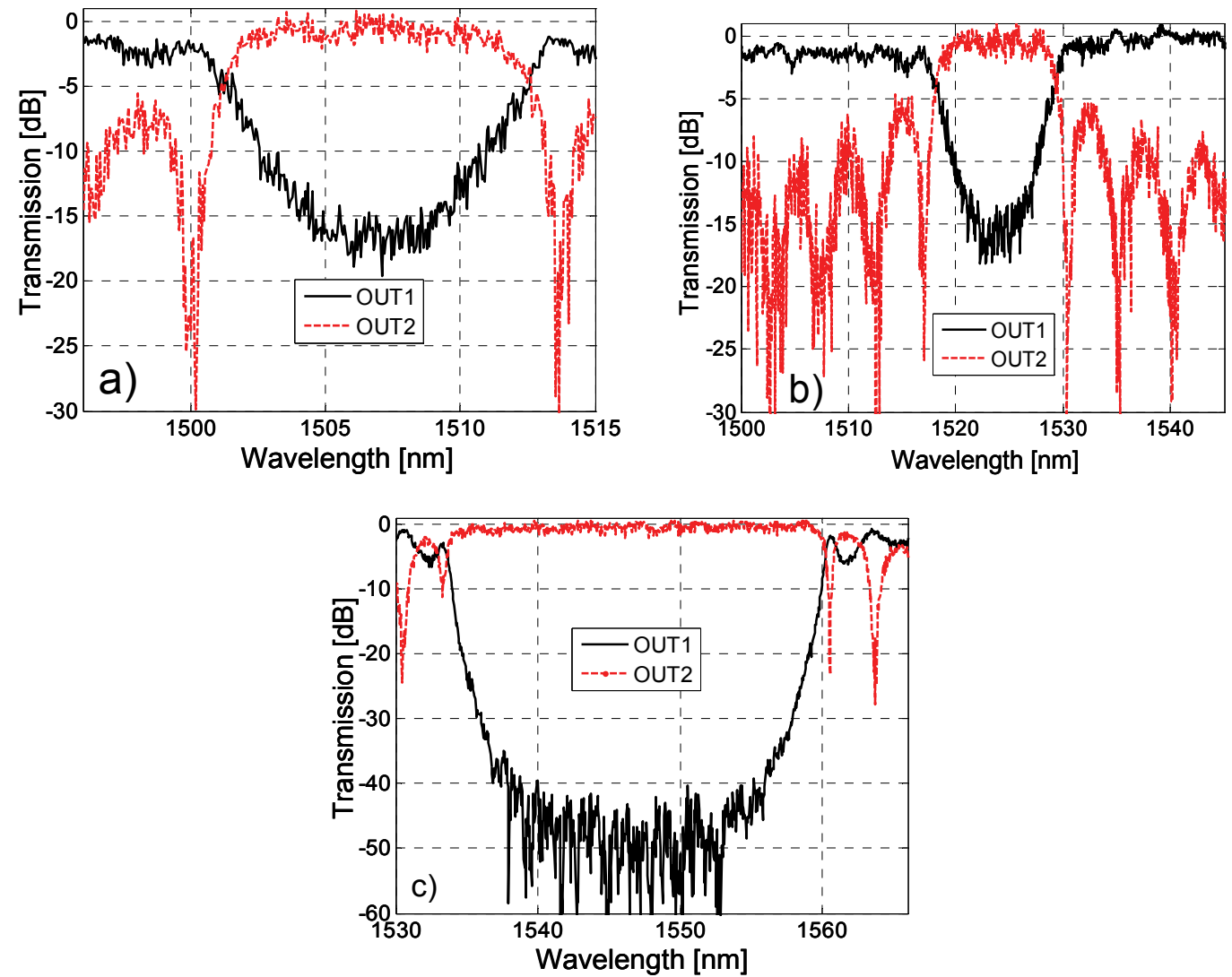

Fig. 23. Measured transmission spectra of different grating-based wavelength routers. a) Device that has a weak grating (width variation $20 \mathrm{~nm}$ ). b) Same as in a), but due to a different exposure dose the width of the waveguide structures is some $10 \mathrm{~nm}$ larger (see main text for details). c) Strong grating with a width variation of $60 \mathrm{~nm}$ (see section 2.2 for details of the gratings). 


\subsubsection{A ring resonator}

In a ring resonator, see Fig. 24a, a fraction of light is coupled from the input waveguide to the ring waveguide, using a directional coupler section, such as discussed in section 3.2.1. A part of the light that is coupled to the ring waveguide travels back to the point where it was coupled in. During this propagation the light accumulates a phase difference and if it arrives this point in phase match with respect to light in the input waveguide, due to constructive interference enhanced coupling of light to the ring occurs, leading to a resonance build up at the wavelengths that fulfill the phase match condition. In a steady state light power coupled to the ring at the resonance wavelength equals the power that the ring loses. If the ring is coupled symmetrically with two waveguides, as shown in Fig. $24 \mathrm{a}$, the light at the resonance wavelength is coupled from IN port through ring to OUT2 port. A detailed discussion about ring-resonators is given e.g. in [34].

The resonance wavelength of a ring resonator can be obtained from the following equation:

$$
\lambda_{\text {res }}=\frac{L_{\text {ring }}}{m} n_{\text {eff }},
$$

in which $L_{\text {ring }}$ is the round trip length of the ring and $m$ the cavity mode order ( $m=1$, $2 \ldots)$.

We have implemented racetrack-type ring resonators, coupled to adjacent waveguides, with varying coupling lengths $\left(l_{c}=12 \mu \mathrm{m}, 14 \mu \mathrm{m}\right.$ and $\left.16 \mu \mathrm{m}\right)$ and $\operatorname{ring}$ radii $(R=5 \mu \mathrm{m}$ and $10 \mu \mathrm{m})$. The waveguide is a single mode $\mathrm{Si}$ photonic wire waveguide and in the coupling region the gap between the waveguides is designed to be $200 \mathrm{~nm}$. A typical transmission spectrum measured for a 193-nm DUV-lithography-fabricated ringresonator is shown in Fig. 24b. The chosen coupling lengths and radii result is FSRs from 5 to $13 \mathrm{~nm}$, with Q-values better than 10000 and resonance dip depths of $>8 \mathrm{~dB}$.

The area required by the ring-resonator is approximately $40 \mu \mathrm{m} \times 30 \mu \mathrm{m}$, while for $\mathrm{PhC}$ cavity devices it is about $20 \mu \mathrm{m} \times 10 \mu \mathrm{m}$. The downside of the ring resonator is that it does not selectively drop only a single wavelength or wavelength range, but drops all the wavelengths that fulfill the resonance condition (4). The maximum free spectral range, i.e. the spectral distance between two adjacent resonances, for ring resonators fabricated in SOI technology and operating around wavelengths around $1500 \mathrm{~nm}$ is of the order of $10 \mathrm{~nm}$. The FSR increases for smaller rings, but unfortunately efficient coupling becomes more challenging the smaller the ring is. 

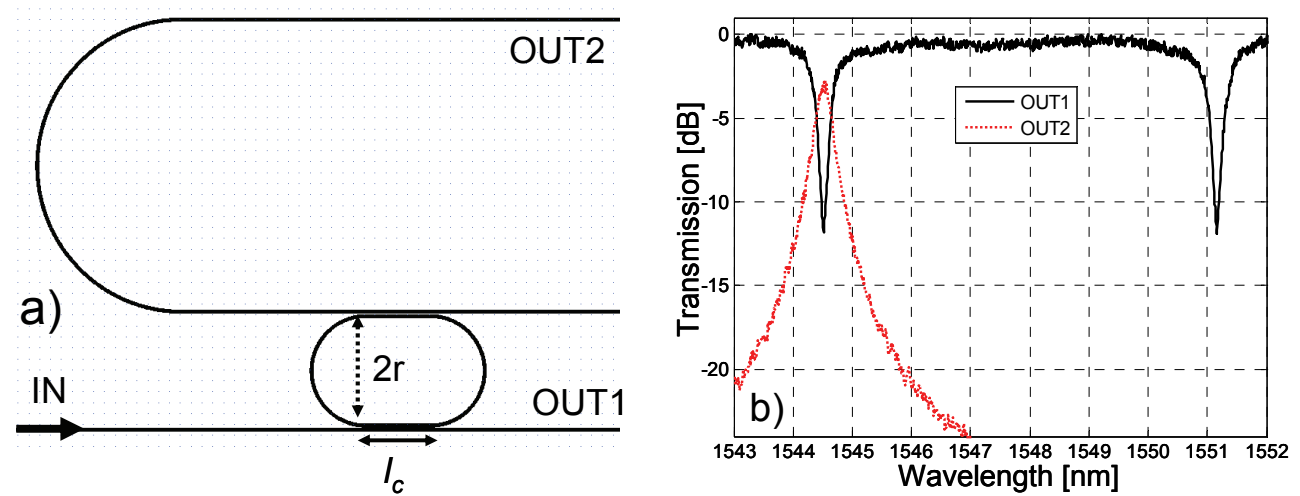

Fig. 24. a) A schematic top view of a ring-resonator-based wavelength router. In this so-called racetrack design, straight waveguide sections of length $l_{c}$ have been inserted in the ring in order to have a wellcontrolled and, if needed, strong coupling to the access waveguides. Light at the resonance wavelengths (4) is coupled through the ring to the OUT2 port. b) Measured transmission spectrum of a ring resonator with $r$ $=10 \mu \mathrm{m}, l_{c}=12 \mu \mathrm{m}$ and a coupler gap of $200 \mathrm{~nm}$. The transmission spectrum to the OUT2 port is measured for wavelengths $1543 \mathrm{~nm}$ to $1547 \mathrm{~nm}$.

\subsubsection{Conclusion}

Optical add-drop multiplexers, based on grated silicon photonic wires, have been designed and characterized. These devices are considerably larger in size than the $\mathrm{PhC}$ based devices discussed in the previous sections. However, the design less critical to process parameter variations and e.g. if the designed waveguide dimensions differ slightly the device still functions, yet at different wavelength (see Fig. 23a and b). In additional the optical loss is much smaller than in the $\mathrm{PhC}$ devices.

At the stopband wavelengths it is possible to get over $40 \mathrm{~dB}$ on/off ratio between the output ports over a wide wavelength range $(25 \mathrm{~nm})$. However, the on/off ratio is poor ( $5 \mathrm{~dB}$ ) outside the stopband region, except for the wavelengths that correspond to resonating Bloch modes. Therefore the spectral shift of the entire stopband does not provide a well defined output signal to OUT2 port. The spectral features of the resonating Bloch modes can be tuned with a narrow cantilever, that is easier to fabricate than a broad one that covers the whole grating (as discussed in chapter 2). Since this type of tuning of the Bloch modes also provides well defined output signals, it is preferred over tuning the entire stopband.

The ring resonator is robust and compact design, although for channel dropping it has the disadvantage that not only one specific wavelength is dropped. Large FSR would require small coupling length and small radii, resulting in shallow and low Q-value resonances. We observed a FSR of $\sim 13 \mathrm{~nm}$ by using a ring that has a radius of $5 \mu \mathrm{m}$ and coupling length of $12 \mu \mathrm{m}$. The loss of the ring resonators is less than $3 \mathrm{~dB}$.

\subsection{Discussion}

The performance requirements for a switch depend on the application. If for example, the switch is used to select a light path to one of several optical sensors, the performance requirements are somewhat different from those needed to perform high-speed packetswitching in an optical telecom network.

In Table 1 we compare the performance of our optical devices with respect to several parameters that are used to characterize the suitability of a switch for its indented 
applications. The PhC-cavity-based channel-drop filter (Sect. 3.1.2) is labeled PhC1, the $\mathrm{PhC}$-cavity-based Y splitter (Sect. 3.1.3) is named $\mathrm{PhC}$ 2, and waveguide grating (Sect. 3.2.2) and ring-resonator-based (Sect. 3.2.3) wavelength routers are shortened to WG and $\mathrm{RR}$, respectively.

The extinction ratio is the output power ratio of the output ports. Insertion loss is the loss that is caused by the optical switching element. The size of a device is its footprint on a chip. For waveguide grating and ring-resonator devices, also the area required by the access waveguides, that are essential part of the device, are taken into account. Bandwidth is the $3-\mathrm{dB}$ spectral width of the wavelength band that is routed by the device.

Table 1. Comparing of different devices designed for MEMS-actuated wavelength selective switching.

\begin{tabular}{|l|c|c|c|c|}
\hline & PhC1 & PhC2 & WG & RR \\
\hline Extinction ratio & $20 \mathrm{~dB}$ & $12 \mathrm{~dB}$ & $15-40 \mathrm{~dB}$ & $12-30 \mathrm{~dB}$ \\
\hline Insertion loss & $5-15 \mathrm{~dB}^{*}$ & $18 \mathrm{~dB}$ & $<2 \mathrm{~dB}$ & $<3 \mathrm{~dB}^{*}$ \\
\hline Size & $100 \mu \mathrm{m}^{2}$ & $100 \mu \mathrm{m}^{2}$ & $6000 \mu \mathrm{m}^{2}$ & $1200 \mu \mathrm{m}^{2}$ \\
\hline Bandwidth & $2-3 \mathrm{~nm}$ & $2-3 \mathrm{~nm}$ & $15-25 \mathrm{~nm}^{* *}$ & $<0.15 \mathrm{~nm}$ \\
\hline
\end{tabular}

*Depends on the wavelength, see section 5.2.4. **Depends on the grating.

Switching speed is one of the key properties of an optical switch. According to [35], the fastest commercially available switches are semiconductor-optical-amplifier-based on/off switches with a switching speed of $1 \mathrm{~ns}$ and lithium niobate electro-optical on/off switch with a switching speed of $10 \mathrm{ps}$. In [36], a switching rate of $8 \mathrm{kHz}$ is reported with an aluminum micro-bridge-actuated ring resonator. The speed of our MEMS-actuated optical switch depends on the micromechanical element; the resonance frequencies of our microcantilevers are in the $\mathrm{MHz}$ range [37], indicating a switching time of $\sim 1 \mathrm{~ns}$.

Another interesting property is the wavelength tuning range that can be achieved with the envisioned micromechanical tuning scheme; electrostatically actuated microcantilever that interacts with the evanescent field of the optical device. For a ring-resonator a wavelength tuning range of tens of nanometers is reported when actuated with an optical fiber tip [38]. The tuning range depends on how strongly the mechanical element interacts with the evanescent field. For waveguide devices this depends e.g. on the interaction length and on how close the mechanical element can be brought to the waveguide in controlled way.

In this thesis we also consider tuning in which the entire optical properties of the device are changed. In this case the microcantilever has submicrometre tips, which will enter the holes of a $\mathrm{PhC}$ and radically change its operation (section 4.6.). This type of tuning is a bit more difficult to realize due to require nanometre scale tips, however much stronger response, than with just evanescent field perturbation, is expected. Experimental tuning results are given in chapter 4 .

\subsection{Conclusions}

Several optical designs for mechano-optically tuned wavelength routing have been designed and realized. The PhC-based devices provide theoretically the most compact and ideal wavelength routing performance. However, the fabrication challenges to accurately produce these nanophotonical devices hamper the device performance. The result is non-ideal channel dropping. In addition, optical loss in $\mathrm{PhC}$ components is 
Compact silicon photonic devices for MEMS-actuated optical switching

relatively high, ranging from 5 to $20 \mathrm{~dB}$. The grating-based wavelength router is suitable to route wide wavelength bands and the optical loss of the device is within our measurement accuracy $\pm 2 \mathrm{~dB}$. Unfortunately, with $120 \mu \mathrm{m} \times 50 \mu \mathrm{m}$, the footprint of the device is quite large. The ring resonator performs well, and, according to our experimental data, is least vulnerable to fabrication process parameter variations. The device is also nearly as compact as the PhC-based routers. It has, however a limited free spectral range, which is disadvantageous for certain applications. 


\section{References}

[1] J. Singh, "Semiconductor optoelectronics: physics and technology", McGraw-Hill 1995.

[2] L.G. Ferreira and M.A.A. Pudenzi, "Waveguiding in a dielectric medium varying slowly in one transverse direction", Journal of the Optical Society of America, vol. 71, pp. 1377-1380, 1981.

[3] O.V. Ivanova, R. Stoffer, L. Kauppinen, and M. Hammer, "Variational effective index method for 3D vectorial scattering problems in photonics: TE Polarization", in Proc. PIERS 2009 Moscow pp. 10381042, 2009.

[4] J.D. Joannopoulos, S.G. Johnson, J.N. Winn, and R.D. Meade, "Photonic crystals: molding the flow of light", Princeton University Press, 2008.

[5] S.G. Johnson and J.D. Joannopoulos, "Block-iterative frequency-domain methods for Maxwell's equations in a planewave basis," Optics Express, vol. 8, pp. 173-190, 2001.

[6] C.G. Bostan, "Design and fabrication of quasi-2D photonic crystal components based on silicon-oninsulator technology", PhD thesis, University of Twente, Enschede, 2005.

[7] W. Bogaerts, "Nanophotonic Waveguides and photonic crystals in silicon-on-insulator", PhD thesis, Universiteit Gent 2004.

[8] S. Hughes, L. Ramunno, J.F. Young, and J.E. Sipe, "Extrinsic Optical Scattering Loss in Photonic Crystal Waveguides: Role of Fabrication Disorder and Photon Group Velocity," Phys. Rev. Lett. vol. 94, 033903, 2005.

[9] L.O'Faolain, T.P. White, D.O’Brien, X. Yuan, M.D. Settle, and T.F. Krauss, "Dependence of extrinsic loss on group velocity in photonic crystal waveguides," Opt. Exp., vol. 15, no. 20, pp. 13129-13138, 2007.

[10] Y. Akahane, T. Asano, B-S. Song, and S. Noda, "High-Q photonic nanocavity in a two-dimensional photonic crystal”, Nature, vol. 425, pp. 944-947, 2003.

[11] A. Yariv, Y. Xu, R.K. Lee, and A. Scherer, "Coupled-resonator optical waveguide: a proposal and analysis", Optics Letters, vol. 24, pp. 711-713, 1999.

[12] P. Sanchis, J. Marti, B. Luyssaert, P. Dumon, P. Bienstman, and R. Baets, "Analysis and design of efficient coupling in photonic crystal circuits", Optical and Quantum Electronics, vol. 37, pp. 133-147, 2005.

[13] P. Sanchis, P. Bienstman, B. Luyssaert, R. Baets, and J. Marti, "Analysis of butt coupling in photonic crystals", IEEE Journal of Quantum Electronics, vol. 40, pp. 541-550, 2004.

[14] S. Fan, P.R. Villeneuve, J.D. Joannopoulos M.J. Khan, C. Manolatou, and H.A. Haus, "Theoretical analysis of channel drop tunneling processes", Physical Review B, vol. 59, pp. 15882-15892, 1999.

[15] E. Drouard, H.T. Hattori, C. Grillet, A. Kazmierczak, X. Letartre, P. Rojo-Romeo, and P. Viktorovitch, "Directional channel-drop filter based on a slow Bloch mode photonic crystal waveguide section", Optics Express, vol. 13, pp. 3037-3048, 2005.

[16] S. Fan, P.R. Villeneuve, J.D. Joannopoulos, and H.A. Haus, "Channel drop filters in photonic crystals", Optics Express, vol. 3, pp. 4-11, 1998.

[17] A. Adibi, R.K. Lee, Y. Xu, A. Yariv, and A. Scherer, "Design of photonic crystal optical waveguides with singlemode propagation in the photonic bandgap", Electronics Letters, vol. 36, pp. 1376-1378, 2000 .

[18] Silicon Photonics Platform, http://www.epixfab.eu/

[19] M. Notomi, A. Shinya, S. Mitsugi, E. Kuramochi, and H-Y. Ryu, "Waveguides, resonators and their coupled elements in photonic crystal slabs", Optics Express, vol. 12, pp. 1551-1561, 2004.

[20] S.Y. Lin, E. Chow, J. Bur, S.G. Johnson, and J.D. Joannopoulos, "Low-loss, wide-angle Y splitter at $\sim 1.6-\mu \mathrm{m}$ wavelengths built with a two-dimensional photonic crystal," Opt. Lett., vol. 27, no. 16, pp. 1400-1402, 2002.

[21] H. Takeda and K. Yoshino, "Tunable light propagation in Y-shaped waveguides in two-dimensional photonic crystals utilizing liquid crystals as linear defects,” Phys. Rev. B, vol. 67, 073106, 2003.

[22] K. Inoue, Y. Sugimoto, N. Ikeda, Y. Tanaka, K. Asakawa, H. Sasaki, and K. Ishida, "Ultra-small photonic-crystal-waveguide-based Y-splitters useful in the near-infrared wavelength region,” Jpn. J. Appl. Phys., vol. 43, no. 4A, pp. L 446-L 448, 2004. 
[23] T. Takahata, K. Hoshino, K. Matsumoto, and I. Shimoyama, "Transmittance tuning of photonic crystal reflectors using an AFM cantilever,” Sens. Actuators A 128 , pp. 197-201, 2006.

[24] W.C.L. Hopman, K.O. van der Werf, A.J.F. Hollink, W. Bogaerts, V. Subramaniam, and R.M. de Ridder, "Modeling and experimental verification of the dynamic interaction of an AFM-tip with a photonic crystal microcavity," IEEE photon. Technol. Lett, vol. 20, pp. 57-59, 2008.

[25] M. Tinkham, Group theory and quantum mechanics. Dover Publications 2003.

[26] K. Sakoda. Optical properties of photonic crystals. Springer Series in Optical Sciences. Springer, 2nd edition, 2005.

[27] K.M. Leung and Y.F. Liu. "Full vector wave calculation of photonic band structures in face-centeredcubic dielectric media," Phys. Rev. Lett., vol. 65, pp. 2646-2649, 1990.

[28] A. Farjadpour, D. Roundy, A. Rodriguez, M. Ibanescu, P. Bermel, J.D. Joannopoulos, S.G. Johnson, and G. Burr, "Improving accuracy by subpixel smoothing in FDTD," Opt. Lett., vol. 31, pp. 29722974, 2006.

[29] D. Taillaert, F. van Laere, M. Ayre,W. Bogaerts, D. van Thourhout, P. Bienstman, R. Baets, "Grating couplers for coupling between optical fibers and nanophotonic waveguides," Jpn. J. Appl. Phys. vol. 45, pp. 6071-6077, 2006.

[30] A. Yariv, “Optical Electronics in modern communications”, Oxford University Press, 1997.

[31] C. Pollock and M. Lipson, "Integrated photonics", Kluwer Academic Publishers 2003.

[32] H. Yamada, T. Chu, S. Ishida, and Y. Arakawa, "Optical add-drop multiplexers based on Si-wire waveguides", Applied Physics Letters, vol. 86, 191107, 2005.

[33] W.C.L. Hopman, H.J.W.M. Hoekstra, R. Dekker, L. Zhuang, and R.M. de Ridder, "Far-field scattering microscopy applied to analysis of slow light, power enhancement, and delay times in uniform Bragg waveguide gratings", Optics Express, vol. 15, pp. 1851-1870, 2007.

[34] E.J. Klein, "Densely integrated microring-resonator based components for fiber-to-the-home applications", PhD thesis, University of Twente, 2007.

[35] R. Ramaswani and K.N. Sivarajan, "Optical networks: a practical perspective”, Morgan Kaufmann, 2002.

[36] G.N. Nielson, D. Seneviratne, F. Lopez-Royo, P.T. Rakich, Y. Avrahami, M.R. Watts, H.A. Haus, H.L. Tuller, and G. Barbastathis, "Integrated Wavelength-Selective Optical MEMS Switching Using Ring Resonator Filters", IEEE Photonics Technology Letters, vol. 17, pp. 1190-1192, 2006.

[37] S.M. Chakkalakkal Abdulla, J.W. Berenschot, M.J. de Boer, L.J. Kauppinen, R.M. de Ridder, and G.J.M. Krijnen, (2009) "Optimisation study of micro cantilevers for switching of photonic band gap crystals", In: Proceedings of the International Conference on Photonics in Switching 2009, pp. 15-19, 2009.

[38] P.T. Rakich, M. A. Popović, M.R. Watts, T. Barwicz, H.I. Smith, and E.P. Ippen, "Ultrawide tuning of photonic microcavities via evanescent field perturbation", Optics Letters, vol. 31, pp. 1241-1243, 2006. 


\section{Mechano-optical actuation}

In this chapter we present the experimental results of mechanically actuated integrated optical devices. The basics of electrostatic actuation of a cantilever are discussed followed by a section that briefly describes the fabrication process to monolithically integrate a microcantilever on an SOI photonics chip. The electrostatically actuated microcantilever was used to tune the optical properties of a ring-resonator and a photonic crystal waveguide. Experimental results from this tuning are given in sections 4.5 and 4.6. The chapter ends with conclusions.

\subsection{Introduction}

The mechanical actuation of integrated optical (IO) devices promises broad wavelength tuning range, low power consumption and rapid actuation cycles. These are particularly attractive properties when compared to commonly used thermo-optic (TO) based actuation [1]. Although this method provides a wide actuation range with moderate actuation speed $(700 \mathrm{kHz}$ in silicon [1]), the TO method is power hungry, making it less attractive for devices where many actuation elements are required.

Electro-optic modulation is another commonly used method to actuate IO devices. In silicon the Pockels or E/O Kerr effects are not practical and often the plasma dispersion method is used (see section 1.3.2.). In this method free carriers are injected to increase the conductivity that in turn causes the refractive index to change. Very fast modulation with this method can be achieved; signal rise time $<1 \mathrm{~ns}$ is reported in [2]. Although this method allows low voltage operation, the increased conductivity results in increased optical loss due to free carrier absorption. For this reason the method is not suitable to e.g. tune a resonance wavelength of a resonator.

In recent years the mechano-optical actuation of IO devices has been intensively investigated. Micromechanically actuated on/off switching of a ring resonator has been demonstrated with an aluminum microbridge that, when pulled close to the ring, kills the resonance due to increased optical loss [3]. Ultrawide tuning of a ring resonator has been demonstrated in [4] by using an optical fiber tip as a movable dielectric load that interacts with the evanescent field of the ring resonator. In addition there have been demonstrations of the interaction of the scanning tip of an atomic force microscope with nanophotonic structures $[5,6]$.

Such devices have potential applications in wavelength division multiplexed (WDM) telecom networks, where waveband switching is desired [7]. Potential future applications include modulation or switching in on-chip optical circuits, e.g. in optical interconnects between integrated electronic circuits. Particularly for on-chip applications, CMOScompatibility, low actuation voltage, and compact size are key properties.

In this chapter we demonstrate tuning of two different devices: a compact ring resonator and a photonic crystal waveguide, both fabricated on SOI. The tuning of the devices is obtained by perturbing the evanescent field with a monolithically integrated silicon nitride microcantilever. The actuation of the cantilever is done electrostatically, which has the advantage that the power required for actuation can be very low as the capacitance of the microcantilever is typically small, of the order of picofarads. 


\subsection{Electrostatic actuation of microcantilever}

In this section we briefly describe the physics of electrostatic actuation of a microcantilever. In a simplified approximation the mechanical properties of a cantilever can be modelled as a spring to which the electrostatic force is applied. For this modelling we consider a system as depicted in Fig. 1.
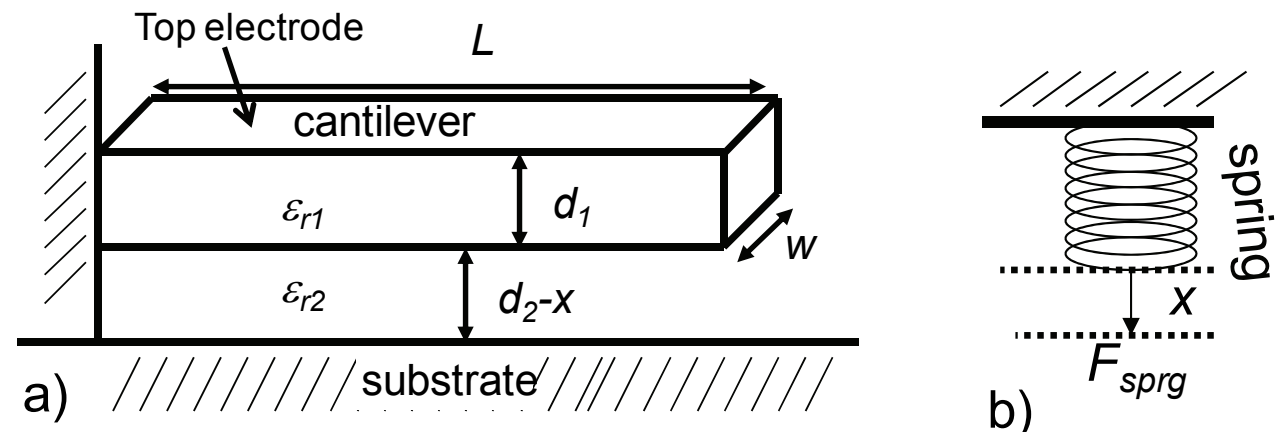

Fig. 1. a) Cantilever suspended above a substrate. The initial gap (without applied force) between cantilever and substrate is $d_{2}$. b) Spring model of the cantilever.

For this type of cantilever the spring constant can be calculated from the following equation [8]:

$$
k_{\text {sprg }}=\frac{Y w d_{1}^{3}}{4 L^{3}},
$$

in which $Y$ is Young's modulus, $w$ is the width, $d_{1}$ is the thickness and $L$ the is length of the cantilever. To stretch a spring distance $x$, see Fig. 1, a force of $F_{\text {sprg }}=k_{\text {sprg }} x$ is required.

To actuate the cantilever electrostatically, a voltage difference between the cantilever and the substrate is applied. From the electrical point of view the cantilever, shown in Fig. 1, can be seen as a capacitor to which the applied voltage $V$ causes a force $F_{c}$ according to:

$$
\begin{aligned}
& E_{c}=\frac{1}{2} C V^{2}=\frac{1}{2} A \frac{\varepsilon_{r 1} \varepsilon_{r 2} \varepsilon_{0}}{\left(d_{2}-x\right) \varepsilon_{r 1}+d_{1} \varepsilon_{r 2}} V^{2} \Rightarrow \\
& F_{c}=\frac{\partial E_{c}}{\partial x}=\frac{1}{2} A \varepsilon_{r 1} \frac{\varepsilon_{r 1} \varepsilon_{r 2} \varepsilon_{0}}{\left(\left(d_{2}-x\right) \varepsilon_{r 1}+d_{1} \varepsilon_{r 2}\right)^{2}} V^{2},
\end{aligned}
$$

in which $E_{c}$ is the energy stored in the capacitor, $C$ is the capacitance, $\varepsilon_{r 1}$ and $\varepsilon_{r 2}$ are the relative permittivities of the cantilever dielectric and the material in the gap, respectively, $\varepsilon_{0}$ is the vacuum permittivity, $d_{2}$ is the initial gap between the substrate and the cantilever, $A=w L$ is the surface area of the cantilever, and $x$ is the deflection caused by the applied voltage.

The spring force $F_{\text {sprg }}$ will be equal to the electric force $F_{c}$, arising from an applied voltage $V$ :

$$
\frac{1}{2} A \varepsilon_{r 1} \frac{\varepsilon_{r 1} \varepsilon_{r 2} \varepsilon_{0}}{\left(\left(d_{2}-x\right) \varepsilon_{r 1}+d_{1} \varepsilon_{r 2}\right)^{2}} V^{2}=k_{\text {sprg }} x
$$


Here we have assumed that the gravitational force can be neglected (due to the small mass of the cantilever). From (3) it is possible to calculate the required voltage to deflect the cantilever by an amount $x$ :

$$
V(x)=\sqrt{\frac{2 k x\left(\left(d_{2}-x\right) \varepsilon_{r 1}+d_{1} \varepsilon_{r 2}\right)^{2}}{\varepsilon_{r 1}^{2} \varepsilon_{r 2} \varepsilon_{0} A}} .
$$

It turns out that up to a critical voltage the deflection increases with voltage. However, the function $V(x)$ has a maximum $V_{p}$ where $\mathrm{d} V(x) / \mathrm{d} x=0$. At $V=V_{p}$, a pull-in phenomenon occurs: the cantilever becomes unstable and its deflection keeps increasing, even if the voltage is decreased. This typically results in catastrophic failure where the cantilever is pulled to the surface. As a rule of thumb, the deflection can be changed in a controlled way only over a range not exceeding $1 / 3$ of the total initial gap between the electrodes.

Fig. 2 shows the calculated voltage, from equation (4), as a function of the deflection $x$. For the cantilever we have used $k=3.24 \mathrm{~N} / \mathrm{m}$, which corresponds to the value that is found for a $1-\mu \mathrm{m}$ thick silicon nitride $\left(Y=280 \mathrm{GPa}, \varepsilon_{\mathrm{r} 1}=7.3\right)$ cantilever, having a length of $40 \mu \mathrm{m}$ and a width of $10 \mu \mathrm{m}$. The initial gap $\left(d_{2}\right)$ is chosen to be $400 \mathrm{~nm}$. It can be seen that a voltage of $6 \mathrm{~V}$ is required to defect the cantilever by $150 \mathrm{~nm}$, which is close to the maximum amount the cantilever can be deflected in controlled way.

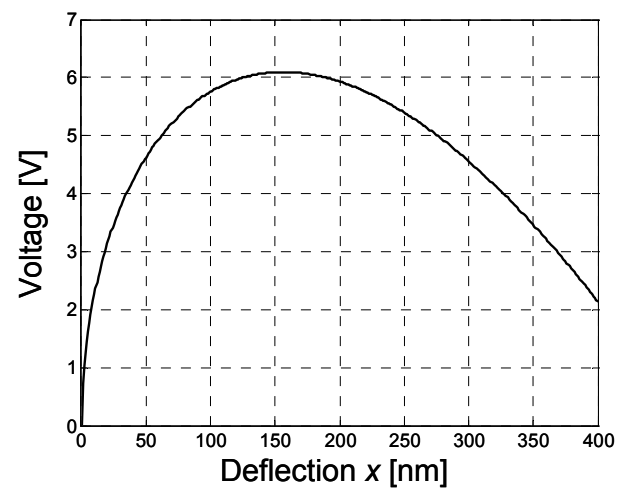

Fig. 2. Deflection response of cantilever (see text) to applied voltage.

\section{The actuation speed}

The speed of the cantilever can be estimated from its resonance frequency [8]:

$$
f=\frac{1}{2 \pi} \sqrt{\frac{k_{\text {sprg }}}{m_{0}}},
$$

in which the $m_{0}$ is the mass of the cantilever.

As a rule of thumb, the maximum actuation frequency is about half of this. However, there are factors that can slow down the speed. For our device the dominant limiting factor is the so-called squeeze force [9]. This force arises from the air trapped in the narrow gap between the cantilever and the substrate. This effect can be modelled as a damper-spring system. For a given cantilever it is possible to calculate damping ratio [10]: 


$$
\Gamma=\frac{\mu w^{2}}{4 \rho d_{2}^{3} d_{1} \pi f}
$$

in which $\mu$ is the air viscosity in the ambient atmosphere $\left(1.79 \cdot 10^{-5} \mathrm{Nsm}^{-2}\right)$ and $\rho$ is the density of the cantilever. From this damping ratio the time response of the system can be calculated. A highly damped system $(\Gamma>1)$ can be described in the Laplace domain by [10]:

$$
H(s)=\frac{K}{\left(1+s \tau_{1}\right)\left(1+s \tau_{2}\right)},
$$

in which $K$ is a constant, $s$ is a complex number and the time constants can be expressed as:

$$
\begin{gathered}
\tau_{1}=\frac{1}{\omega}\left(\Gamma+\sqrt{\Gamma^{2}-1}\right), \\
\tau_{2}=\frac{1}{\omega}\left(\Gamma-\sqrt{\Gamma^{2}-1}\right) .
\end{gathered}
$$

For the underdamped situation $(\Gamma<1)$, similar expressions can be found [11].

With the same cantilever parameters as used for calculating the results given in Fig. 2, and with $\rho=3440 \mathrm{~kg} / \mathrm{m}^{3}$ for silicon nitride, we calculate the step response of the system with two different gap values $d_{2}$. It can be seen from Fig. 3 that a smaller gap results in a slower response. From the graph we estimate switching frequencies of $25 \mathrm{kHz}$ (for 200 $\mathrm{nm}$ initial gap) and $225 \mathrm{kHz}$ (for $400 \mathrm{~nm}$ initial gap). The effect of the squeeze damping can be reduced by reducing the air pressure or designing the cantilever such that air can escape faster from the gap. This correspondingly increases the speed of the system. Based on the experimental data presented in [12-13] we expect orders of magnitude faster operation in low pressure conditions.

The other factor that can limit the speed of the actuation is the electrical RC delay that originates from charging and discharging of the capacitor through a contact resistance. As the capacitance of the microcantilever is typically small (of the order $1 \mathrm{pF}$ ), the effect of the contact resistance would need to be of the order $10 \mathrm{M} \Omega$ for the RC delay to become of the same order of magnitude as the squeeze film damping delay at atmospheric pressure.

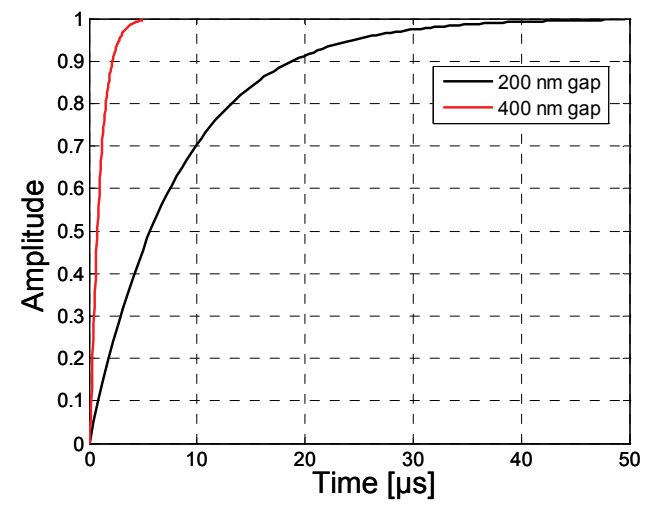

Fig. 3. The step response of a silicon nitride cantilever at two different initial gaps. 


\subsection{Monolithic integration of the microcantilever}

The optical devices, described in chapter 3, have been fabricated at the silicon photonics platform ePIXfab, established at IMEC, Leuven. The devices are fabricated on SOI wafers, containing a $220-\mathrm{nm}$ thick silicon device layer on top of a $2-\mu \mathrm{m}$ thick $\mathrm{SiO}_{2}$ layer. The ring resonator was formed by etching $200 \mathrm{~nm}$ into the Si device layer (leaving a thin Si slab in the etched areas); for photonic crystal devices, the device layer was completely etched through with a 220-nm etch depth. The smaller etch depth of the ring resonator was due to fabrication error.

A cross-section of the integrated mechano-optical structure is shown in Fig. 4. The microcantilevers were fabricated later on top of the optical structures by post-processing at the MESA+ labs. This post-processing starts with a deposition of 40-nm thick siliconrich silicon nitride ( $\mathrm{SiRN}$ ) layer, that functions as a protective layer during the etch steps that are performed later. For PhC devices, this layer is locally removed from the $\mathrm{PhC}$ holes, and at locations that will act as windows for underetching the $\mathrm{PhC}$ device, so that a membrane structure can be fabricated. Next follows the so-called sacrificial layer, for which we used $\mathrm{SiO}_{2}$. The thickness of this layer partially determines the initial gap between the optical device and the cantilever. A thickness of $200 \mathrm{~nm}$ was used for the ring-resonator, while $100 \mathrm{~nm}$ was selected for $\mathrm{PhC}$ device. The thinner layer for the $\mathrm{PhC}$ device is motivated by the desired formation of self-aligned tips that can enter the $\mathrm{PhC}$ holes (see section 4.6). The microcantilever is patterned into a $1-\mu \mathrm{m}$ thick SiRN layer that is deposited on top of the sacrificial layer. For electrostatic actuation a $58 \mathrm{~nm}$ thick chromium-gold layer is deposited on top of this $\mathrm{SiRN}$. The cantilever is patterned using photolithography, in which the pattern is transferred from a mask to the photoresist. The metal layers and the top SiRN layers are then etched according to these patterns (Fig. 4a). In a final step the cantilever is released by etching the sacrificial layer in buffered hydrofluoric acid (BHF) (Fig. 4b). The cantilevers that were used in the experiments are $10 \mu \mathrm{m}$ wide and $40 \mu \mathrm{m}$ long.

The main challenges in the cantilever fabrication are the control of the initial stress of the cantilever and of the etch processes that define and release the cantilever. A detailed discussion on the fabrication process and the mechanical properties of the cantilever will be published in [12-13].
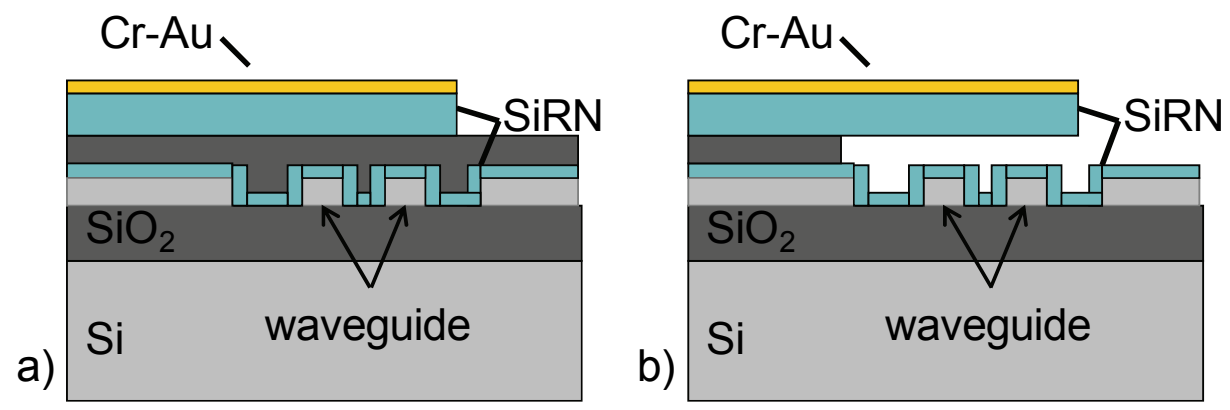

Fig. 4. Schematic cross-section of an integrated mechano-optical device. a) after cantilever patterning and before sacrificial etching. b) After sacrificial etching showing a cantilever suspended above an optical waveguide. 


\subsection{Measurement set-up}

Part of the measurement set-up is shown schematically in Fig. 5. The light is coupled to the device using grating couplers [14]. Transmission spectra were measured using a tunable laser and a photodetector module (Agilent 8164B and 81634B). The dynamic response was recorded by connecting the photodetector output to an oscilloscope. Contact probes, connected to a contact pad on the top surface of the cantilever and to the bulk silicon of the wafer, allowed electrical actuation of the cantilever. The contact resistance of the bulk silicon wafer was measured to be $\sim 10 \mathrm{k} \Omega$.

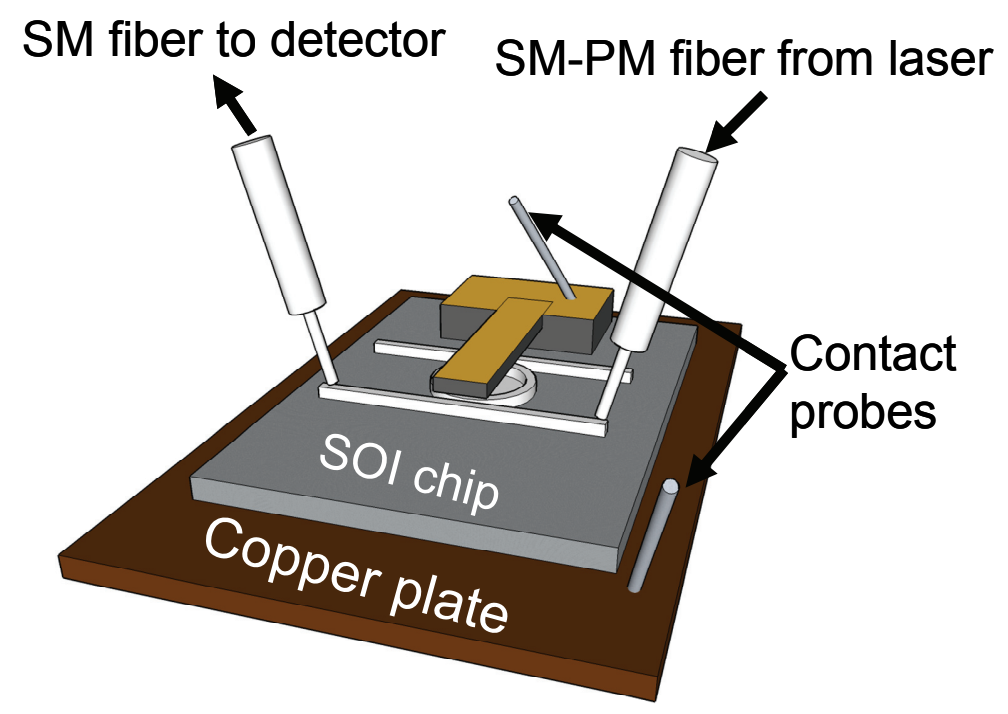

Fig. 5. Schematic of the setup used for optical transmission measurement and electrostatic actuation of the cantilever. Grating couplers, not shown in detail, couple light from a laser into the optical structure through single-mode (SM) polarization maintaining (PM) fiber, and from the structure to a photodetector.

\subsection{Micromechanically actuated ring resonator}

The resonance wavelength of the ring resonator, discussed in section 3.2.3, is changed by perturbation of the evanescent field of the ring waveguide, which increases the optical path length of the ring. If the interaction of the cantilever with the modal field causes a change $\Delta n_{\text {eff }}$ of the effective index over a perturbation length $L_{p e r t}$, this results in a change in the optical path length of $L_{\text {pert }} \Delta n_{\text {eff. }}$. For an optical ring resonance of order $m$, this leads to a shift of resonance wavelength $\Delta \lambda$, given by

$$
\Delta \lambda=\frac{L_{\text {pert }}}{m} \Delta n_{\text {eff }} .
$$

The magnitude of the effective index change depends on the overlap of the cantilever with the evanescent field. We have modelled the perturbation effect by using a twodimensional modesolver [15] to calculate $n_{\text {eff }}$ as a function of the air gap between the cantilever and the waveguide for TE-polarized light at a wavelength of $1550 \mathrm{~nm}$. As expected from the exponential tail of the evanescent field, the response is strongly nonlinear as shown in Fig. 6. It should be noted that due to pull-in phenomena the cantilever can be actuated in a controlled way only over $1 / 3$ of the initial distance between the electrodes (see section 4.2). This emphasizes the need to reduce the initial 
gap to obtain a large actuation range with a small cantilever deflection. If the initial gap between the cantilever and the waveguide is too large, the actuation effect is negligible (Fig. 6).

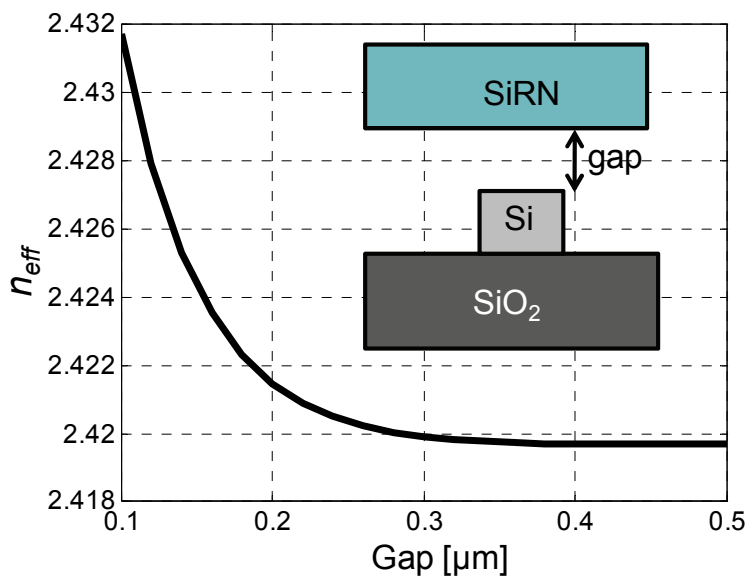

Fig. 6. Calculated effective index as a function of the air gap between the cantilever and the waveguide. Inset: cross-section used in the simulation.

We used 3D FDTD [16] to calculate the effect of the cantilever on directional coupling. In the calculation we considered a structure shown in Fig. 7. In this structure there is a pair of Si photonic wire waveguides (with a $200 \mathrm{~nm}$ gap between them), and a SiRN cantilever, modelled as a dielectric block above the waveguide pair. Wave propagation through the structure was simulated with and without the cantilever. We then compared the output powers of the ports out 1 and out2, at a wavelength of $1550 \mathrm{~nm}$. When the cantilever is present, with a $200 \mathrm{~nm}$ air gap between it and the waveguide pair, $2.435 \%$ of the input light is collected at out 2 port. Without the cantilever the number is $2.360 \%$. According to the FDTD results, $0.18 \%$ of the input light is lost due to the cantilever.

As a check for radiation loss induced by the cantilever, the extreme situation of the cantilever in direct contact with a single waveguide ( $0 \mathrm{~nm}$ gap) was analysed; $96.77 \%$ of the light was transmitted through the waveguide.
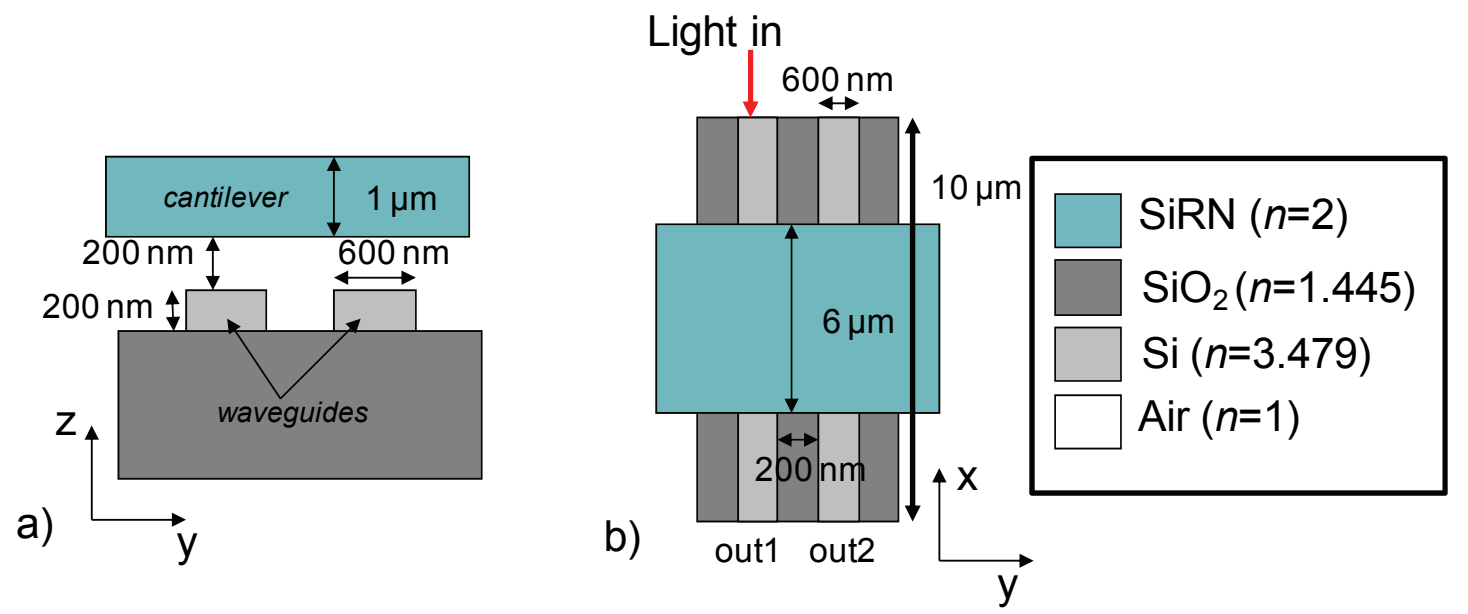

Fig. 7. Model of the structure used in 3D FDTD calculations to evaluate the effect of the cantilever on coupling. a) Cross-section; b) top view. 
The waveguide has been designed to be single mode for TE-polarized light at a wavelength of $1550 \mathrm{~nm}$. For single mode operation and efficient coupling, the waveguide width and the gap between the waveguides (in the coupling region) were designed to be $550 \mathrm{~nm}$ and $240 \mathrm{~nm}$, respectively. The ring resonator is a so-called racetrack design with a radius of $10 \mu \mathrm{m}$ and straight sections of $12 \mu \mathrm{m}$ length. The free spectral range of the ring is $6.6 \mathrm{~nm}$ (at $1550 \mathrm{~nm}$ ).

The microcantilever was monolithically integrated following the steps described in section 4.3. A scanning electron microscope (SEM) image of the integrated device is shown in Fig. 8.

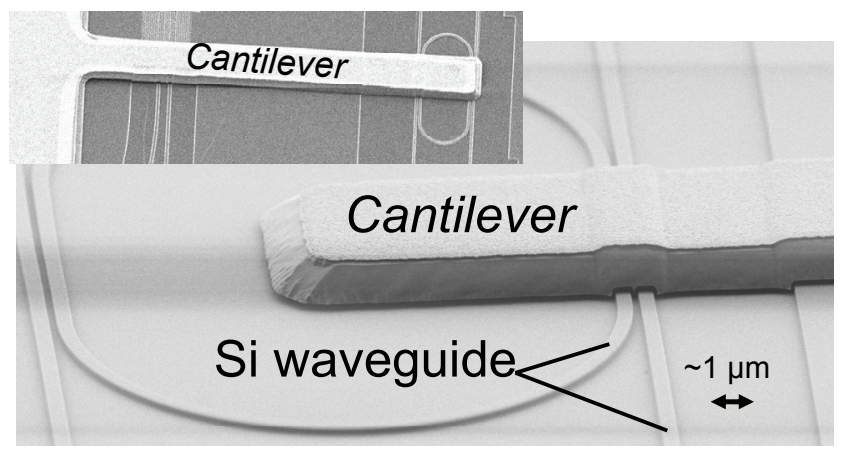

Fig. 8. a) SEM image of the fabricated device. Inset: top SEM image of another device variation.

Fig. 9a shows the measured transmission spectra for two different applied voltages, $0 \mathrm{~V}$ and $8.5 \mathrm{~V}$. It can be see that the resonance wavelength shifts as the cantilever moves closer to the waveguide. The quality factor of the resonance remains high; i.e. the presence of the cantilever does not appear to significantly increase loss in the resonator. However, as shown in Fig. 9b, when the cantilever is pulled in at $9.2 \mathrm{~V}$ (and touching the waveguide surface) a noticeable change of the quality factor; from 10000 to 5000 , was found.

Fig. 9c shows the measured resonance wavelength shift as a function of the actuation voltage. It can be seen that the response is strongly nonlinear with the $20 \mathrm{pm}$ wavelength shift corresponding to voltage change from $8 \mathrm{~V}$ to $8.5 \mathrm{~V}$. The maximum reversibly attainable wavelength shift was found to be $50 \mathrm{pm}$, at an applied voltage of $8.5 \mathrm{~V}$. The energy required for obtaining this shift is given by the energy required to charge the $(\sim 1$ $\mathrm{pF}$ ) capacitance of the cantilever to this voltage, which evaluates to $\sim 36 \mathrm{pJ}$. 


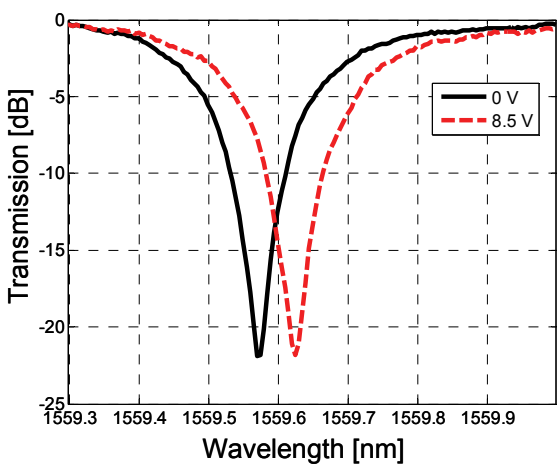

a)

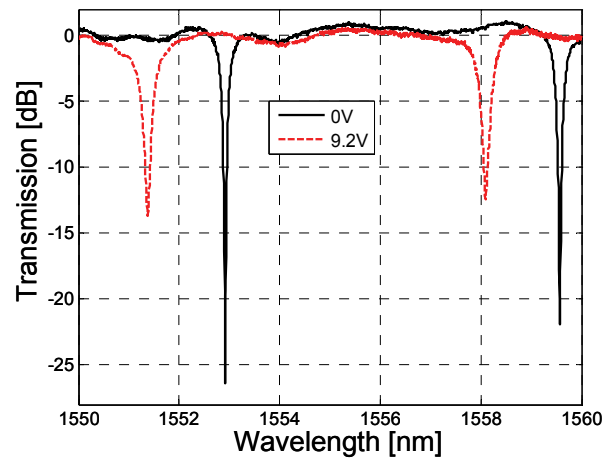

b)

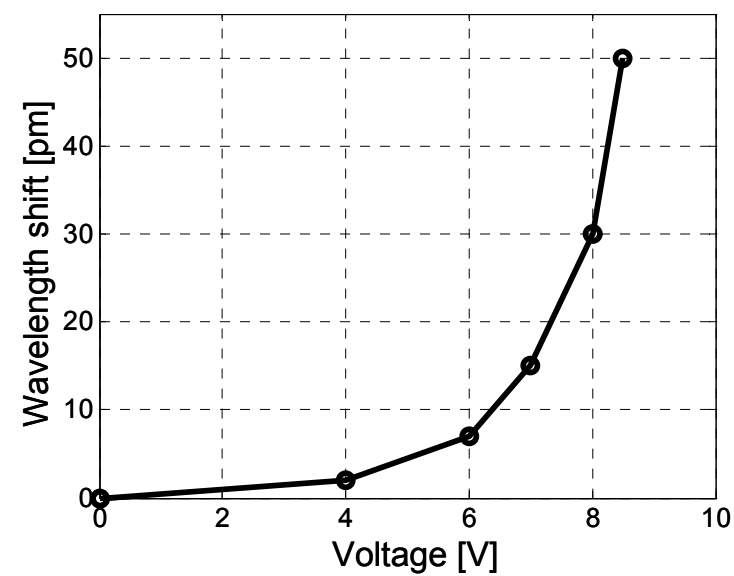

c)

Fig. 9. a) Transmission spectrum of a ring resonator measured for $0 \mathrm{~V}$ and for $8.5 \mathrm{~V}$ actuation voltages. b) Transmission spectrum of a ring resonator measured for $0 \mathrm{~V}$ and for $9.2 \mathrm{~V}$ actuation voltages. At $9.2 \mathrm{~V}$ the cantilever is pulled-in that results in large $(\sim 5 \mathrm{~nm})$ resonance wavelength shift and lowers the quality factor of the ring. Note that due to measurement noise and the used normalization procedure, the transmission is (unphysically) slightly above $0 \mathrm{~dB}$ in some regions. c) Measured resonance wavelength shift as a function of actuation voltage.

The required actuation voltage to obtain a certain resonance wavelength shift depends on the initial gap between the cantilever and the waveguide. We estimate the gap between the cantilever and the waveguide to be $305 \mathrm{~nm}$, resulting from $200 \mathrm{~nm}$ thick sacrificial layer, $40 \mathrm{~nm}$ protective SiRN layer and $65 \mathrm{~nm}$ upward bending of the cantilever due to tailored residual stress. Further on, based on the experimental data the cantilever can be tuned by $150 \mathrm{~nm}$ before pull-in occurs. As can be seen from Fig. 6, much stronger optical tuning could be achieved with a smaller initial gap. It should be noted that fabricating the cantilevers with a good yield becomes increasingly more challenging for smaller initial gaps. However, by optimizing the fabrication process, we expect to be able to reduce the initial gap to approximately $200 \mathrm{~nm}$ with acceptable yield, thus increasing the modulation strength by a factor of 2 or 3 .

To demonstrate dynamic operation of the switch, we have actuated the cantilever with a square wave signal (from 0 to $6 \mathrm{~V}$ ) while recording the optical transmittance through the device at a fixed wavelength. Fig. 10 shows the result for a $2 \mathrm{kHz}$ actuation signal. The 
signal rise time $(110 \mu \mathrm{s})$, shown in Fig. 10, is due to slow photodetector. From the preliminary results, obtained with a fast photodetector (see the appendix), we estimate a signal rise time of $16 \mu \mathrm{s}$, while the fall time is $4 \mu \mathrm{s}$. The maximum actuation frequency is thus limited to approximately $30 \mathrm{kHz}$.

Although higher frequencies can be used to modulate the optical signal, the peak value of the signal is cut due to rise time. This could be compensated by having an overshoot in the actuation signal, yet there would still be phase shift between the actuation and the optical signal. Based on the measured mechanical resonance frequency of the cantilever $(800 \mathrm{kHz})$, we expect rise times that are almost order of magnitude shorter than the measured values. We expect that the operation frequency is limited by the squeeze film damping of the cantilever $[9,10,17]$ (see also section 4.2 ).

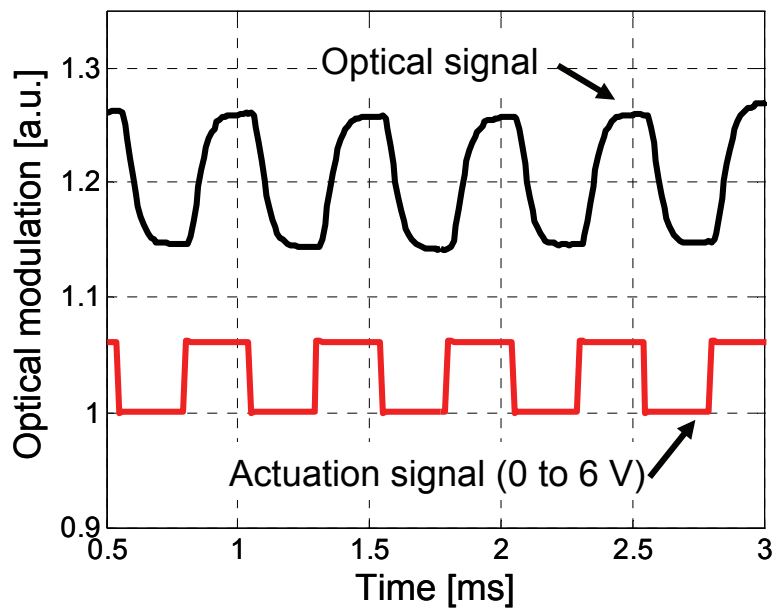

Fig. 10. Dynamic response of the electromechano-optically modulated ring resonator. The upper trace shows the transmission through the device at a fixed wavelength for a $2 \mathrm{kHz}$ square wave actuation signal (lower trace). Measured with a slow photo detector. Preliminary results with the fast detector; see the appendix.

\subsection{Micromechanically actuated photonic crystal waveguide}

Below preliminary results are presented aiming to demonstrate the tuning of a photonic crystal with a specially designed microcantilever. We consider a straight $\mathrm{PhC}$ waveguide that is formed by reducing the hole diameter of one $\mathrm{PhC}$ hole row (changed-hole $\mathrm{W} 1$ waveguide). The optical properties of this $\mathrm{PhC}$ device are actuated with a cantilever that covers the entire top surface of the photonic crystal, see Fig. 11a. The sacrificial $\mathrm{SiO}_{2}$ layer, that partially determines the gap between the $\mathrm{PhC}$ and the cantilever, had a thickness of $100 \mathrm{~nm}$ for this device. As mentioned in 4.3, such a thin layer allows the cantilever to have self-aligned tips that will enter the holes of the $\mathrm{PhC}$ as the cantilever approaches the PhC. A SEM image of the fabricated device is shown in Fig. 11a, the tips are clearly visible in Fig. 11b. 

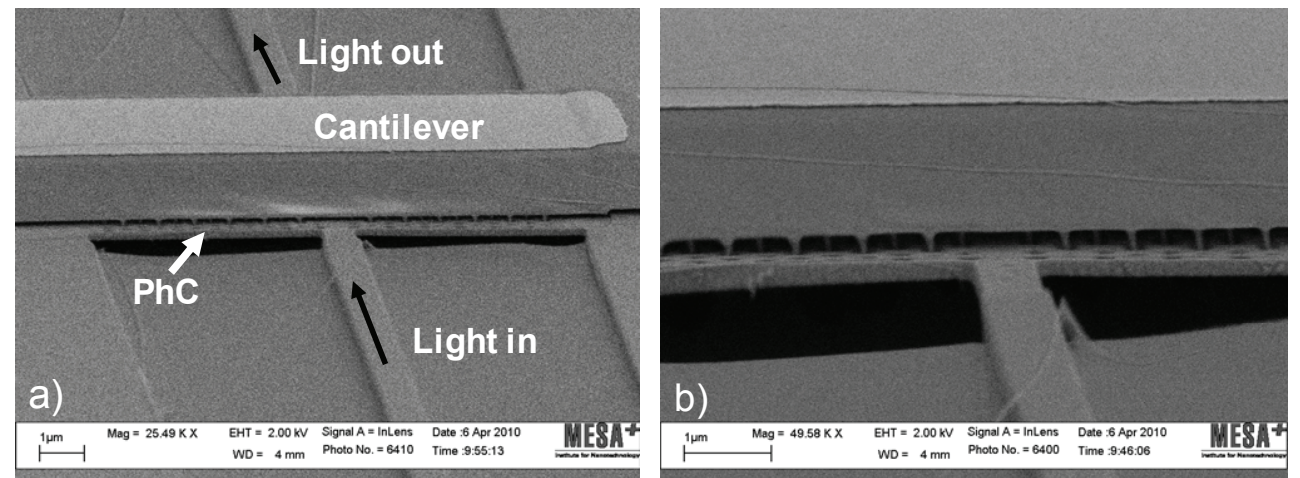

Fig. 11. a) A SEM image of the device. The cantilever covers the entire PhC. b) Detail of the same device. Small nitride tips, self-aligned with respect to the holes in the $\mathrm{PhC}$, protrude from the cantilever.

We have modelled the effect of the tips on the dispersion properties of the $\mathrm{PhC}$ waveguide with the MIT Photonic Bands (MPB) software [18]. Fig. 12a shows the dispersion graph of the $\mathrm{PhC}$ waveguide, as calculated from the structure (a so-called supercell, which is assumed to be repeated periodically) given in Fig. 12b. The slab hole diameter of the $\mathrm{PhC}$ is $270 \mathrm{~nm}$, the reduced hole diameter in the $\mathrm{PhC}$ waveguide is 140 $\mathrm{nm}$ and the period is $440 \mathrm{~nm}$. Fig. $12 \mathrm{~b}$ also shows the nitride tips with a diameter of 180 $\mathrm{nm}$ (grey disks) at the center of each large hole. In the two-dimensional calculations, the presence of the cantilever body was accounted for by changing the effective index of the slab material. The reference case (absence of the cantilever) was modelled with a slab effective index of 2.8495 , providing the reference curve. To model a cantilever without tips, we increased the slab effective index to 2.865. This corresponds to a situation where the nitride cantilever is $60 \mathrm{~nm}$ above the $220 \mathrm{~nm}$ thick Si slab. Finally we included tips into $\mathrm{PhC}$ holes and used the increased slab index (2.865) to model the presence of the cantilever body. From the graph it is clear that the tips have a large effect on the dispersion properties.
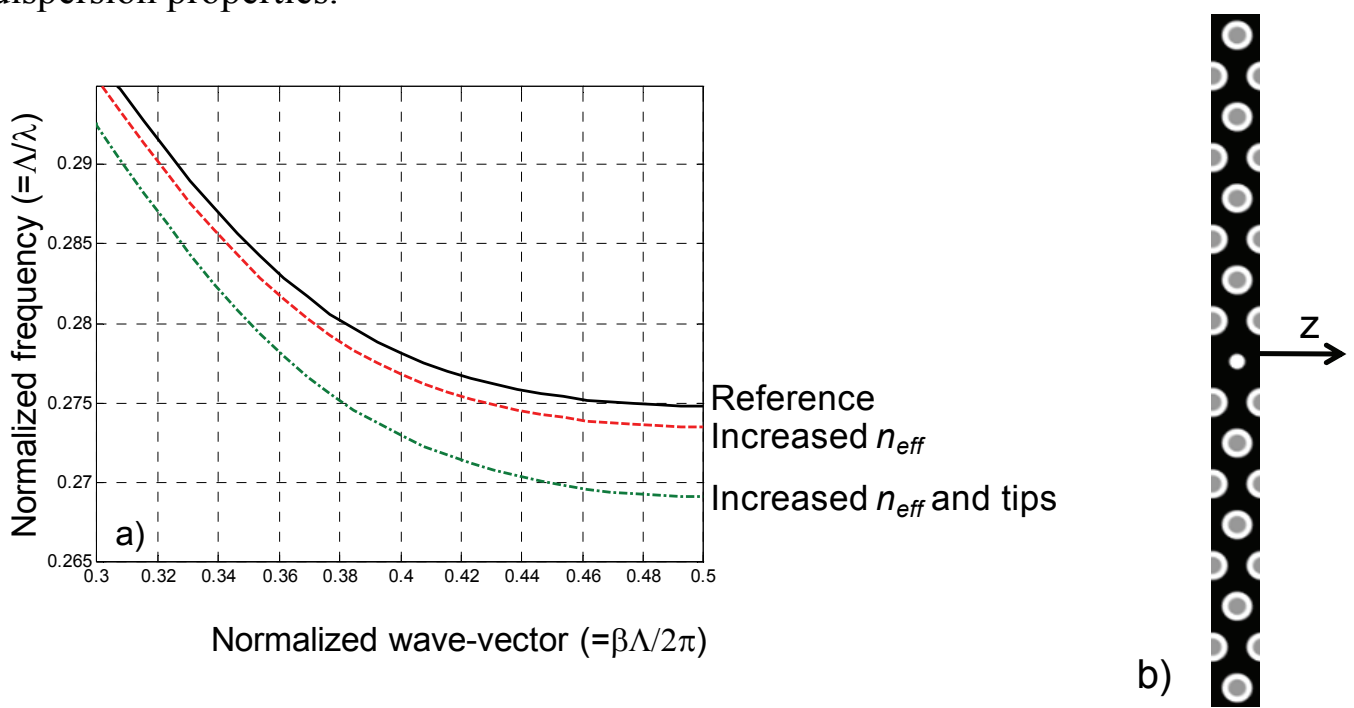

Fig. 12. a) The dispersion graph of the PhC waveguide calculated with MPB using supercell method. b) The supercell structure, to be repeated periodically along the z-axis to represent an infinitely long $\mathrm{PhC}$ waveguide running parallel to the $\mathrm{z}$-axis, that is used to calculate the dispersion properties. 
The optical response of the $\mathrm{PhC}$ waveguide, when actuated with a "tip-cantilever" was measured. The characterization procedure was similar to the one used for the ring resonator (section 4.5). However, here we do not measure a shift of a resonance wavelength, but the change in light transmission (at a constant wavelength) through the $\mathrm{PhC}$ waveguide. The slow-light properties of the $\mathrm{PhC}$ waveguide likely enhance the actuation effect.

Fig. 13 shows the optical modulation (at $1550 \mathrm{~nm}$ wavelength; normalized frequency 0.284 ) as a result of a $1-\mathrm{kHz}$ actuation signal. The modulation depth of the optical signal is approximately $7 \mathrm{~dB}$. The exact reason why the propagation loss increases when the cantilever is actuated closer to the $\mathrm{PhC}$ is not known. Mode cut-off of the $\mathrm{PhC}$ waveguide can be excluded as a potential mechanism, as it occurs at a wavelength longer than 1620 $\mathrm{nm}$ for all cantilever positions; also, as the cantilever moves closer to the waveguide, the cut-off wavelength is moved further away from the operating wavelength. There are two potential reasons: the dispersion properties change such that a part of the light is diffracted from the $\mathrm{PhC}$ waveguide (light line crossing), or the presence of the cantilever close to the $\mathrm{PhC}$ introduces such an abrupt index change that it leads to strong out-ofplane scattering.

When the cantilever is in the off state, the measured propagation loss of the $\mathrm{PhC}$ waveguide is less than $2 \mathrm{~dB}$, which is equal (within the measurement accuracy of $\pm 2 \mathrm{~dB}$ ) to that of a straight waveguide without any cantilever at all.

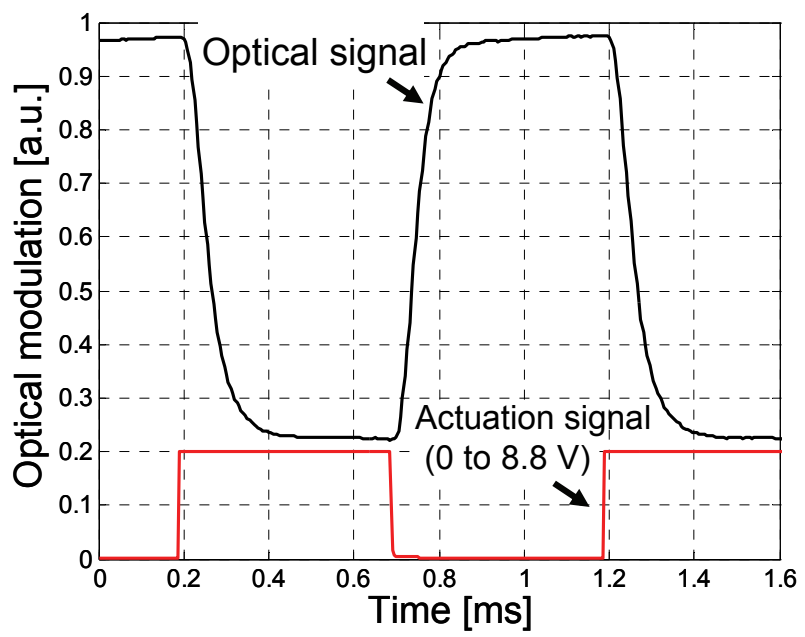

Fig. 13. Dynamic response. Transmission through the device at a fixed wavelength for a $1 \mathrm{kHz}$ square wave actuation signal. Rise (and fall) time of the optical signal is limited by the speed of the photodetector.

\subsection{Conclusions}

Electrostatically actuated microcantilevers were used to tune the optical properties of a racetrack ring-resonator and a photonic crystal waveguide. These cantilevers were monolithically integrated in wafer scale on SOI photonic chips.

The resonance wavelength of the ring-resonator was tuned by $50 \mathrm{pm}$ with an actuation voltage of $8.5 \mathrm{~V}$. The actuation of the resonance wavelength is obtained without notably increasing the optical loss. With a compact $(10 \mu \mathrm{m}$ by $10 \mu \mathrm{m})$ photonic crystal waveguide component a modulation depth of $7 \mathrm{~dB}$ was achieved with $8.8 \mathrm{~V}$ actuation voltage. For both devices the actuation frequency is limited to approximately $30 \mathrm{kHz}$. 
Mechano-optical actuation

Based on the measured mechanical resonance frequencies of the cantilevers $(\sim 800 \mathrm{kHz})$ in vacuum, we expect that the operation frequency is limited by the squeeze film damping of the cantilever. 


\section{References}

[1] G. Cocorullo, M. Iodice, I. Rendina, and P.M. Sarro, "Silicon Thermooptical Micromodulator with 700kHz - 3-dB Bandwidth", IEEE Photonics Technology Letters, vol. 7, pp. 363-365, 1995.

[2] Q. Xu, B. Schmidt, S. Pradhan, and M. Lipson, "Micrometre-scale silicon electro-optic modulator", Nature, vol. 435, pp. 325-327, 2005.

[3] G.N. Nielson, D. Seneviratne, F. Lopez-Royo, P.T. Rakich, Y. Avrahami, M.R. Watts, H.A. Haus, H.L. Tuller, and G. Barbastathis, "Integrated wavelength-selective optical MEMS switching using ring resonator filters", IEEE Photonics Technology Letters, vol. 17, pp. 1190-1192, 2005.

[4] P.T. Rakich, M. A. Popović, M.R. Watts, T. Barwicz, H.I. Smith, and E.P. Ippen, "Ultrawide tuning of photonic microcavities via evanescent field perturbation", Optics Letters, vol. 31, pp. 1241-1243, 2006.

[5] W.C.L. Hopman, K.O. van der Werf, A.J.F Hollink, W. Bogaerts, V. Subramaniam, and R.M. de Ridder, "Nano-mechanical tuning and imaging of a photonic crystal micro-cavity resonance," Optics Express, vol. 14, pp. 8745-8752, 2006.

[6] I. Märki, M. Salt, and H.P. Herzig, "Tuning the resonance of a photonic crystal microcavity with an AFM probe," Optics Express 14, pp. 2969-2978, 2006.

[7] M. Maier and M. Reisslein, "Trends in Optical Switching Techniques: A Short Survey," IEEE Network, vol. 22, pp. 42-47, 2008.

[8] N.V. Lavrik, M.J. Sepaniak, and P.G. Datskos, "Cantilever transducers as a platform for chemical and biological sensors", Review of Scientific Instruments, vol. 75, pp. 2229-2253, 2004.

[9] H. Hosaka, K. Itao, and S. Kuroda, "Damping characteristics of beam-shaped micro-oscillators", Sensors and Actuators A, vol. 49, pp. 87-95, 1995.

[10] G.J. Veldhuis, T. Nauta, C. Gui, J.W. Berenschot, and P.V. Lambeck, "Electrostatically Actuated Mechanooptical Waveguide ON-OFF Switch Showing High Extinction at a Low Actuation-Voltage", IEEE Journal of Selected Topics in Quantum Electronics, vol. 5, pp. 60-66, 1999.

[11] G.N. Nielson, "Micro-Opto-Mechanical Switching and Tuning for Integrated Optical Systems", Massachusetts Institute of Technology, PhD thesis, 2004.

[12] S.M. Chakkalakkal Abdulla, et al., "Tuning of Race-Track Ring Resonators by Integrated Bimorph Cantilevers", in preparation.

[13] S.M. Chakkalakkal Abdulla, PhD thesis, Faculty of Electrical Engineering, Mathematics and Computer Science, University of Twente, Enschede, The Netherlands, in preparation.

[14] D. Taillaert, F. van Laere, M. Ayre, W. Bogaerts, D. van Thourhout, P. Bienstman, and R. Baets, "Grating couplers for coupling between optical fibers and nanophotonic waveguides," Japanese Journal of Applied Physics, vol. 45, pp. 6071-6077, 2006.

[15] OlympIOs Integrated Optics Software. C2V, P.O. Box 318, 7500 AH Enschede, The Netherlands,(http://www.c2v.nl/software/).

[16] A. Farjadpour, D. Roundy, A. Rodriguez, M. Ibanescu, P. Bermel, J.D. Joannopoulos, S.G. Johnson, and G. Burr, "Improving accuracy by subpixel smoothing in FDTD," Optics Letters, vol. 31, pp. 29722974, 2006.

[17] H. Sumali, "Squeeze-film damping in the free molecular regime: model validation and measurement on a MEMS", Journal of Micromechanics and Microengineering, vol. 17, pp. 2231-2240, 2007.

[18] S.G. Johnson and J.D. Joannopoulos, "Block-iterative frequency-domain methods for Maxwell's equations in a planewave basis", Optics Express, vol. 8, pp. 173-190, 2001. 


\section{Summary and outlook}

This thesis describes the design, fabrication, and characterization of compact optical devices for sensing and switching applications. Our focus has been to realize the devices using CMOS-compatible fabrication processes. Particularly the silicon photonics fabrication platform, ePIXfab, has been used for this purpose. There are high hopes that silicon photonics, by utilizing the CMOS processing tools and standardized fabrication processes, can reduce the cost of photonic integrated circuits and open new markets to this technology-driven field of research.

Silicon photonics is currently in a state where separate components and fabrication technologies are actively developed, like the ones described in this thesis. Unlike for electronic CMOS ICs, no global standardized fabrication process exists for photonic integrated circuits, despite great efforts made by the ePIXnet and ePIXfab communities. The logical next step ahead would be standardization of the components and technologies, such that they could be effectively used. Due to the multidisciplinarity and complexity of these technologies it is unlikely that all fabrication will be done in one place. Instead, a distributed fabrication network providing different process options seems to be more feasible.

\section{Sensor work}

In chapter 2 we exploited waveguide-grating-based sensors. The waveguide grating is a simple building block for sensors; essentially it is just a straight waveguide requiring laser source and photodetector for sensor operation. The sensitivity of the grating based can be tailored (taking into account the process limitations) by changing grating parameters like length and the strength of the grating.

Grated silicon photonic wires (GSPWs) were designed and fabricated using the ePIXfab fabrication platform to realize cost effective, compact and sensitive biosensor.

According to our experimental results, cladding index changes as small as $10^{-5}$ RIU can be detected with the GSPWs. The length of the device is smaller than $180 \mu \mathrm{m}$, allowing dense integration of the sensor elements on an optical chip. As a demonstration the sensor was used to monitor the evaporation process of isopropanol from an aqueous solution. Label-free enzyme sensing was also demonstrated with the sensor, after depositing an immobilized enzyme-specific anti-body layer on the sensing element. Such sensors are expected to have applications in, e.g., medical diagnostics and detection of contaminants in the food and feed industry.

The GSPW that was developed and characterized in this thesis is just one part of a sensor. The other important parts are the biochemicals (that enable selectivity), (micro)fluidics, light source, photodetector and electrical read-out circuitry. Particularly for label-free sensing it would be of interested to pattern and deposit the biochemicals (receptor layers) on wafer scale in mass production way. In general it is debatable what the level of integration of these components should be. If all the functions are integrated on one chip (so called lab-on-chip approach), compact devices can be obtained. However, if the integration technology is complex, the cost of the device might be high, especially if the sensor is not reusable. On the other hand, set-ups based on devices with a low level of integration, such as commercial surface plasmon resonance based systems, are neither cheap nor compact. 
A new integrated optical read-out method for microcantilever displacements was developed. The optical read-out of cantilever deflection can for example be used in microcantilever sensor applications. These sensors are based on deflection of a cantilever when exposed to an analyte, such as hydrogen gas. The optical detection of cantilever deflection is a particularly attractive property for measurements in hazardous environments where electric contacts cannot be used.

Our optical cantilever read-out sensor is based on the spatial localization of a resonance in waveguide gratings. High displacement sensitivity is obtained by placing the microcantilever such that it interacts with the evanescent field of this resonance. The properties of the sensors were theoretically evaluated with computer simulations. Based on these results, a sub-nanometer detection limit was found for the deflection of silicon oxide cantilevers. Fabrication aspects of this sensor were studied. For the optical part, a well-established silicon nitride waveguide grating technology was chosen. The emphasis of the technological work was on the integration of the fabrication processes for optical devices and microcantilevers. After selecting a material that provides a high etch selectivity between different layers, a test fabrication was done. We tested isotropic reactive ion etching for releasing the cantilevers, as opposed to wet etching that requires a time-consuming and cumbersome freeze-drying step. The dry-etching method was found to result in high surface roughness of silicon nitride layers, likely ruining the optical properties of the waveguides in this layer. Based on the first fabrication test, the initial bending of the cantilever was indentified as an important factor for further study. This work was continued in a new $\mathrm{PhD}$ project.

\section{Micromechano-optical actuation}

The aim of the research was to implement an electromechanically actuated compact optical switch. The micromechanical actuation could be an attractive alternative to thermo-optical actuation due to its much smaller power consumption, yet providing similar tuning range and speed. In earlier work done in this field use was made of components that are not particularly compact and/or are assembled using bonding techniques; the mechanical part is attached to the optics chip. Our approach was to use a standardized silicon photonics fabrication platform, to design compact optical devices, and then do further processing with CMOS-compatible technology to monolithically integrate microcantilevers on these optics chips.

In chapter 3 we presented several optical devices that were designed to realize a compact mechanically actuated optical switch. Considering the available cantilever technology, the main focus in the design was on devices that could be actuated with a $10-\mu \mathrm{m}$ wide cantilever. For typically sized photonic crystal devices this implies that the cantilever covers the whole PhC. However, we also designed a PhC component that could be actuated by just manipulating one of its holes (with a diameter of only $250 \mathrm{~nm}$ ). Since photonic-crystal components are known to be challenging to design, and are infamous for their high optical loss, we also implemented more conventional, yet less compact, designs based on waveguide gratings and ring-resonators.

In chapter 4 , the micromechanical actuation of a ring resonator and a photonic crystal were demonstrated. We also discussed the basic mechanical properties of an electrostatically actuated microcantilever. From this theoretical discussion we predicted a low actuation voltage $(<6 \mathrm{~V})$, and an actuation frequency of better than $25 \mathrm{kHz}$ (limited 
by squeeze film damping). Actuating a cantilever in close proximity to a ring resonator resulted in a shift of the resonance wavelength by $50 \mathrm{pm}$. The required actuation voltage was $8.5 \mathrm{~V}$. The modulation frequency was limited to $30 \mathrm{kHz}$, likely due to squeeze film damping.

A similar cantilever was used to modulate the intensity of light that propagates in a compact $(10 \mu \mathrm{m}$ by $10 \mu \mathrm{m})$ photonic crystal $(\mathrm{PhC})$ device. For the $\mathrm{PhC}$ actuation the cantilever was designed such that it has small tips that can enter the holes of the PhC. A computer simulation showed that this significantly changes the dispersion properties of the $\mathrm{PhC}$ device. A modulation depth of $7 \mathrm{~dB}$ was measured, with potential actuation frequencies up to $30 \mathrm{kHz}$.

The speed of mechanically actuated optical devices is poor compared to the nearly 1 $\mathrm{MHz}$ actuation frequency that is possible with the thermo-optic method in silicon. Even if the power consumption of the cantilever-tuned device is much smaller, the speed likely limits the possible applications. Assuming that the speed is limited by the squeeze damping, it could be increased by designing the cantilever such that air can escape faster from the small gap that is between the cantilever and the optics chips. This could be achieved e.g. by reducing the width of the cantilever. The narrow cantilever in turn requires optical devices where only very local actuation results in a strong optical response; this can be achieved e.g. with the aforementioned photonic crystal devices. Another straightforward approach is to operate the device at low pressure conditions. 


\section{Samenvatting}

Dit proefschrift beschrijft ontwerp, vervaardiging en karakterisering van compacte optische componenten voor toepassing in sensoren en optische schakelaars. Het werk was gericht op realisatie van de componenten in een fabricageproces dat compatibel is met de processen die gebruikt worden voor de vervaardiging van standaard (CMOS) elektronica. Hiervoor is in het bijzonder gebruik gemaakt van een Europees "fabricageplatform" voor fotonische componenten in silicium, genaamd "ePIXfab". Men verwacht dat het gebruik van de gestandaardiseerde gereedschappen en fabricageprocessen voor CMOS de kosten van silicium fotonica kan reduceren en nieuwe markten kan openen.

Ten tijde van het schrijven van dit proefschrift (2010) wordt actief gewerkt aan de ontwikkeling van losse componenten en de benodigde technologie, zoals die bijvoorbeeld beschreven worden in dit proefschrift. Anders dan het geval is voor CMOS elektronica, bestaat er nog geen algemeen gestandaardiseerd fabricageproces voor geïntegreerde fotonische schakelingen, ondanks de krachtige inspanningen die verricht zijn door de onderzoekers die samenwerkten in het Europese netwerk "ePIXnet" en die worden voortgezet door middel van ePIXfab. De logische volgende stap is de standaardisatie van componenten en technologieën, zodat deze efficiënt kunnen worden ingezet. Wegens de multidisciplinaire aard en de complexiteit van deze technologieën is het onwaarschijnlijk dat alle fabricagestappen op een enkele locatie zullen worden uitgevoerd. Een ruimtelijk gespreid fabricagenetwerk dat een variatie aan procesopties kan bieden lijkt een realistischer vooruitzicht.

\section{Sensorwerk}

In hoofdstuk 2 zijn sensoren besproken die gebaseerd zijn op optische golfgeleiders waarin een traliestructuur is aangebracht. Een dergelijk "golfgeleidertralie" is een relatief eenvoudige bouwsteen voor sensoren, die slechts bestaat uit een recht golfgeleidend kanaal waarin een periodieke geometrische verstoring is aangebracht. Voor toepassing als sensor moeten nog een laserlichtbron en een fotodetector worden toegevoegd. De gevoeligheid van de sensor kan -binnen de grenzen die door het fabricageproces worden gesteld- worden aangepast aan de specifieke toepassing, door tralieparameters zoals lengte en sterkte van de periodieke verstoring te kiezen.

De golfgeleidertralies in dit werk, die zijn gebaseerd op optische kanalen met dwarsafmetingen kleiner dan $1 \mu \mathrm{m}$, zogenaamde "fotonische draden" (photonic wires), zijn ontworpen en vervaardigd op het ePIXfab platform. Hiermee zijn relatief goedkope, compacte en gevoelige biosensoren gerealiseerd.

Onze experimentele resultaten laten zien dat veranderingen van de brekingsindex in het medium grenzend aan het golfgeleidertralie van $10^{-5}$ brekingsindexeenheid kunnen worden gedetecteerd met deze golfgeleidertralies. Het actieve deel van de optische component heeft een lengte kleiner dan $180 \mu \mathrm{m}$, waardoor in principe grote aantallen sensorelementen in grote dichtheid op een enkele optische "chip" kunnen worden geïntegreerd.

De werking van de sensor is gedemonstreerd door het verdampingsproces van isopropanol uit een waterige oplossing in de tijd te volgen. Ook is de concentratie van een enzym gemeten zonder dat dit met van een extra chemisch "label" werd voorzien (het zogenaamde "label-free sensing"). Hiertoe werd het optisch gevoelige element voorzien 
van een dun laagje biologische antilichamen met een specifieke affiniteit voor het te detecteren enzym. Verwacht wordt dat dergelijke sensoren toepassing zullen vinden in bijvoorbeeld de medische diagnostiek en voor de detectie van verontreinigingen in de voedsel- en voederindustrie.

Het silicium golfgeleidertralie dat in dit werk werd ontwikkeld en gekarakteriseerd is slechts een onderdeel van een complete sensor. De andere belangrijke onderdelen zijn de biochemicaliën die de selectiviteit van de sensor bepalen (de zogenaamde "receptorlagen"), het microfluïdische systeem dat de te analyseren materialen aan het gevoelige element toevoert, de lichtbron, de fotodetector en de elektronica voor signaalverwerking en presentatie van de meetresultaten. In het bijzonder voor de massafabricage van elementen voor label-free sensing is het interessant om diverse receptorlagen selectief te kunnen aanbrengen en structureren op de schaal van complete siliciumplakken (de zogenaamde wafers die als basis dienen voor de fabricage van zowel elektronische als optische schakelingen). De meest wenselijke schaal van integratie van optische componenten is niet onomstreden. Zeer compacte microsystemen kunnen worden verkregen door integratie van alle functies op een enkele chip (de zogenaamde "lab-on-a-chip"-aanpak). Als echter de integratietechnologie complex is, wordt de prijs van de microsystemen te hoog, in het bijzonder voor sensoren die niet herbruikbaar zijn. Anderzijds zijn systemen met een laag integratieniveau goedkoop noch compact, zoals de huidige systemen gebaseerd op resonantie van oppervlakteplasmonen.

Een geheel ander type sensoren maakt gebruik van microbalkjes die verbuigen onder invloed van een te meten grootheid, bijvoorbeeld de concentratie van waterstofgas. Optische detectie van de verplaatsing van zo'n balkje maakt het mogelijk sensoren te ontwikkelen die geen rechtstreekse elektrische verbindingen nodig hebben en die daardoor bijzonder geschikt zijn voor toepassingen in ruimten met explosiegevaar.

Een nieuwe optische uitleesmethode voor de positie van een microbalkje is gebaseerd op de ruimtelijke lokalisatie van een resonantie in een golfgeleidertralie. Een grote verplaatsingsgevoeligheid is verkregen door het microbalkje in het evanescente optische veld van de tralieresonantie te plaatsen. De eigenschappen van de sensoren zijn theoretisch onderzocht door middel van computersimulaties. Op basis van die resultaten is gevonden dat de detectielimiet voor verplaatsingen van een siliciumoxide-balkje kleiner is dan $1 \mathrm{~nm}$. Op grond van een studie naar de vervaardigingsmogelijkheden van een dergelijke sensor is ervoor gekozen om het golfgeleidertralie te realiseren in een beproefde siliciumnitride-technologie. Het technologische werk concentreerde zich op de integratie van de optische structuren met de microbalkjes. Hierbij was de keuze van materialen belangrijk die een hoge mate van selectiviteit bieden bij de noodzakelijke etsprocessen. Vergelijkende tests zijn uitgevoerd van een isotroop reactief plasmaetsproces en een nat chemisch proces. De plasma-etsmethode bleek een grote ruwheid van de optische siliciumnitridelaag te veroorzaken, leidend tot onacceptabele optische verliezen. Daarom werd de voorkeur gegeven aan het natte proces, ondanks het daarvoor vereiste omslachtige en tijdrovende vriesdroogproces. Een eerste testfabricage leidde tot de conclusie dat meer onderzoek nodig is naar de initiële kromming van de microbalkjes t.g.v. interne spanningen die door het fabricageproces veroorzaakt worden. Dit werk is afgesplitst en voortgezet in een nieuw promotieproject. 


\section{Samenvatting}

\section{Micromechano-optische actuatie}

Het doel van dit onderzoek was de realisatie van een elektromechanisch bekrachtigde compacte optische schakelaar. Micromechanische actuatie kan een aantrekkelijk alternatief zijn voor thermo-optische actuatie wegens het veel geringere energieverbruik bij een modulatiebereik en -snelheid die van gelijke grootteorde zijn. Eerder werk in dit veld heeft componenten opgeleverd die niet bijzonder compact zijn en/of gebruik maken van een hybride techniek waarin optische en mechanische onderdelen van de component op aparte substraten worden vervaardigd, die later op elkaar worden bevestigd. In onze benadering daarentegen wordt eerst het optische deel van de component vervaardigd op het ePIXfab platform, waarna de elektromechanisch te bekrachtigen microbalkjes hier in een CMOS-compatibel proces monolithisch mee worden geïntegreerd.

Hoofdstuk 3 beschrijft verschillende optische componenten die ontworpen zijn voor toepassing in een compacte mechanisch bekrachtigde optische schakelaar. Op basis van fotonische kristallen kunnen uiterst compacte optische structuren ontworpen worden. Op grond van de beschikbare vervaardigingstechnologie voor microbalkjes werd het optisch ontwerp hoofdzakelijk gericht op structuren die bestuurd kunnen worden met $10 \mu \mathrm{m}$ brede balkjes. Gezien de typische afmetingen van fotonische kristalstructuren die werken bij infrarode golflengten, zal een balkje het fotonisch kristal nagenoeg volledig bedekken. We hebben echter ook een optische structuur ontworpen waarmee het schakeleffect wordt bereikt door selectief de vulling van een specifiek gat (met een diameter van ca. $250 \mathrm{~nm}$ ) in een fotonisch kristal te manipuleren. Aangezien ontwerp en voldoende nauwkeurige fabricage van fotonische een aanzienlijke uitdaging vormen en dit type structuren relatief hoge optische verliezen vertonen, zijn ook conventionelere doch minder compacte ontwerpen gemaakt, gebaseerd op golfgeleidertralies en ringresonatoren.

Hoofdstuk 4 beschrijft de experimentele resultaten van elektromechanisch bekrachtigde ringresonatoren en fotonische kristalstructuren. Daarbij worden tevens de elementaire mechanische eigenschappen van elektrostatisch bekrachtigd microbalkjes besproken. Op grond van deze theoretische beschouwing konden een actuatiespanning van minder dan 6 $\mathrm{V}$ en een actuatiefrequentie van meer dan $25 \mathrm{kHz}$ worden voorspeld. De actuatiefrequentie wordt theoretisch begrensd door het dempende effect van een dun luchtlaagje tussen het balkje en de onderliggende optische structuur (de zogenaamde "squeeze-film"-demping). Experimenteel werden een actuatiespanning van $8.5 \mathrm{~V}$ en een maximale actuatiefrequentie van $30 \mathrm{kHz}$ gevonden.

Met een balkje van overeenkomstige afmetingen is de lichtintensiteit gemoduleerd die wordt doorgelaten door een fotonische kristalstructuur met afmetingen van $10 \mu \mathrm{m} \times 10$ $\mu \mathrm{m}$. Het balkje is zodanig ontworpen dat het kleine uitsteeksels bezit die precies in de gaatjes van het fotonisch kristal vallen. Uit computersimulaties is gebleken dat hiermee de dispersie-eigenschappen van het fotonisch kristal sterk beïnvloed kunnen worden. Een modulatiediepte van $7 \mathrm{~dB}$ is gemeten.

De snelheid van mechanisch bekrachtigde optische componenten blijkt aanzienlijk lager te zijn dan de bijna $1 \mathrm{MHz}$ die mogelijk is met thermo-optische actuatie in silicium. Ook al is het energieverbruik van de mechano-optische actuatoren veel geringer, toch zal de geringere snelheid de mogelijke toepassingen beperken. Indien de snelheid door squeezefilm-demping wordt beperkt, kan het helpen om het balkje zo te ontwerpen dat de lucht gemakkelijker kan ontsnappen, bijvoorbeeld door het balkje smaller te maken. Een optische structuur die ook met zeer smalle balkjes een sterk schakeleffect vertoont is 


\section{Samenvatting}

reeds ontworpen. Een andere voor de hand liggende, doch wellicht minder praktische, oplossing is het bedrijven van de schakelaar in vacuüm. 
Appendix: Dynamic response of cantilever

\section{Appendix: Dynamic response of cantilever}

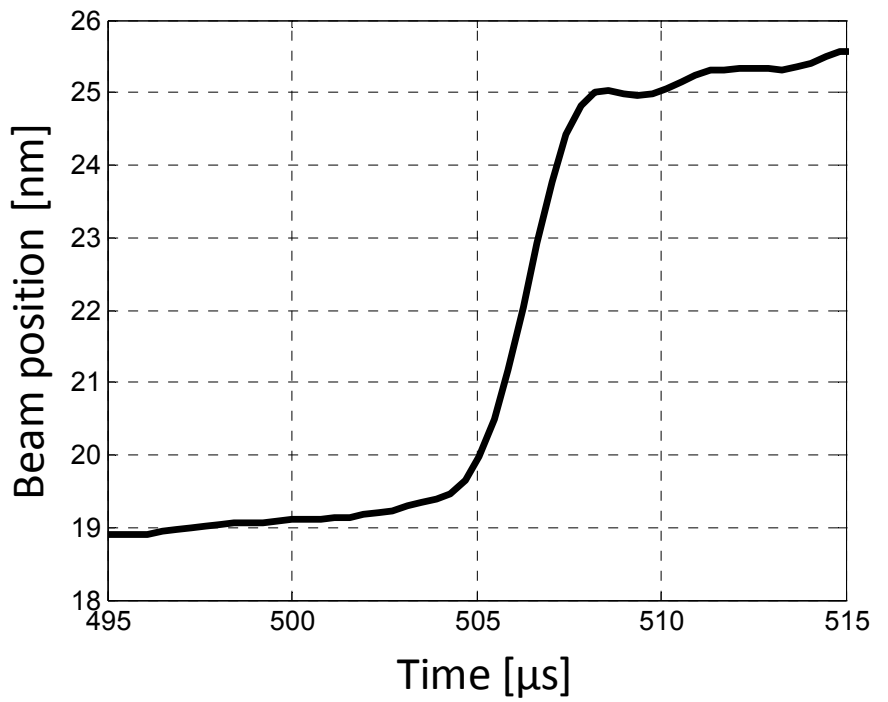

Fig. 1. The rise time response of a $40 \mu \mathrm{m}$ long cantilever as measured with a vibrometer. The actuation signal (not shown) is a $2 \mathrm{~V}$ square wave.

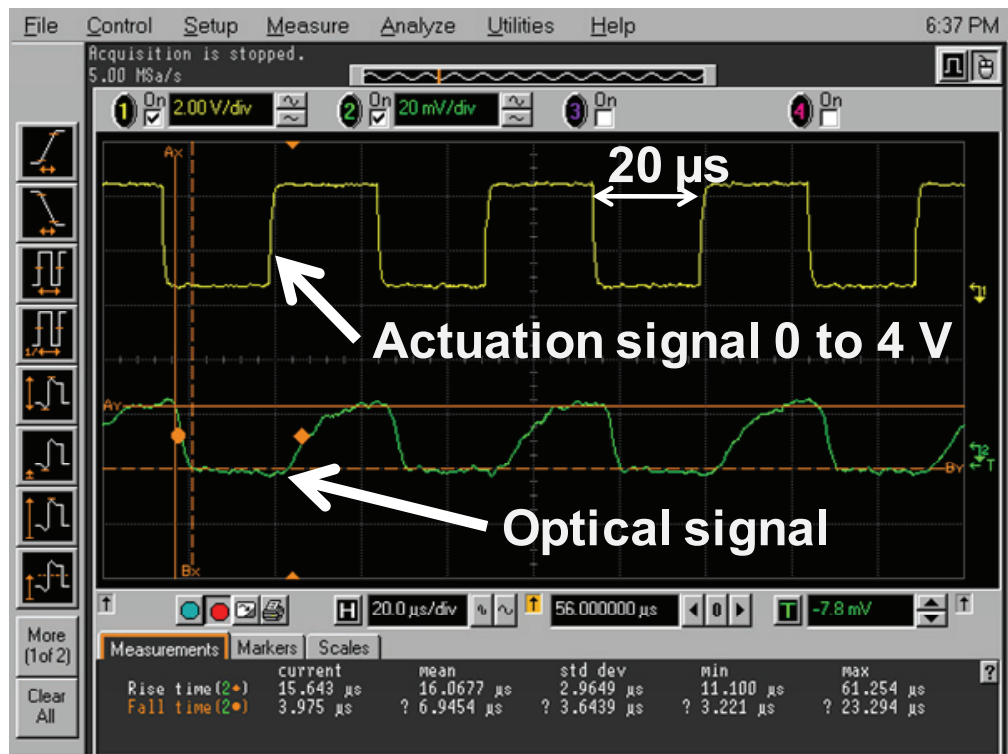

Fig. 2. Dynamic response of the electromechano-optically modulated ring resonator (with a $100 \mu \mathrm{m}$ long cantilever). The lower trace shows the transmission through the device (see section 4.5) at a fixed wavelength for a $25 \mathrm{kHz}$ square wave actuation signal (upper trace). 


\section{List of Publications}

\section{Peer-Reviewed Journal Articles}

1. L.J. Kauppinen, T.J. Pinkert, H.J.W.M. Hoekstra and R.M. de Ridder, "Photonic crystal cavity-based Y splitter for mechano-optical switching", IEEE photonics technology letters, vol. 22, pp. 966-968, 2010.

2. L.J. Kauppinen, H.J.W.M. Hoekstra and R.M. de Ridder, "A compact refractometric sensor based on grated silicon photonic wires", Sensors and actuators B (Chemical), vol. 139, pp. 194-198 2009.

\section{Conferences \\ Oral presentations}

3. L.J. Kauppinen, T.J. Pinkert, H.J.W.M. Hoekstra and R.M. de Ridder, "Photonic Crystal Cavity-Based Wavelength Routers", In: Proceedings of the 15th European Conference on Integrated Optics (ECIO), 7-9 April 2010, Cambridge, United Kingdom. ThG6, London: IET.

4. L.J. Kauppinen, M. Dijkstra, H.J.W.M. Hoekstra and R.M. de Ridder, "Label-free Enzyme Sensing with Grated Silicon Photonic Wire", In: Proceedings of the 2009 Annual Symposium of the IEEE Photonics Benelux Chapter, 05-06 Nov 2009, Brussels, Belgium. pp. 41-44. VUBPress Brussels University Press.

5. L.J. Kauppinen, H.J.W.M. Hoekstra and R.M. de Ridder, "Refractometric sensor based on silicon photonic wires", In: 3rd EOS Topical Meeting on Optical Microsystems (OMS09), pp. 27-30 Sep 2009, Capri, Italy. 2118. European Optical Society (EOS).

6. L.J. Kauppinen, H.J.W.M. Hoekstra, M. Dijkstra, R.M. de Ridder and G.J.M. Krijnen, "Grated waveguide optical cavity as a compact sensor for sub-nanometre cantilever deflections", In: Proceedings of the 14th European Conference on Integrated Optics (ECIO), 11-13 June 2008, Eindhoven, The Netherlands. pp. 111-114. Eindhoven University of Technology.

\section{Poster presentations}

7. L.J. Kauppinen, H.J.W.M. Hoekstra, M. Dijkstra, R.M. de Ridder and G.J.M. Krijnen, "Grated waveguide-based optical cavities as compact sensors for subnanometre cantilever deflections, and small refractive-index changes", In: Book of Abstracts 9th European Conference on Optical Chemical Sensors and Biosensors (Europtrode IX), 30-3-2008-02-04-2008, Dublin, Ireland. P084. Dublin City University. 


\section{Contributed}

8. F. Ay, L.J. Kauppinen, J.D.B. Bradley, K. Wörhoff, R.M. de Ridder and M. Pollnau, "Focused ion beam milled on-chip resonator nanostructures for applications in rare-earth-ion-doped A12O3 active waveguides", In: LEOS Annual Meeting Conference Proceedings, 2009. LEOS '09, IEEE, 4-8 Oct. 2009, BelekAntalya, Turkey. pp. 379-380. IEEE Photonics Society.

9. S.M. Chakkalakkal Abdulla, J.W. Berenschot, M.J. de Boer, L.J. Kauppinen, R.M. de Ridder and G.J.M. Krijnen, "Optimisation study of micro cantilevers for switching of photonic band gap crystals", In: Proceedings of the International Conference on Photonics in Switching 2009, 15-19 Sep 2009, Pisa, Italy. pp. 1-2. IEEE Photonics in Switching.

10. O.V. Ivanova, R. Stoffer, L.J. Kauppinen and M. Hammer, "Variational Effective Index Method for 3D Vectorial Scattering Problems in Photonics: TE Polarization", In: PIERS 2009 Moscow Proceedings, 18-21 Aug 2009, Moscow. pp. 1038-1042. The Electromagnetics Academy.

11. V.S. Pham, L.J. Kauppinen, M. Dijkstra, H.A.G.M. van Wolferen, R.M. de Ridder and H.J.W.M. Hoekstra, "Photonic crystal waveguides integrated with microcantilevers as a novel platform for gas sensors", In: Proceedings of the 7th International Symposium on Modern Optics and Its Applications (ISMOA), 10-14 August 2009, Bandung, Indonesia. pp. 68-68. Bandung Institute of Technology.

12. V.S. Pham, L.J. Kauppinen, M. Dijkstra, H.A.G.M. van Wolferen, R.M. de Ridder and H.J.W.M. Hoekstra, "Fabrication of mechano-optical sensors for hydrogen gas", In: Annual Workshop on Semiconductor Advances for Future Electronics and Sensors (SAFE), 26-27 Nov 2009, Veldhoven, the Netherlands. pp. 44-47. Technology Foundation STW.

13. V.S. Pham, L.J. Kauppinen, M. Dijkstra, H.A.G.M. van Wolferen, R.M. de Ridder and H.J.W.M. Hoekstra, "Simulation and fabrication of a mechano-optical sensor for nano-displacements", In: 1st Nano Today Conference, 2-5 Aug 2009, Biopolis, Singapore. pp. 33-33. Institute of Bioengineering and Nanotechnology, Singapore.

14. V.S. Pham, L.J. Kauppinen, H.J.W.M. Hoekstra, M. Dijkstra, H.A.G.M. van Wolferen, G.J.M. Krijnen and R.M. de Ridder, "Fabrication of microcantileverbased IO grated waveguide sensors for detection of nano-displacements", In: Proceedings of the 13th Annual Symposium of the IEEE LEOS Benelux Chapter, 27,28 November 2008, Enschede, The Netherlands. pp. 155-158. IEEE LEOS Benelux Chapter.

15. H.J.W.M. Hoekstra, R. Dekker, M. Dijkstra, C. Dongre, M. Hoekman, L.J. Kauppinen, P.V. Lambeck, M. Pollnau, H.P. Uranus, "Integrated optics sensors for multi-sensing platforms", In: Proceedings "The Sense of Contact", 4 April 2007, Zeist, The Netherlands. 5. Technology Foundation STW. 


\section{Acknowledgements}

I am grateful for Dutch Technology Foundation (STW) and the nanotechnology investment program NanoNed, by the Dutch Ministry of Economic Affairs, for financing the research work reported in this thesis.

Many people have contributed to this thesis and I would like to take the opportunity to thank all those people.

I thank my promoter Markus Pollnau and my supervisors Hugo Hoekstra and René de Ridder for the opportunity complete my Ph.D in the IOMS group.

As my daily supervisors Hugo and René deserve big thanks for their kind and supportive guidance during the work. I particularly appreciated the patience in reading my paper manuscripts and providing valuable feedback.

I would like to express my appreciation to the rest of the IOMS scientific staff, including Alfred Driessen, Kerstin Wörhoff and Paul Lambeck.

I thank IOMS group sectaries Rita ter Weele-Stokkers and Annitta David for their patience and good administrative support.

I like to thank all the members of the graduation committee: prof. dr. M. Pollnau, dr. ir. R.M. de Ridder, prof. dr. K.J. Boller, dr. H.J.W.M. Hoekstra, prof. dr. G.J.M. Krijnen and prof. dr. H.W.M. Salemink for their time and efforts in reviewing this thesis.

During the first 18 months I had the privilege to participate in many fruitful technical and scientific discussions in biweekly "multi-sensing" meetings with Hugo Hoekstra, Gjis Krijnen, Marcel Hoekman, Henri Uranus and Meindert Dijkstra. I truly appreciate these meetings in helping me to build my expertise in integrated optics.

I also want to acknowledge the optics theory meetings with Manfred Hammer, Remco Stoffer, Milan Maksimović, René de Ridder, Hugo Hoekstra and Alyona Ivanova as very useful insight into theoretical and modelling aspects. I am grateful for all the tips and advices I got from you during these meetings.

I am grateful to Anton Hollink for his technical support and his phenomenal talent in keeping the lab up \& running.

Meindert Dijkstra deserves big thanks for all the cleanroom work and all the technical advices he provided.

I also want to acknowledge the technical help of Meint de Boer and Erwin Berenschot, particularly in cantilever process integration.

I thank Silicon photonics platform staff Pieter Dumon and Jin Guo for advices and help in platform use and mask design. 
I wish to thank Shahina Mumthaz for co-operation in the "optical switch" project. I appreciate her willingness to spend countless hours in the cleanroom to get the devices, only to join me in the lab for more countless hours in measuring the devices :-)

During his M.Sc. thesis work Tjeerd Pinkert initiated the photonic crystal Y-splitter work, reported in chapter 3. I thank Tjeerd for all the nice scientific and non-scientific discussions we had, as well as for his active participation in publishing the results of the Y-splitter device.

Special thanks for Jing Yang and Chaitanya Dongre for being in a same situation; that is being a final year $\mathrm{PhD}$. I appreciate all the conversations we had about finishing the $\mathrm{PhD}$, job hunting and life after the book is done.

I highly appreciate the efforts of Dimitri Geskus for being the spirit and organizer of the (P)IOMS group.

I thank Muralidharan Balakrishnam for accompanying me for *real* warm lunch every day during his stay in UT.

I thank René de Ridder for translating the summary into Dutch.

Saara-Maarit Reijn, kiitokset monista suomen kielisistä keskusteluista!

I also wish to extend my thanks to the other current and former members of the IOMS group. Fei Sun, Nur Ismail, Edward Bernhardi, So Van Pham, Fehmi Civitci, Imran Akca, Edwin Klein, Ronald Dekker, Gamar Hussein, Wico Hopman, Laura Agazzi, Jonathan Bradley, Abu Aravazhi, Marko van Dalfsen, Henry Kelderman, Feridun Ay, Gabriël Sengo and Henk van Wolferen. You made my stay enjoyable and memorable, my sincere thanks!

I wish to thank my roommate Irene for shearing a flat with me and all those fun times we had.

Last but not least I wish to thank my family for giving me the strength to go on during the hard times. I thank my brother Juha, my sister in law Anu and my niece Sara for many nice moments that we have had. I want to thank my parents Veikko and Pirkko for the love, support and encouragement. 

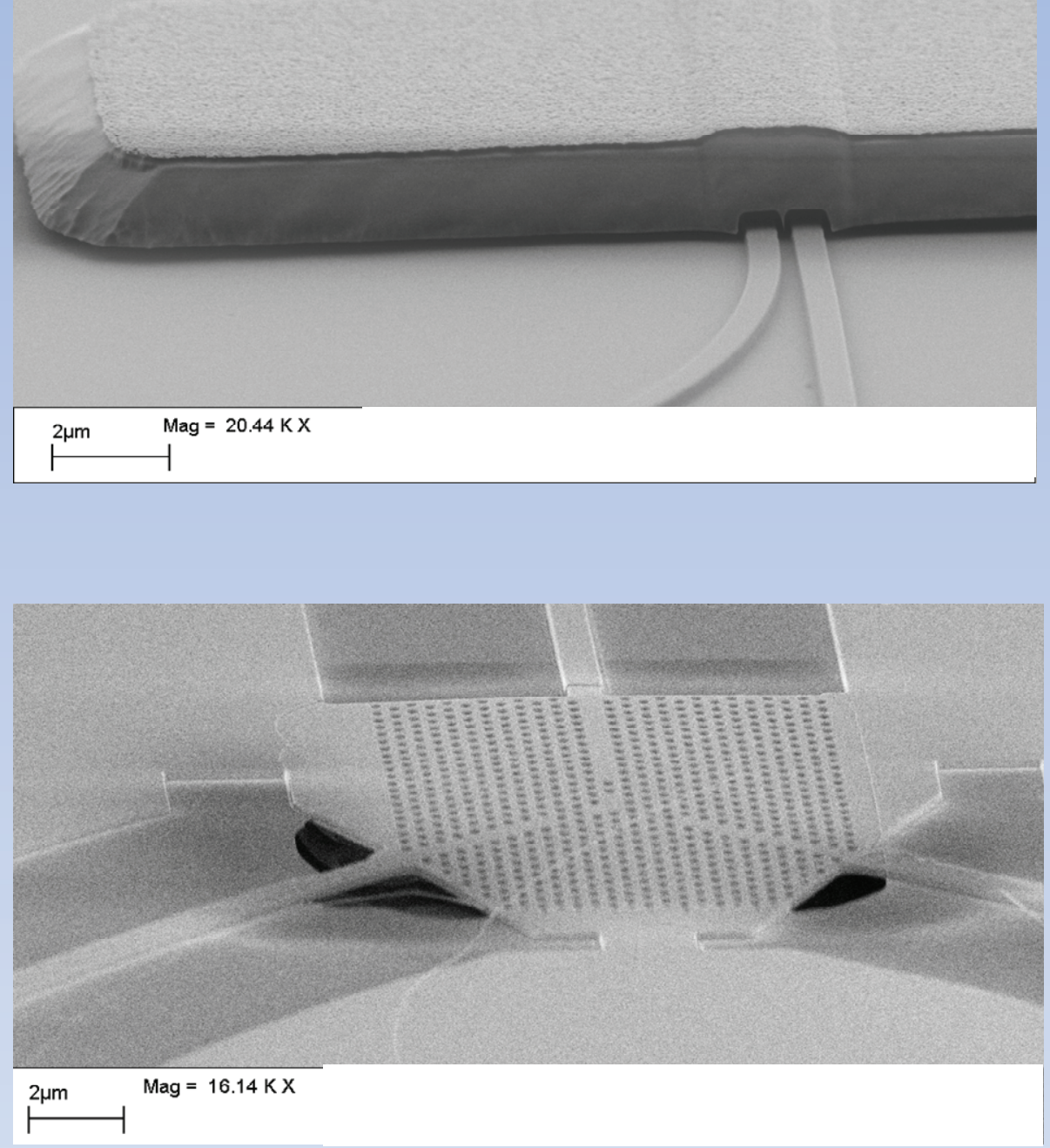

ISBN: 978-90-365-3088-0 